


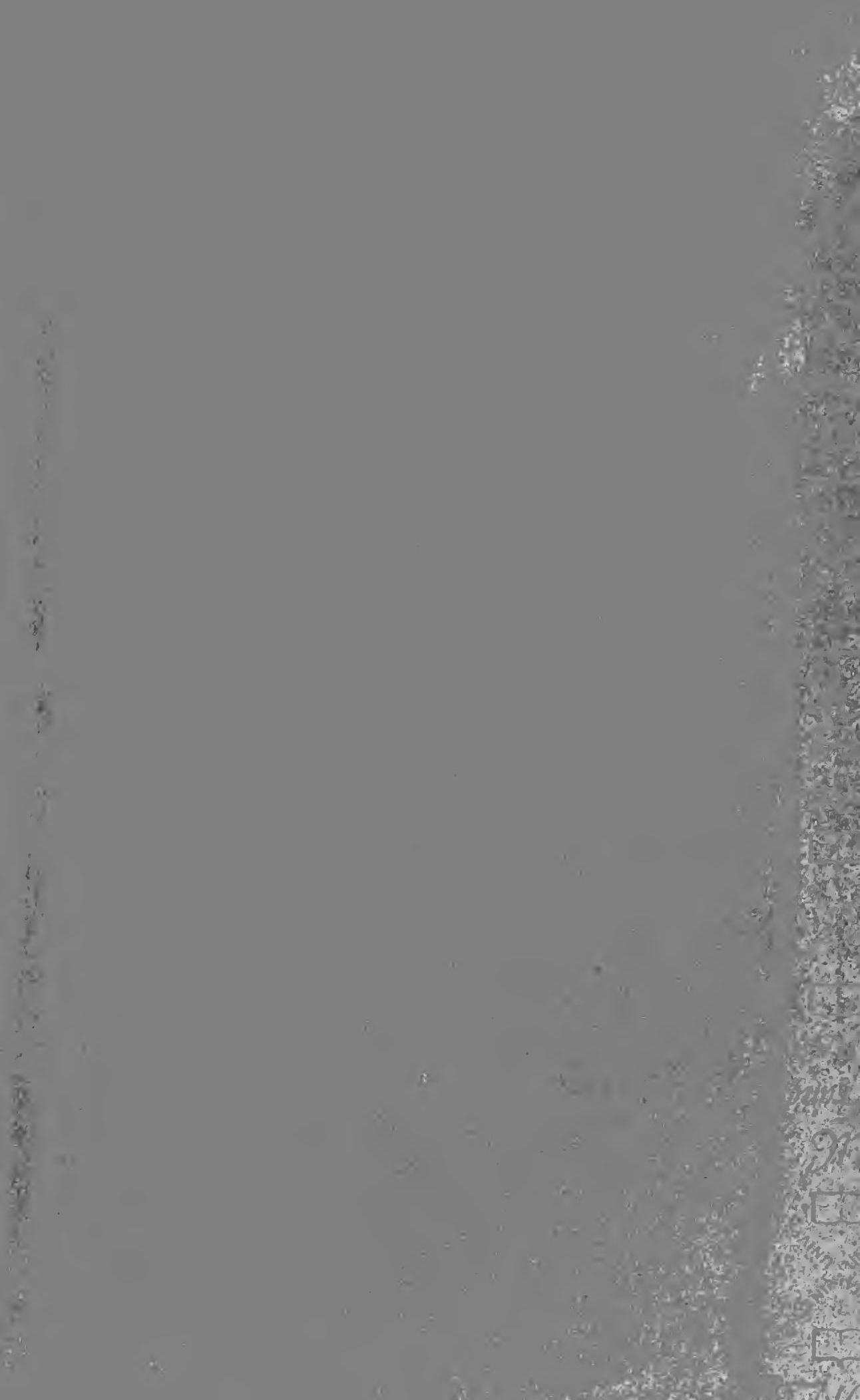




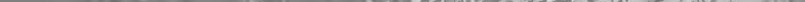




\section{Digitized by the Internet Archive in 2007 with funding from Microsoft Corporation}





\title{
THE MECHANISM OF THE BRAIN
}

AND THE

\author{
FUNCTION OF THE FRONTAL LOBES
}

Northwestern University Medical School Llbrary 



\section{THE MECHANISM $\mathrm{OF}$ THE BRAIN} AND THE

\section{FUNCTION OF THE FRONTAL LOBES}

\section{BY \\ Professor LEONARDO BIANCHI}

Senator of the Kingdom of Italy, Professor of Psychiatry and Neuropathology in the Royal University of Naples, Ex-Minister of Public

Instruction, Honorary Member of the Medico.

Psychological Association of Great Britain and Ireland, etc., etc.

AUTHORISED TRANSLATION FROM THE ITALIAN

BY

JAMES H. MACDONALD

M.B., Cir.B., F.R.F.P.S.(GLasG.)

MacKintosh Lecturer in Psychological Medicine, Glasgow Lniversity; Medical Superintendent, Govan District Asylum, Hawkhead, Glasgow

WITH A FOREWORD BY

C. LLOYD MORGAN, L.L.D., D.Sc., F.R.S.

Emeritus Professor in the University of Bristol

$$
22852
$$

ED I N B U R G H

E. \& S. L I V I N G S O N E 
PRINTED IN GREAT BRITAIN

BY THE RIVERSIDE .PRESS LIMITED

EDINBURGH 


\section{AUTHOR'S PREFACE}

THIs volume, which I now offer to physicians and others interested in the study of cerebral functioning, is the outcome of a series of experimental investigations expressly undertaken and covering a considerable period of time, commencing with the year 1888. Indeed, from the time I first applied my mind to the problem of functional localisations in the cerebral cortex, ${ }^{1}$ I had become convinced of the need for this special research.

In 1881 I had occasion to observe a remarkable feature relating to dogs which had undergone extirpation of the sigmoid gyrus of both sides. I was struck by the fact that there was an appreciable difference in behaviour between those animals in which the extirpation had been limited chiefly to the sigmoid gyrus, affecting either the whole or a part of that convolution, and other animals in which the operation had involved more than the sigmoid gyrus, having extended forwards, in front of the presylvian fissure. I had performed these operations with the special object of elucidating, as far as possible, the problem of functional compensations within the cerebrum and the anatomical limits within which cortical compensation was possible. The experiments had perforce to be numerous, and it was also necessary that I should keep the animals under observation for a long time after the operations. Thus it came about, apart from any stimulus to further investigation to be found in the scanty literature of the subject, that there arose in my mind a strong desire to attack the problem of the function of all that cerebral area which is situated in front of the presylvian fissure, a function that was wrapped in utter darkness. Afterwards came the great discussion between Hitzig, Munk, and Goltz, and thenceforwards I applied myself seriously to this particular inquiry.

During the session of the International Congress, in Rome, in 1894, a committee, composed of eminent authorities, including amongst others

${ }^{1}$ L. Bianchi. "Sui centri motori del cervello." Communication to the Associazione dei Naturalisti e Medici, 21st February 1878.

"Sul significato della eccitazione della zona motrice." Movimento Medico Chirurg. Anno xii. 1880.

"Sulle compensazioni funzionali della corteccia del cervello." La Psichiatria, la Neuropatologia e scienze affini. Naples, 1883 ; and Rivista Sperimentale di Freniatria. 1882.

"Ancora sulla dottrina dei centri corticali motori del cervello." La Psichiatria, etc. 1885 .

"Le degenerazioni sperimentali nel cervello e nel midollo spinale." In collaboration with Professor D'A bundo. La Psichiatria, etc., 1886, and Neurologisches Centralblatt, 1886. 
two highly esteemed friends, the late Professor Hitzig and Professor Henschen of Stockholm, was appointed for the special purpose of examining, and reporting upon, the monkeys I had operated upon, and still retained under observation. Although the judgment pronounced was distinctly favourable it was at the same time hedged around by many reservations. The conclusions I had outlined were afterwards subjected to criticisms by a number of physiologists in Italy and elsewhere, criticisms that were not always dispassionate and unprejudiced. The arguments adduced by these opponents, however, were never cogent enough to overwhelm me. Additional experiments and new methods only served to deepen my conviction of a physiological reality that withstood all the weightiest and best directed attacks. My subsequent communication to the Congress at Madrid had this result, that it opened up more practical paths for the investigation of the functions of the frontal lobes. Later on, the rigidly conducted investigations of two distinguished physiologists, Shepherd and Bechterew, giving results that were uniform with those obtained by me in a new and contemporary series of experiments, convinced me that the time had now arrived when I might opportunely publish the synthetic results of all my experiments, along with the conclusions that had been maturing in my mind.

My first intention was to publish a work that would appeal only to medical men, and with that object in view I had collected a number of clinical cases. On continuing my inquiries and subjecting the published clinical observations to close examination, it became clear that a considerable number, if not indeed the majority, of these could not be utilised, for many cases had been recorded both for and against the doctrine of the high, psychic function of the frontal lobes which, in their ultimate analysis, were destitute of any real value. I therefore abandoned the idea of dealing with the clinical aspects of the subject in this volume. With the outbreak of the Great War I could foresee that a rich store of human material would become available for the advancement of the physiology and pathology of the brain, and I therefore decided to put into print only the present volume, devoted to general doctrines, to the experimental aspects of the subject and to an initial phase of anatomical psychology, postponing the analysis of clinical cases to a more opportune time.

I have thought it particularly important to set forth clearly the outstanding points in the physiology of the cerebral mantle, to review the doctrine of the localisations, which appeared for a time to have been rudely shaken by the views expressed by Loeb ${ }^{1}$ and Monakow, ${ }^{2}$ and to define as clearly as possible the functional relations between the cerebral mantle, properly so-called, the zone of language, and the frontal lobes. I have also considered it necessary to lay down certain rules which may

${ }^{1}$ Loeb. Report of the Congress of Geneva. 1909.

2 Monakow. Les localisations cérébrales. Wieshaden, 1914. 
serve as reliable and useful guides to clinical investigations, and so counteract the tendency to encumber the clinical literature of the subject with superficial observations and with conclusions that are too often one-sided and hasty, and, consequently, of little or no real value for the progress of science, more especially of anatomical psychology.

If it be claimed for psychology, now so full of flattering promise and suggestion, that it is well that it be not restricted to one sole path of progress, and that it may even be permitted to indulge occasionally in a new form of transcendentalism, ingenious and fantastical though that be, it nevertheless remains the constant duty of the biologist to safeguard the one sure pillar to which it may be fastened, like a ship to its moorings.

Human and comparative anatomy and histology, comparative psychology, experiments upon the brains of the more highly developed mammals, aided by psychological and anatomo-pathological research, and the pathology (including psycho-pathology) of the human brain, are the most reliable, if not the only, fields of study which provide us with facts of a positive character, fields which can be made to yield a rich harvest to psychology, which to-day dominates the modern spirit of inquiry.

Such are the criteria which have inspired the work summarised in the present volume.

L. Bianchi. 



\section{'TRANSLATOR'S NOTE}

When I had completed the translation of Professor Bianchi's Trattatto di Psichiatria I did not anticipate that I should again undertake a similar task. The translation of a scientific work entails much careful thinking and painstaking labour, if it is to be presented in language which is intelligible, precise and, at the same time, a faithful interpretation of the author's thoughts. Nevertheless, I have experienced a sincere pleasure in the translation of this latest work of Professor Bianchi, because I am convinced that it has come at an opportune time and that, notwithstanding the enormous increase of psychological literature in recent years, there is ample room for another work which will enable us to develop our notions of the relations that exist between mind and brain, without transgressing biological limits.

The great impulse which has been imparted to the study of psychology and psychological problems in recent years is due, in part, to the influence of the leaders of psycho-analytical schools of thought, but it is also the inevitable result of modern discoveries in the realm of physical science. The psychologist is indebted to the physicist for those facts he has brought to light which go far to prove the inseparability, the oneness, the continuity of matter and force. From these later conquests of physical science the psychologist will draw inspiration which will help him to confront, with renewed energy, the obscure problems of psychic life.

To the masters of psycho-analytical research we are indebted for much, and not least for the enthusiasm they have awakened in many new quarters in the pursuit of psychological studies. It is true, however, that not a few of the many who have come forward as exponents of the new psychology have failed to give evidence of that length and breadth of experience which would entitle them to be ranked with the authorities. That cannot be said of the author of this work. The doctrine of the higher functions of the frontal lobes herein advanced is the outcome of many years of study and research on the part of one whose long record of scientific activity and achievement must claim our admiration and respect.

I sincerely hope that the author may not have to say of the translator : "Traduttore-Traditore."

To my colleague, Dr D. McKinlay Reid, I am indebted for much valuable assistance in the revision of manuscript and in the correction of proofs.

James H. MacDonald.

Glasgow University,

15 th February 1922. 



\title{
FOREWORD
}

\author{
By C. Lloyd Morgan, LL.D., D.Sc., F.R.S. \\ Emeritus Professor in the University of Bristol
}

Professor Bianchi is a stalwart champion of naturalism; and I suppose that I have been requested to contribute a Foreword to this translation because I too believe that all attempts at scientific interpretation which are other than naturalistic are worthless.

Let me start (Professor Bianchi's opening chapter justifies such a departure) from a comprehensive world-scheme here briefly outlined as a basal hypothesis within the framework of which (as I think) any naturalistic interpretation should find a place. At a very early stage of evolution there were physical events only. Protons and electrons existed in such whirls of changing relatedness as to constitute atoms. Higher kinds of relatedness than those we name physical were not yet in being. At a later stage the atoms were grouped into molecules of increasing complexity under the new kind of relations we call chemical. With the advent of lowly organisms a higher kind of relatedness (purely natural), which we call vital, comes into the picture. At a yet later stage, when a critical point in the development of the nervous system is reached, quite new kinds of relation, which we commonly speak of as conscious, supervene. Not less natural than their predecessors, they permit of profiting by previous experience. For the present we need carry the outline sketch no further.

What are the salient features in such a scheme? They are (1) that new kinds of natural relation among pre-existent things, or groups of events, appear at succeeding stages or levels in the course of evolution; (2) that their successive appearance is to be accepted "with natural piety," as Professor Alexander puts it ; (3) that it is part of the business of science to ascertain the environing conditions which contributed to their appearance; but (4) that it is no part of the business of science to dally with such explanations as metaphysical philosophers may, from time to time, suggest in terms of some supra-natural agency through the operation of which, it is said, they so appear. Such explanations may well be true; but they have no place in natural science.

The position may perhaps be rendered clearer by distinguishing two kinds of implication under which the phenomena may be interpreted. In any given natural system what takes place at any given level involves concurrent changes at lower levels. In their absence it does not occur. Thus, conscious profiting by experience involves physiological changes in the brain ; these again involve (within the system of the organism) less specialised vital 
changes; and these, in turn, physico-chemical events. One cannot invert this order of involution. But when, in the course of world-progress, a new kind of relatedness supervenes at any given level, the way in which concurrent events on the level below it run their course (what Maxwell, as a boy, called their "particular go ") depends on this new kind of relatedness. Thus all vital changes in the organism "involve" physico-chemical events and transformations of energy; but the particular way in which these events and these transformations occur "depends on" the presence of vital relations. No vitality without changes of energy (involution) ; no such changes of energy without vitality (dependence). In concrete fact both kinds of implication reciprocally obtain ; but, even if they be inseparable, it conduces to clearness of thought to distinguish them. Progressive development of novelty is the keynote of evolutionary progress. New orders or kinds of relation successively emerge at ascending levels (chemical, vital, mental); but on their emergence much novelty in the course of events at lower levels thereafter depends.

Now apply this to the naturalistic thesis which Professor Bianchi consistently advocates. There is an order of relatedness which we call mental. But its very existence involves physiological changes-or (more specifically) the establishment of sets of permeable neurone-routes-in the central nervous system. Let us so far follow Semon as to give the name "engram" to a permeable system of neurone-routes. Then the naturalistic thesis may be summarised thus: Every psychological complex involves a neural engram. This, of course, does not mean that a complex is an engram. One might just as well say that a tune that I hear is a gramophone record. None the less that tune which I so hear (with some paucity of higher overtones) involves such a record, and can have no being without it. But the making of the record depends on reflective thought. So, too, the establishment of an engram may depend--that of some engrams does depend-on acquired permeability of neurone-routes-permeability which would not come into being in the absence of mental relations. So runs the naturalistic hypothesis.

If, then, we may say that, for evolutionary naturalism, every psychological complex involves a neural engram, inherited or acquired-or (if it be preferred) that all mental events involve physiological correlates-can we follow up the scheme of implication (involution and dependence) somewhat further?

Professior Bianchi deals in careful detail with two cortical levels : $(a)$ the frontal, and $(b)$ that which we may call the post-frontal, including the temporal, parietal, and occipital centres. Development of the frontal region marks a relatively late stage of evolutionary progress (e.g. in mammals), and this development, in proportion to that of the post-frontal regions, is far more extensive in man than in the monkey, and a good deal more extensive in the monkey than in the dog. In the intact animal functional action in the frontal region always involves that of the post-frontal and perhaps other subordinate centres; and the way in which these centres then play their part 
depends, in some measure, on the mental complex correlated with frontal engrams. Now the manner in which an organism behaves is held to afford (1) from the physiological point of view an index of engrammic permeability of neurone-routes, and (2) from the psychological point of view a basis of inference to the mental complex which is correlated with neuro-physiological process, frontal or other.

What, then, does Professor Bianchi feel justified in inferring from the behaviour of monkeys from whose brains the frontal cortex has been ablated ? That they are reduced to the level of unconscious automata-at any rate in one sense of the word "unconscious." In what sense? Professor Bianchi in his concluding chapter discusses the concept of consciousness; and he favours the hypothesis that two stages may be distinguished, a lower and a higher. The reactions which we can observe in animals "warrant us," he says, " in speaking of the dawn of a higher consciousness ; and, if we trace the various stages in the evolution of the brain, we find that they first show themselves coincidently with the first appearance of the frontal lobes." If this be so, we may say that consciousness of the higher order-i.e. that kind of consciousness which we human folk enjoy--involves the integrity of the frontal lobes. Hence one seems justified in speaking of the mutilated monkey as in this sense an unconscious automaton. And one may hope, on humanitarian grounds, that this is so ; for, if it be so, then with the destruction of the frontal cortex, consciousness, as the analogue of what we commonly mean by the word, ceases, though the behaviour of an automaton is still open to observation.

The question then arises: What characterises the lower stage of consciousness-that, let us say, which is correlated with post-frontal function? The answer must be inferential and in large measure conjectural. It may be epiphenomenal in the behaviourist sense. In other words it may be the otiose accompaniment in sentience of post-frontal process. If so, no part of the observed behaviour of the mutilated monkey depends on its presence; for sentience is then, in Huxley's metaphor, merely the steam-whistle of the postfrontal engine, exercising no influence on its mode of running. That, however, is clearly not Professor Bianchi's view. The mutilated monkey, as he infers, retains much capacity for the simpler forms of perception. Furthermore, it is in the post-frontal centres that "images" are in some sense retained. A difficult matter is here opened up. Any such expression as the retention, storage, or preservation of images is highly elliptical. It should, in my opinion, be avoided. It involves the introduction of a psychological term into the physiological universe of discourse. What I suppose to be retained, physiologically, is not an image but a set of permeable neuroneroutes radiating forth from any given sensory centre. It is, however, no part of my purpose to enter into a question which admits of different answers (apart from the connotation of the words we use) in accordance with divergent interpretations of the observed facts.

In accordance with the scheme I have outlined as a basis for reference, we may regard frontal structure and function as affording evidence of 
a higher level or stage of physiological evolution than does post-frontal structure and function. A superior order of consciousness is correlated with the former; an inferior order is correlated with the latter. Now all the evidence goes to show that language depends on the higher mental processes correlated with the functional activity of the frontal lobes. But all the evidence goes also to show that speech-centres are localised in certain parts of the post-frontal regions. Is it a valid criticism to urge that the evidence is therefore contradictory? Surely not. There is nothing contradictory in saying (1) that speech depends on higher mental process, and (2) that language involves the appropriate functioning of post-frontal centres. Nay, rather it is in strict accord with the general principles, that (1) what takes place at a higher level, in which new relations obtain, involves lower-level events; and that (2) the particular way in which these events run their course depends on the new relations which obtain at the higher level.

If Professor Bianchi is right in correlating the higher mental processes which may be inferred from the behaviour of mammals with the functional activity of the frontal lobes, then, since this part of the brain is so highly developed in man, it falls well within his province fully to discuss the part which intellect, sentiment, and human sociality have played in the advance of civilisation. In any case his treatment of these matters will awaken wide interest, and will stimulate further inquiry. The position is, however, somewhat peculiar. There are, broadly speaking, three sets of people. There are the supra-naturalists (if I may so call them), who assert that Mind, in its proper and disparate order of being, with its prerogative of " pure memory," does not "involve" any brain-changes, frontal or other, save in so far as it acts on, or into, or through, the organism it has created for its use and has to some extent automatised. On the other hand there are the behaviourists (pur sang) who say that conscious relations count for nothing in the course of events. At most they are luxurious adjuncts devoid of business capacity. Behaviour gets along quite comfortably, they say, without any supposed dependence on the kind of mental relations which introspective psychologists have invented. To neither of these sets of people does Professor Bianchi belong. As man of science he rejects supra-natural influx; but he accepts mental relations as counting for all they are worth in the progress of man. $\mathrm{He}$, with his associates (and most evolutionists are here his associates), says that mental development always involves development of the brain. He has now adduced cogent evidence in support of the further contention that higher mental development is correlated with the activity of the frontal brain. It follows that the mental processes of which civilisation is the expression have physiological correlates in the frontal lobes. But when we turn to the fuller discussion of these mental processes, we find that the activity of the frontal lobes is rather tacitly taken for granted than called on as an aid to the interpretation of human thought. Not much, as yet, has the neuro-physiology of the frontal lobes been able to contribute to the elucidation of the psychological matters on which Professor Bianchi so ably discourses. That the 
frontal region is in functional activity during all mental process is no doubt taken for granted; but just how it so functions in detail remains in large measure an unsolved problem. It is, however, those who work on the careful lines that Professor Bianchi indicates in this work who are most likely to contribute to its solution. And no one who approaches in a scientific spirit the problems which Professor Bianchi discusses, can fail to appreciate the value of the light that he has thrown on the Mechanism of the Brain. 



\title{
TABLE OF CONTENTS
}

\author{
CHAPTER I
}

Evolution of the Nervous System and the Cerebral

Localisations

Mind and brain-The brain a transformer of the cosmic energies - The applicability of physical theories to mind-The reduction of the phenomena of life and the energies whence they are derived to universal laws-Process of composition and decomposition of the images and thoughts-Chemical, physical, and mechanical nature of the reactions of the lower animals-Tropisms, differential sensibility and associative memory-The mechanism of the reactions increases in complexity with the development of the nervous system and of the brain in particular-The origin of the higher mental processes to be sought for in the natural history of life and in the evolutionary phases of the nervous system-The optic lobes and optic thalamus-The thalamus prepares the sensory waves destined for the further elaboration of the cerebral mantle-The functional products of pre-existing nervous organs are indispensable for the elaboration of the more complex products of the organs of more recent development-Functional differentiation of the cerebral mantle and cerebral localisations -Modern conception of the doctrine of the localisations-The sensory areas-Are there cortical organs which subserve the function of the higher mental processes ?-The associative zones of FlechsigCriticism of the doctrine of Flechsig-The function of that part of the posterior associative zone of Flechsig known as the inferior parietal lobule-What we learn from clinical syndromes depending upon lesions of the left inferior parietal lobule-Clinical cases-The function of the temporal lobe (anterior part of the posterior associative zone of Flechsig)-Clinical and anatomico-pathological observations-The cortical motor area and the intermediate or evolutionary motor area

\section{CHAPTER II}

\section{History and Evolution of the Doctrine Concerning the} Functions of the Frontal Lobes

Hypotheses bearing upon the function of the extensive cortical field situated in front of the motor zone-Gall and Spurzhein, Fleurens, Gratiolet, Hitzig, Ferrier, Bianchi, Munk, Goltz, Moolman, Loeb, Luciani, Wundt, Meynert, Sciamanna, Libertini, Fano, Polimanti, Shepherd, Roncoroni, Lugaro, Monakow, Rossolino, Rechterew 


\section{CHAPTER III}

\section{Evolution, Morphology, and Structure of the Frontal Lobe}

The law of development of the cerebrum and especially of the cortex is confirmed by the evolutionary story of the frontal lobe-Brains of fishes, amphibians, and reptiles-Volumetric relations between the optic lobes and the fore-brain-Brains of rabbit, dolphin, and dogOnly in the higher mammals do we begin to find an appreciable frontal lobe-Frontal lobes of the $\mathrm{dog}$, horse, and ruminants-The author's investigations of the brain of the sheep-Frontal lobe of the macaque, cebus, and the orang-utang-Proportion of the cortical surface, total volume of cortex, and richness in cells in the middlegrade monkey, the orang-utang, and man-The frontal lobe of manThe inexcitable precentral area-The intermediate precentral areaThe frontal area of the frontal lobe-The pre-frontal area, properly so called-The morphology of the human frontal lobes-The cytotecture of the frontal lobe-Indications of evolution of the frontal lobe in man - Functional differences between the right and left frontal lobes

\section{CHAPTER IV}

\section{METhods OF INQUIRY}

The difficulties of investigation justify criticism-Critical observations of Schäfer and Monakow-Shepherd's investigations carried out out on the lines laid down by Schäfer-Functional compensation and degenerations consccutive to experimental mutilations are sources of errors-Objections raised against the use of mammals in investigations bearing upon the functions of the frontal lobes-Experiments reported in this work furnish sufficient confirmatory data of the capacity for precise perceptions, judgments, comparisons, memory and new adaptations in the cebus-The intensity of the current employed to excite the cortex may be the cause of error-Errors may arise from the fact that limited extirpations of one frontal lobe do not produce noteworthy disturbances - Relation between the extent and the effects of mutilation

\section{CHAPTER V}

\section{Cuinical and Experimental Histories of Dogs, Foxes and} Monkeys, including Controls.

$1,2,3$, control experiments -4, 5, removal of the frontal lobes of the dog and the fox $-6,7,8,9,10,11,12,13$, experiments on monkeys - Summary of the experiments-Modifications of the perceptive, attentive, and associative capacities, of the sentiments and of the conduct in monkeys as the result of the mutilations 
The Excitable Cortical Area of the Frontal Lobe and its SignificANCE

Literature of experiments showing the existence of small motor fields in the frontal lobe for movements of the eyes, pupils, and earsDisposition and topography of these areas-The author's experiments -Difficulty in interpretation-Are there special fields for the motor concomitants of the higher mental processes ?-Experiments on the human subject lend probability to such a hypothesis-Visual disturbances in dogs and monkeys that have suffered mutilation of the frontal lobes-Tentative interpretations of the phenomenon-Is it a question of diaschisis (disturbance at a distance)?-Monkeys mutilated in both frontal lobes lose the alert and mobile glance characteristic of these animals and assume an appearance that reminds us of human dements

\section{CHAPTER VII}

\section{Associative Paths between the Frontal Lobe ani) the Sensory}

\section{Area of the Cortex}

Superior or arcuate longitudinal bundle, the occipito-frontal bundle, the cingulum, their origin, course, and termination-Does the internal capsule receive projection fibres from the frontal lobe ?-Does there exist a fronto-pontine bundle and a fronto-ponto-cerebellar bundle ?Relation of the frontal lobe with the caudate nucleus, the putamen, and the claustrum - The results of the author's investigations confirm the existence, in monkeys, of bundles establishing reciprocal relations between the frontal lobes and the rest of the cerebral mantle

\section{CHAPTER VIII}

\section{Intelligence and LANGUAGE}

Intelligence should be examined by the naturalist exclusively from the biological point of view-A more realistic conception of life, intelligence, and morality is to be derived from the examination of facts than can be obtained from ideative abstractions, however brilliantIntelligence is not exempt from the general laws that apply to cosmic energy, including the laws of quantity-Intelligence with its power of penetration into the cosmic and social environment displays a biophylactic function-The whole mental edifice rests upon the mechanism of the associations-Consciousness and the unconscious-Language - The law of evolution is the supreme law of thought and speechOrigin and evolution of language-Physiological fusion of the concrete images of things with those of the words which symbolise themAnatomical substratum of both of these-Abstract conceptions and language-Intrinsic constituent elements of speech and their anatomical basis-The evolution of the cortical fields of language-Increasing complexity of the intellectual and linguistic mechanism with the advance of knowledge-Part played by the frontal lobes in the construction and expression of thought and in human conduct-Parallelis $m$ between human imbeciles and animals mutilated in the frontal lobes-Logic-The evolution of logic coincides with the evolution of 


\section{CHAPTER VIII-continued}

the brain and of language-Logic and the norms of exact knowledge PAISE -Defects of logic due to ovolutionary defects or to morbid processes in the brain-Logic as a biophylactic function, a thesis confirmed by cerebral pathology

Appendix. Logic



\section{CHAPTER IX}

\section{Emotions and Sentiments .}

General conception of emotions-Physical agents and their influence - The kinesthetic sense-All the organic functions are represented in the cerebral cortex-Experimental proofs-The fundamental emotions-The higher or intermediate emotions-The anatomical field of the organic phenomena accompanying the fundamental emotions is situated posteriorly to the frontal lobe-Psychosomatic characters of pleasure and of pain-The James-Lange doctrineIts vulnerable side-Sentiments represent a higher evolutionary grade of the fundamental and intermediate emotions-Desire-The sentiments and not the emotions disappear after mutilation of the frontal lobes-Sociality-Its evolution-Imbeciles are asocial -Is sociality an instinct?-Elements which contribute to the more rapid development of sociality amongst civilised peoplesHuman solidarity-The sentiment of sociality makes its appearance coincidentally with the development of the frontal lobes in mammals-It disappears after frontal mutilations, although the primary and intermediate emotions remain, sometimes exalted

Appendix. The Social Sentiment

\section{CHAPTER X}

\section{Consciousness}

Modern conception of consciousness-Its limits with regard to unconscious thought-Its evolution-Constituent elements-Its orientation in time and space-The two hypotheses regarding the extent to be assigned to the conception consciousness-The utilisation of experience which contributes to the evolution of consciousness coincides with increase of the nervous mass-The sum of the mnemonic traces and the conflict occurring in the case of actual perceptions or representations with their impulses appear to be characteristic features of an enlightened or higher consciousness-Insufficiency of the hind brain in relation to the development and manifestation of the higher consciousness-This coincides with development of the frontal lobes-The study of retarded or arrested evolution of the human brain allows us to retrace the story of the evolution of consciousness-The movement ef ideas and of emotions in consciousness - The uncol scious and the subconscious-Inhibitory power, higher consciousness, and the frontal lobes-The physical law of inhibition -Conduct, the resultant of impulsions and inhibitions-Anatomical psychology of conduct-Applications of Anatomical Psychology to pedagogy 


\section{THE}

\section{MECHANISM OF THE BRAIN}

\section{CHAPTER I}

\section{Evolution of the Nervous System and the Cerebral Localisations}

Psychic facts compel us to presume the existence of organs of a particular structure whence emanate energies which we conclude to be intrinsic in their substance, and laws regulating these energies. The brain is the great factory of thought. To it are directed all the forces of nature, forces which, for thousands of years, have been expending themselves upon it and impressing on it a slow and continuous motion of evolution.

The brain in turn transforms these cosmic forces into another form of energy which possesses analogous properties, which, indeed, summarises, accumulates, integrates and utilises them all in the process of thought and for the purposes of life.

The great problem that confronts us is to determine in what manner and by what means the transformation of these energies (external stimuli) takes place. How are vibrations of ethereal matter transformed into psychic products, or, in other words, what is the process of mentalisation or spiritualisation of matter to which the elementary phenomena of mind may be reduced? That question, however, cannot even to-day receive a plausible reply unless it be in the form of hypothesis, or even of pure and simple conjecture.

The marvellous progress attained in physical science provides us with data that are full of value and hope for psychology. The doctrine of the electrons has thrown a flood of light on this vexatious and tormenting problem, and as a result it would seem legitimate to inquire whether the nervous, and therefore also the psychic processes, at least the more elementary of these, are not to be regarded as the product of transformation of a special localised condition of the universal ether into a still more localised and specialised one which we call nerve-waves, which, in turn, are resolved into sensations and images (percepts) and might be considered the equivalents of atoms of matter launched from afar. Admittedly, this is all very hypothetical, but one cannot help thinking there is in it a germ of truth.

Many physicists after Helmholtz (amongst the Italians, Rhigi ${ }^{1}$ ) are

${ }^{1}$ Rhigi. La moderna teoria dei fenomeni fisici. Bologna, 1904. 
inclined to favour the view that electrons-" those atoms of negative electricity, provided with mass of an electro-magnetic nature and varying according to velocity " (Cantoni) - do not become fused in one homogeneous whole but preserve their individuality. If this were the case it would be reasonable to suppose that the groups of electrons constituting an image are not blended into one uniform whole, nor the innumerable images that go to make up the mind into another uniform product, but preserve their individuality in each brain and correspond in different brains according to fixed laws. This much is certain that, casting a rapid glance over the development of the human mind, we seem to behold a progressive assimilation of nature on the part of the psyche and a progressive penetration of the collective intelligence into the world and its forces, so that with these it becomes integrated and rises essentially to the consciousness of the universe.

There is an analogy that is worthy of mention in this connection. If we make a close examination of evolved mental life we seem to behold a continuous motion of composition and decomposition, of integration and disintegration of images and thoughts (hence of states of consciousness), differing in no way from what happens in the material interchange in living organisms and in brute matter-the continual transformation of the component elements as the result of reciprocal chemical action.

All impressions, indeed, excite a process of composition that varies extremely in men of different structure. There are associative fields which behave in a thousand different ways in different individuals under the influence of identical stimuli and undergo many variations in the same individual. The sight of the sea, for example, awakens in the poet the melancholy song of the distant navigator who has bade Adieu to his loved ones. In the merchant it arouses a series of notions concerning lines of navigation, merchandise, exchange values and economic intuitions, and aspirations after wealth. The sight of a woman-i.e. the vibrations which, proceeding from a body illuminated in space, reach the retina-produces in one man a simple image which remains photographed in the brain more or less distinctly. In another man it gives rise to a series of æsthetic emotions which arise from harmony of form, along with a tendency to draw near. To the ordinary man it is the infatuation of woman, to the poet the more felicitous picture of a night of love, which the whole world may behold. Now, when we bear in mind the fact that not only very simple mental formations, the sole acquisitions of men of inferior calibre, but also complex ones, such as those that scintillate from superior brains, sometimes of unexpected importance and unforeseen consequence, are prepared in obscure cerebral workshops from elements in the greatest variety and diversity of combinations, elements which in most cases are pre-existent and identical in different brains and, further, that the material furnished by the whole cerebral mantle, or a great part of it, is derived from a process of decomposition and recomposition (association), the ultimate products of which rise to the light of consciousness and of the universal intellect, it seems to me one might suggest a tentative interpretation on the lines of that already employed 
by physicists in the case of the theory to which allusion has been madeviz. the deformation of, and the elastic vicissitudes special to, ether, or, again, electric dissociation consisting in the separation of negative electrons from neutral atoms, a separation which affords an explanation of chemical mutations in bodies. The writer is not sure if this conception, which he put before a gathering of scientists in Naples in $1909,{ }^{1}$ harmonises with the idea of Loeb, who would adopt the term associative hysteresis as corresponding to associative memory, by analogy with the posthumous facts defined by physicists as hysteresis. In any case there is something to be said for having taken the luminous impressions that go to form visual images as an example, because Maxwell already had regarded luminous waves as electro-magnetic waves, a doctrine which found further support in the experiments of Hertz, Lorentz and Rhigi. Then again, the fact that, by varying the disposition of apparatus designed to produce electro-magnetic waves we can reproduce ordinary luminous waves, allows us for the moment to embrace the hypothesis that luminous waves are transformed as soon as they reach the retina into the equivalent of the electro-magnetic waves. These we may regard as the nervous waves, as already supposed by Dubois-Reymond.

To-day more than ever there is a tendency on the part of physicists to bring the phenomena of nature and the energies whence they are derived under identical laws and to recognise in them all one single force. We know, in fact, that the particles which, with their rapid motion, constitute the cathodic rays are nothing else than negative electrons, and that a body exposed to the action of the ultra-violet rays emits electrons. It is known also that there is probably a correspondence between the chemical valences and the function of certain electrons contained in the atom--those less tied to the positive nucleus--and, therefore, that electrons are the intermediaries between matter and the cosmic ether (Cantoni). If it be true that the Röntgen rays are only manifestations of ethereal waves generated by abrupt variations in velocity of the electrons (which seems to be the conclusion to be drawn from a series of investigations that have given marvellous results) we might conclude with modern physicists, as did Matteucci a good few years ago, that there is but one only force which assumes different appearances and behaves differently according to the special circumstances of the means by which and of the bodies whence it is displayed.

The researches of Loeb ${ }^{2}$ are of great value in this connection. The phenomena of tropism so diligently investigated by this physiologist, who is inclined to connect them with the colloidal substances, regarded by some as the engine of the vital phenomena, throw a new light on the genesis and essence of psychic phenomena. No doubt the continual motion of ideas in the brain corresponds to the constant transformation of matter, to the perennial motion of the energies which bodies emit and absorb at all

1 Bianchi. "La meccanica del cervello e la doppia coscienza." Atti del Congresso delle scienze. 1910.

${ }^{2}$ Loeb. Comparative Physiology of the Brain and Comparative Psychology. 
temperatures, and to the continuous metabolism of things in general, such as the motion of the stars.

Any conception can be divided into its constituent elements by a process of analysis, just as a chemist does with any organic or inorganic substance when he reduces it to its elementary components, the syntheses of which he expresses in more or less complex formulæ. Even what appear to be very simple images are composed of several elements with different and extensive anatomical bases, elements to which we can apply the atomic or ionic laws in so far as affinities are established between them, so that in all men the same psychic compounds are met with (perceptive uniformity), while at the same time an infinity of other components are combined with one another in an incalculable variety of ways, giving rise to products which differ in different individuals either in some slight modality or in actual constitution. It is in some such fashion that we must conceive the Law of Variation which is the more pronounced, the more we proceed from the simple to the complex, in the structure of the mental products.

All mental components, in their gradations from the most simple to the most complex, are products of the work of an enormous number of histological elements distributed throughout the human brain, elements which apparently have gradually become specialised for particular forms of work, capable of furnishing specific products with different materials. It is therefore necessary to admit the existence of anatomical relations between those morphological groups the products of which are combined together so as to give rise to mental syntheses which, in their turn, are transmitted into the sphere of consciousness, sometimes to be launched out again to shed light on the zenith of the collective and universal consciousness.

The number of these paths of communication between one cell and another, or between some cell groups and other groups, between convolution and convolution, between certain cortical areas and other cortical areas, near or remote, defies all attempts at calculation. If we could estimate the number of all the paths that have been constructed between different communities, provinces and nations; if we could have a purview of all the network of telegraphic wires extended over the surface of the world or traversing the depths of the ocean, of all the railway lines, and ocean paths ploughed by vessels great and small, of the entire network of telephones through which circulate infinite thoughts and cares which arouse and agitate an incalculable number of interests, which in synthesis give the value of the life of a social group, of a country, of entire humanity, we should yet probably fail to form the faintest idea of the connections which morphology and histology have shown to exist between the cell-groups of the cerebral mantle where are formed the syntheses that give the measure of the intellectual value of each individual and which serve as the workshops that animate the collective life in the different and varied fields of human activity.

The writer feels tempted to suggest a notable analogy between the world and the brain in this regard, without compromising himself by affirming whether the world in its process of civilisation is modelled on the evolution 
of the brain or whether the latter becomes evolved along with the physical and social world. Further, he is not wholly prepared to deny the hypothesis that the discharge of electrons within the brain, between remote or neighbouring groups of cells, may happen independently of the nervous paths of association.

$$
* *
$$

The natural history of the evolution of the nervous system, which is the latest and most complex form of organic nature, offers confirmation of the law long recognised by chemists in dealing with the affinities of brute matter - viz. that bodies found to be of a very complex atomic constitution lead us to infer the existence of simpler bodies with less complex atomical combinations as well as the possibility of splitting a complicated atomical formula into simpler formulæ. This law, indisputable in chemistry, is strictly applicable to the forms of life and to the apparatus that is constantly giving to life new characteristics and varying lines of action as well as ever-increasing energies. This apparatus is the nervous system.

If we consider the nervous system from the point of view of its chemical constitution or of its structure or its functions, it impresses us as being a most complex work of nature, summing up all the cosmic energies in one vast combination and giving a new expression to our ideas of force.

The lowest representatives of animal life, such as the proto-amœbæ, show a remarkable homogeneity of their protoplasm. One and the same substance digests, assimilates, excretes, breathes, feels and moves.

Differentiation of protoplasm is first noticed in the rhizopods and infusorians. The first muscular fibre is derived from epithelial elements by a progressive differentiation giving rise, in the hydra, to the so-called neuromuscular cells (Kleinenberg ${ }^{1}$ ). These cells may be regarded as the precursors or germinative elements of the muscular fibre and of the nerve-cells, which we find quite differentiated from one another in the higher metazoans. The common origin of the muscular fibre and of the nerve-cell is not admitted by 0. \& K. Hertwig, ${ }^{2}$ who attribute an exclusively muscular significance to the so-called neuro-muscular cell, otherwise known as the musculo-epithelial cell. According to these and other investigators, the nervous substance develops quite independently of the muscular tissue. The two authors above mentioned maintain that nervous tissue appears with certainty in the colenterates and is composed of sensory and ganglionic cells (the latter according to Havet ${ }^{3}$ being true motor-cells). The musculo-epithelial, the sensory and the ganglionic cells, in cœlenterates, are derived and simultaneously differentiated from the epithelial elements.

\section{Leipzig.}

${ }^{1}$ Kleinenberg. Eine anatomisch-Entwicklungsgeschichtliche Untersuchung.

${ }^{2}$ O. \& K. Hertwig. Das Nervensystem und die Sinnesorgane der Medusen. Leipzig.

${ }^{3}$ Havet. "Contribution à l'étude du système nerveux des Actinies." La Cellule, t. 18. 
Parker ${ }^{1}$ has demonstrated the presence of true sphincters in sponges belonging to the group of the lower metazoans (Stylotella), these being formed of muscular fibres without any trace of nervous elements. This would go to prove that these muscular fibres contract quite independently of the intervention of any nervous elements and that in the course of evolution of the neuro-muscular system, the muscular fibre appears before the nerve-cell. If we trace the progressive development of the nervous system up to that of man, we are led to conclude that in the process of evolution, under the action of the energies of nature, the nervous tissue becomes increasingly developed in the central organ, being differentiated in accordance with the variations assumed by the forces of nature and that, by summarising and transforming these forces, it attains the dignity of an organ fitted for the spiritualisation of the world.

Between the earliest appearance of nervous substance and its latest grade of development there is only a morphological and numerical progression of nervous elements (even though it be not constant) and a proportionate progression of the reactive capacity of the living being, or, in other words, of corresponding adaptations to the environment.

The unfolding of the nervous system follows the same plan as the evolution of life. To begin with, the cells which receive the external energies and distribute them to other parts of the small organism are few in number. Later, we have small groups of cells, almost independent one from the other, more or less regularly distributed amongst equal parts of the larger organism which now has become less homogeneous, as in the medusæ, which are provided with a sensitive ectoderm innervated by a specialised nervous reticulum.

The nervous system undergoes a slow but progressive evolution from that of the medusæ and the echinoderms to that of the higher insects, the ants and the bees, in which we find a noteworthy feature in the relative increase of the nervous mass, with greater concentration and more complete functional differentiation of the cellular groups (ganglia), as compared with what we find in worms, crustaceans, arachnids and cephalopods.

Parallel with the development of the nervous tissue we have that of the organs of the body and in particular the organs of the senses. The visual organ, e.g., makes its appearance in some protozoans (Pouchet, Engelmann) with the so-called ocular spots, composed of pigment cells which have the property of fixing light.

In some planarians there appears a group of nerve-cells which may be regarded as a rudimentary brain, and at the same time we have the very simplest form of eye, represented by cells containing granular pigment and, behind these, retinal cells from which an optic nerve runs to the rudimentary brain just mentioned. In the higher worms the anterior cell-group or ganglion is more developed. New parts have been added to the eye of the lower planarians for there now appears a refractive medium (nemertines and

${ }^{1}$ Parker. "The Reaction of Sponges, with a Consideration of the Nervous System." Journal of Experimental Zoology, vol. viii. 
some nematodes) which suggests the commencement of the lens; also two anterior nervous ganglia which even here are united by a commissure.

These eyes, still of a simple kind, are thus in relation with small ganglionic groups which, after successive stages of development, are found in insects to have become larger and more complex in structure, appearing in them as peri-œsophageal ganglia, and as optic lobes in fishes, batrachians and birds.

We know well what stage of perfection the eye reaches in the vertebrates, especially in mammals, and the degree of development that the corresponding nervous system has attained with the superimposition of the optic thalamus and the cerebral cortex, and we know that the numerous eyes of some insects do not permit such perfect vision as that possessed by the higher mammals. Sight in insects is of a very low order and probably the power of accommodation (Lubbock) and of distinguishing the contours of objects (Forel) is absent. Wasmann considers that ants possess visual acuity and the faculty of perceiving the form of objects but this is not very likely. The perfection of the eye, writes G. Bohn, ${ }^{1}$ has permitted the formation of a world of images and with this there has occurred a true psychic revolution.

$$
* *
$$

Amongst the lower living orders, reactions assume rather a chemical, physical or mechanical character. If we pass an electric current through water in which some ciliates are swimming these all turn their heads towards the positive pole. ${ }^{2}$ Again, heat accelerates or suspends the ciliary movements according to its degree. A capillary tube containing certain acids or salts induces these small organisms to rotate around it and then to enter within it whilst other acids or other salts in that tube will make them migrate to a distance from it (chemical action). These small organisms can be attracted by chemical substances that may yet be poisonous in nature, and repelled by substances that are actually nutritive ( Pfeffer $^{3}$ ).

Loeb has described analogous phenomena in starfishes and he explains the righting movements as due to the force of gravity or to stereotypism. It has been proven that, even after the appearance of the nervous system, many phenomena regarded as of psychic nature are essentially tropisms. ${ }^{4}$

Anna Drzewina has shown that if a crab alters its direction when confronted by a rock this does not happen as the result of reasoning or judgment followed by will, as Pieret would wish us to believe; it is, rather, to be regarded as a mechanical reaction, for the simple reason that this animal behaves in identical fashion when the eyes and the brain (ganglia) are removed.

Many manifestations of this kind are due to chemical or physiogenetic variations and to mechanical or kinetogenetic variations, and accordingly

${ }^{1}$ Bohn. La naissance de l'intelligence. 1909.

2 Thornton. Paper rend to British Association. 1909.

${ }^{3}$ Pfeffer. Untersuchungen aus dem Botanischen Institut zu Tubingen. Bd. 2.

${ }^{4}$ Max Werworn. Psychologische Studien der Protisten. 
many observers exclude the idea of any truly psychic process. To-day much discussion centres around the question as to where psychism really begins. Many support the doctrine of Iamarck, who would attribute psychic action only to animals possessing a well-differentiated nervous system. Others, amongst them Bechterew, even go so far as to admit the existence of very low forms of psychism in orders void of a nervous system. If an animal with a differentiated nervous system-e.g. an ascidian-has its nervous ganglia removed, as in the experiments of Loeb and, notwithstanding, continues to perform movements like those executed under normal conditions, one must admit that even in those creatures the so-called phenomena of psychism are but the results of reactions of the living matter under the direct influence of the forces of the external surroundings. Bohn ${ }^{1}$ introduces another factor between tropism and psychism-viz. differential sensibility. Variability of reactions is a condition characteristic of psychism. This is distinguished by the association of diverse sensations with one another and of present with past sensations. Bohn insists that this new feature of variability of reaction is founded on difference in sensibilities (light, contact, gravity, chemical action, etc.), and in degrees of intensity. To this is to be added another factor in the form of associative memory, mentioned by Loeb. ${ }^{2}$

As the evolution of organisms proceeds apace, more especially in the matter of their nervous systems, the internal. variations come to exercise a strong determinative influence over the reactions.

The reactions of tropisms and of differential sensibility are mechanical, physical or chemical in nature; they induce internal variations and are isolated in character. With the intervention of association of sensations and associative memory we see emerging the features of psychism. Ants, e.g. , return to their nests as a result of visual, tactile, olfactory and mechanical stimuli, the associated memory-residua of which constitute individual experiences that are utilised in the varying circumstances of their lives. We can also conceive a new factor to come into play here, inasmuch as the memory-traces of previous sensations may represent not only an experience related to the actual sensation but a latent dynamism and a tendency toward determined reactions.

It serves no good purpose to discuss the particular theories of this or that author (Dantec, ${ }^{3}$ Radl $^{4}$ and others) concerning this question of mechanical, chemical or physical action; it is more important to lay stress on the notion that the earliest manifestations of psychic life__in other words, those reactions that present the features of volitional or reflex attitudes-are very probably the effect of chemical or physical actions. This would serve to strengthen the hypothesis that with the appearance of the nervous system the same physical and chemical forces intrinsic in the protoplasm (biological

1 Bohn. Loc, cit.

${ }^{2}$ Loeb. Loc, cit.

${ }^{3}$ Dantec. Traité de Biologie. 1903.

4 Radl. Untersuchungen über dem. Phototropismus des Thiere. 1903. 
equivalents) become gradually transformed into nervous equivalents and with progressive development of the nervous system into psychic equivalents.

In vertebrates, concentration of the small nervous masses (ganglia) becomes a notable feature. The two chains of separate ganglia that we meet with in the annelides are approximated and reincorporated in the spinal medulla. The antennal, optic, supra- and sub-œsophageal ganglia become increasingly larger and are brought into more intimate associative relations with one another, whilst to these there becomes added another nervous mass -namely, the anterior brain.

The cerebrum of vertebrates in its earliest manifestation seems to be substituted for the antennal lobes of insects-which in them are quite distinct tacto-olfactory lobes - and the other ganglia above mentioned, and it presents a new formation, the anterior brain, the cavity of which communicates with that of the olfactory lobe in the case of fishes.

The first representatives of the latter are furnished with a cerebral cortex which is merely a thin covering consisting of epithelial cells (Edinger, ${ }^{1}$ Jakob ${ }^{2}$ ):

Throughout the different classes of vertebrates the cortex goes on develop. ing and becoming richer in nervous elements till it reaches that surprising degree of development which we find in the higher mammals, where it is more or less superimposed on the representatives of the old ganglia which were the only nerve-centres of the higher invertebrates.

The development and differentiation of the cells of the cerebral cortex proceed almost uniformly from fishes to the higher mammals, reaching the highest grade in man, whilst at the same time the cells assume much closer relations with one another and acquire a higher functional dignity.

With the increase of the nerve-elements there coincides an incessant assimilation of the energies of nature, which we commonly speak of as stimuli, these giving rise to that functional differentiation whereby the number of notions and adaptations is multiplied. We might almost say that nature becomes revealed to itself through the intermediary of the nervous system. A wide and rigorous application of the experimental method enables us to detect the various stages of development of the nervous system and of the mind throughout the animal series (phylogenesis) as well as in the individual (ontogenesis), and gives good general grounds for stating that the development of mind is co-extensive with the development of nervous substancei.e. with the number of differentiated elements-or, in other words, with the successive differentiation of notions and adequate adaptations.

Loeb's statement ${ }^{3}$ that neither the weight of the brain nor the number of nerve-cells has any influence on the degree of intelligence, though supported by some anthropological researches dealing with the weight of the human brain in civilised and non-civilised races, does not seem sufficiently well 1905.

${ }^{1}$ Edinger. Vorlesungen über den Bau der Nervösen Centralorgane, etc., chap. iii.

'Jakob. Vom Thierhirn zum Menschenhirn. 1911.

${ }^{3}$ Loeb. Loc. cit. 
founded when we take into consideration the natural history and comparative anatomy of the brain.

Growth takes place in greater proportion in those parts of the brain which are last to appear-i.e. in the hemispheres or rather the neopallium, which by degrees assumes an enormous size in comparison with the mesencephalon and the posterior brain.

On the other hand the number of nerve-elements increases progressively if we take into account the fact that not only is the grey substance of the hemisphere thicker in man than in all other species, but it is also incomparably more extensive owing to the formation of convolutions and sulci of considerable depth, whilst at the same time it is much richer in cells. ${ }^{1}$

$$
*^{*} *
$$

The origin of the highest mental processes coexisting with that modality of mind which we call consciousness is not to be sought for in the laborious and exhaustive effusions of metaphysicians nor amongst postulates founded on the analyses of logicians nor by following the abstractions derived from introspection but rather in the natural history of life and in the phases of evolution of the nervous system. According to some naturalists, living things are so many chemical machines. If this be so, it naturally follows that mental activities are within the pale of that vast ocean of ether in which the universe swims, and are subject to the laws that regulate the energies which penetrate and move the world. In a later chapter this matter is discussed in greater detail. Meanwhile it is sufficient to state that the majority of physiologists and naturalists are agreed that manifestations of thought and feeling are bound up with the dawning of life and, in a progressive manner, with reflexes and automatisms, corresponding with the development of the nervous system.

Intelligence in its progressive ascent, manifesting itself as a vital force, is a later product which seems to be almost superimposed upon the reflexes and automatisms just as these succeed and are intermingled with the phenomena of tropism. Tropisms, reflexes, automatisms, intelligent reactions, represent phases of evolution which follow one upon the other without interruption, The point where one merges into the other cannot be defined because there is no distinct or essential difference in their nature. Although automatisms lose their conspicuous identity and become blended in the mechanism of intelligent reactions, yet, if looked for, they can still be detected in the complicate structure of these mental products which are manifested in the most varied reactions of living beings, in their physical and ultimately in their social environment.

The first cell-groupings of nervous elements, in organisms with a nervous system now differentiated from other tissues, may be regarded as organs for the transformation of the forees of nature into nervous waves which find resolution in reflexes associated with phenomena of tropism, and next in

1 See Chapter III. for greater detail. 
automatisms which become ever more complex and regulate the adaptations of living creatures to their environment.

With the further development of additional groups of cells and new paths of communication between them, the earliest reflexes become complicated with others that are less simple in character. In the reactions of creatures with a more evolved nervous system new nervous organs come into play, and these, in turn, acquire new and more complex co-ordination.

In the lower vertebrates-e.g. in Necturus and in the frog-the mid-brain assumes great importance as a centre for the reception and elaboration of acoustic, visual and tactile nerve-waves and the corresponding reflexes. The optic thalamus, now in course of formation, still receives a few optic fibres directly and has no well-defined function. As, however, new paths become developed and new cell-groups come into existence in the optic thalamus of the more evolved species, the reflexes, or, rather, the reactions become more complicate and of a higher order than the reflexes of the midbrain. The feature that is now gradually coming into evidence is the wider extent and greater variability of the associations, so that by degrees animal reactions lose their former characters of simplicity and relative immutability, and this is especially the case when the cerebral cortex makes its appearance.

The optic thalamus is an organ which prepares sensory waves for further elaboration in the sensory cortex, which meanwhile is developing and becoming superimposed on the optic lobes and the optic thalami. In the higher fishes and also in the lower batrachians the cerebral mantle is, in the main, an olfactory formation. Its simplicity is a very characteristic feature in fish and the lower amphibians, especially when we compare it with what we find in birds and to a much more marked extent throughout the scale of mammals up to man (vide Chapter III.). To the hippocampic formation (archipallium) there is added, by progressive development, the neopallium which is evolved from what is called by Johnston the somatic area (internal part of the hemisphere). It is from this part of the neopallium that we have the gradual development and differentiation of the non-hippocampic areas of the cortex, which does not receive fibres directly from the sensory organs but rather from the centres that immediately preceded it in evolution-i.e. the various nuclei of the optic thalamus. These prepare and modify the nerve-waves for the formation of the images which are the immediate and necessary components in the structure and evolution of intelligence.

Alongside of the development of the cerebral cortex we have a growing complexity of the system of thalamo-cortical connections, and while the thalamus preserves its reflexes through the peduncle (from the medial grey substance and the hypo-thalamic region) we have a gradual development of the neothalamus (Edinger) which comprises the anterior, lateral, and ventral nuclei, the pulvinar and the lateral and medial geniculate nuclei. In primates and in man these assume great importance because of their relations and connections with differentiated areas of the cerebral cortex. 
The neothalamus is, as it were, the vestibule of the cerebral cortex (Henrick $\left.{ }^{1}\right)$.

Its nuclei, we have said, are organs for the preparation of the nerve-waves of all the senses except smell, which has its analogous preparatory nucleus in the anterior perforated substance. It is from these nuclei that the nervewaves provoked by stimuli from the external world reach the cerebral cortex where they are spiritualised (images). The exogenous material of consciousness is furnished by the images.

If the nuclei of the optic thalamus are united with one another by means of associative paths we can readily conceive that they may give rise to a series of adaptations and adequate movements (the psychic facts which Luciani attributes to the subcortical ganglionic masses).

These reactions are almost identical in each animal species according to the more or less complex structure of the optic thalamus, although it may not be easy to distinguish what part of the reactions of these beings belongs to the optic lobes and what to the thalamus or to the cortex now in course of formation.

With the development of the cerebral cortex we have not only new cellmasses superimposed on pre-existing ones but at the same time these become more numerous, more complicated in structure and more closely associated with one another, so that we no longer have the invariability (relative) that is characteristic of the reactions of lower creatures and all those reactions included amongst the automatisms, which arise mainly from the subcortical centres. We have, instead, remarkable variability of attitude in the individual and in the species proportionate to the capacity for receiving a greater or less number of stimuli from the external world and for transforming, conserving, and associating these so as to give rise to the actual notion which becomes combined with past experience (memory) of the effects of reactions connected with previous notions (memory images). It is to this complex of facts that we must attribute the origin of the new adaptations and the variability of the movements of each individual in the changing circumstances of the environment.

The reflexes and automatisms dependent upon the subcortical centres become confused in varying degree with the more complicated, variable and co-ordinated movements that issue from the sensory and motor areas of the cerebral cortex.

One can readily understand how the coming into play of this new cerebral formation, under the influence of stimuli much greater in number than those that succeeded in elaborating the subcortical centres, must lessen, if not suppress, the autonomy of the latter, for the subcortical centres become subservient to the cortex and transmit their products to it for further transformation. The function of elaboration and transmission, the substitution of receptive and reactive processes, determined by that sum of stimuli, for the thalamic automatisms now transferred to the cortex, takes away from

1 Henrick. "Some Reflections on the Origin and Significance of the Cerebral Cortex." Journal of Animal Behaviour. 1913. 
the power of these automatisms, albeit these are only variable within very narrow limits, automatisms in which is summed up the mind of the lower vertebrates.

The automatisms centred in the subcortical organs are not altogether suppressed although they are reduced and under particular conditions they exhibit a certain contrast with the higher powers.

Moreover, the subcortical centres with their appropriate reflex arcs (sensory-motor) contribute to the surety, precision, co-ordination and energy ${ }^{1}$ of all the movements brought together in the motor area of the brain under the constant influence of actual sensations and the mnemonic residua of previous sensations and movements as well as the most varied combinations of these. Now, as the evolution of the optic thalamus proceeds alongside that of the cerebral cortex we cannot ascertain how, or to what extent, the thalamic automatisms develop in man, because it is quite impossible to distinguish with precision where the thalamic function ceases and the cerebral function begins and to what extent the thalamus contributes to automatic responses, although it is agreed that mental processes contain a conspicuous coefficient of automatisms (unconscious and subconscious), which in any case are of a much more complex nature than the simple thalamic automatisms.

The more simple structure and the more circumscribed field of the thalamus as compared with the extent and the varied and complicated structure of the cortical field, furnish a very reliable anatomical proof of the great differences that exist amongst the various categories of products which by analogy we include under the term automatisms. These, in the case of the thalamus, resolve themselves through nervous elements and paths of association that are incomparably scantier than in the case of the cortex; they come into play outside the field of consciousness, whilst cortical automatisms have most often been effected within the domain of consciousness in the past, under opportune circumstances come within it again and can be taken under its control. The thalamic automatisms always reveal themselves in identical fashion owing to the structural simplicity of their anatomical mechanisms, whilst the cortical automatisms undergo variation determined by the variety of stimuli that can be received by the anatomical mechanism which is itself complex and variable. The former are based upon organic memory, the latter derive their elements from experience and hence have as their basis psycho-organic memory. The "organic circuit concept" of Dewey ${ }^{2}$ is not applicable to the subcortical automatisms.

This subject is discussed in a succeeding chapter. What is necessary to bear in mind, at this point, is the fact that direct acts of intellect involve numerous sensory and motor complexes besides others of an unconscious or automatic order, so that it is more than probable that the subcortical mechanisms also come into play.

There are those who hold that some of those manifestations that go to.

1 Bianchi. "La emicorea sintomatica." Ann. di Nev., ann. xxvii.

2 Dewey. "The Reflex Arc Concept in Psychology." Psych. Rev., 1893; and Journ. Philosoph. Psychol., 1912. 
make up the complex phenomenology of the unconscious or subconscious have their origin in the subcortical organs (Herrick). It would be more correct to say that in the cycle of conscious and unconscious cortical reactions, corresponding with psycho-motor circuits, the automatisms originating from the subcortical organs play their part. Here we have to deal with various circuits superimposed upon and intimately bound up with one another so as to constitute a single, but very complicate, mechanism.

With the superimposition of new formations of nervous substance and the assumption by these of more numerous intercortical and cortico-thalamic relations, or, in other words, with the intervention of a much greater number of nerve-elements, the product is much more conspicuous and variable because it is the work not only of the part most recently developed but also of preexisting nervous organs.

This statement should leave no room for doubt because in every evolutionary phase of the nervous system we find this constant phenomenon, that the anatomical integrity of pre-existing organs is an indispensable condition of the evolution and functional perfecting of new nervous organs. In other words, the functional products of pre-existing nervous organs are indispensable for the elaboration of the more complex products of work of the organs of most recent development. If the earlier products appear to be less appreciable it is because they are absorbed as necessary material in the production of the organ last evolved. It is the products of later development that contribute directly to the growth of consciousness.

$$
* * *
$$

The progressive and specific functional differentiation of the cerebral mantle from its earliest manifestation up to what we find it to be in man proceeds contemporaneously from the general to the particular, from the simple to the complex. No true functional differentiation has yet been detected in the cortex of the batrachians. It displays no well-defined action upon the vital functions of the batrachians and it may even be removed without any remarkable loss of activity. Schrader ${ }^{1}$ has shown that if the cerebral hemispheres are removed from the frog it loses little or nothing of its ordinary manifestations of life. It behaves as if it were whole and sound. It catches insects and changes its domicile from land to water, and vice versa. No doubt the cortex of the frog is too simple and uniform to dethrone the optic lobes which are still the real higher nervous organs, apart altogether from the question as to whether or not it has commenced to exercise an exhibiting influence over certain reflexes $\left(\right.$ Goltz $\left.^{2}\right)$.

In the case of frogs, the optic lobes (mid-brain) exercise almost the whole directive influence over the life of these amphibians. They still enjoy a good deal of autonomy. The cortex, now in course of formation, presents no feature of specific function, nor does it seem to have any directive influence over the subcortical organs.

1 Schrader. "Zur Physiologie der Froschhirn." Pflüger's Archiv., Bd. xli. 1887.

${ }^{2}$ Goltz. Beiträge zur Lehre von den Nervencentren des Frosches. Berlin, 1868. 
When we come to birds a sensible differentiation of the cerebral mantle has now made its appeance. The function of the posterior cerebral hemisphere differs in some degree from that of the anterior part. Nevertheless, Schrader found that decerebrated birds still had initiative, were able to see, and wandered about the room, avoiding obstacles.

Complexity of movements and attitudes is progressive. The jumping and swimming of the frog are much more uniform than the movements of the chaffinch, and these again are much less complex than the movements of the mouse, dog, elephant and of the monkey, in progressive series. And what a variety of attitudes there is in man himself according to the degree of culture attained either by the individual or by the social group as the result of cerebral development !

$$
*^{*} *
$$

With the anatomical development of the cerebral cortex there coincides, as already mentioned, a functional differentiation. This can be demonstrated in higher mammals by electrical excitation of the cortical surface and by the effects of experimental destruction of the cortex. In the case of man, clinical observation in particular has contributed to our knowledge. It has furnished a valuable mass of evidence, which, in the main, goes to confirm the results of experiments on mammals. All the senses, all the muscular groups, are represented in more or less distinct areas of the cerebral cortex in apes and in man.

Functional differentiation and localisation in the cerebral cortex proceed in accordance with that same law of evolution that we meet with in the social life, and in the structure of living things. In the cortex we find a histofunctional differentiation which reminds us of the differentiation of the nuclei in the optic thalamus but on a larger scale and in a much wider sense.

The whole nervous system of the more highly evolved beings is highly. differentiated-e.g. the bundles composing the spinal medulla differ greatly from one another as regards origin, time of myelinisation and function. There is differentiation amongst the various parts that go to make up the medulla oblongata, the pons, the cerebral peduncle and the optic thalamus. The last-named has a number of nuclei, as already mentioned, and these in turn are developed at different periods. ${ }^{1}$ The same rule applies also to the cerebral cortex. The law of differentiation and co-operative association should be regarded as fundamental. It corresponds with the various aspects of the energies of nature assimilated by the brain. Each of these develops its own appropriate cortical organ, for touch, smell, taste, hearing, sight.

Doubts have been expressed by some neuropathologists and physiologists as regards the doctrine of localisation. No doubt this is due to ambiguity in terms. Some authors speak of localisation of intelligence and of consciousness. Loeb, ${ }^{2}$ e.g., does not make himself perfectly precise and clear

${ }^{1} \mathrm{~V}$. Bianchi. "Anatomische Untersuchungen über die Entwicklungsgeschichte der Kerne des Talamus opticus des Kaninchens." Monatschrift für Psychiatrie. Bd. xxv.

${ }^{2}$ Loeb. Physiology of the Brain and Comparative Psychology. 
in formulating his conception of functional localisation in the cerebral cortex when he speaks of "visual conscience" or of "consciousness of a limb." He still adopts the terms of Hitzig. The consciousness of a limb is not localised, but, rather, the psychic components-i.e. the memory-residua of the movements of the limb-which contribute in combination with all other memories to the elaboration of consciousness. We cannot speak of visual consciousness but only of the seat of actual visual formations (images) which are assimilated by the consciousness or of the mnemonic traces of previous visual perceptions, which have already become part of the mental patrimony and are capable of being made use of for intelligence and conscience. What are actually localised are the specific and individual products furnished by the work of the cerebral cortex, distinguished into areas that have become differentiated in the course of thousands of years, under the influence of particular categories of stimuli (manifestations of the energies of nature). Whether or not these areas have definite boundaries is a question of little consequence. All of them furnish components for the development of consciousness. The case of Laura Bridgmann does not support the view of Loeb, because, if a sense organ and its respective cortical organ are awanting their function may be compensated by greater development of other senses and by a great associative power in other parts of the brain that are more developed. Again, the will is not localised although the motor area whence are discharged the impulses animating the muscular movements is specialised. Volitional motor reactions are the result of mental processes which often involve the entire brain. Who would dream of localising what we call the will?

Thought, even when it appears simple, is always the effect of widely distributed cerebral activity. It is not localisable. It is only the elements from which it results that are capable of localisation.

$$
\text { ** } *
$$

The patient and laborious work of the past fifty years has afforded ample proof of the soundness of the experimental and clinical basis on which is founded the doctrine of the localisation of the functions of the cerebral cortex. This is now understood to apply not in an absolute sense but only to the isolated and differentiated sensory and motor processes. The precise limits of each area have not been mapped out, with the exception, perhaps, of the motor area and, even if they could be, there are good grounds for believing that they may vary in different men. Not only so but we know that each function is bound up and co-related with others. Clinical experience, physiology and, to some extent, histology, have now demonstrated the functional complexity of the relations subsisting between each sensory and motor area and all the others.

One might almost say that the specific waves transmitted by all the sense-organs are projected upon distinct areas of the cerebral cortex. These will now be briefly indicated.

The tactile zone is represented by the posterior Rolandic or ascending 
parietal convolution, limited in front by the Rolandic fissure, behind, presumably, by the interparietal sulcus (Fig. 1). The precise limits, above and

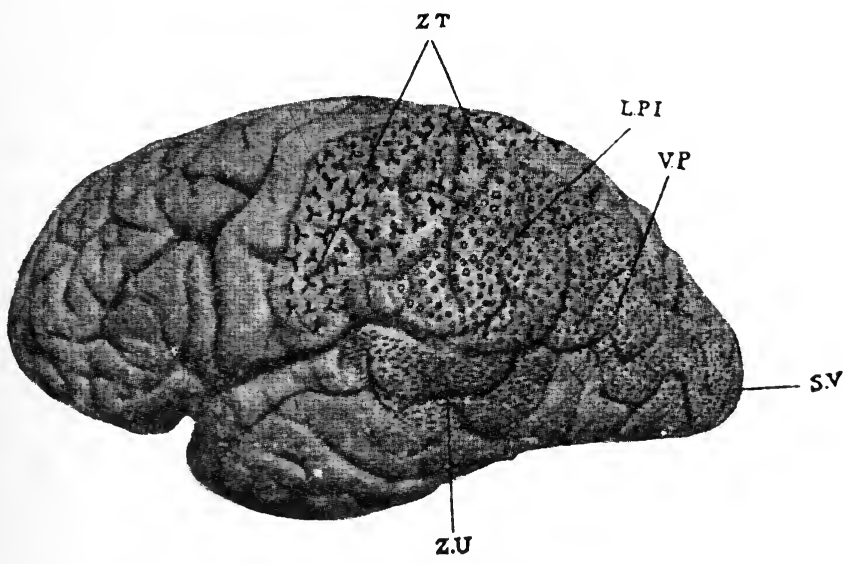

Fig. 1.-External surface of cerebral hemisphere

ZT. Tactile zone.-LPI. Inferior parietal lobule.-VP. Visuo-psychic area (supposed anterior limit). - SV. Visuo-sensory area.-ZU. Anditory area

below, in the superior parietal lobule cannot be defined on the basis either of experimental research or of clinical observation. On the interhemispheric surface the tactile area extends to the median aspect of the ascending parietal convolution (Fig. 2). Certain experimental investigations and

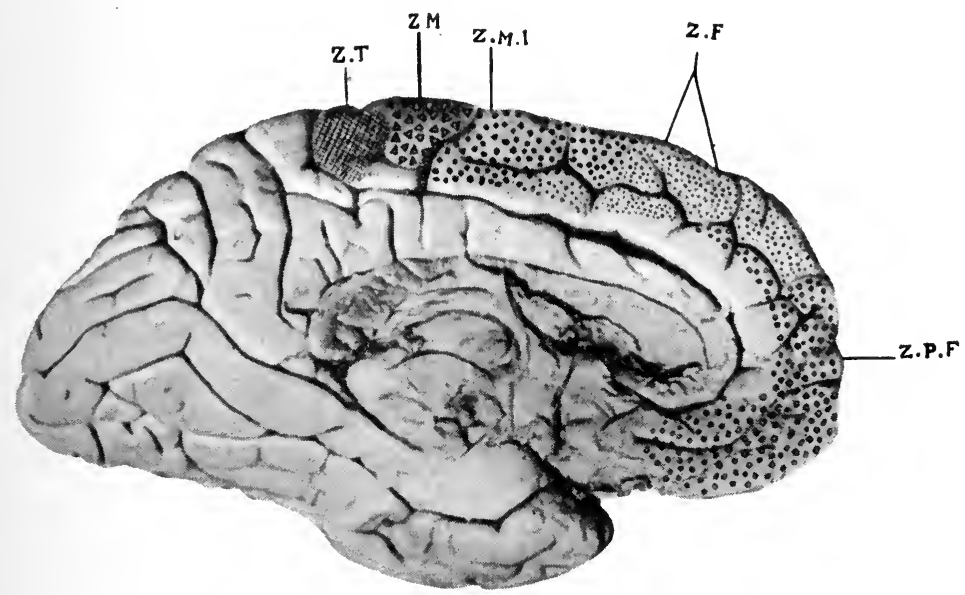

Fig. 2.-Median aspect of cerebral hemisphere

ZT. Tactile zone.-ZM. Motor area.-ZMI. Intermediate motor area.-ZF. Frontal area.ZPF. Pre-frontal area.

clinical observations (Ferrier, Starr) would seem to point to the convolution of the corpus callosum (Fig. 3, L1) and even the hippocampus-i.e. a part of the limbic lobe, as a cortical organ of tactile sensibility.

The study of cell-structure, as well as certain clinical observations which 
will be detailed further on in this chapter, would point to the necessity for distinguishing, in the ascending parietal convolution, an anterior part which would be tacto-sensory and a posterior part, bordering the interparietal sulcus, which should be regarded as a tacto-psychic or intermediate tactile zone. As a matter of fact, the majority of asteriognoses and apraxias have been due to lesions limited to this area. It is beyond the scope of this work

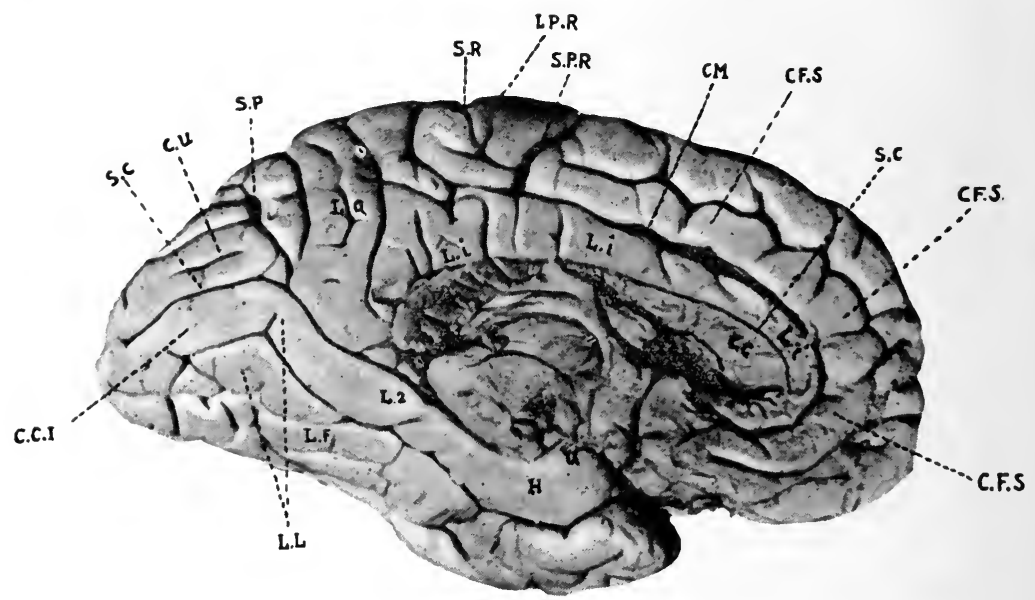

Fig. 3

CFs. Superior frontal convolution.-SC. Callosal sulcus.-CM. Calloso-marginal sulcus.SPR. Para-Rolandic sulcus.-SR. Fissure of Rolando.-SP. Parieto-oceipital tissure.CU. Cuneus.-SC. Calcarine tissure.-CCI. Inferior calcarine convolution.-LL. Lingual lobule.-L1. Limbic convolution.-L2. Inferior limbic convolution.-H. Hippocampus.U. Uncus.-CC. Corpus callosum.-LQ. Quadrate lobule or precuneus

to enter into minute details regarding the various functions of the tactile area and the question as to whether or not it extends forwards on to the anterior Rolandic convolution, as Horsley has maintained, on rather slender grounds, as we shall see.

The visual area comprises the convolutions surrounding the calcarine fissure (Fig. 3), the cuneus, a part of the lingual lobule, the external surface of the occipital lobe and almost all the inferior parietal lobule (Fig. 1). Here, again, experimental evidence, clinical experience and cytotectural studies compel us to distinguish a visuo-sensory area limited to the convolutions of the calcarine fissure and the occipital pole, and also another larger area surrounding the former and comprising the remainder of the cuneus, a part of the lingual lobe, the external surface of the occipital lobe, and the inferior parietal lobule. The last-named is to be regarded as the most evolved in as much as it is the parietal organ for reading-i.e. for visual perception of the graphic symbols of the images of the external world, formed and conserved in their own particular sensory areas.

The auditory zone may be held to embrace two-thirds (middle and posterior) of the temporal lobe. Like the visual, it can be divided into three portions. First, there is the fundamental or acoustic-sensory area limited approximately to the transverse temporal convolution. Next, a much 
larger acoustico-psychic area surrounding the latter, and concerned with the formation and comprehension of the acoustic images of speech. This area is confined to the posterior portions of the first and second temporal convolutions, although its limits are not precisely defined (ZU, Fig. 1). Around it lies a third, more extensive, area, representing a greater evolution of language in its grammatical and syntactical inflexions, allowing the expression of thought as a reflex of history, of personal and human experiences or, again, of the imagination when the mind projects itself into the future, following the laws of association, on the basis of experience (phantasy).

The olfactory and gustatory areas are unfortunately not so well defined but they are no doubt less important. The olfactory area is situated in the olfactory trigone on the median aspect of the hemisphere and in the limbic lobe (L1 and L2, Fig. 3) in both its anterior portion and the hippocampus $(\mathrm{H})$. The gustatory area comprises the uncus and the temporo-sphenoidal pole. Whilst the limbic lobe is concerned with the sense of smell it has probably also to do with the tactile sense. This mingling of functions reminds us of the condition of the cerebral mantle in its earliest manifestation when the senses of smell and taste were both represented in the hippocampal area.

To sum up, recent investigations go to prove that each sensory area is divisible into two or three parts, one of which prepares material for the service of another. One we describe as purely sensory, another as psychosensory or intermediate. The latter only functions when the former is active-i.e. when the area that preceded it in the process of development is sound and whole. Metaphorically speaking, the first area prepares the raw material for transmission to the factory, where it is converted into concrete images of the things (of the stimuli) that go to make up the intellect. In each cerebral area we have a reproduction of the relations which subsist between the cerebral cortex and the optic thalamus. Take the case of the calcarine area in its relations to the visuo-psychic area. We can put it thus: when the organs of lower order suspend operations, work ceases in higher organs, even though they are in perfect working order, simply through lack of necessary material. This law permits no shadow of doubt. We have an excellent example in the function of language, in the relations of the images of speech to the concrete images of things.

The acoustic images of speech serve as the symbol not only of the objects themselves found in the external world but also of the visual, tactile, and other sensory images of these. The recall of the acoustic image of the word pen, e.g., is subject to this condition, that the visual and tactile images of the pen are available. If these are lacking, then, even although the object itself confronts us, the acoustic word image that symbolises the object cannot be evoked or is only with great difficulty recalled.

It is clear, then, that localisation is not to be understood in its ordinary and restricted sense. Rather should we think of it as involving a continuous chain of processes, each having its own organ and all being closely bound up with one another functionally and anatomically. This is particularly true 
when we come to speak of intelligence or, if you like, Mind, which is a functional product of the whole cerebrum. One must admit that Munk had good reason on his side when, in the course of his memorable debate with Hitzig, he maintained that intelligence had no circumscribed seat in the cerebral cortex but involved the entire brain. If intelligence emanates from play of sensory images of the external world elaborated by the respective sensory areas, and of infinite combinations of these and other images not sensory in character, it is very evident that all these areas contribute in varying proportion to the content and play of intellect. It is also manifest that experimental destruction of one or more of these areas must have a distinct effect upon the intellectual function which is now deprived of the contribution from the area or areas destroyed, always taking into account the degree of cerebral development in the animal that is the subject of experiment. The function in this case is impoverished because intellect is the result of association and synthesis of the simple or complex images and representations furnished by the respective areas. The actual amount of damage will depend in some measure upon the seat, extent and depth of the lesion.

Such, then, is the conception of the doctrine of the localisation which the data at our command warrant us in accepting, and it is the conception which the author has supported for many years. In its essential features it may be summed up in the functional differentiation of the cortex into as many areas as there are special senses. To these we must add not only the motor area-which represents in the cortex, on a higher scale, what the anterior cornu is in the spinal medulla-but also those areas which are neither sensory nor motor in their essential characters, areas which now demand special consideration. ${ }^{1}$

$$
*^{*} *
$$

First of all, it is to be remarked that the phenomena that go to make up what we call intelligence are not co-extensive merely with the sensory areas of the cerebral cortex (the areas already described as factories where the images are formed and their memories stored) and the excitable motor area.

1 The arguments that have been used from time to time against the doctrine of cortical localisations strike us as being a species of mental gymnastics rather than sound views controlled by experimental findings and elinical observations. Some seem to regard the doctrine of the localisation of the functions of the brain as still very involved. The want of correspondence between functional areas and cytotectural areas, the presence of motor cells and motor paths in the sensory areas, and the motor phenomena induced by excitation of sensory areas, have been brought forward as arguments against the doctrine. So far as the want of correspondence between the areas that have been mapped out by histologists on the basis of eell-arehitecture with those assigned by experimental and clinical researeh to a particular function is concerned, it is to be pointed out that these histologists have admitted that it is necessary to make certain reservations in applying the data of histology to physiology and to clinical findings. The presence of motor cells and paths in the sensory areas brings to mind the old notion that these were sensory-motor areas, a point raised by the author in 1882. (Riv. Sper. di Fren. 1882.) 
The accident wards of our hospitals, as well as experimental, morphological and histological investigations, have demonstrated the existence of extensive areas of the cerebral cortex which do not possess sensory characteristics in the ordinary sense of the term nor yet the features of motility. The evolution of the brain, as manifested throughout the scale from the lower mammals up to man, has greatly enlarged the cortical field of intellectual function by the appearance and development, in apes (primates) and more particularly

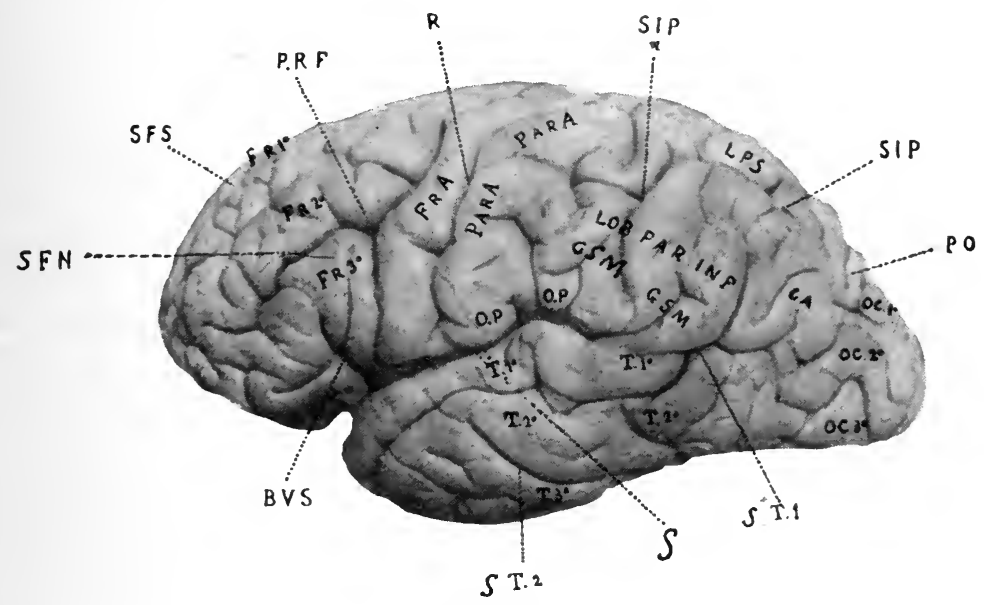

Fig. 4

FR1, FR2, FR3. Frontal convolutions, superior, middle and inferior.-FRA. Ascending frontal convolution.-PARA. Ascending parietal convolution,-OP. Parietal operculum. SIP. Interparietal sulcus.-I.PS. Superior parietal lobule.-IOB, PAR, INF. Inferior parietal lobule.-GS.I. Supramarginal gyrus.-CA. Angular gyrus, posterior extremity.T1, T2, T3. Superior, middle, and inferior temporal convolutions.-OC1, OC2, OC3. Superior, middle and inferior occipital conrolutions.-SFS. Superior frontal sulcus. SFN. Inferior frontal sulcus.-BVS. Ascending branch of fissure of sylvius.-PRF. Prefrontal sulcus. - R. Fissure of Rolando._SIP. Interparietal sulens. -ST1, S'T2. superior and inferior temporal sulci.-S. Fissure of Sylvius

in man, of cortical regions, the function of which is neither sensory nor motor in the ordinary acceptation of the term.

This fact gave rise to the conception of the existence of cortical organs in which the higher mental operations take place, a conception that has been gradually developing.

Hitzig advanced the hypothesis in favour of a centre of this nature. Some time later, the author, in the course of some experimental investigations, was led to infer the existence of an organ in which there takes place a confluence of images re-evoked in other parts so that a process of coordination is arrived at thereby. This would be a centre of centres, giving rise to a more extensive association of the products of perception (mnemonic images) and, consequently, to more complex mental products (mental syntheses). Flechsig put forward the same theory. Subsequently many others have admitted the existence of this high function, consisting in the power of associating and combining the mental products of the perceptive areas and recomposing them into psychic complexes of a higher order. At 
the same time it is to be remarked that all are not agreed as to the precise cortical region that is to be regarded as the seat of this very superior function nor as to the exact mechanism concerned therein. Some authors (Hitzig, Bianchi) regard the frontal lobe as the centre of this association and coordination; others have located it in the so-called posterior associative centre or parieto-temporo-occipital region of Flechsig. ${ }^{1}$ The latter view is supported by not a few physiologists and psychiatrists. At the same time, Flechsig's doctrine has met with a strong and consistent opposition on the part of many neurologists and it has been warmly contested in almost all the discussions that have taken place on this subject on the occasion of International Congresses.

Before proceeding to discuss the thesis which I desire particularly to put forward as regards the function of the frontal lobes, it will be well to clear the ground by giving a rapid review and criticism of the ponderous doctrine of Flechsig and the anatomical basis on which it is founded.

The idea of the existence of associative areas or zones of co-ordination had already been clearly expressed by me in 1883 as the following quotation from my work dealing with experimental investigations into the functional compensations occurring in the cerebral cortex shows ${ }^{2}$ :-

"Sight, regarded as the perception and cognition of objects falling within the visual field, is not to be considered as the result of a physiological process of a simple nature nor of processes that merely follow one another in time and space. Rather is it to be regarded as the complex and co-ordinated result of several processes, varying in nature, following one upon another and extended over the cerebral surface. Perception of an object, in order to furnish data for its cognition, requires: (1) a luminous stimulus ; (2) a series of ocular movements the effecting of which delineates, as it were, in the corresponding pereeptive centre, the features of form, extension and spatial relations ; (3) the co-ordination of these two factors, bringing about objective unity and giving rise to the image of the object in the perceptive centre. If there is no morphological difference between the elements concerned in these various physiological moments of vision, no one will yet care to deny that these elementary facts differ in nature from one another and may accordingly be manifested in different nervous elements and in different sites. Electric stimulation of different portions of the extensive visual area of the dog affords evidence of the differentiation of its component parts. In fact the anterior portion of the second external convolution (excitation of which is followed by contraction of the orbicularis palpebrarum) shows close relations with intensities of luminous impressions as such and may be regarded as the field of light and colour impressions. ${ }^{3}$ The central and most

${ }^{1}$ Flechsig. Die Localisation der Geistigen Vorgänge, etc. Leipzig, 1896.

${ }^{2}$ Bianehi. La Psichiatria, la Neuropatologia et le scienze affini. 1883.

3 The author was induced to regard the anterior part of the visual zone as a luminous centre because of the spasmodie movements of the orbicularis palpebrarum which he looked upon as light-reflexes, but he would not maintain that view to-day. 
prominent part, electric excitation of which produces bilateral and coordinated movements of the ocular bulbs, is the site of formation and registration of the features of form, size and spatial relations of the object, for these depend upon movements of the ocular muscles. The occipital lobe does not respond to electric stimulation but extirpation in mammals (dogs) is followed by the same symptoms as are produced by extirpation of one of the afore-mentioned sections. Its function is to co-ordinate the two more elementary factors of visual perception, thus completing the image of the object."

The results of the author's investigations, even at that date, induced him to affirm the existence of an area for the co-ordination of the elements of visual perception, and there seemed to be good grounds for believing that the same thing held good in the case of the other sense-perceptions. The term "area of co-ordination" was only another way of expressing what is now more generally known as " association area."

Since that date this additional fact has been brought to light_- that the sensory fields are rather extensive and their limits ill-defined. All functions referable originally to the same sense should be regarded as being comprised within the same sensory field for they represent grades of evolution or subsidiary functions closely bound up with the primitive formation of the special images referable to that particular sense.

On the basis of clinical observations, and apparently also of cortical histology, one can formulate the general law that as each sensory area evolves it assumes different histological characters and furnishes products of higher value for the development of intellect. The old conception of evolutionary areas, formulated by the author many years ago, has been confirmed by later clinical and histological researches, and this is true as regards both the sensory spheres, which especially have been the objects of investigation, and also the motor spheres. An outstanding example is furnished by the development of our knowledge regarding the visual zone.

Amongst the clinical investigations that have rendered valuable contributions to the physiological and clinical doctrine of the cerebral localisations the numerous and convincing researches of Henschen, ${ }^{1}$ and those of Vialet, are especially worthy of notice, for on them was based and formulated the doctrine, fully confirmed by subsequent clinical researches, that in the human brain the cortical visual area is represented by the convolutions bordering upon the calcarine fissure and by the occipital pole. This area is the field of cortical perception of luminous retinal impressions. Anatomical observations support this hypothesis. In point of fact the fibres of the optic tract, after being interrupted in the optic thalamus (in the external geniculate body and the pulvinar) proceed onward again as the "bundle of Gratiolet" or thalamo-cortical bundle, and terminate precisely in those convolutions forming the lips of the calcarine fissure and in the occipital pole. This area

1 Henschen. Die Pathologie des Gehirns, vol. ii., 1892; also Klinische und anatomische Beiträge zür Pathologie des Gehirns. Upsala, 1911. 
represents the projection of the retina upon the cerebral mantle and can be divided into sectors corresponding to the sectors of the retina, always taking into account the refraction of the luminous rays. Such was the doctrine advanced by Henschen. It has been confirmed by many others, including Pierre Marie, ${ }^{1}$ who recently examined a number of soldiers wounded in the occipital lobe and found that the visual field showed blind sectors with the characters of hemianopsia, whenever the injury fell within the limits of the zone of cortical projection of the retina.

The old ideas expressed by the author have been amply confirmed by the later clinical, experimental and histological researches, for all now agree that the visual area is not strictly limited to the region of the calcarine fissure and the occipital pole. Another more extensive region has been gradually formed around the former. It has been named by Campbell the visuopsychic zone so as to distinguish it from the other, the visuo-sensory. It is in the visuo-psychic zone that the concrete visual images are formed with the concurrence of the essential and necessary products furnished by the visuo-sensory calcarine area. If the latter is destroyed we get blindness of the visual field of the retina as represented in its various sectors or quadrants. It is evident, then, that this area plays an essential part in the visual function because the element of light is a component sine qua non of the visual function (perception), but it is not the only anatomical field concerned with perception. The images are formed in the peri-calcarine area, and on the external aspect of the occipital lobe, and perhaps also in the parietal lobe, as will afterwards be seen. There are other components that must be combined with the element light so as to permit the formation of the visual images (on the external aspect of the parieto-occipital lobe). I refer to the motor components. It is the fusion of these with the luminous elements that gives rise to the concrete visual image. Clinical and experimental data find support in the facts of anatomy, for the lips of the calcarine fissure are connected wth the external surface of the occipital lobe and with the inferior parietal lobule by means of certain well-recognised bundles of fibres. I refer particularly to Wernicke's vertical occipital bundle, Vialet's transverse bundle of the lingual lobule, and the transverse bundle of the cuneus. ${ }^{2}$

The statement made by some physiologists that the images are formed in one cortical field and conserved in another from which they can then be evoked is no doubt ambiguous but it is not without foundation, for the primary sensory zones, such as the calcarine and other analogous areas, are not the fields of formation of concrete images of the external world and of spatial relations. They merely furnish some essential elements which have to be combined with the motor components before they can give rise to the concrete image. Destruction of the primary area-e.g. the calcarine-causes complete blindness. Destruction of the secondary area spares luminous perception, to some extent, but destroys the capacity for perception and recognition of objects (Munk's psychic blindness). Regarded in this light, it is clear that a perceptive zone is necessarily an associative zone. The ${ }^{1}$ Pierre Marie, Revue Neurologique. 1916. aDejerine, Anat.descentresnerveux. Vol. i. 
combining of different elements so as to give rise to visual images becomes more evident when we trace the development of sight in the infant.

In the first days after birth, the infant perceives only the light, not the form of objects, nor distance, nor spatial relations. It is only later that it perceives these other features, as it commences to measure distances and to form its first notions of space. The researches of Brodmann, Campbell and Bolton have demonstrated the fact that the visuo-psychic zone is developed at a later period than the visuo-sensory area and presents marked differences in structure as compared with the latter.

All that has been said above with regard to the visual images is equally applicable to the other senses.

Flechsig in several of his publications has expressed practically the same idea in another form. He asserts that around the projection zones, properly so called, there are formed intermediate zones which would not really be associative areas in the strict sense of the term, but centres of secondary sensibility as distinguished from the centres of primary sensibility. ${ }^{1}$ They would contain a smaller number of projection fibres than the primary centres and a greater number than the terminal zones. It thus appears that Flechsig has now reduced the extent of his association centres to almost a third of that ascribed to them in his original publication, whilst, at the same time, the field of the centres of sensibility is correspondingly increased.

The first areas, those of primary sensibility, are projection areas of a receptive order; the second-i.e. Flechsig's centres of secondary sensibilitymay be more appropriately described as perceptive. The fibres received by the latter get their myeline sheaths some time after the fibres of the first system and in a progressive manner from the periphery of the receptive zones towards the terminal zones, which is the name Flechsig gives to the outermost areas of the centres of secondary sensibility. The nerve-fibres that are late in becoming myelinised come for the most part from the receptive areas, so that it seems a very likely inference that in the so-called associative areas we have an elaboration of the products of work of the receptive projection areas.

$$
* * *
$$

According to Flechsig ${ }^{2}$ there are three associative areas on the external surface of the hemisphere; an anterior or frontal situated in front of the motor or, as he calls it, the somæsthetic or tactile zone; a much smaller middle area consisting of the insula situated between the frontal lobe, the olfactory, somæsthetic and auditory areas; a third, considerably larger than the two preceding, the great posterior or parieto-temporo-occipital

1 To avoid academic discussion dependent upon variation in terms, it is well to mention that Flechsig's centre of secondary sensibility corresponds to the perceptive zone, such as the previously mentioned visuo-psychic and acoustico-psychic areas, and to the author's " field of co-ordination of the components of the concrete images" as conceived in 1883 . Loc, cit.

${ }^{2}$ Flechsig. Die Localisation der geistigen Vorgänge. Leipzig, 1896. 
associative zone which includes a wide surface limited in front by the postrolandic convolution and the interparietal sulcus, in front and below by the insula, the olfactory zone (according to some also acoustic) and the auditory

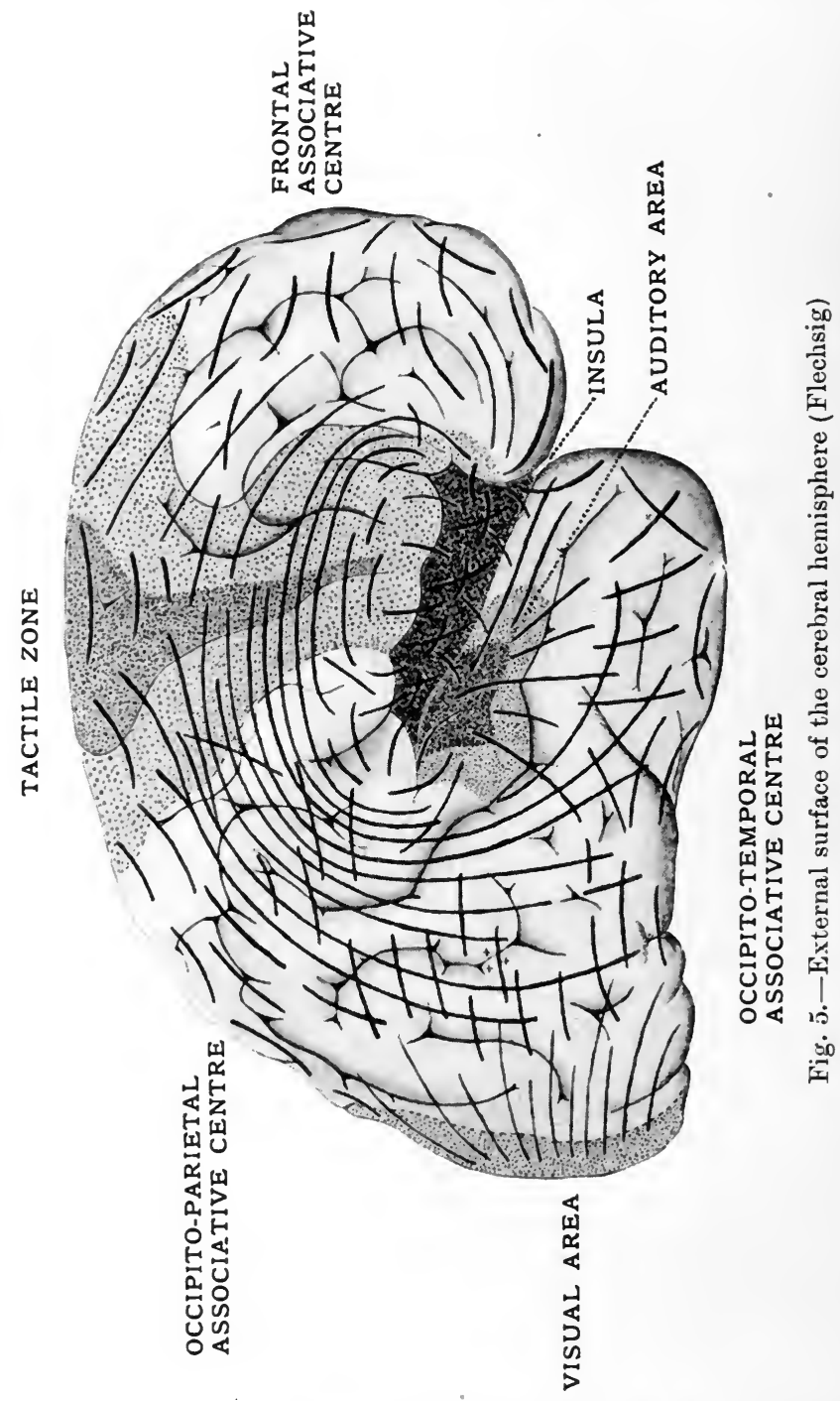

area, behind by the optic zone. It is continued on to the inferior aspect of the hemisphere as far as the hippocampus (Figs. 5 and 6).

In a later publication Flechsig, ${ }^{1}$ as the result of further investigations, has described a much larger number of special areas called by him embryological cortical territories. Of these he distinguished forty (Figs. 7 and 8) and this number was not necessarily final. This large number of cortical territories

${ }^{1}$ Flechsig. Neue Untersuchungen über die Markbildung in dem menschlichen Grosshirnlappen. 1898. 
is divided into three groups : $(a)$ primordial territories which normally are formed before the fœetus reaches maturity (Nos. 1 to 8 in his system) and correspond to genuine projection zones ; $(b)$ intermediate territories the fibres

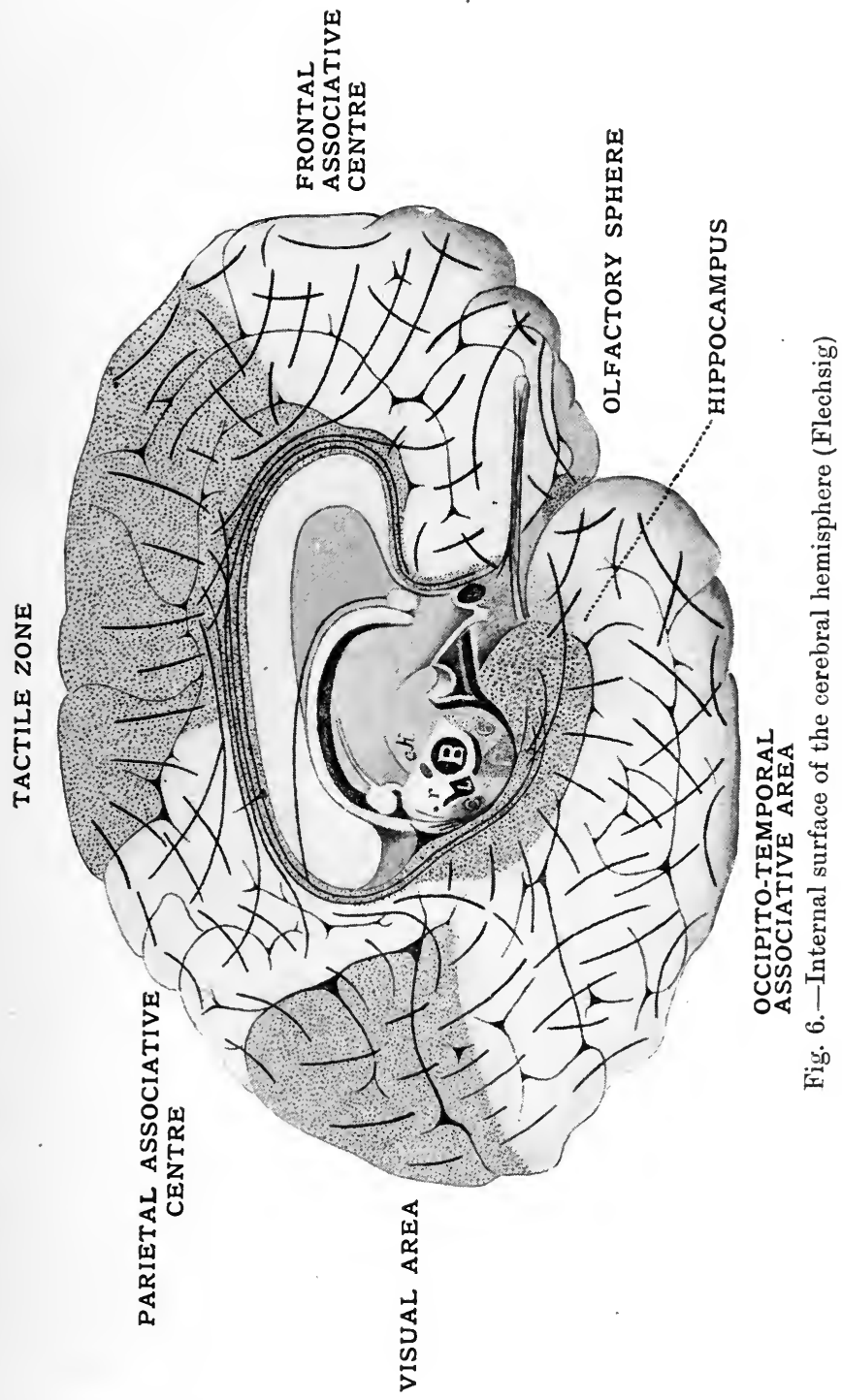

of which get their myeline sheaths up to one month after birth at full time (Nos. 9 to 32); these areas are situated in the immediate vicinity of the projection zones; $(c)$ terminal territories the fibres of which mature from one to four months after birth at full time (Nos. 33 to 40). In a subsequent communication to the International Congress of Physiologists held at Turin, Flechsig reduced the number of these areas from forty to thirty-six, as the result, he said, of further investigations. 
At the International Congress of Psychology held in Rome, $1905,{ }^{1}$ he

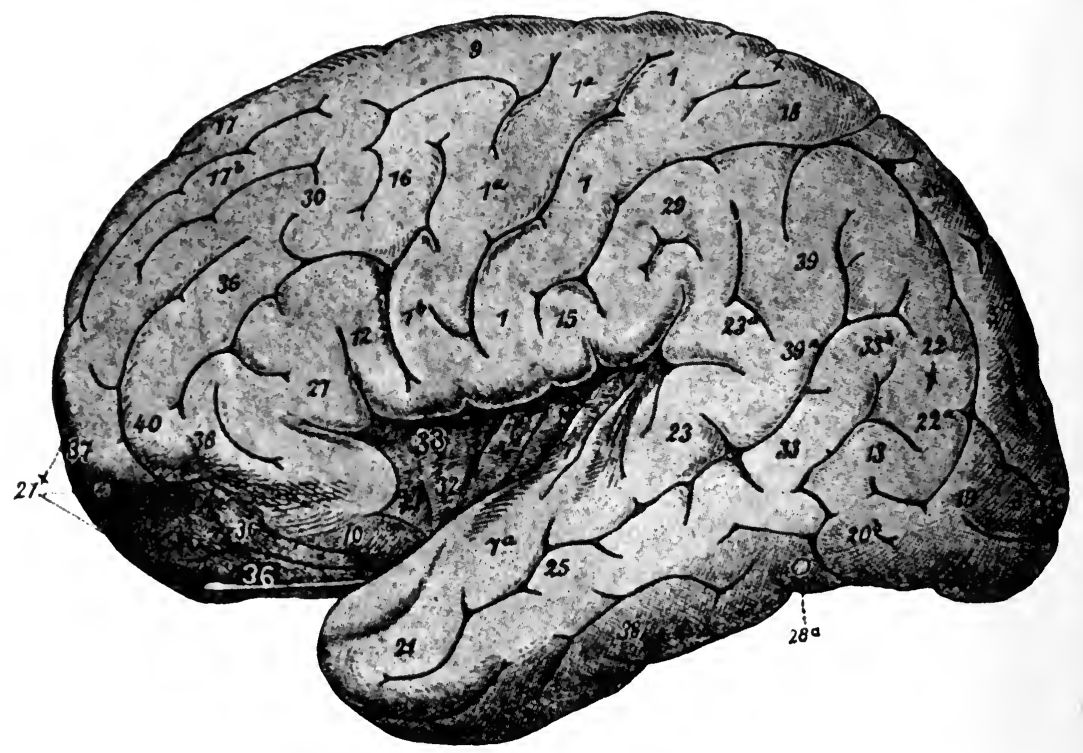

Fig. 7

spoke of thirty-five areas and expressed his agreement with the views the author put forward at the Congress in Madrid, ${ }^{2} 1903$, concerning the

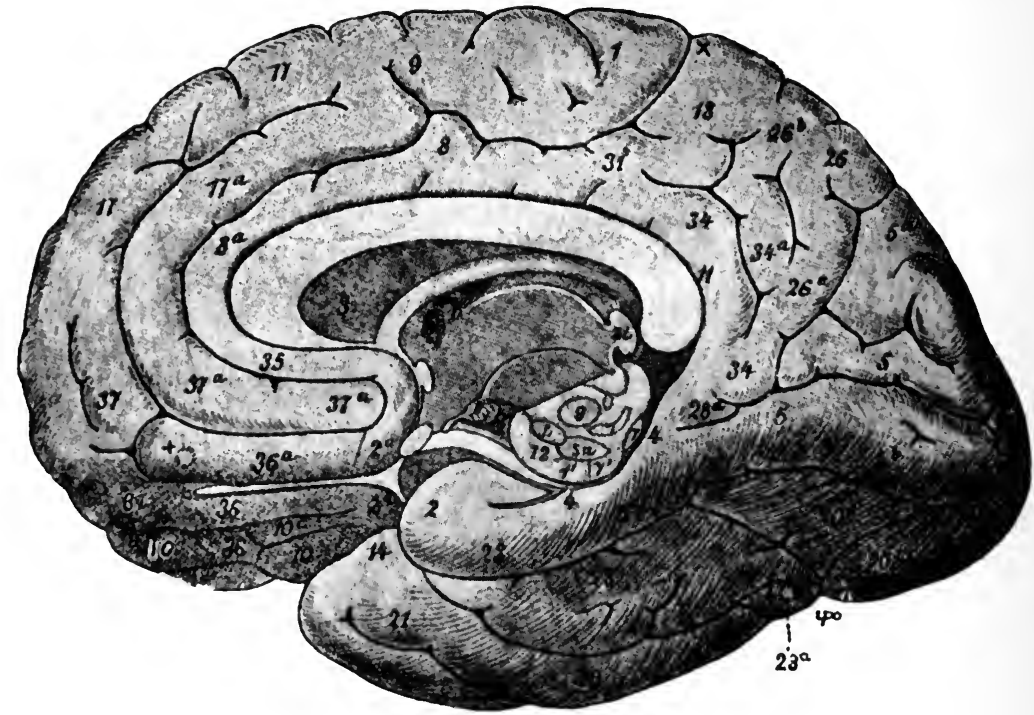

Fig. 8

intellectual value of the language zones, and thus detracted, to some extent,

${ }^{1}$ Flechsig. "Hirnphysiologie und Willentheorien." Atti del Congresso Internazionale di Psicologia. Rome, 1905.

2Bianehi. XI I'e Congrès International de Médecine. Madrid, 1903. 
from the importance he originally ascribed to the posterior associative zone, giving instead, to language and the cortical area of speech the value the author had assigned to it in the intellectual mechanism, as long ago as 1887.1

A problem of anatomy and psychology so important as this, considered without prejudice, cannot be solved solely by histological inquiries such as those of Flechsig because it is proven that myelinisation of the endohemispheric fibres does not follow a constant law, and even if it did, it could not be inferred, as a corollary without exception, that this geography of evolutionary anatomy should be regarded as a sure foundation for a species of psychological geography. Even granting it were true, there would still be no difficulty in accepting the author's view, for the intermediate and terminal territories would fall to be regarded as fields of extension of the receptive and perceptive functions of the corresponding primordial territories and would represent evolutionary phases of the original functions.

Siemerling, Monsieur and Madame Vogt have shown that the various systems are not developed in their entirety at one and the same time. Both in the projection and in the association territories there remain undeveloped areas that have anatomical relations with one another, where myelinisation does not follow a constant law of development. According to O. Vogt, ${ }^{2}$ the fields of myelinisation do not exhibit the same boundaries in different subjects nor do they coincide with the cyto-tectural areas. It is more likely that they correspond with the various subcortical nuclei, especially those of the optic thalamus. The myelinisation or, in other words, the development of the fibres of different sections of the cerebral mantle, traced from the fourth month of fœetal life until complete development, after birth, shows no great difference between the frontal, parietal (Balkensegment) and parieto-occipital segments. Expressed in figures, the relative degrees of myelinisation in these segments would be 20 to 25 per cent. in the frontal lobe, 40 to 46 per cent. in the parietal (Rolandic) segment, and 29 to 38 per cent. in the parieto-occipital segment.

Dejerine and Monakow, following the method of descending degenerations, arrived at results that do not support the assertions of Flechsig. ${ }^{3}$

If we follow the inductive method of reasoning we are obliged to conclude that myelinisation of the fibres leading to the so-called associative centres has nothing to do with the evolution of intelligence, for the simple reason that whilst the fibres of the terminal territories are developed in the fourth and often as late as the tenth month after birth, intelligence is not developed until a much later period. There is no correspondence between the development of mental phenomena and that of the anatomical territories assigned 1887.

${ }^{1}$ Bianchi. "Un caso di sordità verbale," ecc. Rivista Sperim. di Freniatra.

${ }^{2}$ Vogt. Myélinisation des Hémisphères Cérébraux. Paris, 1900. Also O. \& C. Vogt. "Markreifung des Kindergehirns." Neurol. Arbeitens, etc. Jena, 1904.

${ }^{3}$ Hitzig. "Les centres de projection et les centres d'association." Le Névraxe. 1900. 
to them. The histological factor, as Brodmann has remarked, remains to some extent detached from the psychological factor and does not offer a satisfactory explanation of the latter. Flechsig sets out from an anatomopsychological point of view that is open to discussion when he holds that the memory-centres are distinct from those of perception. He expresses his view in these words: "The spheres of sensibility are, according to the degree of their connections with the organs of the senses, so many centres of perception, so that it is in them that one should seek for the essential bases of the conceptions of time or of space. Vice versa they appear to be incapable of reproducing in an independent manner large numbers of images and memories." 1

Speaking of tactile sensibility he states that the corresponding cortical sphere serves to a large extent as the medium of our relations with the external world ; that paralysis of touch, caused by a lesion of this sphere, would not depend upon loss of memory-images but upon the impossibility of associating, in the cortex, the tactile impressions, and that this then betrays itself in loss of the faculty of co-ordination. Practically speaking, Flechsig regards the association centre as having the task of associating and reproducing the states of excitation of the different spheres of sensibility, connecting these spheres indirectly with one another by systems of association-fibres. Nevertheless, it is not proven nor is it probable that there exist separate centres of memory around the centres of perception. If we admit the identity of the anatomical substratum of the perceptions and the memory-records of sensations, representing the stimuli that have excited our senses, we must also admit that the zone of perception is at the same time the area of memory. Flechsig's diction is very obscure. To the author's mind the only intelligent conception of the psychological mechanism is that which he himself has put forward since 1883-viz. that perception is a process of association of the various components whence it results. The product of perception (the concrete image of things) arises from the physiological fusion of sensory and motor elements furnished by separate portions of the same sensory area considered in its entirety. It is evident that in the attempt at interpretation the receptive has been confused with the perceptive area. Destruction of a cerebral area, if it suppresses one of the components of the image which perception should furnish or if it prevents its physiological fusion with others, leads to suppression of the perceptive process ; but the fact remains, beyond dispute, that we can still have reproduction of the particular images already stored in each normal perceptive area.

If a so-called association-area were located in the occipito-parietal zone, in front of the area of the visual perceptions, keeping in mind Flechsig's idea that the association area is the intermedium which permits the process of recognition and localisation of the perceptions, which in the first instance are furnished by the sensory areas, and if in this zone there should meet together not only the products of visual perceptions but in addition the

${ }^{1}$ Flechsig. "Gehirn und Seele," 1896; and "Localisation," etc. Neurol. Centralb. 1898. 
auditory, olfactory and tactile memory-images, so as to give rise to series of psychic compounds of a higher order, no doubt Flechsig's hypothesis regarding such a zone would be at once more probable and intelligible. Yet this vast cerebral area fulfils functions that are undoubtedly visual in character in its parieto-occipital portion and auditory in the temporal part. In several points of the occipito-parietal region electrical stimulation is followed by ocular movements. Total or partial destruction of the said area, if it affects only the cortex, gives rise to fleeting disturbances of vision and nothing more. If the destruction is deep we have permanent hemianopsia and, if it is bilateral, psychic blindness, in which case objects are seen but not recognised. The results of the writer's investigations are in close agreement with those of Munk, Ferrier, Luciani and Tamburini, Hitzig, Ferrier and Yeo, Horsley, and others, and show that after destruction of the cortex of the occipital lobe the conditions necessary for the formation and registration of new images of objects seen for the first time also disappear, whilst auditory and tactile perceptions and memories remain normal.

The anterior extremity of the occipito-parietal zone in man also performs a function that is exclusively and essentially visual, though on a higher level in the psychological hierarchy, for it is concerned with reading, in which process we have the formation and conservation of the visual images of the graphic signs of words. From a psychological point of view, the area of the graphic visual images is more complex and represents a higher grade of evolution than that of the visual images of the objects themselves. It must receive a greater number of associative fibres and it has a higher physiological value because the function of reading is much more complex without ceasing to be at the same time perceptive and visual in nature.

It thus appears that the visual function is evolved from the simple luminous perception, with its seat in the calcarine fissure, a part of the cuneus, and the occipital pole (Figs. 2 and 3), by the addition in the first instance of oculo-motor elements, these giving rise to the formation of the concrete visual images of objects (the dotted area on the external occipito-parietal area, Fig. 1, VP). Having attained this stage, in itself rather complex, it passes by further evolution of another part of the same visual area to the formation of the visual graphic signs of the objects themselves and their sensory images. This portion of the visual area is developed towards the anterior limits of the said area (Fig. 1, LPI, area covered with small circles). Bilateral lesions of the occipital cortex, besides producing a more or less severe psychic blindness, give rise to a variety of dementia which can reasonably be regarded as resulting from the loss of a great part of a man's intellectual capital-viz. his store of visual images of the external world, for these form a considerable proportion of the sensory elements that contribute to the structure and manifestation of intellect.

A good deal of value has been attached to Flechsig's doctrine in the field of psychiatry, for various forms of dementia have been attributed to anatomical lesions of his posterior associative zone. As a matter of fact, statements of this kind have not been properly controlled or confirmed by accurate 
neurological and neuro-pathological investigations. The observations that are most likely to help us in settling this question are those that have been made on the zone of language. We know that word-deafness, which is generally due to a destructive focus in the posterior portions of the left superior and middle temporal convolutions, causes a particular variety of dementia. We know, too, that this part of the language-area is comprised within the limits of the auditory sensory area or perceptive area of Flechsig (intermediate territory No. 23, Fig. 7). We all understand what psychic and sensory elements come into play in the formation and comprehension of auditory word images. These, in fact, synthetise not only the auditory and kinæsthetic elements of speech but also the numerous and complex series of visual, tactile, olfactory, muscular, etc. images formed by all the other sensory areas, inasmuch as these images, which reproduce the external world, are expressed in words which reconstitute in the form of phonetic symbols the objects recognised and their relations, rendering them sensible in all their details and all their qualities as furnished by the different senses. To the author's mind there are few regions of the cerebral cortex that compare with the left superior temporal convolution in its numerous and extensive relations with the rest of the brain, relations very intimately bound up with thought and all the psychic manifestations. It is in this region that ideas assume the plastic form of speech, which is ever tending to give fuller expression to their essential characters and all the changes they undergo in consequence of an infinite and perpetual succession of external and internal stimuli. Flechsig regarded the auditory area of speech as a "primordial" and "intermediate" zone of perception, and hence assigned to it a less importance than that attributed to his great occipito-parietal zone. The fact remains that a small destructive focus in this area destroys the mnemonic capital of speech. At the same time it is to be noted that Flechsig has gradually altered his opinion, as is evident from his communication to the International Congress of Psychology in Rome, in 1905.

For many years the writer has attempted to define the syndromes of the different functional areas of the human cerebral cortex, and his investigations may serve to throw some light on this fundamental question.

\section{**}

Let us turn our attention first of all to the inferior parietal lobule, a part of the great posterior associative zone of Flechsig.

Symptoms due to lesions of the parietal lobe have been recorded by various writers. They have been described, though incompletely, by Monakow. ${ }^{1}$ 'They are outlined in various descriptions of the optic variety of sensory aphasia (word-blindness), and Beduschi," in particular, has collected the scattered records in a monograph. Up till 1909, when the author published his views on the subject, ${ }^{3}$ there was no work that embraced

${ }^{1}$ Monakow. Gehirnkrankheiten. 1905.

${ }^{2}$ Beduschi. Afasie. Milan, 1909.

${ }^{3}$ Bianchi. "La sindrome parietale." Ann. di Neurol. 1910. 
the entire symptomatology of lesions of the parietal lobe and provided a feasible conception of the physiology and pathology of this part of the brain.

Human pathology offers the one and only means of control over physiological doctrines of the nervous system founded on experiments and histological investigations. It is the only means of adjusting these and bringing them to their proper level. The difference between lesions of the parietal lobe and those of the motor area or the area of general sensibility lies in this. Motor and sensory paralysis of one side or of a limb is a fact that is not only quickly noticed by the patient but also falls readily under the immediate observation of the neuropathologist, and from ancient times has claimed the attention of the clinician and even of the layman. These paralyses being very frequent have been more thoroughly examined, distinguished and classified and it has been possible to establish definite relations between the symptoms and the lesions.

It is only within comparatively recent times that the parietal lobe has received much attention, and lesions therein are not recognised unless the clinical examination is particularly directed to the investigation of the special symptomatic complexes involved, which are sometimes very vague. The physiology and pathology of this cortical area are so ill defined that only a very accurate, acute and detailed investigation will be of much service in the advancement of our knowledge of this part of the physiopathology of the brain.

In this connection it is to be observed that the results of experiments upon animals cannot be presumed to be applicable to man. There are many facts that are referable only to the human being, and it is those that must be singled out and carefully investigated both in the living subject and in the cadaver.

The histological researches of Campbell, ${ }^{1}$ Brodmann ${ }^{2}$ and others provide no uniform and conclusive data respecting the function of the parietal lobule except this important fact that it is later in development than the visuo-sensory zone. It has been established that the visuo-psychic area extends forwards, merging into that part of the cortex which is regarded as the inferior parietal lobule. The area of this lobule is limited above, in front, and a little behind by the inter-parietal sulcus and comprises the supra-marginal convolution and the anterior portion of the angular gyrus (Figs. 1 and 4). Posteriorly it is limited by an imaginary line drawn vertically from the occipito-parietal fissure, but for the purposes of this work it is unnecessary to enter into details regarding the posterior boundary. Inferiorly it is limited in its front and middle portions by the fissure of Sylvius, in its hind portion by an imaginary prolongation of this fissure in a horizontal direction to the point where it meets the vertical line above-mentioned. This clinico-morphological boundary, as already observed, does not coincide

${ }^{1}$ Campbell. Histological Studies on the Localisation of Cerebral Function. 1905.

2 Brodmann. "Beiträge zur histologischen Lokalisation des Grosshirnrinde," etc. Journ. f. Psych. und Neurol. 1906. 
with that which is drawn on the basis of cytotectural studies. Clinical observations have furnished data of a much more conclusive character.

Lesions of the supra-marginal convolution and the anterior part of the angular gyrus (the area LPI indicated with small circles, Figs. 1 and 4) give rise to disturbances that must be regarded as belonging to the visual function. Permanent auditory or tactile disorders were never observed by the author.

Auditory disturbances are only apparent at the outset of the condition and are fleeting in character; or they may make their appearance if the lesion invades also the superior temporal convolution. Disturbances of tactile sensibility are only observed when the lesion extends from the parietal field to the post-central convolution.

The area we are dealing with is not distinctly and precisely delimited, and it seems clear that the variations in the symptomatology are to be explained by its indefinite continuation and merging into other areas, which have fairly well-defined functions.

The disturbances of tactile and auditory sensibility which complicate the clinical picture in parietal lesions do not depend upon the fact that the inferior parietal lobule forms a part of the posterior associative zone but upon the extension of the lesion into anatomical fields that involve the function of hearing or of tactile sensibility or of both.

Both the supra-marginal convolution and the anterior portion of the angular gyrus form a part of Wernicke's zone, but the writer is convinced, from the material he has been able to examine, that it is necessary from the pathological point of view to divide Wernicke's zone into two areas, the temporal (Fig. 1, ZU) and the parietal (Fig. 1, LPI).

If the lesion involves the whole temporo-parietal region of language one can understand that in this case the syndrome will be very complex. Undoubtedly this is the chief reason why this region of the neopallium was considered as one area, after Wernicke and Kussmaul ${ }^{1}$ had established therein the seat of sensory aphasia. One must take into account this other fact that a lesion of the temporal part alone of Wernicke's zone gives rise to a very complex syndrome, just as if the lesion extended also to the parietal portion, so that it is not easy to distinguish between symptoms depending on lesions localised in the temporal lobe and those affecting also the inferior parietal lobule. The complex character of the symptoms produced by a lesion limited to the temporal lobe goes to prove that the function of the parietal area of language is in this case merely interdicted (inhibited) by the lesion limited to the temporal portion of the zone in question.

So, too, in the case of foci limited to the parietal portion of Wernicke's zone, we get, during the initial period, a complex syndrome, which includes loss of the function of the posterior part of the superior temporal convolution, though this is of short duration.

It is only by systematic observation of these patients over a considerable

'Kussmaul. "Disordini della favella." Ziemsen. Patole Terap, vol. xi., parte 3 . 
period of time that we can follow the gradual disappearance of some symptoms whilst others remain unaltered so as to constitute a syndrome that is fairly uniform and directly attributable to the lesion of the parietal area with which we are dealing.

The thing that stands out clearly is the fact that small cortical or subcortical foci in the superior temporal convolution give rise to symptomatic complexes which embrace the function of all, or a large part of, the zone of language and become simpler as time goes on, always leaving out of account secondary lesions. The explanation lies in the intimate nature of the anatomical and physiological relations subsisting between the various elements that go to make up language, a point that the writer has insisted upon. Monakow applied the term diaschisis to this relationship.

The syndrome produced by a lesion of the left inferior parietal lobulefor we are dealing for the moment only with the left side-consists of certain symptoms belonging to the attack itself and of others that only become evident after the disappearance of these. 'The attack may assume either a vertiginous or an apoplectic form. It may come on whilst the patient is in the full enjoyment of health. As a rule, the patients do not fall but are conscious of a violent stunning. In some instances, the onset of the malady is in the form of an ordinary apoplexy.

The paralysis or paresis of the right side need not be dwelt upon. If present, it soon disappears, though in some cases it persists, in varying degree, for some time. In a good number, if not in the majority, of cases, hemianæsthesia is found. Of this symptom we shall treat later on.

After the attack of vertigo, the majority of patients remain as though interdicted, they are dysorientated, know nothing and have no memory. If they were talking at the time, conversation is suddenly interrupted and they do not understand the words addressed to them. If they attempt to speak they utter only mutilated, strange, distorted words without connection. They have no clear insight into their condition. Some smile and continue to speak in unintelligible words and show no great self-concern. Others, again, have a confused notion of what has happened to them. If addressed by those around them as if nothing had occurred they reply in whispering incomprehensible language. I remember a lawyer giving me to understand, many weeks after his attack, the great surprise he felt in being no longer able to read or to understand anything that day when the attack came on whilst at work at his desk. Immediately after the onset, all patients present symptoms of complete or incomplete word-deafness, and paraphasia, but none shows any great amount of self-concern or of grief such as is generally exhibited by patients who losel the power of one side after an apoplectic attack without loss, or with only a transient clouding, of consciousness.

At the outset, the somatic complex embraces a number of symptoms that are not immediately dependent upon the part destroyed but are due to functional paralysis of areas immediately surrounding the parietal lobule or even situated at some distance away. In the motor field, e.g., we have hemiparesis of the right side, though not in all cases. This hemiparesis is 
fleeting in character and presents no special feature apart from the lesser degree of the paralysis and the slighter accentuation of the tendinous reflexes of the paralysed side, as compared with the exaggeration of the reflexes that we find in hemiplegia due to direct involvement of the rolandic system. Babinski's sign is absent and the author has never observed paralysis or paresis of the ocular muscles.

In the sensory field, tactile and muscular hemianæsthesias have been frequent, the latter being the more marked. Heat-sensibility is fairly well preserved. Astereognosis is very frequent. In the majority of cases the hemianæsthesia has been persistent.

In none of the author's patients was there any disturbance of taste or of smell.

Homonymous bilateral hemianopsia was present in almost every case, in quite classic form, for in one only was the line of separation of the blind field of vision found outside the point of fixation and in one only was it wanting.

Signs of dysorientation are hardly ever absent at the beginning. By degrees they disappear. Only one of the author's patients had complete loss of memory for places and was quite unable to make his way about when outside, so that he was obliged to have someone with him or else he had to ask passers-by the way to his home. ${ }^{1}$

At first we have all the signs of complete sensory aphasia-worddeafness (inability to understand language spoken by others), loss of the capacity to give grammatical and logical expression to ideas, mental confusion, paraphasia, amnesia of names and places, alexia and agraphia.

In the course of a few weeks or months the patient by degrees begins to re-establish normal relations with his surroundings. He still has great difficulty, however, in understanding what is said to him, but if the phrases are repeated in a loud voice he finally succeeds in grasping their meaning. As time goes on the patient improves definitely, the word-deafness diminishes and he gradually regains his power of comprehension. He formulates his thoughts better and expresses correct phrases. The paraphasia disappears, and even if the patient still distorts some words he is able to correct himself. The most persistent symptom is alexia (word-blindness) and with this there is associated a certain amount of verbal amnesia, a torpid state of intelligence, a certain degree of incoherence and not infrequently apraxia, which may last for some time.

If the patient should happen to be a highly educated person, the picture he presents from the beginning is much more serious than in the case of an illiterate or poorly educated individual. The loss at once appears enormous. The man of ready speech, clear and correct in his diction, becomes mute, stupid and void. He looks with questioning eye at bystanders, and utters strange phrases consisting of mutilated and distorted words. He fails to understand the words addressed to him or replies with dysphrasic remnants

${ }^{1}$ L. Bianchi. "Contributo clinico ed anatomo-patologico alla dottrina della cecità verbale (afasia ottica)." Ann. di Nev. 1891. 
of language. Wherever he is, he loses his bearings. Often he smiles in a stupid fashion and remains irresolute, so that it is necessary to lead him away like a lost infant.

The symptoms are incomplete word-deafness, verbal and syllabic alexia, inability to write any normal word, or at the most he may write his own name or some few words that are often deformed or written in separate syllables ; inability to write from dictation or to copy; total or partial inability to repeat words pronounced by others, and impoverished intellect without evidence of any regularly formulated thought. Right hemiopia and right hemianæsthesia are very frequent, in this, as in the preceding, group.

These patients often show marked mental confusion. As a rule, in the course of weeks or a few months, when the lesion is circumscribed, the mental condition becomes more satisfactory and the personality seems partly re-established. In those eases where there has been paralysis of the right side at the beginning of the attack, this disappears. Hemianæsthesia, unlike the hemiparesis, does not disappear entirely, though it may be much diminished.

Word-deafness also passes off, sometimes incompletely, but the patients understand most of the questions addressed to them provided these are nct too complicate nor put to them too rapidly, one after another.

Along with the word-deafness the paraphasia disappears. Sometimes, however, the patients still distort a few words, not always the same, but words suggested can be understood and repeated correctly. Within certain limits, the power of writing to dictation is regained. The other symptoms remain and there is great intellectual impoverishment.

In the illiterate, the syndrome is quite insignificant.

The following cases fell under the author's personal observation and they are summarised here because they are in many respects demonstrative and conclusive :-

Case 1.-E. G. Aged 28. Married. Butcher. Illiterate. Drank wine to excess. Mother died of apoplexy. Admitted to the elinique, 29th December 1909.

About a year and a half before, whilst at work, he had an attack of dizziness, followed by loss of conseiousness. When he came to himself, two hours afterwards, he was unable to speak and had paralysis of the limbs of the right side.

A few days after the attack he regained voluntary movement of the right lower limb, and shortly afterwards of the upper limb. He was able to return to his work as a butcher.

Objective examination elicited the following:-Diminution of tactile sensibility over all the right half of the body, slight contacts not being noticed and stronger contacts only confusedly, and badly localised. This finding was confirmed by the use of the æsthesiometer. Heat and pressure sensibilities were almost normal. Muscular sensibility and the sense of position of the limbs on the right side were markedly altered. There was no ataxia but with his eyes bandaged the patient often could not find his own hand.

The stereognostic sense was abolished, to the extent that the patient could not recognise any common object put into his hand.

Bilateral homonymous hemianopsia on the right side. All other speeial sensibilities normal. 
Visceral, genital and kinæsthetic sensibilities were normal, as was also that of space and the relative sense of orientation.

Cutaneous and mucous reflexes weak or absent on the right side and lively on the left.

Patellar and tendo-achillis reflexes somewhat exaggerated on the right side. No Babinski reaction.

No paralysis of the right lower limbs. The patient walked well. There was no paresis of the face but, when the patient laughed, facial movements appeared more limited on the right side. Paralysis of the right upper limb was present, with atrophy of the museles, especially of the trieeps, the muscles of the fore-arm and of the thenar eminence. Electrical tests showed diminution of faradic and galvanie excitability in these museles. After the cerebral attack, a polyneuritis extraneous to the cerebral disorder was superadded.

No disturbanee of speceh and no alteration of intelligence. For all purposes of life, this patient had preserved and was able to make use of the capital of thought and of langnage that he had acquired.

Neither in the elinique from the time he was admitted, nor in his own home, aecording to the statement of his wife, an intelligent woman, did he manifest any mental disorder or disturbanee of speech, for he had regained the power of speech very soon after the attack.

Apart from the paralysis of the upper limb, which followed upon a gastrointestinal febrile disorder, (which evidently caused a polyneuritis in the upper arm in an individual predisposed thereto by alcoholism) the syndrome presented by this patient, much more extensive immediately after the attack, became reduced to disturbances of sensibility on the right side, especially astereognosis and bilateral right homonymous hemianopsia. The inerease of the tendon-reflexes on the right side was a remnant of the hemiplegia from which he had suffered, a remnant which is very common after a destruetive focus located at some distance from the motor area and the corona radiata.

This foeus, given the presenee of hemianesthesia and hemianopsia, must have been situated in the inferior parietal lobule, must have extended towards the ascending parietal convolution and reached in depth to the optic radiations of Gratiolet (thalamo-ealcarine fibres).

This view of the localisation is strengthened and eonfirmed by other cases now to be reeorded, some of them followed by autopsy.

Case 2.-L. L. Aged 55. Professor of Literature in the Lyeeum. Was admitted to the elinique, 6th Novomber 1901. Family history negative. The personal history did not exclude the possibility of syphilis as a youth. On 22nd October, while conducting his elass, he was seized with vertigo and was unable to continue speaking. According to the statement of the person accompanying him, he was condueted home by one of his seholars, in an almost unconscious condition. There it was found that, along with serious disturbance of speech, there was paresis of the limbs of the right side. This paresis disappeared in the course of a few days. The confusion and disturbance of speech persisted and was so marked on the days on which he was subjected to examination that it was impossible to decide with eertainty whether or not there existed any disturbance of sensibility on the right side.

Later on, one could say that tactile sensibility was normal but even after some weeks it was still impossible to make any definite pronouncement as to the state of the stereognostic sensibility, owing to the serious disturbance of language and intelligence. Hearing was normal on both sides; not so, sight, for as soon as it was possible to examine him with the perimeter a bilateral homonymous hemianopsia with marked restriction of the visual field was detected. The motor paralysis had disappeared, so that there was no indieation of the loss of power so noticeable on the 22nd October. 
The patient was a man of culture, knew several foreign languages, a lover of Dante, and could repeat from memory a great part of the Divina Commedia, but now remembered nothing of these.

He could not repeat a single verse of the Divina Commedia, nor could he give a quotation from any of the numerous authors with whom he had been familiar. Not a word could he say of the languages he had learned. When taken into the lecture-room there was a murmur of surprise amongst the students, some of whom recognised in him their old Master of Literature. To them it was a cause of great surprise that, when asked to repeat something from Dante, he could no longer remember anything of what he had taught. He gave us, in a way, to understand how great was the misfortune that had befallen him and how he lamented the loss of his intellectual capital. The amnesia, however, embraced something more than matters of culture alone. The associative process was no longer so well ordered, so that he made mistakes and fell into dysphrasias and dyslogias. If, e.g., he was asked: "How old are you?" he replied: " $22 \ldots 23 \ldots 26 \ldots 30 \ldots 10 \ldots$. . . I could begin ...200." Perceiving he had said something foolish he then commenced to count from 1 upwards, and stopped at 55, which was the figure nearest to his age. He did not show any word-deafness for he understood all the questions put to him and that without much delay in time. When in the course of my lecture I was talking of him and of the signs and symptoms he manifested, he frequently caught the significance of what I was saying and smilingly nodded if it truly corresponded to what he felt and appreciated. He understood single questions and phrases but was unable to follow a long discourse. Although a highly eultured man, he remained there during the lecture like one in a dream, with a smile playing around his lips. When it was over, and I asked him if he knew anything of what I had been saying, he replied, or, rather, implied, with a slight shrug of the shoulders and a questioning face, that he had not understood. Here it was a case not so much of true word-deafness as of interruption of the paths of intellect or a breakdown of one of the essential mechanisms of comprehension.

Word-blindness was complete. He recognised neither words nor syllables. These, to him, had become so many hieroglyphics with hidden meaning.

Word-blindness without word-deafness persisted for some years, then slightly improved and became less complete. Alexia passed into paralexia. From time to time the patient returned to the clinique for examination and it was only after a long period and many hours of daily exercise that reading beeame less arduous work; but he forgot immediately what he read as well as what he heard. As for his writing it was greatly altered and furnished a tangible proof of the mental decadence of this poor man.

In 1905 he had improved considerably so as to be able to read and eopy. Regular and methodical examination of this"patient proved that, as he regained the function of spoken language, he also became able to write spontaneously and intelligibly. It might with reason be held that the partial restoration of the function of reading was the result of the vicarious development of the homonymous region of the right side or of the portion of the parietal lobe that had been spared by the lesion.

During all the time this patient remained under observation it was definitely ascertained that he was always affectionate towards his wife, preoccupied with his family, and had not committed any strange, incoherent or dangerous actions, if we leave out of account the first stage of his illness, during which he was dysorientated.

In this case, as in the preceding, two features stand out clearly in addition to the parietal syndrome : (1) well-preserved affectivity; (2) consciousness of the loss of intellectual capital and of a difference between the present state and that which existed prior to the attack. The patient's consciousness of his past intellectual personality and the well-preserved affectivity are characteristic, one might say, of this variety of aphasic dementia. 
Case 3.-On 4th May 1903, C. M., a priest, was sent to the Psychiatrie Clinique. In January of the same year he had had an attack of apoplexy followed by a right hemiplegia which disappeared in two months. It appears that, following upon the cerebral attack, delusions of persecution (not uncommon in aphasies in the first stage) arose, with the rosult that he went to the Police Station to make a eharge against his landlord. There it was not difficult for the offieials to recognise that he was profoundly disordered in intellect, and they had him sent to the asylum. In the reception-room he had the appearance of one stunned. He paid no heed to his new surroundings, passed rapidly from an angry to a eomposed state of mind and did his best to make us understand that ho had been robbed by his land. lord for no reason. His talk was confused, paraphasie and paraphrasic.

The examination revealed tactile, algesic, thermal, muscular and stereognostie hemianæsthesia of the right side; bilateral right homonymous hemianopsia and slight hypocophosis, more marked on the right side.

Facial movements normal on both sides. No disturbance of movements of the eyes, tongue or limbs. Muscular force was diminished, but equal on both sides, the dynamometer indicating 15 kilos with each hand and 22 kilos with both hands.

Plantar reflexes absent on the right, lively on the left side. Cremasterie and abdominal reflexes absent on hoth sides. Patellar reflex stronger on the right. Tendo-achillis reflex absent on both sides. No Babinski sign. No ankle-elonus. Gait and general behaviour quite normal, taking into account the advanced age of the prtient.

The state of intelligence ean be summed up as follows:-Patient generally exhibited a happy frame of mind, was garrulous and liked to talk with all near him. He comprehended perfeetly well the nature and uses of the various artielos around him. He recognised all the persons who attended to him, showing great regard for the doctors and especially the ehief of the elinique. He understood the questions put to him, provided they were spoken in a loud voice, in short, detached phrases. Sometimes, however, he did not understand the question but remained in an attitude of interrogation so that the question had to be repeated.

He showed that he possessed a eertain experience of life, but in speaking did not succed in expressing his thoughts fully. His talk ended by being rather dysphrasic. Closer examination led us to the eonviction that he had suffered a real loss of words correspon ling to the things he wished to $\mathrm{n}^{\mathrm{n}} \mathrm{me}$ and had before him in his mind, whilst his pronunciation of many words was so transformed and deformed that often he sought to make himself understood by means of eireumlocutions and gestures and hence he became rather incoherent.

The most marked defieit consisted especially in amnesia of proper names and names of objects, even the very commonest. This defect was rendere 1 very evident when he was asked to name various objects handed to him one after another. When told the name of an object, he sometimes was able to keep in mind for a short time a sort of phonetic image of one of the more accentuated syllables of the word, and with this he would eompose a name which bore some slight resemblance to the proper word, but, taken as a whole, was quite a new and meaningless production. Thus, he would say laps for lapsis, amello for anell, calamita for calamaio, rita for riga, inghiorno for inchiostro, callina for cartolina, etc. On other oceasions, when he was unable to pronounce the name of an object, he tried to make himself understood by indicating its use or by mentioning one or other of its main features. For example, on showing him a pen-holder, he said: "What you put a pen near. I have a lot at home." On showing him an ink-bottle, he said: "Where you put the imp or ging." On handing him a watch, he said: "I have one too, of gold. What time is it?" etc.

On showing him various objects mixed together on a table and inviting him to take up one after another by merely mentioning the names, he did so with 
promptitude and made no mistakes and knew the use of each object perfectly well. (Normal visual perceptive processes.) If, however, he was asked to tell the name of an object, he failed. As sight alone was not sufficient to reawaken the verbal image of any of these objects, he often made with his hand the movements one generally makes when using a particular object, and in this way, especially after the long exercises he was made to undergo in the clinique, he sometimes succeeded in pronouncing the names, key, ring, pen, etc.

It is important to note that the patient succecded more rapidly in pronouncing the name of an object that he took in his left hand, with eyes elosed-i.e. when he made use only of the tactile and muscular images - than he did with the eyes open, in which case visual images were associated with the tactile.

Reading was almost entirely forgotten (word-blindness). It was only after the patient had been a considerable time in the clinique that he succeeded in

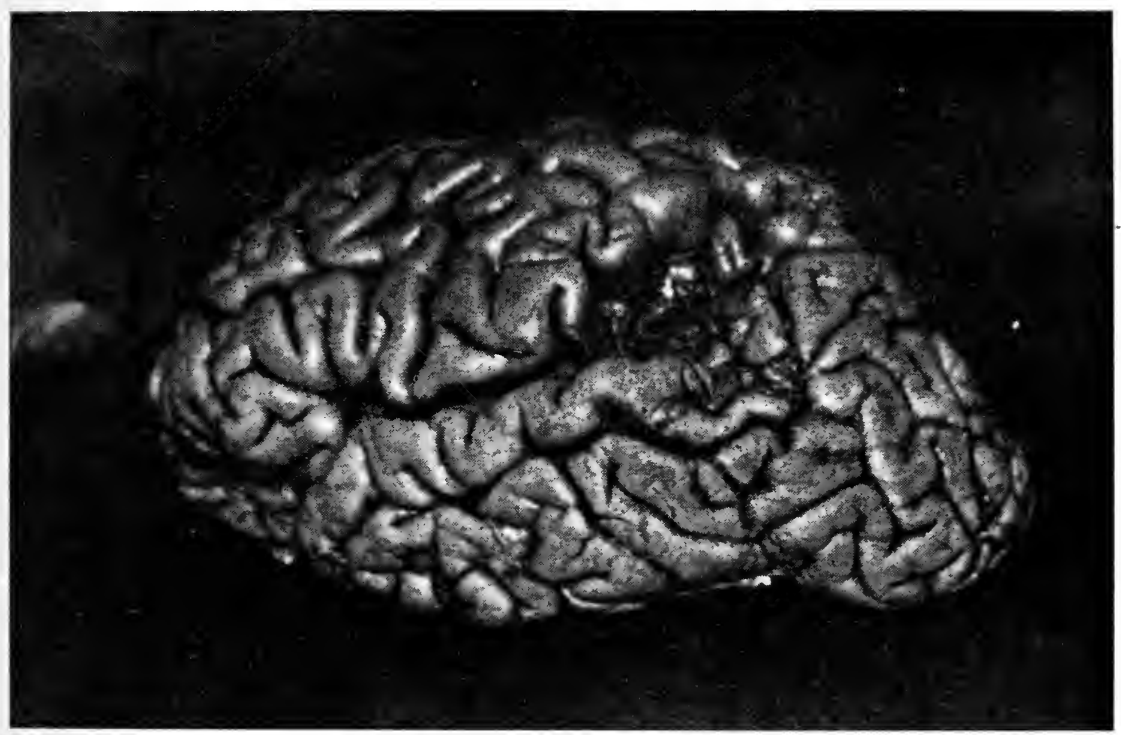

Fig. 9

recognising letters and syllables, and then a few words, but in reading aloud he substantially altered the words (paralexia) and he never quite understood the significance of any writing placed before him.

Spontaneous writing was limited to his own name, and numbers up to ten. He could not write a single phrase from dictation nor could he even copy, in a recognisable manner, the two words "provincial asylum."

He remained in this state till 17th June 1905. He was composed, correct in his behaviour, respectful and sociable, especially with the doctors. Death occurred, 19th June 1905, after an apoplectiform attack.

At the autopsy a single large focus of softening was found, along with a falling in of the cortex, in the region of the left inferior parietal lobule (Fig. 9).

The focus was limited in front by the anterior branch of the interparietal sulcus. On opening up the sulcus it was seen that the lesion extended obliquely downwards and forwards, reaching the grey substance of the posterior aspect (in the sulcus) of the ascending parietal convolution. The lesion was afterwards found to extend in depth to the corona radiata and the middle and inferior part of that convolution. Above, it was limited by the interparietal sulcus following the curve of the sulcus but remaining a few millimetres below it; behind, it reached a line drawn vertically in the direction of the prolongation of the occipito-parietal fissure. Below, it 
invaded the posterior end of the superior temporal convolution and extended backwards on to the angular gyrus.

A vertical section through the posterior part of the aseending parietal convolution shows elearly the lesion of the corona radiata of this region (Fig. 10).

Another section through the centre of the focus parallel with the first shows quite distinetly that it reaches in depth to the ventricular ependyma, interrupting the course of the optic or thalamo-cortical fibres and even those of the tapetum (Fig. 11).

Serial sections through the site of the lesion show that the posterior surface of the ascending parietal convolution is involved in the area of softening. At this same level one ean also observe the lesion of the superior temporal eonvolution.



Fig. 10

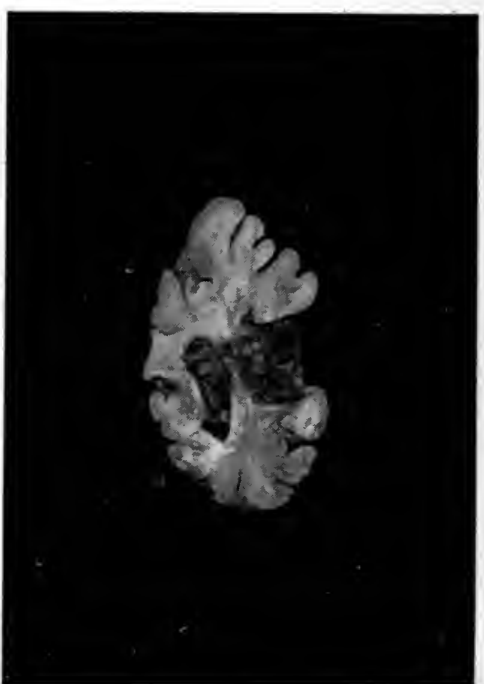

Fig 11.

It is seen to be limited to the upper portion of this convolution, the remainder of the convolution remaining sound (Fig. 12).

The lesion becomes gradually more restricted as it extends backwards, as shown by a series of sections made in the neighbourhood of the anterior limits of the visuopsychic area.

The rest of the hemisphere is normal.

To sum up, the foeus in this ease is very extensive, for it involves all the inferior parietal lobule within the limits above mentioned and also extends forwards to the ractiations of the tactile area, below to a small part of the superior temporal convolution, and in depth as far as the tapetum.

This finding fully explains symptoms observed during life. The disturbanees of tactile sensibility, of hearing, speech and sight, should not bo deseribed as dependent upon a lesion occupying an associative area in the sense of Flechsig (interruption of the paths for intercortical association). Rather should we say that the tactile disturbances depend direetly upon a lesion of the ascending parietal convolution and its eorona radiata ( $p a$, Fig. 12) (the cortical organ of tactile sensibility); the visual disorders were due to interruption of the optic radiations of Gratiolet (destroyed along the line marked E), the word-deafness that existed at the beginning and the verbal amnesia were evidently due to a partial lesion of the superior temporal convolution (Fig. 11 and $t s$ of Fig 12).

The disappearanee of the word-deafness and the general restoration of the psychic 
personality can be attributed to the fact that the superior temporal convolution was in great part spared and able, in the course of time, to resume its functions (after disappearance of the symptoms of diaschisis), whilst the word-amnesia remained, as usual, to testify to the functional weakness of the organ where wordimages are conserved and reproduced-viz. the superior temporal convolution.

Case 4.-Z. P., aged 67 years, a man of fairly good education. Admitted to tho clinique, January 1906.

In August 1905 he had had an apoplectic attack of which the only sign that remained after a few weeks was a slight dragging of the right leg, as he walked.

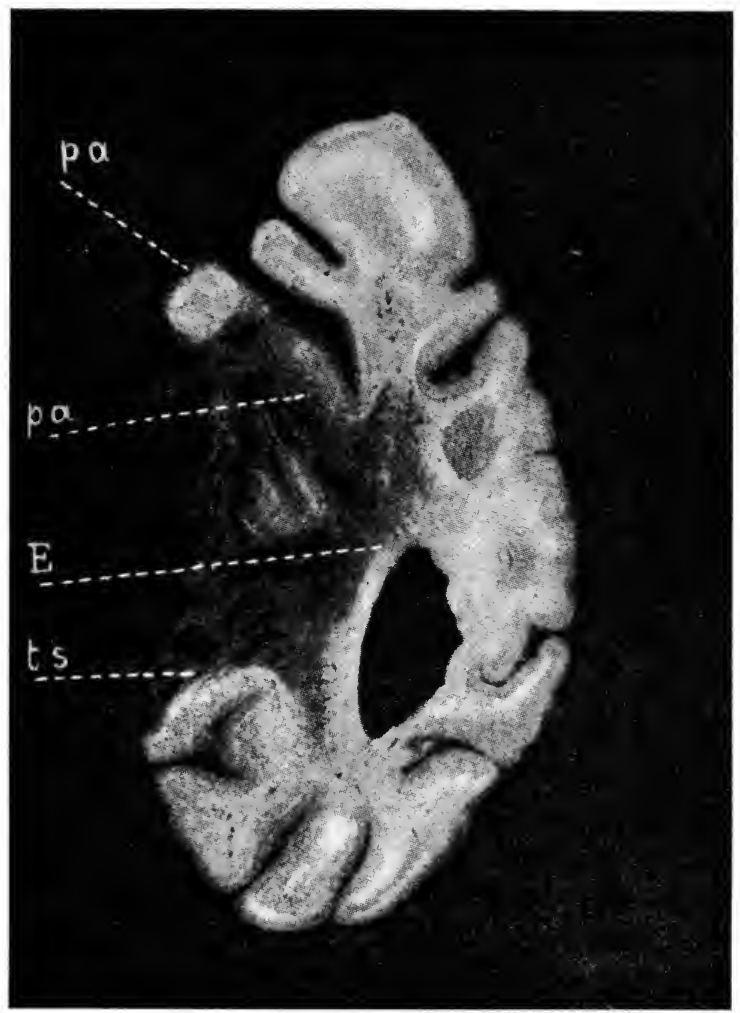

Fig. 12

pa. Ascending parietal (damaged).-E. Ependyma, with a portion of the fibres of the tapetum. - ts. Superior temporal convolution, damaged in part

Movements of the right arm appeared normal but the dynamometer-reading on the right side was $12 \mathrm{~kg}$. and on the left $17 \frac{1}{2} \mathrm{~kg}$.

The deep reflexes were exaggerated on the right side, rather weak on the left. There was also anæsthesia on the right side, not only of tactile sensibility but also of pressure and heat and at the same time diminution of the museular sense and abolition of the stereognostic sense. With eyes elosed the patient recognised none of the common objeets put into his right hand although the movements of his fingers were normal. All the special senses were normal. No bilateral homonymous hemianopsia. At the examination immediately after admission there was noted a marked mental weakness. He had no idea where he was, did not recognise it to be an asylum, and generally appeared to be very confused. 
He had lost the eapacity to read. He saw the written words quite well, but although he was able to reeognise the majority of the letters composing each word, he could on longer recognise words as a whole.

He heard and understood perfectly well words and phrases addressed to him. There was never any paraphasia. He could write only his name and surname, nothing else. He was unable to write from dictation. With great diffieulty he suceeded in copying short words, reproducing them better from the printed form, as from a design. He was quite incapable of transeribing printed letters into handwriting.

He had forgotten a remarkable number of nouns so that his speech beeame slow and he was incapable sometimes of expressing his thought owing to this defeet in voeabulary. He was dysphrasic and void. He showed no initiative in conversation with the doetors. He had difficulty in giving expression to a single thought

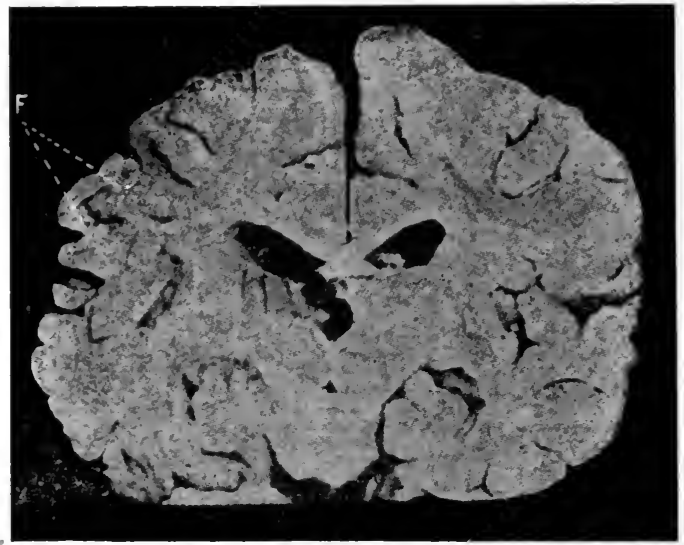

Fig. 13

F. Destructive focus

or desire and for months he remained mute yet quite eonseious of his insuffieieney and of his inability to formulate and give expression to thoughts.

This patient, when re-examined several times during the years 1906-1907, presented always the same syndrome. Towards the end of 1907, his mental eondition beeame worse and he showed symptoms of word-deafness and all the features of auditory aphasic dementia.

Dementia and word-deafness by degrees beeame very profound. Death oceurred 2nd February 1909.

The results of the post-mortem examination wore as follow :-

On external examination the hemispheres appeared normal. No foeus was visible on the cortex; only an area of depression about $3 \mathrm{~cm}$. in diameter, in the upper and anterior part of the inferior parietal lobule, near the interparietal suleus. The posterior part of the left hemisphere seemed smaller than the right, especially the temporal lobe.

There are here reproduced three transverse-vertical sections of the brain. The first section was made through the ascending parietal convolution. In its upper limits it cuts also the aseending frontal and, in its lower portion, the anterior part of the inferior parietal lobule, eorresponding to Nos. 86 and 89 of the transverse-vertical seetions deseribed by Dejerine. The left hemisphere is here seen to be smaller than the right. There is shown a focus of softening apparently limited in front by the interparietal suleus but in fact extending forwards and downwards to the radiations of the aseending parietal eonvolution. In depth it extends to a point 3 or $4 \mathrm{~mm}$. above the ependyma of the lateral ventriele. At this point 
a good part of the upper portion of the supra-marginal convolution is also involved (Lob. par. inf., Fig. 4) and there is evident injury to the tactile radiations of the ascending parietal convolution. There is no direct injury to the temporal lobe (Fig. 13).

The next section, made further behind, shows the focus to be much more circumscribed. It involves the grey substance and the radiations of the supra-

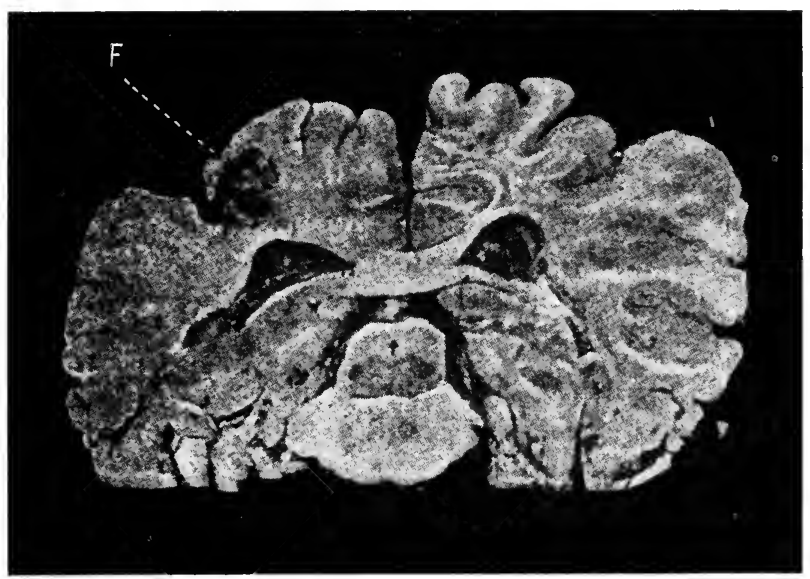

Fig. 14

marginal gyrus in its anterior superior aspect, bordering on the interparietal suleus, and dips down as a tunnel into the white substance of the ascending parietal convolution, at this point considerably removed from the temporal lobe, which nevertheless seems somewhat shrunken. The lesion reaches in depth to the centrum

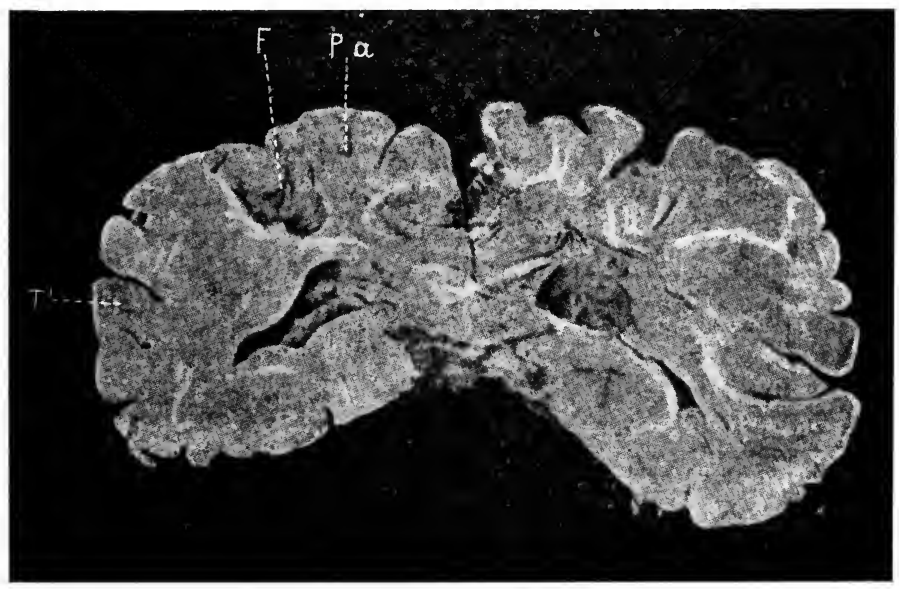

Fig. 15

ovale, to witnin a few millimetres of the ependyma, but remains much above and in front of the path of the optic radiations of Gratiolet.

The third section (Fig. 15), made a few millimetres behind the preceding, still shows the focus quite distinctly, but here it is much smaller. It involves a small tract of the radiations of the ascendin parietal convolution above and in front and the anterior-superior part of the supra-marginal gyrus behind and below. It dips down deeply into the centrum ovale but remains above and in front of the path of the radiations of Gratiolet. 
Apart from the atrophy of the left hemisphere, and partieularly of the temporal lobe, atrophy that was certainly seeondary and late, on which, too, depended the late word-deafness with symptoms of dementia, the great interest of this ease lies in the pure form of alexia along with a partieular form of dementia, the conspicuous symptoms of verbal amnesia, and the absence of hemianopsia from the onset of the attack.

The absence of word-deafness was due to the fact that the focus was at some considerable distanee from the superior temporal convolution and did not even destroy the whole of the supra-marginal.

Hemianopsia was absent beeause the focus did not extend behind and deep enough to affect the paths of the thalamo-oceipital radiations. The syndromealexia and amnesia - can only be related to the partial subeortical destruction of the supra-marginal convolution, whilst the eomplieating hemianasthesia, with loss of the stereognostie sense, must be attributed to injury to the radiations of the aseending parietal eonvolution.

It is thus proven that alexia is a symptom associated with lesions of the parietal zone independently of any lesion of the thalamo-occipital visual paths.

If lesions in the posterior part of this parietal zone produce alexia and hemianopsia (when they are deep, as in Cases 2 and 3) it is because the bundle of Gratiolet is involved. Classical hemianæsthesia and hemiagnosis are only produced when the lesion extends to the corona radiata of the ascending parietal convolution or, at least, to the anterior branch of the interparietal sulcus, because the area of tactile hemianæsthesia and agnosis is situated in the cortex of the antero-superior branch of the interparietal sulcus, and especially in the precincts of the ascending parietal convolution and its corona radiata. Again, intellectual disturbances in educated men are to be put down directly to the alexia, and hence to the lesion of the inferior parietal lobule, inasmuch as this is the visual organ of language, in educated men an organ of high value.

$$
\text { ** }
$$

From the point of view of intelligence the patients above-mentioned can be divided into two groups, one being formed of those individuals who had acquired a fair degree of culture and the other consisting of illiterate individuals or those who had learned to read and write, but had hardly cultivated those faculties to any extent. The clinical histories of some of the patients whom the author has been able to keep under observation for a long time, following them even to the post-mortem table, support this distinction. We are here dealing with different levels of mental development according to the degree of culture, and the syndrome varies in accordance with the richness or otherwise of the mental capital acquired by means of reading, and with the extent and depth of the focus.

With regard to the amnesia and the conspicuous intellectual impoverishment that are displayed as a result of more or less extensive lesions of the inferior parietal lobule it may be asked: "Are these due to the fact that this area forms a part of the language-zone or are we to account for them independently of the function of language by saying that the area in question 
forms a part of the posterior associative zone of Flechsig?" The cases above mentioned do not furnish arguments in favour of Flechsig's doctrine.

If the parietal area were the zone of passage, or the meeting-place of paths for cortical association of visual, auditory and tactile images, a sort of laboratory where images of various kinds are combined (associated) so as to furnish intellectual products of a higher order, then the symptoms dependent upon lesions of this area would be more uniform in all the patients afflicted. It is to be noted, however, that in some men, as in the priest in Case 3, even very extensive lesions give rise to symptoms that are relatively insignificant so far as the intellect is concerned whilst the functional deficit (alexia) is always of a visual order, yet, in other cases, although the lesions are comparatively slight, the loss is enormous. It is thus evident that the loss finds its explanation not in the fact that the lesion destroys a part of the brain that is anatomically and physiologically identical in all men, but in the fact that the function of that area, essentially visual, is not equally developed in all alike. If, in the case of the priest, C. M., the symptomatic complex at the beginning of the attack and a considerable time after was serious, taking into account the nature of the intellectual disturbance (extensive amnesia and confusion), that was because the lesion of the parietal lobule extended to the superior temporal convolution. This patient was word-deaf and we know that along with word-deafness, in consequence of lesions of the superior temporal convolution, we have symptoms of an imposing nature referable to the intelligence (mental confusion and, not infrequently, hallucinations and delusions). The word-deafness, indeed, was not the effect of mere solidarity of the various areas of language but was the direct result of the lesion of the auditory area of speech.

It can reasonably be held that the exemption of the greater part of the auditory zone of language from injury (vide Figs. 9, 11 and 12) explains why this was paralysed and interdicted in the first stage but was able gradually to regain its function whilst at the same time the personality and intellect became recomposed, inasmuch as many images and memories of things were able to come into the field of consciousness associated with their respective verbal images. The personality was enabled to display itself again in all the manifestations of mental life and with the old normal characteristics, though no doubt limited in potentiality and in extent.

Further, although it is true that our priest was rather ignorant and from the time of his youth had not opened a book nor read a journal except the Office which he had repeated for years in an automatic fashion, and a popular dream book by the aid of which he sought to interpret the dream that should bring him fortune, it is not at all improbable that in his case the word-visual sphere had been sufficiently exercised in his youth in the course of the studies necessarily associated with his ecclesiastic career. Had he never opened another book after he first took Mass nor again exercised his visual area, but had centralised the dynamism of language, and hence of thought, wholly or to a great extent in the auditory area alone, one could not deny that his visual area was yet to some extent more active than that of an uneducated 
and illiterate person. In this, I believe, is to be found the reason why the dementia in his case was marked at the outset and why it gradually disappeared as the auditory area, which was almost completely spared, gradually resumed its linguistic function.

This explanation does not exclude the possibility of compensation on the part, also, of the right hemisphere.

In the patient mentioned in Case 4 the syndrome was almost identical, but at no time was there ever word-deafness or paraphasia because the lesion was situated at some considerable distance from the superior temporal con. volution, which undoubtedly exercises a strong regulative influence over the motor centre of speech (wherever that may be). The alexia was absolute and word-amnesia very marked, although the focus in the inferior parietal area was much smaller. Here the explanation lies in the fact that this man, though an artisan, was a habitual reader and spent his evenings in teaching children.

If we accept the doctrine of Flechsig and take the anatomical fact of the existence of a preponderating number of associative fibres mingled and interlaced with a smaller number of projection fibres, as Dejerine afterwards demonstrated, as a proof of the high, intellectual function of this area of the cerebral cortex, we should still have to explain the very marked difference that occurs in the case of educated as compared with illiterate individuals ; and we should always expect to find the same symptomatology following destructive foci in that region, just as we expect in the case of lesions of the motor or the occipital area. The enormous difference between the effects of the large focus found in the brain of the ignorant C. M. and the very serious effects suffered by the well-read patient of Case 2 , and the comparatively minor disturbances in Case 4, must point to a functional difference which would seem to be the result of long exercise and of the differentiation of the area in question, giving rise to a special function which is altogether absent in the illiterate individual and not more than faintly outlined in those who have merely learned to read and write, but have failed to acquire a linguistic and intellectual capital by a regular course of reading.

So far as sight and touch are concerned, the difference in the symptomatic complex must be attributed to the precise site and extent of the focus, a fact of which there is ample available proof.

To the writer these observations prove that the images of the graphic symbols are formed in the evolutionary area of the visual cortical field and are reproduced there in situ. Destruction of this area renders impossible the perception, recognition (word-blindness) and reproduction of those symbols because the whole of this vast associative mechanism becomes interdicted and this applies both to the images of the various external objects that have been gradually formed in the different perceptive areas and to conceptions compounded of these. It is clear that this area becomes an associative field by a specific evolution of the visual function without on that account losing any of its characteristies as a perceptive area. What may seem a common meeting-ground and an area of interlocking, using the 
terminology of Luciani, is simply one field made up of functionally differentiated areas situated close to one another so that a destructive focus produces a syndrome that is more or less complex, dependent not only on the evolutionary dignity of the particular area or areas concerned but on the material injury to one or more of the surrounding areas or their projection-bundles, according to the site, extent and depth of the focus.

In the case of individuals who have read a great deal, one can reasonably suppose that the visual images of things-and in aphasics these persist and are perfectly capable of being recalled-reawaken the corresponding graphic images more readily than the phonetic images of these objects. It is clear that in the case of educated and cultured men the area of the graphic images may become a mechanism of great importance in the formation and movement of thought, and consequently, when this mechanism is destroyed, the intellectual capital can no longer be reproduced in the language with which it is essentially bound up, language which is the one thing indispensable for its sensible manifestation, although that intellectual capital may yet be in existence like so many deposits of constructive material. If we break the pendulum of a clock we stop the movement of the entire system of wheels, yet the mechanism of the clock remains sound and is capable of performing its work, provided a new pendulum is substituted. What happens in the case of lesions of the language-zone is quite analogous.

Just as the auditory cortical area, during the very long period that has been required for the formation of language, has been undergoing a gradual process of differentiation so as to give rise to a specialised area for the formation and conservation of the phonetic images of things and their relations, so too, in a much later epoch we have had in the case of the visual zone-i.e. in the area destined for the formation and conservation of the visual images of things - a process of differentiation giving rise to a special area where are formed, registered and conserved, the images of the graphic symbols of things and their relations. In other words, a part of the visual area becomes transformed into an organ which gives products that are analogous (because they are essentially visual) but of a higher order.

Our contention that the area of formation of the sensory symbols of thought may be an associative centre of the first order is based on the notion that the products of work of the cerebral cortex must in turn be reflected towards the sensory organ of speech inasmuch as they all tend to find their respective verbal symbol and to become fused with it. It is through the operation of this centre and by means of the graphic symbols that consciousness extends and comes into relationship with the whole of humanity. It is an indisputable fact that destruction of the language-zone does not merely suppress the register of words but puts out of gear the whole functional mechanism of the brain. Hence the very marked disorder and enfeeblement of mind associated with word-deafness and paraphasia, conditions which on post-mortem examination we find to be related to a destructive focus in the left superior temporal convolution. From this point of view, Flechsig's first conception would appear to be an unnecessary complication, incapable 
of proof, not based on clinical and anatomical findings nor yet essential for the interpretation of the mental mechanism.

Pierre Marie inclines to the view that the inability of the word-deaf to understand spoken language is a consequence of their dementia. I do not see how this can be proved, nor is there any apparent reason for the inversion of the formula.

The view that the patient who suffers from word-deafness is demented because the lesion which produces the sensory aphasic syndrome destroys a centre that is essential for the formation and flow of thought is one that is readily capable of proof. The analogy of the broken pendulum comes in here.

Now, the reasoned views we have put forwàrd with regard to the auditory area of language are equally applicable to the visual area, with this limitation, that in the former case they apply to men in general and in the latter only to some, for it must ever be borne in mind that, in the case of the illiterate, the visual area remains undifferentiated. In those in whom it has been developed, it also acts as a meeting-place for many mental products, and hence is an associative zone of inestimable intellectual value, though not in the sense of Flechsig.

$$
\text { ** }
$$

Let us now turn our attention to the temporal lobe which, with the occipital lobe and the inferior parietal lobule, completes the great temporoparieto-occipital, or posterior associative zone of Flechsig. We will pass in rapid review the really reliable observations made on this region, few in number as they are, and seek to arrive at some conclusion as to its function.

It is needless to reproduce the old, fantastic ideas concerning the function of this as of other areas of the brain. To give one example only, one might instance Burdach, who said of the temporal lobe that "It belongs to the motor activity of the mind." Again, "Its relations with the cingulum (organ of imagination), with the optic thalamus (organ of general sensibility and of will), with the anterior commissure (organ of sensible intuition and of the corporal will)," with the occipital lobe, the anterior lobe and the island of Reil, gave rise to the notion that its function was eminently psychic. ${ }^{1}$

More recently, thanks to the researches of Hitzig, Ferrier, ${ }^{2}$ of Luciani and Tamburini, ${ }^{3}$ and of Munk, ${ }^{4}$ a better founded idea of the function of the whole temporal lobe has been arrived at.

On exciting with electricity the superior temporal convolution in the monkey and in the dog, the most constant response is movement of the ear. With a stronger current there is produced, in addition, widening of the palpebral fissure, movements of the head and eyes towards the opposite side and dilatation of the opposite pupil.

${ }^{1}$ Soury. Système nerveux central. Paris, 1899.

${ }^{2}$ Ferrier. Los. cit.

${ }^{3}$ Luciani and Tamburini. Rivista Sperimentale di Freniatria. 1889.

4 Munk. Über die Functionen des Grosshirnrinde. 1890. 
The experimental destruction of this area led Ferrier, and Luciani and Tamburini, to conclude that it was the cortical centre of hearing, because this function was abolished after extensive bilateral destruction of the area concerned.

Meanwhile the syndrome of sensory aphasia (word-deafness) was becoming gradually defined and brought into relation with a lesion of the posterior part of the superior and middle temporal convolutions in man. The literature of this subject has grown enormously from the time of Wernicke, Kussmaul and Charcot, up to the present day. Experimental and clinical data now available prove clearly that the temporal lobe is the organ of hearing and comprises the organ of fundamental hearing in its acousticosensory area (the transverse temporal convolution, Fig. 5, auditory sphere), and the organ for the audition of speech, and hence for the formation and conservation of verbal and musical ${ }^{1}$ acoustic images, in the surrounding acoustico-psychic sphere, the boundaries of which are indefinite.

I. give here several unpublished observations that are useful in helping us to form a notion of the physio-pathology of the temporal lobe. The scope of this volume does not permit a full review of all that is to be found in the literature of the subject.

I have no intention of dealing with destructive foci limited to the language zone (posterior parts of the superior and middle temporal convolutions, ZU, Fig. 1 and T1, Fig. 4). The symptomatology of this area coincides with the story of sensory aphasia (word-deafness) concerning which there is a very rich literature in all languages. Small foci, occurring not in Wernicke's zone (temporal section) but in a more or less distant point of the same temporal lobe may reveal themselves in the symptomatic complex of the so-called amnesic aphasia. This, however, is due not to destruction but rather to functional paresis of the area of aphasia owing to a lesion in its neighbourhood (a small focus or commencing tumour ${ }^{2}$ ).

Case 5. - A man of about forty years was admitted to the asylum in a state of advanced dementia. Repeated observations revealed the existence of complete word-deafness with suppression of all manifestations of thought either spontaneous or induced. The patient did not reply to a single question. There was complete mental arrest so far as one could judge from the entire absence of any form of language. There was no sign of paralysis. There was blindness with choked dise and secondary neuro-retinitis. He remained in this state for about fifteen days, when pulmonary complications set in, followed by death. On post-mortem examination, the whole left temporal lobe from the sphenoidal extremity to the occipital pole was found transformed into a hard mass which had no longer the appearance of cerebral tissue. On section the new growth was seen to be of a sareomatous nature.

Case 6.-A woman of seventy years was admitted into the asylum suffering from severe dementia. There was no physical sign of function aijl distrap bance in the field of sensibility, neither was there disturbance moveineni in any par tof the body nor alteration of the reflexes. Theses well-marked deafness on she

${ }^{1}$ Ingenieros. Le langage musical. Paris, 199 \% . .

2 Bianchi. "L'afasia amnesica." Ann." di Nev. 1914. 
right side. It was impossible to learn whether or not she had suffered from an ordinary apoplectic attack. The mental examination revealed very severe worddeafness. She did not understand a single word addressed to her. Her attention could be attracted by ealling her in a loud voice or tapping her with the hand, but she very rapidly relapsed into unintelligible talk. She chattered constantly but few of her words had any resemblance to those found in the vocabulary of the Italian language. They were almost entirely fragments of words or words quite altered in structure (paraphasia). Never a phrase or proposition did we hear formulated by her on grammatical lines. She called things by the most extravagant names, composed. of a confused englomeration of syllables. There was no sueh thing as grammatical inflexion; at most, her talk consisted of a few distorted words and these were pronouneed ad infinitum. To sum up, we had here worddeafness, annesic aphasia, paraphasia, akataphasia, dysphrasia and alogia. She

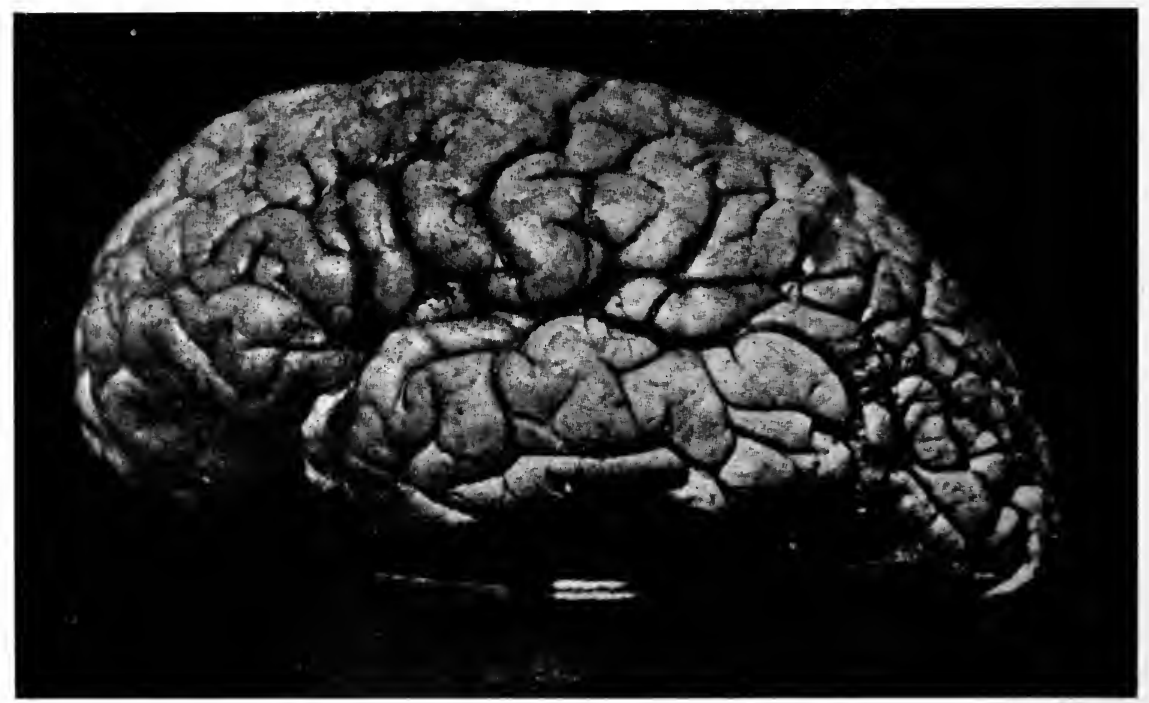

Fig. 16

showed indifference towards everyore and everything and was sometimes filthy in her habits (aphasie dementia).

It so happened that there was another woman of the same age in the elinique at the same time. She too was illiterate, and displayed similar symptoms, though less severe. 'This patient died and on post-mortem examination we found a large destructive foeus involving the posterior half of the superior and middle temporal convolutions and a good part of the inferior parietal lobule. I was therefore induced to make a similar diagnosis in the present case-i.e. a large destructive focus in the zone of Wernicke. The patient died from pulmonary complieations.

Post-mortem examination revealed the existence of very advanced primary sclerotic atrophy of the whole left temporal lobe, which had become reduced to little more than half the size of the right temporal lobe. Histological examination revealed the presence of a magnificent growth of neuroglia, with dense network and bundles of fibres, atrophy and disappearance of the nerve-cells. ${ }^{1}$

Case i- w wortan of forty-eight. Her illness commenced with attacks of rertigo" and confusion. "Thón carne an attack of classical epilepsy, which com-

1 Frańmeschí." "Giliosi-porivasiolare, in un caso di demenza afasica. Annali ci Necrolsyia: ! 908 
menced in the right side. After the lapse of two months there was another epileptic attack, and this was followed by headache, localised in the left side of the head, and by a fleeting disturbance of speech. Later on there was paresis of the right

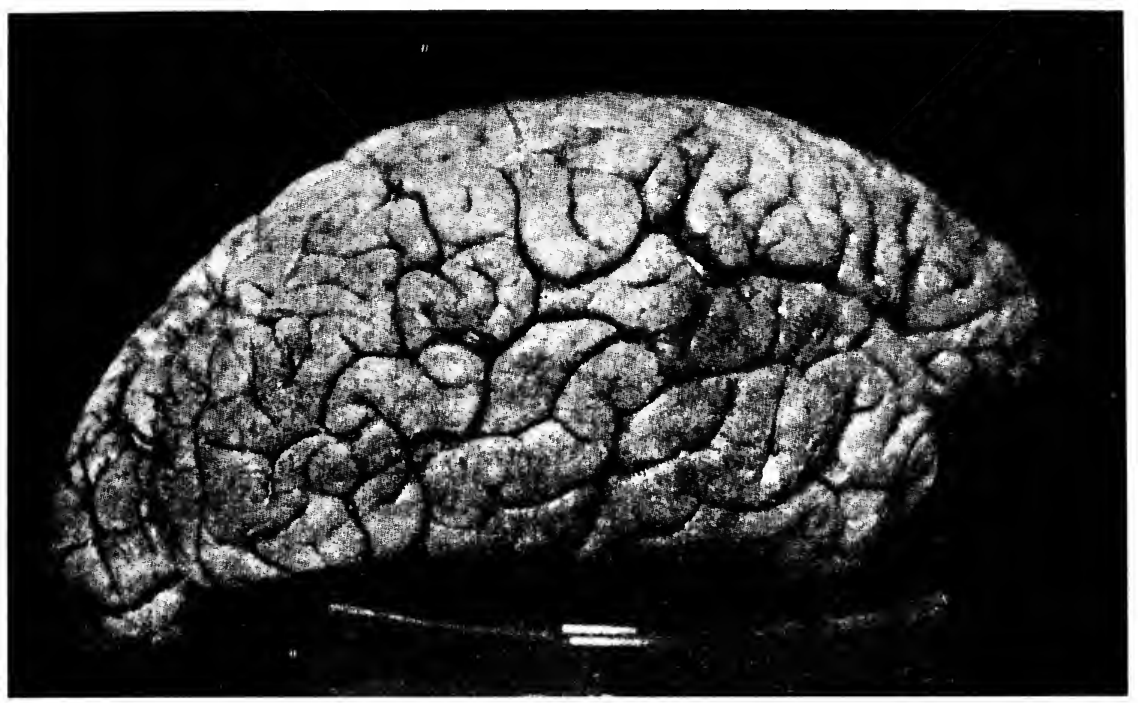

Fig. 17

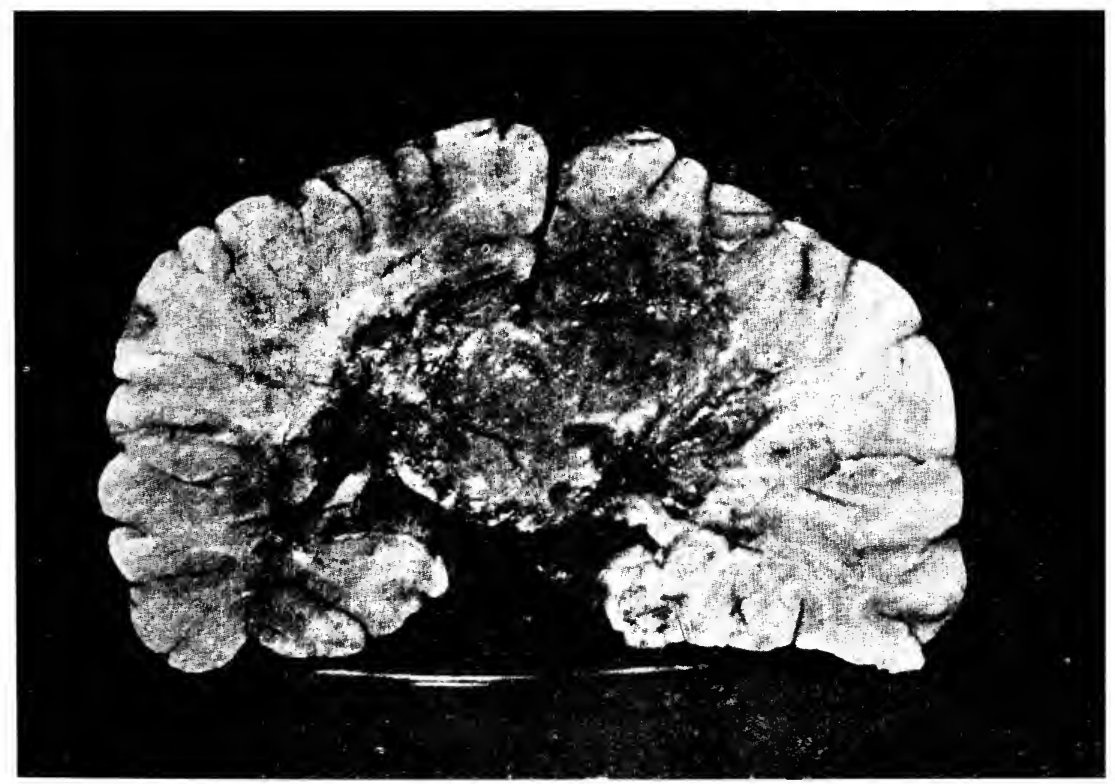

Fig. 18

side, which afterwards developed into hemiplegia whilst, at the same time, a state of dementia developed and became very severe in the eourse of a few weeks. She showed great diffieulty in eomprehending words and phrases spoken by others. There was amnesia not only of words but of things, persons and events. Visual disturbances supersened, and later on complete blindness, with choked dise. The 
dementia made such rapid progress that, with the physical blindness and the worddeafness, the patient lost all relations with her surroundings. Difficulty in deglu. tition set in. There was conjugate deviation of the head and eyes. Speech was entirely absent. The patient did not understand a single word addressed to her. Sometimes she would turn her head slowly towards the side of the speaker if he addressed her repeatedly in a loud voice, or she would open her eyes, or groan, or give a ery.

On post-mortem examination, the left superior temporal convolution was found to be about half the breadth of that of the right side. Measured in the same

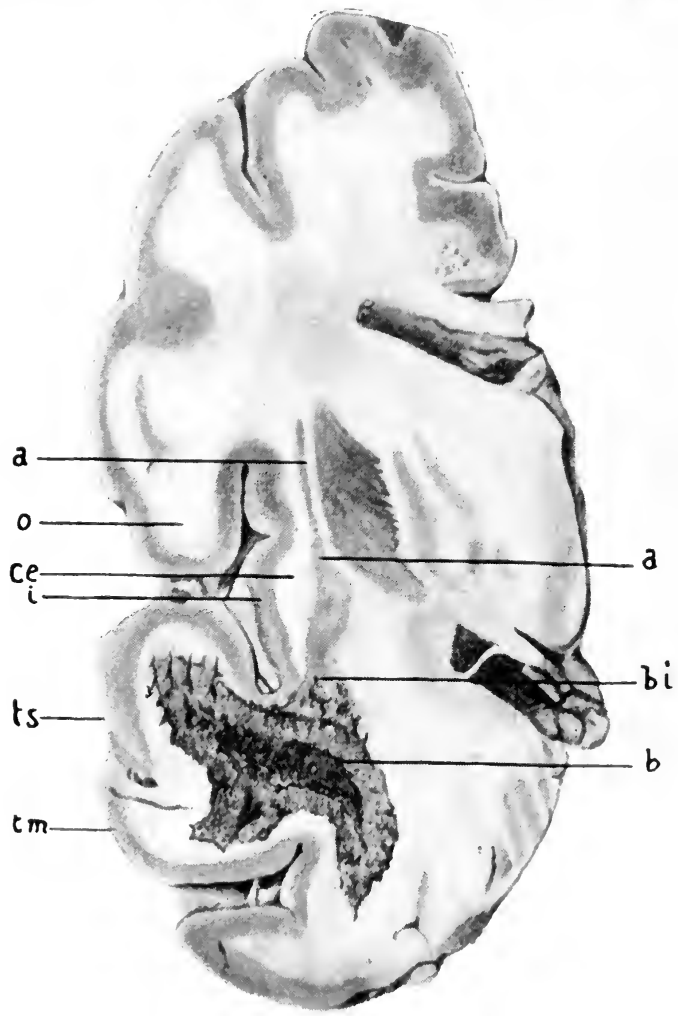

Fig. 19 (cross-section)

a. Claustrum. - o. Operculum. - ce. Ontermost capsule.- $i$. Island of Reil.-bi. Limits of focus in neighbourhood of Island of Reil. - b. Focus. $-t s$. Superior temporal convolution. $-t m$. Middle temporal convolution

vertieal plane, the height of the left temporal lobe was $45 \mathrm{~mm}$., and of the right $50 \mathrm{~mm}$. (Figs. 16 and 17). After immersion of the brain in fixing fluid, parallel antero-posterior sections were made and a large cancerous mass was found occupying the position of the corpus callosum, no trace of which remained. The mass had grown particularly towards the left, in a direction outwards and backwards, destroying first the sensory and motor paths then all the basal ganglia (corpus striatum and optie thalamus), and infiltrating behind into the corona radiata of the upper two temporal convolutions (Fig. 18). The last-mentioned finding explained the atrophy of the superior temporal convolution, the word-deafness and the severity of the dementia as eompared with what has been found in other eases of tumour of the corpus eallosum. ${ }^{1}$

${ }^{1}$ Bianchi. Atti dell'Accademia medico-chirurgica di Napoli. 1915. 


\section{EVOLUTION OF THE NERVOUS SYSTEM}

Case 8.1-C. D. was admitted to the asylum on 1st March 1894, and died there fourteen days later. From the time of admission till his death he could not express a single thought in proper words.

His language was reduced to a veritable jumble of words, these being quite wasted, broken and distorted. His words were composed in strange and varying fashion, new syllables and various vocal sounds being introduced and letters added here and there without order. For example, he would say "onno managgio" for "voglio mangiare," etc. He seemed to be in despair because unable to express his own thoughts, and to be conscious of the mistakes he made. He tried to correct

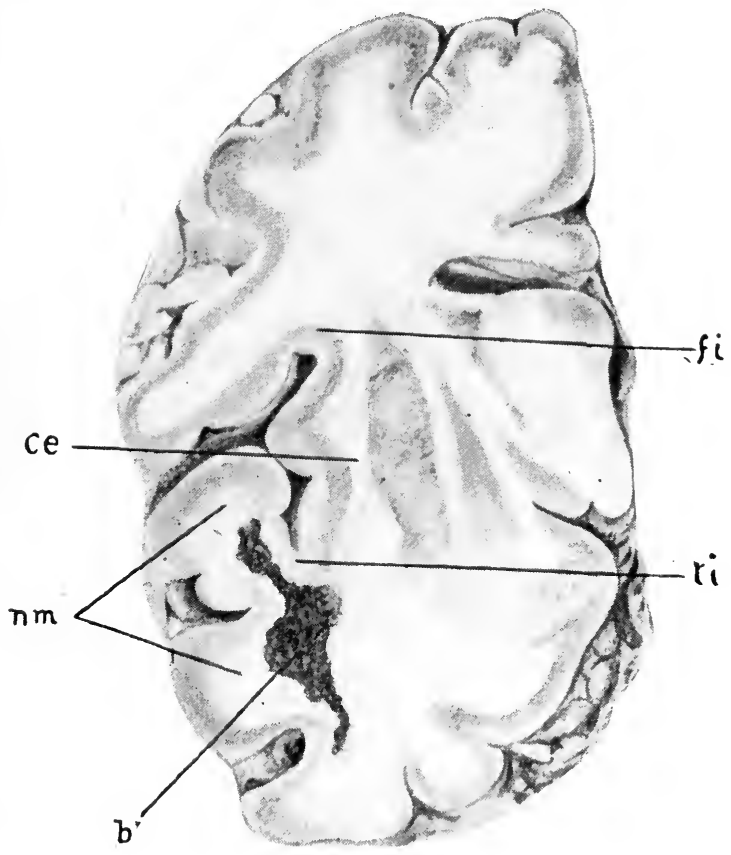

Fig. 20

fi. Fronto-insular angle.-ce. External capsule. $-n m$. Corona radiata of the two superior temporal convolutions, the tibres of which must pass by $t i$ and $f i$, through the outermost capsule and the external capsule, ce

himself, only to fall into worse error. He retained, however, a certain power of mimicry corresponding to his thoughts and emotions, and so one was able to some extent to decipher his jargon and have it confirmed by him. He would try to say: "I am very distressed because I eannot speak," and many were his exclama. tions of despair at his futile attempts.

He understood the brief questions that were put to him, and when told once or twice to make a certain movement, did so. Sometimes he made use of normal words, but they were out of place and quite incoherent. Once when asked: "What is wrong with you?" he replied: "First of first I have done." To : "How old are you?" he replied: "What you want! Yes! Four days!" Another man who lay opposite him used to address him in a disdainful manner. Our patient, listening, would frown at him, then give vent to an oath very common among Neapolitans, his voice and pronunciation in this case being decided and correct (as often happens with aphasics).

"Bianchi. "Trattato delle malattie del eervello." Patologia, by Cantani and Maragliano. Vol. ii., parte 2. 
He recognised letters, syllables and words, but jumbled them in reading almost as he did in speaking.

He could write spontaneously but rather slowly, in a large and ehildish hand, but the words were distorted. He could copy very well.

At the post-mortem examination an old focus of white softening was found in the left hemisphere. The whole of the anterior part of the temporo-sphenoidal lobe resembled a sack filled with a milky, whitish, pultaceous material which escaped on making a fronto-peduncular section of the brain, this section going through the anterior extremity of the temporo-sphenoidal lobe.

The next section was made through the line of junction of the frontal convolutions with the ascending frontal, and is represented by Fig. 19. The space $b$, from which the liquid material has escaped, is seen to occupy the position of the white substanee of the anterior extremities of the first, seeond and part of the third temporal eonvolutions. It extends considerably inwards and also upwards

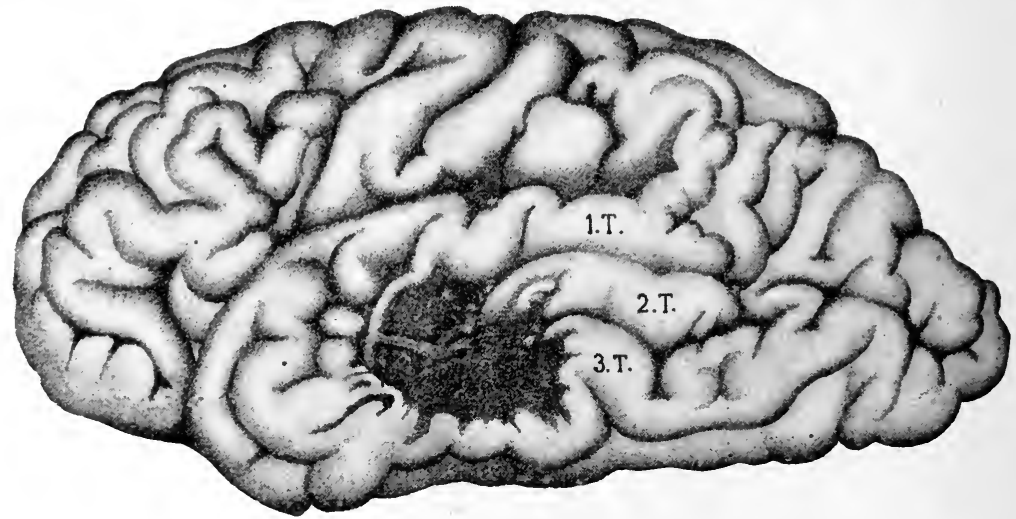

Fig. 21-Left hemisphere

1.T. Superior temporal convolution.-2.T. Middle.--3.T. Inferior temporal convolution.$y$. Destructive focus

to a point close to the white substance of the insula $b i$, so that the long stretch of fibres running from the temporal lobe to the insula is destroyed.

On making another vertical section, one centimetre behind the former but still in front of the supposed anterior limit of the auditory area of speech in the superior temporal convolution (Fig. 20), the position of the focus of softening $(b)$ is seen to be such that it interrupts the nervous paths leading from the superior and middle temporal convolutions to the frontal operculum. The focus, in fact, runs in a longitudinal or antero-posterior direction for half the length of the first and second temporal convolutions and extends inwards into the white substance of the temporal lobe, so that all communication between the latter and the operculum of the ascending frontal and the inferior frontal convolutions must have been destroyed.

The fibres $\mathrm{nm}$ ean only run through a certain tract in the two temporal convolutions, gradually leaving which they curve round the temporo-insular suleus $t i$, passing into the grey substance of the insula or into the outermost eapsule (ce, Fig. 19), some perhaps into the external eapsule (ce, Fig. 20). They then bend round the fronto-insular suleus $f i$ to reach the foot of the inferior frontal convolution, represented in the figure by the anterior part of the Rolandic operculum, the foot of the frontal convolution being in front of the section.

The posterior limit of the focus of softening remains in front of the posterior third of the temporal convolutions. This localisation affords an explanation of the clinical picture. The patient heard and understood because the auditory centre was sufficiently sound and in relation with the peripheral organs and the subcortical stations of hearing and also with a good part of the rest of the cerebral cortex, 
so that intelligence was in great part preserved. He could articulate syllables and words because the kinæsthetic centre of spoken speech was untouched. $\mathrm{He}$ could copy because the visuo-cheiro-kinæsthetic (Bastian) and audito-cheirokinæsthetic paths, as well as the corresponding centres, were undamaged. Classical paraphasia and paragraphia were present because the motor centres of spoken and of written language respectively were withdrawn from the control of the acousticosensory centres. The lesion disturbed the whole linguistic mechanism by reason of the interruption of the associative paths between the sensory centre and the motor centre of spoken language (in $b i$, Fig. 19 and $t i$, Fig. 20), and also by suppression of the grammatical inflexions. Indeed, the most important feature was the dysphrasia and the alogia, closely related as these were with the absolute in. capacity for the inflexion of verbs and the intuition and expression of the tenses.

Case 9. ${ }^{1}$-P. G., born 1863, subject to convulsions from infancy, associated with a nervous temperament and a distinctly epileptic character.

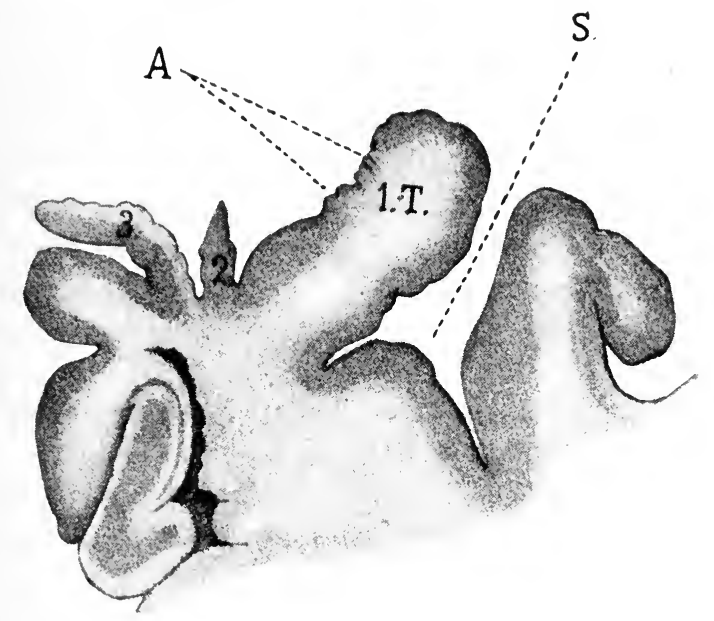

Fig 22. - Vertical section through the centre of the destructive focus

S. Fissure of Sylvius. - 1.T. Superior temporal. - 2. Middle temporal.-
3. Inferior temporal convolution (almost entirely destroyed).-
A. Iesion of grey substance of superior temporal convolution

He had learned no trade but, being obliged to earn his own living, found employment as a domestic. He was fortunate in finding mistresses who put up with him and he was able to get along for a time because his convulsions were not frequent, and he passed as quiet and respectable though really captious and turbulent. In the course of time, his convulsions became more frequent and were followed by mental disturbances that became more prolonged and more severe. His psychic life, abnormal to begin with, now underwent a process of constant deterioration, and at last he had to be sent for eare and treatment to the asylum.

In the later stages of his trouble his convulsions became exceptionally severe, and were followed by agitation and delirium lasting for several days. When the post-convulsive confusion passed off he talked constantly. Sometimes, indeed, he was very talkative immediately after the convulsion. The rapid deterioration of his already weak intellect and the meekness that took the place of his former impulsiveness were remarkable features of the last stages. The clinical picture was the very common one of the epileptic character with recurrent accessions of

${ }^{1} \mathrm{~L}$. Bianchi. "Su di un caso di lesione destruttiva del lobo temporosfenoidale sinistro." La Psichiatria, etc. 1888. 
epileptic psychosis, ending in post-epileptic dementia. Death followed upon a convulsion at a time when he was a physical wreek.

A post-mortem examination was made. In addition to pulmonary and eardiae lesions and senile changes, an old apoplectic cyst was found in the left hemisphere, exactly in the middle part of the temporo-sphenoidal lobe little more than $4 \mathrm{~cm}$. in length and $3 \mathrm{~cm}$. in breadth (Fig. 21). The second temporal convolution was completely destroyed, along with the neighbouring part of the grey substance of the superior convolution in the superior temporal sulcus. The greater portion of the inferior temporal convolution was also destroyed, only a narrow strip of grey substance remaining at its lower edge.

Fig 22. represents a vertical section through the eyst in the temporal lobe and shows elearly the extent and depth of the lesion.

No doubt a lesion such as that met with in the case above described may well have been expected to have given rise during life to particular symptoms of dementia, because it did damage to a conspicuous portion of the posterior zone of Flechsig both on the surface and in its depth. Yet the fact remains that classic aphasic dementia did not exist, very probably because the seat of the focus was in front of and below Wernicke's zone and wholly in the posterior associative zone of Flechsig. Not even verbal amnesia was present.

In the light of Flechsig's doctrine we ought to have found complex disturbances of intelligence in this as in the previous case. On the contrary, the dementia exhibited by this patient was late in appearance and was not attributable to that old cyst. It had all the features of classic dementia described by psychiatrists as post-epileptic dementia, common enough in all asylums-i.e. an enfeeblement of mind in toto, superimposed upon the purely epileptic character such as the patient had shown from his early youth. The patient was left-handed, so that the absence of any disturbance of speech could be attributed to the fact that he spoke with his right hemisphere. But this very fact goes to prove that the particular intellectual disturbances that accompany lesions of the temporal lobe are in relation with the physiopathological mechanism of language, a function which in this case was discharged by the right hemisphere. In other words, they depend upon destruction of the archives of the word-images (and hence of the vocabulary) formed and conserved in loco and in anatomico-physiological relation with the whole sensory cortex, and upon damage to the grammatical mechanism which would appear, from the facts at our disposal, to be located in that region.

In some cases of tumour of the temporal lobe reported by Kennedy ${ }^{1}$ the most important symptoms met with were some alterations of language, frequent amongst these being inability to recall the names of objects. In other cases the aphasic syndrome was more or less conspicuous.

The paraphasia observed in some of these patients is often due to the defective representation of substantive names which are necessary for the formation of phrases and propositions.

Paralogia, like dysphrasia, depends partly upon amnesia of nouns and verbs, which causes the patients to lose the thread of their discourse and to

${ }^{1}$ Kennedy. "The Symptomatology of Temporo-Sphenoidal Tumours. Archives of Internal Medicine. 1911. 
give utterance to phrases that have nothing to do with the premises, and partly upon the lack of ability to give words their proper inflexion. Agrammatism is a frequent condition in those who suffer from word-deafness but it also is due to deficient grammatical inflexion of verbs. There is reason to believe that this grammatical deficiency has a considerable influence upon the orientation of consciousness in time, because, after all, grammatical inflexions correspond to a mental disposition which transports the actual moment of the psychic process into the past and into the future.

Intuitions of time; the duration of periods of time and rhythm, are intimately bound up with hearing and with auditory perceptions. Indeed this function, in addition to constituting a part of the mental patrimony, furnishes ontogenetic experimental data in the evolution of the personality for abstract intuitions of time, for the orientation of the person in time and for the grammatical inflexions which express the corresponding psychic situations.

It is apparent that the temporal lobe contributes largely to the formation and development of thought, but its contribution is very different from that provided respectively by the occipital lobe and the inferior parietal lobule and it is of an exclusively acoustico-perceptive character.

The great variety of symptoms and the differences in the clinical pictures met with as the result of destructive lesions of the inferior parietal lobule and of the temporal lobe do not harmonise with the notion of functional uniformity, such as has been ascribed, more or less, to the great posterior associative zone. We are probably much nearer the truth if, like Bohn, we say that many regions of the brain, and especially those that surround the bottom of the fissure of Sylvius, are new and unexplored regions, full of possibilities for the future, regions which one day will be the centre of a marvellous human advancement. That Flechsig attributes a single function to this zone, as a whole, can also be inferred from Hitzig's communication to the Congress at Paris. ${ }^{1}$

In formulating his conclusions regarding the great posterior associationcentre Hitzig wrote as follows:- "Flechsig regards it as the organ which forms and re-unites the representations of external objects and the phonetic images of words and their combinations, and if this be so, it would therefore be the organ of positive cognition properly so called, as well as the organ of imaginative activity of representation, the organ for the preparation of speech according to the thought-content and the organ of verbal form-in short, the organ of the most essential elements of what in ordinary language is specially designated as thought." 2

This, however, is surely a remodelled interpretation of Flechsig's idea. It agrees, in part, with the view the author put forward very explicitly in a communication to the Congress at Madrid, ${ }^{3}$ when, in dealing with disturbances of intellect following upon lesions of the region in question, he

${ }^{1}$ Hitzig. "Les eentres de projeetion et les eentres d'association," ete. Névraxe, vol. $\mathrm{i}$.

${ }^{2}$ Hitzig. Loc. cit.

${ }^{3}$ Bianchi. Loc. cit. 
maintained that these were purely aphasic disorders. Yet Flechsig adds that it is the organ of positive cognition-which, on the contrary, has its organs in the sensory zones-and of the imaginative activity, thus reaffirming the old conception, which we will deal with in the following chapters.

Lesions of the temporal lobe do not give rise to disturbances of movement nor do they cause disorders of tactile and muscular sensibility. In all those cases in which the lesions were limited to the temporal lobe, though at the same time fairly extensive, tactile sensibility was found to be normal. These lesions do not produce visual disturbances unless they extend in depth to the optic paths of Gratiolet. The visual and tactile areas always furnish their normal products (positive cognition), with this difference, that they cannot combine with their respective words, the register of which is destroyed, a fact which explains the special behaviour of the intelligence in aphasics.

The temporal lobe contributes to the formation and manifestation of intellect not only by means of the simple auditory images, which represent one of the modes by which the world is revealed to us, but especially by means of language. It is by means of language that we are enabled to recompose in our minds our world of cognitions, along with the ever-growing and manifold network of our social relations, which offer an ever-increasing scope for the exercise and development of intellect.

When we speak of language we mean language with all its inflexions, and these are intellectual in character. Undoubtedly language exercises an enormous influence upon the structure and power of intellect.

$$
\text { ** }
$$

The various questions we have thus far dealt with have a distinct bearing upon the fundamental problem which it is proposed to elucidate in this volume.

One cannot arrive at a clear conception of the function of the frontal lobe unless one knows something of the contribution rendered by other definite areas of the brain and unless one is, at the same time, conversant with all the facts that hitherto have been ascertained regarding the complex and intricate function of the cerebral cortex. Proceeding along these lines one may hope at least to lay the foundation of a more promising anatomical psychology, more promising because founded on facts, for in the past it has suffered from the prevalence of conceptions that have been rather one-sided.

$$
*^{*} *
$$

We come now to the motor area. If it is to be considered as a projectionzone one can readily understand that it must receive all the nerve-waves from the tactile and the other sensory areas so as to render possible the relative reactions upon the external world. It is to the cortex of the brain what the anterior cornua are to the posterior cornua and the sensory paths of the spinal medulla. In each case centripetal waves arrive at the motor centres and after traversing these are reflected upon the muscular apparatus. The fibres of intercommunication between the tactile area (ascending 
parietal) and the motor area (ascending frontal) are exceedingly numerous. As compared with the sub-cortical and medullary motor centres we have here a difference only in the degree, dignity and number of the components. The motor area refleets upon the external world the number and nature of the single or eombined psycho-sensory products which excite it.

It is always the first to be myelinised and it is marked No. 1 in Flechsig's scheme. There is evidence to show that the fibres first myelinised are not the only ones which arrive at the motor zone, so that this area really serves as an associative area of considerable value.

At the front of the motor zone, almost forming a part of it, there is an area which extends towards the bases of the frontal convolutions and is

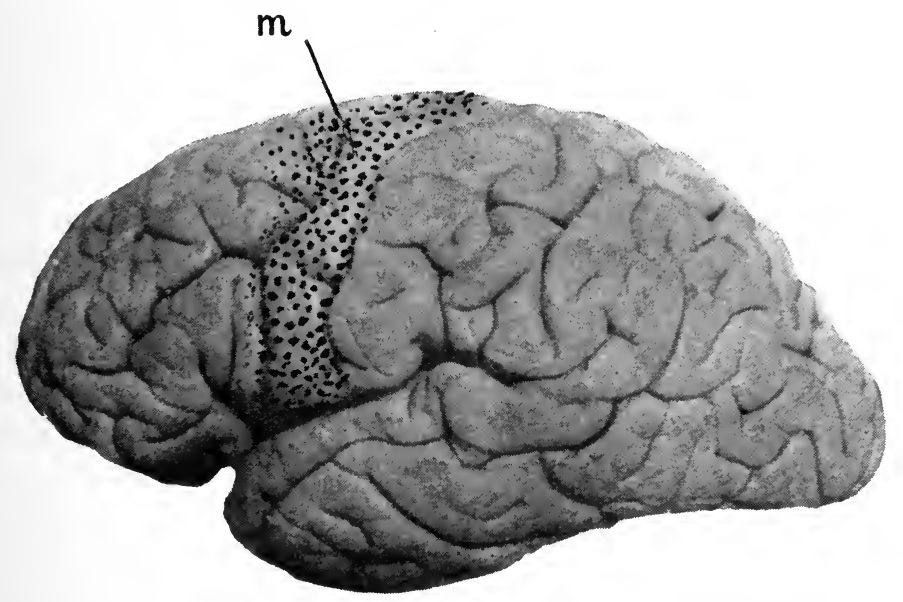

Fig. 23

m. Motor area

regarded by some authors as an integral part of the motor area. De Boyer and Pitres ${ }^{1}$ and, generally speaking, Charcot's sehool regard the motor zone as extending somewhat in front of the aseending frontal convolution.

Several reliable observations go to prove that this narrow zone of extension and evolution of the motor area, and in particular the middle portion of it, serves as the field of extension of the motor area of the upper limb for the complex movements involved in particular professions, such as writing. The histological researehes of Campbell, Bolton, Brodmann and Flechsig go to confirm this view. Further confirmation has been afforded by physiological and pathological investigations (Chapter III., intermediate motor zone). Indeed, it would seem that any new work, if intense and prolonged, leads not only to the perfecting of the primary function but to a further extension of the functioning surface of the cortex. Doubts have been thrown upon the existence of a centre for writing, especially by Dejerine and Mirallie, ${ }^{2}$ but it is probable that prolonged practice, such as is involved

${ }^{1}$ De Boyer and Pitres. Études topographiques sur les lésions corticales des hémisphères cérébraux. 1879.

${ }^{2}$ Dejerine and Mirallie. De l'aphasie sensorielle. 1896. 
in the automatism of writing with the right hand or the automatism of the violinist or the pianist, must bring about the formation of a centre or cortical area adapted for a particular co-ordination of movements, a centre which is distinct from that of the ordinary movements of the upper limb, movements involving, e.g., prehension, defensive actions and the grosser occupations directed towards the preservation of the individual and towards industrial production.

No doubt one can write with the left hand, or with a pen held in the mouth or with the foot, or fixed even on the trunk of the body. These are facts that have been amply demonstrated by the results of re-education anongst those who have suffered loss of limbs during the great war. They prove, at most, that new motor co-ordinations are possible in all muscular fields as the result of exercise, but they do not warrant us in excluding the idea that new work and new adaptations may enlarge the motor field and that new functional co-ordinations, once they become strongly constituted by repeated exercise, may represent a differentiation of labour in the anatomical field concerned.

The physiological researches of Sherrington and Grünbaum, ${ }^{1}$ of Sherrington, Mott and E. Schuster, ${ }^{2}$ agree with the cytotectural investigations of the above-mentioned histologists in this respect that they localise the area of general motility in the ascending frontal convolution, this area being distinctly limited behind by the fissure of Rolando, but not well defined in front. In the gibbon, which makes use of its arms and hands, and moves about by preference on its posterior limbs, experimental excitation of the motor area has shown a displacement downwards and forwards of the area for the movements of the body and especially of the eyes. On the other hand, the motor area for the movements of flexion and rotation of the wrist extends to the foot of the middle frontal convolution. Histological investigation has confirmed the existence of the precentral intermediate area of Campbell and of the areas defined by Brodmann; but the experimental investigations above mentioned go to show that, in the gibbon, the intermediate area extends forwards to some considerable extent, both on the external and on the mesial surface of the hemisphere, so that the prefrontal area would in this case be reduced to a comparatively small region of the frontal pole. Then again, if we accept the findings of Brodmann and Campbell, the frontal area, properly so called, becomes similarly much reduced, to the advantage of the intermediate precentral area. These experiments of Sherrington, Mott and Schuster give rise to considerable doubt because, if the initial use of the hands brings about such an extension of the intermediate motor area in the gibbon, this area should be much more extensive in the case of man. In this connection, the conclusions arrived at by Pitres and De Boyer as the result of clinical observations seem more consistent. These authors limit the extension of the motor area in man

1 Grünbaum. Loc. cit.

2 Schuster. "Motor Localisations in the Brain of the Gibbon, correlated with Histological Examination." Proc. Roy. Soc. 1911. 
to the bases of the frontal convolutions. The writer's personal observations, which led him to conclude for the existence of an evolutionary motor zone, ${ }^{1}$ and the observations made by surgeons on living patients (such as those of Kocher, ${ }^{2}$ who agrees that all the motor points with the exception of those of the thumb and of the shoulder are to be found on the anterior Rolandic convolution and concludes that the chief point of rendezvous in the motor region must be the precentral sulcus), warrant us in admitting a forward extension of the motor zone.

This argument certainly applies to the foot of the inferior frontal convolution, which must still be regarded as the motor zone of spoken language, notwithstanding the revolutionary doctrine of Pierre Marie ; it is unnecessary from this point of view to make any distinction between the functions of the two hemispheres. This area is placed immediately in front of the motor zone for the movements of the lips, face, tongue, larynx, etc., in fronti.e. of the area for all those groups of muscles employed in mastication, deglutition, whistling, shouting, etc.

The writer puts forwards the hypothesis that the centre of writing, about which there has been so much discussion, stands in relation to that of the arm, for movements of prehension and defence, just as the centre of articulate speech stands in relation to the centres for mastication, phonation, the facial and lingual muscles, etc. Just as the anterior portion of the visual area can be regarded as exhibiting a much higher grade of evolution as compared with the rest of the visual zone, so the pre-Rolandic intermediate area, considered as a part of the motor zone, may be regarded (even provisionally) as an evolutionary motor zone, in the sense that it represents the field of extrinsication of motor activities of a higher, more spiritual order, in men of a more advanced type. To-day, notwithstanding Marie's brilliant criticism of the old doctrine, one can hold firmly to the idea that the motor area of articulate speech is found on the foot of the inferior frontal convolution and its neighbourhood, as, e.g., the anterior part of the insula. I hold that these are areas in course of evolution and they are not differentiated alike in all men nor do they give fibres directly to the pyramidal tract.

In writing we make use of the same limbs and the same muscles as in the coarser and more elementary movements requisite in the manifold needs of life. The more elementary movements of the upper limbs are those by which we take hold of objects that are necessary for the satisfaction of the corporal needs and the individual instincts. It is only at a much later period in the course of human evolution that the progressive and specific functional differentiation for reading takes place and, therefore, this cortical field is less surely and generally established than the cortical motor area of articulate speech.

The view the writer here puts forward can be aptly illustrated by two

${ }^{1}$ Bianchi. Lieçoes sobre as Localisaçôes cerebraes e a Physiopathologia de Linguagem. Trans. of Dr de Souza. Rio de Janeiro, 1899.

${ }^{2}$ Kocher. Hirnerschuttering, Hirndruck und chirurgischen Eingriffe bei Hirnkrankheiten, 1901, and L'écorce cérébrale. Bonne, 1910. 
concrete examples. Confronted by an enemy, the savage may employ a club in self-defence, whilst the eivilised man of to-day, skilled in fencing, may, under similar circumstanees, make use of his sword. Both bring into action the same muscles of the right upper limb, and they have the same objeet in view. The savage performs a series of movements that are coarse in character and involve a useless dissipation of his energy. The fencer, on the other hand, more composed, performs a series of very delicate movements each of which has its raison d'être, movements that are co-ordinated in due and proper proportion and in perfect harmony with the two objectives of offence and defence.

Whilst the savage is unable, because he has not learned how, to perform other movements more adapted for the purpose he has in view, the fencer can, if he choose, make use of the movements employed by the savage, although he recognises the great inferiority and incoherence of these.

In the ease of the fencer, a differentiation has taken place in the structure of the eerebral cortex of the right upper limb, thanks to which a more complex and better eo-ordinated product is available.

Suppose, now, the fencer lays aside the sword and takes up the pen in order to deseribe his encounter, or let us go further and suppose that he makes use of the pen to attack his enemy or to defend himself from the attacks of the latter. Here we are witnesses of a combat on a much higher plane. What a marvellous cerebral advancement is involved in this process ! How much more delicate is the muscular co-ordination, compared with the movements of these same museles as employed by the savage and the fencer !

Here we must necessarily have the intervention of a larger number of co-efficients. Amongst these, the conceptual new-formations and the auditory and visual images of the words, by means of which the writer seeks to give expression to his particular notions, assume a new importance. These images are furnished by the new cerebral fields ; they have no place in the movements of assault and parry of the fencer and much less in those of the primitive man. This, surely, goes to prove that to arrive at a much wider co-ordination of movements and to rise to a much higher level of functional dignity requires not only the differentiation of the cortical motor centres themselves, but perhaps also the extension of the motor area into a field eapable of differentiation, as well as the indispensable auxiliary in the shape of other factors, or, as one might say, the work of special workmen. It is a case of division of labour and eo-ordination of the working forees.

$$
\text { *** }
$$

Flechsig would have it that in the tactile sphere we have organically concentrated the centre of our intellectual existence in as much as this region is in direct and indirect anatomical relationship with all the other regions of the cortex, as well as with numerous other areas (mostly centripetal) which come into touch with it through the intermedium of the optic thalamus. Not only does this tactile sphere serve as the path for relations with the external world, in so far as these are established by means of the tactile 
sense, but, if the author interprets Flechsig's complicated conception rightly, it is only by means of the tactile sphere, which embraces the sensory zone in relation to the body, and also the field of innervation of the circulation, respiration and other organic sensations, that all the other perceptions become assimilated with the consciousness.

Bearing in mind the fact that according to the most recent investigations the tactile sphere embraces only the post-central, and does not extend to the precentral, convolution (Figs. 1 and 4), one can agree with Flechsig in regarding the tactile sphere as the field of all the sensory impressions coming from the organs composing our body and that it may consequently be the principal component in the formation of the idea of the ego or, in other words, the organ of the central nucleus of the sense of individual existence (kinæsthesis).

The author's pathological studies have induced him to admit, too, that the organic me is always or almost always made aware of and modified by external stimuli no matter what the area where the relative image is formed. In other words, every perception is accompanied by a resonance in the kinæsthetic consciousness and an emotional reflex of varying degree, a fact which implies the existence of direct and indirect anatomical paths between the posterior Rolandic convolution and all the other spheres of sensibility. We know that the tactile sphere - which in opposition to llechsig's view is now limited to the post-Rolandic convolution as far as the interparietal sulcus behind and above, and, on the interhemispheric aspect, to the part corresponding to the para-Rolandic lobule-contains points excitation of which causes modifications in the functions of the viscera, of the circulation, respiration, digestion, secretions and excretions. It is well established that analogous modifications of these functions occur under the influence of more or less powerful impressions and accompany the emotions, inasmuch as all impressions furnish, by one path, the cognition, and by another path the awareness on the part of the ego, of the stimulus which has acted on the senses, vibrations of which have reached the sensory fields of the cortex. Some physiologists and psychologists hold that emotion consists essentially in the organic manifestations of respiration and of circulation, those same manifestations which, under the influence of stronger stimuli, give rise to pallor or reddening, to palpitation and respiratory oppression, to dryness of the mouth, to trembling, the desire to urinate, etc.

Further reference will be made to this question in a succeeding chapter. Meanwhile we are agreed that the nerve-waves from every part of the organism are directed to this organ or cortical area and there become blended in the sense of individual existence, with attributes of well-being and strength, or of malaise and weakness, and on it depend variations in the tone of mind, which may be gay and active, or sad, irritable and negativistic.

When all parts of the organism perform their functions harmoniously the ego manifests interest in the external world (activity, courage). When, on the contrary, any region of the body is diseased or out of order, or all the organs perform their functions feebly owing to defect or alteration of the 
material intended for their nourishment and necessary for the maintenance of life, a vague feeling of malaise, pessimism, negativism, discouragement, a prevailing sense of difficulty, are significant of the altered internal conditions in the vital sphere. In some illnesses there occurs a kind of dissociation between the percept and the me which perceives. The author has observed this in some cases of melancholia and severe neurasthenia, where the kinæsthetic consciousness, which is the basis of the affectivities, is quite dormant, so that the patient perceives the external world yet has no feeling of an ego which is perceiving. For example, a mother said to me: "I see my sons but have no longer any feeling for them. I seem to be another person and this causes me great distress." Other patients are capable of identifying their ego historically yet maintain that they do not feel they are perceiving although they recognise objects and persons, and this causes them much concern. This condition induces in some a true metamorphosis of the individual personality. One lady, whose story I have given in my Text Bool: of Psychintry, ${ }^{1}$ said to me: "I seem to recognise you as Professor B. but I am not the Mrs A. whom you say I am. That lady does not exist and I who am speaking have no existence," etc. In this state one must recognise something akin to a profound sleep of the organic ego which is the nucleus of the personality and normally should receive notice of all sensory processes occurring in the other areas of the cortex, but in the cases under consideration these products fail to be linked up with the irresponsive ego. Here we have a state of paresis of the cortical field of the kinæsthesis. The ego is profoundly modified and loses its historical bearings owing to a break in continuity. The cortical fields of the other senses are in active working order but their products are missing links in the clain of historical continuity provided by the somatic ego, because the latter fails to identify them.

If we have succeeded in proving that the conception of a great occipitoparieto-temporal intellectual centre, in the sense of Flechsig, is not tenable and are agreed that this region should be regarded as an extensive area of evolution of the primary sensory areas, visual, auditory and tactile, and that these preserve their functional individuality and their respective characteristics, we are in a position to attack the problem of the function of the frontal lobe.

What does this very conspicuous part of the neopallium represent in the evolution of the brain? What part does it play in the harmony of the cerebral activity?

${ }^{1}$ Bianchi. T'rallato di Psichiatria. 1st and 2nd edit. Naples, 1915. Eng. Trans., 1906. 


\section{History and Evolution of the Doctrine Concerning the Functions of the Frontal Lobes}

ThF question now to be confronted is one of the most abstruse and debated in cerebral physiology or, rather, in anatomical psychology. The function of the extensive cortical field situated in front of the motor zone has given rise to one of the very keenest discussions. The problem can, and should, be stated in precise terms. Does the anatomical substratum of the psychic processes consist exclusively of the sensory and motor areas and the language areas, or are there other cortical areas which, whilst neither sensory nor motor nor organs of language, yet co-operate in a particular manner towards the formation and development of thought and the psychic personality? These were the terms in which the writer put forward the problem in his first communication on this subject to the National Congress of Medicine in Rome, 1892, when he introduced a discussion on the functional localisations of the cerebral cortex ${ }^{1}$ and afterwards to the International Congress at Rome in 1894, and they were surely sufficiently explicit to have prevented any misunderstanding. ${ }^{2}$

In the preceding pages we have mentioned Flechsig's attempt (which seemed for a time to have succeeded completely) to assign the highest functions of association and mental syntheses to the great temporo-parietooccipital area, and we have demonstrated that Flechsig's doctrine does not accord with fact. These areas are the anatomical substratum that is gradually developed through pereeptions and processes of perceptual differentiation and they represent organs for the perfecting of products from simpler processes.

From ancient times anatomists, physiologists and poets have spoken of the frontal lobes as the organ of intelligence.

The doctrines of Gall and Spurzhein, which Hufeland, though a strong opponent, declared to be "the most important event of the eighteenth century (Soury) and one of the boldest and most conspicuous advances in the domain of natural science," can be condensed in the following propositions: (1) the cerebral convolutions and especially the grey substance are the organs of the intellectual faculty and should be recognised as "the parts where the instincts, sentiments, tendencies, talents, affective qualities and, in general, the moral and intellectual forces, are exercised." (2) There exist particular forms of intelligence, memory, imagination, and tendencies, and

${ }^{1}$ Bianchi. Atti del Congresso della Società Italiana di Medicina interna. 1892.

${ }^{2}$ Bianchi. Atti del Congresso Internazionale di Medicina e Chirurgia in Roma. 1894. 
each cerebral organ has its own particular intelligence, memory, imagination; etc. (3) "The qualities common to man and to animals have their seats in the lateral and posterior parts of the head (brain). In proportion as animals have been endowed with a share of certain anterior and superior encephalic parts, so they enjoy certain intellectual faculties. In man, when the organs of the posterior and inferior part of the brain are excessively developed and those of the anterior superior portion are compressed, the animal inclinations must have the ascendancy. . . . Man is the more intelligent, the more the anterior superior brain is developed." 1 This, of course, is an intuitive, subjective manner of regarding the part played by the anterior ?brain in the extrinsication of life, but one cannot deny that the proposition contains a germ of truth, since later clinical and experimental researches have, to a certain extent, confirmed the proposition in a general way.

The work of Gall and Spurzhein was much exalted by Flechsig, but there was one other part of the doctrine which had also a foundation of truth, and survives to-day, as mentioned by that ardent student of neurology, Ireland ${ }^{2}$ - viz. the localisation of the faculty of language in the orbital part of the anterior lobe.

According to Burdach ${ }^{3}$ the frontal lobes would be related particularly with psychic activity and objective cognition. He had observed the feeling of tension (already noted by Lancisi, who had designated the frontal lobes as the factory of thought) in the frontal region when thought is intense. $\mathrm{He}$, too, attributed a great importance to the particular form of the forehead in relation to the intellectual faculty and maintained that the two poles of the brain, anterior and posterior, do not act independently but manifest a common activity, because "in cognition we have predominating at one time objectivity, at another time subjectivity."

Fleurens ${ }^{4}$ cast these notions aside as fantastical, and maintained that " there is no such thing as a different site for the different faculties and the different perceptions." The faculty of perceiving, or judging, or willing one thing resides in the same locality which perceives, judges, and wills another. According to this view, there would be no differentiation in the cerebral mantle for different sensations, so that destruction of any one part of the cortex would lead to weakening of all, and it would follow that all the intellectual faculties, all the perceptions, all the instincts, constitute essentially one single faculty, for they would reside in the same organ and occupy the same site. True, Fleurens distinguished the organ which pereeives and wills from that which co-ordinates and that which excites, but these organs were respectively the cerebral hemispheres, the cerebellum and the spinal medulla, with its nerves. That doctrine is now a thing of the past.

${ }^{1}$ Jules Soury. Le système nerveux central. Paris, 1899.

2 Ireland. Journ. of Mental Science. 1898.

${ }^{3}$ Soury. Loc. cit. and Baue u. Leben des Gehirns. Leipzig, 1819-1826.

${ }^{4}$ Fleurens. Recherches expérimentales sur les propriétés et les fonctions du système nerveux dans les animaux certébrés. Paris, 1842. 2nd edit. 
Gratiolet ${ }^{1}$ was strongly opposed to the doctrine of the localisations, yet, in the light of to-day, when we review the results of clinical and experimental researches and the debates upon the views advanced by Broca concerning the localisation of language, he appears as one of the founders of the doctrine of localisations. He admitted the possibility of " supposing the cerebral hemispheres to be divided into as many different regions as there are organs of different sensations in the periphery," also "the possibility of allocating a memory and an imagination " to each of these brains ; and although he was prepared to admit the likelihood of a seat for the "reason which commands" and to recognise in the frontal lobe " the majesty of the human brain " he supported the doctrine of Fleurens, especially because he found it difficult to understand why there should be so many cerebral organs. "Of what assistance would one be to the other? How would the brain of the ear be able to come to the aid of the brain of the eye?" With good reason, he assigned great importance to the centrum ovale because here, if anywhere, "the functional synergy of the brain could find its anatomical condition-viz. in the multiple commissures which, uniting together all the convolutions of one hemisphere in the most complex manner, prove to the hilt the functional unity of the brain." Gratiolet was undoubtedly possessed of superior judgment and great penetrative capacity, but he was unable to cast aside the old prejudice that favoured the doctrine of the unity of thought. It is to his credit, however, that he recognised the various possibilities, objective proofs of which have, since his time, come to hand.

Experimental and clinical investigations have now overcome the barrier of prejudice which confronted all attempts to found a psychology based on the anatomy of the brain. As an example of this prejudice one may cite the statement of Lelut," who wrote: "The question of the relations to be established between the brain and the higher acts of thought is very obviously one of those questions condemned by their very nature to perpetual indetermination."

To Hitzig must be given the credit of having placed the question upon an experimental basis. Starting out with the data of comparative anatomy bearing on the evolutionary parallelism between the frontal lobes and intelligence, he initiated experiments on higher mammals which confirmed the supposition previously deduced from the literature of the subject and from comparative anatomy-viz. that the frontal lobes were really the seat of the higher mental faculties. ${ }^{3}$ It also stands to the credit of Hitzig that, in his controversy with the German physiologists, Munk and Goltz, he was able to bring forward experimental proof of the facts that, in dogs, removal of the frontal lobes was never followed by any motor disturbance, ${ }^{4}$ and, in

${ }^{1}$ Gratiolet. Observations sur la forme et le poids du cerveau. Paris, 1861.

${ }^{2}$ Lelut. "Formule des rapports du cerveau à la pensée." Annales medicopsychologiques. 1843.

${ }^{3}$ Hitzig. Untersuchungen über das Gehirn. 1874.

${ }^{4}$ Hitzig. "Achte Wanderversammlung d. W. D. Neurologen." Neurologisches Centralb. 1883. 
monkeys, resulted în loss of recent acquisitions and those that were the result of training.

In 1884, in his dispute with Munk and Goltz, Hitzig ${ }^{1}$ wrote as follows :"I adhere to-day to the hypothesis I put forward in 1870 that the cortical centres are merely collecting centres. I can support the opinion, which has often been expressed, that deep or very extensive lesions affecting the central mechanism necessarily interrupt a multitude of bundles which unite different cortical regions of the brain with one another, and must consequently give rise to symptoms that are susceptible to a relatively rapid improvement. To this category belong the transitory disturbances of sight which follow deep lesions affecting different regions of the hemispheres. I find myself in opposition to Munk as regards the nature of the higher intellectual functions and their relations with the anatomical substratum. Munk holds that there are no such things as special organs for these functions and that there is no necessity for them. I believe with him that intelligence, or more correctly, the treasury of ideas, is to be sought for in all parts of the cortex, or rather, in all parts of the brain ; but I hold that abstract thought must of necessity require particular organs and those I find in the frontal brain."

Ferrier, ${ }^{2}$ contemporaneously with Hitzig and perhaps even before him, attacked the question of functional localisations by means of experiments on higher mammals. In the first edition of his work he located the centre for movements of the head and eyes in the frontal lobes and attached great importance to these movements in attentive attitudes, regarding them as an essential part of attention. He makes the frontal lobes the organ of attention, and so we can regard him as having arrived, by a different path, in the company of those who attribute a higher psychic function to the frontal lobes.

In the second edition of his book Ferrier reaffirms the view that in perception there is included a muscular element, which plays its part in the re-awakening of the correlative idea. When we recall particular ideas to memory, we really execute, in a more or less suppressed manner, the movements with which these ideas are respectively associated in a form of organic cohesion. In representing a thought we reproduce, in a more or less rudimentary manner, the articulatory movements involved in the words in which the thought has been symbolised. ${ }^{3}$ In the same way, just as our attention, when attracted by an object, determines an accommodation of the eyes, associated with such movements of the head as serve to bring the object on the central point of the retina, so, when engaged in attentive ideation, we are making precisely the same movements of the head and eyes as are necessary for clear, actual vision. "... Destruction of the frontal lobes according to the degree of its complexity impairs or paralyses the

1 All Hitzig's works and the relative disputes were eollected in one volumeUntersuchungen über das Gehirn. 1904.

${ }^{2}$ Ferrier. The Functions of the Brain. 1877.

${ }^{3}$ Stricker. Du langage et de la musique. 1885. 
lateral movements of the head and eyes. Although some ocular movements may be excited reflexly by retinal impressions, there appears to be loss of power of locating or directing the gaze towards objects which do not fall spontaneously within the field of vision. Correlative with this immobility of the head and eyes there is the aspect of uninterest and stupidity, the absence of that active curiosity which is normally manifested by monkeys, and the mental degradation which seems to depend on the loss of the faculty of attention and all that it implies in the sphere of intellectual operations." 1

Ferrier accordingly makes the frontal lobes a centre for the movements of the head and eyes, which are the motor equivalents of attention. These results were confirmed in part by Grïnbaum and by Sherrington, ${ }^{2}$ who, in the brain of the chimpanzee, more especially at the posterior extremity of the middle frontal convolution, found a frontal area, electric stimulation of which induced movements of the eyes.

In point of fact, observation of animals deprived of their frontal lobes shows that movements of the head and eyes persist in all respects, neither more nor less, just as we find them in human imbeciles. In the case of monkeys it is only during the first few days after the operation, when the lesion falls too near the motor zone or directly affects the motor area of the muscles of the head and trunk, that motor disturbances along with a certain fixity of the gaze are observed. After days or weeks these symptoms disappear, so that the absence of that active curiosity normally exhibited by monkeys cannot be the result of paralysis of these movements which, after all, are the somatic elements necessary in the attentive process, but rather the effect of a particular mental condition which will be dealt with in a subsequent chapter.

The author's first experiments on dogs warranted the opinion heexpressed at the time ${ }^{3}$ that unilateral destruction of the prefrontal lobe of the dog was not followed by any noteworthy symptom, whilst bilateral destruction brought into evidence a distinct alteration of character, marked especially by weakening of all the psychic manifestations: defect in perceptive judgment; exaggerated fear, resulting from defective critical capacity and inability to make use of the physical powers although these were well preserved ; amnesia and, generally speaking, a psychically blind behaviour; defect in initiative and resource; lack of finality in the complex movements, as betrayed by incoherent conduct and diminished vivacity (lowering of the psychic tone). In fact, any ordinary individual would have classed these dogs as weak-minded.

How were these phenomena to be interpreted in the light of the then

${ }^{1}$ Ferrier. The Functions of the Brain. 2nd edit. 1887.

2 Sherrington. "Observations on the Physiology of the Cerebral Cortex of some of the Higher Apes." Proc. Roy. Soc. 1901.

${ }^{3}$ Bianchi. "Ist die Vernunft eine auschliesslich den Empfindungsbereichen der Hirnrinde zukommende Thätigkeit?" Untersuchungen zur Naturlehre des Menschen und der Tiere; herausgegeben vom Moleschott. Bd. xix., Heft 4. 
current knowledge? The sensory areas had escaped injury. The associative fibres running between them and between the two hemispheres had not been interrupted at any point. The sensory functions, including the tactile, were preserved intact, as were also the movements. Yet the dog in question had suffered no small damage to its psychic life! The author's explanation ran thus: "We are bound to admit that the process of perception is the product of the co-ordination of indispensable elementary factors, which cannot separately furnish the concrete images of objects, no matter what sensory apparatus is involved. The co-ordination of simple perceptions, either actual or representative, by means of associative paths and laws, gives rise to more complex products, the intellectual exponent of which, in man, augments enormously, up to the highest abstract conceptions, which are the outcome of still vaster co-ordinations. Now, I believe it is permissible to suppose that this vast co-ordination, whence result the finality of conduct and the homogeneity, to use a Spencerian phrase, has its seat in an organ distinct from the organs of perception.'

In support of this view the author adduced the example of the progressive complication of the reflexes. The stimulus which reaches the cells in the posterior cornua of the spinal cord and, without proceeding further, finds resolution in a simple reflex movement, furnishes an example of co-ordination. The passage of the stimulus from the lower to higher stations such as those in the mid-brain, and, still higher, in the optic thalamus, and the much more complicated and co-ordinated movements which result therefrom, provide examples of other co-ordinations which are effected in organs that are quite distinct from the preceding, and at the same time, much more complex. This being so, there were grounds for the argument that the more extensive associations, the more delicate co-ordinations of the sensory products whence result the complex conceptions which resolve themselves into conduct (in the broad sense of the term), possess their own particular organ, of recent evolution, in close and immediate relation with all those others that have to furnish it with the sensory material which it is destined to elaborate. " This organ would be to the sensory and motor centres what these are to the optic thalamus and subcortical nuclei, and these in turn to the spinal cord. The natural history of the evolution of the nervous system throughout the whole animal series testifies to the probable truth of my argument."

In an article published in Brain, ${ }^{1} 1895$, the author gave the results of a series of experiments he had performed on dogs and monkeys which were shown to, and examined by, the members of a committee of the International Congress of Medicine held at Rome in $1894 .^{2}$ The findings were summarised as follows :-

1. Restlessness and inquietude of the animals which had suffered mutilation of both frontal lobes. The animals were always moving about aimlessly, hither and thither.

1 Bianchi. "The Functions of the Frontal Lobe." Brain. 1895.

${ }^{2}$ Bianchi. Attidel Congresso Internazionale di Medicina e Chirurgia. Roma, 1894. 
2. Suppression of curiosity and of the attitudes of observation so common in monkeys, and hence a certain degree of indifference to all that occurred or existed in their surroundings.

3. Defect of affectivity towards other monkeys and other animals, and also towards persons in whom they had manifested a lively interest prior to operation.

4. Greater emotivity, especially a strange fear induced by noises, or by the sight of other animals, such as a dog, the presence of which prior to the operation did not at all disturb the monkey, and difficulty in restoring a state of calm (result of decadence of the power of criticism and of the perceptive capacity).

5. Defect of reflection, judgment and memory, and an incapacity for new adaptations.

6. Weakening of certain instincts.

7. Stereotypisms and automatisms.

These findings have been confirmed by other investigators but they have also met with a good deal of severe criticism, to which attention will be directed.

When we say that intelligence or, rather, mind, as Hitzig put it, is not a function of the whole brain (in the sense of Fleurens), and that some psychic functions depend upon particular regions of the cerebral cortex that have been more or less well defined, we lay ourselves open to criticism. The writer agrees with Munk, as he already clearly stated in his first communication, that the entire brain contributes to the formation and extrinsication of intelligence, not in the sense of Fleurens, but in the sense of a division of labour between the various cortical areas, and of a co-ordination and association of the various products on the part of a particular cerebral organ. This notion harmonises with all the data that have been furnished both by experimental investigation and by clinical observation.

The writer has never asserted that the frontal lobe is the organ of the intellect. What he did say was that it is an organ of intellect and that the work of the entire brain contributes to this, its highest manifestation. He expressed the problem in terms that were quite clear and distinct. It may be put as follows:-Does there exist a cerebral organ which has the faculty of utilising the mental products of the sensory areas of the cortex for the construction of mental syntheses more suited for the spiritualisation, and hence the cognition, of nature, an organ giving rise to reactions upon the world which, on the basis of individual and collective experience, permit a higher adaptation of the individual to his physical and social environment, an organ which renders possible the unfolding of a long process of logically connected thought?

There was no ground for the misconstruction put upon the author's views. In the article referred to in Brain, page $519,{ }^{1}$ apropos of the formation of the psychic personality, will be found these words: "The whole of the

1 Bianchi. Loc. cit. 
nervous system takes part in it" ; and on page 521: "my hypothesis is that the frontal lobes are the seat of co-ordination and fusion of the incoming and outgoing products of the several sensory and motor areas of the cortex." Those who quote the author as having said that the frontal lobes are the organs of intelligence make a great mistake and distort an idea that was expressed clearly and precisely.

Munk ${ }^{1}$ and Goltz have taken a very prominent part in the debate on this subjeet, and both authorities have ranged themselves against the doctrines formulated by Hitzig.

Munk strongly opposes the views both of Hitzig and of Ferrier and maintains that there are no relations subsisting between intelligence and the frontal lobes any more than with any other region of the brain. In his opinion, the disturbances of intelligence that follow extensive lesions of the two occipital lobes are incomparably more severe than those that occur after removal of the frontal lobes. In one of his works he writes: "Intelligence has its seat everywhere in the cortex of the brain and in no part in particular. It is, in effeet, the sum and resultant of all the images or representations derived from sensory perceptions. Any lesion of the cerebral cortex whatsoever alters the intelligence, all the more severely the more extensive the lesion, and this is always due to the loss of its groups of images or representations, simple and eomplex, which had their foundations in the perceptions that belong to the injured cortical area. The intellectual disturbance will be definite if : (1) the pereeptive elements are destroyed; (2) there remains no more substance which can become the new seat of the notions lost. Blindness, deafness, and motor paralysis, complete or incomplete, produce, each on its own account, a restriction of the field of intelligence, and, if several areas are.injured at one and the same time, the intellectual breadth and aetivity diminish all the more with the restriction of the field of the remaining notions, whilst there is an inereasing obstacle to the formation of new ideas, so that sooner or later the animal appears to us as though it were affected by imbecility." One cannot find fault with Munk so far as dogs are concerned, but one cannot agree with him when he states that in man, as well as in monkeys, the region (cortical) of the nape of the neck has, to a great extent, the same functions as the frontal lobe.

Munk found that he could excite the frontal lobe by means of the faradic current, and so attributed to it a senso-motor function, controlling the nape of the neck and trunk. Hitzig, on the other hand, found the frontal lobe could not be excited, and with good reason blamed Munk for having used too strong currents. Hitzig, again, admitted having met with visual disturbances on mutilation of the frontal lobes, a finding confirmed by Goltz. Munk, however, exeluded this and stated that he had never observed it. The truth is that in the dog the frontal lobe is still relatively small, and electric currents of even moderate strength excite the neighbouring sigmoid

'Munk. Über die Functionen des Crosshirnrinde, ece. 1890. (Many of Munk's communications on the functional localisation of the brain, up to 1890 , are collected in this volume.) 
gyrus and the area for the musculature of the neck and trunk, unless strict precautions are taken.

Munk ${ }^{1}$ extends what he called the Fühlsphäre (feeling sphere) to the frontal lobe and locates there the centres for the muscles of the nape of the neck and trunk, maintaining that motor paralysis of the muscles of the trunk follows mutilation of the frontal lobes in dogs, so that the animals were unable to turn to the paralysed side. The results of the author's experiments on monkeys are in direct opposition to this finding. In dogs, this symptom is only present at the beginning, or when a part of the sigmoid gyrus has been excised. When a lesion is bilateral, the deviation of the trunk and the circus movements are of brief duration and towards the more injured side. In these cases, along with the circus movements, which tend to disappear, there are also mental disturbances or changes in the character and conduct of the animals operated upon, features observed also by Goltz. The insistent affirmations of Munk were severely criticised by Ferrier, Goltz, Bechterew, and by Shepherd, who declared that in the monkeys and cats on which he had experimented he found no disturbance of the muscles of the trunk. The cats could jump and run about, and the monkeys walk and climb, precisely as they did before they underwent the mutilation of the frontal lobes:

That the views of Grossglick, Munk, Luciani and others, who have maintained that the frontal lobes are the centres for the musculature and the sensibility of the nape of the neck and of the back, are erroneous, has been amply proved by unprejudiced and reliable observers. It is certainly a fact that even strong excitation of the area in question, in monkeys, is rarely followed by movements of the neck, even when the state of narcosis in the animal operated upon is very light. It is to be added that destruction of this area never gives rise to prominent disturbances of sensibility in the limbs, the neck, the trunk, or the head, much less to permanent limitation of movements. Werner ${ }^{2}$ maintains that strong and prolonged currents are necessary in order to excite the frontal lobe, and he blames other investigators for having employed very weak currents, and so having arrived at what he considered to be an erroneous conclusion regarding the inexcitability of the frontal lobe. In this respect Werner commits an undoubted error in technique, and the absurdity of the position he takes up is proven by the fact that the prolonged application of strong currents to any part whatsoever of the brain gives rise to disturbances at a distance, for very obvious reasons. Werner ought to come into line with Unverricht. ${ }^{3}$ This investigator provoked an epileptic attack by means of prolonged and severe excitation of the occipital lobe, similar to what took place when he excited the motor zone. The convulsive attack was caused by excitement in the motor area

${ }^{1}$ Munk. Sitzungsber. Berlin Akademie d. Wissensch. 1889-1896.

${ }^{2}$ Werner. "UUber elektrische Reizversuche in der Rumpf und Nackenregion d. Grosshirns beim Hunde." Allgemeine Zeitschrift f. Psych. 1895. Vol. lii.

${ }^{3}$ Unverricht. "Experimentelle und klinische Untersuchungen über die Epilepsie." Arch. f. Psychiatrie u. Nerventer. Bd. xiv. 
produced from a distance by a powerful current applied to the occipital lober Many years ago, Penta and the author repeated Unverricht's experiments with confirmatory results. The test consisted in making a deep incision into the cerebral hemisphere between the point excited and the sigmoid gyrus (the epilepto-genetic centre in dogs, according to Albertoni), immediately the first contractions characteristic of the epileptic seizure occurred. This incision at once arrested the convulsive seizure, because it interrupted the paths of propagation of the strong currents from the occipital lobe to the sigmoid gyrus.

If a faradic current of moderate strength, applied to the precentral convolution of a monkey, properly prepared and anæsthetised, provokes distinct contractions of determined groups of muscles in the limbs, and if the electrode is then removed to a point in front of the motor zone of the limbs, and movements of the head, eyes, pupil and ear are then induced owing to stimulation of the respective areas, and if, again, the electrode is moved to another point farther forward in the frontal or prefrontal area and now no muscular response is obtained, even although the intensity of the current is somewhat increased, we have no right to maintain that the last-named area is a motor centre. If movements are provoked only by greatly increasing the strength of the current or by prolonging its application, we are not in a position to draw legitimate and reliable conclusions from the experiment, because it is a fact that strong electric excitations of any point of the cortex give rise to convulsive seizures. The excitations, either by contiguity or by means of the direct and indirect nervous paths of communication, reach the motor zone, whence eventuate single contractions to begin with, and afterwards an epileptiform convulsion.

After a lapse of many years we find Munk ${ }^{1}$ reappearing on the scene with renewed ardour, bringing forward fresh arguments in support of his old doctrine, and attacking the hypotheses of Ferrier, Bianchi, and of Goltz. He attributes the intellectual disturbances observed in dogs that have suffered mutilation of his so-called musculo-sensitive zone, partly to a rather extensive lesion of the cerebral cortex, partly to shock, and partly also to subsequent inflammatory processes. In his criticism of Flechsig's doctrine he stoutly denies the existence of a cortical field for the higher psychic functions. To him, the cerebral mantle is the sphere of the senses and hence an aggregate of fields, each related to a particular sense. Each sensory sphere is an organ for a specific perceptive function, for representations and for sentiments (Empfindungen).

Goltz ${ }^{2}$ refuses to accept the involved conception of Ferrier and of Hitzig concerning the intellectual function of the frontal lobes-i.e. that they are the seat of intelligence-and expresses views similar to those of Munk, maintaining that the whole brain is the organ of the higher psychic functions.

1 Munk. "Ưber die Ausdehnung des Sinnesphäre in der Grosshirnrinde." Sitz. Berl. d. Preuss. Akad. 1901.

${ }^{2}$ Goltz. Uber die Verrichtungen des Grosshirns. 1881. 
He maintains that it is not possible to localise in any particular part of the brain any one of the higher manifestations of psychic life such as we understand by intelligence, thought, sentiment, passion, will. He insists that every part of the cerebral cortex is a point of departure for those higher manifestations. Nevertheless, in dogs which had both frontal lobes mutilated he observed certain phenomena similar to those described by Bianchi in his first communication, such as clumsier movements, delayed sensations and changes in character (one of Goltz' dogs bit his own paw when excited). He further declares that after extensive and deep lesions of the two occipital lobes there is also loss of voluntary energy. 'This he inferred from the defective resistance exhibited by the animals to passive movements. ${ }^{1}$

He maintains that intelligence has no relations with the anterior lobes of the brain that differ from the relations subsisting between it and any other part of the brain; that the disturbances of intelligence which follow removal of the occipital lobes are incomparably graver; and that Hitzig and Ferrier had been unable to free themselves from the old prejudice that the anterior lobes of the brain were the organ of intelligence. He writes as follows:"I look upon the demonstration that the cortex of the brain is the organ of the higher psychic functions-i.e. the organ of intelligence, in all its partsas the most important result of my researches. By intelligence I mean the faculty of elaborating the perceptions of the senses by aid of reflection, with a view to action suited to attain a definite object. I am not sure if philosophers will be satisfied with this definition. It is sufficient for the physiologist."

Moolman ${ }^{2}$ and Goltz ${ }^{3}$ observed two facts which the writer was able afterwards to confirm :-

(a) Lesions confined to the frontal lobes are followed by consequences that are less significant than those due to lesions of other parts of the brain.

(b) Lesions limited to one frontal lobe produce no appreciable effect.

It is to be noted, however, that there is a great divergence between this finding and the assertion of Goltz to the effect that extirpation of both frontal lobes gives rise to disturbances similar to those which follow upon removal of the parietal lobes.

Goltz had certainly not failed to observe the symptomatic complex, at least in its more obvious details, which one generally finds after removal of the frontal lobes, such as general excitability, absence of control over movements, and the incoercibility of certain reflexes, in dogs. These symptoms, however, were always complicated by disturbances of general sensibility and of certain movements because, as a rule, the posterior limit of the lesion fell upon the sigmoid gyrus or even on a point behind this. On

1 Goltz. "Zur Physiologie der Grosshirns." Arch f. Psychiatrie, xv. 1884; Pfliiger's Archiv. 1884. Bd. 34; Id. Bd. 42.

${ }^{2}$ Moolman. Researches carried out in the laboratory of Goltz.

${ }^{3}$ Goltz. Loc. cit. 
the other hand, it is to be remembered that these phenomena were judged in the light of a doctrine which, for a long time, even up to the present day, had obtained possession of the minds of many physiologists, the doctrine of arrest or inhibition. If a dog has its frontal lobes removed and afterwards performs marked circus movements, running in a mad fashion round a very wide circle - a symptom observed not only by Goltz but also by Polimanti and the author-the conclusion is that there is a defect in the power of arrest or inhibition, as the result of which the animal obeys an internal impulse_one might almost say a mechanical impulse - which in itself is irresistible. Goltz even went further. In his opinion the sensory and motor paralyses which followed removal of the frontal lobes were not the direct effects of the lesion of the cerebral centres of motion and sensation, but indirect effects, in this respect that the lesion produced a transitory state of arrest of the motor centres at various lower levels in the subcortical organs.

Loeb ${ }^{1}$ denies the psychic functions attributed by others to the frontal lobes. Admittedly he is right when he asserts that cerebral lesions, if of slight extent, do not produce psychic disturbances, because they do not affect the associative memory (another definition, perhaps unnecessary, but useful) - and that it is only extensive bilateral lesions that cause more or less conspicuous disorders of the associative memory. ${ }^{2}$ This distinguished physiologist, however, was unfortunate both in his observations and in his conclusions, if, in his experience of a number of cases of mutilation of both frontal lobes of dogs, he never noticed any difference in mental behaviour between sound, healthy dogs, and those operated upon.

Luciani ${ }^{3}$ is one of the physiologists who have offered very strong opposition to the author's views concerning the function of the frontal lobes. In the last edition of his classic work * he strongly supports the doctrine of Flechsig. He admits that the associative centres fulfil higher psychic functions, but does not ascribe to the frontal lobes any such higher functions, founding his argument upon the brief and transitory character of the symptoms observed in animals deprived of their frontal lobes. He writes as follows:- "Neither from Munk's experiments nor my own, nor from those of Horsley and Schäfer, does it appear that, after destruction of the prefrontal lobes, dogs or apes differ in any appreciable way from intact animals, so far as intelligence is concerned." He attributes the changes of character, observed by Goltz in animals which had suffered mutilation of the fore-brain, mostly to elimination of the sensory-motor zone, and to a slight

${ }^{1}$ Loeb. "Beiträge zur Physiologie des Grosshirns." Pflüger's Arch. Bd. xхxix. 1886.

2 Loeb. Loc. cit.

${ }^{3}$ Luciani. T'rattato di Fisiologia. 1912. Vol. iii.

* The author puts aside the harsh and not altogether courteous judgment expressed by Professor Lueiani in the first edition of his book, because in a renewed friendship the author maintains a sincere regard for his work as a physiologist of very high standing. 
extent to destruction of the prefrontal area. "No one," he writes, "who has been long occupied with the effects of partial destruction of the brain in dogs or monkeys, can fail to note the insignificance and brief duration of the symptoms presented by animals after removal of the prefrontal lobes." It is worthy of note that whilst Professor Luciani does not attach much importance to the monkey shown by the author at the International Congress in Rome, in 1894, he assigns a greater value to the monkey presented by Sciamanna, whose conclusions are invalidated by the result of the postmortem examination, which revealed only an insignificant loss of brain substance, as indeed was to be anticipated from the statement made by Sciamanna himself, when he described the process he had followed in his experiments. The technique employed in his excision of the frontal pole was inadequate. The frontal pole is but a small portion of the prefrontal lobe and a still smaller part of the frontal lobe, taking into account the whole extent of the latter, up to the limits assigned to it in front of the pre-Rolandic convolution.

Luciani attaches much more importance to the parietal area so far as the psychic powers are concerned, and in this respect agrees with Flechsig, although he bases his argument upon different grounds. He states that removal of the parietal area (parieto-occipital and parieto-temporal) does much greater damage to the intelligence of dogs. In the dog, each sensory sphere not only has its own particular territory but also possesses an area in common with those around it, an area which constitutes what he calls a zone of interlocking or overlapping of the various sensory regions (visual, auditory, olfactory, somæsthetic). Owing to this overlapping we get the marginal zones (Flechsig) of the various sensory areas confused as one areathe parietal lobe-regarded by Luciani as the most important region of the dog's hemisphere, as the centre of centres on which the normal association of percepts and their memory images depend. This conception has its foundation in the fact that the sensory areas are not neatly and precisely delimited, from the experimental and clinical points of view. They are certainly not well defined in man, although they have attained a much greater differentiation than in the dog, and still less in the dog, which is the animal preferred in these experiments. One must always bear in mind the fact that, in the dog, the frontal lobes are but slightly evolved, whilst temporo-parietal lesions do damage to the mnemonic perceptive field of sight and hearing, perhaps also of touch, in its full functional efficiency.

Luciani points to the results of the researches of Kalischer, ${ }^{1}$ Horsley and Schäfer, in support of his views.

It remains to be shown that experimental removal of the parietal zone of Luciani does not give rise to disturbance of the perceptive process in any sensory field. A dangerous ambiguity is perpetuated in believing or in making believe that those who ascribe higher intellectual functions to the frontal lobes regard the cortical fields of the different sensibilities as not

${ }^{1}$ Kalischer. "Berichte Kgl. Preuss. Akad. d. Wissenschaf." Berlin, 1907. Arch. Anat. u. Physiol. 1909. 
possessing functions of a psychic nature. If we lay it down as a fundamental truth that the sensory spheres are organs which compose and conserve the images which are the material necessary for the higher mental constructions, we can understand that when the temporal or parietal or occipital lobes on both sides are removed, the mental capacity must be diminished to the extent to which these areas, with their sensory content, contribute to the working and development of the psychic processes. The consequent degradation, to a greater or less extent, of the entire mental capacity of the animal experimented upon is surely very obvious. There are good reasons why we should accept this view, all the more when we remember that in dogs the frontal lobe has not assumed control of the mental life, which revolves mostly in the sensory cortex. In the relations between the frontal lobe and the cerebral mantle, when the former is still in process of development, as in the case of dogs, we have a repetition of what we previously found in the relations subsisting between the cerebral cortex and the optic lobes in fishes, and even in batrachians, when the mantle is in its first stage of evolution. The same argument applies in the case of man, as the casualty wards of our hospitals show.

We, for our part, hold that the mental defect that follows removal of the frontal lobes affects all psychic manifestations in the case of the dog or of the monkey, notwithstanding the fact that the senses and their respective sensory areas are all normal and fulfil their functions in a regular manner, and, in the case of man, notwithstanding the fact that the areas of spoken and written language likewise remain normal and functionate regularly.

The terms of this problem cannot be altered. We still await a reply to the question: "What is the function of that large inexcitable mass of brain situated in front of the motor zone?"

According to Wundt ${ }^{1}$ the frontal lobes are the centre of apperception. Like most modern psychologists, he distinguishes what one might call a sensory perception- the noticing or simple perception of some object which excites our senses-and an attentive perception.

External stimuli, in order to furnish mental elements of judgment, must be perceived with the aid and intervention of the attentive faculty. The perceptions must be arrested in the field of consciousness, and recognised. Attentive recognition is what Wundt calls apperception. It is easy to understand that recognition can only happen when similar, dissimilar, or analogous images are reawakened, when, as James would say, they are called up into the associative fields, or, as the writer would put it, recalled from their respective perceptive fields.

It is evident, however, that to assign the faculty of apperception to the frontal lobes is much the same thing as ascribing to them the capacity for recalling from the sensory centres those images conserved therein and necessary for recognition, so that in this sense, although Wundt does not say so, the frontal lobe becomes an evocative centre-i.e. a centre regulating

${ }^{1}$ Wundt. Grundzüge der physiolog. Psychologie. Bd. 1. 
the mnemonic function of all the sensory centres. Flechsig appears, recently, to have accepted this view, as we shall afterwards see.

Meynert ${ }^{1}$ expressed the opinion that Hitzig's hypothesis exceeded the limits of physiological induction, and only served in a provisional way to fill a blank in our knowledge. In other words, it lacked a scientific foundation. On the other hand, Meynert believed that there was some ground for the view put forward by Munk, because he thought the upright posture of the body, the human gait with trunk erect, the manual labour of man (since his upper limbs are no longer employed in walking) would explain the existence of a large cortical motor area such as the frontal lobe, provided with a great mass of bundles of white substance which would constitute the anatomical substratum of the mental processes of the respective associations. This view is, of course, contradicted by the fact that the extensive prefrontal zone is not excitable, whereas all the muscles of the body are represented in the motor area, much behind the field of these experimental mutilations which, as a matter of fact, do not give rise to any paralysis.

Flechsig's ideas regarding the function of the frontal lobe are neither clear nor yet precise. He starts out with the fundamental notion that an associative zone is first and foremost an area to which are transmitted and in which are associated the products of the surrounding areas of sensibility. As the anterior associative zone (frontal lobe) is found between the tactile area (centre of sensibility of the organic ego) and the olfactory area, he infers from this that its function is, at least in part, that of transmitting the memorytraces of corporal (somatic) phenomena, so that this centre would play a predominant part in the formation of the ego, in so far as it is composed of feelings and voluntary acts and mnemonic traces of these. Nevertheless, he supposes that the sphere of activity of the frontal lobe is not confined to this function alone. He does not express any opinion as to whether or not its functions are essentially different from those of the great posterior centre of association. The function which this centre would have, in common with the other associative centres, would be that of combining various internal and external percepts, as well as calling up and combining their memory. traces. From this point of view the centres of association dominate the intellectual life and are the true organs of thought.

That Flechsig attributes a very high intellectual value to those centres can be inferred from the position he takes up, unsupported though it be by much clinical and experimental proof, when he asserts that the sensory image-records can only be reproduced by bringing into action the centres of association, without allowing for the intervention of the centres of sensibility. In the first chapter we have seen that all the clinical data in our possession are at variance with this mode of conceiving the centres of sensibility and centres of association. The sensory aphasic, e.g., has no longer the memory of words though he certainly has that of objects. He perceives,

${ }^{1}$ Meynert. Wanders. d. Sudwest d. Neurol u. Innerärate in Baden. 1883. 
imagines, desires and recognises objects with sight, touch, etc., but does not name them, because the centre, where are formed and conserved the phonetic images of the words expressing the respective objects, is destroyed. He has conserved the tactile, visual and auditory images of these objects and makes use of these as he did before he became aphasic. He remembers and recognises the places towards which he directs his footsteps. If his thought is interdicted, it is because he lacks nouns and verbs and the relative grammatical inflexions.

In this case the images of things themselves are capable of being recalled in a regular manner. When, however, there is an injury to the occipital lobes, the patient speaks, forming phrases and sentences, although he cannot reproduce the visual images of objects and places seen. Even here we are faced with a mental disorder, inasmuch as the conceptions are more difficult and imperfect, because the visual images, which are such a large part of the mental content and so necessary for recalling the respective words, are wanting.

Clinical observation, unbiassed and unprejudiced, goes to prove that the various special functions of sensibility and motility have their origin in different cortical areas, which are functionally and anatomically co-ordinated with one another. Flechsig ascribes the most important rôle in the development and display of the higher mental processes to the posterior associative zone, and he goes on to say that the height of the forehead depends on the volume of the sensory sphere, and this in its turn on the volume of the body. The height of the forehead is, in his opinion, not a direct index to mental power. ${ }^{1}$ He embraces a part of Munk's idea and puts forward the hypothesis that the frontal lobe is the greater kinæsthetic centre. He speaks of the Körperfuihlsphäre (body-feeling sphere) as the organ to which come the nerve fibres from the lower centres of circulation, respiration, hunger, thirst, well-being, etc. Critically examined, however, this statement appears rather arbitrary and improbable. Monkeys that have suffered mutilation of the frontal lobes behave, up to a point, like healthy monkeys. Not only do they move about and perform movements with a definite object, measuring distances and taking hold of things, but they also run after food stuffs and satisfy their appetite in a normal manner. Their chief instincts are preserved (hunger, thirst, sexual instinct). There is no experimental proof to show that the frontal lobes are a kinæsthetic centre and accordingly a centre of somatic consciousness (the organic ego, which is the nucleus of the personality).

The frontal lobe certainly plays a part in the formation of the ego in so far as it contributes, as we shall see, to the development of consciousness. This does not mean to say that the organic ego is formed in the frontal lobe, because all our experience goes to show that the cortical area, excitation of which modifies the organic functions, is that of the Rolandic convolutions. A few experiments with electric excitation of the frontal lobes, which seem

${ }^{1}$ Flechsig. Gehirn und Seele. I.eipzig, 1896; Dic Localisation der geistigen Vorgänge insbesondere der Sinnesempfindungen des Menschen. Leipzig, 1896. 
to have furnished proofs to the contrary, are open to considerable doubt and criticism, as will be seen when discussing the hypothesis put forward by Sciamanna.

Flechsig's view has been gradually modified. Briefly put it is as follows :-

"Granted that in lesions of Broca's area we have to deal essentially with particular disturbances of memory, this special form of amnesia concerns the frontal lobe in particular." These phenomena " are directly related to those internal phenomena which Wundt has grouped under the term apperception. I therefore speak of the frontal lobe simply as a centre of apperception." Since the apperceptive process is essentially one of evocation, and as he admits, a connection between the frontal lobes and the visual, auditory, etc. spheres, the frontal lobe thus becomes also a centre of attention and an evocative agent. On the other hand, the union of the nucleus of the somatic consciousness with the fields of the external impressions would be, as a whole, an exclusive function of the pre-frontal lobe, "and in this," he continues, "I find myself in full agreement with distinguished Italian investigators such as Bianchi, Sergi, Tamburini." By a process of interpretation, which it is needless to give in detail, he finds the bridge, " in Bianchi's demonstration of the relations of the frontal lobe with sociability and the social sentiments." He is induced to admit that "the most important factors of will are connected with the frontal associative centres," although, he adds, "it would be going beyond experimental facts did we seek to locate the seat of voluntary activity and of attention exclusively in the frontal lobe."

An attempt will be made to clear up this point in a succeeding chapter. Meanwhile, it is comforting to note the fact that such an acute and persistent investigator as Flechsig is coming into line with the conclusions which the author deduced from his first experiments.

Sciamanna ${ }^{2}$ set out to inquire whether there was any region of the brain the integrity of which was necessary and sufficient for the fulfilment of the more strictly intellectual functions. This is an erroneous way of putting the question and serves only to perpetuate ambiguity. It is further complicated by Sciamanna's hypothesis that in the frontal lobe "there reside centres of projection which are conjoined with the bulbar centres of organic innervation by means of ascending and descending paths." This hypothesis merely repeats Flechsig's first assertion in another form, all the more since, according to Sciamanna, emotional states would exercise a great influence over the associative dynamics, and, having regard to the organic basis of the emotions, one could thus understand the importance of the pre-frontal lobe in conscious and voluntary attention. ${ }^{3}$

1 Flechsig. " "Hirnphysiologie und Willenstheorie." Atti del Congresso Intern. di Psicologia. 1905.

${ }^{2}$ Sciamanna. "Le funzioni della corteccia cerebrale," ecc. Clinica Moderna. Anno 3a, 1897.

${ }^{3}$ Proceedings of the International Conyress of Psychology. Rome, 1905. 
The most reliable experimental findings do not bear out the truth of Sciamanna's hypothesis any more than that of Flechsig.

In the attempt to assign a large measure of importance to the frontal lobe in the mechanism of the emotions, Sciamanna transgresses the limits of well-founded experimental facts. Indeed, Luciani ${ }^{1}$ states that stimulation of any point of the motor zone excites the organic phenomena of emotion, if a weak or moderate current is employed. Mislawski and Munk obtained arrest in the inspiratory phase by exciting a point of the frontal lobe in front of the motor zone. W. G. Spencer, Langelaan and Beyermann obtained similar results. The two last-mentioned investigators defined an area, in the dog, situated at the extremity of the sigmoid gyrus where the coronary meets the presylvian fissure, excitation of which, with a weak current, produced respiratory acceleration followed by inspiratory arrest. ${ }^{2}$ The observations of Bochefontaine, François-Franck and Pitres, of Bechterew and Mislawski, of Sherrington, of Mosso and Pellacane, who have demonstrated vesical reactions following excitation of the motor zone, and those of Pfungen who provoked intestinal movements, do not bear out the truth of Sciamanna's conclusions. All these investigators have provoked the organic phenomena of emotions by exciting the sigmoid gyrus in animals or the bases of the superior and middle frontal convolutions in man (Langelaan and Beyermann). These phenomena are not elicited by exciting the pre-frontal lobe, provided strong currents are not employed.

It is true that some emotions reinforce the associative powers, but these are the higher emotions, the sentiments, not the primary and inferior emotions.

If the terms used by Sciamanna are to be interpreted in the sense of interest, to which Smith and others ascribe great importance in the determinism of human conduct, one might agree to accept the hypothesis mentioned. As we shall see hereafter, interest depends on the individual manner of feeling and on the sum and colouring of the ideas representing an object to be attained or a danger to be avoided. We know that ideas contain a mnemonic residuum of the emotions accompanying the sensations which originally went to form the content and essence of mind. Nevertheless, it is necessary to draw a distinction between interests of an inferior order (arising from organic desires, appetites, and pressing instincts ; those, in fact, most closely associated with the organic phenomena of the primary emotions) and social, moral, political, religious, economic and scientific interests, with which are bound up an infinite series of intellectual syntheses and relative resonances in the consciousness.

Sciamanna reproduces the author's view but expresses the opinion that clinical experience has not confirmed it, or at least has not given constant results. He writes as follows:- "In 1900 Bianchi stated his views thus: 'The frontal lobes serve for the conscious fusion of the two great activities

${ }^{1}$ Luciani. Loc. cit.

2W. Langelaan and H. Boyermann. "On the Localisation of a Respiratory and a Cardio-motor Centre in the Cortex of the Frontal Lobe." Brain, 1903. 
of the mind, the somatic-emotive and the intellectual, and are thus the organ of physiological connection of all the sensory and motor products of the other regions of the cortex. In disease of the frontal lobes the capacity for operations of intelligence of the higher order is lost, although the sensory functions themselves remain uninjured.' Having regard to the fact that clinical experience has not categorically demonstrated my assumption, and admitting that 'explicit experimental proof' is also lacking, one may none the less hold, on the basis of the inductive criteria above mentioned, that even if the frontal lobe by its influence on feeling can exert in man, more than in animals, an indirect influence over the mental manifestations, intelligence must nevertheless be the result of the work of the entire brain.'

From a practical point of view Sciamanna's experiments are of little value when it is considered that the portions removed from the frontal cortex of his monkeys were quite insignificant : 0.99 gramme from one lobe, 1.29 grammes from another, and a maximum of 2.73 grammes, which represent a very small fraction of the weight of the vault of the frontal lobe of either side in a small monkey. In point of fact, it was shown at the autopsy that the frontal lobe had been, in the main, preserved.

The same criterion should be applied to clinical experience. Only extensive bilateral destructions (and more particularly destruction of the left lobe) produce considerable and appreciable modifications of intelligence and character.

It is dangerous to draw anatomical and physiological conclusions from imperfect observations such as those of Langelaan, with the intention of locating the seat of any function in a particular part of the cerebral cortex. To maintain that the frontal lobe plays a part in the essence and mechanism of the emotions, as an organ which receives kinsesthetic images which would come into the field of conscious attention and thus exert an influence over the associative dynamics, is a bold hypothesis in which there is a good deal of mere conjecture and certainly no basis of proof.

The view that the frontal lobes are essentially an inhibitory organ has been put forward and maintained by eminent Italian physiologists. Indeed, it should be mentioned that the results of the author's investigations and the conclusions which he considered he was justified in drawing from them, met with very strong opposition in Italy.

Libertini ${ }^{1}$ stated the problem in accurate terms and attacked it with rigorous methods which afforded fairly definite results. He found shortening of the time of the tendonous reflexes after mutilation of the frontal lobes more in the anterior than in the posterior limb of the opposite side. This was more pronounced after extirpation of the left than after that of the right frontal lobe.

The notion that this shortening of the time of the reflexes was due to loss of the inhibitory function of the frontal lobes was rather contradicted by

${ }^{1}$ Libertini. "Sulla localizzazione dei poteri inibitori nella corteecia cerebrale. Ricerche sperimentali." Arch. per le Scienze mediche. Vol. xix.; e Arch. Ital. de Biologie. 
the investigations of Fano. ${ }^{1}$ This investigator excited the frontal lobe with electricity for five seconds and at the same time provoked a reflex by faradic excitation of the skin. He found that whilst the time of reaction was notably lengthened in the opposite anterior limb it was shortened in that of the same side, the myographic curve being at the same time lowered.

Oddi ${ }^{2}$ confirmed this finding by another method. He excited the fifth lumbar motor root with a faradic current, a metronome being interposed in the circuit, and produced rhythmical contractions of the gastrocnemius which were registered on a revolving drum. With another induced current, he then excited the pre-frontal lobe (previously laid bare) on the opposite side, and found that the myographic curve underwent a significant lowering. As the result of these experiments, Oddi denied that the frontal lobes possessed any activity of high psychic value. He stoutly maintained that they were centres of inhibition and also indicated the paths by which this inhibitive activity influenced the spinal medulla.

Polimanti ${ }^{3}$ arrived at pretty much the same conclusions. As the result of a series of experiments upon dogs he concludes that the most striking phenomenon following upon removal of a frontal lobe is the circus-motion of the animal towards the side of the injured hemisphere. These circusmovements are in a small circle during the first few days after the operation, and take in a large circle after several weeks. Both types of movement last only a certain time, then disappear. The longer or shorter duration depends on the extent of lesion; the more extensive the lesion, the longer the duration of the symptom, more especially when the lesion involves the "boundary-zones of the.frontal lobe" (i.e. the sigmoid gyrus).

Given the irresistible character of these circus-movements, Polimanti (who, a priori, favoured the rloctrine of the inhibitory function of the frontal lobe) accounts for them by the absence, to a greater or less extent, of restraining influence on the part of the boundary-zones. This eminent physiologist attributes to the frontal lobe what is essentially the function of motor innervation of the muscles of the nape of the neck, the head, and trunk of the opposite side (in this supporting the doctrine of Munk). He infers from his experiments that the loss of function of the frontal lobe destroyed is compensated by the surrounding areas when (as is generally the case) the circus-movements disappear, some days after the operation. This functional compensation he attributes to the substance bordering zone $1^{\text {a }}$ (frontal lobe) of the corresponding hemisphere and to the analogous area in the opposite hemisphere, a point in which he seems to agree with the views previously advanced by the author in regard to functional compensation. ${ }^{4}$

Leaving aside the question of compensation, it is worth while examining this author's views in some detail. He prefers to use the word restraint.

${ }^{1}$ Fano. Arch. Italiennes de Biologie. 1895.

2Oddi. Atti della Reale Accademia dei Lincei. Roma, 1895.

3 Polimanti. Contributo alla fisiologia ed all'anatomia dei lobi frontali. Rome, 1906.

"Bianchi. "Le compensazioni funzionali." La Psichiatria. 1883. 
After removal of the frontal lobes " there is wanting, at least during the first few days, that restraint which we must ascribe to zone $1^{\text {a }}$ (frontal lobe) and the parts bordering it."

It is a fact, however, that more intense and more prolonged circusmovements are always observed in dogs when a part of the sigmoid gyrus adjoining zone $1^{a}$ is destroyed, and this area is the most conspicuous part of the Fühlsphäre of Munk and, in the dog, is both motor and sensory.

If, then, very limited lesions of zone $1^{a}$ in the dog give rise to circusmovements of brief duration, if these movements are more marked and lasting when the lesion extends backwards to the sigmoid gyrus, and if, again, the same symptoms are obtained when we spare the frontal lobe and limit the experimental lesion to the sigmoid gyrus alone (not to mention other regions of the brain, injury of which gives rise to similar circus-movements), we have no right, strictly speaking, to infer therefrom that zone $1^{\text {a }}$ is the centre of inhibition of the movements of the head and trunk. It is well to bear in mind that one cannot judge the function of a given cerebral region from the symptoms that present themselves immediately after an experimental lesion, because these symptoms include the effect of circulatory and functional disturbances of neighbouring, and sometimes also of distant parts which have functional and perhaps also anatomical relations with the area destroyed in the experiment. One should not, it is true, exaggerate the significance of the phenomena of diaschisis, upon which Monakow lays a good deal of stress. At the same time, one must accept it as a fundamental rule that the investigator who seeks to determine the function of separate parts of the brain, by means of experimental lesions, must not judge the function of the part destroyed from the symptoms that appear immediately. He must wait for days, and often weeks, before he can arrive at a just appreciation of the permanent functional deficit, at the same time taking into account functional compensations and substitutions.

Polimanti draws attention to two other phenomena: (a) ataxia on the side opposite to the lesion; $(b)$ exaggeration of the deep reflexes.

1. The ataxia on the opposite side is more evident in the anterior than in the posterior limb and it disappears first in those cases in which the lesion has been limited or incomplete.

In another place we will deal with frontal ataxia associated with tumours of the frontal lobe in man, but here it is to be mentioned that, in the dog, the form of ataxia to which Polimanti draws attention is precisely that observed after destructive lesions of the sigmoid gyrus. This symptom, which the author has frequently observed, ${ }^{1}$ was also insisted upon by Schiff, ${ }^{2}$ who held that the ataxic symptoms presented by dogs after removal of the sigmoid gyrus were due to sensory disturbances, and resembled the ataxia produced after section of the posterior columns of the spinal cord.

${ }^{1}$ Bianchi. Sui centri motori corticali. Communication to the Association of Naturalists and Physicians. Naples, 1878.

${ }^{2}$ Schiff. "Dei pretesi centri motori negli emisferi cerebrali." Riv. Sper. di Fren. 1895. 
2. Exaggeration of the knee-jerks is a symptom that is constantly associated with a lesion of the motor area or pyramidal paths or parts surrounding the motor area. It is, indeed, one of the fundamental rules in the semeiology of cerebral affections that lesions situated in the neighbourhood of the motor zone or in the pyramidal paths give rise to exaggeration of the deep reflexes.

We also know that, apart from destructive lesions, any irritative process in the motor cortex causes exaggeration of the deep reflexes.* What is the use, then, of labouring the question of " a real and actual restraint . . . or a pronounced inhibitory effect" in the frontal lobe of the dog ?

The simple fact, which Polimanti does not dwell upon, is that after extirpation of both frontal lobes, dogs perform movements which have the appearance of irresistibility inasmuch as they are in constant motion or very nearly so, the pauses being of very brief duration. We will see afterwards what is the psyehological significance to be given to this symptom, already observed by Goltz, and described and interpreted by the author in Brain, 1895. Whether, as Polimanti thinks, the frontal lobe exercises a regulating and reinforeing action upon the movements of the limbs of the opposite side, like that ascribed to the cerebellum, save for a difference in degree, is a point that is very doubtful, as is also the question whether or not this influence is exercised through the paths that are supposed to exist between the frontal lobes and the cerebellum, the so-called fronto-pontocerebellar tract, which Polimanti failed to find in the dog. From his experiments upon monkeys he concludes that, in these animals, the frontal lobes "have no outstanding influence upon mentality, such as would warrant us in regarding them as the seat of the higher psychic manifestations." "To speak to-day," he continues, "of particular centres and organs presiding over intelligence as we speak of motor and visual centres, etc., is a very risky thing and would only be taking us back to the views of years long past."

Ivory Shepherd ${ }^{1}$ expresses a very different view. He is one of the few investigators who took such precautions as would enable him to detect alterations in the mental manifestations of cats and monkeys resulting from mutilation of the frontal lobes. In his investigations he followed the methods adopted by Thorndike. ${ }^{2}$ The animals to be experimented upon were trained to acquire new associations, and their mental capacity was then re-examined after mutilation of the frontal lobes. The same plan had already been followed by Hitzig and the author, but Shepherd's method was much more ingenious. After extirpation of the frontal lobes, cats were found to have lost the memory of what they had learned with regard to the food box, although, to an observer unacquainted with all the details, the cats seemed to be quite normal. In point of fact, some of the cats had lost their recent

* Because the pyramidal fibres originate in the deeper eell-layers.

'Shepherd. "On the Funetions of the Cerebrum." The Frontal Lobes. 1907.

2 'Thorndike. Animal. Intelligence and Food Boxes, and an article by this author in The American Journal of Physiology. August 1902. 
acquisitions (experimental), whilst their impulses, habits and inherited tendencies were preserved, as were also their emotional reactions.

So far as emotions are concerned, Shepherd made a special study of these in the monkeys and cats upon which he operated and found that they underwent no noteworthy alteration, although he observed a greater domesticity and friendliness in some of the animals.

Whether sentimentality and emotivity are or are not modified by removal of the frontal lobes is an open question. Shepherd carried out certain experiments with the express purpose of settling this point and he declares that the emotional reactions after removal of the frontal lobes remained the same as they were prior to the operation. Many of his cats showed the same familiarity as before the operation and were displeased when treated harshly. In monkeys, no difference was observed in the emotional responses. T'his keen and unprejudiced observer, however, does not hesitate to place before us certain doubtful points. "We cannot distinguish," he says, "between emotion, properly speaking, and emotional reaction, and it is consequently difficult, indeed impossible, to say that the emotions were not altered. We are only justified in concluding that the reaction remained the same; the emotions may or may not have been different."

It should be mentioned that separation of the frontal lobe was incomplete. In most cases it remained in relation with the brain behind, and it is not to be wondered at if the resultant changes in the affective reactions of the animals operated upon were slight. Nevertheless, from the sum-total of numerous experiments, to which further allusion will be made in a subsequent chapter, he arrived at conclusions which almost entirely confirmed the results of the writer's previous experiments, amongst them the following :-

(a) When the frontal lobes are destroyed recent acquisitions are lost.

(b) The loss of associations does not result from lesions of other parts of the brain.

(c) The loss of associations is not due to shock.

It seems that, in cats, mutilation of the frontal lobes may have some influence upon the organic metabolism, although he did not observe this in monkeys. Here it may be remarked that Ferrier found a marked alteration of nutrition in monkeys, after lesions of the occipital lobes but observed no appreciable difference after extirpation of the frontal lobes.

Roncoroni ${ }^{1}$ attempted to arrive at a judgment as to the function of the frontal lobes by examination of the intimate structure of the frontal cortex. In the writer's opinion, however, any conclusion as to the functions of the frontal lobe that is based on histological investigation is premature. When we remember that the myelogenetic geography of Flechsig does not agree with that of other investigators and that remarkable differences exist between the results obtained by Brodmann, Vogt, Campbell, and Bolton, and that

${ }^{1}$ Roncoroni. "Le funzion dei lobi prefontali," ete. Rivista di Patologia nervosa e mentale. 1911. 
a good deal depends upon the exact methods employed, upon the strict details of technique, and on the chemical state of the brain, it will be realised that we cannot found a doctrine upon cytotecture. Investigations in this direction are not calculated to throw light upon the question of the function of the frontal lobe.

Roncoroni is opposed to those who locate the higher mental activities in the frontal lobes. "We cannot admit," he writes, " that the more highly evolved faculties have any limited seat, nor can we accept as admissible those theories which localise intelligence, memory, will or consciousness in the frontal lobes."

This distinguished neurologist of the University of Parma makes use of the old notion, renewed by Monakow under the term diaschisis, that as the frontal lobe takes part in the associative overlapping with many or all of the other parts of the brain, a lesion of the frontal lobes disturbs the function of the whole brain. "If it is true," he writes, "that higher intelligence and emotivity require for their full development the integrity of all the psychic organs, it is clear that these will be generically altered by lesions of the frontal lobes just as they would by injury to any other part of the cortex." The frontal lobes, he thinks, are composed of several different cytotectural fields, fulfilling elementary functions which represent phases in the psychic reflex arc, the structure of which does not correspond either with that of the motor fields or with that of the sensory areas. "These," he writes, " have to do with the elementary functions of that part of the psychic reflex arc corresponding to the associations which manifest themselves on the threshold of the conscious act, in the pre-emissive period of the psychic reflex arc."

Roncoroni is not at all clear on this point. What, indeed, would be the elementary functions of that part of the psychic reflex are? The associations, it seems ; and associations of what? Of images, memories, experiences, emotions and actions! Now, the frontal lobes must either form these or evoke them-i.e. recall them from their respective areas. If this is his view, we can claim to be in agreement, to a certain extent, but agreement is impossible with the preconception of diaschisis, and this again is irreconcilable with the conception of elementary functions as applied to the frontal lobes.

If lesions of the frontal lobe disturb the psychic life, this, according to Roncoroni, is due to the fact that higher intelligence and emotivity (these high functions) require for their full development the integrity of all the psychic organs, " so that these functions would be altered in a generic fashion by lesions of the frontal lobes just as they would by injury to any other part of the cortex," for the simple reason that the prefrontal lobe, like any other part of the cortex, is a collection of fields and areas. From this point of view the frontal lobes become deprived of their great dignity. They are regarded as possessing no special function, and as merely providing an additional number of psychic fields and elementary functions, like any other part of the cerebral cortex. In its origin this conception has a physical basis. Suppose for a moment that intelligence emanates from the cortical fields (ares) like 
electricity from electric batteries; it becomes more intense and reaches a higher potential as the number of areas increases, just as the intensity of the electric current, ceteris paribus, increases with an increased number of batteries. It is difficult, however, to understand what Roncoroni means when he says that the frontal psychic arcs are precisely those which manifest themselves on the threshold of the conscious act in the pre-emissive period. It is a pity that Italians should adopt the involved form of language employed by some Germans who aim at originality. Unless we are mistaken, however, his " threshold of the conscious act in the pre-emissive period " would be the moment of deliberation in the volitional act which, again, is based on evocation and association of the memories of the experience of life that are the patrimony of the personality, comprising the knowledge of social obligations and the social sentiment, in which may be summed up the fiat of deliberation. Now, if destruction of other parts of the brain gives rise, as we know it does, to quite another kind of deficit, then the functioning of the brain is not to be likened to that of a group of electric batteries, or even an orchestra, for in the latter case the addition of new instruments serves to raise the musical art into the sphere of the highest and most wonderful harmonies.

We can agree with Lugaro, ${ }^{1}$ who partly accepts Flechsig's notion and puts forward, in another form, the view we have held for many years-viz. that the frontal lobes are the organ which "registers the story of the acts of all one's life (experience), the organ which feels the most intimate impulses of the organism, and elaborates the particular individual mode of reaction to external stimuli." Lesions of the frontal lobe do damage to the personality and alter the individual character. That is the constant finding. Not only animals operated upon but also many men who suffer from extensive lesions of the frontal lobes exhibit a profound alteration of character. The actions of those who have suffered damage to the frontal lobes are impulsive in character, whilst the actions of healthy, well-developed men are deliberate and based on experience which, again, is bound up with representations and imaginations. The historical experience of the ego is the result of representations of the modifications undergone under the influence of stimuli, in the first place, and then of actions performed and the consequences of these. If we are not mistaken, this representative power is based on evocation, in which case the actions are always the result of the impulse of the ego, according to its constitution, and also of the inhibition of impulses, which is generated by representations. Mental life in all its manifestations is the product of collaboration, in synthesis, of the representative and emotional contents of the personality, a point which we shall seek to make clear in another chapter.

Monakow ${ }^{2}$ joins issue with those who hold that the frontal lobes are organs for the higher mental processes. His conclusion is based on the

${ }^{1}$ Lugaro. Atti del Congresso della Società di Neurologia in Napoli. April 1908.

${ }^{2}$ Monakow. Gehirnpathologie, 1905, e Über der gegenvärtigen Stand der Frage nach der Localisation in Grosshirn. 1902. 
assertion that it is not the case that the development of the frontal lobe is a feature characteristic of the higher mammals. He states that in ungulates (horse, ox, goat) the frontal lobes form no less than 30 per cent. of the entire volume of the fore-brain, whilst the white substance of the frontal group of convolutions is much less developed. In the following chapter we shall see what truth there is in this statement.

In Monakow's opinion clinical observations do not furnish material sufficient to prove the functional rôle of the frontal lobes. This neurologist of Zurich admits a certain agreement between some of the results obtained in the old investigations of Goltz, Ferrier, Hitzig and Bianchi, but suggests that the operative technique did not fulfil the necessary aseptic requirements, and that it is consequently necessary to take into account secondary inflammatory processes which would not be without some effect upon the mentality of the monkeys operated upon. In support of his view, he mentions the results of the latest researches of Munk, ${ }^{1}$ Grossglick, ${ }^{2}$ and Horsley and Schäfer, ${ }^{3}$ which he regards as positive, in the sense that extirpation of both frontal lobes in the monkey did not give rise to any essential psychic alterations, nor did it affect the behaviour of the animals operated upon. Against the conception that the frontal lobes are exclusively an associative centre in the sense of Flechsig, he brings forward anatomico-experimental investigations in dogs and macaques in the brains of which, following mutilation of the frontal lobes, there was found undoubted secondary degeneration of the anterior and middle nuclei of the optic thalamus (Monakow and Rutishauser). This would prove that the frontal lobe possesses, at least in those animals, projection bundles which bring it into direct relation with subcortical ganglia.

Rossolimo (reported by Bechterew ${ }^{4}$ ) found sensory and motor disturbances as well as alteration of the psychic functions in dogs which had suffered mutilation of both frontal lobes. The former consisted in a weakening of the musculature and a kind of ataxia of the movements, the latter in a variety of psychic excitability, dysorientation, general diminution of the reflexes, enfeeblement of intellect, and marked lowering of the power of judgment.

Bechterew ${ }^{5}$ maintains that all questions regarding the localisation of the higher psychic functions can only be settled on the basis of experience and clinical observation. After a critical review of Flechsig's doctrine and a clear exposition of the author's researches and deductions regarding the function of the frontal lobes, he arrives at conclusions in perfect agreement with the results of the author's old and recent investigations.

1 Munk. "Ausdehn. d. Sinnes. in der Grosshirnrinde." Sitzberichten d. Akad. d. Wissensch, 1899, 1900, 1901; Id. "Folgen des Sensibilitätsverlustes der Extremitäten für deren motilitat." Sitzungsberichte d. König Preus. Akal. 1903.

${ }^{2}$ Grossglick. "Physiologie der Stirnlappen." Archiv. für Anat. u. Physiol. 1895.

"Horsley and Schäfer. "Functions of the Cerebral Cortex." Philosoph. Transactions. 1888.

4 Ioc. cit.

"Beehterew. Die Functionen der Ncrencentra. Heft 3. Jena, 1911. 
The alteration in character observed in dogs operated upon by Bechterew and by Zulowski in Bechterew's institute was very pronounced. At the outset the animals exhibited a condition of marked psychic arrest, laziness, somnolence, and reactive indifference as compared with what they exhibited prior to mutilation of the frontal lobes. With the disappearance, however, of symptoms observed during the early stage, there were observed other important symptoms related to the psychic field, and these exhibited the feature of persistence, for the animals were kept alive for one or two months. Two of them exhibited a high degree of irascibility and all showed signs of inaptitude, a lack of consideration, fear, amnesia, and incapacity to make use of previous experiences. Their habits and methods of eating were also markedly altered. They made no response to the calls of the attendant, and they hardly reacted to caresses or to threats. When caressed they manifested no friendliness; instead, there was trembling of the body and lowering of the tail. As a rule they were apathetic, although an occasional animal was restless, irritable and aggressive.

During the week following the operation, sight, hearing, and sometimes also the tactile sense, remained normal. The animals certainly recognised objects but had entirely lost that higher faculty of recognition which consists in associating the actual impression with that of past experience, and of acting in accordance therewith.

This higher faculty of recognition depends to a great extent on the process known in subjective psychology as judgment and consequential conclusion, a process which is very closely connected with attention and choice of action and, on the whole, is to be regarded as a regulative psychic activity.

Bechterew has long been recognised as an investigator and psychologist of undoubted authority, and one can reasonably assign great value to the results of these researches which were carried out in an unprejudiced manner. His experiments were very rigorously conducted and shed new light on the results of previous inquiries into this subject. 


\section{Evolution, Morphology, and Structure of the Frontal Lobe}

THE law of the progressive development of the brain, and of the cortex in particular, is confirmed by the evolutionary story of the frontal lobe.

It is well established that the frontal lobe makes its appearance very late in the evolution of the central nervous system. No trace of it is to be found in the lower vertebrates (fishes, batrachians, reptiles). In these the anterior extremity of the brain is represented by an extension of the hippocampic formation. Moreover, the structure of the cerebral cortex, when it first appears, is very simple. In the lower fishes, the cerebral hemisphere is

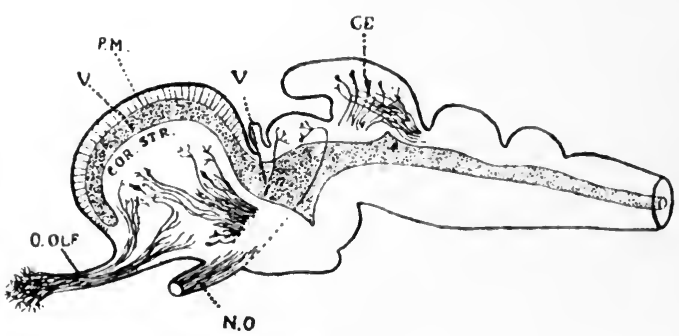

Fig. 24

O. Ol,F. Olfactory lolse.-N.O. Optic nerve.-V. Jateral ventricle.-P.M. Pallium membranosum.-CE. Cerebellum.-COR. STR. Corpus striatum (after Jacob)

represented by a mere membrane, in the structure of which there is as yet no true nervous substance (pallium membranosum). Even when nervous structure commences, the principal part of the hemisphere consists of the corpus striatum, more especially the caudate nucleus which, phylogenetically, may be regarded as an important motor organ, because it receives bundles of fibres from the olfactory apparatus. The corpus striatum, which is highly developed before the neopallium assumes its functions, receives not only olfactory fibres but probably also others coming from the visceral organs, and transmits nervous waves which are resolved into movements of locomotion as required in the search for nourishment, or respiratory movements, or those belonging to the sexual functions. In the lower fishes, the membranous pallium encloses a space, filled with lymph (cerebro-spinal fluid), which is the lateral ventricle, and the surface of the corpus striatum projects freely into this cavity. The membrane may be regarded as a protective organ (Fig. 24). By degrees it becomes replaced by the cerebral cortex. This occurs even in the higher fishes, but to a much larger extent in the higher vertebrates. Up to that point in the scale of fishes where the pallium 
membranosum begins to acquire a nervous structure, the corpus striatum is the sole organ which functions. In the myxine the pallium is composed of two layers of cells the structure of which is mainly hippocampic.

In amphibians we have the development of an episphere. The dorsal portion of this belongs to the olfactory sphere, which by degrees becomes

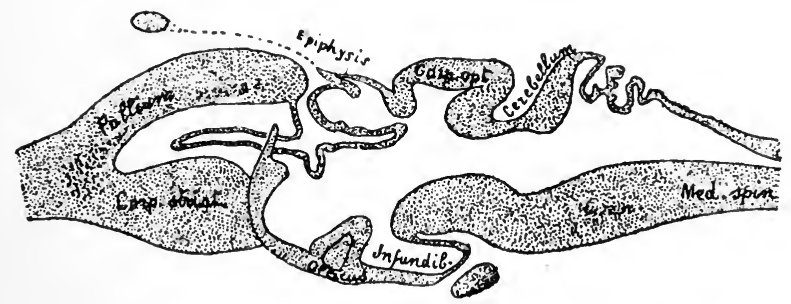

Fig. 25. - Brain of amphibian ; sagittal section (after Edinger)

circumscribed within the hyposphere. There are, however, points in the cerebral pallium of amphibians (as also, in that of the myxine and the petromyzon, though less numerous) where the cellular elements have no relations with the olfactory apparatus. In amphibians and, to a greater extent, in reptiles the cerebral mantle presents specially characteristic features in the cell-structure. The nerve-cells are at the same time more highly developed.

Figures 24, 25, 26, represent sagittal sections of the brains of a lower fish, an amphibian and a reptile, and show clearly that in these vertebrates

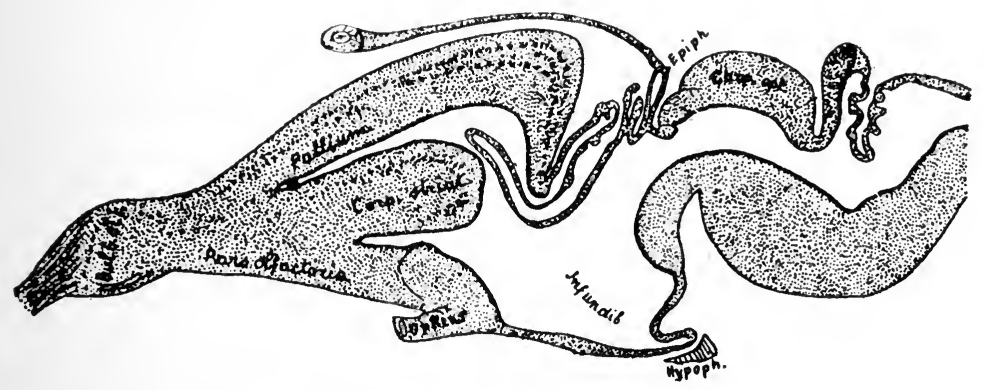

Fig. 26.-Brain of reptile ; sagittal section (after Edinger)

the fore-brain becomes gradually thicker, and at the outset seems, as it were, a prolongation of the central olfactory apparatus.

In birds, we find the whole mass of cortical substance much more developed. This development coincides with the commencing differentiation of the functions of the cortex, for experiments on the brain of the pigeon demonstrate the existence of an area, in the anterior and upper part of the hemisphere, which is capable of being excited with electricity.

In Figures 27, 28, 29, one can observe, at a glance, the progressive development of the fore-brain from fishes to batrachians and from these to birds, as well as the inverted volumetric relations between the fore-brain and the optic lobes. 
In the lower mammals we find an excitable area already distinctly differentiated and delimited. This is the motor zone. It occupies the anterior and external part of the hemisphere, on which now appears the forerunner of the crucial sulcus. In rodents this is located well forwards towards the anterior extremity of the hemisphere. It is short and shallow and is surmounted in front by a small rudimentary mass.

Figure 30 represents in diagrammatic fashion the brain of a rabbit. The area within the circle is approximately the motor zone, according to Ferrier's

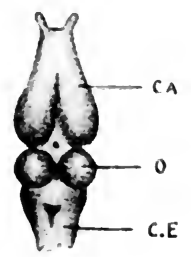

Fig. 27 Brain of fish

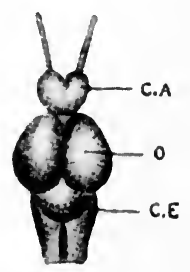

Fig. 28

Brain of frog

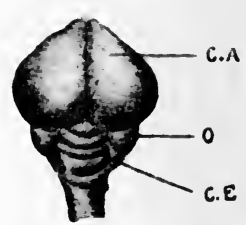

Fig. 29

Brain of bird

CA. Fore-brain.-O. Optic lobes.-CE. Cerebellum

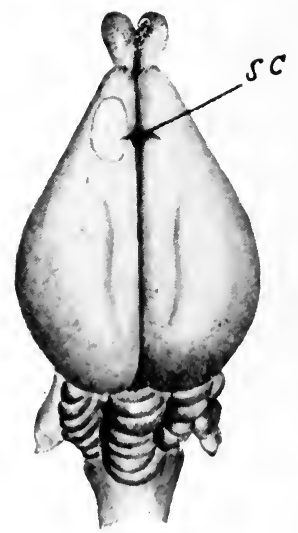

Fig 30.-Brain of rabbit

sc. Crucial sulcus

The circle represents approximately the motor area

experiments. ${ }^{1}$ The anterior extremity represents, as it were, an evolutionary outgrowth of the frontal pole of the pallium. In the higher mammals this anterior or frontal part is more or less developed, although it does not always bear an evident relation to the development of intelligence, so far as one can judge from the scanty observations hitherto made. For example, it is comparatively small in the brain of the rat, which manifests intelligence, but is relatively much smaller still in that of the dolphin, which is a stupid animal, although in it the parietal lobe has attained enormous development (Fig 31). ${ }^{2}$

1 Ferrier. The Funtions of the Brain. 1876.

2 Vine. Bianchi. "Il mantello eerebrale del delfino." Atti della R. Accademia delle scienze fisiche e chimiche di Napoli. 1905. 
It is only in the higher mammals that we find a more appreciable development of the frontal lobe. In these as, e.g., in the dog, we find the frontal extremity of the hemisphere not only more developed but also more distinct from the sigmoid gyrus owing to the appearance of the presylvian sulcus.

In the brain of the $\mathrm{dog}$, the hemispheric mass is already very large, the convolutions are more distinct, the sulci deeper and the frontal lobe well outlined (Fig. 32).

Amongst different members of the cat family (fam. felid $x$ ) the frontal lobe

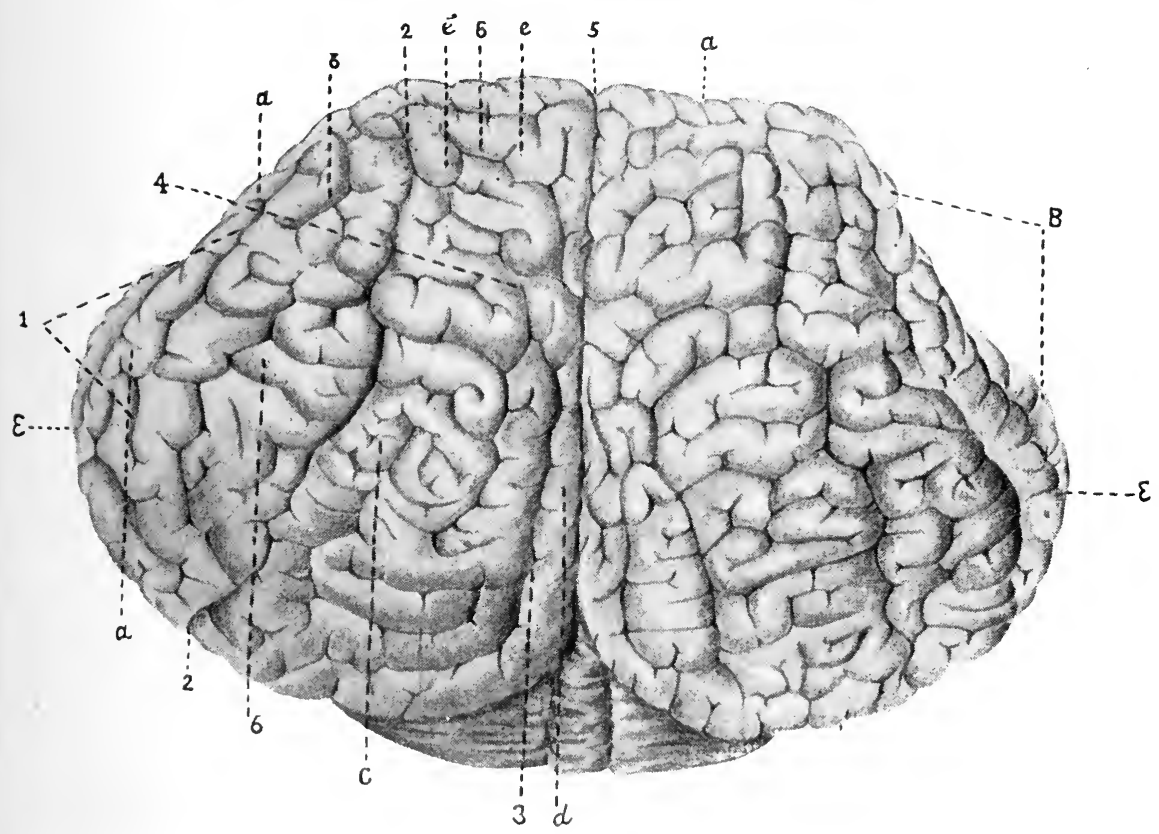

Fig. 31.-Brain of dolphin

1. Ectosylvian fissure.-2. Suprasylvian fissure.-3. Lateral fissure.-4. Ectolateral sulcus.5. Interhemispheric fissure.-6. Crucial sulens.-e. Anterior division of sigmoid gyrus. $e^{\prime}$. Posterior division of sigmoirl gyrus

shows considerable differences in size. In the ordinary cat, again, it is much less developed than in the dog. ${ }^{1}$

Some writers hold the crucial sulcus to be the analogue of the fissure of Rolando and the two branches of the sigmoid gyrus to represent the two central convolutions in monkeys and in man (Munk, Leuret). Others, again, including Broca, Meynert, Eberstaller, regard the presylvian fissure (the vordere Hauptfurchen of Pansch) as corresponding to the Rolandic fissure in man and in monkeys. In the former case the frontal lobe, though still fairly small, is quite appreciable and is continuous behind and above with the sigmoid gyrus. In the latter case one should have to regard only that part of the mantle lying in front of the presylvian fissure as pre-Rolandic, so that the frontal lobe would be represented by a very small portion indeed.

${ }^{1}$ See the excellent study of C. Winkler and Ada Potter. An Anatomical Guide to Experimental Researches on the Cat's Brain. Amsterdam, 1909. 
Eberstaller ${ }^{1}$ declares that in carnivorous mammals the frontal lobe is still very small if not rudimentary. Now, as the presylvian fissure is placed in front of and not above the sylvian fissure one can understand how the frontal lobe in this group of mammals is still small in size, as compared with the parietal lobe, which is much more developed.

Much discussion has taken place apropos of the development of the frontal lobe of the ox, the sheep and the horse, in relation to the intelligence of these animals.

In ruminants the frontal lobe would appear to be relatively more developed than in the dog, although, from what we know, the intelligence of the sheep

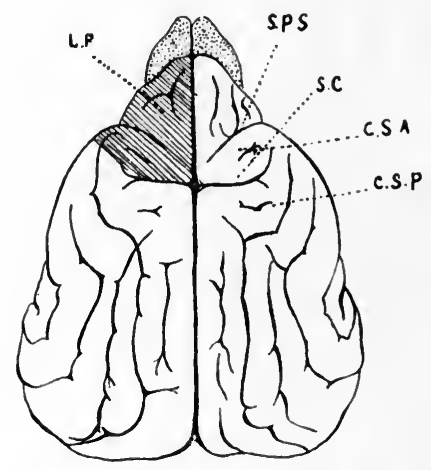

Fig. 32.-Brain of the dog

(From Ellenberger and Baum. Anat. deser. du Chien)

LF. Froutal lobe.-SC. Crueial suleus. -SPS. Presylvian fissure-CSA. Anterior signıid or pre-crucial convolution.-CSP. Posterior sigmoid or post-crucial convolution

and the ox is much inferior to that of the dog, leaving aside the intelligence of the horse, which some regard as still sub judice.

Tenchini and Negrini ${ }^{2}$ found a marked analogy between the cerebral cortex of man and that of the horse and the ox. These two investigators regard the convolutions of the frontal lobes as organs of perfecting. On the other hand, the study of mentality, as exhibited by the ox, the sheep and the goat, does not support the judgment founded on anatomical observation. How is it that these organs of perfecting should give such poor results in ruminants, which would appear to have well-developed frontal lobes? Either the conclusion is unfounded or the premises are wrong. The truth is that the frontal lobes are not so highly developed as has been affirmed.

It is of the utmost importance that this question of fact should be cleared up, all the more since Monakow points to the marked development of the frontal lobes in the domestic ruminants and to the notorious stupidity of

1 Eberstaller. “Das Stirnhirn." Ein Beitrag zur Anatomie der Oberflache des Grosshirns. Leipzig, 1890. One may profitably compare the valuable atlas of Jakob.

2Tenchini and Negrini. Sulla corteccia cerebrale degli equini e dei bovini, studiata nelle sue omologie con quella dell'womo. Parma, 1899, 
these animals as a strong argument against the physio-psychological dignity which I assign to these lobes.

With this object in view I have examined the brains of oxen and of sheep (Figs. 34, 35, 36, 37), the examination being conducted on the lines followed by Zimmerl. ${ }^{1}$ My results have been so confirmatory of the findings of this investigator that I venture to say the erroneous notion as to the great

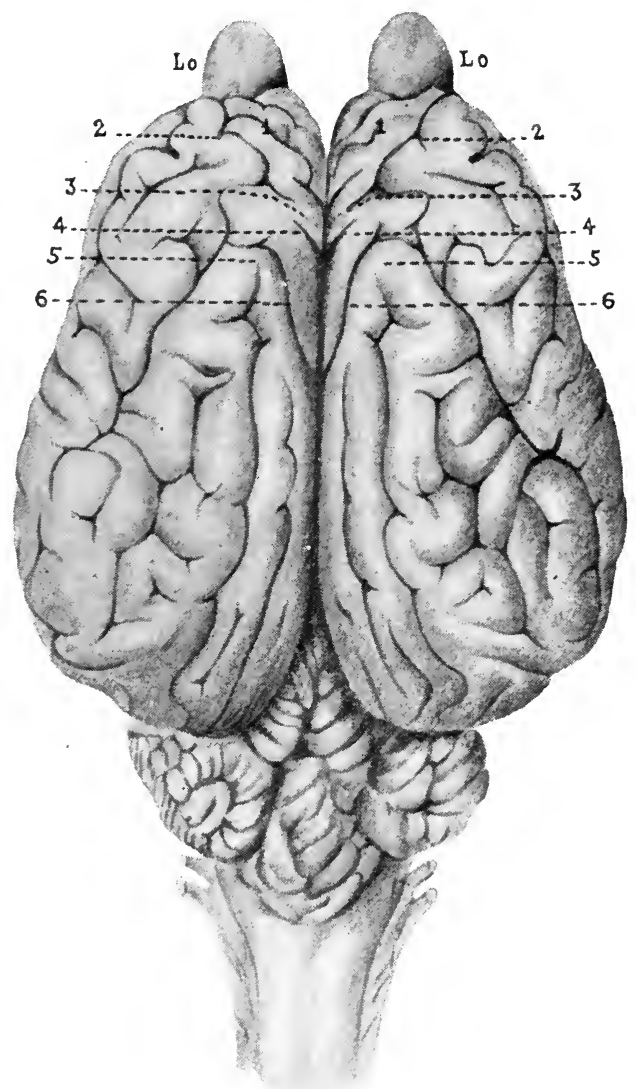

Fig 33. - Brain of horse

LO. Olfactory lobe. - 1. Orbital lobe.-2. Presylvian fissure.3. Sigmoid gyrus, anterior branch.-4. Crucial sulcus.5. Sagittal pole.-6. Accessory crucial sulcus

development of the frontal lobes in these mammals can be definitely disposed of, once and for all.

In Figure 33 I reproduce from Zimmerl's work the figure of the brain of the horse, and I have compared it with the brain of a horse which I secured for this study. If the crucial sulcus should correspond to that marked 2 in the figure almost nothing would be left of the frontal lobe, for the so-called orbital lobe, marked 1 in the figure, would have to be regarded as a part of the motor area. If the crucial sulcus corresponds to that marked 4, then,

${ }^{1}$ Zimmerl. Trattato di anatomia veterinaria. Vol. iii.: Sistema nervoso. 
when we take into consideration the fact that in the dog the sigmoid gyrus extends to the presylvian fissure and that it is more than probable that the

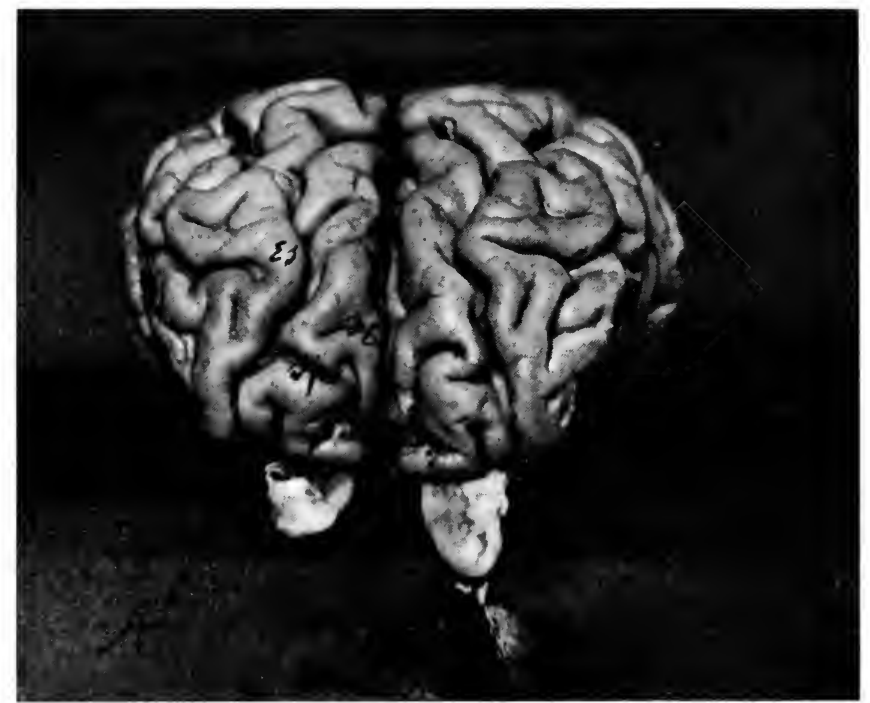

Fig. 34.-Sitperior surface, brain of ox, photographerl from above and in front

Es. Eetosylvian convolution,_sc. Crucial sulcus.-sp. Presylvian fissure

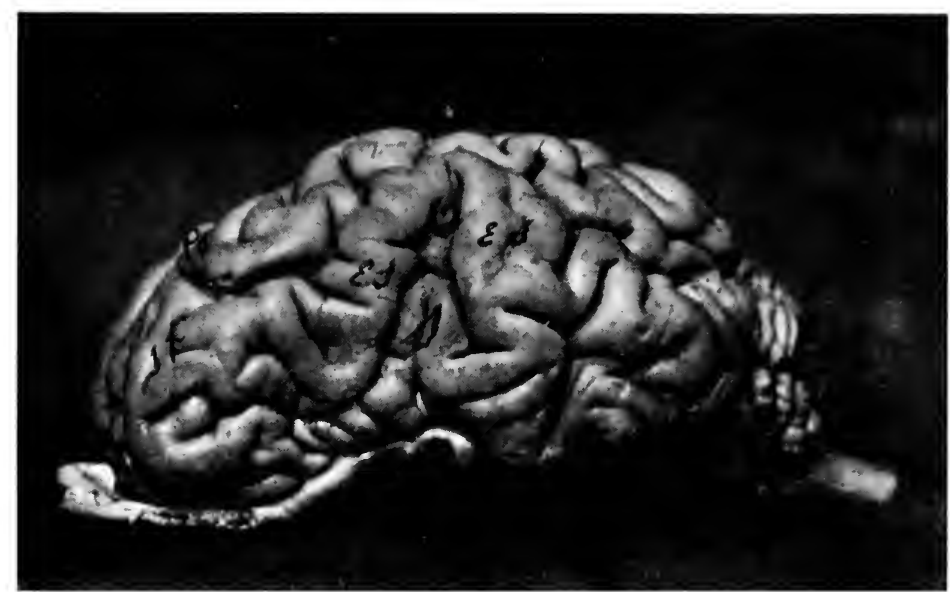

Fig, 35.-Brain of ox, external surface

$\therefore$ Fissure and convolution of Sylvius, - Fs. Ectosylvian convolution.-p,s. Sagittal pole.sf. Sylvio-frontal sulcus

same thing happens in the horse, which has an enormously developed musculature, the frontal lobe still remains very small, as compared with the great development of the occipito-parietal mass of the horse's brain.

The same thing holds good with regard to the brain of the ox.

In Figure 34, which is the photograph of the brain of an ox, taken from 
the front and close at hand, the crucial sulcus $(s c)$ is situated in front of the sagittal pole. Now, if we consider the extent of the motor area surrounding the crucial sulcus, we will be convinced that what remains in front representing the frontal lobe or, according to Zimmerl, the orbital lobe, is a very small part of the cerebral mantle, in fact only a portion of the convolutions 1 to 9 in the figure taken from Zimmerl (orbital lobe and anterior common convolution). It is further to be observed that the disposition of the convolutions of the cerebral mantles of the ox and sheep, which resemble that of the horse in almost every respect, is quite different from the plan of architecture in the brain of the dog or the monkey. The Sylvian fissure $(S)$ is

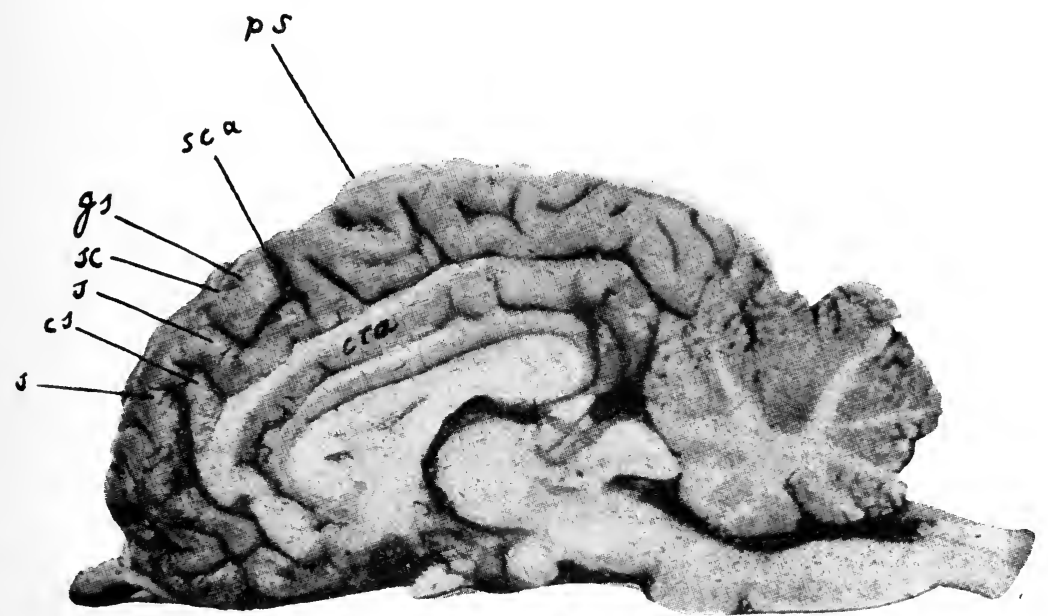

Fig 36. - Interhemispheric surface of the brain of the ox

Note the anastomosis of the convolution of the corpus callosum with the median surface of the sagittal convolution $c s$

ps. Sagittal pole.-sce. Accessory crucial sulcus. - gs. Posterior branch of the sigmoid gyrus.$s c$. Crucial sulcus. $-s$. and $s$. Median surface of orbital lobule.-es. Accessory calloso-orbital anastomosis

almost vertical (Fig. 35) and is found in front of a line dividing the cerebral hemisphere into two equal parts. The ectosylvian convolution, $E s$, is large, and is prolonged backwards as well as forwards where it is separated from the anterior common convolution (9, in Zimmerl's figure) by a sulcus which I would call Sylvio-frontal $(s f)$. The frontal lobe would thus consist of a part of the hemisphere situated in front of the presylvian sulcus (a part of the orbital lobe ${ }^{1}$ and a part of the anterior common convolution (9)).

On the external aspect, the crucial sulcus is not visible in Figure 35 but is situated in front of the sagittal pole, ps. When we take into account the extent of the motor area in front of $p s$, the development of the frontal lobe is very obviously rudimentary. One has to bear in mind that the sagittal convolution is. separated from the ectosylvian convolution by the parietal fissure which is very deep and prolonged forwards almost to the frontal pole, distinctly separating the ectosylvian convolution from the supposed motor area and from the orbital lobule, and it is this fact that has 
given rise to considerable doubt as to the extent respectively of the motor zone and of the frontal lobe.

On the anterior part of the median or interhemispheric aspect (Fig. 36) the crucial sulcus is marked $s c$. Behind it is the accessory crucial sulcus $s c a$, and behind that the sagittal pole, $p s$. The posterior division of the sigmoid gyrus on the median aspect is represented by $g s$. What remains in front of the sigmoid gyrus is the continuation on the median aspect of the region marked 1 (Fig. 33) on the external surface (orbital lobule). The convolution of the corpus callosum cca is strongly developed in ruminants and anastomoses to a variable extent (vide $c s$ ) with the orbital convolutions of which only a portion, in my opinion, goes to form the frontal lobe.

In any case the frontal lobe would be represented by the convolution which is the prolongation, to the pole, of the sagittal convolution which, on

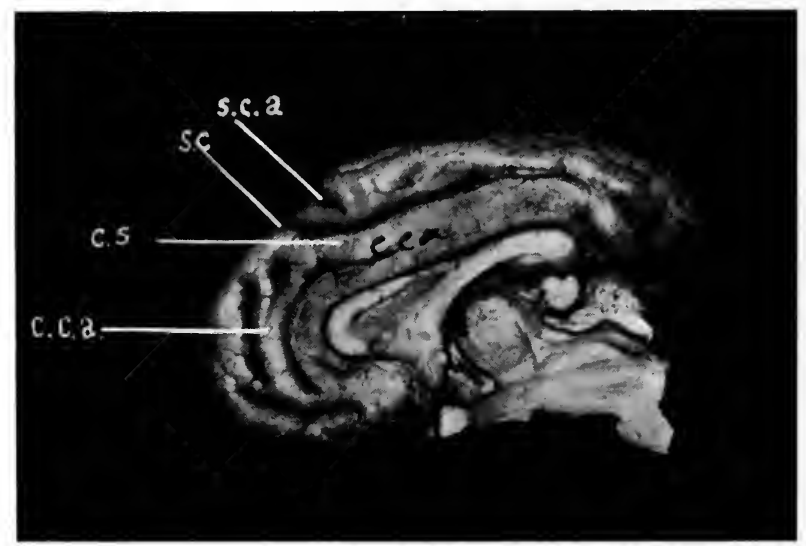

Fig. 37.-Brain of sheep, median aspect

sce. Accessory crucial sulcus. - sc. Crucial sulcus, - cec. Callosal convolution.cs. Calloso-sagittal anastomosis

the external surface, is distinctly separated from the ectosylvian convolution by the deep parietal fissure. This convolution, curving underneath, becomes the gyrus rectus and forms a hollow which receives the large olfactory bulb. Everything points to that part of the anterior lobe being an olfactory organ, all the more because this single convolution, along with the large olfactory bulb, constitutes the base of orbital surface of the frontal pole.

On the median aspect of the brain of the sheep, as shown in Figure 37, the crucial sulcus $s c$ is situated well forwards; $s c a$ is the accessory crucial sulcus. The convolution of the corpus callosum is enormously developed. It is divided by a superficial sulcus into two portions, the upper of which anastomoses with the sagittal convolution so that it is very difficult to decide how much of a frontal lobe there is in these animals.

I had hoped to carry out a series of experiments, with the object of defining the limits of the motor area in the sheep by means of electric excitation, but the absence of the hospital staff on military duty since 1915 prevented this. Still, when we take into consideration the relatively great size of the 
callosal convolution, $c c a$, and the fact that the sagittal convolution (which in front of the sulcus $s c$ curves underneath to become the orbital lobule) assumes considerable relations with the olfactory lobe--a fact which would lead us to believe that it is there mainly a hippocampic cortical organ-it

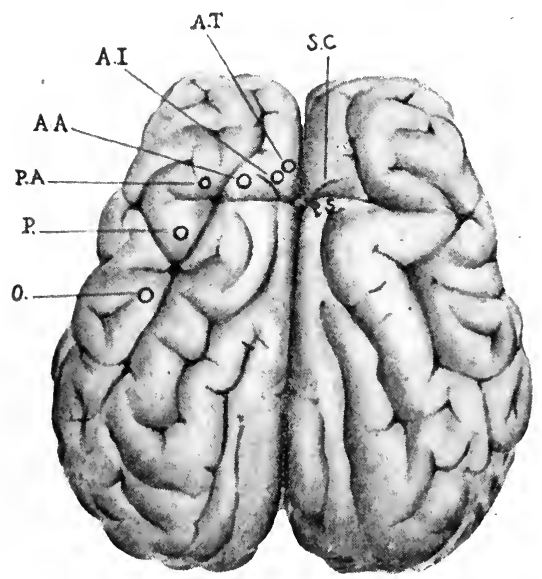

Fig. 38

PS. Sagittal pole.-SC. Crucial sulens,-AT, Exeitable area for muscles of neck and trunk,-AI. The same for hind limb.AA. Fore limb with less marked movements of posterior limb.-PA. Opening of eyelids. - P'. Closing of eyelids.O. Movements of ear

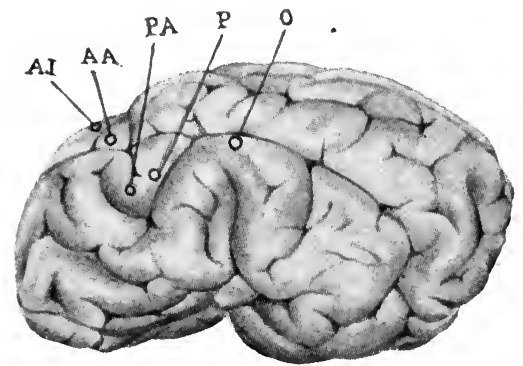

Fig. 39

O. Area for movements of ear.-P. Closing of eyelids.PA. Opening of eyelids.-AA. Movements of anterior limb and to a less extent of the posterior limb.AI. Movements of posterior limb and to a slight extent also the anterior

seems clear that the remaining part, which we can rightly regard as the frontal lobe, is a small portion.

Owing to the difficulties experienced during war-time I was able to carry out only one experimental inquiry, the results of which, however, I consider fairly conclusive. In a lamb three months' old I laid bare a large area of the brain. As usual, I measured, with a sledge, the minimum faradic current necessary to excite movements of the limbs and of the head, and with small platinum-tipped electrodes, provided with a hand interrupter, I was able 
to mark out with certainty, after repeated tests, the points of motor excitation indicated in Figures 38 and 39.

If these findings are correct and if it be admitted that the anterior extremity of the ectosylvian convolution has no part in common with the frontal lobe, the disproportion between the development of the parietooccipital portion and that of the part that can be assigned to the frontal lobe is so apparent that it is surprising how writers of authority still persist in bringing forward the weak argument that there is remarkable development

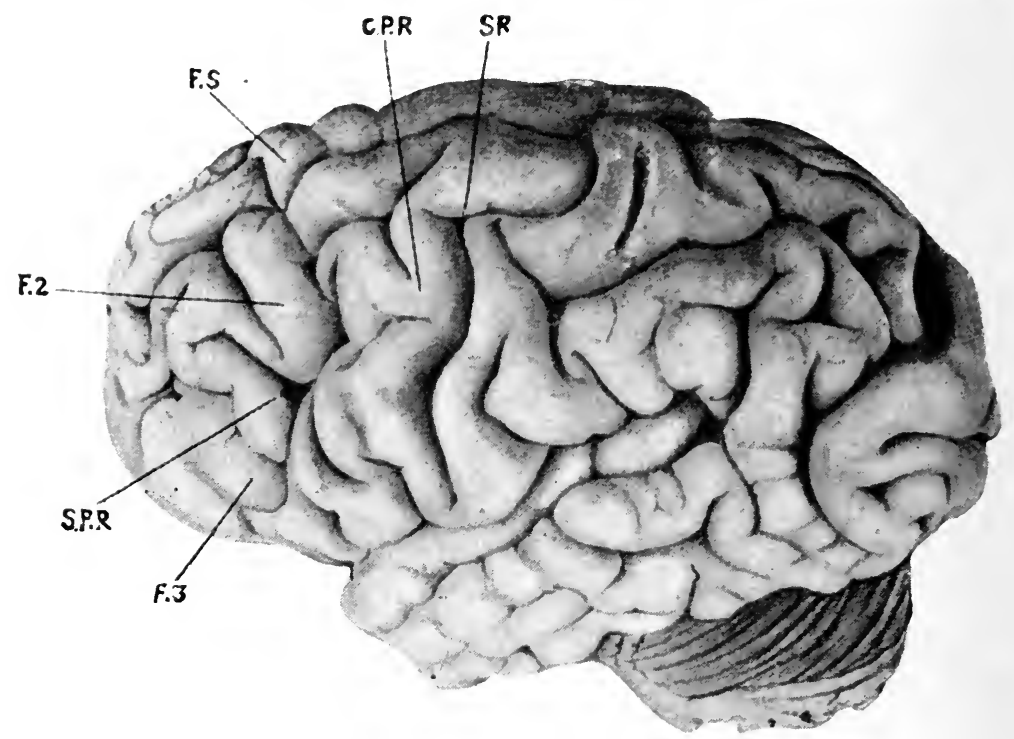

Fig. 40.-Brain of orang-outang

SR. Fissure of Rolando. - CPR. Pre-Rolandic convolution. - Fs. Superior frontal convolution.-F2. Middle frontal convolution.-F3. Third or inferior frontal convolution. SPR. Pre-frontal sulcus

of the frontal lobes in the ox and the sheep which are stupid animals, and that the parietal lobes are those which show a development more parallel with intelligence.

In monkeys and especially in apes (primates) the development of the frontal lobe attains a high degree. The brain presents the same architectural plan as in the human being, and at the same time its weight is much greater relatively to the weight of the body. Elementary cerebral functions are better localised, and the functional differentiation more pronounced, so that the functional areas are better defined, as compared with those in the cat and the dog, and correspond more closely to the plan of functional differentiation of the human cerebral cortex. In point of fact, it is on the frontal lobe of the monkey alone that it has been possible to arrive at a precise distinction of areas which behave differently under electric stimulation, or present differences in histological structure. These are, as we shall shortly see, the pre-Rolandic convolution, the pre-central intermediate area, the frontal area properly so-called, and the pre-frontal zone. 
It is well at this point to make reference to a delicate question concerning the morphology of the frontal lobe in monkeys. Is there a third (inferior) frontal convolution in apes? This is an important question because it would be wrong to judge a priori of the absence of an organ to which, in man, many ascribe a special function, simply because that function does not exist or is supposed to be completely absent in monkeys. Marchand ${ }^{1}$ has emphasised this point.

Whatever be the function performed by the third frontal convolution in man, be it or be it not the motor area for spoken language, it represents, in the monkey, an evolutionary phase of what it is in man. The fact remains that in the frontal lobe of the higher monkeys the third frontal convolution does exist. Marchand (loc. cit.) singles it out in the orang-outang. Beevor

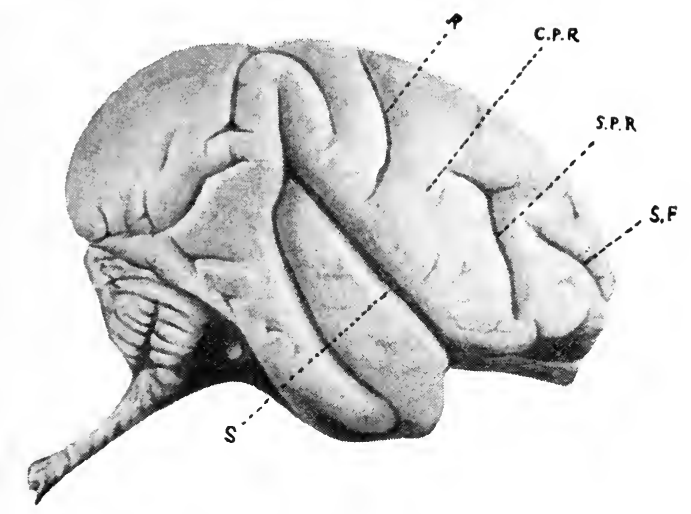

Fig. 41.--Brain of macacus (after Jakob)

S. Fissure of Sylvius. - R. Fissure of Rolando.-CPR. Pre. Rolandic convolution.-SPR. Pre-Rolandic sulcus. -SF. Frontal sulcus which separates the superior from the inferior frontal convolution

and Horsley found it also in the gorilla and the chimpanzee. In the two last named the third frontal presents the same features as in the human brain. It anastomoses with the inferior part of the ascending frontal and with the second frontal convolution. In those anthropoid apes the inferior frontal sulcus is clearly present, although it is much more superficial in the brain of the orang-outang (Fig. 40) than in that of the others.

The fact that in the foot of the third frontal convolution the layer of the large pyramidal cells is more distinct and thicker than in the first and second frontal, and the presence of a greater number of Betz cells (though this is denied by Brodmann) would support the doctrine of the motor function of the foot of the third frontal convolution. Its structural details are always Rolandic in nature, like those of the intermediate frontal area described by Campbell and Turner.

In tracing the phylogenetic development of the brain we observe a progressive increase of the frontal lobe which, almost throughout the scale,

${ }^{1}$ Marchand. Die Morfoloyie des Stirnlappens, etc. Jena, 1893. 
maintains a certain parallelism with the growth of intelligence. Even in the different species of monkeys we find a gradual development of the frontal

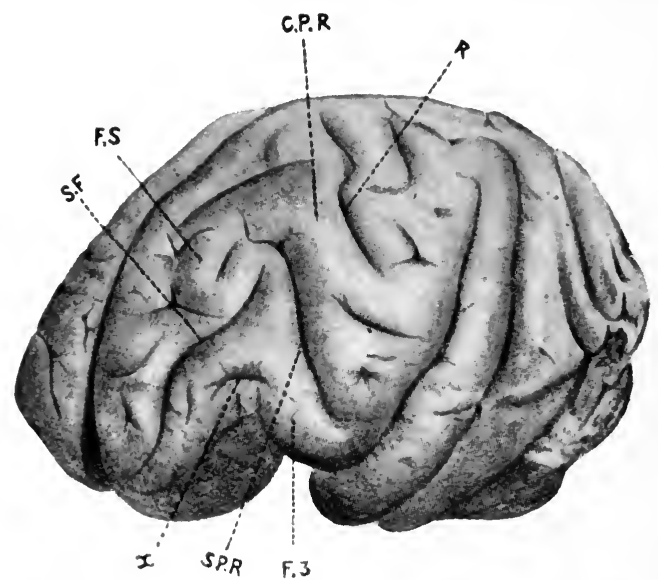

Fig. 42.-Brain of one of the cinocephalida which were the subjects of experiment

R. Fissure of Rolando.-CPR. Pre.Rolandic convolution.FS. Superior frontal convolution,-SF. Superior (1) frontal sulcus. $-x$. In ferior (?) frontal sulcus more marked lere than in the illustration taken from Jakob.-SPR. Pre-frontal sulcus.-F3. Embryo of the third frontal convolution, or orbital operculum of the frontal lobe

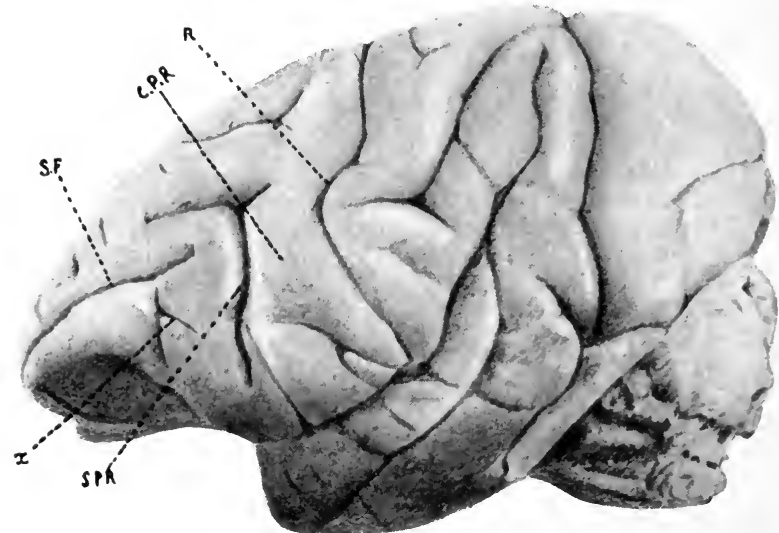

Fig. 43.-Brain of cinocephalus (Jakob)

R. Fissure of Rolando,-CPR. Pre-Rolandic convolution.-SF. Frontal sulcus. $-x$. Commencing of inferior frontal sulcus, - SPR. Pre-frontal suleus

lobe, from the macaques to the cebidx, then to the anthropoid apes, and finally to man.

The brain of the lower cibus (Fig. 41) is very simple. The pre-frontal sulcus is situated well forwards, the pre-Rolandic convolution is very broad, there is no trace of a second frontal sulcus. In the brain of the higher cibus we find a conspicuous number of small sulci on all the convolutions, including 
those of the frontal lobe (Figs. 42 and 43). Altogether, the frontal lobe has attained a relatively marked development. On the lower frontal convolution there appears a new sulcus which assumes different forms in different members of the same family (Figs. 42 and 43). There is every reason to believe that this is the forerunner of the inferior frontal sulcus, and that it is significant of the commencing distinction of a third frontal convolution, which is then found more developed in the orang-outang, the gorilla, and the chimpanzee.

Taking into account what we have learned of the crucial sulcus and the architectural plan of the convolutions related to it, it seems a very suggestive hypothesis that the development of the frontal region of the human brain has proceeded upon lines already distinctly laid down in the lower apes. ${ }^{1}$

The superficial extent of the cerebral mantle in the middle-grade monkey cebus), in the orang-outang, and in European man, respectively, is in the proportion $1: 5: 17$. The total volume of cortex is as $1: 5: 24$. In richness of cells the ratio is $1: 8$ in the cibus and orang-outang, and $10: 100$ in the orang-outang and man. ${ }^{2}$

The total number of cells in the cortex of the orang-outang is one thousand millions as compared with ten thousand millions in man. The entire brain participates in this enormous development, but the great mass of the frontal lobe more than any other part. As a matter of fact, if we take into consideration the entire length of the hemisphere between the two poles of the human brain, the frontal lobe from its anterior tip to the centre of the fissure of Rolando occupies 40 to 41 per cent., and in some cases even more, of the length.

There is no lack of proof which controverts the statements of Flechsig and Luciani. If we trace the development of the brain in mammals it is precisely the anterior part which develops, as one might say, ex novo. The frontal lobe of lower mammals-e.g. the dolphin-contains a smaller number of cells, and these are less differentiated as compared with the cells of other regions. $^{3}$ At most it may be said that the development of the temporal lobe is also very conspicuous in apes and in man, so that the fissure of Sylvius, which is vertical in the brain of the horse and of the ox, becomes inclined at a considerable angle in the brain of the anthropoid apes, and much more so in the case of man in whom, indeed, it becomes almost horizontal. The extraordinary development of the temporal lobe, which remains separated from the parietal by the fissure of Sylvius, gives an illusory appearance of great size to the parietal lobe, although it, too, undoubtedly shows development.

My conclusions are drawn from comparative anatomy. From the cat to the dog, from the latter to the macacus, the cebus, the gorilla, and finally to man, there is a constantly increasing prominence of the frontal lobe, which

"Sydney Cole. "The Comparative Anatomy of the Frontal Lobe." Journ. of Ment. Science. 1911.

2 Jakob. Loc. cit.

${ }^{3}$ Vin. Bianchi. Loc. cit. 
assumes the appearance of a new region in relation to the other parts of the cerebral mantle, although these all develop and increase in size, especially, as has been remarked, the temporal lobe.

That the parietal has not attained a development proportional with that of the frontal lobe had already been noted by Broca, ${ }^{1}$ who laid stress upon the contrast between the frontal lobes and the parietal and limbic lobes in point of development, at the same time ascribing to the frontal lobes the higher faculties predominant in intelligent mammals.

Meynert maintains the view that the great proportions attained by the frontal lobes are more apparent than real, being due in great measure to the growth in height of the corpus striatum, the insula and the temporal lobe. There is some measure of truth in this so far as the influence exercised by the temporal lobe is concerned. With regard to the corpus striatum it is to be observed that a great part of the substance of the pre-frontal lobe is found in front of the anterior pole of the corpus striatum, as will be seen from the figures in Chapter IV. showing vertical sections of brains that were subjected to experimental mutilations.

It is needless to enter into great detail or to review at length the literature dealing with the limits and conformation of the frontal lobes in man. The position may be summed up thus: On the external aspect, the limits of the frontal lobe are easily defined. They are the fissure of Rolando behind, the fissure of Sylvius below, the interhemispheric fissure above and within, and the free extremity of the pole in front. It is only when we seek to define the limits on the lower or orbital surface and on the median or interhemispheric aspect, that difficulties arise.

As regards the external surface, it is well to clear the way of all discussions as to whether or not the ascending frontal convolution belongs to the frontal lobe. The arrangement and implantation of the frontal convolutions are such that, at a glance, one is persuaded that, from a morphological point of view, it is the fissure of Rolando, and not the pre-frontal suleus, which forms the posterior limit of the frontal lobe and that the ascending frontal convolution forms a part of the frontal lobe.

The morphological features of the surface of the brain do not coincide with the functional facts. In other words, the convolutions and sulci neither constitute nor limit, in any regular fashion, organs with specialised and determined functions. The detailed study that has been made of the convolutions and sulci forms a rich and brilliant chapter in morphology, phylogenesis and ontogenesis of the cerebral mantle; it has been utilised as a ground for contending views regarding mental pathology, criminal anthropology and general anthropology, but it has not been a profitable chapter in the physiology and pathology of the brain. All attempts based on these grounds have ended in failure and have hindered a truer conception of the functions of the cerebral cortex. Each individual lobe may very well be composed of parts having different functions and, further, each single

${ }^{1}$ Broca. Localisations cérébrales. Recherches sur les centres olfactives, et mémoires sur le cerveau de l'homme et des primates. 1888. 
convolution may have different parts with varying eytotectural features and different functional and psychic arcs. Indeed these two propositions can be put forward with a fair amount of assurance: (1) no function of the cerebral mantle coincides strictly with any particular region that is defined on a purely morphological basis; (2) the indications for functional areas can be determined only by experiment and human pathology, though in the future they may be provided also by cytotectural studies. When, therefore, we say that the frontal lobe is limited behind by the fissure of Rolando we merely speak of an organ the different parts of which have different functions. The ascending frontal convolution, as already mentioned, is a motor area. It extends behind into the fissure of Rolando and, in front, near the feet of the three frontal convolutions, it merges into the area now known as the precentral intermediate area.

According to histologists the precentral intermediate area occupies a strip of the pre-Rolandic convolution in front of the motor area, and at its upper portion it extends forwards on to the superior frontal convolution for about $15 \mathrm{~mm}$. in front of the pre-Rolandic sulcus. Thence it describes a curve with convexity behind to a point near the centre of the pre-Rolandic convolution, curving forward again on to the third or inferior frontal convolution (Figs. 44, 45, area 6 ; and Figs. 46, 47, area MI) and extending underneath, on the orbital aspect of the frontal lobe, to a part of the transverse portion of the third frontal convolution. It thus embraces the foot of the superior and middle frontal convolutions, a part of the ascending frontal, and a fairly large portion of the inferior frontal, including Broca's area and the orbital part of the frontal operculum. These limits are not absolutely fixed and determined, but, according to Campbell, are fairly easily recognised. Campbell insists that the area in question coincides with the experimentally excitable area in monkeys and that its limits correspond to some extent with the anterior limits of the so-called somæsthetic or tactile zone of Flechsig (Figs. 5 and 6). In monkeys (cebidos) I have found the anterior portion o, this area inexcitable when employing a current just sufficiently strong, when applied to the ascending frontal or pre-Rolandic convolution, to induce movements of the limbs, except in the case of the first frontal convolution where, over a considerable area, electrical excitation induces movement of the trunk and head (Fig. 51). Campbell supposes that all this area, which in structure closely resembles the motor zone, serves for the more specialised and delicate movements in which consciousness and will play no small part. This, he thinks, explains why the foot of the third frontal convolution is the central organ of spoken language in man, and that of the middle frontal, the organ of other more delicate and complex movements of the fingers such as those of writing.

The view which has just been expressed and which agrees with that of Mott ${ }^{1}$ and with the view put forward by the author in $1883^{2}$ when he regarded

1 Mott. "On the Physiological Significance of the Convolutional Pattern in the Primates." Brit. Med. Journ. 1906.

${ }^{2}$ Bianchi. Le localizzioni cerebrali. Ed. Pasquale. Naples, 1883. 
that area as a zone of evolution, is not supported by experiments upon the gibbon. This is owing to the fact that, in the brain of the gibbon, the area in question covers a wider extent than in that of man, especially on the foot of the third frontal (experiments of Sherrington, Schuster and Mott ${ }^{1}$ ), a fact which takes away a good deal of value from the physiological interpretation

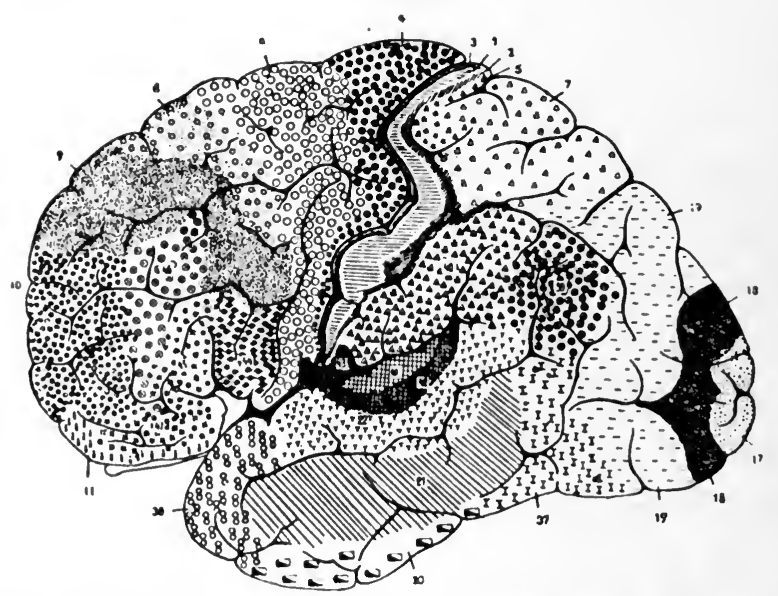

Fig. 44.-Histotectural geography of the cerebral cortex. External aspect (after Brodmann)

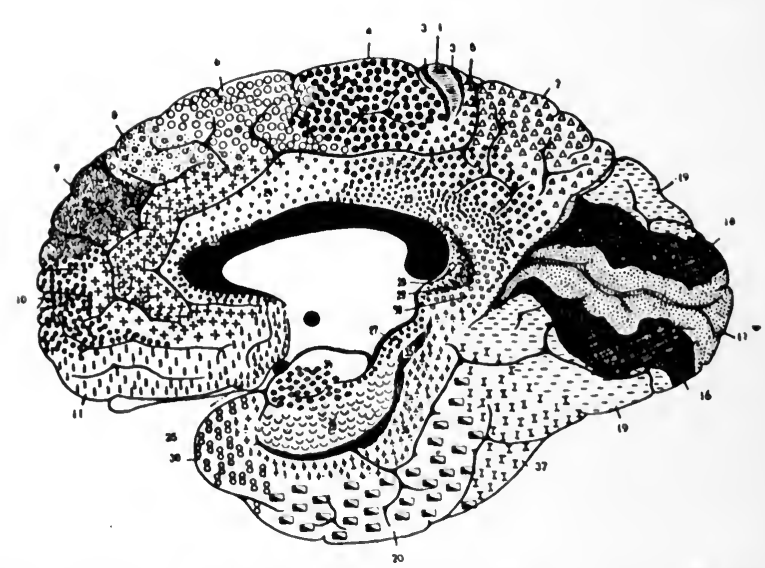

Fig. 45. - Histotectural geography of the cerebral cortex. Median aspect

given to this area by Campbell, because it is obviously in man, if anywhere, that one should expect to find the excitable zone attain its greatest extent.

It is sufficient to compare the great extension forward of the motor zone in the brain of the gibbon, which has been explained by the remarkable use it makes of its hands (Fig. 48), with the corresponding area in man who makes ever so much more use of his hands than the gibbon. In man, the motor zone in one sense extends in front of the limits of the ascending frontal, although in another sense it does not. The contradiction is striking.

${ }^{1}$ Sherrington, Schuster and Mott. Loc. cit. 
In Figure 48, e.g., the larger dots, starting from the fissure of Rolando, indicate the motor area which contains giant pyramidal cells and would correspond to the true motor zone or Brodmann's histotectural type No. 4

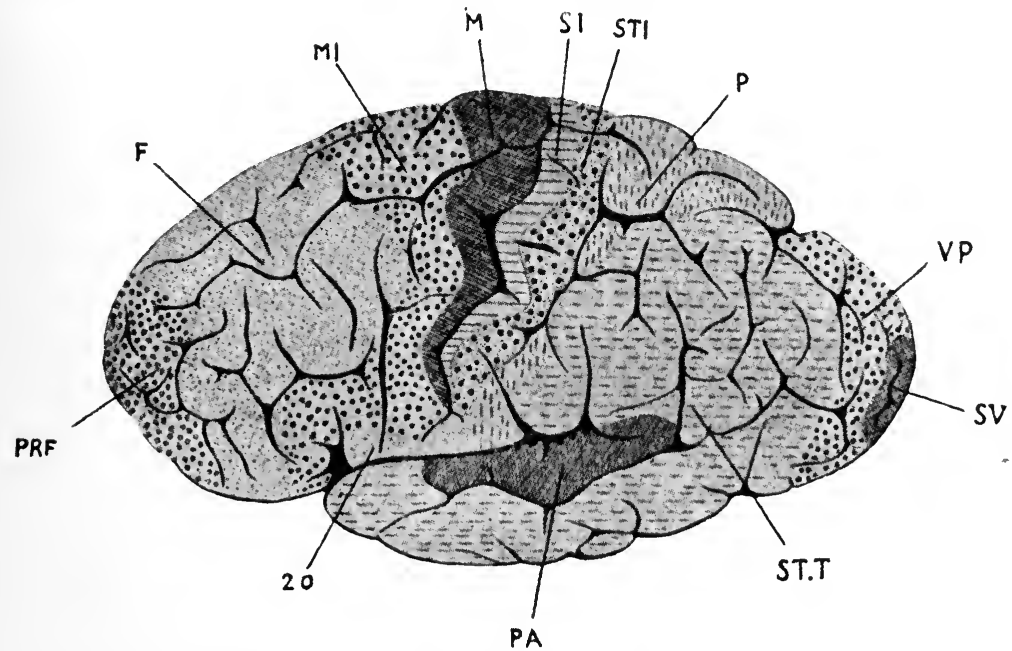

Fig. 46. - Histotectural geography of cerebral cortex. External aspect (after Campbell



Fig. 47.-Histotectural geography of ccrebral cortex. Median surface

(Fig. 44). ${ }^{1}$ The smaller dots indicate the intermediate motor area which would correspond to the type marked No. 6 in Figure 44.

I have personally been unable to satisfy myself regarding these distinctions even in my most recent investigations.

In all my researches I have investigated the whole of that part of the

1 Brodmann. " Beiträge zur histologischen Lokalisation der Grosshirnkinde." Journ. für Psych. und Neurol. 1905. 
frontal lobe which remains in front of the pre-Rolandic convolution, including a part of the intermediate motor area. This region comprises the two areas distinguished by histologists as the frontal and prefrontal, besides a part of the intermediate motor zone. The structure of the frontal area differs somewhat from that of the pre-frontal area, properly so called. On the external surface of the hemisphere, commencing at the limits of the intermediate zone, the frontal area extends on to the anterior half of the superior frontal convolution, embraces a good part of the middle frontal, and takes in the anterior extremity of the inferior frontal, as well as a small area on the orbital surface. On the interhemispheric aspect it occupies the upper portion of that part of the superior frontal or marginal convolution which lies above the genu of the corpus callosum (Fig. 48a). It differs in structural

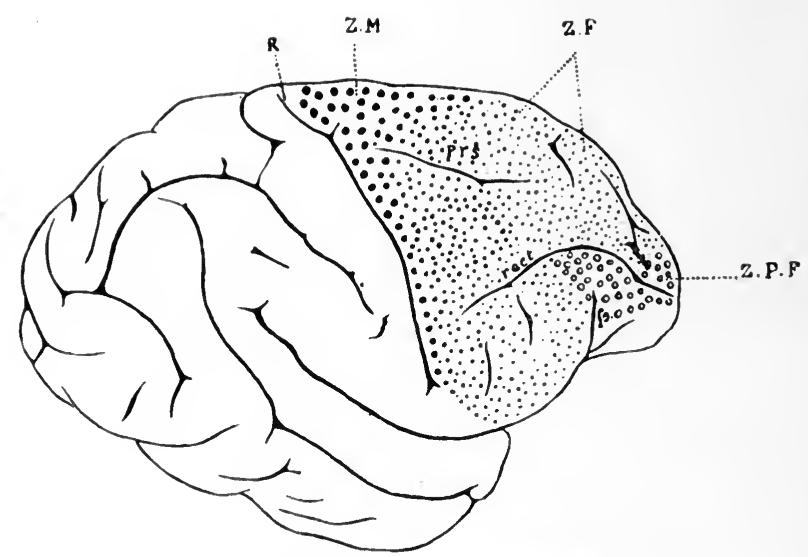

Fig. 48. - Brain of gibbon

R. Fissure of Rolando.-ZM. Motor zone.-ZF. Frontal zone in continuation of the intermediate area. $-Z P F$. Pre-frontal zone

details from the intermediate area for there is an almost complete absence of fibres of large calibre, although it contains some of medium calibre. According to Campbell, the difference is most marked in the layẹ of large and small pyramidal cells, these being fewer in number and smaller in size.

The pre-frontal zone includes what one might call the polar part of the superior frontal convolution on the interhemispheric surface, the polar extremity of the second frontal and all the orbital surface situated in front of the transverse orbital sulcus (Fig. 47). According to Campbell, this area is even less developed than the frontal area and the fibres it contains are very few in number and very fine in calibre. The association-fibres are especially deficient. The pre-frontal area might be said to be still in process of evolution, its development being very late as compared with the other areas of the frontal lobe. There is a marked difference between primates and man in the structure of this part of the brain, and one would be justified in admitting also a considerable difference in development in different men. 
Bolton, ${ }^{1}$ on the other hand, maintains that the structure of the frontal lobe closely resembles that of the visuo-psychic area. There is the same number of cell-layers but the superficial layer shows a considerable complexity of fibrils. The second layer, that of the pyramidal cells (the smaller external and the large internal), shows a wealth of cells and fibres as do also the lower strata. The columns of Meynert are especially in evidence and contain many large fibres. Bolton arrives at the conclusion that, so far as richness in neuro-fibrils is concerned, the prefrontal region probably excels all other regions of the cortex. The fact that the frontal lobe is the last region of the

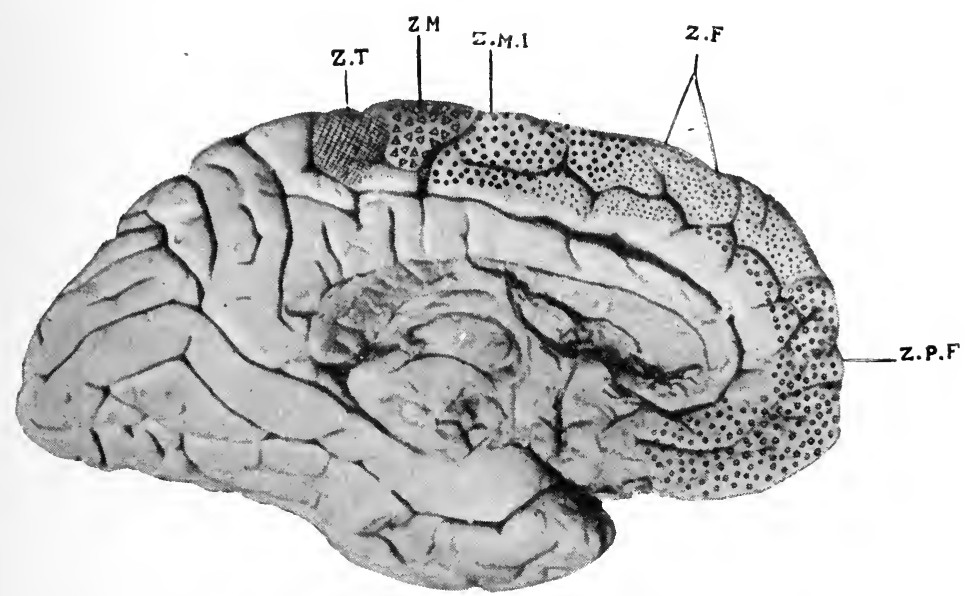

Fig. 48a.--Median aspect of the cerebral hemisphere

ZT. Tactile area.-ZM. Motor area.-ZMI. Intermediate motor area.-ZF. Frontal area.ZPF. Pre-frontal area

brain to develop gives ground for regarding it as an organ of great importance for intellectual functions.

Bolton thus obtained results very different from those of Campbell, whose methods he considered defective. His findings, however, agree with those of Turner, who also found a remarkable richness of fibres and cells in the area in question.

These results seem all the more worthy of consideration in respect that the layer of pyramidal cells in the frontal lobe is the last to be developed, a finding which agrees with the results of the investigations of Watson ${ }^{2}$ and Brodmann. ${ }^{3}$

It is a remarkable fact that the layer of pyramidal cells is found more or less sub-evolved in phrenasthenics in proportion to the degree of imbecility or idiocy, and it is the first cerebral organ to undergo the degenerative process in the various forms of dementia, a fact which is in agreement with the

1 Bolton. "A Contribution to the Localisation of Cerebral Function." The Goulstonian Lectures. 1910.

${ }^{2}$ Watson. "The Mammalian Cerebral Cortex with Special Reference to its Comparative History." Arc. of Neurol. 1907.

${ }^{3}$ Brodmann. U̇ber den allgemeinen Bauplan des Cortex Pallii bei den Mammaliern und zwei Homologe Rindenfelder in Besonderen, etc. Mitteilungen, 1906. 
general law that dissolution proceeds in the inverse order of evolution. The first organs to degenerate are those which were the last to appear in the evolutionary scale. Flechsig's researches, which have demonstrated the lateness in maturity of the fibres of the frontal lobe, are in agreement with those of Campbell, Bolton and Brodmann.

The orbital surface of the frontal lobe is in part taken up with anatomical relations with the olfactory tract and bulb. The first frontal or internal convolution on the orbital surface, or, rather, that part of it known as the

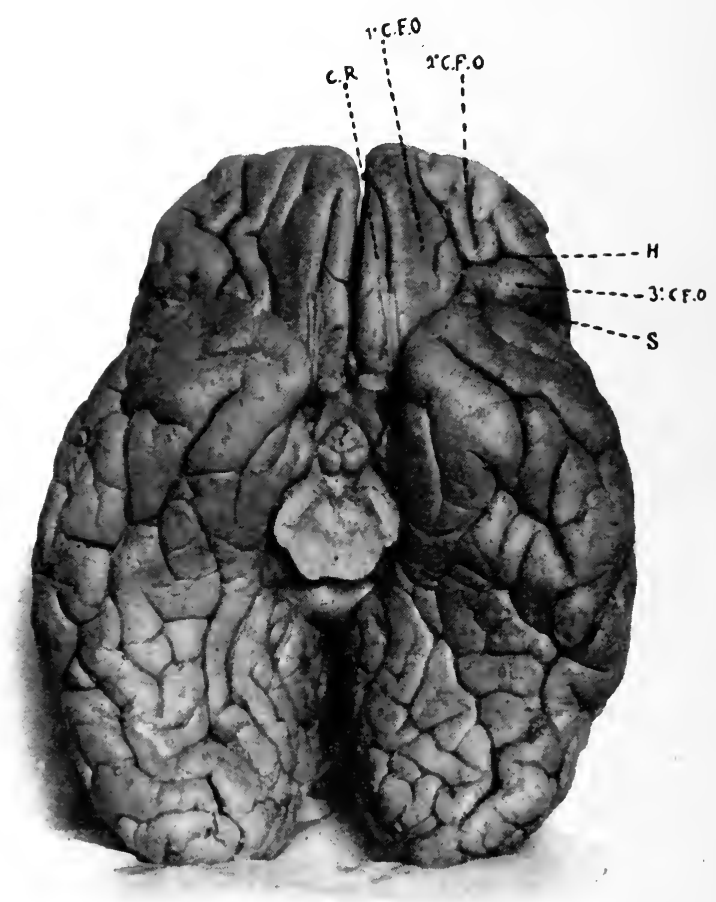

Fig. 49. - Inferior orbital surface of the frontal lobe

gyrus rectus (CR Fig. 49), is limbic in its structure. With regard to the orbital portions of the other two frontal convolutions (the middle or second frontal 2: CFO and the third frontal 3: CFO), which are regarded by anatomists as the prolongations of the second and third frontal convolutions on the orbital base (Dejerine), ${ }^{1}$ nothing is definitely known of their functions, either from experiments or from the study of human pathology. They exhibit a structure similar to that of the external surface of the frontal and pre-frontal areas.

From the morphological point of view, the second fronto-orbital convolution is limited behind by the " $\mathrm{H}$ "'-shaped fissure (Fig. 49). The third convolution is placed horizontally, bordering the second and forming the transverse branch of the third fronto-orbital convolution $(3: \mathrm{CFO})$.

${ }^{1}$ Dejerine. Anatomie des centres nerveaux. Vol. i. 
The superior frontal convolution with a part of its orbital surface is separated from the second or middle frontal by means of the superior frontal sulcus, and, just in front of the point where it curves round to the orbital surface, it is incised by the fronto-marginal sulcus of Wernicke (sulcus anterior transversus). It then proceeds to the orbital surface, where it forms the fronto-orbital convolution $(1: \mathrm{CFO})$. The part of this internal to the olfactory sulcus is known as the gyrus rectus. Behind and above, it reaches the para-Rolandic lobule on the interhemispheric aspect (Fig. 48a) and from this it is separated by the para-central sulcus which anastomoses with the callosomarginal sulcus. As the latter is not prolonged upwards in many brains, the interhemispheric margin of the first or superior frontal convolution is continued, without morphological distinction, into the para-Rolandic lobule (Figs. $48 a$ and 3). In the brain photographed (Fig. 48a), it happens that the para-Rolandic sulcus is prolonged upwards to the interhemispheric margin so that the superior frontal convolution appears definitely separated from the para-Rolandic lobule.

The middle or second is separated from the superior frontal convolution by the superior frontal sulcus above, from the inferior or third frontal convolution by the inferior sulcus below. Behind, it is implanted upon the ascending frontal from which it is often partly separated by the inferior branch of the pre-frontal sulcus. In front, it curves round to the orbital surface, going as far as the transverse branch of the third frontal. Like the superior, it also is incised transversely by the fronto-marginal sulcus at the frontal pole. It is often divided into two by a sulcus parallel with the two frontal sulci.

The third or inferior frontal convolution forms many folds around the horizontal and the vertical branch of the fissure of Sylvius. On the orbital surface, it is found situated transversely behind the sulcus of the " $H$ ". shaped fissure. It begins at the posterior extremity of the olfactory sulcus, curves upwards and is folded around the branches of the Sylvian fissure to be implanted on the ascending frontal. Often it receives above, in one of the " $M$ "-shaped foldings, the inferior pre-frontal sulcus. It is divided into three parts, the inferior comprising the horizontal orbital portion (from the olfactory sulcus to the anterior horizontal branch of the fissure of Sylvius) ; a triangular portion on the external aspect of the hemisphere (Broca's convolution) comprised between the anterior horizontal branch and the vertical or ascending branch of the fissure of Sylvius; a third part situated behind the vertical branch of the fissure of Sylvius and forming the so-called foot or pars opercularis of the third frontal.

It is to be observed that the plan of distinguishing two sulci separating three convolutions on the external surface of the frontal lobe, though adopted in almost all the more recent text-books of anatomy of the nervous centres; is only schematic. Often there exists only one very distinct sulcus, the superior or the inferior, and groups of sulci or segments of sulci in various situations. The latter mode of disposition of the sulci and of the convolutions of the frontal lobe has been observed in the brains of Herero and 
Indians, but one is not warranted in regarding it as the rule. In several hundred brains of Campani ${ }^{1}$ examined by the writer in the Naples asylum, where post-mortem examinations were made in every case, he almost always succeeded in singling out the two chief frontal sulci. The third sulcus which divides the second frontal convolution into two was also frequent, as well as groups of secondary and tertiary sulci running in different directions.

The author's particular field of study, so far as this work is concerned, has comprised all the frontal lobe in front of the motor zone, properly so called, including the orbital surface of the second frontal, the external orbital portion of the first frontal; and sometimes the transverse portion of the third frontal. To avoid ambiguity and complications the writer is bound to admit that he has hardly ever included the orbital plane of the frontal lobe in his experimental destructions, nor has he taken account of the histological fields.

We can speak of all that region situated in front of the ascending frontal convolution as pre-Rolandic. It is divisible, according to experimental indications, into three areas. We can take the pre-frontal sulcus as the line separating the Rolandic region from the pre-Rolandic area, which in monkeys would include a part of the intermediate zone; but this line, passing as it does through the points of insertion of the frontal convolutions into the preRolandic, is only an ideal one. The pre-frontal sulcus has not always the same length, nor the same inclination. It is found farther behind or farther forwards in different individuals of the same species, as is well exemplified in the case of the cebidæ (vide Figs. 41, 42, 43).

In monkeys from the Red Sea littoral, the pre-frontal sulcus is placed much farther forwards than in the primates and in man. Between the motor area and the frontal area, properly so called, in which we find points of electrical excitability for the eyes, pupils and ears, there exists in the majority of cases an inexcitable strip or zone. In these monkeys this area is found, as a rule, behind the pre-frontal sulcus. It is the area which, on experimental grounds, can competently be called the intermediate motor zone. Thus, proceeding from behind forwards, we meet with $(a)$ the zone for the limbs (anterior Rolandic); $(b)$ an intermediate motor zone which is inexcitable with weak electric currents; $(c)$ a frontal zone, properly so called, which has some excitable points and apparently also, some cytotectural peculiarities; (d) the pre-frontal area.

Taking a general survey of the development of the cortex, the frontal lobe presents certain peculiarities, as is evidenced by Bolton's studies. The development takes place from within, outwards - that is to say, from the deeper layers to the surface. As a matter of fact the superficial layer and that of the pyramidal cells (external), in a fœtus of six months, have only half the thickness they attain in the adult. The growth is due to fission of the neuroblasts in an upper and a lower part, a fact which is of some considerable significance. The development of the various layers of the cortex

${ }^{1}$ Inhabitants of the Campania-i.e. the district comprising the provinces of Naples, Caserta and, in part, Salerno. 
differs in different functional zones, and Bolton maintains that he has good grounds for his contention that development takes place in the visuo-sensory area before it occurs in the visuo-psychic area, and in the latter before the pre-frontal region. He insists on the importance of the granular layer and maintains that in the visuo-sensory area individual differences are related to the power of receptivity, whilst the layer of pyramidal cells would be the physical basis of the associative and voluntary processes. When this layer in the frontal lobe is thinner than the mean normal, this would signify sub-evolution of this lobe, a condition which is found most marked in amentia (phrenasthenia). The thickness of this layer in the frontal lobe varies directly with the degree of imbecility or idiocy. In normal evolution this layer is last of all to be evolved. Further, it is very poorly developed in lower mammals and shows a progressive evolution as we rise in the mammalian scale. ${ }^{1}$ Contrary to what we observe in the evolution of the cortex (from within outwards), in dissolution the process makes its appearance first in the pyramidal cell layers, thus confirming the general law that dissolution begins first in those organs that developed last in the evolutionary series.

Apart from the little the writer has reported in the preceding pages, the study of cytotecture has not furnished us with positive data on which we can build and support any hypothesis. Without entering into great detail one may briefly review all the more interesting findings.

Schäfer, ${ }^{2}$ from the histological point of view, divides the frontal lobe into three regions. The first is the region next to the ascending frontal and includes the intermediate zone of Campbell and a part of the frontal area, properly so called. The second is that of the pole, which extends backwards to the area called by others pre-frontal (inexcitable), and this region he found to be poorly provided with fibres. The third is the basal or orbital region which is rich in fibres.

Hammerberg ${ }^{3}$ found the intimate structure of the frontal convolutions to vary as he proceeded forwards from the bases towards the frontal pole. He found cell-layers becoming thinner whilst the pyramidal cells became smaller and fewer in number.

According to Betz, the orbital part of the second frontal convolution does not differ in structure to any great extent from the external surface. The gyrus rectus, however, he found to resemble the limbic lobe in structure.

Brodmann ${ }^{4}$ distinguishes eight areas in the frontal lobe, in the human subject, each of which can be subdivided into several myelogenetic areas.

Vogt obtained results which agree in some fundamental respects with those of Brodmann yet differ in many points.

Jakob $^{5}$ distinguishes three or four sectors in the frontal lobe excluding

1 Watson. "The Mammalian Cerebral Cortex, with Special Reference to its Comparative Histology. Arch. of Neur. 1907.

${ }^{2}$ Schäfer. Mentioned by Bonne. L'Écorce cérébrale. 1910.

${ }^{3}$ Hammerburg. Studien über Klinik u. Pathologie der Idiotie, etc. Upsala, 1895.

"Brodmann. "Über den allgemeinen Bauplan des Cortex Pallii bei den Mammaliern," etc. Journ. für Psychol. und Neurol.

5Jakob. Vom Tierhirn zum Menschenhirn. 1911. 
the pre-Rolandic convolution-the fronto-polar, the anterior, middle and posterior sectors. He is of opinion that the process of differentiation in the majority of sectors of the neopallium is simultaneous with the progress of intelligence.

Roncoroni ${ }^{1}$ agrees neither with Vogt nor with Brodmann.

This brief reference to the present position of cytotecture and myelotecture is sufficient to convince us that we are still far from possessing precise knowledge as to the intimate structure of the frontal lobe. There is no agreement or correspondence in point of form, number or disposition of the cells of the various zones. Myelogenesis and cytoarchitecture are not in a position at present to define the histological fields of differentiated function. They are alluring and promising sources of study and suggest new fields for the further investigation of mental affections on which it may yet be possible to build up a psychology, normal and pathological, on an anatomical basis.

The frontal lobe is still in process of evolution, even in man himself. This has been borne out by some recent researches. In a comparative study of the sagittal sulci of the frontal lobe in the brains of Indians, Japanese, and Herero, the anastomoses of the principal sulci with accessory or secondary sulci at the upper and lower borders of the frontal lobe were found to be most numerous in the Japanese, whilst in Indians these anastomoses occurred only in the upper portion, and in the Herero were entirely lacking. From these observations Sergi ${ }^{2}$ concludes that the frontal lobe of the Japanese is more evolved than that of the Indian or the Herero.

The measure of the distance from the superior and inferior extremities of the Rolandic fissure to the frontal and occipital poles, taken on the average, has demonstrated a greater absolute development of the frontal lobe, always upwards on the left side and downwards on the right. Further, the develop: ment of the frontal lobe in toto is greater in males than females. There thus exists a marked variability in the morphology of the superior and inferior zones of the frontal lobe in different races between the two sides and the two sexes.

Furthermore, the greater development of the frontal lobe upwards on the left and downwards on the right side holds good in both sexes (Italians, Germans, ete.). The author himself has not carried out investigations in this direction but the measurements taken by $\mathrm{S}$. Sergi from the superior and inferior extremities of the fissure of Rolando to the frontal and occipital poles in brains of Indians, Japanese and Javanese, due allowance being made for individual as well as well-marked racial variations, indicate the remarkable development of the fronto-Rolandic mass in man. It has been shown, too, that not only is there variability in the development of the left frontal lobe as compared with the right, but also the oscillations to right and to left are wider in females than in males, and that the development of the frontal

${ }^{1}$ Roncoroni. Loc. cit.

"Sergi. Note morfologiche sulla superficie metopica dei lobi frontale in cervelli di indiani edigiapponesi. 1913. 
lobe is more marked on the left side in males of the Indian, Japanese and Javanese races.

It is not improbable that the index of variability of the frontal lobe differs in different ethnic groups as well as in the sexes. According to Sergi the development of the frontal lobe takes place by growth in front of the fissure of Sylvius, more on the left than on the right side. This would go to confirm the view that the frontal lobe is still in course of evolution.

Clinical observations support the conclusion that the left frontal lobe is of much more value in the mental processes than the right. Phelps holds that there is an entire absence of intellectual or other function in the right frontal lobe. Years ago Hughlings Jackson postulated the existence of a power of control on the part of the anterior brain over the highest mental functions, and maintained that the anatomical substratum of these mental operations might be the left pre-frontal lobe. One of the first clinical indications in this regard is to be found in the observations of Starr, who, as a matter of fact, had not specially directed his attention to the mental symptoms presented by patients suffering from tumour or abscess of the frontal lobes.

In dealing with this subject we are surrounded with difficulties because it is not easy in all cases to estimate the precise extent of the primary lesion, the presence or absence of secondary lesions, the existence of vascular alterations (especially in cases of syphilitic tumour of the frontal lobes), the degree of compression etc. (cases reported by Mills). Phelps supports the view that the left frontal lobe fulfils a high mental function, because of the important relations between high intellectual function and language, which is a function of the left hemisphere. He reasons as follows:- "If articulate speech is localised in the left frontal lobe and only exceptionally in the right and if articulate language represents one of the mechanisms for the expression of thought, one need have no difficulty in ascribing to the left frontal lobe one of the highest cerebral functions such as that of the control of thought." This argument is contradicted by the fact that the functioning of the intellect does not depend upon normal conditions of the cortical motor organ of language (taking the existence of this as incontestable), but on its cortical sensory organs, auditory and visual, as already mentioned. Thus it is that in the few cases of true motor aphasia reported in the literature of the subject there was complete mental integrity, in so far as internal diction (the motion of thought) remained possible.

The writer has had no means of confirming this hypothesis in the course of his experiments. The differences met with after experimental destruction of the right and left frontal lobes, in monkeys of the same family, do not warrant him in giving unconditional support to this view. Shepherd has been more fortunate than the author in finding a decided difference between the results of the destruction of the left and of the right hemisphere, such a difference as has warranted him in stating very definitely that mental disturbances are much more frequently a result of destruction of the left pre-frontal lobe than of the right. A slight difference was also observed by 
the author, but subsequent post-mortem examination of the animals experimented upon did not always convince him that the extent of the mutilation permitted a comparative judgment. Even admitting it to be an actual fact, it can be explained because of the functional differentiation of the left as compared with the right hemisphere, and not because the frontal lobe is the organ of language and indirectly therefore an organ regulative of intellect, for the motor area of language does not exercise any real regulative power either on the formation or on the movement of thought. The hypothesis has had its origin in the fact that the mental disturbances are probably due, at least in part, to the presence of a tumour which, owing to compression or vascular disturbances, affects all the left hemisphere. In such cases there is a great liability to disturbance and disorder of the auditory sphere of language, which is one of the main wheels in the logical movement of thought. 


\section{CHAPTER IV}

\section{Methods of Inquiry}

THE investigation of a problem so abstruse and delicate as that which concerns the function of the frontal lobe, a problem of the highest interest to physiology and psychology and the applications of these to clinical medicine and to pedagogy, demands a calm and dispassionate mind to ensure that facts will have due control over aprioristic conceptions.

Realising that, even in the case of men of science, who sometimes import a great deal of passionate enthusiasm into their studies, the mental organisation is such that preconceptions often give rise to illusory visions of proofs, it has been the desire of the author to avoid this error as far as possible and to retraverse the ground of inquiry on repeated occasions. He has interrupted his experiments for one, two or more years, and taken them up again in series, taking stock of all criticisms and modifying his methods so that he might be better able to state the facts of the case positively, at the same time giving the greatest possible consideration to the objections, sometimes rather formidable, put forward by physiologists, who, by reason of their authority, succeeded in having the author's views rejected.

The investigator who performs a large number of experiments with fairly uniform results experiences a calm confidence which it is useless to expect from a few experiments with hasty conclusions, of which there are not a few examples in the literature of this subject.

Many objections have been urged against the author's views, and his experimental methods have been so criticised that the conclusions drawn from them have been robbed of a good deal of value. It is necessary to take some brief notice of these criticisms.

Schäfer, ${ }^{1}$ Monakow ${ }^{2}$ and others have objected that, after experimental lesions of the brain, the effects are not limited solely to the injured region. Besides the local effects, those strictly related to the function of the injured region, the injury makes itself felt also at a distance, producing in other parts of the brain circulatory disturbances and functional arrest. There is some truth in this criticism, but when Schäfer asserts that the intellectual disturbances observed secondarily in animals mutilated in their frontal lobes are dependent on the operation itself, one may assuredly reply that this is an assertion which is incapable of proof. I recognise that destruction of the cerebral substance to any extent in any region of the brain gives rise in some cases to secondary functional disturbances of circulation and nutrition at a distance. As a rule, however, these secondary symptoms are of

${ }^{1}$ Schäfer. T'ext Book of Physiology. Vol. ii.

${ }^{2}$ Monakow. Gehirnkrankheiten. 1905. 
short duration and can even be diagnosed by methodical and detailed examination of the animals experimented upon, if these are re-examined, as they should be, for weeks and months after the operation. Even from the time of my earliest experiments I have taken special note only of those symptoms that were observed a considerable time after the cerebral mutilation.

Schäfer has laid stress on the fact that I removed the frontal lobes from the cranial cavity, and expressed the opinion that this complicated matters by introducing mechanical and physical disturbances as well as vascular disorders and disturbances of relations, a state of affairs, he declares, "which may lead to erroneous conclusions." This, if I may say so, is rather a subjective mode of judgment on the part of that eminent English physiologist.

As a matter of fact, in many cases the frontal lobes were not removed in toto. As a rule they were decorticated, sometimes the pole was removed, but the orbital surface was almost always spared. On rare occasions. I succeeded in making a very extensive excision of the frontal lobe, including the interhemispheric and a part of the orbital surface. The space which remained after removal of the cortex became filled up with transudate, by blood and later by exudate, as happens in all analogous reparatory processes. The animals completely recovered, almost all of them living for many months and even more than a year. Symptoms ex vacuo were never observed. In formulating conclusions I left out of account, in every case, the symptoms presented by the dogs and monkeys during the first few days. The necessity for this was shown by the behaviour of the animals, which revealed always the same deficit so long as they were kept alive, a deficit afterwards controlled by post-mortem examination. Many of the animals experimented upon were quite lively from a few hours after the operation.

Symptoms at a distance and lesions at a distance may be present, but there is no need to confuse either one or the other with the definite effects of extirpation.

Monakow ${ }^{1}$ introduces a formal element of criticism which tends to depreciate to some extent the doctrine of localisation.

He starts out with the view that psychic manifestations are the resultant of a co-ordinated complexus of factors which have their origin in different cortical areas with different functions (fields or phases of elementary psychic actions). It seems a probable hypothesis, he maintains, that when any one of the sensory areas becomes destroyed, this is followed by interdiction and dysorientation of other areas which function in concert with it in consequence of an anatomical overlapping, the latter being damaged by the experiment in some essential part or else interrupted in its paths of communication with the various mechanisms co-operating with it. In diaschisis, the term which Monakow applies to this mode of action, we recognise an old friend in a new guise.

\footnotetext{
${ }^{1}$ Monakow. Loc, cit.
} 
There is, in fact, such a network of fibres between neighbouring and also distant convolutions that a lesion circumscribed to any one convolution does injury also to the function of neighbouring convolutions. A classical example is provided by Horsley. ${ }^{1}$ This distinguished surgeon laid bare the motor zone in a patient affected by athetosis and severe convulsions of the upper limb. By means of electrical excitation he delimited the area for movements of the upper limb in the pre-Rolandic convolution. He then removed the cortex from this area and the result was cessation of the athetotic movements which were now replaced by paresis and, associated with this, disturbances of sensibility in the limb. As the sensory disturbances had almost disappeared after the lapse of a year and the few that remained could be ascribed to actual lesions of the fibres of the post-Rolandic convolution, it was unnecessary to attribute to the pre-Rolandic convolution both motor and sensory function, as Horsley concluded, basing his conclusion merely upon the fact that disturbances of sensibility were conspicuous soon after the operation. Horsley desired to adhere to his old conviction and, unfortunately, did not compare it with the sound observations of Sherrington. The hemianæsthesia was evidently a symptom of diaschisis, and there is good reason for supposing that what remained of the disturbances of sensibility in that patient was due to an actual, though limited, lesion of the ascending parietal convolution.

Remote lesions which complicate the symptomatic picture are, as a rule, recognisable by control experiments, when they are not mere symptoms of arrest or vaso-motor symptoms, which generally are very fleeting. This certainty in diagnosis, though not absolute, depends upon the methods followed, more especially upon the accurate and systematic investigation of symptoms in the animals experimented upon, and in addition upon the method of successive excitations, with measurement of the electric current employed, controlled by successive and graduated destruction of determined cortical fields. Above all, it depends upon collateral experiments. Proceeding in this manner and comparing the results of the destruction of different areas, one is able to separate out the symptoms that are strictly due to the lesion from the entire syndrome that immediately follows upon the operation and from the further symptoms that result from subsequent operations.

The permanent deficit imprints a particular character on the animal operated upon, and it is this that is to be put down to the mutilation. The author has always had recourse to post-mortem examination in order to determine whether the symptoms observed during life depended solely upon the experimental lesion or upon lesions which had developed in other parts of the brain and had complicated the symptomatic picture observed during life. One of the monkeys upon which he operated presented at the postmortem examination, which followed three months after the operation, an extensive and symmetrical softening of both occipital lobes. In a dog there

${ }^{1}$ Horsley. "The Function of the So-called Motor Area of the Brain." The Linacre Lectures. Brit. Med. Journ. 1909. 
was found complete softening of the hippocampal convolutions on both sides. Conditions such as these are rare but can be diagnosed.

The effects of disturbances ex vacuo have certainly been exaggerated and it is unnecessary to dwell upon them. To speak of symptoms ex vacuo being present one or two months after the operation is nonsense.

Another physiologist, Shepherd, ${ }^{1}$ anxious to avoid the objection raised by Schäfer against the method of excision and removal of the frontal lobe adopted by the author, made a more or less extensive and deep incision into the frontal lobe, separating it from the remainder of the brain, but leaving it in situ. Yet, from the analysis of all his experiments and the examination of all the diagrams of brains of monkeys operated upon by him, it seems clear that the lesion was only partial.

His diagrams, $8,9,10,12,15,16,17,18$, etc., show lesions limited to the foot of the superior or that of the middle frontal convolution. The prefrontal area was certainly not completely incised and detached from the rest of the cerebral mantle, but remained in continuity with the Rolandic zone by means of the second frontal or the superior frontal convolution or parts of both and by that region of the frontal cortex which, on the external surface, forms the frontal operculum and may be considered as the rudiment of the third frontal convolution, in middle-grade Abyssinian monkeys. Yet the results obtained by the physiologist of George Washington University were conspicuous and confirmed those obtained by the author. It is, however, just because a good part of the frontal lobe was spared, in one series of experiments, that this investigator found that in some monkeys and cats there was, to some extent, a recovery of attitudes and actions which they had previously learned, but had forgotten after the operation. If there was any re-education it could only be attributed to the part of the frontal lobe spared by the operation; in every case re-education was incomplete. Shepherd's investigations serve as a very important control of the author's conclusions.

It is worthy of note that actions and attitudes that have become habitual in particular animals are neither lost nor notably altered after mutilation of the frontal lobes, and this is especially true as regards the simpler actions and those of emotive origin. This explains why one of Shepherd's monkeys which lost the faculty of opening the lid of a food-box nevertheless retained that of jumping on the shoulder of the investigator, just as he had done before the operation. The capabilities of jumping, of walking through the room, of tearing at the door in an effort to escape, of climbing, of seeking food, of eating with more or less rapidity, and the sexual instinct (somewhat modified in the mode of satisfaction), are retained after frontal mutilation. It is different in the case of faculties that have been acquired as the result of education. That is an important fact demonstrated by Shepherd, and it confirms once again the difficulty of arriving at a judgment as to the precise loss suffered by animals that have been experimented upon.

1 Shepherd. "On the Functions of the Cerebrum." The Frontal Lobes. 1907. 
The difference in the syndromes according to the areas destroyed, and the proportionality of the effects of serial destructions, offer strong arguments against the views of those who have criticised the results of destruction of the frontal lobes, maintaining that many of the symptoms observed have been due to post-operative shock. It is to be remarked that many animals present no symptoms of shock after the operation, more especially if, cceteris paribus, they have not been too deeply narcotised. In this connection it is worthy of note, as the author has pointed out since 1889, that destructions of the occipital lobe, the motor zone, the temporal lobe, and the parietal lobe, give rise to syndromes that differ greatly from one another. Control experiments carried out both by the author and by Shepherd have confirmed this view. Shepherd explicitly declares: "The last four experiments coupled with those that were previously described on cats, indicate clearly that the loss of association in animals consecutive to lesions in the frontal regions is not due to the general shock effect of the operation." The fact is that many investigators are not yet convinced that the symptoms observed during the first few days, and even more particularly during the first few hours subsequent to the operation, possess no real value for or against the doctrine of the cerebral localisations. One has to keep these animals alive for many weeks and months in order to be able to define the true functional deficit that can justly be attributed to the part of the brain destroyed.

It is a cardinal principle in clinical medicine, in cases of cerebral hæmorrhage or thrombosis, not to make an immediate pronouncement as regards the exact seat and extent of the lesion, and consequently as regards the physical and psychic loss that will be permanent, because the syndrome is very complex, not only during the first few hours but also during the first few days and even for some weeks after the attack. The physician who, at the bedside of the patient on the first or second day after an apoplectic attack, when coma has passed off, pronounced a judgment as to the extent and gravity of the focus (finding, e.g., right hemiplegia and sensory aphasia), and expressed his opinion that the patient was in a very serious condition and unlikely ever to attend to his business again, would find himself in an awkward situation after a few days or weeks when the patient was again capable of understanding what was said to him and of expressing correctly his own thoughts. If more rational methods had always been followed in experimental investigations upon the brain, we should have been spared many useless discussions, and the physio-pathological literature of the cerebral localisations would have been less encumbered, more sincere, and at the same time more convincing. Fortunately, clinical medicine has had its conquests and has accumulated material of incalculable value not only in its own provinces but more especially in its practical application to anatomical physiology, psychology, semeiology and surgical therapy.

It has to be borne in mind that in nine out of ten animals operated upon symptoms of shock are non-existent, or else insignificant as compared with the results of apoplexy or cerebral trauma. Further, animals that have 
suffered mutilation of different cortical areas exhibit marked differences in behaviour. Especially noteworthy is the variance between the mental picture presented by a dog mutilated in the anterior brain and that by others mutilated in the parietal or temporal or occipital lobes.

Sciamanna ${ }^{1}$ unwittingly provided a strong argument against the shock hypothesis. He had denied the existence of any special psychic function in the frontal lobes but it was found at the post-mortem examination that he had removed only small portions of these. This fact could be utilised to explain the absence of marked disturbances of intelligence, but at the same time it provided evidence to show that the symptoms of shock consecutive to the operation had been greatly exaggerated. More recently, Shepherd has again taken up the question and has given further proof of the fact that a surgical operation, consisting of trephining of the skull and incision of the dura mater, carried out under all the ordinary rules, avoiding injury to the cerebral substance, does not give rise to any of those disturbances that generally follow lesions of determined parts of the brain. The results of Bechterew's researches in dogs led to the same conclusions. The author's control experiments have demonstrated the fact that lesions of one lobe only, and also very limited lesions of both frontal lobes, in monkeys, do not give rise to appreciable damage, although the operation itself is equally serious in all cases.

Functional compensation provides another source of error, for we cannot altogether deny the fact that symptoms of compensation may enter upon the scene. The disappearance of some of the symptoms produced by a spontaneous lesion of the brain, or by experimental lesion in the brain of a dog or monkey, is due in part to the compensating action of analogous areas of the healthy hemisphere or of the areas surrounding the focus. ${ }^{2}$

There are functions in the expressive mechanism of which both hemispheres co-operate. This co-operation varies with different functions and different animals. For example, compensation of experimental paralysis is much slower and less marked in monkeys than in dogs, whilst in the human subject compensation of paralysis arising from a spontaneous cerebral lesion is still much more difficult. Indeed, the less differentiated is the cerebral mantle, the readier, cateris paribus, is the compensation of a destructive lesion. In the case of the human brain, the higher functions, such as that of language, can be compensated in some measure more readily than the motor function. This is to be accounted for, to some extent, by the fact that the language-area is very extensive. Partial compensation always occurs when only one part of the entire sensory area of language is destroyed. Thus it happens that, in the long run, the sensory or motor aphasic readjusts

${ }^{1}$ Sciamanna. Loc. cit.

${ }^{2}$ L. Bianchi. "Le compensazioni funzionali del mantello cerebrale. La Psichiatria, la Neuropatologia e le Scienze affini. 1883. Also "Le malattie del cervello" in Trattato di P'atologia Medica, by Maragliano and Cantani. Vol. ii., part 2. 
his relations with the social world by means of language, as the result of a fairly strong compensatory action of the parts surrounding the focus in the left hemisphere, or of the homologous parts in the right hemisphere. So true is this, that if, after improvement in the function of language has taken place, the patient becomes the victim of another destructive focus in the symmetrical region of the other hemisphere or the surrounding parts of the same hemisphere, language may be suppressed for all time.

Besides symptoms of diaschisis, dependent upon disturbance of functional equilibrium, and symptoms of compensation, we may, after a lapse of time, get secondary degenerations starting in the area destroyed (Dejerine, ${ }^{1}$ Bianchi junior $\left.{ }^{2}\right)$. Degenerative processes complicate the clinical picture and may lead us into the error of attributing to the experimental lesion what is really an effect of the secondary degenerations in the brain. Between the first stage, that of shock and diaschisis, the signs of which, if present at all, are very early manifested, and the last stage, the signs of which are late in appearance, there is a period during which the clinical picture is constantly the same in its general lines, and the signs then met with must be put down to the effect of the experimental lesion itself.

Notice must be taken of another prejudice. It has been objected that the mental functions of animals, even of the higher mammals, are not to be confused with those of man and that no serious conclusion can be drawn from the symptoms observed in them, such as would allow us to ascribe analogous functions to determined sections of the cerebral cortex in man. The objection seems a formidable one: When we confront the problems of intelligence we should not seek to solve them except by study of man himself, normal and pathological. I am of opinion, however, that no biologist who attacks these problems can to-day afford to neglect the splendid material that comparative psychology has succeeded in accumulating. This, surely, is only possible in the man whose outlook is absolutely restricted by animistic doctrines which belong to the past. Undoubtedly one should take by preference those mammals in which intelligence is most developed, animals in the common life of which we can, by suitable experiments, detect undoubted signs of judgment, memory, comparison, emotion and new adaptations. These signs are derived from a combination of mental processes of remarkable complexity, reduction or suppression of which can be brought about by means of localised cerebral destructions.

Kalischer, ${ }^{3}$ in particular, has expressed grave doubts as to the applicability of the results of experiments upon animals, more especially dogs, to the psychic functions of the human brain. He is seconded by Roncoroni, ${ }^{4}$

${ }^{1}$ Dejerine. Anatomie des centres nerveux. Vol. ii. 1909.

${ }^{2}$ Bianchi jun. "Alterazioni istologiche della corteccia cerebrale in seguito a focolai distruttivi ed a lesioni sperimentali. Ann. di Nev. 1912.

${ }^{3}$ Kalischer. "Zur Function des Schläfenlappens des Grosshirns. Experimentelle Physiologie des Grosshirns." Handbuch der Neurologie. Teil 1.

${ }^{4}$ Roncoroni. "Le Funzioni dei lobi prefrontali in rapporto ai dati architettonici." Rivista di Patologia nervosa e mentale. 1911. 
who also expresses the view that one has to be very careful in drawing deductions from experiments on animals and applying these to mental conditions in man. The warning is justified, but one cannot agree with Kalischer when he asserts that the whole cerebral cortex of the dog can be removed without the animal showing any fundamental alterations in its behaviour. One has to admit that the psychological analysis of animals is a very difficult matter. Kalischer's views certainly contrast with those of Munk and Goltz, both of whom expressed a very different opinion.

Many, if not the greater number of, experiments that have been directed towards the investigation of the functions of the frontal lobes, have been made upon dogs and cats. Many too have been made on monkeys, and some on rabbits. Now it is evident that the only mammal which lends itself advantageously to the investigation of the frontal lobes is the monkey. Experiments carried out on dogs and cats also give promise of fairly useful results, as has been shown by those performed in the laboratories of Bechterew, Shepherd and the author. They serve the more delicate purposes of control, and in any case, should be included in the series of experiments of any investigator who sets out to inquire into the function of the frontal lobes.

Intelligence and emotions with their relative associative mechanisms are markedly developed in dogs.

Pavlov ${ }^{1}$ and some of his pupils succeeded in establishing the existence of associations between the fundamental sensations by examining the salivary secretion. A gustatory stimulus such as meat powder placed on the animal's tongue, after a fistulous opening into Stenson's duct has been made, determines a marked increase of the salivary secretion. If, after a number of educative experiments of this kind have been made, the animal is allowed to see the same substance at a distance, a like increase of salivary secretion generally, though not invariably, occurs. In these cases an association is formed between the tacto-gustatory cortical centres and the visual centre, excitation of which by the sight of the meat brings about the cortico-gustatory salivary reflex. Subbok's experiments also are well known. This investigator wrote words upon sheets of paper and trained his dog to recognise these by the spoken word. The dog, as a rule, obeyed the command and selected, from amongst the papers, that on which was written the word pronounced by his master. One need only be reminded of the extent to which dogs can be trained for public exhibition and how they understand a number of words corresponding to determined actions performed by them.

Zèliony, ${ }^{2}$ using the method introduced by Pavlov, carried out numerous and patient experiments in dogs and demonstrated associations between meat powder placed upon the tongue and acoustic stimulations (harmonium, trumpet, etc.). Having made a fistulous opening into Stenson's duct, the drops of saliva were allowed to fall upon a small gutter with a registering lever, from which they ran into a graduated vessel, so that he was able to

1 Pavlov. Atti del Congresso di Madrid. 1904.

2 Zèliony. Archives des Sciences Biolngiques. 1909 (referred to by Bohn). 
register the number of drops that fell into the gutter and to measure exactly the quantity of saliva obtained. As in the case of the visual stimuli, associations were found to have been formed between the acoustic excitation and the reflex salivary secretion when various instruments were used. Without discussing these experiments in detail it can be definitely stated that new associations can be developed by means of education. Dogs, and to a greater extent, monkeys, can form many new visual images of form, colour and distance, as well as auditory and musical images, variously associated with one another and capable of giving rise to reflexes or motor orientations or to inhibitions. At the same time it is to be remarked that, so far as concerns the function of the frontal lobes, experiments on dogs do not in themselves furnish legitimate bases for conclusions, because the frontal lobe in these animals is still poorly developed. Further, it is, as we have seen, so near to the anterior branch of the sigmoid gyrus that even limited extirpations can hardly ever be carried out in it without at the same time doing injury to that branch, where there are also motor centres. Thus it happens that, if the lesion is exceedingly limited, either no conspicuous mental disturbances are observed, or, on the other hand, these, when they occur, are complicated by motor and sensory disorders, in which case no legitimate conclusion can be drawn.

Besides doing injury to the motor zone, especially that part concerned in the musculature of the trunk and neck (which in dogs is prolonged fairly far forward, although it does not involve the entire extent of the frontal lobe, as Munk maintained), one is liable to do damage to the olfactory bulb, which is very close to, or in actual contact with, the frontal lobe. In all his experiments the writer made it a rule not to injure any motor or sensory centre or any sensory path, because, if the animal becomes deprived of a series of perceptions of the external world (especially olfactory perceptions in the dog), the psycho-reactive function is disturbed or reduced, and one is apt to put down any disturbance in the mental behaviour of the animal to destruction of the frontal lobe, whereas in reality it depends upon destruction of a sensory centre or its projection paths.

The wide gulf that appears to separate man from other mammals is being gradually narrowed as the result of incessant research. The writer cannot here enter upon any discussion of Darwin's doctrine of descent, or the relative views of any other writer such as G. Bohn, who maintained that man appeared upon the earth by abrupt mutations and was a species of large-brained monster who, with the aid of intelligence, dominated the animal world surrounding him. At the same time one has to admit from what has already been accomplished that psychological investigations are full of promise.

Thorndike, ${ }^{1}$ in an experimental study of the associative processes in platyrrhines, which are not the most intelligent of monkeys, proved that these quadrumanes were capable of acquiring new adaptations when placed under entirely new circumstances. As compared with other animals the

1 Thorndike. "Animal Intelligence : an Experimental Study of the Associative Processes in Animals." Suppl. to Psychological Review. 1908. 
associations were more rapid, more numerous and complex, and, at the same time, there was a greater perceptive fineness and a higher capacity of attention and memory, which enabled these animals to turn to account all that they had learned. The present writer, as the result of his studies of many individual monkeys as well as small tribes of cebidx, was able to confirm this verdict as to the monkey's intelligence. These animals exhibited a much higher perceptive capacity than the dog, rapid judgment, fairly extensive associations, a good use of memory, educability, and a capacity for forming new adaptations when placed under strange conditions. An account of these observations is given in the following chapter.

It has been said that chimpanzees do not distinguish colours. The researches of Thorndike, Jennings and Kinnaman have proven that even the lower monkeys-such as Macacus Rhesus upon which Kinnaman experimented-perceive at a distance the form of an object, its size, its colour, and its spatial relations. The animal is capable of making a selection from amongst several objects, and in the various phases of its conduct it is not difficult to detect motives for its actions, for these are based on precise perceptions and on tests which evidently have for their purpose the avoidance of errors. Bohn would recognise in all these the elements of a superior consciousness. ${ }^{1}$

The experiments detailed in the following chapter furnish confirmatory evidence of precise perceptions, judgments, comparisons, of memory with trials and tests, and of new adaptations. Cebidæ certainly distinguish yellow from green and red from blue. I have never been able to detect in cebidce kinocephalides any articulation of syllabic sounds, although I have observed many inflexions of vocal sounds, along with some mumbling, in the course of their erotic manifestations, and movements of the lips suggestive of the kiss. Garnier, as recorded by Ingenieros, ${ }^{2}$ under very special conditions in the virgin forests of tropical Africa, was able to detect a certain number of articulate sounds. Apart from this finding, which the author had no means of confirming, there are vocal inflexions expressing various emotional states, which are rather more numerous in the cebus than in the dog.

It is not within the scope of this volume to attempt to trace a parallelism between the mental life of various species of animals and that of the human being in his successive phases of development. The author trusts that it will not be said of him that he has been caught in the net of anthropomorphism, but he is compelled to admit that in the more intelligent monkeys one can even perceive the rudiments of human sociability - i.e. of love, friendship, protection, maternal sentiment, feeling of injured dignity, jealousy and ridicule.

Shepherd, following Hitzig's example, has studied the symptoms resulting from mutilation of the frontal lobes in trained monkeys such as those taken about by showmen. The writer also at first preferred trained monkeys for his experiments, and eertainly the symptoms that resulted from extirpation

${ }^{1}$ Bohn. La Naissance de l'Intelligence. 1907.

${ }^{2}$ Ingenieros. Principios di Psicologia Biologica. Madrid, 1913. 
of the frontal lobes were very impressive. The loss of what is a product of education, however, may be a source of error, in view of the following facts. In all the severe and diffuse encephalopathies in the human subject, what is most certainly lost is the product of education; further, all dementias consecutive to psychopathies which do not depend very largely upon diseases of the frontal lobes, and consecutive to diffuse degeneration or inflammatory processes, are contra-distinguished by the loss of the most recent acquisitions. Hence, the writer has considered it preferable to experiment upon monkeys brought in their savage state from the Red Sea littoral. He has subjected these monkeys first of all to an accurate psycho-somatic examination, investigating, one by one, all the senses, the rapidity and mode of perceiving and reacting, the habits, the emotions, the affections, as well as new attitudes and adaptations, so as to be able to formulate a sort of psychic equation for each animal. By adopting this method one was in a better position to estimate the loss of mental capacity in each animal after the mutilation.

To detect, measure and appraise the changes and the reduction in cerebral action caused by the mutilation is indeed the most difficult part of the experiment. At the International Congress in Rome in 1894, the Committee appointed for the purpose of pronouncing judgment as to the mental condition of the mutilated monkey which the writer presented before it was impressed by the fact that the animal recognised objects and executed adequate movements of prehension like other members of the same family. Its behaviour seemed to be quite in keeping with the normal mentality of these quadrumanes. Indeed, it exhibited abundant evidence of perceptive capacity and of coherence in action.

As a matter of fact the animal operated upon sees objects, recognises them, shows emotional activities (desire and sometimes repulsion) and executes appropriate movements.

The writer has no hesitation in saying that, in some cases, he has found himself in a difficulty very similar to that in which the alienist finds himself when he is called upon to express an opinion as to the civil and juridical capacity of a man in a condition of slight imbecility.

There can be no question as to the fallacy involved in the method adopted by some investigators, that of examining, merely en passant, the monkeys operated upon, throwing to them a sweet, a coin, a fruit, and then judging that their behaviour differed in no way from the normal inasmuch as they perceived, picked up and made use of, these objects. When one thinks of the time that is required in order to define the mental capacity of a monkey before as well as after mutilation, or of a human imbecile, one cannot help feeling surprised that anyone should venture, after five minutes, to express an opinion upon the mental condition of a monkey that has suffered mutilation of the frontal lobes, without first starting out from a comparative examination, which in every case requires method and time.

With regard to the question how far the motor zone extends in front, more especially that part of it concerned in the movements of the neck and 
trunk, there is a lack of agreement. Independently of his own observations the author is convinced, from examination of the facts adduced, and the methods employed, by different authorities, that the difference in opinion depends, in part at least, upon individual differences and on diversity of the methods followed. How otherwise can one explain the fact that observers like Hitzig, Munk, Ferrier, Horsley, Schäfer and Luciani located the centres for the muscles of the neck, head and trunk of dogs and monkeys in points of the brain that do not at all coincide? How are we to explain the fact

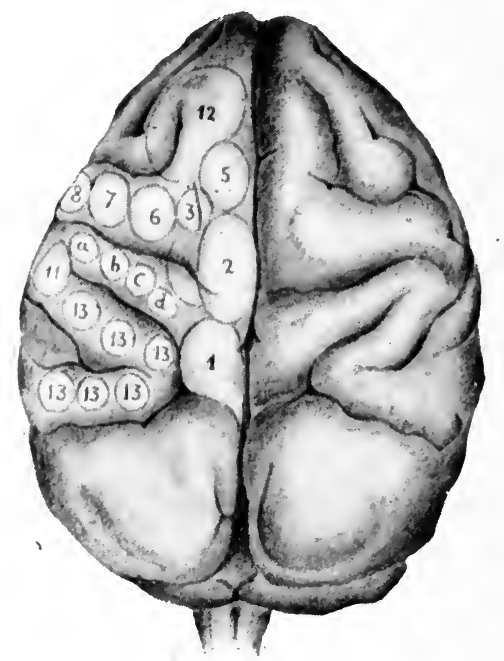

Fig. 50

(after Ferrier)

1, 2, 3. Areas for movements of the lower. limb and tail.$4(a, b, c, d), 5,6$. Areas for the varions segments of the upper limb. $-7,8,11$. Areas for movements of face and tongne. -12. Area for opening of eyelids, dilatation of pupil, and movements of head and eyes to the opposite side.-13. Movements of the eyes (angular gyrusvisual area).- The area for movements of the ear is on external aspect, below 13

that Munk, Glosglik and others have also maintained in later works that, in dogs, the frontal lobes in front of the sigmoid gyrus are nothing else than sensory and motor centres for the muscles of the neck and trunk, forming a part, i.e., of the Fühlsphäre? An examination of their respective works shows that Munk and others have employed very strong currents, and that the extirpation was effected at a point rather far back in the frontal lobe, so that electric excitation gave rise to muscular contractions, and extirpation to paralysis or paresis of the muscles of the neck and trunk and also to sensory disturbances, facts which the author frequently confirmed in his control experiments upon dogs and monkeys.

The difference in situation between the area for the movements of the head and trunk defined by Ferrier (area 12, Fig. 50) and that defined by the writer can only be explained by the greater strength of the currents used by Ferrier, who is nevertheless one of the most experienced investigators. If a small area of the posterior part of the frontal cortex of the monkey be laid 
bare and then strongly excited, movements of the head and eyes are provoked. If, without displacing the electrodes, weaker currents are used we get movements only of the eyes. If the strength of the current is again increased the movements of the head again appear upon the scene, without any displacement of the electrodes. An argument that is sometimes brought forward in favour of Munk's thesis is this, that, whilst these fairly strong excitations of the frontal zone provoke movements of the head, they do not give rise to movements of the upper limb, although the centre of the upper limb is in closer proximity to the point excited (as, e.g., when the frontal centre for movements of the ear is stimulated).

The reason for this will be evident if we take into consideration the synchronicity of the movements of the functional association, and reflect that the centre for movements of the head must be closely connected by means of associative fibres with the frontal centres for the eyes and ears. In attentive states in dogs, especially hunting dogs, but even more particularly in monkeys, which are suspicious, alert and cautious, the movements of the eyes, ears and head form one inseparable whole, taken along with that particular psychic state which we would describe as attentive and observant (suspicious and expectant attention). If there are numerous associative paths it is easy to understand that the waves of electric excitation may be transmitted along these to points somewhat more distant, like those concerned with the head and eyes, whilst there may be difficulty in provoking movements of the upper limb (although the areas concerned with the latter are nearer to the point of excitation) because associations between the area of the limbs and the frontal zone are not so often called into play and consequently not so well established. This is confirmed by the fact that in states of attention the limbs take no part in the particular movements of the eyes, head and ears, but rather are inhibited.

The intensity of the current must be measured on a sledge by an assistant, and estimated by the experimenter by means of the effects produced, in the same way as we test the electric excitability of a muscle which we suppose to be affected by atrophy with degeneration. We apply a small electrode, with a hand interrupter, to the point of excitation of the muscle, and move along the sledge regulator until we notice the first-i.e. the weakest-contraction, and from this, after due control, we judge as to the state of the muscle. No clinical neurologist would commit the error of employing strong currents when he wishes to learn the state of individual muscles or nerves. It is necessary to proceed in a similar manner when we seek to test the excitability of the various points of the cerebral cortex.

Some remarks are due as to the extent of the field of operation. At the International Congress of Psychology in Rome, Sciamanna, ${ }^{1}$ who reported upon the effects of removal of the frontal lobes, believed he had extirpated the frontal lobe, whilst, as a matter of fact, he had succeeded in excising only a small portion of it. It is well that we should be clear upon this point.

\footnotetext{
1 Sciamanna. Loc, cit.
} 
'I'le frontal field of experiment comprises that portion of the brain which lins in front of the convolution of Rolando and includes a part of the intermediate motor areas.

In the anthor's experinents there was destroyed or isolated not only

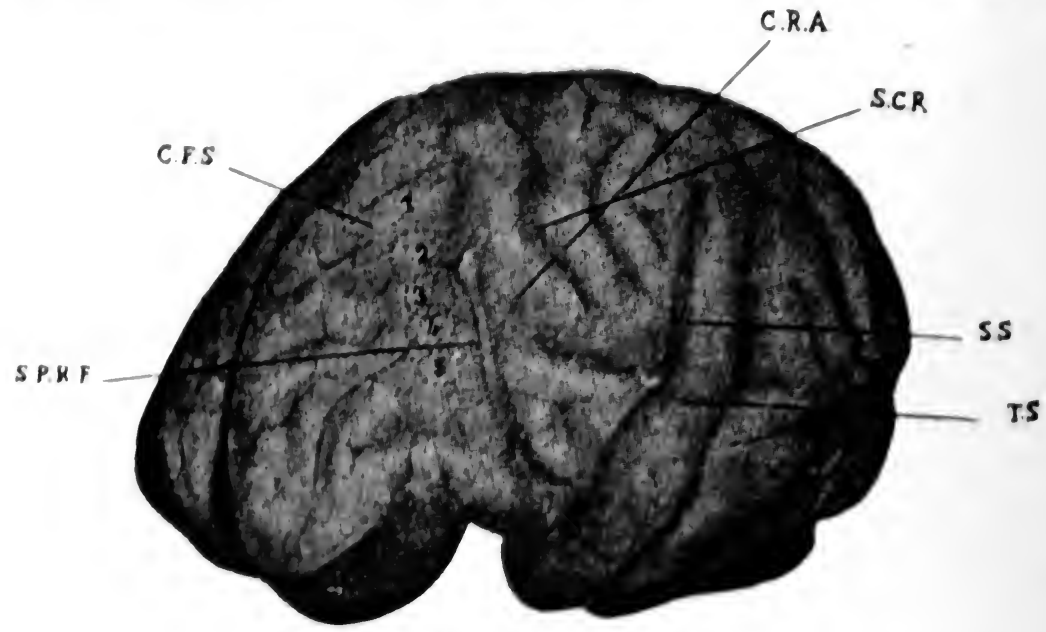

fig. 51. - Ibrain of celous

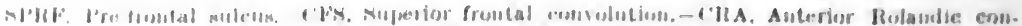

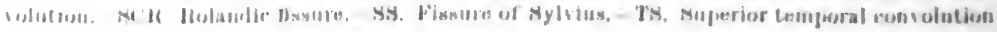

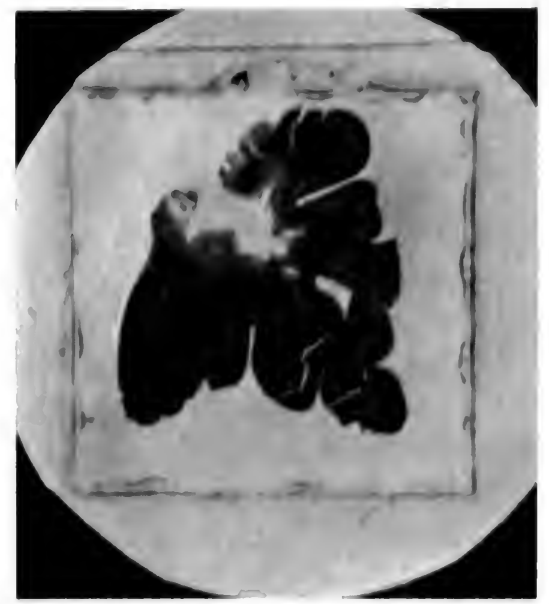

I. ig. fin. Vertieal section thromgh the frontal area slown ing soflening of the whites substance of the frombal lobes of colum, which is arrested in frosic of the muter area

Hoe prefrontal area, but also the fromtul and sometimes a part of the intermediate your. In most instances the excituble part of the frontal rone, indicated hy the numbers 1,2, 3, 1,5, Figure 51, wns included in the field of extirpation.

'lie experimental inymiries thus far completed go to establish a relation 
between the effects and the extent of the mutilation. Very limited extirpations of the frontal lobe, even when bilateral, do not produce appreciable effects. It is to be added that in few cases did the writer succeed in excising all the external surface of the frontal lobe. Very often there remainer somer

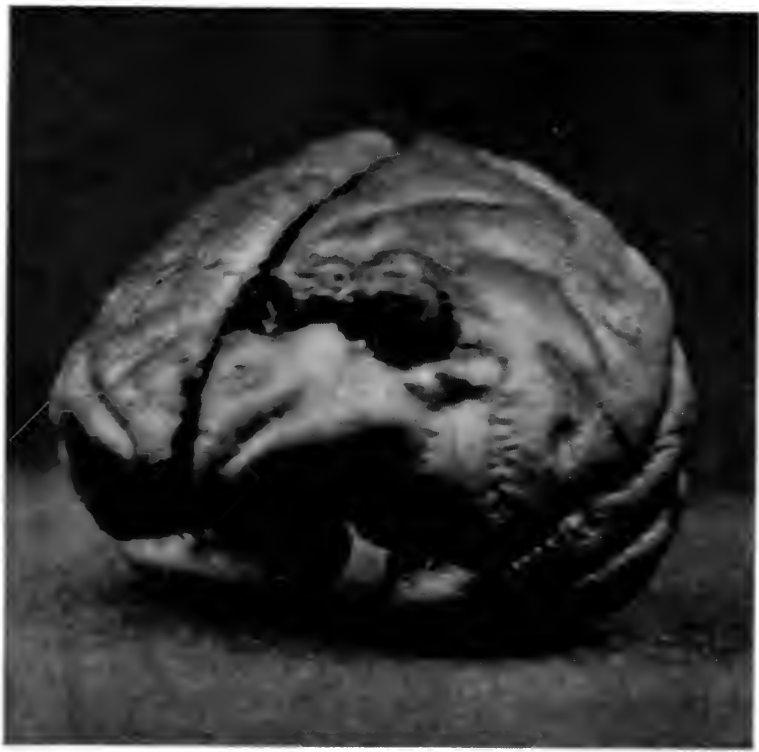

Yig. i3. - Frontal lobes separatel from the motor area with the exception of the operculam, and left in wing

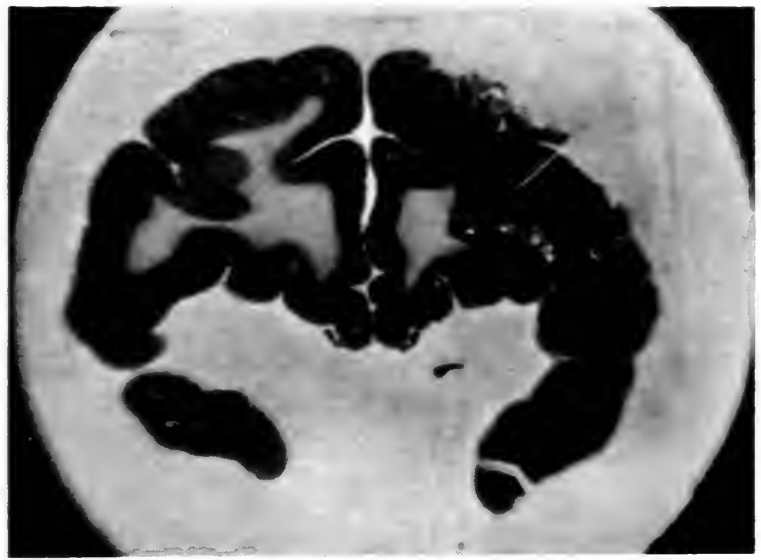

Fig. 54.-Decortication of frontal lote, left in wiens

small bridge, either of the superior frontal convolution or of the opercular part of the frontal zone.

The author has hardly ever removed the orbital surface, which also forms a part of the frontal lobe. This is another fiell for experiment and has scarcely been explored.

The figures here reproduced (Figs. il, i2, 5;, it, in) are serial sections 
showing the extent of mutilation or decortication, and also the parts of the frontal lobes that were spared.

The electrically excitable frontal zone forms a part, along with the prefrontal area, properly so called, of the whole area, excision of which does not give rise to any sensory or motor disturbance, and must be included for the present in the study of the functions of the frontal lobe, for reasons which will be explained further on. The constant experience has been that excision

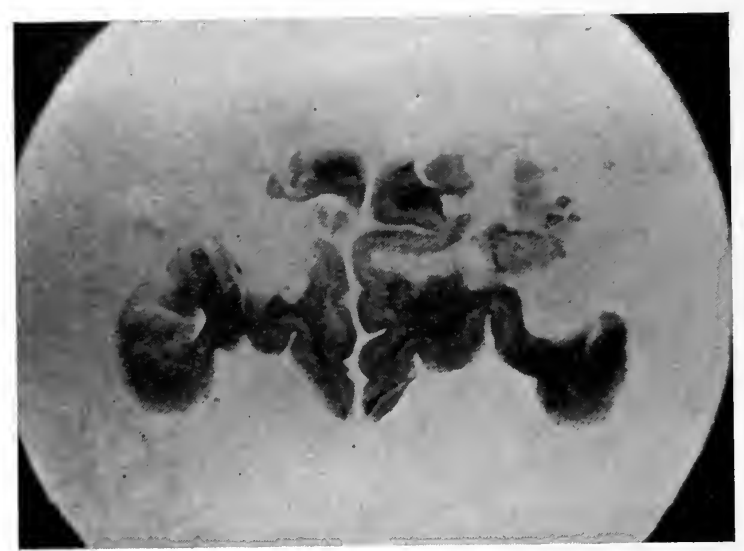

Fig. 55. - Section through the posterior limit of the experimental lesion in front of the motor area. The orbital surface and, on one side, the operculun, are spared

of one frontal lobe alone does not produce appreciable effect. It is only when a large part of the left frontal lobe is extirpated that the effects are perceptible. All objections based upon methods that are not really suited to furnish proofs of the function of the frontal lobes ought to be put to one side.

These preliminary considerations prepare the way for the following summary of experiments upon dogs and monkeys, some of which have not previously been published.

It is superfluous to add that in his researehes the writer paid the strictest attention to aseptic methods, and for excitation of the brain used small bipolar electrodes with a hand interrupter, the electrodes being formed of two little shafts of platinum with clubbed points, a little less than one millimetre in diameter, and two or three millimetres apart from one another. A good sledge of the Du Bois Reymond type permitted sufficient accuracy in the measurement of the faradic current employed in the excitation of the various points of the cortex. 


\section{CHAPTER V}

\section{Clinical and Experimental Histories of Dogs, Foxes and Monkeys, including Controls}

Experiment 1.-11th June 1889.-An adult dog, alert, healthy, docile. Examination prior to operation showed perfect integrity not only of motility but also of all kinds of sensibility. It was intelligent, obedient, quiet, affectionate. Anæsthesia with morphine and chloroform. Skull opened with trephine, of about $2 \mathrm{~cm}$. diameter, in occipital region on both sides, $1 \mathrm{~cm}$. in front of the occipital protuberance and $\frac{1}{2} \mathrm{~cm}$. from the median line on each side, leaving a bridge of about $1 \mathrm{~cm}$. between the two openings. Moderate hæmorrhage. Exposed the upper part of the second external bifurcated convolution and a portion of the first. Removed neatly the exposed cortical disc after making a circular incision into the cortex, with the bistoury following the edge of the opening. Destroyed and removed another part of the cortex on both sides by slightly extending the lines outwards and posteriorly. Arrest of hæmorrhage followed by lavage of the surface, asepsis, suture of the dura mater and of the soft tissues, and antiseptic dressing.

14th June 1889. - Three days later the dog was found seated on his hind quarters quite alert, with head raised and eyes open and turned towards the door. The ears were raised in the attitude of attention. A second dog, previously operated upon, was allowed to enter the room. It barked. Dog No. 1 rose, darted forward in the direction of the new-comer, turning to right and left through the sector of a circle whose radius would be represented by the chain which confined him. He was full of strength, had his tail up-turned, the head erect, and the opened eyes fixed in the void. On attempting to frighten him by pretending to strike him with a stick or waving a hat to and fro just in front of him, he remained immobile till all was quiet, when he immediately turned his gaze in the direction of the second dog and fixed it in the void, or, again, if the air was agitated, with the hat or the hand very close to him, he beat a retreat, in an attitude that suggested something between fear and observant suspicion.

The first examinations showed hearing and tactile sensibility to be very fine, but he did not see a sheet of white paper placed in front of his nose. Only on touching the nose with the paper did he throw the head back in an attitude of surprise, directing his gaze towards the nose.

He heard the slightest noises, moving the ears and directing the gaze in the direction of these. If a vibrating tuning-fork was brought near to any of the ear's he became afraid and retreated.

He reacted to the lightest tactile stimuli, equally on both sides. On drawing a feather or a pencil lightly across the ears, back, nose or paws he shook himself and turned in the direetion of the stimulus. He seratehed himself wherever there was any parasite, with equal readiness and strength on either side. If, in a dark room, a lamp was lit in front of him, he seemed to perceive the sudden light. He turned his gaze towards it and the pupils reacted.

He was suspicious. If one came upon him unawares and touched him lightly, he turned cautiously away to hide himself in a eorner of his bed. When taken out into the passage, he knocked against the walls, now to the right and then to the left, then threaded his way along the passage and arrived at the exit. Here, however, as the white of the passage ended, he knocked against the wall, first on 
the right then on the left, then, sniffing and ealling to his aid the sense of toueh, he felt his way cautiously and finally succeded in passing through the doorway. If, on the way, he came to a step, he put forward his paw and, finding no resistance, he withdrew it again, as though afraid of falling into space, then thrust forward his nose and tried again with his paw. If, in this way, he became convinced of the presence of steps he then descended briskly. In a similar way he succeeded in mounting the steps.

18th June 1889. - He was always alert, in good humour, knew quite well the voice of the attendant who brought him food, ate well, and exhibited erotic tendencies (he attempted to cover another dog that had also suffered mutilation of the frontal lobes). The wound was healing by first intention without trace of suppuration.

28th June 1889.-Healed by first intention. Mental condition identical. Sight was improved so that he knocked less against obstacles. An unsuccessful attempt was made to measure the visual field. He was always alert, sometimes full of strength; had marked erotic tendencies; well cared for, he was kept alive for several months in the same condition.

18th January 1890. - On this date epileptic eonvulsions came on the scene and were repeated with Jacksonian eharacteristies (rhythmieal contractions of left side). If one ealled to him during the attack he was able to raise his head languidly. For some days he remained paretic on the left side, then regained his ordinary condition but was not so alert nor so spirited as previously. He walked with a slightly ataxic gait, the left anterior limb in particular being raised rather high from the ground. Taetile sensibility when tested was found to be normal. If touched on the ear, the side of the nose, or any of the limbs, he always turned to that side. There was no difference in this respect between the two sides. There was, however, diminished power in the limbs, more especially on the left side, so that sometimes his paws bent under the weight of his body, especially when making any effort. Resistance to passive movements was diminished. Smell was found, when tested, to be somewhat diminished. He was kept fasting for about twenty. four hours and then taken to the observation-room into which a plate of meat was brought. He became quite animated again and went sniffing around. He did not always find the place where the plate lay, not recognising it until it was pushed quite close up to him as he went around so that he knoeked against it with his nose, or happened to touch a piece of meat, which he seized in his teeth and ate with evident enjoyment. If pieces of bread and meat were mixed together and thrown in front of him he made no seleetion, but, nosing around, ate indiscriminately whichever piece he first came across. As a rule, when walking, he avoided any objects or obstacles in his way, but if he quickened his pace he often came against them lightly with his nose, which he always kept extended in front of him to aid him in avoiding them. Hearing was good. When called he turned round, but in most instanees did not guess correctly the direction whence came the voice. If the tuning-fork was approximated to one of his ears he shook himself and retreated in surprise at the strange noise, the eyes remaining fixed as though seeking to discover the cause of the unusual sensation; this was true of both ears. It was evident, however, that he still looked into space just as before. The pupils were dilated and reacted to light but there were no movements of aceommodation even when a lamp was passed at various distanees in front of his eyes in a dark room. It seemed, however, that he was aware of the light, because, if he was taken by surprise, and a ray of light was allowed to penetrate the eye from any direction, he showed evidence of fear, turned his head round and hid it in the arms holding him. Intelligence, though well preserved, was not what it formerly was. By means of his senses, other than sight, he recognised places, persons and things. He was very affectionate towards those who were in the habit of attending to him. He was quiet, rarely playful, often indifferent, and when left quietly to himself 
his attitude suggested melancholia, with a distant, uncertain look in his eyes which soon lit up again if anyone called or fondled him. He had lost his former vivacity and often showed an extraordinary fear of noises.

3rd March 1890. - When taken into the observation-room his condition was found to be unchanged. For some minutes he jumped about the room in a joyful way, then commenced to perform some movements as though he saw a fly and wished to catch it. Yet sometimes, in addition, there were movements of the head, upwards, backwards, and to the left, movements of the mouth, which he opened and closed as though in the act of biting (lateral, visual hallucinations?). One noted, too, spasmodic movements of the left orbicularis palpebrarum (Jacksonian contractions).

5th March 1890. - Condition unchanged. The brain was exposed on the left side at a point corresponding to the anterior portion of the second external convolution and a small part of the sigmoid gyrus. Electric excitations in the latter area (sigmoid gyrus) produced the usual movements of the opposite limbs, rather exaggerated and not easily circumscribed, notwithstanding the fact that a very slight intensity of current was employed. Electric stimulation of the exposed part of the second convolution produced only slight contractions of the orbicularis palpebrarum of the opposite side. This part of the cortex was then destroyed, the sigmoid gyrus being spared, and the wound sutured. On examining the animal next day he was found fairly lively but more unsteady in his gait, and he made his way through the doors less easily than before. Circus movements towards the left were present.

11 th March 1890.-Circus movements continued; they were somewhat more marked than before, and always towards the left. When the right eye was elosed they diminished, and the animal was able, to some extent, to avoid obstacles. On the other hand, when the left eye was closed he remained fixed in one place, with the head turned to the left, and, when pushed to make him walk, constantly knocked against things with his right side-i.e. on the side of the open eye. When the left eye was uncovered he went about the room and followed those walking therein, sometimes mistaking the direction and at other times knocking against obstacles (he was evidently unable to measure distances and recognise objects), but when the right eye only was allowed to remain open, it was with great difficulty he could be induced to walk about, and he was constantly knocking against things.

11th A pril 1890.-Killed with chloroform. On post-mortem examination the brain seemed diminished in size. On the occipital lobes two cicatrices, about the size of a two-centimes coin, were observed, with retraction of the surrounding tissue. The first external convolution on the right side, as far as the sigmoid gyrus, was of a dirty whitish colour, at one point rather hard like a cicatrix, at another softened. On the left side the cicatrix was less retracted, though fairly symmetrical with the other. All the anterior part of the occipital focus was more normal in colour, and the convolutions in general were better nourished. On the right side, the upper third of the anterior branch of the sigmoid gyrus up to the presylvian sulcus was softened, and there the dura mater was adherent. A similar but much smaller focus was found on the left side, corresponding exactly in site to that of the other side. On the inferior surface, the hippocampi were symmetrically diminished in size, being reduced to two cysts of a yellowish white colour. The brain was preserved in alcohol.

17th April 1890. - A vertical section in front of the two eicatrices, at a point almost midway between the presylvian sulcus and the occipital extremity, showed marked dilatation of both lateral ventricles. The caudate nucleus is diminished on each side. Below, the two hippocampi are transformed into two small sacs occupying part of the substance proper of these convolutions.

The autopsy provides an explanation: (1) of the ataxia-the result of the lesions of the sigmoid gyri ; $(2)$ of the circus movement to the left-resulting from 
the second operation performed on the left hemisphere; (3) of the hyponosmiabecause of the bilateral hippocampal lesions; (4) of the mental decadence-the result of the multiple, experimental and spontaneous, lesions and of the secondary cerebral atrophy.

The post-mortem examination likewise shows the possibility of non-experimental lesions in other parts of the brain in consequence of the primary experimental lesion, and the necessity of following the animal with close attention, and registering all subsequent and new symptomatic manifestations that are not to be attributed to the primary experimental lesion. This case also shows that although the dog was psychically blind (it failed to recognise food with the aid of sight, apart altogether from the diminution of smell dependent on softening of the hippocampi, and apart from the lesions of the sigmoid gyri and the spherical restriction of intelligence), yet the charaeter, one might almost say the personality, was recognisable. In particular the affective activities, the attention, the interest aroused by stimuli falling upon its body, were fairly well preserved.

Experiment 2.-A fairly young biteh; sharp, intelligent, lively, obedient, good-natured, educable; for several days past she has been affectionate and grateful towards those who had charge of her. Examination of all forms of sensibility reveals nothing abnormal and no difference can be detected between the two sides.

8th May 1889. - Prepared for operation. Anæsthesia by morphine and chloroform. Seetion and reflection of the left temporal muscle followed by trephining in the temporal region, at a point corresponding to the Sylvian fissure but slightly above it. Enlargement of the opening, below and behind, with bone forceps. No disturbing incident during operation. Section of dura mater. Slight hæmorrhage arrested by ordinary hæmostasis. The third external convolution, upper part of the fourth, and part of the second, exposed. Faradic excitation (with a current hardly noticeable on the point of the tongue) in the posterosuperior area-the second external convolution-produces dilatation of the palpebral fissure and of the pupil (previously, markedly contracted by the action of the morphine); further forwards, on the same convolution, stimulation gives no results; below, on the third convolution, it gives rise to movements exclusively of the opposite ear, movements which continue in a clonic fashion even after the current is withdrawn, and which spread to the ocular bulbs, the face and the limbs, causing a classic epileptic convulsion. This having ceased, the exposed part of the third eonvolution, the lower margin of the second, and the exposed upper margin of the fourth are removed. Hremorrhage, which is fairly profuse, is controlled; lavage with sterilised water; suture of dura mater.

9th May 1889. - The bitch is slightly depressed; she is lying stretched out on her couch, but raises her head on hearing the noise of the opening door, looks round, and wags her tail. When called, she turns her head in the direction of the voice but does not move from her position. When lifted and placed in the middle of the room she returns to her couch, walking well. Taken to a path in the garden she moves around, here and there, in an aimless fashion, leans her head on the herlge on the right-hand side but does not lose the path. She walks at a good pace, only occasionally knocking against things and always on the right side.

20th May 1889. - After some suppuration, which commenced on the fourth day, with a discharge from the wound, the bitch is gradually reviving, is putting on flesh and regaining strength. She likes to lie stretched out on her couch. If called, she comes slowly towards the person ealling her, wagging her tail as usual, or, on the other hand, she shows a good deal of fear and walks in an almost crouching fashion. Left to herself in the garden, she returns to her couch. When carried to some distance in the garden and put down where the paths intersect, she stops a little, as though to regain her bearings, then threads her way along the path which 


\section{EXPERIMENTAL HISTORIES OF DOGS, FOXES \& MONKEYS}

leads directly to her kennel. On the first occasion, when she finds herself in front of steps down which she ought to go, she turns to the left (the side on which she sees), proceeds for some distance, stops, turns round, retraces her steps, comes to the stair, descends, and walks straight on. On the second occasion, instead of going straight on, she again turns to the left, but, as before, turns back and goes down the steps in order to reach her kennel. She does not show her former vivacity, but sometimes, squatting on her hind limbs, she makes some movements as though to catch flies, but this she does only towards the left side and never towards the right (unilateral visual hallucinations?). Sight, examined by the method usually adopted in the Institute (application of a vulcanite eye-shade under the eyelids), seems abolished in the right eye ; the bitch does not react to any luminous stimulus falling on the right eye; not even from the internal angle of the eye does she appear to perceive a light brought close up to her, as was often observed in other dogs. There is a central corneal ulcer on the left eye, with superficial epithelial exfoliation. The visual field of the left eye, apart from the defect arising from the corneal spot, seems normal; it presents none of the signs of bilateral hemianopsia. The biteh does not react to the tuning-fork brought elose up to the right ear as she does when it is approximated to the left ear, in which case she lowers her head as though afraid, and shakes her ears. There is no noticeable disturbance of sensibility. She reacts to punctures equally well on either side, but if light contacts be made on the nose with a piece of paper or the head of a pin, now on the right, now on the left; she is seen to react more briskly on the left than on the right side.

There is no disturbance of motion.

3rd June 1889. - On entering we find her on her couch, seated on her hind quarters in an attitude of attention. She has lost some of her former liveliness. She recognises the persons who have had charge of her. Taken out on to the garden paths she makes her way back to the old outhouse, goes round it one way, then the other, then again in an opposite direction, as though looking for the door. She raises the right paws rather higher than the left. She knocks her nose against the wall on her right. She persuades herself it is not the door, takes the path leading to a kennel whieh is not the one assigned to her, and here accommodates herself. Within the kennel, which is rather spacious, she walks cautiously about, as though looking for a place which will suit her best. On waving a pieee of paper elose up to her right eye, she behaves as though nothing were there. On doing the same before the left eye she gets angry, snarls, shows her teeth, and tries to bite. Her behaviour is similar if the other dog is brought near her. She appears not to form an image of what is before her at first sight. It is noticeable, however, that her character is decidedly altered. She likes to remain at peace, is melancholic and irascible, preferring solitude and rest.

She continues to be decidedly deaf in the right ear. On testing sensibility again it is found to be normal on both sides.

21 st June 1889. - Her condition is precisely the same as last recorded. She prefers solitude, quiet and repose. She does not move from her bed, seems melancholic and does not come out, even when called, so that from time to time it is necessary to remove her forcibly from her couch in order to examine her (an abscess with induration of the vulva is present and may possibly be accountable for this state). She walks well and returns to her eouch from any quarter, showing neither error nor uncertainty in direction.

3rd July 1889. - Suitably treated, the vulvar abscess is cured. The cornea, rendered turbid by the ophthalmia, is now quite clear, except for a slight opacity in a small central point. Taken out and left to herself on the garden paths, she walks or runs back to her couch. On the way she knocks against the hedges on the right from time to time, but much less frequently than before. On again covering the left eye with the vulcanite eye-shade, she does not wink when an object is passed rapidly in front of her eyelids. If, however, the room is almost 
dark and a small lamp is lit in front of her right eye she is surprised by the light and draws back her head.

She has had nothing to eat sinee last night so we make the following experiment. While she is seated quietly, with head erect, we suspend a piece of meat on a thread and move it along, from right to left, on a level with her eyes. She perceives the odour of the meat and makes some slight movements of the nose as dogs do when sniffing, but she does not see the meat in the temporal sector of the visual field at a distance of $25 \mathrm{~cm}$. from the eye. As the piece of meat reaches the line of the central meridian of the eye she sees the object, draws back her head as though to recognise it, sniffs more strongly and makes to put her nose up to it. The meat has meanwhile been moved further on, so she gets up and follows it. Finally she reaches it and eats it greedily.

If pieces of meat and bread are scattered on the floor, she sniffs the air, then brings her nose up to the food and eats with avidity, first the pieces of meat, afterwards the bread, beginning with those to the left of a vertical plane passing through the visual axis, then, sniffing and nosing about, eating all that remains, including those that have fallen much outside and to the right of the visual field. Sight is certainly improved as compared with its eondition during the first few days after the operation but the visual field has a decidedly right hemiopic character.

Both eyes being covered, her hearing is now examined. When the tuning-fork is brought close to her left ear she reacts as before, but when it is brought up to the right ear (the side opposite to the field of operation) there is no reaction at all; it seems as though she notices nothing.

Light contacts on the head, ears, back, chest, groin and tail are noticed perfectly and equally on both sides; moreover, this bitch has often been deteeted getting rid of annoying parasites with her teeth and her paws, both fore and hind.

25th July 1889. - Re-examined to-day. She has again beeome alert, as she was prior to the operation. In all her attitudes there is no difference to be seen as compared "with what she was before. No sensory or motor disturbance. Sight also is improved in the right visual sector, apart from an insignificant uncertainty in reeognising morsels of food. Hearing remains almost abolished on the right side. When, however, the tuning-fork is brought elose to the right ear, a vague movement of the ear is observed and the bitch assumes an attitude of attention.

Killed by means of ehloroform. Autopsy. On removal of cranial vault a large thiek cicatrix is seen; it is adherent to the decorticated surface of the left hemisphere. It is now detached. The brain is quite normal apart from the left temporal region. The parts involved are a portion of the fourth convolution, the middle part of the third, and the lower border of the middle part of the second external convolution.

After hardening in formalin solution serial sections were made. No further alterations were visible to the naked eye, save for a slight dilatation of the left lateral ventricle.

In this case, the hemi-hypoxsthesia and amblyopia were phenomena of diaschisis and were very slow in disappearing, first the hemi-hypoxsthesia, then the amblyopia, which passed into bilateral homonymous hemianopsia. Then followed phenomena of compensation, first of all in the visual function, in so far as she saw, though she did not recognise, objects in the right (hemiopic) visual field; and finally the deafness of the right side lost the absolute character it had exhibited for about two months. One can understand the compensation in the visual function, because only a portion of the cortical visual field had been injured. Intelligence and character rosumed their former conditions.

ExPERIMENT 3.-A biteh of medium sizo, adult.

She walks well, jumping motions are precise and there is no disturbance or defect of movement. She is very intelligent and lively. 
Sight is normal. If pieces of meat and bread are placed promiscuously on the floor at various distances, she selects and takes, with precise movements and neat direction, first the pieces of meat, and then, as she is still hungry, she takes and eats the pieces of bread one by one in the same manner.

Smell also normal. If pieces of bread and chalk of the same colour are thrown before her on the ground she takes and eats with rapidity and precision the pieces of bread, but as soon as she brings her nose up to a piece of chalk, she draws back her head and does not go near it again.

With vulcanite eye-shades applied, she hears the slightest noise, and always turns her head and eyes in the direction whence it comes, in a listening attitude.

The muscular sense is well preserved, as can be inferred not only from her movements in jumping and walking but also from the precision with which she seizes a piece of meat in the air as it is thrown to her.

In two days she has learned to recognise places and persons and she shows affection to those who pet and make much of her.

Taken for the first time into the laboratory she shows herself suspicious, paws the attendant and wishes to follow him alone. Called by me, she hides herself. If the attendant goes away she creeps into a corner and remains there like one afflicted and suspicious. If the attendant comes back into the room she becomes playful again. If she hears a noise, especially when alone with us in the observationroom, she pays great attention to it and turns her gaze in its direction, and sometimes makes to go out. She seems docile.

4th March 1890.-Fairly deep narcosis with morphine and chloroform. One attempt only at vomiting.

The cranium is trephined on the left side at a point corresponding to the middle part of the second external convolution. Incision and retraction of the dura mater: On electrification of the anterior portion of the exposed area, we get limited contraction of the orbicularis palpebrarum of the opposite side. On using a stronger current and displacing the electrode a little in front and below, but still keeping within the limits of the second convolution, we get contraction of the other muscles of the right side of the face. We can observe a slight indication towards closing of the eyelids on the left side (the same side as the excited hemisphere). Behind this very excitable area comes another, where even a comparatively strong excitation produces no effect. Displacing the electrodes yet further back we find, still on the second convolution, a third area which is excitable with a weaker current, its effects being visible on the extrinsic muscles of the eye and to a slight extent also on those of the iris, for medium currents induce displacement of the right (opposite) eye towards the right, and of the left eye also towards the right, though to a much less extent; and further, slight dilatation of the pupils. I now demarcate the central inexcitable zone of the exposed area, making vertical incisions with a small bistoury, and then, with a small spoon with cutting edges, I scoop out the bridge between the anterior and the posterior excitable areas. The wound being now sutured with all the usual precautions, and the animal liberated (already awake, for the experiment has lasted over twenty minutes), she begins to walk about with a slow but certain gait. On closer examination it is distinctly noted that she no longer winks the right eye. The examination is now deferred to another day.

7 th March 1890. - The bitch is awake and in her usual good humour. She eats with appetite and recognises the persons who bring her food, exchanging caresses with them. If from the right side one attempts to frighten her with a flash-lamp, a stick, or in any other way, passing these in front of the right eye, she takes no notice and does not wink. It appears, however, that she may notice in an indistinct way, for she will turn her head so that the impression falls within the visual field of the other eye or on the extreme left of the right eye itself. If, standing in front of her, we place on the floor two pieces of meat, one to the right and one to the left at equal distances from the vertical of the nose, she takes and swallows 
with avidity only that on the left side, although she is hungry through having been made to fast, and she looks in my hands for other pieces. If instead of two pieces of meat I put down in the same manner a piece of bread to the left and a piece of meat to the right, she pounces upon the bread and does not see the meat. If I take a piece of meat in each hand and pretend to throw them to her simultaneously, the right to the right and the left to the left, she turns round seeking the piece on her left but never that on her right. If, however, I gradually bring a piece of meat elose up to the median line, then move it beyond that towards the internal angle of the right eye, she seizes it with a sufficiently precise movement. Examination of tactile sensibility, her eyes being covered with the gutta-percha eye-shades, gives negative results. There is no disturbance of motion or of hearing on either side. Intelligenee and humour are perfectly well preserved.

2nd A pril 1890. - Healing by first intention. If, while she is seated on her hind quarters with head erect, a person standing at her shoulders advances the hand or any objeet into the visual field from the side of the lesion, she notices it immediately and turns towards the object in question. If the experiment is repeated on the other side-i.e. the side opposite to the lesion-she does not notice the hand or any small object or even a black hat, until the object is advanced beyond the middle line. During the examination she has made use of the hind paw of the side opposite to the lesion, with adequate movements, in order to remove some irritation from the ear of that side.

The bitch is always quick and active, walks well, avoids all obstacles, except that now and again she knocks against the edge of the doorway on the right side (opposite the lesion). She recognises the persons who take to do with her, paws them and shows affection. She has preserved the instinct of decency, for she neither urinates nor messes in the large cage where she is kept unchained, but, on putting her out, she runs to the extreme end of the garden and there urinates at length and voids the bowels. She has the sense of appetite, for she paws and jumps up on, and makes a great fuss with, the man who is in the habit of bringing her food. The tactile sense, re-examined with eare, is quite normal. She scratehes herself equally well with the hind paw of each side so as to remove cutaneous stimuli, and with her muzzle she seeks for lice, now to the right, and now to the left, with precise movements.

Ocular movements and tactile and painful sensibility of the ocular conjunctiva are normal and equal on both sides.

Hearing is well preserved, judging from her reaction to the tuning-fork brought more or less closely up to the ear. In all the tests she withdraws her head from the acoustic stimulus and turns herself in its direction. This action is even more evident when both eyes are covered with vulcanite eye-shades.

When the left eye is covered, she is rather more cautious in her movements. She avoids all obstacles but as though she has difficulty in recognising them. She seizes pieces of meat thrown to her and runs after them, but her movements are less precise. Her movements are greatly aided by touch and smell.

$10 t h$ May 1890. - She is quick and active, full of spirit, affectionate to her attend. ants, and is domesticated even with the doctors. She is docile, she paws those about her, she runs and jumps, showing no difference in the two sides. She turns her head now to this side, now to that, and curves herself up in every fashion and every direction, using now one paw now another to rid herself of troublesome insects. No difference is to be detected in the two sides, however delicate the stimuli applied to various points of the body.

Sight is restored. Employing the usual method and covering the right and left eye alternately, a proceeding to which she is now accustomed, she recognises persons and foods and runs after little balls as they are thrown away, no matter which oye is open.

She is killed with ehloroform. The brain on removal shows a lesion limited to 
the second external convolution, hardly encroaching on the corresponding lower border of the first external convolution. The lesion is limited in front at a point where the convolution curves round below following the bend of the sigmoid gyrus.

Antero-posterior vertical sections reveal no other lesion to the naked eye, apart from the fact that the left lateral ventricle is dilated as compared with the right.

Experiment 4.-16th April 1889.-A male adult dog, robust, lively, quick, agile, affectionate, sociable and good-natured. Senses all normal.

I expose the right hemisphere towards its anterior extremity laying bare part of the sigmoid gyrus and the frontal extremity of the hemisphere. The usual movements of the limbs are provoked by faradic excitation of the sigmoid gyrus. In front of the anterior branch of this gyrus the same slight electric excitation produces no results. On increasing the strength of the current, movements of the head and eyes to the opposite side are induced.

With a small bistoury I make a deep cut into the grey substance and scoop out all that remains of the hemisphere in front of the presylvian sulcus, leaving in situ only a part of it which it is difficult to remove, without interfering with the olfactory bulb which I desire to spare. The wound is cleansed of blood and washed with sublimate solution. The edges of the dura mater are brought together and sutured; asepsis and suture of the overlying tissues.

18th April 1889. $-\mathrm{He}$ is brought into the observation-room and allowed to go free. He walks well, but shows slight paresis of the left anterior paw. He also exhibits very great fear, and on going near those in the room he crouches and cringes, so that his abdomen touches the floor.

24th A pril 1889. - He eats regularly from the usual bowl. Left at liberty in the garden he at once makes off, going through a jumping performance in the middle of the path. After proceeding a few yards he stops, takes a turn to the right, looks back, wanders here and there, showing great indecision as to which direction to take, as though arrested by a sudden remembrance. He finally makes off, running in one direction, then suddenly stops, seems undecided, turns back, then returns in the same direction, behaving very differently from what he did prior to the operation. Not a single moment does he remain quiet, as was his habit. Called in the usual way by me, he hears and runs towards me, but if on the way he comes across a pool of water or any other object on which his glance may fall, he stops and noses around it, forgetting to come up to me. He goes into a fur.ow in the garden and bites off some leaves as though he no longer recognises them as such, things to which he previously paid no attention. Never a moment at rest, he comes to a rubbish-heap, jumps up on it, noses around and carries off one or other of the leaves or bits of rubbish which he finds there. He does not readily return to his kennel, so that one has to get hold of him and lead him back on a leash. He does not react to visual stimuli equally on both sides, for he does not recognise objects with the left eye. Methodical and patient examination of tactile sensibility shows a slight diminution on the side opposite to the mutilated hemisphere. He hears equally well on both sides, shaking his ears whenever the vibrating tuning-fork is brought near. When he walks slowly, accurate observation enables one to detect a slight paresis of the opposite side and a slight sinking of the pelvis, with the convexity upwards and rather to the left. Priapism has been practically constant for ten days.

27 th A pril 1889. - He does not wink the eyelids on threatening him with a stick or with the hand, after bandaging the right eye. He does not readily recognise a piece of meat held between the fingers, although he directs his gaze towards it. As he walks he avoids obstacles, but displays a manifest uncertainty and perplexity in his movements, sometimes rubbing his left side against a wall.

3rd May 1889. - He is much improved, more cheerful and playful ; he jumps up and paws now one, now another, of the persons in charge of him. He runs hither and thither without ever knocking himself. From a distance he sees a bitch that 
has been operated upon some time before and runs to play with her; though chased away, he returns again, although he makes no attempt at copulation, so that the caresses he bestows on the bitch differ in no way from those he bestows upon the man who brings him his food. In all his ceaseless movement and rushing about there is an evident agitation which seems to point to a total want of that direction and finality which we observed in unmutilated dogs. Although improved, the animal seems unable to form a precise judgment of anything that falls under his senses, as he formerly did, so that not only does he walk hither and thither in an aimless way, but he puts his nose here and there, to right and to left, to substances which formerly never arrested his attention (a leaf, a medlar, a twig, garbage and filth of any kind, etc.). The paresis of the opposite side has almost disappeared, and very rarely does he support himself on the dorsum of the paw. There is no difference between the two sides as regards tactile and painful sensibility. The vibrations of the tuning-fork are heard equally on both sides. He reacts less intensely with the eye of the side opposite to the mutilated hemisphere. On examining his sight with the usual methods there is no evidence of hemianopsia, although there is a manifest defect in the visual recognition of objects (foods) which, when his eyes are bandaged, he can recognise with the aid of his sense of smell.

12th May 1889. - The dog seems completely recovered. He is brisk and lively, recognises and paws the persons who look after him and is continually jumping up on them. No disturbance of motion is to be detected even when he is lifted up and suspended by the thorax. There is no disturbance of sensibility, for he reacts to the slightest stimuli on both sides equally. He hears equally well with each ear. The only thing to be noticed is a certain difference between the two eyes in the manner of reacting, in the sense that he winks the left eyelids less than the right. On bandaging one or other of the eyes, and passing objects or the hand in front of the eye of the side opposite to the mutilated hemisphere, he shows himself markedly uncertain and confused when only this eye remains open, and not when it is bandaged.

Three facts are worthy of note:-(1) Restlessness, uncontrollable and incoherent. (2) The tendency to take up articles which did not before attract his attention (dry leaves, twigs, pieces of wood and filth). (3) The priapism, with curvature of the trunk, which quite disappeared after a couple of weeks.

16th May 1889. - The conditions mentioned in the preceding observations continue as before, except that the difference in sight between the two eyes has gradually diminished until it has practically disappeared.

Employing the same methods, I again open the cranium on the other side. With a faradic current various points of the anterior convolution of the sigmoid gyrus are excited, provoking the usual movements of the limbs and especially of the trunk and head. Excitation of the posterior extremity of the frontal lobe provokes only movements of the head and eyes. Further forwards, excitation gives no reaction. With a sharp bistoury I divide off all the cerebral hemisphere which lies in front of the sigmoid gyrus. With a small spoon the cortex is removed and the wound then elosed.

The dog is very helpless and quite paralysed in both fore limbs. He remains for hours with his nose on the floor as though in a forced position, with the fore paws curved under, the hind limbs extended and raised, as though he were trying to poke his nose into a hole in the ground. No matter when or how we remove him from this position he takes it up again, always returning to press his muzzle on the ground.

21 st June 1889. - He has gained greatly in strength. When the door of the room where he is kept is opened, he threads his way out, walks with eyes cast down, the head inclined forwards, almost touching the ground with his nose. He no longer paws or caresses those who look after him. He wanders here and there in a stupid, awkward, aimless fashion. When called, he turns and walks for a little in the direction of the voice, then seems to forget and takes another direction. If we 
obstruct his path with a hat, he is afraid, and stops, and does not seek to escape from the obstacle. He sees equally well with each eye. He hears, and feels tactile impressions on both sides. The things that are outstandingly defective are vivacity, perceptive promptitude and courage. For example, he exhibits very great incoherence in his movements, and a tendency to take into his mouth anything he comes across, which next moment he leaves aside with equal readiness. He wanders round a basin like one famished, first in one direction, then in the other. $\mathrm{He}$ stumbles over a chain holding another dog which has undergone an operation on the occipital lobes. The latter is blind, and, feeling himself jerked and not knowing the reason, bites the first dog, which, in a state of fear, merely doubles himself up, without seeking to escape from the violent attacks of the other, so that it is necessary to remove him forcibly from his awkward situation. Soon after, he returns to the same place and a similar scene is enacted. Still, a third time, he goes back, and on this occasion the other dog attempts to mount on him whilst he, instead of making off or defending himself, merely takes to howling.

5th July 1889. - The same condition of things persists-incoherence, errors of judgment, restlessness, stereotypism, indifference. If taken out to the garden paths, he runs about stupidly and without signs of tiring, stooping now and again to pick up pieces of dry grass and filth. If called, he turns back in the direction of the voice or whistle, but at a certain point forgets and goes his own way. If he reaches a space where there is a fountain, he runs round it many times in succession, stops for a little, and then without any reason runs off again. If left to himself in the spacious garden, he does not again return to his kennel, but wanders about here and there in an aimless manner. He does not follow anyone. The sense of smell is unaffected; with his eyes bandaged he picks up at once, if hungry, pieces of meat thrown 20 to $30 \mathrm{~cm}$. in front of him. Sight is normal; the visual field, measured by the usual methods, is normal. He picks up everything with precise movements. All trace of paresis of the limbs has disappeared. He can move his head in all directions and raise it high, as is evident when shown a piece of meat suspended 30 to $40 \mathrm{~cm}$. above his head.

20th July 1889.-He remains in the same condition. Killed with chloroform.

At the post-mortem examination the whole brain appears normal except in front, where, at a point corresponding with the trephine-opening, there exists a thick cicatrix which has to be detached gradually with the scalpel in order not to tear the normal margins of the brain. The crucial sulcus is normal on both sides. The anterior and posterior branches of both sigmoid gyri are normal, except a small portion of the anterior branch of the left sigmoid gyrus which has been injured in the operation and is included in the cicatrix.

This brain was kept and prepared for the investigation of degenerations secondary to the extirpation of the frontal lobes.

$$
* * *
$$

In this case we have had very definite evidence of serious alterations of character, affecting all the intellectual and emotional processes. These changes made themselves apparent from the outset and were persistent. They could not be attributed to paralysis of the trunk and limbs, because that was fleeting; nor to disturbances of sight, because this, too, disappeared, although the psychic conditions in no way improved, and this could only be the effect of the lesion of the anterior lobes.

Summing up the results of all the experiments we have carried out on dogs, it is to be observed that in almost every case we had occasion to note changes in character and limitation of the mental powers as the result of mutilation of the frontal lobes. With these we shall deal subsequently. In addition, we had nearly always to notice visual disturbances, consisting of bilateral hemianopsia, much more evident in the eye of the opposite side. These disturbances were aiways 
transitory, except in one case in which we found, at the autopsy, softening of the occipital lobes. There was also observed paralysis of the trunk with lumbar curvature and drooping of the head, but this was fleeting. The paresis of the opposite fore $\operatorname{limb}$, sometimes also of the posterior limb, was transitory, and on each oceasion the autopsy showed a limited lesion of the anterior branch of the sigmoid gyrus, of which, however, the greater part was well preserved. Disturbances of general sensibility were very rare and of a fleeting character (the area of sensibility was almost always spared). We had no disturbances of hearing, hunger or thirst. So far as the sexual instinct or appetite is coneerned, it seemed not to be abolished in the dogs experimented upon, although sexual boldness, which is quite another thing, was absent.

Experiment 5. - 15th August 1889. - A young vixen of $4 \frac{1}{2}$ to 5 months, well developed. It has been found impossible to tame her; so wild is she that she has proved quite refractory to the various plans we have tried to make her docile. Though still small, she can bite like anything. Confined in a large iron cage, she showed herself quite indifferent to all kindly attentions and to the preferential treatment meted out to her, as compared with the other animals in the institute. More than once when taken out of her cage, she nearly strangled herself in her attempts to escape, tugging madly and obstinately at the chain or leash attached to her neek.

Very fond of meat and milk. She made her diet almost exelusively of these. Inside and outside her eage she appeared to have only one instinct-that to escape. Every time the cage was opened, she spied around to see how she could jump out, and in this no doubt she would have succeeded had she not been secured inside the eage with a chain. Her eyes were always wide open, suspicious, stealthy, and her ears always on the alert. The slightest noise brought her to attention. Quite unsociable, she has never shown any sign of gratitude or recognition towards the person who has charge of her, but rather a most obstinate diffidence; nor is she any better disposed towards other animals, dogs and monkeys, in her vicinity. She has always been quite indifferent and has never shown the slightest semblance of sociability or adaptability during more than two months.

15th August 1889. - She is anæsthetised. Following the usual methods, the frontal pole and sigmoid gyrus on both sides are laid bare, the dura mater having been incised and turned aside. Electric exeitation is now employed with the following results :-

(a) All the part lying in front of the sigmoid gyrus is not excitable, with the Du Bois Reymond sledge at $10 \mathrm{~cm}$. (a current which was left fairly strongly on the tongue and lips).

(b) On the anterior branch of the sigmoid gyrus the same strength of eurrent produced, at a point above; dilatation of the pupil of the opposite side, and a slight, but strong and rapid, movement of the head towards that side; below, rapid and marked dilatation of both pupils, ceasing immediately the current is broken, and movements of the trunk and tail; with a stronger current, slight movements of the limbs of the opposite side are also induced.

(c) On the posterior branch of the sigmoid gyrus we find arranged in succession, almost as in the sigmoid gyrus of the dog, the various centres of the muscles of the limbs and of the trunk.

It is unnecessary to enter into further particulars in this respect. Having repeated the exeitations, and made sure of the eonstaney and uniformity of the results of the electric stimulation, and especially of the fact that excitation of the posterior branch of the sigmoid gyrus produces no effect upon the irises, we scoop out, following an inclined plane, all that remains in front of the sigmoid gyrus and a small portion of the anterior branch of the sigmoid gyrus, leaving the most of it uninjured, with the sole ohjeet of avoiding damage of any kind either to the cortex 
or the projection fibres of the motor centres arranged mostly on the posterior branch of the sigmoid convolution. Very scanty hæmorrhage ; closure of wound; antiseptic dressing.

After six hours, having revived from the stupefaction produced by the morphine and the injury, the vixen gets up, runs about all over the cage with head straight and erect as usual, the eyes wide open, the ears attentive. No deviation of the trunk is detected, no disorder or defect in the movements of the limbs, no bending of the paw or resting on the dorsum, no doubling of the limbs under the weight of the body.

Tactile sensibility.-If, from behind, we put a very thin wand through the eage and touch her lightly on any point whatsoever of the body on either side, she reacts, either turning her head towards the side on which she is touched or rising and recommencing her round of the cage.

Hearing.--If the tuning-fork is brought up to her ear on either side, she shows surprise. If at some distance away we make a noise on the terrace as by a slight shaking of the chain, or if in the room, at 10 metres distant, we mako a sudden bang on the table she immediately stands erect, turns her head and eye towards the point whence came the noise and remains on the alert.

Sight.-The eyes of this little beast have lost their former vivacity; they have no longer the savage, inquiring, suspicious glance; they secm like artificial eyes implanted in the orbits of a living face. The gaze is directed into space. If we threaten her or bring a stick or the hand close up to her eye, as though to strike her, she never winks. If, however, we lightly touch the eyelids and more especially the conjunctiva, either on the right or on the left side, the reflex movements of the lids are found to be ready and lively.

Sometimes, as she is going around in the cage, she knocks her nose against the iron bars, especially on the left side.

Slight winking is got in the right eye, if, taking her by surprise, a fairly large body such as the closed fist is brought up to the eye from that side. Corresponding with this difference in the behaviour of the two eyes is the fact that, in order to see, she almost always turns towards the right, and she prefers to go (or automatically is forced to go) towards the right, reminding one of a circus movement.

17th A ugust 1889. - There is no trace of swelling nor of suppuration. The animal exhibits agitation and restlessness while in the cage and is extremely terrified. We set her at liberty in a room, in the middle of which there are several chairs scattered about, giving her a hen for a companion. She at once makes off, skirts the walls, stops at the corners and jumps as though attempting to get out, clambering up the walls where there is neither window nor opening of any kind. She avoids all the obstacles, but wherever there is an opening she forces herself into it at all costs, knocking aside the obstacles as though she were being chased. She is agitated and terrified and pays no attention to the fowl. She rests steadily on her legs, which sometimes are crossed and sometimes are wide apart; for the rest, she is very agile and runs around in an effort to escape, jumping and clambering up the walls.

Tactile sensibility is well preserved. If touched anywhere, especially if taken by surprise, she reacts by shaking herself and running away. She hears the slightest noises. Her eyes still remain immobile and vacuous as though staring into space, especially the left.

22nd August 1889.--Paresis has almost disappeared. Hearing and cutaneous sensibility are normal as before. Her glance is less vacuous; she seems to direct it better and accommodation is more perfect. She does not wink yet, when threatened with a stick, or when the hand is suddenly brought up to her eye, but she certainly notices the approach of any person or object, because she directs her gaze thereto and recoils in terror, puffing and blowing.

She is extremely restless. For hours together she goes from one corner to 
another of her cage in the same identical fashion, reminding one of a stereotyped movement.

1 st September 1889. - Wound healed by first intention.

She takes her milk and meat as before. She recognises foods placed in a corner of the room and eats regularly.

She is always restless and indifferent. She shakes with fear at every noise and at every pretence of hurting her, soon afterwards recommencing her automatic movements hither and thither, almost always in the same direction, whether in the eage or in the room. This automatic monotony is only broken by any noise which frightens her for the moment.

12th September 1889. - All the nervous functions are again accurately examined. Tactile and painful sensibility, examined in the usual fashion, is normal. Hearing perfect; her eyes being covered with caoutchouc eye-shades, she hears the slightest vibrations of the tuning-fork slowly approximated to her ear, and equally on each side. On removing the vulcanite eye-shades she makes movements with her paws over her eyes as though to remove some stimuli, just as men rub their eyes after a troublesome foreign body is removed, then turns her head around and recommences the ste:cotyped movements. She does not knock against the wall nor against any chair or other piece of furniture. She avoids them all. She sees her milk in a corner of the room and goes to drink it. Pieces of meat are thrown to right and left at some distance in front of her; she sees them and takes them up with precise movements then begins again her stereotyped motions. If her head and trunk are maintained in a fixed position in a dark room and we now enter with a bull's. eye lantern, she at once notices the light and turns her eyes towards it, to the right or to the left, to the extreme external limit of the visual field.

On this occasion we have been able to observe the pupillary reaction very distinctly, and especially the marked contraction when the light of the lantern penetrated direetly into the eye.

17th September 1889. - Found dead from strangulation, with her chain twisted around her neck.

Experiment 6.-Ciacma cynocephalus porcarius, adult, female, intelligent. She walks on all fours or on the hind limbs alone, at the word of command. She gives the military salute, understanding the command without any sign being given. She takes up or lays down her stick as ordered by word of mouth and without signal. She also shows a good deal of intelligence in arriving at new and spontaneous adaptations in the various new positions in which she finds herself-e.g. raising or lowering a roller-shutter which gives access to a balcony. She distinguishes various food materials and shows an evident and distinct preference for some rather than others. She displays the faculty of observation and fairly well developed sentiments of affection, as evidenced by her behaviour towards another male monkey, operated on several days previously, for it was clear that it was not always characterised by merely erotic tendencies, even during her menstrual periods. She often embraces hin and shows rudimentary kissing movements, holding him close to her breast in an attitude almost of ecstasy and evident satisfaction. This sentiment of affection is even more evident with two puppy dogs to which she has been much attached from the moment she first saw them and has tended like a mother. The following observation was made six days before the operation.

23rd May 1892. - To-day, whilst tied, a small puppy dog happens, by chance, to come near her. She looks at him with an air of surprise and curiosity, then takes him up carefully in her arm, bestowing on him a thousand caresses and showing all the care and anxiety that a mother might feel for her young offspring. She sets the puppy down and follows him as he walks, but if he begins to go too far, so that she cannot follow him because confined with her ehain, she takes him up gently in her arm, carries him back and, from fear of losing him, keeps him embraced, then 


\section{EXPERIMENTAL HISTORIES OF DOGS, FOXES \& MONKEYS}

sets him on her lap, keeping him slightly raised with one arm passed under his chest. It is impossible, either with eajolery or with threats, to make her leave him. If, when at last she does set him down on the ground, we try to call the puppy in the ordinary way but without giving any sign that would make the other suspicious, she immediately understands the whistle and at once takes him up again and hugs him to her breast. If now we seek to intimidate her with a stick, matters become worse. She shouts and becomes angry, and, holding the puppy closely to her, but so that she shall not hurt him, she clambers up the iron railings of the terrace in order to keep him in safety as far as possible.

With great difficulty we succeed in taking him from her. After a little I take him back again to her. She manifests great satisfaction and several times she comes up to me as though in gratitude and recognition, although there are several people looking on, and she puts out her hand to me so that I may stroke it.

We now bring in a second puppy dog. He begins to walk around her. She looks at him, smells him, touches him, but does not take him in her arm, rather does she seem quite indifferent. As soon, however, as the puppy is about to get out of her reach she grasps him by the tail and slowly and gently draws him back towards her. This scene is repeated several times. When she rises to go from one point to another she takes the first puppy in one arm, and with the other hand draws the other puppy by the tail. She emits guttural, mournful-like sounds. Sometimes she places them on her knees, and with the fingers of both hands she seeks for lice amongst the hair of the neek, in almost human manner.

It has been impossible to take them from her at close quarters. If anyone approaches her she seizes both, one in each arm, and seeks as far as possible to put them into safety.

In order to make her lay them down it has been necessary to have recourse to a jet of water. Of this she has a great fear, and it is sufficient to let her see the hose pipe, when she will commence to cry and make a great noise. Notwithstanding the stream of water she has kept the two puppies in her embrace for quite a long time, trying to protect them as well as possible. Failing in this she has put down the second puppy, and, with the other in her arm, has jumped up on the iron railing. At length, able to bear it no longer, she has come down again, has carefully and slowly put the puppy on the ground, and has again clambered up on the railing.

29th May 1892. - To-day we have operated on the frontal lobe of the left side, employing the usual method already described, and removing about $3 \frac{1}{2} \mathrm{grm}$. of cerebral substance, covering an area a little more than $2 \mathrm{~cm}$. sq. in front of the feet of the first and second convolutions, leaving the frontal pole in situ. Slight hæmorrhage during the operation.

30th May 1892. - In the morning hours she has been fevered, but towards 2 P.M. her temperature has again become normal. During the entire day she has been rather low and depressed, but it has been possible to give her some nourishment.

2nd June 1892. - There is paresis of the left side. In walking she makes use of her four limbs, and has sufficient strength to avoid falling on the paretic side. Loss of power on the right side is, however, quite distinct. When she jumps it is evident that she supports herself chiefly on the limbs of the left side, and to a slight extent on the right hind limb, which is stronger than the right fore limb. In the position of rest, the paralytic attitude of the extremity of the right fore limb is very evident. She is no longer able to give the military salute, and threats do not help matters. She cannot make use of the right fore limb to take hold of any object, and if we force her to employ this limb by holding the other, it requires considerable effort, the object readily falls from her hand, and she fails altogether to bring it to her mouth.

She can raise herself on the flexed hind limbs, but cannot stand erect on the feet as she formerly did with ease.

Vision is, at least, much weakened in the external segment of the visual field of the right eye, and the internal segment of that of the left eye, so that she cannot 
recognise the nature of an object presented to her in those parts of the two visual fields, although she can recognise it readily in the other segments. The test has been made in the usual way by suspending to a thread a fruit of whieh she is very fond, and bringing it horizontally from without inwards and from within outwards before each eye. Her favourite fruit in the month of June is the cherry, which she immediately seizes as soon as she recognises it.

5th June 1892. - She walks well. There are no eireus movements. She does not make use of the right hand, and, if offered a cherry, takes it only with the left. If she takes hold of a table in the attempt to see what is on it, or if she tries to open the door, it is always with the left hand. She never offers anyone the right hand. She no longer salutes with the right hand as she formerly did, raising herself on her hind limbs, grasping her tail with the left hand, and bringing the right to the forehead in a military fashion.

If we hold her left hand and offer her a eherry, she repeatedly makes a great effort with the right and finally succeeds in taking it, but, lacking the power to raise it to her mouth, she waits passively till her left hand is liberated, and with it immediately puts the cherry in her mouth.

She hears the tuning-fork and reacts equally on both sides.

She does not see the cherry well until it is brought into the visual field from without inwards to a point corresponding to the median vertical axis of the right eye.

She seratehes the right side of her nose and occiput with the right lind limb and the left fore limb.

She shows, as formerly, a capacity for new adaptations and sentimonts. On again presenting to her the puppy towards which she formerly showed great affeetion, she takes hold of him gently, embraces him, hugs him, takes him up tenderly in her left arm, begins to seareh amongst the hair for lice, and, as she cannot move her right hand as formerly, she makes adept uso of her left hand and her teeth in this operation. Woe to anyone who takes the puppy away. She becomes angry, eries out, and is desperate. Being unable to jump up on the ehair with the puppy in her arm she first places him on the chair and jumps up after him. The second puppy, which had also previously been presented to her, has not met with the same favour, rather does she avoid him. Of four puppies she shows a liking for her first aequaintance alone. If we call the four puppies around her she takes up her favourite and hugs him all the more elosely to her breast.

28th June 1892, - The paretic limbs have gained still more in strength, and, although she prefers to use the sound arm for small movements, and when she has free choice, yet if eompelled by necessity, she can also make use of the other arm, and with it perform movements with eertainty.

Sight on the side opposite to the operation has improved. Vision, however, is not yet quite distinct, for a!though the monkey notiees anything which falls within the blind visual field and turns her gaze towards it, she does not recognise it and makes no attempt to grasp it; the process is limited to the turning of the head and gaze towards the object, the light from which falls within the temporal visual field of the right eye or nasal of the left.

16th August 1892. - Second operation. - This time a trephine opening is made over the right frontal lobe, at a point corresponding to the feet of the frontal convolutions. Faradie exeitation of the upper and posterior part of the superior frontal convolution provokes contraetions of the nape of the neek. The head is turned towards the left. At a point some millimetres below, we obtain movements of the head and eyes to the opposite side. Still further below, we provoke move. ments of the eyes alone, with dilatation of the pupil of the opposite side. Still lower down, we get movements of the pericranium of the opposite side. Movement of the pavilion of the ear alone is given by a small area slightly removed from that which awakens contractions of the pericranium and of the pavilion simultaneously. 
We remove about four grammes of cerebral cortex. The incision falls preeisely in front of the feet of the first and seeond frontal eonvolutions, and we endeavour to remove all that portion of the frontal lobe lying in front of the incision. Considerablo loss of blood.

About two hours after the operation the pationt is able to drink a glass of milk.

19th August 1892.-A large swelling has formed over the site of operation. No fever. The animal is able to stand erect on the hind limbs, but her support on the left limb seems less secure. When she walks on all four paws she drags the left fore limb. She shows a marked tendeney to cireus movement, this being displayed towards the right side. Reflex exeitability is markedly increased on both sides; fairly light taps provoke tumultuous jerks. Almost complete blindness in the left eye.

24th August 1892. - Cireus movement continues though it is less marked. Restlessness, almost vertiginous, is persistent. She jumps up on, and down from, a ehair placed near her. She knocks it down, pulls it after her or pushes it away, or shakes it and grasps it in various ways. She opens and shuts the door on the balcony, tugs repeatedly at the chain which confines her, or, if not performing any of the acts mentioned, she again goes through the eireus movement. These actions resemble stereotyped movements. She is sufficiently accurate in her movements to prevent the chair from falling on her head, and to avoid knocking herself against other objects, but she immediately goes through the same performance again and exposes herself to the same inconveniences. She shows no concern for anyone approaching her, and no interest in what is happening around her. Often, when called repeatedly, she does not respond but, rather, shows an extraordinary fear, such as she never exhibited before. She often shows herself afraid of another monkey if the latter goes near her, whilst formerly she would clasp him to her breast in ecstasy, perhaps erotic, perhaps simply tacto-emotional. She shows an indifference towards many things in which she formerly evinced a lively interest; thus, e.g., she cares nothing for the puppy towards which she was formerly so affectionate, and even shows fear of him when he is placed beside her.

The reflex hyper-excitability, so marked in the first few days, is diminished.

The right ear seems more sensitive than the left to the sound of the tuning-fork.

With the object of examining the sense of taste, we place side by side on the palm of the hand a piece of sugar and a piece of chalk, taking care that they should resemble one another in form and volume as far as possible, and offer them to her to eat. As the piece of chalk is placed a little nearer her she takes it, puts it in her mouth, and one cannot detect in her physiognomy any sign either of pleasure or disgust, for she masticates it and finally swallows it. She is then given the piece of sugar to eat. This, like the ehalk, she masticates automatically and then swallows without exhibiting any sign of pleasure. The experiment is now repeated, and even this second time her hand is directed to the piece of chalk, which is a few centimetres nearer her, and without looking at; it or examining it in detail, as was formerly her habit when offered any eatable to which she was not accustomed, she gobbles it up, just as she does, soon after, with the sugar also.

Examination of sight, carried out by the usual methods, reveals blindness, or at least a very marked diminution of visual power, in the left eye. With two figs the following experiment is made:-One is brought a certain distance into the outer half of the visual field of the left eye, and is then placed quite close to the left upper limb, whilst the other eye is eovered with caoutchouc; under these conditions the monkey makes no attempt to take the fig although it is quite within her reach. The second fig is now brought inwards from her right-hand side, and as soon as it reaches the right side of the left visual field, she immediately makes efforts to reach it, although it is some distance from her.

Visual perception is now tested in all meridians of the visual field. Perception 
is only arrived at when the object enters into the visual ficld of the right eye, when this is left uneovered, or into the internal half of that of the left eye.

20th October 1892. - In all the psychic and psycho-motor manifestations of this monkey automatism seems but slightly diminished. The circus movement towards the right side is well marked. When called loudly she trembles. After long practice she is beginning to attempt to give the military salute when ordered, and she shows a certain pleasure when caressed. She shows also an avidity to obtain possession of any eatable shown to her, but this is always very transitory and flecting, for her habitual state is one of indifference, and soon she repeats her automatic, aimless movements, evineing no interest in her surroundings. In new positions she is incapable of new adaptations as formerly and, indeed, the majority of these pass unobserved or, at any rate, make no impression. She no longer plays with the other two monkeys who are her companions. She is indifferent if they go near her, or, if she does dally with them for a little, she soon tears herself away. She is no longer jealous if any of them are petted. If threatened, she is afraid but not rebellious; she does not react or defend herself or threaten in turn, as she formerly did with great energy. A sense of indeterminate and baseless fear seems to overtake her whenever anyone approaches her, or if one breaks in upon her automatic restlessness with a sudden call, or when any new impression strikes her.

The movements of the limbs of the left side are more precise, not yet completely free, however, although she makes use of these limbs to take articles of food, and as her principal support when making the salute, ete.

Vision in the left eye is largely restored, and although it is not easy to moasure it precisely, it is certain that vision in the inner part of the visual field of the left eye is more distinct.

She lived in this state up till the 21st January 1893.

21st January 1893. - She maintains the same behaviour as in the preceding months. She always shows great restlessness and instability. She still exhibits a certain tendency to go round from left to right. She makes little use of the left hand. She walks fairly smartly so that no paralysis of any kind is noticeable. In walking, however, she makes good use also of the left upper limb. All forms of sensibility are well preserved, as previously recorded, especially touch, hearing and taste. She is to-day killed with chloroform.

The left hemisphere seems much larger than the right. The inferior frontal convolution of this hemisphere is destroyed (except a small part of the operculum) up to its insertion into the anterior Rolandic convolution, which is wholly spared. The superior frontal convolution is almost entirely spared up to its anterior extremity. The right hemisphere shows destruetion of the superior frontal convolution up to its base. The extreme tip of this convolution is, however, spared, and it communicates with the rest of the brain by a very thin bridge of about $2 \mathrm{~cm}$. in diameter. The second frontal convolution is destroyed to a larger extent. Of a rudimentary third frontal convolution there is destroyed only the central part, the foot and anterior extremity remaining normal. ${ }^{1}$

Experiment 7.-A female monkey, cinocephalus porcarius, fairly large, an alert and lively animal. She often walks erect and gives the salute, bringing the right hand to the forehead. On careful examination no sensory or motor disorders or defect of any kind is detected. She is full of curiosity, perspicacious, astute, familiar with and affectionate towards persons who have charge of her. Menstruation is regular. At the period of ovulation she is excited and rather shameless, judging from the fact that she raises her tail and exposes her genitals.

20th May 1893.-Anæsthesia with morphine and chloroform. Two trephine

${ }^{1}$ In this brain a branch of the pre-frontal sulcus running horizontally and at right angles allows the distinetion of a third convolution. 
openings are made over the frontal lobes, $1 \mathrm{~cm}$., or a little more, in front of the Rolandic line. Hæmorrhage slight. The brain being laid bare, with a sharp knife I make curved incisions with eonvexity behind, as nearly as possible equal on both sides, anterior to the feet of the frontal convolutions and just immediately in front of the pre-frontal sulcus. The faradic current, applied repeatedly to various points on that portion of the brain situated in front of the ineisions, produces no reaction. The observation is repeated under profound ehloroform-anasthesia and also under the condition of simple morphinic lethargy. With a small scoop I remove all, or almost all, those portions of the frontal lobes situated in front of the incisions on both sides. Hæmorrhage being controlled the surface of the wound is washed, the strictest aseptic precautions being adopted. The edges of the dura mater are sutured, and finally the cutaneous wound is sutured and treated with iodoform dressing. The patient is now placed and fixed on a suitable bed.

21st May 1893. - In a somnolent eondition; great reflex exeitability is inclueed by the slightest noises. She laps up a little milk with great difficulty, immersing her nose in it. Temperature in vagina, C. $38: 6^{\circ}$.

22nd May 1893. - She continues in a very low state and sometimes seems to be in a semi-conscious condition. Reflex exeitability is very extreme so that her whole body shakes at the slightest noise. She is eating a little more; remains in bed; enemati.

27th May 1893. - She is a little more lively but her head is all swollen and cedematous as far as the oceiput. Nevertheless, the first examination is now undertaken. Taetile and painful sensibilities are exaggerated, rather more on the left than on the right. The slightest contacts, even on the points of the hairs, are sufficient to provoke not only a reflex action sueh as shaking of the whole body but also a movement on the part of the head and eyes, which she turns towards the part stimulated. The slightest prick, or pinehing, or pulling of the hairs in any part of the body, makes her ery aloud.

On bringing near the vibrating tuning-fork, from either side, she is surprised by the strange noise, moves her ears slightly, and turns her head to the side whence comes the noise, or lowers her head as though to get away from the strange and annoying sensation. If she is sleeping, or, as often happens, resting with her eyes closed, she opens them if any noise is made in the room or if the tuning-fork is approximated to one of her ears.

If the hand or any object whatsoever is brought elose up to her eyes from any point in the periphery of the visual fields, she winks the eyelids and withdraws her head as though in fear. If shown a cherry, of which she is very fond, she fixes her gaze upon it. If it is suspended to a thread and allowed to fall from above, or brought from without inwards, into the visual field, she sees it, recognises it, and with adequate and eo-ordinated movements tries to take it between her lips or with her left hand.

Seldom does she get up to walk, and then she staggers; her head is somewhat bent, there is slight curvature of the trunk, she drags the right lower limb and makes little use of the right hand.

She hardly ever moves from her eouch and she shows no interest in anything. She trembles at the slightest noise and at every toueh even whilst being tended, and then eries out with manifestations of fear, annoyance and anger.

3rd June 1893. - The swelling of the head has disappeared but the wound is suppurating at one point, and it is necessary to reopen it to elear out a small superficial abscess and to dress it with every antiseptic precaution. The behaviour of the monkey remains the same as already deseribed, only she eats more and stands more ereetly on her legs, and can walk, although with some uncertainty, for she drags the right lower limb, which seems almost completely paralysed.

7th June 1893.--Suppuration has ceased. The wound is granulating and is 
being dressed with sublimate and iodoform. There is no fever nor has there been any sinee the first few days after the operation. The behaviour of the monkey is rather surprising and altogether now. She remains in any position in which she is placed and shows no euriosity or interest in what is happening around her. For hours together she sits with head bent or remains eurled up, often asleep. If any noise is made her whole body trembles and of ten she gives a ery, raises her head, and looks around, only to fall again into her former position. The same seene oecurs three or four times in succession, as the noise is repeated.

Nothing exeites her curiosity or induces her to move except the sight of some favourite fruit, such as a eherry, which is thrown down a short distance in front of her. In this ease she rises and runs forward to take it. If, however, the cherry is thrown rather farther away, she does not run after it, and it is difficult to say if it is owing to the fact that she does not see it or if it is due to inertia or laziness or indifferenee. She seratehes herself now here now there, especially on the left side, but does not seareh for parasites with that promptitude and facility which were apparent prior to the operation. If one wishes to remove her from the position she occupies she resists and if she can lay hold of anything she elings to it obstinately: If taken by the neck and foreibly driven from her position, she walks a certain distance, rubbing by preference along the wall till she comes to a corner, where she stops, remaining there for a considerable time. If called loudly she raises her head in a listening and attentive attitude, then sinks back with the head bent forward, and in this position saliva frequently runs from her mouth. If she wishes to empty her bladder or bowels she does not move off to some other spot as she used to do but evacuates them wherever she may happen to be, wetting and messing herself like a stupid dement.

She meets another female monkey of the same species. This animal is menstruating and in a highly erotic condition. Monkey No. 1 stops automatically but remains quite indifferent to the many earesses bestowed on her by monkey No. 2. The latter exposes her genitals, touches the other's nipples, and uses her hands, in a laseivious manner. Monkey No. 1 stands like an automaton, betraying no sign of instinct aroused, nor the slightest eommunity of interest or feeling with the other animal.

Meanwhile a noise eauses her to shake and to show fear. On threatening her with a stick she becomes much more terrified than the other monkey, which evidently notices the sham attitude of the experimenter, and so remains indifferent to those same threats which frighten monkey No. 1.

Fruits, sugar, or other eatables of which she is fond exeite her rather more, and so she darts forwards with a eertain avidity to get hold of cherries and pieces of sugar, in doing which she shows no discrimination. No more certain proof of her loss in discrimination can be furnished than her conduct in comparison with the other monkey. I open two cherries, put a drop of solution of quinine into both of them and give one to each monkey. The healthy animal, after a few moments of examination, rejects it from her mouth with a movement of impatience, makes faces, looks at me in a disdainful manner, eleans her tongue several times with the back of her hand, then rubs and squashes that deceptive eherry on the ground with her hand, till nothing is left of it. Monkey No. 1, however, masticates her eherry, but stops after a time and commenees to pour out a great quantity of saliva from her mouth; for some time she cannot decide whether to swallow the cherry or to spit it out, but finally ends by swallowing it. I offer them other good cherries, which are eaten with great relish by both of them. Afterwards I give each of them a eherry that has been dipped in solution of quinine. The healthy monkey puts hers in her mouth but immediately rejeets it and, as she throws it away, makes faces and exhibits anger. Shortly afterwards, seeing it lying sound and whole, she takes it up again, serutinises it earefully, opens it, eautiously eats the pulp and throws away the skin. Monkey No. 1 keeps her cherry in her mouth 
for a while, is undecided whether to masticate it or reject it, and after a certain time, during whieh much saliva has poured from her mouth, ends by swallowing it.

To the healthy monkey I now offer a piece of sugar, and shortly afterwards a piece of chalk of the same colour and appearanee, which I take from amongst other pieces of sugar in my hand. When I throw in front of her the piece of chalk she takes it up, examines it earefully, raises it to her nostrils, smells it and, without even tasting it, throws it away. After she has eaten some eherries I offer her a piece of sealing-wax of the same shape and eolour as these. She takes it, examines it, re-examines it, turns it round in her fingers as though surprised, smells it, lets it fall on the ground as though to test it by the sound and ends by squashing it on the floor and abandoning it. These experiments are repeated with the monkey which has been operated upon and in each ease she shows a surprising eritieal defeet, in this respeet, that without any discrimination she takes the piee of ehalk just as she took the piece of sugar, masticates and swallows it. Later, on repeating the experiment with the cherries, she again gives proof of her deficiency, inasmuch as she takes the piece of sealing-wax, puts it in her mouth although it is different in shape, masticates it, and only by dint of eajolery are we able to extract it from her mouth.

20th June 1893.-No ehange in her eondition.

15th July 1893. - We have kept this monkey under observation with the object of studying better her eharaeteristic tendencies, instinets and intelligenee in comparison with the known eondition of these prior to operation. Without the slightest doubt her behaviour is altered and her physiognomy more stupid. She is torpid. Her glanee is rather uneertain and betrays no flash of roguery nor does it light up with euriosity. The instinet of sociability seems abolished. Fear and terror charaeterise the mental state of this animal. She is perturbed and afraid, eries out, and grinds her teeth at every feigned threat and every painful stimulus, but she never reacts in an aggressive sense. For some time past she has been rather restless. When in a large room with all exits closed, she erosses backwards and forwards, always in the same directions, without stopping near any object or person in the room. There is an evident absence of objective in her movements; or, if she does give some indieation of objective, it is soon forgotten, is transitory and elusive. Thus, e.g., she sometimes runs towards the door, stops near it, then turns back and retraces her steps, running towards the opposite door, and so on many times in suceession.

She no longer shows any sentiment either of affection or gratitude towards those persons to whom she was formerly mueh attached, whose legs she used to embrace and with whom she used to ehatter, as is the way of these animals. Indeed, it seems as though she were absolutely indifferent to them although they bestow no less care and kindness on her than before. There is not merely an indifference, but a ready reaction eharacterised by fear every time anyone comes near her, even when one shows a desire to pet her. If she sees one of the attendants carrying her bowl or a piece of fruit in his hand, only then does she approach him, giving him no time to offer the food, but tearing from his hand, without any restraint, whatever has awakened her violent appetite. It is no longer possible for her to be petted by me or my attendants at the Institute, with whom she was formerly very familiar. She is no longer sociable with the other monkeys, nor does she ever play with them. She shows no resouree in front of small difficulties, no new adaptations. She has learned nothing new and has regained little or nothing of what she lost. She gathers up and puts in her mouth anything she comes across, but is less filthy than formerly.

The sexual instinct seems to have returned, but the menstrual periods are less regular and the flow less abundant. Sometimes she displays an impulsive cruelty that is foreign to her kind. One day, whilst she was menstruating, another female cynocephalus came near her. She at once manifested her desire by lifting up her 
tail and exposing the vulva; but finding the other unable to satisfy it she attacked her eompanion so ferociously that she would have killed her had it not been for the presence of the keeper who, armed with a stick, drove her off.

She suffers from a psycho-motor tic which has first appeared during these past few days. Every ten or fifteen minutes, whilst walking, she suddenly sits down and, placing the foot on the hip, bites the right buttock (sometimes also the left). Oceasionally she proceeds to bite also one heel, then the other, after which she quietly resumes her walk. In the regions which she bites there is no local factor to be detected which may explain these movements, which are always identical, stereotyped, almost rhythmical. It is possible that some abnormal sensation has first of all given rise to them, in an effort to remove a source of irritation, and the movements have afterwards remained fixed, obeying the law of automatism, and withdrawn from all control.

15th August 1893. - The mental state of this animal remains unchanged. Her movements have a stereotyped character, but are otherwise perfect. It has not been possible for her to acquire any new adaptation. She walks up and down, sits and rests from time to time, and searches for parasites all over the body. She bestirs herself a little when she sees her customary bowl of food, otherwise she is indifferent to everyone and everything. She often puts the parasites in her mouth, a thing which she never did in the past. Occasionally she collects and eats dried flowers and leaves. Menstruation is still irregular. Since the brutal sexual episode referred to in the previous note she has not again exposed herself either before or during menstruation, nor has she expressed any desire or invitation by lascivious chattering or gesture as she did on that oecasion. Indeed her voice has not been heard since she underwent operation.

Her movements are precise, and there is no sign of any sensory disturbance or defeet. On that point there is no doubt whatever. Diserimination, however, and that higher co-ordination of sensory factors which gives rise to more complex psychic manifestations, and to actions more suited to the conservation of the individual and of the species, are reduced to quite rudimentary proportions. For example, we give her a sweet; she takes it and eats it with relish. Then we offer her a piece of chalk. This time she notices that it is not a sweet, but puts it in her mouth, breaks it with her teeth, and reduces it to a great many small pieces, which she spits out on the floor. Then with each fragment she commences a series of stereotyped movements. She takes up one fragment, lays it down, takes it up again, raises it to her mouth, replaces it on the ground, takes it up again and eats it greedily, and so on with the second, third, tenth, until not one of the smallest fragments of that piece of chalk remains on the ground. The white colour of these fragments and their resemblance to those of the sweet have been her guide, and have prevailed over her sense of taste. Her rudimentary power of arrest has promptly been overcome by the visual image of the fragment alone. There has been no intervention of other factors for the judgment of identification, similarity, dissimilarity or analogy. She is certainly capable of experiencing single sensations, but she cannot co-ordinate the individual representations so as to arrive at more complex movements and judgments of a higher order. As a matter of fact, a normal monkey either takes the piece of chalk, looks at it, examines it, smells it, and discards it, or, if it resembles a sweet very closely, puts it in her mouth, but spits it out immediately it is broken, and pays no more attention to the fragments. In this case the judgment of comparison is sufficiently sure, and the memory of it suffieiently lasting to spare her from the sequel of movements exhibited by the other monkey, in whose case there prevails a more elementary representation which can only give rise to movements that are inadequate and impulsive in eharacter. In the monkey operated upon, the utilisation of past experience is absolutely wanting.

23rd August 1893. - Her condition being stationary, and the Congress at which it was intended to demonstrate this monkey having been postponed, it has been 
decided to proceed to the autopsy. After anæsthesia by chloroform, the right hemisphere is laid bare. The existence of all the motor centres of the limbs and of the face is ascertained by electric excitation, and afterwards the animal is killed with chloroform.

Experiment 8. - 2nd August 1893. - A young female monkey of the family of cinocephalidæ (higher Cebus), which has been tended with care for some time in the Institute, and has become fairly domesticated. She has had her first two menstrual periods. She is good-natured, affectionate towards me, and the attendants of the Institute, to all of whom she shows grateful recognition. She is docile, obedient, lively and full of curiosity.

I have had this monkey under observation for a long time and have kept her near the entrance to my laboratory so that I might have her under my eyes as much as possible. Along with my assistants I have been able to make many observations and experiments upon her, to some of which reference is now made.

On certain occasions she has been given a small bottle with a narrow neck, full of water and sugar. She has taken it in both hands, has drunk the contents with relish, and then exercised her ingenuity as best she could in order to get the sugar lying at the bottom of the bottle. First she introduced one or two fingers into the bottle as far as she could, then withdrew and licked them. Next she thrust her tongue into the bottle and licked the inside. After having done this several times she seemed to become irritated because unable to succeed in her design, so she squeezed the bottle between her hands and against her chest in an attempt to break it. Convinced at length that in no other way could she satisfy her eager appetite, she forcibly threw it on the ground so as to break it, then eagerly licked the sugar from the fragments of the bottom of the bottle.

She recognised perfectly the persons who were in the habit of giving her anything, and, in acknowledgment, she would sit on her ischial tuberosities, stretch out her hand, seek to grasp their clothing, and then go through some movements as though seeking for lice, to the accompaniment of a special vocal sound, which, by its constant repetition in presence of persons with whom she was friendly, might almost be characterised as a manifestation of contentment, friendliness and devotion. If anyone bent down so as to bring his head within reach of her hands, she acted in a similar way, apparently seeking for insects.

If anyone poked at her with a stick, or made as though to strike her, or turned a jet of water on her, she became irritated, stood on her lind legs, leapt forwards, ,contracted her lips strongly, displayed and ground her teeth, gave a loud cry and, being unable to reach the person molesting her because of her chain, she hurled at him or thrust towards him anything she could lay hold of.

On several occasions we had occasion to lunch in the laboratory, and were in the habit of throwing her something to eat, as she was tied up opposite the entrance to the laboratory. If it happened that by chance she was tied up a little farther away than usual, so that she could not be seen by us, and she knew that we were at lunch, we could hear her shaking her chain noisily and emitting her usual grunts, as though determined to make us aware of her presence. If we pretended not to hear her, she became irritated and threw her milk-tin, which was lying near, or any other article she could get hold of, with violence towards the door of the laboratory.

She was very fond of cigarette ends, and if any, still alight, were thrown near her she rubbed them on the ground with both hands so as to extinguish them, and then ate them. Again, if anything was thrown on the ground in front of her which she could not manage to grasp with her outstretched fore limbs, she stretched out her body and attempted to lay hold of it with the extremities of the hind limbs, using these like forceps.

When offered a piece of coffee-ice she did not grasp it in her hands, but took it with her tongue and lips, apparently to avoid the unpleasant sensation of cold on 
the finger-tips, or to prevent the ice melting. If, however, she was given a glass with some coffee-ice in it she took it in her hands, as any person would do.

During menstruation her genitals became so greatly swollen that the tension of the parts must have caused her great pain and discomfort. She then assumed such positions as afforded her some measure of relief, at the same time applying some pressure to the parts with her hands.

She was greatly averse to the presence of women, and if by chance she saw one, she was apt to throw at her anything that lay within her reach (jealousy ?).

The experiment with the sugar and the ehalk was repeated on several oecasions with this monkey. She ate the sugar, but, recognising the ehalk, either from its form or its tactile impression, threw it away.

If she saw anyone washing his hands with soap she at once imitated his movements.

She nourished no hatred or rancour towards anyone who molested her. Indeed, if anyone who had irritated her afterwards eame up to her and petted her, she very soon entered into a treaty of peace with him.

If anyone began by playing with her and then eommeneed to molest her, one eould notice the gradual passage of her voice from the sound before mentioned to a very piercing ery.

She manifested very great affeetion towards another monkey, destined for experiment, and onee when she happened to be near her embraced her in a veritable transport of joy.

One day I gave her a plum, a fruit of which she was very fond, and she ato it eagerly. On another occasion I injected a little solution of quinine into a plum by means of a syringe, and gave it to her. As soon as she put her teeth into it, she drew back her head, horrified. She examined it carefully from every point of view, then, as though her mind were made up, nervously detached all the pulp with her fingers, broke the stone, ate the kernel and threw away all the rest. What she threw away, however, she pieked up from time to time in her greedy desire to eat, and after having examined it minutely, and smelt it, and eauticusly tasted it, threw it away again.

Memory.-A long time afterwards I again gave her some plums, which she ate very circumspectly and only after she had examined them minutely, and smelt them, and tested them on the tip of her tongue.

The left anterior brain is now exposed through a large opening. On electrie excitation, employing a Du Bois Reymond sledge, no excitable point is detected in front of $a$ and $b$ (these are two points of rendezvous, one above and one below, in a slightly eurved line situated in front of the excitable area of the limbs). Excitation of $a$ provokes movements of the museles of the trunk and pelvis which need not be further referred to. Exeitation in $b$ provokes combined movements upwards and forwards of the pavilion of the ear, widening of the palpebral fissure, with elevation of the upper eyelid, and dilatation of the pupils (more marked on the same side). This excitation is repeated several times, always with the same result. On slightly increasing the strength of the eurrent or on displacing the electrodes one or two millimetres backwards, movements of the hand (extension of the fingers chiefly) are obtained.

With a sharp eurved blade I make a deep incision into the frontal lobe in front of the points $a$ and $b$, which $I$ just graze but leave undamaged. I remove abont six grammes of brain tissue. Hrmorrhage seanty. The surface of the wound is now washed and all blood and detritus removed. Rigorous antiseptic precantions. Suture of the edges of the dura mater and of the overlying soft parts.

3rd August 1893. - She is dosing almost the whole day. She sits on her hind quarters and does not lie down. No deviation of the head or eyes. Makes use of her hands, preferably the left. Goes from one point to another in the large eage 
without showing any disturbance of gait. She takes a little milk. The head is erect and mobile.

4th A ugust 1893. - Same condition; is sick once.

7th August 1893.-Has quite revived from the operation. The edges of the wound are adherent, and healing by first intention is almost complete.

Apart from a certain degree of fear, and hemianopsia of the outer half of the right visual field (which can only be detected by minute and careful examination), this monkey now seems completely restored to her former condition. To look at her one would hardly imagine that she had lost a part of her brain.

She walks perfectly and has never shown any circus movement. She can move hor head and trunk in all directions without any difficulty and without any sign of preponderance of one side over the other. She can move about with the same promptitude and with the same ability as before.

When taken unawares, contacts in the region of the face and the ear provoke the same degree of notice on either side.

Punctures with a very fine needle produce lively reactions, in the form of cries, agitation and flight, especially when taken by surprise.

On obstructing vision by the application of sub-palpebral eye-shades of caout. chouc, after previous application of cocaine, she displays some excitement and uncertainty for a time, but soon afterwards shows, by the precision of her movements, that she possesses a normal muscular sense. If, e.g., a peach is placed within her reach she immediately grasps it, bites it, peels it, and puts pieces of it in her mouth with either hand, always with precise and certain movements.

She hears the sound of the tuning-fork at an equal distance on either side and is afraid of it when it is brought up to the ear.

A methodical examination of the senses of taste and smell has not been carried out, but it has been observed that she shows a preference for peaches rather than pears, for the latter rather than bread, and for sweets more than pears.

From the day of the operation she has presented a defect of vision in the opposite eye. An assistant holds her in his arm on the table. Another assistant stands opposite and attracts her attention with a peach or a pear. Standing behind, I bring a piece of peach to within 10 to $25 \mathrm{~cm}$. of one or other eye, moving it from without or above, so as to test the various ocular meridians. When the piece of peach, proceeding from without and behind, arrives at a point corresponding to the external angle of the left eye, she immedianely notices it, turns her head and eyes towards it and, sometimes, with a rapid movement of the hand, seizes it and puts it in her mouth. When the same experiment is repeated at the external angle of the right eye, it is always necessary to advance the piece of peach much farther forward, to a point corresponding to the median vertical axis of the eye, so that she may notice it and behave in a similar fashion.

12th A ugust 1893. - The examination is repeated and a certain difference between the two eyes is still noticeable, although it is not so marked or so definite as before. With the right eye the monkey notices anything but does not recognise it until it has arrived almost at the median line and then she grasps it with rapid and certain movement; whilst as soon as any object enters into the visual field at the external angle of the other eye, she immediately recognises it and seeks to take hold of it with rapid and precise movements.

11th September 1893. - She is always lively, agile, quick and observant. She unties a cord which secures the door of the cage, draws out an iron pin, then makes her exit, without going far from the establishment.

She is very fond of tobacco. I throw in front of her half a cigar still alight. She goes to pick it up with her left hand, which she burns, withdrawing it in great haste and thon licking the fingers which are sore.

She waits for a little, pioks up the cigar again very cautiously, taking hold of 
the end that has not been lit, examines it, smells it, strips the leaves off it, and eats them one by one.

She remains under observation until January 1894, showing no ehange, apart from the fact that she has become rather more irritable.

31st January 1894. - Anæsthesia by morphine and chloroform. The anterior part of the right hemisphere is now laid bare. The ascending frontal and posterior part of the first frontal convolution remain unexposed. No movements of any kind are provoked by faradic excitation of the cortical surface lying in front of the feet of the frontal convolutions, except in the inferior, opereular, part of the second convolution, where, in front of the facial centre (contraction of the muscles of the lower half of the face), one can determine a vertical rectangular area, extending upwards and embracing all the second frontal convolution, where excitation constantly provokes slight widening of the palpebral fissures, slight raising of the eyebrows, and dilatation of the pupils, more marked on the side opposite to the exeited hemisphere. At the foot of the first convolution and also a little further forward, exeitation produces movement of the head and eyes to the opposite side. It has not been possible to induce any other manifestation.

Repeated electric excitation of the pupillary centre induces a more prolonged dilatation of the opposite pupil, lasting about ten minutes.

I now incise the brain immediately in front of the centre for the face and behind the centre for the dilator iridis. I remove as much as possible of what remains of the frontal lobe in front of the incision, only the posterior third of the first convolution being spared.

Slight hæmorrhage. Lavage of the resulting cavity with sublimate solution ( 1 in 2000). Aseptie dressing.

After the operation the animal remains depressed and somnolent for several hours, but when called opens her eyes. No vomiting, no fever.

2nd February 1894. - She remains in a sitting position with the head bent, the eyes closed, and drowsy. When called, she raises her head, slowly opens her eyes, looks around, then resumes her former position. If any part of her body is touched she wakens up, sometimes giving a sudden jump or emitting a cry, but does not run off or show any sign of fear.

Driven from her couch and left at liberty in the room, she paces the floor in every direction, with measured steps. No deviation of the trunk can be detected, nor paralysis, except in the left side of the face (lower half), where it only becomes evident when she grinds her teeth or cries out.

9 th and 14th February 1894. - The records of these two days are given as one, because identical.

Facial paresis on the left (opposite) side continues. It is less evident than before, but there is no doubt as to its existence. In walking, the lower part of the trunk is seen to be slightly arched. She turns to right and left, runs and gallops about, curls herself up if frightened, and betrays no trace of paralysis. There is no difference in the two sides, but she has lost the elasticity, the suppleness, and the darting, jumping movements so characteristic of monkeys. She has gained in weight and is inclined to fall and be awkward in her movements when chased. She makes use preferably of the left hand but also grasps objects with the right, and does so with fair precision though with less resolution and certainty than with the left hand. She can raise and lower her head and turn it to right and to left. There is no trace of paralysis in the muscles of the neek and back of the head. She takes her food very well and eats it with relish, swallows well, and ealls out as before.

'Tactile sensibility is well preserved. If, taking her unawares, we touch any part of the body, she notices the contact at once, withdraws the limb or shakes herself, or eries out, or turns her head and eyes towards the side touched. Feeling is less acute on the left ear, as one may judge from the fact that on touching or tickling that region she does not move the ear nor shake herself, as always happens 
in the case of the right ear. She scratches herself and seeks for parasites equally readily on either side, using both hands with fairly precise movements. At the same time, it is evident that the muscular sense is well preserved, for no sign of ataxia is evident in her gait, or in movements of prehension, or even in more delicate actions. The right eye being covered (same side as recent extirpation) the monkey walks and runs about without ever knocking against any of the obstacles in her path, such as a table, chairs, a stick, etc. She avoids them all with precision.

If, however, she is now held at rest on a table, and a piece of sugar suspended by a thread is brought from without inwards into the left visual field, she does not perceive it until it reaches the line of the visual axis, and then only does she grasp it with a rapid movement of the right or of the left hand, snapping it up and putting it into her mouth. This experiment on several repetitions has always given the same result. It is different in the case of the right eye, when the left eye is completely covered. In this case, when the piece of sugar passes beyond the external physiological limit of the visual field she turns her gaze on it, makes a grab at it, and, if one does not promptly remove it, takes hold of it. There is no marked difference between the two eyes in the extent of the visual fields upwards and downwards. Owing to the excited condition of the animal it has not been possible to examine the internal part of the right visual field.

Intelligence.-Stupid in her general attitude; changed and expressionless in her physiognomy. Like the previous monkey, she runs from one end of the room to the other in an incoherent manner; arrived at one wall, she stops a little, turns back and goes towards the opposite corner; half-way she sees a spot on the wall where plaster has come off, she goes and sits opposite it, and with her teeth or hand picks off pieces of lime, which she masticates and swallows; or she notices little pieces of dirt on the floor and takes them up and puts them in her mouth. She is now offered a piece of chalk. She immediately grasps it and, without any examina. tion, puts it in her mouth, masticates and swallows it, although not quite in the same way as she does with a piece of sugar. It seems as though she does not fail altogether to distinguish sugar from chalk, because she masticates the chalk for a long period, and sometimes looks as if uncertain to continue masticating or not; but she seems unable to resist the temptation of the image of sugar which the chalk, by its similarity, has reawakened, and so she ends by swallowing the chalk and afterwards picks up any little fragments that perchance have fallen from her mouth. There is, as one can argue, a defect in the perceptions. These seem elementary and not completed with the help of all the other factors (association) which ought to intervene so that a more complete judgment may be arrived at.

She puts the chalk in her mouth apparently because it is of the same shape and colour as a piece of sugar, these factors determining that combination of movements which she is accustomed to perform when she sees a piece of sugar in front of her. Possibly there is also a defect of inhibition, because though she does not find it to be sweet, yet she does not decide to reject the chalk from her mouth.

Her psychic life evidently manifests itself through the lower ares.

Reflex excitability is increased; any noise makes her shạke. She is less courageous and does not show to the same extent that attitude of confidence and certainty of which she formerly gave proof.

If one makes a pretence of assaulting her, she runs off, sometimes voiding urine in her fear, or she hides herself in a corner of the room and cries out in despair. She shows no resource in defence. Sometimes she does not make off, nor seek to hide, making no attempt either at offensive or defensive. What is more remarkable is the fact that when these tests are repeated several times after brief intervals she appears not to remember that the aggression was pretended, and always behaves in the same way. Formerly she used to clasp and oling to the leg of her attendant in token of affection, and pawed him, and rested her head on him with almost human confidence and disregard, like a lover in sweet abandonment. This no 
longer happens. Men and things are alike indifferent to her. She no longer pays any attention to the objects about her, or else she stops at everything, though only for a second. Everything awakens her senses, but her pereeptions are incomplete, erroneous and fleeting. She no longer formulates complex judgments, nor does she make use of past experiences.

Experiment 9.-27th May 1907.-Monkey A.-A Cebus, female, medium size. ${ }^{1}$

When she is taken from the cage which forms her particular section of the monkey-house, all the other monkeys come forward to the front of their cages, shaking the iron railings and calling out as though in protest.

The monkey $A$ is nervous and restless. She wishes to return to her den, and it is with no small difficulty that we manage to take her to a room in the laboratory. She attempts to clamber up every possible object on the way, and secks to escape, heedless of calls.

She is entirely taken up with the voices of her companions, and looks back with great anxiety towards the place where they are. As we are going through a gateway some distance away, she clambers up to the very top of the iron gate and spies out the abode of her companions, with whom she seems to try to communieate by means of special cries.

In the laboratory she seems somewhat irritated. On giving her a piece of sugar wrapped in red paper she takes the packet, smells it for a second in a nervous manner, then throws it away and pays no more heed to it. Sometimes she grasps the whip in my hand and bites it nervously.

She is now given a piece of sugar. She does not eat it but throws it away.

She is offered a pod of beans. She takes it quickly, opens it, takes the beans out one by one, puts them in her mouth, and eats them.

I put a small piece of white chalk in the empty pod and present it to her. She opens it, is surprised at the white content, takes it out, smells it, puts it in her mouth with diffidence, and immediately afterwards rolls it eight or ten times between her fingers as though, impatient and irritated, she wishes to destroy something which has choated her desire. Afterwards she throws it away and pays no more heed to it.

I now offer her a small radish, which other monkeys eat very readily. She takes it unwillingly, and, after keeping it under her eyes for a short time, throws it away. She seems to be greatly disturbed beeause taken away from her companions.

$30 t h$ May 1907. - As soon as she is removed from her cell, all the other monkeys become excited. She goes with great reluctance to the experiment-room, the others meanwhile coming forward to the front of their cages and making a din.

I offer her two eherries, one after the other, and she eats them with relish. I next take the stalk of a cherry and wrap red paper round one end, making it as nearly as possible of the same size and appearance as the real article. I offer it to her. She looks at this imitation cherry with indifference and does not even put out her hand to take it. Evidently she notices the unevenness of the surface, which is not that of a cherry (rapid perception). At length, after it has been kept dangling under her eyes for some time sho takes it, undoes the paper wrappings, tears them and throws all away. I next offer her another real cherry. She looks at it greedily and darts forward to take it.

I give her a bean. She takes off the outer skin and eats it. I drop a little solution of quinine into a bean pod. She commences to take out the beans, notices the bitter taste, takes the beans one by one, skins them cautiously with her fingers,

1 A small tribe of eleven monkeys of the genus Cebus, middle grade, was sent to the author in 1906 by the Governor of Italian Abyssinia. They were lodged in separate cages on a terrace in the Institute at Naples. 
although in the habit of shelling them with her teeth, and munches the beans wherever the quinine has not penetrated.

I offer her a cherry dipped in solution of quinine, then dried and wrapped in blue paper. She sees me wrapping it up, so that, after brief hesitation, she takes it, unwraps the paper, puts the cherry in her mouth, then, finding it bitter, squashes it between her fingers and on the floor with evident impatience and anger.

I give her another dipped in quinine. She eats the pulp very cautiously, leaving the skin.

9th June 1907.-I offer her a cherry wrapped in blue paper. She does not take it, but turns her head and eyes to the other side. She quite refuses to take it (evidently she remembers the trick played upon her nine days previously with the cherry wrapped in blue paper).

12th June 1907. - She refuses to go to the experiment-room, being evidently unwilling to detach herself from her companions. With the chain fixed round her sides she is brought along, but is always turning and looking back towards the terrace where the other monkeys are. As the chain does not allow her free movements she tries with her hands and teeth to liberate herself, and, annoyed at her failure, finally gives it an impatient tug.

Flies annoy her, and whenever one rests on her head she tries to get hold of it and chases it. I set her at liberty in the experiment-room. I put some fruit on my desk, at a certain distance from where she is. She looks at it, measures the distance with her eyes, but makes no attempt to lay hands on it, being uncertain whether she can reach it with one jump.

Noticing me busy writing, she has gradually come a little nearer. She takes up her pole and, as I make no attempt to correct her, seems reassured, clambers up cautiously and spies the eherries on my desk. Seeing I have made no attempt to chase her off, she jumps on to the desk with great precaution and takes some cherries. Having succeeded in this attempt she repeats it; gradually gaining in confidence but watching me all the time, she returns several times until she has taken them all.

She appears to have no musical sense, or, at most, a very rudimentary one. She shows fear at the sound of the bugle. She gets excited and shows her teeth. She has also a great dislike for the sound of a small stringed instrument. She is, however, indifferent, indeed seems rather animated, when she hears the sound of a sweet wind-instrument.

19th June 1907. - She is now quite at home with me. She goes without any difficulty to the expcriment-room. She spies on to the table where fruit lay on former occasions, and clambers up to see if there is any there; then she comes up to me, and looks at me, as though she wanted something. She searches my hands to see if there is any fruit there.

She is irritated by the strident tones of the bugle. She is indifferent or comes nearer if wind instruments are played upon. Some fruit is now brought in and placed on the table. I give her some, which she eats with great delight. After. wards, she jumps up on the table, grasps the whip with one hand, and, with a rapid and very stealthy movement of the other, makes a dive at the cherries, seizing as many as she can. I run after her to take them from her. She puts as many as she can into her mouth and eats them greedily (evidently she goes through a somewhat long reasoning process, in so far as she safeguards herself by getting hold of the whip before darting at the fruit).

A male monkey (which we will call B), is now brought into the room for the first time. He is excited, tries to escape from his chain, and makes a great din. He quietens down shortly afterwards as monkey A, now reassured, comes up to him. They exchange vocal sounds with one another, sotto voce. Next they caress one another. The female begins to look for insects all over the body of her male companion. B scems to enter into a state of ecstasy and abandons himself to the attentions of the female. Erection ensues, but he makes no attempt at copulation. 
After a time, as the excitement continues, he attempts to copulate, heedless of my presence. He is prevented by the ehain, and gives up the attempt. The female never touches the genital organs of the male although her cleansing attentions are directed mostly to the thighs and buttocks. The male afterwards repeats the insect-hunting operation on the female, accompanying it with vocal murmurings and cadenees that remind one of the caresses bestowed upon an infant. All the while, the female remains in a state of ecstasy, closing her eyes and raising her hands to allow him to search the mamme. She abandons herself completely to his attentions as he prosecutes his search in the inter-femoral regions.

If, during these episodes, I throw down a cherry, there is no show of generosity on one side or the other. Whichever can grab it swallows it, heedless of the other. There has bcen no sign, however, of vindictiveness, or anger, or tendency to reaction on the part of either. Whichever remains with empty hands soon begins a friendly search for insects on the more fortunate or elever neighbour. I leave the room for a moment and on returning find them in close embrace, nose to nose, and breast to breast. Whether or not copulation took place I do not know, but they immediately separated and recommeneed the insect-hunting operation. One might almost suspect a rudimentary sense of shame.

29th June 1907.- She is even more domesticated and readily comes near. I wrap some cherries in red and some in blue paper. Those in red paper are normal, those in blue paper have been passed through a solution of bisulphate of quinine or injected with a little of that solution. I give her first those in red paper and she eats them with relish. Next I give her those wrapped in blue paper. Immediately the cherry touches her lips or tongue she takes it from her mouth, opens it, nibbles a little here and there, takes out the stone, breaks it, eats the kernel, and throws the rest away.

The experiment is repeated a second and a third time. On the third occasion the monkey does not taste the cherry wrapped in blue paper. She merely unwraps it, opens it, takes out the stone to get the kernel, and throws away the pulp (distinction of eolours and judgmerit based on memory).

The experiment is now repeated with Japanese medlars. In the ease of those wrapped in blue paper, she neglects the pulp and takes only the kernel. Those wrapped in red paper she eats in the usual way.

From these experiments one ean infer:-(1) That the monkey distinguishes red from blue; (2) that she is able to associate the bitter taste of the fruit with the blue colour of the paper wrapper; (3) that she is eapable of remembering the association formed between the colour and the taste, and of formulating the judg. ment (imagination) that the kernel is not affected by the bitterness, and that consequently she ean, without risk, eat the kernel, which indeed she at once breaks, shells and eats without any hesitation.

A male monkey is now brought in. They exchange compliments. The female begins to look for insects on the male. The latter is in a state of erection, but makes no attempt at copulation, the presence of four persons apparently having a restraining influence (shame?) although there has been a considerable exchange of earesses, especially on the part of the female. The scene on separation after half-anhour is distinctly painful. The male elings tightly to the female, and howls desperately when forcibly removed. The female, rather more indifferent, looks longingly after him.

I set a looking-glass before monkey A. She is greatly surprised. She follows all the movements of her image with great curiosity. She approaches the glass cautiously and soon afterwards tries to waylay the image behind the looking-glass, peeping round now to the right, now to the left. She exhibits a great power of observation.

At other two sittings on 10th and 15th July similar results were obtained. On the second experiment with the looking-glass, she seems persuaded that no other 
monkey is present and is no longer surprised, nor has she again looked for the other monkey behind the glass.

25th July 1907. - She is prepared and fixed on the operation-table. Is very agitated. An injection of chloral and morphine $(3 \mathrm{mg}$.) is given. After a few minutes, profound narcosis. Whilst the hair is being shaved, respiration becomes feeble and then suspended. Artificial respiration. She revives but respiration is still superficial, and nareosis remains profound. The animal is prepared with all aseptic precautions. With the trephine I make two openings, about $2 \mathrm{~cm}$. in diameter, in the frontal region, one on each side, $8 \mathrm{~mm}$. above the orbital ridge and $3 \mathrm{~mm}$. external to the median line. Slight hæmorrhage from the diploë. I cut around the dura mater and reflect it to one side. Electric excitation, repeated here and there all over the anterior part of the exposed area, gives no results. Only prolonged and fairly strong excitation of the upper and posterior part of the exposed area (the base of the superior frontal convolution) produces movements of the head. and eyes to the opposite side. On exciting the interhemispheric aspect of the margins of the pre-frontal sulcus there is produced a slight dilatation of the opposite pupil. Even on increasing the strength of the current and displacing the small bipolar electrodes upwards and backwards, one succeeds only in provoking a slight movement of the lower limb of the opposite side. Similar results are obtained on exciting corresponding points on opposite sides of the brain.

I incise each frontal lobe at a point corresponding to the pre-frontal sulcus. On one side I scoop out the cortex and remove over $3 \mathrm{gm}$. of brain tissue. On the other side I incise the cortex with a bistoury after the manner of Shepherd, making a vertical section, the knife penetrating in depth to the white substance, but taking eare not to go so deeply as to reach the base. Very slight hæmorrhage. Hæmostasis. Lavage.

The dura mater being stretched, and its edges sutured, I stitch the overlying soft parts with silk thread, fixing the free margins of the muscles to the perioranium. The entaneous wound being closed, it is painted with collodion. Narcosis lasts until the following night. On the morning of the 27 th the monkey is quite conscious. She takes a little milk. She is paretic in the left lower limb, and walks on the dorsum of the foot. It is evident that she does not recognise persons or things.

In order to avoid confusing the deficit producerl by the operation and the narcosis with that due to the removal of the frontal lobes, I postpone methodical examination until another day.

1st August 1907. - When the cage is opened she remains indifferent, and makes no attempt to come out. On removing her from the eage she makes some cireus movements, four or five times, from right to left. On offering her a plum she looks at it for a long time before recognising it, and then comes forward to take it. Movements of prehension, however, are badly directed, and she does not reach the object with precision. Finally she takes the plum, eats it, then breaks the stone, eats the kernel, and afterwards nibbles also the hard pericarp. She has no initiative: remains beside her eage; makes no attempt to escape, and no longer responds to the voices of ber companions, towards whom she appears to be absolutely indifferent.

She comes to the front of the cage and stands there for a long time, rubbing her nose and licking one of the iron bars in a very mechanical and automatic fashion. I dangle a pear before her eyes at a distance of one metre or more. For a long time she seems not to notice it. I throw it on the ground, however, and after some time she feels it, recognises it by the touch, takes it with satisfaction and eats it with avidity. I suspend a slice of pear by a thread and let it swing like a pendulum in front of her, within the visual field. It is only when the pear reaches the middle line of vision that she notices it. Although she sees it and turns her gaze on it, recognition only takes place when, after seeing the pear for some time, she at length puts her hand on it with some hesitaney, or when it is brought so near her nose that 
she can smell it. She never fails to notice contacts on the head or trunk, on either side.

I now set her at liberty. She leaves the cage and comes into the porchway walking well, except for some paresis of the left lower limb. An abscess has formed on the left buttock. Whether this is due to the cerebral lesion (decubitus) or to the injection of a syringeful of ether on the day of the operation is uncertain. She walks, however, as though she had no idea of direction, and sometimes knocks against large obstacles, as, e.g., a large flower vase. She keeps her head erect, as under normal conditions, and moves it in all directions. The dorsum presents a slight inward curvature. She has great difficulty in raising herself on the lower limbs, in standing erect, and in walking like a biped as she did before the operation.

Hearing is well preserved. Indeed, on taking her by the neck to lead her back to her cage, she has emitted a cry which has evoked calls from her companions, which are at some distance off, and she has immediately replied with vocal utterances, in the usual monkey fashion. The tuning-fork test, with the eyes closed, gives proof of the integrity of hearing on both sides.

6th August 1907.-Examination of sight. One eye being covered in the usual way, the other eye is tested. In this way it is found that the visual function has been completely restored, the visual field being normal for each eye (recognition of objects and persons in all sectors of the visual ficld). I take four pieces of a pear and wrap them in red paper. I take four similar pieces, dip them in a solution of quinine, and wrap them in bluc paper. I throw a picce wrapped in red paper to her. She unwraps the paper and eats the contents. I now throw her a piece wrapped in blue paper. She throws it away. A little later I throw down before her a piece wrapped in red paper and a piece wrapped in blue paper. She takes the red but does not even attempt to unroll the blue packet (she evidently remembers the bitter taste of the previous experiments).

She has adapted herself to her surroundings in the eage. Even when it is opened she makes no attempt to come out She exhibits great fear and has become timid. If one brings a cane near her, she crouches and ereeps off to a corner of the cage. She has an excessive fear of the humming noise of the vibrating tuning-fork, which she hears perfectly on both sides. Her eyes are now covered with sub-palpebral eye-shades of caontchouc. After a short period of restlessness she remains quite still. Under these conditions slight contacts on the ears, nose, back, chest and hands are noticed equally well on both sides. The wound is perfectly healed.

15th August 1907. - Identical conditions. No change. She always shows very little enterprise ; rather is she full of fear. She remembers fairly well all she had learned. After being kept without food for a considerable period she succeeds in finding a mode of opening a box containing a pear. Altogether she is more timid, less agile, less enterprising, but still fairly intelligent.

19th August 1907.-Killed with chloroform. The brain is removed after detaching the dura mater which is adherent to the cortex and quite one with the cicatrix on the left side. The left hemisphere has been deprived of a part of the pre-frontal lobe by a section which has fallen $2 \mathrm{~mm}$. in front of the pre-frontal sulcus. The superior and internal part of the frontal lobe has been spared, so that from the margin of the section there is a distance of about one centimetre and a half to the interhemispheric margin. The pole of the frontal lobe is spared, and is continuous below with the orbital surface and externally with the opercular part of the first Rolandic convolution.

The right hemisphere is almost intact. The incision is limited to the middle area. The first frontal convolution, which is uninjured, and the inferior part of the frontal lobe, are continuous with the rest of the brain. The incision has fallen $\frac{1}{2} \mathrm{~cm}$. in front of the pre-frontal sulcus. The brain is immersed in Muller's fluid, for subsequent examination by Marchi's method.

This case serves as a good control, inasmuch as it shows that partial lesions, 
even although they affeet both frontal lobes, do not produce the great frontal syndrome. The symptomatology is reduced to a change of character which is represented at most by indifference, inactivity, fear, and lack of interest, euriosity, initiative and reactivity.

Experiment 10.-4th September 1907.-Monkey B.-Male, slightly larger than Monkey A, lively, intelligent, daring, artful, very agile and circumspect. He goes around the room, jumps on chairs and other articles of furniture, takes various objects in his hands, examines them, lays them down, sometimes carries them off, so that it is neeessary to run after him in order to get them. He takes papers, unfolds them, refolds them, crumples them up and tears them. He dips his index finger into the ink-bottle, then looks at it surprised, gazes at me and does not know what to do with the finger, wet and black at the tip, but finally decides to clean it by rubbing it on the hair of his body.

He jumps up on the window-ledge and calls to his companions, who respond in turn. During four days he has learned to recognise, with equal facility, red and blue packets, the former containing pieces of pear or small plums in good eating condition, whilst the latter contain the same fruit, but made bitter. On the fourth day, those wrapped in blue paper had not even a glanee bestowed upon them, even when the monkey had been rendered hungry by long fasting.

When left alone in the laboratory and observed through a small spying aperture, he makes a tour of inspection all around, jumps on chairs and tables, takes up objects, examines them, lays them down, takes up some waste papers left there on purpose and tears them, apparently finding pleasure in the tearing sound, as happens with infants of three or four years. He makes several attempts to open the well-closed drawer from which the fruit given to him was generally taken. He fails in the attempt, jumps up on the ledge of the window and calls to his companions.

10th September 1907.-He has been kept apart in a large cage for several days and $\mathrm{I}$ now put a female monkey $\mathrm{C}$ into the cage beside him. The meeting is very cordial and effusive. They embrace and hug one another. The female more particularly seems to feel the need of protection and remains elinging to the male, grasping him preferably from behind. Occasionally they hug one another, face to face, and almost seem to be kissing. They search one another for insects and seem to enjoy the operation immensely, for they assume attitudes of ecstasy.

The male exercises the right over prey (food). If I put a pear at the front of the cage it is always the male, never the female, who takes it. On one occasion the female monkey has attempted to take half a pear which has fallen into the cage, but the male has prevented her with the energetic and clomineering gesture of a master. I lay down two pears beside one another at the front of the cage. The male takes one, then, when the female (C) attempts to take the other, he brusquely pushes her aside with an incomprehensible gesture. To make sure that he will get the second pear, he lays down half of the one that he took first, puts his foot over it, and uses his free hands to get hold of the other, which does not readily pass through the space between the iron bars of the eage. The female then brings her nose near to that of the male, who meanwhile eats greedily away without giving any share to the female. The latter, in full subjection to her male companion, has to play the role of spectator, and content herself by gathering up any morsels that may drop. During the whole day there has not been a single action of generosity on the part of the male. Even when the female takes up any morsel that he has let fall, she does so timidly, as though awaiting her eompanion's consent. Three or four times in the eourse of an hour the male has made attempts at copulation, sometimes apparently on the invitation of the female, who has held up her tail and exposed her genitals. The sexual aet is preeded by lengthy tactile preparations but is not eompleted, because the female twice makes her escape. 
1Sth September 1907.-To-day he is again brought into the experiment-room. He comes without any reluctanee, possibly because he has a pleasant reeollection of the previous experiment. Experience has now taught him to recognise sugar and distinguish it from ehalk. I take a piece of each from the drawer in my desk and show them to him at some little distanee, the sugar in my right and the chalk in my left hand. He greedily puts out his hand towards mine with the sugar but I quickly close my hand before he can take it. I put both hands behind my back and then bring them forward again in front of him. I open them and again he easts a glanee of envy at the sugar in my right hand, and a second time, as he is making a rapid movement to take it, I forestall him by elosing my hand. I repeat the experiment but, whilst my hands are at my back, I change the sugar from my right to my left hand. I now bring the hands forward, keeping them closed and quite near him. He seems to be quite ravenous and tries in every way to force open my right hand, in which he has twice seen the sugar. After a time I open the hand and he quickly seizes the white eube but, immediately recognising it as chalk, throws it away and at once tries to open the other hand, which all the time has been kept elosed before him. I take a roasted chestnut, of which he is very fond, attach a thread to it, and move it before him in such a way that I can withdraw it each time he puts out his hand to take it. Indeed, he makes many efforts to grasp it, but I remove it in time, so that he is disappointed. Sometimes the movement of prehension is so rapid or the apparent inattention of the monkey so well feigned that I have not been quick enough, and he has managed to get it. This has happened when, after several vain attempts to grasp it, he has pretended to pry no moro attention to it and sometimes has turned his head away with an air of refusal. If, whilst in this attitude, I brought the chestnut rather near, then, with lightning rapidity and precision, he sueceeded in snatching it before I had time to withdraw it. There was evidently, here, an attempt at dissimulation with the object of deceiving (memory and imagination).

24th September and 4th October 1907.-Experiments repeated in similar fashion.

16th October 1907.-Mutilation of the left frontal lobe, following the usual practice. Operation without noteworthy incident. Hremorrhage readily controlled. Cross seetion of the dura mater, and reflection of the four segments on the edge of the breach. Electric exeitation of the feet of the frontal convolutions. The areas of excitation for the museles of the neck, eyes, pupils and ear are defined in the same order as before, in the neighbourhood of the pre-frontal sulcus. I scoop out the cortex of the frontal lobe (superior and inferior convolutions). The weight of cerebral substance removed is six grammes and a fraction. Hæmostasis. Lavage of th surface of the wound and of the breach with sterilised water; suture of the lips of the dura mater, and then of the perieranium and overlying soft parts; dressing with iodoform and eollodion. The monkey, alrcady partly awake, is now plaeed upon a coueh. He lies quite limp.

I omit the notes of the first few days because they are of little use.

19th Oclober 1907. - He walks well. One ean just detect a certain difference in the movements of the limbs of the right side. He seems restless; walks about the room like one dysorientated; now he jumps up on a window, now on a chair. He has a preference for corners, but remains only a short time in one position. He turns round his head and eyes like one in a dream. In any case one does not find the usual air of curiosity and craftiness in his attitude and look. His glence is more lifeless; he has an air of indifference. He approaches, without any suspicion, people of whom he was formerly afraid. He has been noticed several times scratehing his head with the right hind limb and taking pieces of apple with the right hand, with fair precision in his movements. No eircus movement. At no time has he shown deviation of the head towards one or other side, no curvature of the trunk or of the neek. He seems to have lost his reactive nature, even when one takes away his food from before him, although, prior to the operation, he took 
possession of it with an air of authority and would not leave it in spite of threats or violence.

1st November 1907. - The examination of the visual field has shown vision to be defective in the external half of the right eye, and at the extreme right of the left visual field. It is needless to repeat here the method followed in measuring the visual field. Suffice it to say that $\mathrm{jt}$ has given fairly precise and reliable results.

10th November 1907.- Torpid and dysorientated. Recognises fruits but does not distinguish the deceptions practised upon him with the same rapidity and precision as before. For example, he was given a sham pear made of bread paste allowed to harden and then painted. When the animal was in good health he recognised the deception after the first trial, and subsequently paid no more attention to it. Since his operation he has persisted in taking it and breaking it angrily with his teeth.

He is indolent, timid, indifferent, has no initiative, and will remain for a long time in a corner of the room.

He eats well, taking food equally well with either hand and carrying it to his mouth with preeise movements. If one holds a small apple firmly in the hand and offers it to him, he will take it if allowed but will not make persistent and foreible efforts to obtain it as he did before the operation. He is more submissive than he was. There seems to be a slight weakness about the right hand.

22nd November 1907.-A second operation, on the right side. No serious incident during the operation. Faradic exeitation; identical results, which are summarised in the chapter following. The incision was inclined from behind forwards, commencing about two miliimetres in front of the pre-frontal sulcus, leaving in situ a part of the cortex at the anterior pole. Suture and aseptic dressing as usual.

23rd November 1907.- Great depression and prostration. He has never moved from his couch since the operation, nor has he taken any food. He displays a profound indifference. If, however, we catch him by the back and attempt to chase him from his couch he clings with his hands to the iron bars. When put on the ground he curls himself up and lies prostrated.

24th November 1907. - He is able to take some milk. His condition is but little altered although there are appreciable signs of commencing improvement. He is able to raise his head, to look around, to rise up and stand on his legs for a few moments.

29th November 1907. - During the past three days he appears to have recovered from the first post-operative phase, which has been fairly severe. His condition is now such that methodical examination can be undertaken.

$\mathrm{He}$ is torpid and indifferent to all that occurs around him. He remains curled up in a corner of the room for a long time without moving, sometimes with the head bent. He passes to another corner of the room, apparently without any object, rather from a pure automatic impulse, and assumes the same attitude. Sometimes he jumps up on the window but does not call out. He eats when food is proffered him - a piece of bread, a chestnut, an apple; but he does not peel the chestnut with the same smartness, nicety and care that he exhibited under normal conditions, and sometimes he even eats the skin. In any case he is rather filthy.

6th December 1907.-Remains in the same condition. He is full of fear. A slight noise is sufficient to make him suddenly jump and show signs of terror. Examination of tactile sensibility in all points of the body is quite negative. Attempts to prick him or pull hairs from his back or head provoke emotions of fear, with tremblings, cries and efforts to escape.

Sight, measured in the usual way, is normal on the right side, though less clear towards the inner angle. On the left side, proceeding from within outwards, elear vision ends at a point corresponding to a vertical line passing through the visual 
axis. Repeating the experiment from without inwards, he sees the object (a roasted chestnut, peeled) clearly, even before it reaches that line.

10th December 1907. - Condition as already described. The following experiment is made. Being rendered hungry by prolonged fasting, he is allowed to see a dish containing pieces of apples and roasted chestnuts. He is agitated at the sight of the food, and extends his hand to take it, but I withdraw the plate and place it on the top of a bookease about two metres high. The monkey begins to jump and clamber up the panels of the door of the bookease. Half-way up, he slips on the smooth surface and falls back to the ground. He takes up a position about half a metre in front of the bookcase, and renews the attempt in the same way with the same result. He repeats this attempt twelve times in the presenee of one of my assistants and myself, without any modification whatsoever, the rhythmic character of the process being indeed surprising, and showing all the characters of a stereotyped movement or a tic.

I now bring into the room the female monkey C. She goes up to B, walks around him, petting and caressing him, and looking at him with much curiosity. $B$ makes but little response to all C's manifestations of friendliness. He remains torpid and indifferent, showing at most some signs of satisfaction when $\mathrm{C}$ touches him about the neck, especially when she strokes and fondles him there. He seems to experience very little sexual excitement, even when solicited by the female. If he does make an attempt at copulation it is futile, wholly lacking in that vigour and boldness that were so marked in him, as, indeed, in all normal monkeys, prior to operation. ${ }^{1}$

The wounds are now completely closed.

20th December 1907.-Conditions identical. His life is solitary, stupid and sterentyped. He crouches in a eorner and only leaves that position to pass to another spot in the room, with movements that are rhythmic and always the same. When along with other monkeys he takes no share in their caresses and merriment. He recognises his food and eats regularly, but is more filthy. Indeed, he makes a dreadful mess of himself if not carefully attended to. If given food in a basin in enmmon with the others, he generally eats nothing. When three of them are together in a room, and pieces of apple or chestnuts are thrown on the ground, he never suceeds in getting any, because the other two are much more alert. Were it not for the care bestowed upon him in giving him food apart from the others, he would

${ }^{1}$ In order to emphasize the anomaly of this frigid and stupid behaviour on the part of a monkey that has suffered mutilation of the frontal lobes, I cite here one of many eontrol-experiments that served as a guide to examination.

Three monkeys which hitherto have been kept in separate cells are brought together in the laboratory-Alpha, a large female, Beta, a smaller female, and Delta, a male monkey larger than either of the females. Hardly have they set eyes upon one another when Delta attempts copulation with Alpha. The latter fondles and caresses the male and embraces him amorously. Soon afterwards they commence insect-hunting operations on one another, each submitting in turn to the other in a voluptuous attitude. Meanwhile Beta remains a melancholy spectator. I throw a cooked potato on the floor. Beta runs to pick it up. The other two are too busy to think of eating. They stand embracing one another; they follow each other around; they caress one another. It is a regular flirtation. Then comes a moment when Alpha leaves her companion to jump up on the window-ledge and call out to her friends in the monkey-house. Beta seizes advantage of the opportunity and offers herself to Delta. Alpha appears to grasp the situation, for she immediately jumps down from the window and rushes at Beta, driving her away and pulling her by the hair, whilst Delta, the courted one, looks on calmly as the two females settle their affair, though perhaps not without a benevolent glance towards Beta, which, being the smaller and weaker, gets the worst of the encounter. 
certainly be victimised by his companions who, on questions of food, are not very generous with one another. In this respect his behaviour and lot resemble those of low-grade human imbeciles.

22nd December 1907. - On post-mortem examination the two frontal lobes are seen to be decorticated over a considerable area, from the pre-frontal sulcus to the pole, rather deeply so in the centre of this area. On both sides the parts spared comprise the whole of the orbital surface along with the external margin of the second frontal convolution and the entire interhemispheric surface along with a part of the superior frontal convolution.

Experiment 11.-28th November 1907.-Monkey C. Female. The same which lived for some time with the male $B$.

Rather small but very intelligent, smart, lively, observant and curious. She is fond of the company of other monkeys, so that she is in great despair, eries and calls out, and is very unsettled, when taken from her quarters to the observationroom. Although there are many intervening objects between this room and the monkey quarters, she peeps out every time the door is opened and does her best to get out. She goes around the room taking stock of the few pieces of furniture which are there. She jumps up on the window-ledge in order to spy better down the garden, and gives a call. She takes apples quickly and eats them with avidity. She does not know the chestnut, for she sees it for the first time, and is not aware that it is good food. I peel one and offer it to her. She examines it, tastes it cautiously at first, and then eats it. She is not afraid of the rod with which she is threatened, for in the past there have been several threats which did not materialise. She seems much less wild than she was, and is commeneing to understand the command "Give me your hand," without me making any sign or putting out my hand. I put an apple on the cornice of the bookease. She elambers up over the door and almost manages to get it, making use of every projection of the wood as a point of support, precisely as a man would do. After two trials, as there are no further points of support, all being smooth, she abandons the attempt.

1st December 1907. - She is offered a chestnut; she recognises it at onee, takes it, strips off the skin, and eats it with great satisfaction. I give her a sham pear. She shows even greater pleasure at the sight of this but, having taken it, she turns and rolls it in her hands, brings it up to her mouth, remembering the pears it resembles, for she has often partaken of this fruit, but changes her attitude and seems vexed. After having felt it many times, scrutinised it, smelt it, and tried it between her lips and teeth and on her tongue, she ends by throwing it away and does not look at it again except with an air of indifference.

3rd December 1907. - I take her into my private room and leave her at liberty. She makes a tour of inspection all over the room as though trying to get her bearings. At first, every time the door is opened she tries to make her exit. She clambers up to the ledge of the window, spying through the window towards the place where, at some distance off, her eompanions are, ealling out repeatedly and never remaining at rest. The others reply, so she peers out more anxiously still, and cries as though she were hailing them.

I repeat the experiment with the apple on the cornice of the bookcase. She clambers up and sueceeds in taking it, exhibiting marvellous precision in her movements. She has jumped up on my writing-table but, as she has knocked over some papers, I rise up and, taking the rod in my hand, assume a threatening attitude. She makes her escape and hides under a couch. I sit down to write again. She remains hidden behind the couch which is directly in front of me, but from time to time clambers up the back of it and craftily protrudes her head to spy what I am doing. If my eyes encounter hers she immediately hides herself. She is very partial to apples, so I now put three of these on my writing-table and, going to an arm-chair, sit down to read. Again she peeps from behind the eouch, and, as soon as 
she feels safe, she eautiously makes one spring forward like a skilful thief, seizes an apple and returns to her hiding-place. I follow her and threaten her with the whip, and then for a long time she refrains from making any further attempt (inhibition, memory). She has allowed the two apples to remain on my writing-table, although still very hungry. Indeed, when, after a time, I eall her to me and offer her another apple, she approaches at once without fear and snatches it from my hand with surprising precision and rapidity.

12 th December 1907. - I have again taken her to the study where I am alone. She is much more domesticated. She has not jumped up on the writing-table, although there are apples there as in the former experiment. Twice she has eautiously elambered up the legs of the table to spy my attitude, but it has sufficed for me to say "No" to induce her immediately to descend and refrain from any further attempt. I now give her a piece of apple that has been dipped in a solu. tion of quinine, and although she is very hungry, having been kept without food, she immediately notices the bitter taste, and seems surprised and vexed. She smells it, licks it eautiously, and then lays it aside. Several times as she has been touring around the room she has gone up to the rejeeted apple, handled it, examined it, smelt it and then left it alone. After a long time, urged by hunger and desire, for she has had no food, she has decided to eat it; she begins studiously at the centre and proceeds gradually towards the periphery until she comes to the bitter part, then discards the outer layer of the piece of apple.

I have placed three apples on the arm-chair near the table. She has made three attempts to approach the place, watching my eyes, but it has been enough for me to say " $N_{0}$ " to induce her to retreat. Next, she has taken a turn round the room examining everything in it, and then she has hidden behind the eouch and several times has peeped over it, to see if I were awake, or if I were disposed to offer her an apple. She has made no attempt to steal them. It is only after the lapse of an hour that she has come forward to me very timidly and circumspectly, meeting my gaze and then turning round towards the apples. I now offer her one and she takes it from my hand with great joy and avidity.

I have given her a piece of apple immersed in solution of quinine. She has im. mediately recognised it and left it alone, evidently having a good recollection of the previous experiment, for she has spent no time over the examination. I now throw in front of her some small packets eontaining sugar, pieces of apple, and chestnuts, wrapped in red paper, and other packets containing pieces of ehalk, pieces of apple dipped in solution of quinine, and artificial chestnuts composed of ehalk and sawdust, wrapped in blue paper. She opens them one after another just as she comes upon them, eating the contents of the red packets and throwing away the contents of the blue packets after a brief examination, paying no more heed to them. After a lapse of one hour I repeat the experiment. I am not convinced that she notices the colour of the packets, because she repeats the examination of their contents in the same way.

13th December 1907. - She has been rendered hungry by prolonged fasting. I now throw in front of her red and blue packets alternately. After two experiments she eommences to show some doubt as to the blue packets. Nevertheless she unwraps them and throws away the contents with an air of disdain.

14th December 1907. - She has not had any fond sinee last night. When brought to-day into the observation-room, I give her a red packet, which she takes with confidence and rapidity, unwraps it and without any diffidence takes the content, putting it at once in her mouth, and eating it with relish. I now offer her a blue packet. She takes it with some diffidence, unwraps it without enthusiasm, recognises the eontent, and without delaying much over it as in the former experiments, throws it away, although there is no doubt her appetite is still unsatisfied.

15th December 1907.-Operation. Nareosis. Bilateral trepannings, half a centimetre above the supraciliary orbital ridges. Strict aseptic precautions. No 
hæmorrhage. Section and reflection of the dura mater is made without the occurrence of any unpleasant incident. Electric excitation reveals a small area in the middle of the pre-frontal sulcus (about half a centimetre in front of the excitable area for extension of the hand and wrist), which gives rise to dilatation of the pupil of the opposite side. On displacing the small bipolar electrodes a little higher on the same line, a combined movement of the eyes to the opposite side and dilatation of the pupil is obtained. Passing still higher to the foot of the first frontal convolution, we get co-ordinated movements of rotation and inclination of the head and of the eyes to the opposite side. At a point still higher up, near the interhemispheric margin, we get movements of the head to the opposite side, slight movement of the pelvis with a tendeney to eurvature inwards, and an upward movement of the tail. With the help of careful assistants these observations were repeated several times with the same results, the animal being kept in a state of slight narcosis. It is, however, to be remarked that symmetrical correspondence between the two sides is not perfect either in the case of the area of excitation for the external ocular muscles and the iris or that of the hand. At a point very close to that of the ocular muscles, but underlying it, excitation produces movements of the opposite ear, and a little further below and behind is a small area, excitation of which gives rise to slight blinking movements of the eyelids, resembling those that occur when the eye is rather irritated by light. With a cataract knife $I$ incise the cortex between the area of the upper arm, and that of the pupil, but much nearer the latter point, making a curved incision with convexity upwards and backwards so as to spare the area for conjugate movements of the head and eyes, and of the trunk, on the foot of the superior frontal convolution. For protection I insert a small spatula and scoop out the exposed cortical area, proceeding from behind forwards, and removing what of the cortex lies in front of the incision. The lesion in the left cortex is deeper than that in the right. The weight of cerebral tissue removed is tive grammes.

16th December 1907. - She is depressed and immobile. She lies on the couch with her head and her arms spread out. The upper limbs are cold and somewhat œdematous. The lower limbs are cold but dry. On being shaken, she opens her eyes and raises her head. She has been without food for thirty-six hours, and now shows a desire to have a slice of apple when it is placed near her. As, however, she cannot take it with her hands, she moves her head and body forwards slowly and without energy so as to reach it with her mouth, but, soon after, exhausted by the effort, lets it drop. She is given a subcutaneous injection of sulphuric ether and is wrapped in warm flannel, after which she revives to some extent.

18th December 1907. - Improvement has been progressive. She has taken some milk and some slices of pear and apple. The swelling has almost entirely dis. appeared from the upper limbs, but she is still very weak and unable to stand up on her hind limbs. The hands seem heavy as she raises them to take a small piece of sugar. Respiration is no longer difficult, but the heart-beats are still abnormally frequent.

23rd December 1907. - General condition still further improved, but she is inactive and depressed. Urged to leave her eouch, she rises and makes a few steps, but is completely dysorientated, does not know where to go, moving now to the right, now to the left in an uncertain fashion. Sometimes her hands or her feet are bent under so that she walks on the back of them (paresis), then, as she continues to walk, she straightens them out. It is not yet opportune to proceed with the methodieal examination of her mental state.

28th December 1907.- She walks well and no longer supports herself on the dorsum of the hands. She turns to right or to left by chance, or when shown something to eat. She presents a slight inward curvature of the trunk, but at times stands erect on her feet. The eyes being covered with sub-palpebral eye-shades of caoutchouc, we proceed to the examination of sensibility, which is found to be well 
preserved in every part of the body. She always shakes herself when touched unawares.

Hearing, tested in the usual way with the tuning-fork, is well preserved.

The examination of sight, one or other eye being covered, leaves some doubt as to its precision as regards recognition of objects placed successively in front of her, and as to the clearness and distance of vision, inasmuch as she makes mistakes in her movements of prehension. On suspending a piece of sugar by a thread, and making it pass from without inwards opposite each eye, on a horizontal plane at the level of the visual axis, she turns her eye upon the object as soon as it enters the normal visual field, but, although hungry, does not always extend her hand to grasp it. She seems torpid and indifferent, and puts out her hand slowly, and does not always measure distance with precision. She has lost her vivacity, her craftiness, and the lightning rapidity of the movements of her right hand. The condition is identical on both sides.

If, when left to her own devices, she comes to a halt anywhere in the room and I throw a piece of sugar very near her, either to her right or to her left, she takes it and puts it in her mouth. If I throw it some distance off she malses a run after it, then seems to lose sight of it, stops half-way, as though she had forgotten about it, gives up the search and takes another direction. Possibly her accommodation is defective, but it is very difficult to decide whether we have to deal with visual accom. modation or with defect of mental accommodation based on memory and attention.

8th January 1908. - Her physiognomy seems altered. It has lost that lively glance, that air of scrutinising and crafty curiosity that was so characteristic of this monkey. She walks about the room, where she is kept under observation, for several hours together. Sometimes she stops and picks up morsels of bread and small picces of chalk thrown down together. She puts everything in her mouth. She halts in front of a yellow spot on the wall, touches it and retouches it, as though she wished to pick out the plaster with her finger. She jumps up on the windowledge as usual, but does not give the call; she remains there, indifferent, with her head bent, for several minutes, then comes down again. She shakes and jumps at every noise and crouches in a corner, where she remains for a considerable time with head bent. Then she gets up, raises her head, and passes and repasses more than a dozen times, in stereotypic fashion, between the wall and the door. She does not trouble about me, nor does she seek to come on to my table, as she was so keen to do prior to the operation. I have given her a small box with one side made of glass through which she ean readily see its contents-viz. two apples. She has taken it, has turned and returned it over in her hands. She has tried to put her fingers through the glass to take the apple, then has put her nose and lips to the glass. She makes off, with the box in her hand, to a corner of the room where she sits on her hind quarters for several minutes, keeping the box in her hands, rotating it between her fingers, but making no attempt to open it.

$24 t h$ January. - Death occurred this evening as the result of an aceident.

Post-mortem examination nine hours after death. On laying bare the cranium nothing of note is observed except the two breaches in the frontal lobes, now closed by cicatrices. Apart from two pearly spots corresponding to the breaches, the meninges are perfectly healthy. The brain, when removed and subjected to an accurate inspection, appears normal in colour, without sign of any lesion with the exception of the experimental injuries to the frontal lobes. These seem almost symmetrical. On the right side, the lesion in the first convolution extends behind to within almost $3 \mathrm{~cm}$. from the upper extremity of the Rolandic fissure. In its middle portion it reaches the anterior margin of the pre-Rolandic convolution. Below, the operenlum of the seeond convolution is injured, but there remains a small bridge by means of which it is continuous, below, with the orbital surface of the frontal lobo, which is perfeetly preserved, and, posteriorly, with the operculum of the anterior Rolandio convolution. 
The pole of the frontal lobe is preserved, and is continuous with the first frontal convolution by means of a bridge which forms, as it were, a vault hiding the lesion which, underneath, reaches the internal surface of the hemisphere, doing injury at one point to the first convolution and the convolution of the corpus callosum just over the genu of the latter, which is intact.

On the left hemisphere the lesion is almost symmetrical. The frontal and prefrontal portion lying in front of the pre-frontal sulcus is almost completely removed. The pole is fairly well preserved, and is continuous with the rest of the cerebrum only on the orbital surface, which appears normal. The first frontal convolution on the interhemispheric aspect is interrupted by the experimental incision, which reaches in depth to the convolution of the corpus callosum one millimetre in front of the germ of the latter. Below and without, the lesion reaches almost to the external margin of the orbital surface.

On each side the whole of the orbital surface of the frontal lobe is spared, along with a zone of the external surface more than one centimetre in length, by means of which the orbital surface is continuous with the pre-Rolandic operculum.

This observation is interesting, because it provides another illustration of the fact that it is very difficult to destroy and remove all the frontal lobe, without making a very large breach and laying bare the whole field of operation. In the latter case, however, the operation is a much longer one, and in my experience small monkeys do not survive it. Even this monkey passed through a phase of exceptional gravity lasting several days, and it was only by assiduous care and attention that we succeeded in keeping it alive. What generally happens is that the opening in the cranium is made rather far in front, so that one can detach the pole and decorticate a tract of what remains behind, in which case a considerable portion of the frontal lobe is left; or, on the other hand, one exposes the excitable frontal area along with a little of the Rolandic convolution suitable for making observations on the electric excitability, and in this case the lateral limits and the pole of the frontal lobe remain covered so that a part of the frontal lobe remains in anatomical relation with the Rolandic brain and what lies behind it. As it is important that the animal operated upon should be kept alive, one has to avoid prolonging the operation to any great extent, or making the cranial opening too large.

Experiment 12.-Monkey D. - The largest of the tribe. Strong, alert and bold. If $I$ approach the cells of the other monkeys and take any of them by the hand, he spies me from his own little cell, becomes agitated and angry, shakes his chain with both hands, and watches me with a threatening air and leonine haughtiness, as though it fell to him to look after the interests of all the other monkeys. If one remains in front of the cell in an antagonistic or threatening attitude he falls back a pace or two, opens his mouth and shows his teeth, or he springs at the gate and shakes it in a threatening and irate manner. If he gets possession of a piece of bread or small stone he hurls it at one. If another monkey is led away to the observationroom, he assumes the air of a protector, peers out in an angry manner, hurls himself against the iron bars, shaking them in his rage, and sometimes calling out.

23rd December 1907. - Along with other pieces of apple I give him one dipped in a solution of quinine. He at once notices the difference. When he takes it he examines it carefully, smells it, looks at me with suspicion, rubs it with some straw, and on the wall of his cell, and after this operation brings it to his lips again, finds that it is still bitter, throws it away, then takes the straw in both hands, tossing it several times into tho air, as though to give vent to his rage. As, however, he has keen kept without food for some time, and is rather hungry, he again picks up the piece of apple, breaks it, and, exercising great care and caution, eats the contral part.

I place a piece of apple on a large stone lying in front of his cell, just beyond reach of his hands. After many futile attempts to reach it he decides to move the 
stone cautiously, with the evident object of bringing it nearer him without allowing the apple to fall. I now fix an apple to a ribbon and suspend it in front of his cell, at a distance slightly greater than the length of his arm. He makes every effort to reach it, but always falls short of it by about 4 or $5 \mathrm{~cm}$. Seeing the futility of his attempts he gives them up, squatting down with his hands crossed, between mortification and irritation.

I now make the apple swing like a pendulum. On the second oscillation he elutches it with marvellous rapidity and precision. He draws in the ribbon with it to the bottom of his cell, and, after having eaten the apple, plays with the ribbon. In vain I seek to get it from him. I hold an apple before him in one hand, and put out the other hand in the attitude of one asking something. After twice calling loudly to him he has rolled up the ribbon and thrown it to me. I piek it up and give him an apple in exchange. I put an apple on the top of a stone, at some distance from him. Strong as he is, he reaches through the bars and tries to shake the stone so as to make the apple fall. Failing in this, he gives up the attempt. I give him a spindle off a chair. He plays with it, turning it round in his hands. Whilst ho still has it in his hand I chase him out of the cell. With it he could have knocked down the apple, but he has not been capable of this reasoning process. He has, however, thrown the spindle in the direction of the apple which, in point of fact, has actually fallen, though I remain in doubt as to whether this was a ehance affair or the result of reasoning.

I wrap pieces of apple in red, and pieces of chalk in blue, paper. After four trials he has not shown any sign of remembrance that the blue contain chalk and the red apple (has he failed to perceive the difference between the colours, or is it that he has not remembered?).

8th January 1908. - I give him a long stick which has been applied to him several times as an instrument of correction. At first he bites it, then he plays with it. As there is an apple on a stone at some distance off, he throws the stick so that it falls on that stone. The apple falls down, and, with lightning rapidity, he throws himself on it and seizes it with his hand. At the same time I dart forward to lay hold of the stick and keep it out of the cell, but the monkey, with the rapidity of a leopard, seizes it with his other hand and draws it away into his cell before I can reach it.

He is next transferred into a large cage made of wooden bars arranged very close to one another. An apple is now placed near the cage and he tries to put his hand and wrist through the different spaces between the bars, trying one space after another until he finds one large enough to allow him to get his hand through and grasp the apple. When he gets it, he finds that the space is not wide enough to let the apple pass through. Ho becomes agitated and tries all the spaces, putting his feet against the wooden bars and trying to forcibly pull the apple through. 'This plan not succeeding, ho bites the apple all round, with the apparent object of making it smaller so as to let it pass between the bars of the eage.

I put pieces of apple in a narrow-necked bottle which I give to him. Immediately he gets it he tries all he can to get out the pieces of apple, showing great interest and anxiety in the process, turning and returning the bottle and biting it. After many attempts he breaks the bottle and eats the pieces of apple.

I put two apples in a little tin box with a little glass window. I have hardly given it to him when, in tho twinkling of an eye, he breaks into it with his teeth.

I relate the psychological data of this monkey because they serve to give a certain measure of the intelligence and emotions of this kind of quadrumane, and to furnish still another proof of the necessity for a full examination of these animals, both before and after the operation. Unfortunately this strong, intelligent and masterful monkey died in less than twenty-four hours after operation.

Experiment 13. - Sth March 1908.-Monkey E. Male. Anasthesia by chloroform after previous injection of morphine. Bilateral trepanning without serious 
difficulty and without loss of blood. Section and reflection of the dura mater. Electric exeitation of a small area, $3 \mathrm{~mm}$. in diameter, situated 2 or $3 \mathrm{~mm}$. in front of the limits of the area of excitation of the anterior limb, produces dilatation of the pupils, more especially that of the opposite side. Further upwards and inwards, excitation produces conjugate movements of the eyes. Still further inwards, on the foot of the first frontal convolution near the interhemispheric margin, excitation gives rise to movements of the head to the opposite side. A few millimetres below the area for dilatation of the pupil there is a small excitable area related to the opposite ear.

With the thermo-cautery at red heat, I incise the frontal lobe (first and second convolutions) along the pre-frontal sulcus, to the depth of at least one centimetre, on both sides. No troublesome incident. Suture of the dura mater and of the other parts, and the usual dressing with iodoform and collodion.

9th March 1908.--No accident.

11 $t$ h March 1908. - He is fairly lively. He can grasp pieces of apple or chestnuts equally well with either hand. He clambers up the bars of the cage. The pupils are equal. Winking is normal. He sees near at hand, and grasps pieces of bread or apple, but he does not see at a distance. He does not see an apple placed on the ground two metres off.

Tactile sensibility is normal.

Both monkey $\mathrm{F}_{4}$ and the female monkey $G$, which had previously undergone operation on one side, are now taken from their cages and left together. E immediately attempts copulation with $\mathrm{G}$, his movements being quite normal, but he seems afraid, and the attempt is hardly begun before it is given up. Shortly afterwards he curls himself up, lowers his head and appears to sleep.

15th March 1908. - The wound is healing satisfactorily by first intention. There is no suggestion of suppuration. There is no sign of paralysis in the trunk, neck or limbs. He walks, jumps, grasps with both hands, and masticates as though nothing had happened to him; indeed, just as he did prior to the operation. He does not perceive objects with precision and certainty at a distance, but this symptom was more marked in the first days after the operation. He shows a fair amount of boldness in looking around the room for food even when chased away from it. Tactile sensibility is perfectly preserved. If, when his eyes are covered, the ear is lightly touched, he immediately shakes his head as under normal conditions. Vision, which in the first few days was very defective, is now much more precise. Fear is always a marked symptom. Clapping the hands, even after numerous repetitions, produces a strong trembling of the whole body.

He goes about the room in a rambling, dysorientated fashion. If given a piece of bread dipped in a weak solution of quinine he notices its bitter taste, is very doubtful about masticating it, then ends by rejecting it from his mouth (the female selects from the bread that part which has not been soaked with the quinine). The bitter taste of a piece of apple immersed in quinine solution is certainly detected by the male, but rather more readily by the female. 'The former has eaten it reluctantly, the latter after two trials has definitely rejected it.

A good deal passes unobserved under his eyes. If he changes his position from one corner to another of the room, it is without aim or purpose. He seems indifferent quite as much as dysorientated. If an apple is thrown on the floor he sometimes runs after it. At other times he loses trace of it, no matter how short its distance from him, as though he either forgot to continue running after it or else did not see it. The female runs after it to any distance and picks it up. E readily recognises the persons who are in the habit of bringing him food, and sometimes follows them as though expecting something to eat, but beyond this shows no other sign of mental activity.

Sometimes he picks up straw or other small and insignificant objeets-e.g. a thread or a piece of crumpled paper-so that he reminds one of the tendency to 
collect uscless objects that is so marked in some dements. It is certainly a thing he never did before. The feature that is most prominent is his loss of courage. He no longer reacts as before, no matter what one does to him-e.g., when one snatches an apple out of his hand, although he formerly reacted with a courage and vigour that were truly impressive, even when shut in his eage. He no longer calls out to his companions as in the past. If offered some morsels of sugar, they must fall within the visual field of the eye and within reach of his hand; otherwise he either fails to see them, or, if he does see them, neglects to take them; once he has taken them, howerer, he eats them with relish. If, on the other hand, one throws him a piece of chalk, he breaks off a bit with his teeth and keeps the fragments a long time in his mouth. He notices the difference, and remains with the other piece in his hand. If, after half an hour, the experiment with the chalk is repeated, he does not remember the previous experience and again he tastes it, biting off a piece, and keeping the rest a long time in his hand, or letting it fall, whilst before the operation it was sufficient for him to taste the chalk once and for all before he threw it into the air, or squashed it in pieces on the floor, never again to fall into the same trap.

8th A pril 1908. - There is no marked sign of defect of motor function or of special sensibility. The visual field is not apparently diminished. The monkey notices a picce of apple suspended to a thread and allowed to come within the extreme limit of the visual field either from the right or the left, and grasps it with rapid and certain movements that betray no noticeable difference between the two sides. Only occasionally has it seemed that vision was less elear on the right side. There is no difference in the size of the pupils. There is a hardly noticeable difference in the motility of the upper limbs, in the sense that he makes more use of the left; but in all operations necessitating the use of both hands, as in forcibly pulling an apple through the space between the bars of the eage, he makes very good use also of the right hand, with which he executes movements that are as precise as those of the left.

25th April 1908. - He is brought into the room without a chain. He is very docile and timid. He has lost the alacrity, vivacity, aggressiveness and boldness which so eharacterised his actions in the past. He moves about the room very indifferent, or remains curled up a long time in one spot, perhaps in a corner, without manifesting care or concern about anything, showing, above all else, a great lack of that spirit of observation, suspieiousness and eraftiness which is so characteristic of the mental life of these quadrumanes.

Visual sensibility is undamaged. If, however, a piece of fruit or bread is placed three or four metres in front of him he gives no sign of having realised that it is there (disattention or lack of accommodative power ?).

I now report a series of comparative experiments made with other two monkeys F, a male, intact; G, a female, operated upon on one side only.

Monkey E remains indifferent, or wanders aimlessly about the room, or performs purposeless movements, paying no heed to the female, which is calling out and making a great noise in her eage on the terrace.

Monkey $\mathrm{G}$ is now brought into the room and $\mathrm{E}$ is taken to his cage. $\mathrm{G}$ is evidently greatly concerned. She jumps up on the window-ledge to see where $\mathrm{E}$ is, and exerts herself in every possible way, spying out of the window with great anxiety.

Monkey $\mathrm{E}$ is restless and excited, and, if he sees a piece of apple or bread, he becomes agitated and elambers up the iron bars of his eage, performing all sorts of anties. I offer him a slice of apple. He puts out his hand to take it, and at the same moment $I$ hide it by shutting my hand, letting him take hold of my forefinger instead. Forgetful of the deeeit so often practised upon him he anxiously seizes my hand, trying to force his own hand into my closed fist, in order to get hold of the apple, and he obstinately persists in the attempt, at the same time trembling all over. The other male monkey, F, acts differently, for onee he is cheated he makes 
no second attempt, despite the fact that I display the apple, holding it between my fingers, so near to him that he could easily get possession of it. His attitude suggests a dignified indifierence, so much so that one might well argue that he feels offended at the deception. No matter how often I offer the piece of apple to $\mathrm{E}$ and cheat him in the manner described, so often does $\mathrm{E}$ fall stupidly into the same trap. He has learned nothing from the deceptions practised upon him at brief intervals.

When the two males are taken out of their cages and left together, the small monkey $\mathrm{F}$ pays court to the big monkey E, running behind him and making attempts at copulation. E accepts and endures the caresses of the other without evincing any sign of affection. Occasionally he seems to go into ecstasy when $F$ and $G$ commence insect-hunting operations upon him, or stroke the skin of his neck.

If now I throw three or four pieces of fruit on the ground quite near to $E$, the other two, F and G, immediately hurl themselves on the booty, using hands and feet to seize as much as they can. E makes an attempt to get hold of a piece which is very near him, but so torpid and slow is he that he is forestalled by his com. panions and remains with empty hands. This experiment brings very clearly into evidence a defective judgment, because he does not foresee that his two companions might get possession of all the fruit before he has managed to secure a piece for himself. He has made no use of past experience (defect of memory, imagination and judgment concerning this form of struggle for life), as compared with the alert rapidity of the other two. There is also evident a defect in the motor discharge, so that his morement is more torpid, slower and all in vain. If not specially taken care of and fed he would have succumbed, owing to his incapacity to compete efficaciously with the othors in assuring for himself the necessities of existence.

Pretty much the same thing happens with human imbeciles (if not cared for and protected), who are quite unfitted for the struggle for existence.

10th May 1908. - E has become aggressive, revengeful and voracious. If, shortly after he has been enjoying the caresses of the female monkey G, I give the latter a piece of apple, he endeavours to snatch it from hor. Failing in this, because $\mathrm{G}$ is in her cage, he seizes her tail and bites it, and then grasps her by the back in order to take the apple from her. G, full of cunning, puts the apple in her mouth, and with both hands liberates herself. This scene has been repeated several times. E also hurls himself against the other male. Every sentiment seems overruled by that of voracity, so that he has become impulsive and aggressive.

I throw to $\mathbf{E}$ a piece of apple wrapped in blue paper. He makes no effort to take it, and does not move from his position. I pick it up and put it just under his nose. He takes it, unwraps it, and eats the apple with relish. Shortly afterwards he takes the blue paper, masticates it and swallows a piece, a thing which none of the normal monkeys under my observation has ever done. I throw a similar packet to $G$, at some distance off from her. She goes after it wherever it lands, unwraps it and eats the content.

I place a plate of apples on the top of a high bookcase. E makes no attempt to reach it. G, on the contrary, makes repeated attempts, and tries in every possible way to reach it. She fails, but this is because of the smooth surface and glass doors of the bookcase. A chair is now brought close up to the bookcase. E pays no heed to this, notwithstanding the fact that he has eaten very little. G jumps up on to the back of the chair and tries to spring up to the bookcase to get the apples.

In general $\mathrm{E}$ is dysorientated, remiss and stupid in behaviour. He exhibits no spirit of enterprise, no boldness. The behaviour of $G$, on the contrary, is that of one who moves with definite aims which are in the domain of actual perceptions, one who formulates judgments on the basis of memory and experience, making use of all new contingencies in order to arrive at the object in view.

26th May 1908.-Monkey G (first operation, 8th March). An opening is now made in the cranium on the other side, the usual technique being employed. Save 
for some little hemorrhage, the operation is free of any incident. Electric excitation in front of the ascending frontal convolution at some eonsiderable distance from the area of excitation of the anterior limb has produced:-(1) Movement of the ocular bulbs towards the opposite side. (2) At a point slightly higher up, movement of the head towards the opposite side. (3) Rather more upwards and inwards, more marked movements of the head to the opposite side and backwards. (4) Lower down, under the area for movements of the eyes, movements of the ears, more marked in the ear of the opposite side.

The cortex of that part of the frontal lobe lying in front of this excitable area is now removed.

Aseptic suture.

30th May 1908. - Four days have been allowed to elapse before taking observa. tions. The monkey is now brought into the observation-room, and allowed complete freedom. She hides under a couch, and there moves backwards and forwards from one end to the other, always in the same fashion, in an aimless way, as though unconscious of, and indifferent to, everything and everyone in the room. At length she comes out, sees the handle of the door which leads on to the terrace where her companions are, and makes numerous obstinate attempts to elamber up the door so as to reach the handle and open it. She jumps and clambers up, seizes the handle, sits on it, and soon comes down again to begin the process anew, always going through the same movements in the same rhythmical stereotyped fashion, perhaps ten or twelve times, reminding one of a tie or automatism.

30th May 1908.-Monkey F, male, intact. Smaller than E. He is brought into the examination-room secured by his chain. He is circumspect, he jumps up on the furniture to see what there is around. He makes a tour of inspection of the room. He even jumps up to the window-ledge to spy out the terrace where the other monkeys are, and to them he makes calls. He sees a piece of hread placed on the top edge of the bookcase, and seeks with adequate movements to jump up to get possession of it. After two failures he gives up the attempt.

He takes hold of pieces of bread or apple with rapidity and precision in his movements, getting at them even in difficult positions, unless they are closely guarded. When one makes evident signs of deterring him, he pretends to have abandoned the undertaking, only to seize any moment of distraction on the part of the person who has made signs of intervention, when he draws near with great cunning, seizes the object of his desire, and retreats with much precaution.

His movements in all their variety show a finality quite closely resembling those of an erethistic imbecile, who overcomes many obstacles so long as the quest of food is involved.

At intervals of fifteen minutes small red paper packets are thrown to him, the frst containing a piece of chalk, the second a piece of apple previously immersed in solution of quinine, the third a piece of chalk. He unwraps the first packet, recognises the chalk after testing it, and rejects it. On the second occasion he tastes and tests the bitter apple, examines it several times, retastes it, and ends by throwing it away. On the third trial, though hungry, he remains indifferent, allowing the packet to lie on the ground, and pays no heed to it, although he passes close by it several times.

Monkey E. Comparative experiments with $F$ (male) and $G$ (female). Those three monkeys are placed together. $\mathrm{E}$ and $\mathrm{G}$ are indifferent to one another, and both show indifference towards the third.

$F$ follows them about for a little as though to make friends. On seeing hinself deceived by the indifference of the other two, he jumps up on the window-ledge, peeps out, and makes calls. Even to these calls the other two remain indifferent. The one passes to and fro from one wall to another along the same track. The second remains near a wall, repeatedly touching a small spot with his forefinger, as though desirous of removing it. I throw some cherries and pieces of medlar on the 
ground. F jumps down from the window, and with both hands and feet gathers up as many of these as he can, stuffing his mouth with them with oxtraordinary rapidity. The other two monkeys run forward, but they are torpid and slow in their movements, and munage only to piek up what is left by F. In fact, had I let fall on the ground only four or five cherries, the two mutilated monkeys might possibly have picked up one, but just as possibly none at all, notwithstanding the fact that $\mathbf{F}$ (the normal monkey) was posted on the window at a. greater distance away. I throw some pieces of plaster on the ground. At the noise and sight of these the two run forward, awkwardly as usual, pick up some pieces and stand there, uneertain whether or not to put them in their mouths. F does not even move from the window-ledge, but looks on with an air of euriosity at the behaviour of the other two (rapidity of perception and precision of judgment). He has perceived the form of the pieces of plaster and has noticed that the noise they make is very different from that caused by the falling of fruit on the ground.

What is particularly surprising is the fact that $\mathbf{F}$ no longer cares to associate with $\mathbf{E}$ and $\mathbf{G}$, who have no dealings with him in the way of fondling and caressing, so customary amongst these cebi, especially in eaptivity. It is pretty much the same thing that occurs in the relations between imbeciles and normal beings, leaving aside the sentiment of pity in the normal man, a sentiment followed by that of protection and guardianship, or the utilitarian sentiment, when the imbecile is capable of rendering some serviee, as often happens, provided he is not of a low grade.

If surprised by the somewhat strident sound of the bugle, which is not new to them, the two mutilated monkeys are startled, and crouch up in a corner of the room or near a wall. F, on the other hand, after the first impression of surprise, turns on me and my instrument a gaze that is half curious, half interrogative, then looks hard and long at the fearful attitude of $\mathrm{E}$ and $\mathrm{G}$ with an air that $\mathrm{I}$ can only describe as one of pity for those two unfortunates. If $\mathrm{E}$ and $\mathrm{G}$ meet one another as they stroll around the room, each on his or her own account, they stop, touch one another and remain for a little while near each other, but there is no enthusiasm and none of those ardent manifestations of sexuality such as we have seen in normal couples. There is an occasional but futile attempt at copulation eharacterised by brutality and stupidity. The attempt is abandoned and each remains quite indifferent towards the other. It is noteworthy that $\mathrm{F}$ manifests no enthusiasm for the now stupid $G$.

Summing up briefly the main points in the psychology of monkeys that have suffered mutilation of the frontal lobes, the following results may be regarded as confirmed:-

1. Defect of the perceptive power consisting in an incomplete perception of the objects of the external world, a perception which is lacking in certain of the specific and differential features and accordingly gives rise to defective recognition of objects already known and of new objects having relations of similarity, analogy, etc., with those. So it happens that some objects are mistaken for others that resemble them only in colour or in form. There is also a notable defect in the perceptive power, in the sense that, whilst intact animals let nothing escape them and are constantly poking about and taking stock of anything that exists in any particular environment, mutilated monkeys, on the other hand, allow a number of things and situations to pass unobserved. Further, whilst the normal cebus distinguishes the reality or pretence, let us say, of a threat, from the attitude of the experimenter, the 
mutilated monkey fails completely to recognise the deception, no matter how often the pretended threat is repeated. A joke falls entirely without the perceptive power of a mutilated monkey. Perceptions are limited to the grosser features of the stronger stimuli. Everything else remains outside the perceptive field.

2. Memory, weak and unreliable, becomes enormously reduced, not only for recent but also for old acquisitions. The mutilated monkey does not utilise past experience; he persists always in repeating the same actions, without profiting from the futility of the previous action, and without making any alteration so as to arrive at a determined object. When he allows himself to be tricked many times in succession, as he is made to take a finger instead of a piece of sugar or fruit with which he has been enticed, that indicates that he does not remember the futility of his actions, and does not notice the ridiculous position in which he places himself, although normal cebi are very susceptible to ridicule. When he takes a cherry embittered with quinine and puts it in his mouth without suspicion, although the same experiment was performed the previous day, this would indicate that there is an immediate reflex to impressions, without the intervention of memories of similar or analogous situations. Mutilated monkeys, incapable as they are of utilising all their experience, have consequently lost that biophylactic power which serves as a guide through life, amongst the difficulties interposed by the physical environment and those offered by fellow-beings living therein.

3 . The associative power is greatly reduced. The controlling power of practical experience and acquisitions, such as a normal monkey exhibits in the varied situations of its existence, especially in captivity, which offers opportunity for new adaptations, is absent or withdrawn from the mutilated monkey. Judgment is poor and immediate, often erroneous, owing to the absence of elements of contrast, as when the mutilated monkey stops to pick up pieces of plaster, or straws or dry leaves, or when it stops in front of a spot on the wall, which it persistently touches with the finger (defect of perception and of association, in so far as recognition is made up of associations).

What is more important is the fact that there is a complete absence of any initiative in those animals upon which the operation has been successfully accomplished. The movements performed lack any evident objective. They are the effect of internal impulses which readily become automatisms, or they are reflexes following immediately upon simple impressions, which do not find a field of association or co-ordination, so as to achieve a determined object. The monkey which used to jump on to the window-ledge; to call out to his companions, after the operation jumps on to the ledge again, but does not call out. The sight of the window determines the reflex of the jump, but the purpose is now lacking, for it is no longer represented in the focal point of consciousness.

Another monkey sees the handle of the door and grasps it, but the mental process stops at the sight of the bright colour of the handle; the animal does not attempt to turn it so as to open the door, but sits on it. 
Evidently there are lacking all those other images that are necessary for the determination of a series of movements co-ordinated towards one end. None of the monkeys operated upon has shown the existence of that regulative and inhibitive power which it possessed prior to the operation. Hiding under a couch for a longer or shorter period, watching for the occasion which offers an opportune moment to seize the fruit lying on the table at which someone is sitting, is based on association and detention of the end in view in the focal point of consciousness. Jumping on to the table and first of all getting possession of the stick, so as to be able to seize the fruit with impunity; is the expression of a series of reasonings calculated to achieve the end in view with the least possible risk.

The attitude of dignified indifference displayed by a normal monkey after it has been tricked once or twice is the result of reasonings and sentiments based on memory and association, of which the mutilated monkey is totally incapable. The self-restraint exercised by a normal monkey after being cheated, even when the experimenter offers it fruit of which it is very fond, contrasts markedly with the behaviour of the mutilated monkey, which goes through the same movements time and again, perhaps ten or twenty times, in order to obtain a piece of sugar or fruit-behaviour which reveals an incapacity to utilise past experience. In the latter case there is a defect of that sentiment of self, which we speak of as dignity, for I can hardly qualify it otherwise in the case of a monkey which, though hungry, turns its head and eyes to the other side when offered something which, utilising its imagination and memory, it believes to represent once again that same insidious deception which has already led to its mortification. Indeed, the normal monkey exhibits a true inhibition that is almost human in character. Again, if he controls that instinctive impulse that is aroused by the sight of eatables lying within his reach-fruit, let us say, of which he is very fond-and calmly awaits an opportune moment to snatch it from the hands of the experimenter with an air of satisfaction, that surely is proof not only of inhibition but also of premeditation (imagination). No doubt one can hardly claim that such behaviour is the outcome of a logical process involving a series of notions, such as can only be manifested with the help of language, yet there is certainly a logical process involved, based on memories, representations and imagination, as comprised within the limited circle in which the mental life of the cebus revolves.

4. Mutilation of the frontal lobes gives rise to a no less remarkable modification in the emotional and sentimental manifestations of the life of these monkeys. It is well here to draw a distinction between the primitive emotions - especially that irrational, illogical fear, which the majority of the mutilated monkeys displayed-and sentiments or emotions of a higher order. The desire for satisfaction of hunger, thirst, and other organic needs, persists. The obstinacy displayed by a mutilated monkey in its endeavours to open the closed fist in which, a moment before, it caught a glimpse of a piece of sugar, along with the failure to adopt a change in method after repeated deceptions practised upon it, shows the intensity of the desire. The fact that 
it runs after eatables shows that the immediate perception of food material excites desire and adequate movements, apart from the slowness of these in comparison with normal monkeys and their consequent inferiority in competition with healthy companions. The defect that is most outstanding after mutilation of the frontal lobes consists in the entire absence of the higher sentiments, which represent a complication of the primitive emotions with numerous new factors. The sentiments of friendship, gratitude, jealousy, maternity, protection, dominion, authority, self-esteem, ridicule and, above all, that of sociality, all these disappear after mutilation of the frontal lobes, whilst the primitive emotions remain, sometimes even intensified, but not adapted for the struggle for existence, in which these now inferior animals succumb. In a later chapter we shall return to this question of the emotions and sentiments.

5. In all cases the conduct is seen to be incoherent. This incoherence is due to defect of imagination and of memory, to incapacity to represent and sustain an objective in the focal point of consciousness. The incapacity to support the objective by calling up the requisite correlative images (in obedience to the hedonistic law which governs conduct), in order to formulate judgments and regulate conduct, explains the incoherence of these mutilated animals. The whole psychic tone is lowered. Torpor and stupidity dominate the scene.

Attention must also be directed to the stereotypes and tics. The history of some of these animals provides several examples of tics and stereotypes, such as we often observe in dements and especially in imbeciles and idiots. We know that tics represent a phenomenon of degeneration, in as much as the lower centres are withdrawn from the regulative powers and the discipline of the higher centres. The fact itself that these phenomena are very frequent in imbeciles and idiots indicates a close analogy in their origin, in respect that they evidently depend upon an insufficient evolution of the frontal lobes in the case of imbeciles, and upon destruction of the frontal organs in the animals experimented upon. 


\section{The Excitable Cortical Area of the Frontal Lobe and its Significance}

The phenomena provoked by electric excitation of the frontal lobe are of very obscure origin and not readily explained.

It is first of all to be noted that between the motor zone (ascending frontal convolution) and the excitable area of the pre-Rolandic lobe there is found, in monkeys, a thin, irregular strip which is inexcitable and variable in extent in individual animals. In front of this strip, posterior to the pre-frontal sulcus, or in some cases at the anterior margin of this sulcus; there is an area which is excitable, in the order shortly to be mentioned.

Hitzig ${ }^{1}$ observed combined movements of the head and eyes on exciting the presigmoid area in the dog. As these movements were combined, he believed that he was warranted in inferring that they were not the effect of particular representations ; that, rather, they depended upon excitation of organs of co-ordination. Reasoning from analogy, he thought that there existed on the cortex of the brain an organ for isolated movements of the eyes, quite as independent as the other cortical motor centres.

Ferrier ${ }^{2}$ defined more clearly, in the brain of the monkey, an area which comprises the neighbouring halves of the two posterior thirds of the superior and middle frontal convolutions, excitation of which provokes opening of the eyes, dilatation of the pupil, and deviation of the head and eyes to the opposite side. Only in three cases did he succeed in provoking isolated movements of the ocular bulbs towards the opposite side, by means of electric excitation in the pre-frontal region (see area 12, Fig. 50).

He obtained like movements of the eyes to the opposite side by exciting the areas marked with the numbers 13 and 13' (the two branches of the angular gyrus), with this difference, that the movements of the ocular bulbs were directed upwards on exciting area number 13, and downwards on exciting $13^{\prime}$.

He observed that there generally was contraction of the pupil on exciting these areas (13 and $\left.13^{\prime}\right)$, just as when the eyes are subjected to the influence of light.

Luciani and Tamburini ${ }^{3}$ occasionally observed dilatation instead of contraction of the pupil.

Ferrier attributed little importance to this fact, because he found he could

${ }^{1}$ Hitzig. Untersuchungen über das Gehirn. Berlin, 1904.

${ }^{2}$ Ferrier. The Functions of the Brain. 2nd edit. 1886.

"Luciani and Tamburini. "Su i centri psico-sensorii corticali." Riv. sper. di Fren. 1879. 
lave either contraction or dilatation, according as the animal experimented upon was in a state of sleep, or was awake and had its eyes open.

Horsley and Schäfer ${ }^{1}$ defined more clearly, on the frontal lobe of the monkey, an area situated in front of the pre-frontal sulcus, excitation of which produced deviation of the eyes and head to the opposite side.

Bechterew ${ }^{2}$ succeeded in isolating, in monkeys, an area of the frontal lobe, excitation of which provoked isolated movements of the eyes with deviation of the head. Further, ${ }^{3}$ he found a particular centre for divergence of the eyes, on the foot of the second frontal convolution in the monkey. Excitation at this point produced a slight elevation of the upper eyelid, dilatation of the pupil and divergence of the eyes. He states that as long ago as 1885-1886 he, along with Mislawski, had succeeded in producing a significant dilatation of the pupil and exophthalmos, in dogs, by exciting neighbouring parts of the anterior and posterior branches of the sigmoid gyrus with a weak faradic current. He subsequently obtained similar results in the monkey by exciting the posterior tract of the second frontal convolution-i.e. he obtained dilatation of the pupil associated with a series of other movements such as opening of the eye and inward movements of the third eyelid, precisely as observed on exciting the sympathetic in the neck.

Levinsohn ${ }^{4}$ has observed dilatation of the pupil following excitation of diverse points of the cerebral mantle- the occipital region, the visual sphere and that of the ocular movements-but he denies the existence of any isolated centre for dilatation of the pupil.

Pearson, ${ }^{5}$ in many of his researches upon dogs and cats, obtained dilatation of the pupil, more on the opposite, but also on the homonymous, side, by exciting either the occipital lobe or the frontal region. Section of the sympathetic in the neck, according to this author, weakens but does not suppress dilatation of the pupil.

The areas mentioned and illustrated by these and other authors do not perfectly correspond, but this does not detract from the value of the fact itself, which remains fairly well established.

Stewart, ${ }^{6}$ in the last edition of his work, locates the motor centres of the eyes, in dogs, on the anterior part of the second external convolution which, curving downwards and forwards, embraces and surrounds the sigmoid gyrus. The present writer ${ }^{7}$ has pointed to the posterior limit of this zone

${ }^{1}$ Horsley and Schäfer. "A Record of Experiments upon the Functions of the Cerebral Cortex." Phil. Trans. 1888.

${ }^{2}$ Bechterow. Über die Ergebnisse der Untersuchungen der Erregbarkeit des hintere Abschnittes des Stirnlappens. 1889. Loc. cit.

${ }^{3}$ Bechterew. Die Functionen der Nervencentra. Heft 3. 1911.

"Levinsohn. "Ưber die Beziehungen zwisehen Grosshirnrinde und Pupillen." Zeitschrift f. Augenheilk. 1902.

"Pearson. "On the Dilatation of the Pupil." Collected Papers of Physiolog. Laboratory. 1903.

"Stewart. A Manual of Physiology. 5th edit.

' Bianchi. "Le compensazioni funzionali del mantello cerebrale. La Psichiatria, la Neuropatologia, etc., 1883, and Riv. sper. di Fren. 1882. 
as forming a part of the visual area of the dog, because excitation thereof produces contraction of the eyelids, the protective organ of the eye, whilst destruction of the same area produces amblyopia of the opposite side. This area almost coincides, certainly is in contact, with the area of the facial nerve. The area for dilatation of the pupil is indicated as lying on the anterior branch of the sigmoid gyrus, near the cruciate sulcus.

In the chimpanzee's brain, Sherrington and Grünbaum ${ }^{1}$ found the area for the eyes situated well in front, on the external surface of the frontal lobe and separated from the motor zone by an inexcitable strip, whilst Horsley and Beevor, ${ }^{2}$ experimenting on the orang-outang, found the area of excitation of the ocular muscles in front of the pre-frontal sulcus, almost in the centre of the frontal lobe.

Schäfer, ${ }^{3}$ in his classic work on physiology, shows great competence in dealing with the majority of those questions that have reference to the nervous system, but he dedicates only a few lines to the effects of electric excitation of the cortex upon the pupil. He accepts the results of the experiments of Ferrier and others and states that weak excitations do not give rise to alteration of the pupil unless they fall upon regions of the cortex connected with the movements of the head and eyes. This statement does not altogether agree with the results of the investigations described by the present writer and others.

There are two regions excitation of which produces movements of the head and eyes accompanied by widening of the palpebral fissure on the opposite side-viz. the area just indicated, situated in front of the motor zone, and another area well known as the visual zone and situated in the occipital lobe. It is to be added that excitation of a point of the first temporal convolution which may provoke movements of the head and eyes (when the current is very strong (Bianchi)) also produces contraction of the pupils.

Conjugate movements of the eyes to one side and upwards or downwards, along with deviation of the head, were much better defined by Schäfer and Mott $^{4}$ as depending upon differentiated areas of the zone in question. Their experiments were carried out upon a species of cercopithecus, the brain of which seems better differentiated than that of the macacus. The results obtained by excitation were more marked when this was applied to the area corresponding to the angle of the pre-frontal sulcus. Stimulation of the posterior margin of this area produced rotation and erection or retraction of the opposite ear, sometimes also of the ear of the same side.

Sherrington ${ }^{5}$ holds that this frontal area also exercises an inhibitive

1 Sherrington and Grünbaum. "Observations on the Physiology of the Cerebral Cortex of the Anthropoid Apes." Proc. Roy. Soc. Vol. 1.

${ }^{2}$ Horsley and Beevor. "A Record of the Results obtained by Electric Excitation of the So-called Motor Cortex in the Orang (Simia Satyrus)." Philos. London. 1890.

${ }^{3}$ Schäfer. Text-Book of Physiology. 1900.

${ }^{4}$ Schäfer and Mott. Brain. 1890.

5 Sherrington. "Further Researches on the Movements of the Eye." The Journal of Physiology, 1894-1895. 
action on the antagonistic ocular muscles, and that in any case the results obtained are not to be attributed either to the corresponding zone of the frontal lobe of the opposite side or to that for conjugate movements of the head and eyes situated in the angular gyrus, in the occipital lobe and in the first temporal convolution.

In the course of the experiments the author has carried out at different intervals upon the frontal lobe of monkeys (cebus), he has always noted, in front of the excitable area for the limbs, another excitable area (almost parallel with, but separated from, it by a thin strip no broader than a few

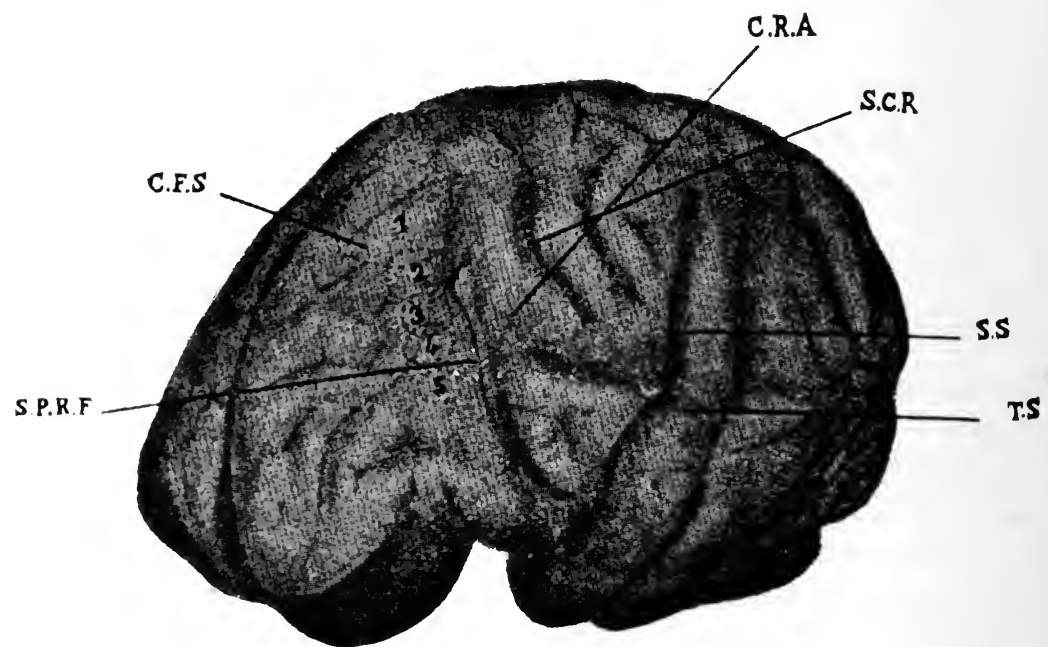

Fig. 56.-Brain of cebus

SPRF. Pre-frontal sulcus.-CFS. Superior frontal convolution.-CRA. Anterior Rolandic convolution.-

SCR. Rolandic fissure.-SS. Fissure of Sylvius. -TS. Superior temporal convolution

millimetres) fairly wide above, much narrower below, the excitable points within this area being disposed in a determined order. These points have not always corresponded exactly in the different animals experimented upon. Differences greater or less in degree have been noted and found to depend upon various circumstances some of which have been ascertained. ${ }^{1}$

The accompanying figure (same as Fig. 51, page 134) shows the disposition and order of these excitable areas and is based upon the more frequent results of experiments upon the higher cebidx, taking into account the total sum of individual differences.

1. Lateral movements of the head; contraction of the muscles of the neck on the opposite side and sometimes of the trunk.

2. Conjugate contraction of the muscles of the neck and of the eyes, on the opposite side.

3. Deviation of the eyes alone, along with dilatation of the pupil.

4. Dilatation of the pupil on the opposite side alone and, if the current

1 L. Bianchi. "Sur la signification de l'aire corticale du lobe frontal." Archives Italiennes de Biologie. Tome LXVI., fase. iii. 1916, 1917. 
is slightly increased in strength, dilatation also of the pupil on the same side, and deviation of the eyes to the opposite side.

5. Movements of the ear on the opposite side (stronger currents give movements also of the ear of the same side but much less pronounced).

Destruction of this area does not produce paralysis of the external ocular muscles nor does it suppress the reflexes of the iris. During the first few days after operation one does observe conjugate deviation of the head and eyes towards the side of the lesion, but if the destruction is not very extensive so that the area of the neck and trunk is spared, this symptom, accompanied as it often is by circus movements, lasts only a few hours, or at most a day. It is only bilateral mutilation of the frontal lobes that produces temporary limitation of the movements of the pupils and of the eyes. This limitation lasts several days so that the physiognomy assumes a certain aspect of immobility, the glance being lifeless, as though the animal were staring into space. It is this aspect, perhaps, which suggested the view expressed by Ferrier.

It thus seems a well-established fact that electric stimulation of certain points of the cortex of the frontal lobe can produce movements of the eyes in various meridians and, separately, dilatation of the pupil. The dilatation of the pupil may be combined with movements of the eyes, or, on the other hand, it may occur independently as the result of excitation of a distinct area, differentiated from that for movements of the eyes.

It is not at all easy to interpret the fact, now well confirmed, that there exist frontal areas where excitation provokes movements of the eyes, pupils and ears, analogous and, so far as comparison goes, similar in every respect to the movements obtained by exciting the respective sensory areas in the occipital lobe, the angular gyrus and the first temporal convolution. It is not to be accounted for by supposing the existence of circles of diffusion of the electric stimulus, because even a slight displacement of the exciting electrode is sufficient to give rise to different results. Further, it has long been proved that isolation of the different zones of excitation by means of a circular incision around each area does not modify the result of the experiment. ${ }^{1}$ It is. also noteworthy that Bechterew, after incising deeply the cerebral hemisphere in front of the visual area of the animal experimented upon, again obtained the same ocular movements and dilatation of the pupil, on exciting the frontal area.

The dilatation of the pupil is not to be attributed to the sympathetic because it persists after section of the sympathetic in the neck, after section of the spinal medulla on the level of the first cervical vertebræ, and even after section through the posterior quadrigeminal bodies. No more is it a question of reflexes, because the only reflex which can reasonably be invoked would be that associated with pain due to stimulation of the meningeal branches of the trigeminal nerve. The reflex hypothesis, however, might acquire a certain degree of probability in any experiments in which very

${ }^{1}$ Bianchi. "Sul significato della eccitazione della corteccia cerebrale." Il Movimento Med. Chir. Naples, 1881. 
strong currents were employed. In this case the law formulated by Franck and Pitres would come into play-i.e. that all currents that are strong enough to invoke epileptic attacks, no matter to what part of the cerebral mantle they are applied, give rise at the same time to dilatation of the pupil.

Our experiments on the other hand show that the current, when of comparatively slight intensity, not only does not provoke epileptic convulsions but does not give rise to dilatation of the pupil, unless the two electrodes fall on a determined small area, whilst electric excitation above this area induces movements of the ocular bulbs, and, below the said area, movements of the ear. Further, the same current, applied farther back, on the precentral gyrus, produces determined movements of the upper limb whilst, if applied farther forward, on the pre-frontal area, properly so called, it produces no results.

It might be argued that what we have to deal with here is the reawakening and representation of visual images projected into space, a sort of hallucination provoked by electric stimulation of that area. This hypothesis, probable as it is, so far as the interpretation of the like symptoms produced by electric excitation of the angular gyrus and occipital lobe in monkeys is concerned, cannot be applied to the frontal lobe because we should then be obliged to admit the existence of a second area of formation, conservation and reproduction of visual images, and this, surely, is markedly in opposition to all that we know to-day of the sensory areas. The two following considerations should be sufficient to dispose of such a theory :-

1. The area of excitation for dilatation of the pupil is very limited. Those authorities who, in their respective publications, have indicated a much larger area on the frontal lobe have certainly employed currents that were too strong.

2. It is difficult to understand the bis in idem. We should have to admit the same thing in the case of the auditory area, since electric excitation in the frontal area produces movements of the ear that do not differ from those provoked by electric excitation of the first temporal convolution, and there is no reasonable explanation for such miniature replicas of the sensory areas in the frontal lobe. The fact remains that, in all cases, destruction of the frontal lobe, including the area for movements of the pupils and the ear, has never produced paresis of the motor apparatus of the respective senses, nor permanent defect of perception or of recognition. No doubt it is true that after removal of the frontal lobes in the monkey and in the dog one does observe visual disorders very similar to those that follow destruction of the occipital lobe. These signs, however, are often fleeting in character, disappearing in the course of a few weeks, so that often there remains no perceptive deficit in the animals operated upon. The theory in question does not withstand criticism.

Bechterew ${ }^{1}$ is inclined to the view that the dilatation of the pupil is the concomitant of an effort at accommodation, coinciding with phenomena dependent upon the sympathetic.

1 Bechterew. Loc. cit. 
Grïnhagen and Bessau found that, even after section of the sympathetic in the neck, excitation of certain points of the cortex still gives rise to dilatation of the pupil. Although the signs, provoked by cortical excitation, resemble those due to excitation of the sympathetic in the neck (dilatation of the pupil, exophthalmos and adduction of the third eyelid), one cannot deny the dilating action of the cortex upon the pupil, independently of that of the sympathetic in the neck.

On the other hand, Braunstein ${ }^{1}$ has maintained that dilatation of the pupil might result solely from an inhibitive action of the cortical excitation upon the nuclei of the ocular motor nerve.

The present writer advances the hypothesis that the movements of the eyes, pupil and ear (possibly there will yet be discovered others connected with the senses of smell and taste, senses that are certainly less intellectual and more inherent in the instincts, and accordingly not so well represented in the frontal lobe) represent motor organs that are closely bound up with another order of psychic processes of a more elevated character, coinciding with the mechanism of attention.

One need not here enlarge upon the findings of psychologists, who, pursuing their inquiries along another path, are often led to draw a distinction between sensory perception that is immediate and involuntary and voluntary perception associated with the mechanism of attention (the apperception of Wundt). In involuntary sensory perception the movement of the head and eyes, or of the eyes alone, as well as the movements of the pupils and eyes, are true reflexes, or movements of perceptive adaptation, that follow immediately upon the modification induced by the stimulus, which reaches its appropriate cortical field by its ordinary centripetal paths. In voluntary perception, on the other hand, a much more complicated mechanism is brought into play. In this the more important elements are : interest in the examination of something which is present or preperception of something which is expected, fixation or immobilisation of the object of perception or preperception in the focal point, re-evocation of the images (image-records) bound up with the object of perception in so far as it reflects past experience, curiosity, preoccupation based upon past experience, etc. When one watches the moviements of a pointer dog as he steps carefully and circumspectly a short distance in front of the sportsman, as he keenly follows up the traces of the prey whose tracks he has now noticed, and sees him come to a dead stop, gazing intently at the game as soon as he has caught a glimpse of it lying hidden in the broken ground or under thick cover, one must be convinced that herein there is involved a much more complex mechanism than the mere erection of the ears and the raising and turning of the head and eyes towards any point from which has come a noise that has broken in upon his repose. The careful curiosity which a monkey displays towards anything new falling under his senses, the minute examination he makes of persons, objects and food stuffs, the study he gives to the overcoming of new 1895.

${ }^{1}$ Braunstein. Zur Lehre von der Innervation der Pupillenbewegungen. Charkow, 
difficulties, along with the keen, mobile, intent glance, full of craft, suspicion and desire, promoted by preperception and accompanied by a surprising mobility of the pupil, are facts that are closely and inseparably bound up with one another.

In all this one cannot pretend to see merely a simple reflex. Rather are we dealing with an attentive perception, with preperception, with new, combined with past, experience, with the evocation of memories, and the representation of similar or analogous images. To my thinking, pupillary movements are inseparable from such a complex of emotions, representations, experiences and will. Just as certain organic phenomena are intimately bound up with a particular representation in the emotions of pleasure and of pain, just as in the minds of some physiologists emotion cannot be conceived as separate or separable from organic phenomena, so the writer believes that the mental processes cannot be separated from certain motor concomitants emanating from that part of the cerebral mantle which is approximate to the field that is more specifically active.

Of course, they are not the same things. Just as the subjective fact of fear and the content of fearful images or precepts are not the same as the tremor, the vascular constriction, the secretion of urine, etc., which are organic phenomena inseparable from the emotion of fear, so pupillary movements first of all, and next ocular movements, are strictly bound up with the movements of thought as it ranges through the infinite tracts from which observation and imagination draw their immense constructive material.

Certain experiments upon men would seem to confirm the existence of a frontal centre for dilatation of the pupil. The writer need only mention the experiments of Haab, ${ }^{1}$ Pilcz, ${ }^{2}$ Bunke ${ }^{3}$ and Bechterew, who attempt to establish mental reflexes of the pupil in relation with voluntary attention, and even claim to have observed voluntary dilatation of the pupil in some cases (Bechterew).

For several years past the writer has been carrying out observations which seem to demonstrate the association of dilatation of the pupil with certain voluntary movements involving the facial nerve. The person observed is made to stand facing the light. The observer, with his fingers, lowers the eyelids and then raises them separately or together. The result is the well-known reflex of constriction of the pupil, less marked when only one, more marked when both eyelids are raised. It is simply a method of reproducing the classic reflex to light. If, instead of bringing down the eyelids with his fingers, the observer asks the subject, still in the same position, to shut his eyes voluntarily and to keep the eyelids tightly and forcibly closed and then to open them quickly and forcibly upon a given signal, the observer can detect, in the act of opening, a marked dilatation of the

${ }^{1}$ Haab. “Der Hirnrindenreflex der Pupille." Arch. f. Augenheilk. 1902.

2 Pilcz. "Uber Ausmerlsamkeitsreflexe der Pupillen." Neurol. Centralb. 1899.

${ }^{3}$ Bunke. Cited by Bechterew.

4 Beehterew. Loc. cit, 
pupils which rapidly contract again to light, but re-dilate immediately afterwards so as to regain the medium diameter which they usually have with that same degree of illumination. The dilatation of the pupil which follows protection from light is not so great as that obtained by voluntary and strong closure of the eyelids or by rapid and forced opening of the eye, this being followed immediately by contraction, due to the action of the light (reflex). ${ }^{1}$

Only in a few individuals does forcible and voluntary closure of the eyelids produce contraction of the pupil but in any case what we have here to deal with are voluntary movements of the pupil associated with voluntary movements of the orbicularis palpebrarum.

Another simple experiment may here be mentioned. The subject is made to face the light as in the previous experiment and the observer stands in front of him with his back to the light. The subject is then told to look at a small object placed at a distance of $30-40$ centimetres so as to fix the diameter of the pupil. The observer next asks him to think of his distant home, still keeping his gaze fixed upon the object. The pupils dilate slightly. There can be no doubt that these movements of dilatation and contraction of the pupil are bound up with mental processes such as attention, imagination and evocation. Naturally, the experimental results are more marked in those subjects who are more intelligent, more willing, more deferent to the investigator, who have at the same time a more lively and ready imagination. Sometimes a certain training is necessary. Individuals with light-coloured are more sensitive than those with very dark irises.

Visual Disturbances in Dogs and Monkeys after Mutilation of the Frontal Lobes

As may be inferred from the preceding experiments, one of the symptoms most frequently observed as the result of extirpation of the frontal lobe consists in disturbance of vision, in the form of amblyopia of the opposite eye, or bilateral hemiopia, the latter condition being more frequent. As

1 Dr A Westphaal ("Über ein bischer nicht beschriebenes Pupillen Phänomen." Neur. Centralb. 1899) states that with energetic contraction of the orbicularis palpebrarum we get contraction (Verengerung) of the pupil of the corresponding eye. The same phenomenon (contraction of the pupil on strong closure of the eyelids and dilatation on reopening) has been observed by Pierre Marie (La Pratique Neurologique) in 41 per cent. of tabetics. I have carried out these experiments in a great number of healthy, or at most neurasthenic, men, and in a high percentage have obtained precisely the opposite result, as already described. In recent times, when examining tabetics, I have found that we do not get inversion of the physiological fact but, rather, a state of pupillary torpor. It is certain that when both eyelids are closed tightly we almost always get a marked dilatation of the pupil on the reopening of the eyelids, a dilatation which is immediately followed by contraction, and then, soon afterwards, by moderate re-dilatation. It is found that voluntary and forcible action of the orbicularis of both eyelids coincides with a stronger dilatation of the pupils. 
to the actual existence of this symptom there is no room for doubt. The debate between Hitzig, who vigorously upheld the existence of visual disturbance after mutilation, and Munk, who denied it with equal obstinacy, was settled by subsequent observations in favour of the existence of this symptom, a view which is supported by the cases previously referred to. It is, however, a relatively fleeting symptom, being rarely prolonged beyond three weeks. It has been found in both dogs and monkeys. The method of investigation preferred by the writer consisted in the application of a sub. palpebral eye-shade of caoutchouc. This caused no annoyance and it allowed of greater certainty in ascertaining the results of the experiments ; not only so, but it entirely removed any doubt as to the actual existence of the symptom. Closing the eye of the animal to be examined by the application of a plaster or small forceps to the eyelids, or by bandaging, causes so much annoyance to the animal that it becomes restless and excited and attempts in every possible way to free itself from the encumbrance. The method employed by the writer is much to be preferred because it permits the animal to remain quiet and also saves time.

If, however, the existence of the symptom is assured, its interpretation is still a matter of some doubt. We can set aside the theory of peripheral blindness, for the simple reason that in most cases we have to deal with bilateral homonymous hemianopsia (both in the monkey and in the dog). I merely mention this theory because Hitzig supported the view that "the commencement of all vision consists in the prompt production of the optic image in the retina. The maintenance of vision consists in the combination of these images with the motor facts and perhaps with another impression in the infracortical centres." 1

How little importance is to be attached to this view can be seen from all that appears in the first chapter of this book. It is not the retinal image which is transmitted but the nervous waves into which the luminous waves are transformed, and these in turn reproduce the luminous image. As for the formation of the concrete image, this results from the combination of various more elementary components. That the cortical visual field comes into play can be argued from the fact that the hemiopia is quite similar to that which results from excision of the occipital lobe.

A peculiar thing is that the phenomenon is more characteristic when only one frontal lobe is excised. Observations upon monkeys mutilated in both frontal lobes show clearly that the mutilated animal sees, often avoids obstacles, and picks up eatables. The only remark to be made here is that if some solid piece of food, like an apple or chestnut, is thrown at some distance from it, the animal runs after it for a certain distance and then stops, either as the result of deficient attention or because it loses sight of the object owing to defect of the attentive accommodation.

I put forward the hypothesis that the bilateral homonymous hemianopsia is a phenomenon of diaschisis. The anatomical and functional relations

1 Hitzig. "Alte und neue Untersuchungen über das Gehirn." Arch fiir. Psichiatrie. Bd. XXXVII. ii. 2, 3. 
between the occipital and frontal lobe are fairly well marked. Attentive vision, which gives rise to a kind of visual apperception in the dog and in the monkey, is a symptom which strikes the eye of any observer. The frontal and occipital lobes normally function in concert. The anatomical relations between the frontal lobe and the occipital lobe of the same side are, as we shall see, numerous, and one can therefore readily understand why it is that a violent lesion of the frontal lobe of one side brings about functional interdiction of the occipital lobe of the same side, with the same symptoms as are observed after removal of the occipital lobe. Vision becomes gradually restored in the mutilated animals and, after a few weeks, all sign of visual disturbance has disappeared. When, however, both frontal lobes are removed the glance of the animal never again acquires that vivacity, that malicious, crafty and suspicious mobility which is so characteristic of the monkey. Those who are familiar with asylum inmates, gross imbeciles and young dements, will more readily appreciate the resemblance between the looks and attitudes of such patients and those of mutilated monkeys. Anyone who compares the physiognomy and appearance of a young girl, before the onset of an illness which has left her demented, with her general appearance and attitude and her lifeless and vacant glance after the dementia has impressed itself upon her, cannot help being struck by the marked analogy between the two states in the two diverse conditions of existence. That this is due not only to interdicted movement of images, to suppressed power of evocation, to loss of interest owing to defect of association, but also to diminished capacity of attention, that complex movement of the eyes and pupils so intimately connected therewith, seems to the writer to permit of no doubt. The destruction of the frontal cortical centres for those movements of the eyes and pupils which are so strictly bound up with perception, evocation, judgments and the relative emotional states, suppresses the organic basis of those phenomena. As already mentioned, the ocular and pupillary movements represented in the frontal lobe stand in the same relationship to apperception as vascular constriction and trembling to fear. We infer the severe mental deficit from the careless and indifferent attitude of the monkey and from the wan, expressionless look, void of that vital spark which we observe in intelligent dogs and in normal monkeys, whose psychology is still far from being completely reconstructed. Over these attitudes we have presiding the function of the frontal lobe with its own immediate centres which are the motor organs of its summarising function, located in definite order one above the other on the frontal zone, these conferring upon the higher intellectual processes that motor expression that is evidenced by mobility of the glance, the pupil and the head. 


\section{CHAPTER VII}

\section{Associative Paths between the Frontal Lobe and the Sensory Area of the Cortex}

There are to be found in the frontal lobe long bundles of association fibres which connect it with neighbouring and remote cortical areas of the brain, and short paths connecting different parts of the frontal lobe itself. The relations between the frontal region and the motor zone are especially very numerous.

The intra-frontal associative bundles are not so distinct as those in the occipital lobe (vide Chapter I.) but at the same time are capable of being traced. Brief mention may here be made of the fibres which run from the internal aspect of the frontal lobe to the orbital aspect and also to the external surface; the bundles of fibres which secure reciprocal communications between the orbital convolutions; other fibres running from the superior and external surface in an oblique but almost vertical direction to connect the respective convolutions with the internal surface; others, again, proceeding from the anterior perforated substance to distribute themselves in the orbital portions of the first and third convolutions. It is unnecessary here to dwell at length upon these connections.

The relations of the frontal lobe with the other parts of the cerebral mantle have been, in the main, confirmed by all anatomists and neurologists.

Van Gehuchten ${ }^{1}$ gives a brief description, along with a clear figure, of three single bundles which would connect the frontal lobe with the other cortical areas :-

1. The superior longitudinal bundle formed of fibres which unite the grey substance of the frontal lobe with that of the occipital and temporal lobes.

2. The cingulum formed of fibres running antero-posteriorly and following the direction of the convolution of the corpus callosum, joining the frontal with the temporal lobe.

3. The uncinate or unciform bundle which unites the inferior frontal convolution (grey substance) with the tip of the temporal lobe.

Dejerine ${ }^{2}$ describes four association-bundles composed of long fibres:

(a) The cingulum is considered as an association-bundle of the rhinencephalon. It follows the curve of the limbic lobe and composes the great mass of its white substance (first and second limbic convolutions). In its course it exchanges numerous fibres with the superior frontal convolution, the parietal lobule, the precuneus, the cuneus, the lingual lobule, the fusiform

1 Van Gehuchten. Système nerveux de l'homme. Louvain, 1906.

${ }^{2}$ Dejerine. Anatomie des centres nerveux. Paris, 1895. 
lobule and the temporal pole. It is generally admitted that this bundle establishes relations between the frontal lobe and anterior rhinencephalon on the one hand (Van Gehuchten, Dejerine, Beevor) and the median part of the limbic convolution, perhaps also the hippocampus, besides the lingual and fusiform lobules on the other hand.

According to Monakow ${ }^{1}$ the cingulum contains fibres of different lengths. The short fibres establish relations between neighbouring cortical fields, whilst the long fibres connect the frontal lobe with the occipital and temporal lobes.

(b) The uncinate bundle is composed of relatively short fibres which unite the temporal pole with the orbital face of the frontal lobe, passing underneath and behind the insula in its anterior segment.

The deeper and more anterior fibres of this bundle reach the third frontal convolution and the orbital part of the other two frontal convolutions.

(c) The superior longitudinal or arcuate bundle runs to the outside of the corona radiata (whilst the cingulum runs to the inside), corresponding to the operculum of the Sylvian fissure. In vertico-transverse sections one can readily observe that this bundle describes a very open curve downwards. The more superficial and inferior fibres pass close by the putamen to enter into the slaustrum, whilst others contribute to the formation of the anterosuperior part of the internal capsule (?). Its fibres spread themselves out into the anterior and posterior parts of the first and second temporal convolutions, the supra-marginal gyrus, the angular gyrus and the external surface of the occipital lobe. This bundle derives its origin from the whole convexity of the frontal lobe.*

(d) The occipito-frontal bundle. This bundle was identified by Forel and Onufrowicz with the arcuate or superior longitudinal bundle, just described. Dejerine looks upon it as a distinct bundle situated to the inside of the corona radiata. It runs somewhat above and a little to the outside of the caudate nucleus and is separated from the lateral ventricle by means of the sub-ependymal grey substance. The fibres composing it come from the white substance of the frontal lobe. From the upper and outer border of the caudate nucleus, where they become collected in a bundle, they proceed to pass between the caudate nucleus and the upper border of the putamen.

This bundle, according to Dejerine, describes an open curve forwards and downwards, is well defined at the level of the head and trunk of the caudate nucleus, curves downwards at a point corresponding to the ventricular carrefour and spreads out in fanlike fashion to form the tapetum. Dejerine thinks it not improbable that some of the fibres of this bundle may penetrate the sub-ependymal grey substance.

\section{Monakow. Gehirnkrankheiten. 1905.}

* According to Schnopfhagen the fibres of the arcuate bundle take origin in the frontal lobe of the opposite side, and would reach the position described after erossing through the corpus callosum. They would thus serve as a path of association of the frontal lobe of one hemisphere with the temporal and occipital lobes of the opposite hemisphere. This view has not been confirmed. 
The fibres composing the bundle in question derive their origin from all the cortex of the frontal lobe, not excluding the orbital surface. They intersect at various angles with the fibres of the corpus callosum and also with those of the corona radiata, and, after having formed the tapetum, they spread themselves out in the grey substance of the convolutions of the occipital and temporal lobes.

This bundle (and the same applies to the other great association paths, the cingulum and the arcuate bundle) is not composed solely of fibres running from the frontal lobe to the occipito-temporal cortex. It also contains fibres which come from the occipital and temporal lobes to the frontal lobe. Lesions both of the occipital and of the temporal lobes produce degeneration of the occipito-frontal and temporo-frontal fibres.

Muratow and Monakow, on the other hand, assert that the fronto-occipital bundle exhibits partial degeneration, in the dog, after extirpation of the motor zone and also after removal of the occipital and temporal lobes. These findings were confirmed, as will be shown, by the writer's observations, and discredit the view that the tapetum is composed of fibres of the corpus callosum.

Section of the corpus callosum (Muratow ${ }^{1}$ ) does not give rise to degeneration of the tapetum, whilst lesions of the frontal lobe of the same side, without doing any injury to the corpus callosum, induce degeneration of the fibres which pass through the tapetum.

The occipito-frontal bundle, which Dejerine thinks presents a fairly uniform picture, has been divided by German authors, such as Anton and Zingerle, into separate bundles. One of these has been specially distinguished as the medial longitudinal bundle of Anton ${ }^{2}$ (sub-callosal of Muratow). The short fibres of this bundle unite the frontal cortical areas with the head of the caudate nucleus, whilst the long fibres reach the tapetum.

Laterally and external to this bundle is found the true fronto-occipital fasciculus of Dejerine.

Monakow's ${ }^{3}$ investigations have induced him to accept the distinction of the fronto-occipital bundle into three well-differentiated fasciculi. One corresponds to the medial longitudinal bundle of Anton, a second to the fronto-occipital bundle of Dejerine and the reticulo-cortical bundle of Obersteiner and Redlich, whilst the third, situated laterally and below the other two, appears to have a more immediate relation with the Rolandic zone, although it was found by Monakow as a separate bundle.

We pass now to the question whether or not the frontal lobe possesses projection fibres and whether these, if they exist, pass through the internal capsule (anterior segment).

Nearly all authorities are in agreement on this point.

1 "Sekund. Degener, nach Durchschneid d. Balkens." Neurol. Centralb. 1892.

2 Anton and Zingerle. "Bau, Leistung der Menschlichen Stirnhirns. Festschrift des Gräzer Universität. 1901.

${ }^{3}$ Monakow. Gehirnkrankheiten. 1905. Vol. i. 
Monakow states that the anterior tract of the internal capsule contains projection fibres from the frontal lobe, so that all the convolutions of the cerebral mantle, including those of the frontal lobe, would be represented in the internal capsule by means of fibres of the respective corona radiata.

Through the anterior tract of the internal capsule there would pass fibres coming more especially from the corona radiata of the first and second frontal convolutions - to be exact, from the basal portions or feet of these convolutions - and these would lead directly to the cerebral peduncle, where they would come to form a part of the mesial tract. Other analogous fibres would proceed to the pons (fronto-pontine paths). A third bundle would consist of fibres leaving the foot of the third frontal and the operculum of the ascending frontal convolution, where are found the cortical areas of innervation of the face, tongue, pharynx and larynx, to pass down in the neighbourhood of the genu of the internal capsule.

Edinger ${ }^{1}$ mentions, although he does not describe, a bundle which he calls the tractus fronto-pontine zur Brücke, which would be analogous to the temporo-pontine, Rolandic-pontine, etc., tracts. He holds that the function of the corona radiata is to connect the individual apparatus of the Neencephalon with the various apparatus of the Paloencephalon. The figures shown by this distinguished anatomist demonstrate clearly what he means. He several times makes use of the phrase : Die Bahnen aus dem Stirnhirn zur Brïcke.

Dejerine, ${ }^{2}$ in the second volume of his Anatomy, repeats the notion that the constitution of the anterior segment of the internal capsule includes fibres which come from the orbital and external surfaces of the frontal lobe. These fibres, he thinks, do not all proceed directly to the anterior segment of the capsule. A small number of them run with the fronto-occipital bundle and surround the head of the caudate nucleus. They form a mixed bundle composed of both projection and association fibres, which degenerate in consequence of destructive lesions of the frontal lobe.

Of the clinical cases reported by Dejerine that of Moriceau is very interesting, but it is to be remarked that the frontal foci which had there caused degeneration in the anterior segment of the capsule were of old standing and, indeed, dated back ten years previously. Schveigoffer's case of partial degeneration of the anterior segment of the capsule is not quite reliable because the focus occupied the Rolandic operculum, and it is generally agreed that the fibres in this area are, in the main, projection fibres.

Another bundle of fibres of the anterior segment of the capsule would appear to be derived from the frontal convolutions and to terminate in the mesial and lateral nuclei of the optic thalamus. The frontal lobe would thus come into relation with the nuclei of the optic thalamus through the intermediary of the internal capsule.

${ }^{1}$ Edinger. Einführung in die Lehre vom Bau und den Verrichtungen Nervensystems. 1909.

2 Dejerine. Anatomie des centres nerveux. 1901. 
N. Schukowski ${ }^{1}$ states that connections are set up between the frontal lobe and the anterior part of the optic thalamus by means of fibres which course through the anterior segment of the capsule and spread out into the stratum reticulatum thalami. This author is convinced that similar connections exist between the frontal lobe, the stratum intermedium and the substantia nigra by means of fibres which pass through the genu of the internal capsule and between the frontal lobe, the internal segment of the cerebral peduncle, and the nuclei of the pons, through the intermediary of the fronto-pontine bundle.

The frontal cortico-pontine bundle, according to Flechsig, belongs to the third system of sensory bundles of the internal capsule, and goes to form the inner third of the cerebral peduncle. This bundle would terminate, in great measure, in the grey substance of the pons. According to this author, however, these fibres do not come from the frontal pole- that is to say, from the true anterior associative centre-but from those areas of the frontal convolutions which form a part of his tactile zone-i.e. from the feet of the frontal convolutions (the intermediate motor zone), in which he, like others, has observed very large pyramidal cells which give origin to the fibres of the fronto-protuberant bundle, and more especially from the feet of the Rolandic convolutions. The fibres of the bundle in question degenerate as the result of destructive foci in this region. They would, therefore, be the motor paths for the muscles of the neck, trunk, face, larynx, etc., and they, by means of the thalamic corona radiata, would unite the cortex to the thalamus, the superior cerebellar peduncles, the island of Reil and the reticulate substance. This bundle would correspond to a large extent with the geniculate bundle.

Quensel ${ }^{2}$ confirms the relations of the frontal lobe with the anterior segment of the internal capsule and with determined nuclei of the optic thalamus, but gives a different interpretation from Dejerine of the course and relations of the so-called fronto-occipital bundle.

From the résumé of his work which he gave to the Congress of German Psychiatrists and Neurologists in Jena in 1909, one cannot form any accurate judgment as to how far his thesis has been demonstrated. The author speaks of the feet of the frontal convolutions and of the operculum of the ascending frontal, which, from physiological and functional points of view, are quite distinct from the frontal and pre-frontal regions with which we are here dealing particularly. The bundle in question has been described as an intellectual or psychic bundle, but according to Dejerine ${ }^{3}$ and Flechsig it has nothing to do with the psychic functions. The fibres which degenerate come from the motor zone, the para-Rolandic lobule and the immediately neighbouring parts of the frontal lobe (evolutionary motor zone).

${ }^{1}$ N. Schukowski. "Ưber anatomische Verbindungen des Frontallappen." Neurologisches Centralblatt. 1897.

2 Quensel. Neurol. Centralb. 1909.

${ }^{3}$ Dejerine. Sur l'origine corticale et le trajet intracérébral des fibres de l'étage inférieur ou pied du pédoncle cérébral. 1894. 
On the question of the relations of the frontal lobe with the optic thalamus there still remains a good deal of doubt, and certainly one has to admit that up to the present there has been no clear demonstration of the interconnections.

The relations between the frontal lobes and the caudate nucleus are more definite.

H. Sachs ${ }^{1}$ describes a fasciculus nuclei caudati which unites the corpus striatum with the cerebral cortex and is composed of fibres derived from the frontal lobe, the parietal lobe and the insula.

Marinesco, ${ }^{2}$ applying Marchi's method to the brains of monkeys and dogs which had suffered mutilation of the frontal lobes thirteen to twenty days previously, found bundles containing degenerate fibres in the caudate nucleus in every case.

These fibres run in the anterior segment of the internal capsule which they then leave in order to penetrate the caudate nucleus. Anatomical relations between the frontal lobe and the corpus striatum have been noted by Kölliker and Meynert. It is to Ramon y Cajal, ${ }^{3}$ however, that we are indebted for the first positive histological proof of the existence of these relations. He writes as follows:- " From all regions of the frontal lobe situated in front and above the head of the corpus striatum there descend nervous fibres which penetrate the corpus striatum and unite there into small bundles. These fibres are axis-cylinders coming from the small and large pyramidal and also the polymorphous cells. . . ."

In this connection the present writer has carried out two series of researches. The first dates back to 1897 . The brains investigated were those of monkeys which had been operated upon many months before. In one case in particular the operation took place eleven months before the autopsy. In all cases the operation consisted of removal of both frontal lobes. ${ }^{4}$ The second series is of more recent date. The brains were those of monkeys operated upon eighteen to twenty days previously. Marchi's method of investigation was employed. ${ }^{5}$

In the first series sure signs of degeneration were found:

(1) In the fronto-occipital bundle above the ependymal substance, just over the head and body of the caudate nucleus, degenerate fibres running in an antero-posterior direction. In this region the degenerate fibres, readily recognisable by Weigert's method, are intersected at an acute or right angle by the fibres of the corpus callosum and the fibres of the corona radiata of the overlying convolutions.

In one of the preparations (Fig. 57) one can observe a pale yellowish grey

${ }^{1}$ H. Sachs. Vorträge über den Bau und die Thätigkeit des Grosshirns. Breslau, 1893.

2 Marinesco. Société de Biologie. Février, 1895.

${ }^{3}$ Cajal. La Cellula. 1892.

${ }^{4}$ Bianchi, L. "Le degenerazioni discendenti endoemisferiche consecutive alle ablazioni dei lobi frontali. Ann. di Nev. 1897.

${ }^{5}$ International Congress of Medicine, Psychiatric Section. London, 1913. 
area at the base of the corona radiata of the first frontal just where it joins with the second frontal convolution, $F o$. A little below this one can see normal bundles of fibres, coloured blue, just at the commencement of the anterior segment of the eapsule. Neither the anterior nor the posterior segment shows any trace of degeneration.

(2) Still further below and more externally, at a point just above and internal to the upper angle of the fissure of Sylvius, there is found a second yellowish area containing a large number of degenerate fibres which belong to the arcuate bundle. These fibres are proceeding, in the main, to the outermost capsule, the claustrum, and some to the putamen of the lenticular

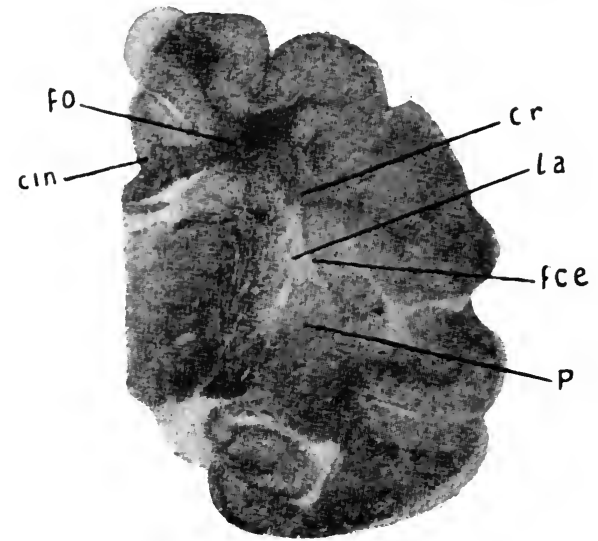

Fig. 57

Fo. Fronto-occipital bundle.-cin. Cingulum, - cr. Corona radiata with marked degeneration.-la. lacuna of disintegration.-Fce. bundle of the ontermost capsule. $-p$. Putamen

nucleus. Underneath is found an empty space closely resembling a lacuna of disintegration, triangular in shape, with the longest line or base looking towards the posterior segment of the internal capsule. It is a true lacuna and it comprises the upper part of the putamen and part of the second segment of the lenticular nucleus. The external wall of this lacuna is composed of the bundle of fibres of the outermost capsule which establishes a connection between the first temporal convolution and the frontal operculum, following the bend around the upper angle of the fissure of Sylvius where one can see distinctly the outlines of the insula, $i$ (Fig. 58). No other lesion is visible at this level except a certain number of degenerate fibres in the cingulum (cin).

The injury was bilateral, and similar lesions of the putamen $(p)$ were found at the same level in the other hemisphere, only much more limited. Degeneration is very marked near the upper angle of the lacuna, outside the anterior segment of the internal capsule, at the highest point of the curve described by the corona radiata of the frontal operculum as it meets with that of the frontal convolutions ( $t a$, Fig. 58).

Another area of degeneration is visible in the fronto-occipital bundle 
$(F o)$ above the head of the caudate nucleus. The anterior segment of the internal capsule $(c i a)$ is quite intact. The same degenerations are present

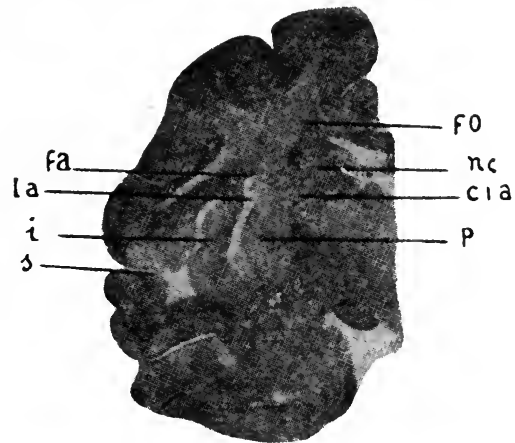

Fig. 58

Fo. Occipital frontal bundle.-Fa. Arcuate bundle.-nc. Candate nucleus.$t a$. Area of greater degeneration.- $i$. Insula. $-s$. Fissure of Sylvius.cic. Anterior segment of capsule. $-p$. Putamen

in the cingulum. In sections posterior to those represented in Figures 57 and 58, a large number of degenerate fibres are found in the tapetum.

In the section represented by Figure 59, taken from the brain of a cebus killed six months after the mutilation and stained by Weigert's method, one can distinctly observe an area of degeneration, intersected by normal fibres

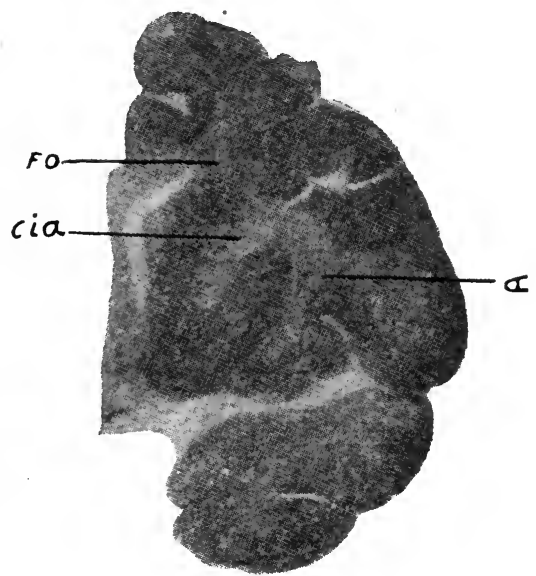

Fig. 59

Fo. Fronto-occipital bundle.-cia. Anterior segment of internal capsule.a. Claustrum

of the corpus callosum, which corresponds with the fronto-occipital bundle $\left(F^{\prime} o\right)$. There is also another area of more marked degeneration in the situation of the arcuate bundle, the fibres of which are proceeding to the claustrum $(a)$, the outermost capsule and the external capsule. In this section, as in others at the same level, stained by carmine, the anterior segment of the internal capsule is seen to be without trace of degeneration.

Investigation by Marchi's method confirmed these findings. 
Degeneration of the cingulum has not been a frequent finding. It was found to be more marked in the case of one monkey in which damage had been done on the internal surface of the frontal lobe, not only to the superior frontal convolution but also to a part of the limbic convolution in front of the genu of the corpus callosum.

Degeneration of the superior longitudinal or arcuate bundle and of the fronto-occipital bundle has been a constant finding in the case of brains in which the destruction of the frontal lobes involved a large extent of the external surface in front of the so-called intermediate motor zone.

In this second series of observations one readily observes the degeneration limited to the two quite distinct bundles above mentioned. One is found

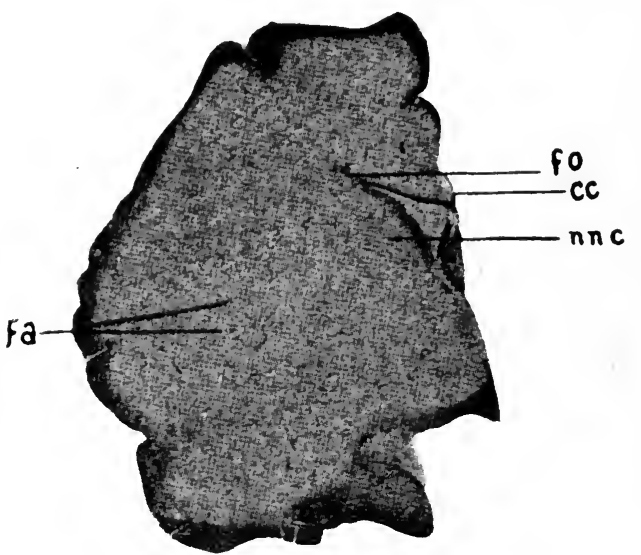

Fig. 60

Fo. Fronto-occipital bundle.-cc. Corpus callosum.-nnc. Candate nucleus.$F a$. Fibres of arcuate bundle which penetrate the claustrum and runs in the external and ontermost capsules

above and to the outside of the angle of the lateral ventricle, hence above the head of the caudate nucleus. This bundle is represented by a dark area about $2 \mathrm{~mm}$. high and $1 \mathrm{~mm}$. broad (Fo, Fig. 60 ), which is seen under the lower powers of the microscope to furnish some fibres which penetrate the head of the caudate nucleus, whilst the superior part of the bundle runs above the head of the nucleus.

The other bundle is found in the course of the external capsule and the outermost capsule and also occupies the grey substance of the claustrum ( $F a$, Figs. 60 and 61$)$. As far as the level indicated in Figure 61 by the line cia, the anterior segment of the internal capsule shows no degenerate fibres.

Figure 61 reproduces a section from the frontal lobe of a cebus which underwent mutilation of the external surface of the frontal lobe in front of the intermediate motor zone. It shows an area of degeneration situated in the fronto-occipital bundle $(F o)$ and a line of degeneration along the length of the claustrum and involving also a part of the external capsule. No degenerative fibres are found in the cingulum $(c)$ (the internal surface of the hemisphere had been spared). Under high magnification one can observe 
small offshoots from $F_{o}$ in the head of the caudate nucleus $(n c)$ at its upper and outer corner. The anterior segment of the internal capsule (cia) shows no trace of degeneration.

Figure 62, which is an exact photographic reproduction, furnishes further confirmation of the fact that the two areas of degeneration mentioned are

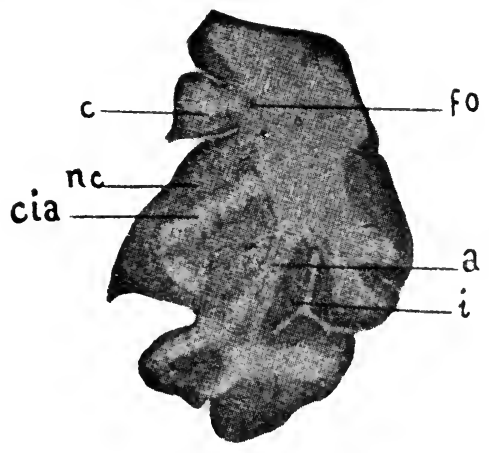

Fig. 61

Fo. Area of degeneration of fronto-occipital bundle. $-c$. Cingulum. $-n c$. Caudate nucleus.-cia. Internal capsule, anterior segment.- $a$. Area of degeneration in the claustrum. $-i$. Insula

the only ones which can be detected by this method of investigation (Fig. $62, F_{o}$ and $F a$ ).

From these researches it seems to the writer to be fairly well established that the frontal lobe, or rather, that part of it which lies in front of the

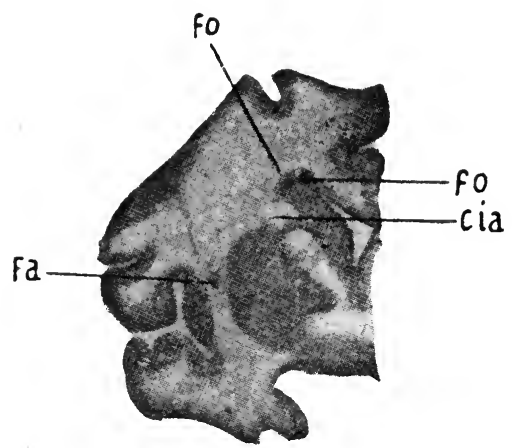

Fig. 62

Fo Fo. Area of fronto-occipital bundle--ciu. Anterior segment of interna capsule. $-F$ c . Area of degeneration of arcuate bundle in site of claustrum

intermediate motor one, has no direct relations with the optic thalamus through the internal capsule. It evidently has distinct relations with the head of the caudate nucleus and it is fairly clear that it has some relations with the putamen of the lenticular nucleus and with the claustrum. Besides being associated with the motor zone it is especially associated with the whole sensory mantle by means of the fibres of the fronto-occipital and arcuate bundles. If, as Dejerine and Monakow claim to have demonstrated, these 
same bundles contain fibres which degenerate as the result of occipital and temporal mutilations, this would afford proof of the reciprocal associative relations that subsist between the frontal lobe and the sensory areas, more especially the tactile, the visual and the auditory. The cingulum and at least a part of the uncinate bundle would establish communications between the frontal lobe, the limbic lobe, and the sphenoidal pole, including the uncus, which is probably a centre of taste. It would thus appear that the frontal lobe has acquired paths of communication (associative) with all the sensory fields of the mantle.

The exchange of fibres is reciprocal, as already mentioned.

The writer's investigations do not confirm the existence of fronto-thalamic fibres, and in the absence of proof he cannot associate himself with those who admit a direct relation with the optic thalamus, except in so far as he has been able to draw certain inferences from preparations taken from a brain in which the lesion had fallen too far behind and had involved the intermediate motor area along with a portion of the anterior Rolandic convolution, in which case he found a small degenerate bundle in the internal capsule.

We do not yet know very much of the function of the nuclei of the corpus striatum, and rather than formulate any hypothesis it is preferable to wait until new experimental and histological studies clear up the nature of these relations.

The relations supposed to exist between the frontal and pre-frontal area of the frontal lobe, with the pons, the cerebral peduncle, and the cerebellum, must still remain fields open to investigation. For the moment, everything tends to show that the degenerations which form the basis of these supposed relations were the effect of lesions of the motor zone or were of too old standing.

Anatomico-histological findings thus furnish us with another nonnegligible proof of the functions of the frontal lobes. The associative paths that unite the sensory cortex with the frontal lobes have a twofold office : first, that of informing the higher consciousness of the modifications of the kinæsthesis and of all the new percepts acquired by the personality by means of the sensory centres ; second, that of permitting the higher consciousness to select and recall those images registered in the sensory cortex that, in the vicissitudes of mental and physical life, are reputed necessary for the purposes of the struggle for existence and for higher reasoning which, directly or indirectly, displays biophylactic virtues and determines particular lines of conduct.

That the function is of this nature can be inferred not only from experimental inquiries but also from reliable clinical observations. Its more intimate mechanism will be made clearer in the following chapters upon intelligence, emotions and consciousness. 


\section{CHAPTER VIII}

\section{Intelligence and Language}

IT is not the object of this work to treat of intelligence after the manner of the philosophers. It is altogether beyond the province of the biologist to push his inquiries into fields of abstract thought. Not by any means that the writer would desire to detract from the value of the monumental, though not wholly eurithmic edifice raised by philosophy to intellect, throughout all the evolutionary phases of philosophic thought from the time of the earliest civilisation until to-day. To others the task of dedicating themselves to that labour, which no doubt has often been the happy creator of mental formulæ; a labour to which, from the earliest dawn of civilisation, the human spirit, represented by the intellectual élite of every epoch, embracing the culture of the time and the genius of each stock, has devoted all its energies in an anxious inquiry into the causes, origin and nature of thought and of the ego. In this field the philosopher is quite free to exercise all the powers of fantasy and of logic, either creative or dialectic. He may follow up the principle of causality and penetrate deeply into the mystic regions of theological metaphysics. He is free to invoke the force that is intrinsic in matter, or, if he care, a creative being. His dominion is the immensity of the unknown, into the obscurity of which he, fervent upholder as he may be of a particular doctrine or system, sheds the light of his intellect, sometimes genial and creative, as he confronts and penetrates the unsolved problems of existence and of man's history.

Comparative psychology with the abundant harvest it has gathered by following up the evolution of mind from infancy to maturity, from the savage to the modern civilised man ; the facts furnished by the process of mental dissolution, caused by the most varied morbid processes in the brain, both localised and diffuse; the rich store of knowledge culled from the study of the evolutionary arrests of the human brain as contrasted with the various phases of normal evolution; all the conquests which biology, and more especially neurology, have won and brought to bear upon the dispute between the ancient dominion of speculation and the inquiring spirit which seeks to penetrate nature, making use of, and perfecting in the course of inquiry, the very instruments (senses and brain) which Nature herself elaborates; all these warrant us in remaining within the domain of scientific positivism, drawing from objective observation those conclusions which best reflect reality, as it is perceived.

It is certainly true, as already mentioned in Chapter IV., that comparative psychology has to-day taken its place of honour amongst our studies and conclusions. 
Some naturalists, caught in the throes of religious sentiment, are prepared to recognise a species of mind in animals, but interpose between the animal and the human mind an insurmountable barrier-the reflex nature of the manifestations of mental life and the absence of language in the higher mammals, highly evolved though they be.

Language is necessary for the more complex and longer reasoning processes, but not for the more immediate, simple and short reasonings. It is difficult to understand why any unprejudiced observer should find himself in difficulty before such a barrier. The idea expressed by Bain ${ }^{1}$ is very applicable to the scale (phylogenetic) of mental life. "We have every reason for believing," he writes, "that there is, in company with all our mental processes, an unbroken material succession."

The naturalist who is so constituted in mind as to be attracted into fields of metaphysical thought must lose the psychic attitude that is characteristic of the biologist. Metaphysical abstraction, the product of fantasy and of logic, is far removed from objective reality and from what appears as such to our senses, and in any case it is insufficient to furnish us with elements that will serve as a key to the positive cognitions. The biologist cannot go beyond abstractions which, being founded on connected series of observations and deductions, still maintain contact with facts and retain their fundamental character of objectivity.

Hence it comes that we have formulated a conception of life, intelligence, morality and sociality that is quite independent of any abstract notions, arrived at solely by a process of reasoning.

As biologists, e.g., we do not feel called upon to examine the form or mode of reasoning in order to draw metaphysical conclusions or lay down the norms that regulate the reasoning process (logic). To us the important points in the argument, points which should be clearly set forth, are as follows:-(1) That the intellect is in continuous evolution; (2) that this is proportionate to the development and perfecting of the senses, in so far as these furnish an ever-increasing number of cognitions which are elements capable of being utilised for new and more complex mental formations; (3) that the perfecting of the senses, which supply the positive cognitions, coincides with the evolution of the brain ; (4) that it follows that intellect is variable and not exempt from the law of time and quantity ; (5) that the mental syntheses themselves have likewise a variable value, according to the greater or lesser number of elements which go to make up the mental constructions, the latter being dependent upon the power of combination and association (conjunctive imagination), which in turn is conditioned by the faculty of reproducing rapidly and faithfully, by unconscious processes, the mnemonic traces of percepts, judgments, psychic complexes and past experiences ; (6) that this associative process is based, as daily becomes more evident, on the number of evolved cells in the mantle, and hence on the multiple paths of association (myelinised fibres and neurofibrils), the number of which far surpasses even the most imaginative limits of calculation.

${ }^{1}$ Bain. Mind and Body. 1873. 
The biologist has no need to fall back upon dialectics in order to support his contention. He does not set out with the express object of bolstering up a preconception with arguments which, however skilful they be, are but excogitations intended to demonstrate the " necessary truth" or " the only possible solution "; nor is he obliged to distort facts in order to make them fit in with preconceived notions. Much less can the biologist be guided by motives of an affective order which, generally speaking, are of a religious nature. As E. Rignano ${ }^{1}$ says, it is always the religious element which is at the bottom of every metaphysical speculation whether we are conscious of it or not. This assertion may be rather absolute, but nevertheless the fact remains that the affective element predisposes to affective reasonings and to a variety of extra-logical deductions (Ribot $\left.{ }^{2}\right)$-i.e. to theses or to preconceptions which do not correspond with objective reality. We find examples of this in the attitude of some religious naturalists, especially in Germany, in their opposition to the Darwinian doctrines (Ecker).

The metaphysical attributes of the Divinity are, as James ${ }^{3}$ says, a pure verbalism or a professionalism, substituted for the sensible images of the real facts of life.

All preconceived dogmatisms of affective origin lie quite without the fields of observation which give scope to the work and thought of the biologist. The dignity of experimental science is in no way lessened by the fact that its teachings alter from time to time and that new doctrines are substituted for old. The doctrines are renewed like molecules within the cells, like cells with the organism. As the cell and the organism evolve and become perfected so the science of life is evolved and perfected, maintaining its individuality all along because it always has this characteristic - that it is pivoted on the rigorous investigation of facts, which enlarge the field of inquiry and involve the modification and perfecting of methods and technique. On the other hand, it is to be pointed out that it is not only biological doctrines which change. New discoveries may demolish a doctrine, but this generally represents a historical point in the evolution of the knowledge of life. Sometimes they represent polemic fields which serve as points of departure for new inquiries by means of which we arrive at higher levels of knowledge. At bottom, we strive to define facts and phenomena, to discover their genesis, to trace their relations with other facts and phenomena, to establish ever more intimate relations with all the other natural sciences, with Physics, Chemistry, Palæontology, Zoology, and especially Comparative Anatomy and Comparative Psychology. The biologist-and consequently the psychologist, who is concerned with the higher manifestations of life-does not pursue pre-established aims or subjective aspirations, and much less does he feel constrained to establish a prime cause or an intelligent, willing author of the creation of the world. Sufficient to the biologist is the point of departure that life is a cosmic phenomenon and intelligence a phenomenon of life.

1 Rignano. Il ragionamento internazionale. Scientia, 1915.

2 Ribot. La logique des sentiments. 1905.

${ }^{3}$ James. The Varieties of Religious Experience. 1916. 
If the facts accumulated by biology warrant the conclusions above mentioned, it necessarily follows that intelligence and consciousness are not exempt from the general laws that apply to cosmic energy and hence to quantity.

Although Bergson, ${ }^{1}$ one of the most acute of modern philosophers, refuses to recognise quantity within the sphere of psychic phenomena, it seems to the writer that it must be regarded as an integral element. One cannot conceive a quantitatively static condition of intelligence, unless under exceptional individual circumstances. The movement of images with their emotional content and the constant succession of external stimuli differing in quantity, nature and intensity must necessarily induce not only qualitative but also quantitative modifications of the intelligence. If, e.g., an individual has a perceptive capacity by which he receives and fixes a number of stimuli equal, let us say, to ten, whilst another individual under the same conditions of environment receives and fixes five, it is evident that the two intellects differ, especially because the quantitative denominator is not the same, and one can understand that with a variation in the quantity of elements there is also a difference in the qualitative exponent.

If the first individual assimilates with his consciousness the contents of the ten perceptions, associating and fusing them with his pre-existing mental capital, elaborating them into more complex mental products which may even appear as inventions, whilst the other individual does not assimilate, but, rather, forgets, all or some of his five percepts, then undoubtedly the two intellects will be qualitatively different, but the reason for this will lie especially in the different quantities. The most irrefutable proof in this connection is furnished by the facts of mental evolution in children. Every year at school, every month, every day, perhaps, supplies new material to the evolving intelligence. New elements develop its creative power, new horizons appear before it, and from its increasing energies there emanate diverse forms of reaction (conduct). It is evident that the quantity of cognitions and adequate reactions emanating therefrom impress a certain intonation on consciousness, which in its growth and development not only exhibits features of quality but is at the same time essentially quantitative. One can agree with Masci ${ }^{2}$ when he holds that mutations of consciousness resulting from intensity of feeling, emotionability, reactivity, in direct or inverse ratio with the quantity of utilisable imaginative content in a given unit of time, cannot be expressed in numbers or in units of space, and that, with the exception of the reaction, they remain subjective facts; but this does not take away the quantitative character from these facts, any more than it takes away the qualitative factor. Works of art offer an argument against the idealistic indeterminism of the Bergsonian philosophy, because in many of these, so long as they are not simply reflex copies of nature but reproduce states of mind, there is projected the quantity of feeling and of the intellectual content.

1 Bergson. La evoluzione creatrice. 1907.

2Macci. "Quantità o misura dei fenomeni psichici." Accad. Reale di Scienze morali e politiche. Naples, 1915. 
Grief which afflicts, pity which tempers and mellows, love which exalts, and jealousy which destroys, are not only qualitative states but also expressive of ideative and emotional quantity.

The muscular human figures depicted on the vault of the Sistine Chapel reveal the strong feeling and immense, historical, mental content of Michelangelo. Lord Byron's description of the embrace in Parisina, and the suicidal jealousy of Othello, represent not only qualities of mind but, at the same time, quantities that may even determine criminal action of the most tragic kind.

Consciousness makes us aware not only of the quality of the emotions but also of the ideative content and of the intensity of feeling. It is of no consequence that we possess no means of measuring these grades of feeling. At the same time, the graphic method employed by Mosso, Patrizi and others, in the investigation of the emotions and the study of morbid conditions, serve to strengthen this view. One may recall, e.g., the affectionate mother mentioned in Chapter I. who, exhausted and anæmic as the result of prolonged lactations and hardships in life, fell into a state of neurasthenia, of which the principal symptom was a profound disturbance of the kinæsthetic sense with the disappearance of the sentiment of affection for her sons. "I would like," she said, "to feel the joy of the immense affection which I had for them, but now I feel quite indifferent. I have a great void in my consciousness. I feel that I cannot live." In this way arose the idea of suicide, symbol of the enormous reduction of the maternal personality.

It is obvious that in a case of this kind we cannot speak solely of quality. We have here to deal with a component which was a part of the personality, and it is needless to confuse the two phenomena by asserting that the quality gives the illusion of quantity.

The dement, whose mental capital we see failing every day, furnishes us with clear proof of the quantitative factor.

The capital of ideas is a quantity and the progressive loss of ideas, such as we observe in Progressive General Paralysis, is a phenomenon of quantity. It is undoubtedly true that this phenomenon is accompanied by qualitative changes ; the ideas are less clear, possess less determinative power, have lost their colouring and their affective tone, so that interest comes to fail ; but all this which seems quality can be reduced to quantity; the colouring and tone of ideas is given by their number, by their association and by the affective element, those all making for a strong resonance in consciousness.

Indifference towards those ideals which once stirred us and excited our enthusiasm to such an extent as to lead us to sacrifice our personal interests and risk our very lives is a matter of intensity, just as quantity of electricity in ratio to resistance gives us a light of greater or less intensity. The qualita. tive factor is by no means to be excluded; it is, rather, the more obvious thing, but if we push our inquiries further we find that it depends upon quantity.

From this point of view it seems clear that psychic energy (intellectual force) is not exempt from the universal laws which regulate the other energies 
of nature. If we indicate quantity by $q$, resistance by $r$, and the product by $n$, we can make the following equation: $-\frac{q}{r}=n$.

The efficacy of the conduct of a man or a country is proportionate to the mental quantity (thought, sentiments, will) available for the overcoming of external resistances (popular habits and the impulses of other peoples) and the internal resistances of all the cerebral and extra-cerebral organs concerned, which are represented in consciousness by doubt, by fear, by difficulties which appear insuperable, and generally end in observance of the law of inertia (the dolce far niente).

Intelligence, then, is a biological fact. Thought is a dynamic phenomenon of life, for it arises essentially from the power which the higher living beings, in whom nervous tissue is developed and differentiated, possess of spiritualising, to a greater or less extent, the world in which they live, the power to fix its images, to construct syntheses of these (from the most simple to the most complex judgments), to combine in the most diverse manner the products of the perceptions and their derivatives with the modifications of the self, and with the reactions upon the world, whence is derived what we call experience. So it comes about that experience becomes ever more the safeguard of existence, which follows the law of adaptation to the environment, accompanied by an incalculable series of reactions. Some of these are stabilised by the physical and social environment itself; others vary ad infinitum in different individuals, in proportion as we get perfecting of the perceptive process, which is the basis of intelligence, elaborated more and more by a perennial flow of old and new material. Thought is not a faculty, it is a complex functional product elaborated by co-operation of various functions and processes-transformation of external stimuli into images (perception) ; fixation and conservation of these images ; construction, with the images, of mental complexes in series (to the constitution and number of which no calculation or mathematical formula is applicable) ; the modifications of the ego under the influence of cosmic and social stimuli (emotions and sentiments).

The biological conception of intelligence conflicts strongly with rationalistic intellectualism, which is founded upon an aprioristic conception. Indeed, when submitted to a methodical process of analysis, intelligence always appears before us as a complex vital phenomenon with an anatomical basis.

It is not a unitary function, as some think, because if we take into account the diversity of the elements and processes from which it results (perceiving, feeling, associating, reacting), we find a corresponding variation in the anatomical bases, which comprise all the brain already differentiated, in the sense explained in the first chapter of this book.

From sensation to intellect, from reflex to voluntary movement and to individual and collective conduct, considered as a whole, there is nothing more than a series of processes which are resolved into movements, identical in nature' but extending from the most simple to the most complicated,

${ }^{1}$ Morselli. Manuale di Semiotica delle malattie mentali. 1894. 
movements which obey the fundamental law of evolution on one hand (genetic psychology), and that of adaptation on the other. Thought, therefore, is not an abstract reality, as has been represented, but a physiological fact, inasmuch as it is the product of the function of an organ which may be regarded as the result of the highest grade of evolution of matter.

$$
\text { ** } *
$$

Life is an impulse and a defence, and intelligence, with its power of penetration into the cosmic and social environment and its powers of defence and adaptation, is also a biophylactic function. To penetrate the world in which we live signifies to know it. To know it, signifies to make use of its energies so as to promote, in every possible way, the integration of the individual and the species, and to defend oneself from all that may be inimical to the one or the other. Reality does not immediately obtrude itself on our senses, nor is it at once perceived; it is not directly and intuitively divined and classified as such by the intellect, but rather does it become known to us through individual (ontogenetic) and inherited (phylogenetic) experience. Experience passes through evolutionary phases, the earliest of which may be traced in the obscurity of the unconscious and elaboration of the nervous apparatus under the repeated action of stimuli, these becoming summed together until they acquire the intensity necessary to overcome the threshold of conscious perception. One can convince oneself of the reality of a preparatory phase if one analyses the evolutionary progress of a civilised people (social phylogenesis), or, again, if one investigates the evolution of the individual (perceptive ontogenesis). Many realities only become apparent after long examination. Repetition of the stimulus and observative analysis are factors which prepare the way for the apperceptive process.

The savage and the untutored member of an advanced society do not e.g., observe the filthy, full perception of which determines eliminating actions that become defensive of the personal and collective integrity and conducive to health, which is the fundamental attribute to which the living organism unconsciously aspires. Herein we see the æsthetic sentiment developing and coming into play as a new component of the consciousness of the individual or of a civilised community. One must agree that thought is not always conscious, although it is often directed by consciousness. If we look upon intelligence not as a faculty of the mind but as the dynamic result of the notions and experiences accumulated through the mechanism of the organs of the senses, it seems quite clear that this product is already logically disposed in the archives of the memory and of the subconscious, according to the natural order of the world perceived and of the experience of the ego which created and is perennially creating it in the same logical order as in objective reality, with all its relations and its successions. If, then, it is true, as Ingenieros ${ }^{1}$ and others have asserted, that the psychic functions are not always conscious, it is equally true that the keeping of an objective firmly in view is a fact of consciousness ; round that, objective thought is developed

\footnotetext{
${ }^{1}$ Ingenieros. Principios de Psichologia Biologica. Madrid, 1913.
} 
in a series of combinations and creations which are unconsciously produced by a process intimately bound up with cerebral activity, animated by the theme that is detained in the focal point of consciousness.

It is of capital importance in the study of thought to recognise the innate creative tendency of the human intellect. As already remarked, nature furnishes only the raw material with which it, or, rather, the brain, constructs the modest products of the simple image or the marvellous and immortal creations of genius. In like fashion, with other material, man constructs a hut if he be a savage, a modest house if he be a poor labourer, or a sumptuous palace if he be wealthy; and so there arise the small hamlets and the great metropolitan cities where men live, associate together, vie with one another, form groups, are active or inert, grow and die.

In the evolution of thought there is a constant process of construction and interchange. Notions and judgments assume different forms, and thoughts, variedly coloured and associated, succeed one another in the most diverse combinations, with a tendency to become manifest by translating themselves into different forms of movement, which, again, vary with altering conditions of time and place. Whilst groups of ideas are being formed and organised, components of one pass into another and yet another, the number of combinations being greater the more simple they are.

In the image of "paper," e.g., we find some components such as that of the white colour and the tacto-muscular components of thinness, pliability and lightness, which belong to many other objects. The acoustic image of paper, as when a leaf is torn from a book, is more characteristic, associated, it may be, with collateral images of an ink-bottle, a pen, with the muscular images of writing and many others. All these images are likewise to be found as components of ever so many other psychic complexes. It is the particular way in which they are combined, so as to confer specificity upon a concrete image, that characterises the selective power of the brain in fixing objective realities. It is the same thing with oxygen, carbon, nitrogen, etc.; those elements enter, in diverse combinations, into an incalculable number of organic and inorganic compounds.

In the fact that simple images form components of a large number of concrete images, and that their combinations may be multiplied ad infinitum, we recognise the sovereignty of the law of creation and evolution. Every hour, every day, man receives new impressions with which he forms new percepts. These enter into combination with pre-existing percepts and increase thought, which always reflects reality. Mental constructions which have no counterpart in reality, and give no indication of verisimilitude or analogy, belong to the realm of pathology or its borderlands.

With increase of the psychic products resulting from continuous action of agents of nature upon the perceptive centres (a point upon which psychologists and even histologists like R. y. Cajal are agreed), and with growth of the psychic personality, the differentiation of the ego from surrounding nature becomes more decisive. 'The greater the number of percepts, the more active 
the process of assimilation and fusion of these for the formation of more complex psychic products reflecting the reality of the physical and social environment, and the more complicated and diverse the reaction on the cosmic and social environment, the more distinct becomes the ego in the world in which it lives and expands, because the more intense and varied is its experience.

The psychic products formed with the percepts are of two ordersaggregates and compounds. The former can be decomposed into elements which are associated with each other in a most varied manner, giving rise to different representations. The latter are also susceptible of analysis, but are more stable, for they represent definite external facts and determined relations, associated with one another and with aggregates in the most varied manner, in the perennial motion of thought, yet conserving an always recognisable individuality.

All the concrete images, and the words which symbolise them, are stable compounds, inasmuch as they express real objects and fixed relations. The concrete image, e.g., of A Book, results from a certain number of more elementary images now fused with one another so that the product is unalterable. This image is a whole, the components of which are inseparable from the concrete image of that particular book. Books, however, differ in many particulars, and the various images are so blended as to give rise to a very variable and unstable ideative compound which is the general idea of Bоoк, quite different from the concrete image of each book. The latter refers to a particular book which I have seen and which has passed through my hands, an image which is invariable and not decomposable.

The general idea does not refer to any concrete image but to a conglomeration of all the images which participate in it, these being synthetised in the word in which the general idea is moulded. All the concrete images from which the general idea results either remain on the first level of consciousness (in the subconscious) in greater or less number, and perhaps on its threshold, indefinite and indescribable, or they are represented in consciousness, more especially those which memory serves up because they are more definite and more coloured, having been given by stronger impressions, and because they have assumed a greater number of associative relations and are therefore more capable of forcing the threshold of consciousness. The general idea becomes stable in the word which symbolises it.

If a man forget the word "book" he may yet be able to picture to himself single books, the images of which are stable, but he no longer possesses the general idea of Book.

Concrete images, like general ideas, have a strong assimilative power which, in a manner, is proportionate to the perceptive and attentive capacity of the individual. The concrete image which the illiterate or poorly educated man holds of a book he has seen is very different from that which the studious man has of each volume in his library. A much smaller number of components enters into the construction of concrete images formed by a weak or undeveloped brain. Not only so, but ill-developed brains are incapable of 
constructing general ideas. Their poor activity stops at the formation of concrete images, and even these are made up of a scanty number of com. ponents. The construction of general ideas falls within the capacity of evolved brains alone. The assimilative power of these is such that every image or psychic complex is constantly becoming fuller and more complete by the incorporation of assimilable elements of the external world.

The general idea assimilates the new images, becoming enlarged and more complex. This fusion is only realisable with the intervention of speech. It is the word which possesses the summarising and synthetic property; it is like a cold matrass into which there fall, like distilled drops, new images which, throughout the course of life, become combined with pre-existing images, in accordance with the apprehensive capacity of the personality. The word, that small and inextensible organism, comprises and expresses them all as one. Thus are formed concepts which, under normal conditions, are inseparable from names. Conceptualism and nominalism are two correlative terms co-existent with two indivisible facts, just as life and organism are indivisible. Life may cease and the mummy remain, just as sometimes the cenceptual content disappears whilst its corpse, the word, remains ; or, again, the form may be moulded without life and without content by the inferior man, like the paper doll made by the infant. The live concept or the general idea exists only through the word. If this be suppressed, the concrete images of each book may remain, but no longer the abstract idea of Book.

Images, even though they have no immediate relations with time, transport us beyond the confines of the immediate circumstances owing to their associative bonds and to the fact that they themselves succeed one another in time. This excursion, in consciousness, beyond immediate and direct perception facilitates immensely the expansion of consciousness in its relations with time and space (memory and imagination).

The concrete images which enter into the structure of concepts are only duplicates. They have no life of their own, but are maintained active by their centres of formation. If a particular formative centre is destroyed the concepts lose their vitality and die, beeause they come to lack a particular category of components. Similarly, the concrete images of individual objects are kept active by the elementary images from which they result, and the more complex products of the mind by the latent life of all the mnemonic traces from which they are formed and which remain behind the threshold of consciousness. The clinique provides us with numerous examples in illustration.

Cerebral activity is conditioned precisely by the continuous flow of specific waves (images) from all parts of the cerebral mantle.

Every nascent thought is sustained by the whole train of images which, through their associative relations, go to compose it.

The whole history of destructive foci in the sensory centres, the evolutionary zones (language) and the associative paths, is a complete and irrefragible demonstration of this law, which animates and governs all the complicated mechanism of the intellect. If one cortical area is destroyed, or 
a path of communication intercepted, the activity of all the other mental fields is diminished and the whole intellectual life feels the effect.

In the constant movement of images the prevailing tone comes from those which have a predominance over the others, because of their number and representative vividness. So it happens that, in the movement and extrinsication of thought, some men appear more visual, if the visual images prevail over the others, whilst others are more auditory, or more conceptual or more sensory, according to individual circumstances.

In no case can it ever be said that only one single form of memory comes into play. The motor memory of singers, the visual memory of painters, or the auditory memory of musicians is always associated with all the other memories. What we have to deal with here is a predominance of one form over others. If certain writers are so constituted in mind that their auditory images have the predominance, so that in reading their productions we are struck by the rhythm and harmony of their phrases, whilst in others there is a prevalence of visual images so that colours, in all their shades, spring before our mind's eye in the most harmonious pictures, yet, in every case the product is still a complex one.

The history of art teaches us that many artists, visuo-motor or auditorymotor, are more sensory than conceptual, just as youth is more sensory than mature age, and the childhood of the race more so than succeeding generations who have advanced in thought and action. Conceptualism predominates in maturity and in a very advanced civilisation, but art, which precedes science and history, is transformed later into historical and conceptual art, responding to the influence of progress in the order of knowing and feeling.

$$
*^{*} *
$$

From all the foregoing, it seems clear that the whole mental edifice rests upon the law of association. Aggregations and combinations (the "fusion" of Wundt) are not possible except through the associative power of all the psychic formations, from the simplest to the most complex, and through the anatomical substratum which supplies the organic and mechanical conditions necessary to the psychic dynamism, regarded in the manner already indicated.

Association consists in the connections that are established between ideas, emotions (Scripture) and movements. These connections are formed between all the images corresponding to objects external to ourselves (James), between the complexes that arise from combinations of these same images and between all the forms of reactions of the self. In this mechanism lie the germ and substance of logic.

Two forms of association are generally spoken of: (1) associations founded on resemblance, and (2) associations founded on contiguity, or, according to Wundt, $(a)$ intrinsic, and $(b)$ extrinsic associations. Intrinsic associations correspond to those founded on resemblance; extrinsic to those dependent upon contiguity. If the physiognomy of one person resembles that of another whom 1 know, so that a chance meeting with the first at once 
calls up the image of the second, in this case the bonds of association are close, some elements of the two personalities coincide and the association is intrinsic. If, on the other hand, I meet a person whom I have seen on a former occasion in some other place, so that I now picture to myself that locality or the image of another person who happened also to be there, such an association between person and place, or between two unlike persons and the place where they were at the same time, and consequently between the respective images, is extrinsic, because here we have to deal only with a coincidence which does not touch the nature or the form of the objects.

Associations are also divided by certain psychologists (Wundt, Kraepelin, Aschaffenburg) into two great classes: immediate and mediate associations. The latter are produced through the medium of a common term without this term itself appearing in consciousness. Language offers a good field for experiment. Immediate associations are divided into two groups, according as the word pronounced is understood, or merely evokes an association through its quality of a simple sound. When the word is understood, we get co-ordinative and subordinative associations. The word "statute," e.g., calls up the word "law," "asylum " suggests "madness," "nightingale" suggests "bird" ; or the relation of the associated words may be predicative, as in "ripe-fruit," "black-ink," or causal, as in "merit-decoration," "alcohol-drunkenness" ; these are internal associations. Associations due to assonance (rhyme) enter into another group. Bourdon distinguishes four forms of verbal associations, but it is needless to enter into details.

External are more frequent than internal associations (Aschaffenburg) and at the same time more rapid. Those of nouns are greatly in excess of those of verbs.

In the case of associations due to co-ordination and subordination we have quality associated with object and object with quality. Similarly, in another field, one letter is associated with another letter, a word with a letter, the name of a colour with a letter, one word with another word, a number with a colour (Calkins).

In some men co-ordinate associations prevail, others again associate more frequently and easily through subordination. This explains to some extent the diversity in syntactical forms of speech. It is the particular nature of the associations that impresses a particular character on the intellect; for instance, the intellect of the reasoner differs from that of the descriptive writer, owing to a relative homogeneity of the mind of the reasoner, compared with that of the descriptive writer (Bourdon).

Associations due to contrast are among the most frequent and most active in the process of thought, and they furnish the most plausible explanations of an infinity of normal and morbid phenomena which would otherwise remain quite obscure. In some intellectual and affective categories the first and last components of the series are more closely associated than all the intermediate components.

In the field of sensation white is more closely connected with black than with the intermediate shades of grey; the image of the heaviest body 
is closely associated with that of the lightest body; representation of smoothness is associated with that of roughness, etc.

In the domain of feelings, pleasure and pain come together, although they are the extremes of a series of gradations of pleasures, pains and indifferent sensations. Friendship supposes enmity, love supposes hatred, fear supposes courage, and so on; as we mount in the scale, the beautiful supposes the ugly, the moral the immoral, the perfect the imperfect, the wholesome the unwholesome, the illiterate the cultured.

Many such contrasts are the negative side of an affirmation. When we judge men to be good, moral, beautiful, cultured or disciplined we at the same time formulate comparative judgments, and we represent to ourselves bad, immoral, ugly, uncultured, undisciplined men, whose images are very closely associated with those of the others, so closely, indeed, that whenever I summon up any one of either series the contrasting correlative of the opposite series is immediately evoked.

The law is that associations due to maximum differences are stronger than those founded upon resemblance or minimum differences.

Contrasts become established from the earliest appearance of mental associations, side by side with associations due to resemblance and contiguity. When an infant stretches out his little hand towards a burning light (tropism?) and feels the pain of the burn, he notes and remembers the contrast between the pleasant aspect of the bright light and the pain that results from contact with it. If, in order to wean her child, a mother puts some bitter solution on the nipple, there arises a strong association of contrast between the pleasure of sucking and the repellent bitterness of the nipple. The smile that inspires the playful attitude of a father towards his infant becomes associated with the crossness that determines the father's severe behaviour.

A great part of the education of infants rests upon the law of psychic contrast. Aspirations, desires, movements, that are opposed and hindered, associated as they are with opposite trends of mind with their correlative ideas, furnish very numerous examples of association by contrast. Love and hate, confidence and diffidence, courage and fear, are the emotions most frequently alternating in consciousness, being ever in contact, as it were, on its threshold. Dumas, unlike many psychologists who either make no mention of psychic contrast or deny it altogether, wrote : "Man is a very strange animal, wholly made up of contrasts." After Bain no others have illustrated the importance of the phenomena of contrast in the succession and association of ideas, emotions and actions, more fully than Paulham and S. di Sanctis. G. Bruno, ${ }^{1}$ however, had previously recognised the law of psychic contrast, maintaining "that the beginning, middle and end, the birth and the perfection of all we see, arises from contraries, through contraries, in contraries."

The intense love that the neuropathic mother has for her child awakens in her consciousness the fear that some illness may carry off the infant, in

${ }^{1}$ G. Bruno. "Pensieri." Raccolta di Breviari intellettuali. 
contrast with the joy of possession; or it may be that when she leans upon a balcony she fears that she will cast down the object of her love. A great number of psychic disturbances are to be interpreted solely through associations due to contrast, and instances of delusions and obsessions due to contrast are more frequent than is generally believed.

As far back as 1886 , in a study of psychic polarisation, ${ }^{1}$ the writer set forth clearly the principle of association due to contrast. "This fundamental logical process," he then wrote, " is the resultant of the associative relations of sensations and ideas, especially relations of antithesis, with which are associated analogous states of pleasure and pain. Hence it comes about that in the process of arriving at a conclusion, in normal mental functioning, every idea bears with it the antithetical idea; only, it happens that, owing to the directing attention, the antithetical idea does not reach the focal point of consciousness but remains hidden, though not inactive, in the unconscious."

Even to-day, after many years, the author is still of opinion that this idea serves as a useful interpretation. The whole doctrine of Freud is supported by this fundamental law.

When an individual proposes to himself to do something, ideas more or less actively in contrast with this soon present themselves to his consciousness. The detailed descriptions of mental phenomena given by Socrates, and attributed to his "daimon " (Lelut), were nothing more nor less than phenomena of psychic contrast. The same thing may be said of the motives and counter-motives that dispute with one another, in the field of consciousness, the government of our actions.

Every neuro-pathologist has experience of individuals, more especially hysterical subjects and neuropathic children, who act contrary to the injunction or the recommendation given them. Some people, when they wish to avoid disturbing their neighbours by sneezing or yawning, feel compelled to yawn or sneeze (contrast arising from inhibition).

The anxious fear of committing some act that is dangerous or repugnant to the conscience drives into the field of consciousness all the images connected with that act; and these images, especially in degenerates, are endowed with an extraordinary impulsive activity. Therein lies the explanation of the fact that those who are greatly afraid of death, and especially of suicide, end by committing suicide (coercion due to contrast). Religious practices and the vow of chastity often excite intensely erotic ideas and desires. The hysterical eroticism which claimed so many victims, even in the monasteries, in the Middle Ages, was the effect of contrast between the desire that arose from the over-excited organic needs and the ideas of chastity which the numerous monastic orders and sects attempted to inculcate in mankind, ${ }^{2}$ in the belief that chastity represented the highest virtue.

1 Bianchi. "Archîvio di Psichiatria, Scienze Penali o Antr. Crim." and Revue Philosophique. 1886.

2 Bianchi. “Il nervosismo di questa fine di sccolo.” F'legrea. Naples, 1899. 
This contrast is depicted in one of the most celebrated paintings of Morelli (The Temptations of Saint Anthony).

Some people who have dedicated themselves to religious practices become tormented by lascivious images, because chastity forms the one aim of their conduct. Cases of this kind have been reported by Lemesle.

Many delusions are hinged upon association due to contrast. Here, again, we have an illuminating example of the fact that our knowledge of the phenomena of normal psychology and of the laws regulating healthy mental life can be completed by analysis of pathological facts.

Associations may undergo a species of interference, an analogy, applied rather inaccurately, if we adhere to the strictly physical sense of the term, by A. Bergstrom. ${ }^{1}$ Interference of associations, says this author, is a fundamental fact of the nervous system and consists in this, that once an association is established between two terms, $A$ and $B$, any new association that we attempt to set up between $\mathrm{A}$ and $\mathrm{C}$ comes into conflict with the former. In general, when we wish to exchange a customary mode of doing a thing for another mode the old habit resists, and when we are wearied or busied with' some other matter the tendency is to revert to the old fashion. The law of interference is just as fundamental as that of habit, which has been interpreted in a masterly manner by James. The reason why we are not inclined towards anything that is new lies in the fact that we are not inclined to make the effort required for new associations. This is referable to another law of life - the law of least resistance or the law of misoneism. Bergstrom's experiments are directly intended to prove the fact that, notwithstanding every effort, a decided interference takes place when we endeavour to associate a new reaction with an old stimulus, just as when something is learned in a new or different fashion. The confusion, writes Bergstrom, that we find in the mind of young students when an argument is presented to them in a different form is a case in point. Much of what has been attributed to failure of memory in advanced age ought to be placed in the category of interferences.

The creative imagination, however, is not specially regulated by any of the known laws of association, nor does it always require a clear memory with a considerable wealth of sensible representations to serve all its combinations. In it there come into play the elements of chance and logical incoherence (Vaschide ${ }^{2}$ ). According to this acute observer the first dreams of young children, like the fibs they tell, are phenomena of creative imagination, based on the lack of precise perception of the real facts and on error of the senses.

Who can tell under how many different aspects things may be regarded ? How different are the judgments pronounced upon things that closely resemble one another ! If little drops of ink are allowed to fall on pieces of cardboard and we press other pieces of cardboard upon these, the ink squeezed out will

1 Bergstrom. "Experiments upon Physiological Memory by Means of Interference of Associations." Amer. Journ. of Psychol. 1892-1893.

${ }^{2}$ Vaschide. La logique morbide. 1902. 
make spots of various shapes. In such spots one child will see a horse, another child an animal with its mouth open, whilst another will find there the head of his teacher or his teacher's wife, all of them noting the strangest resemblances. This is not a case of illusion, but of association due to likeness or analogy of these objective figures with others that are preformed and very readily reproducible in memory. ${ }^{1}$

Protracted mental work exercises a notable influence on the associative power and on the quality of the associations. With the onset of fatigue, the number of internal associations diminishes considerably, whilst the number of external associations, such as those formed between rhymes or sounds of words, increases as the work proceeds. ${ }^{2}$ This phenomenon should have a very great significance for pedagogues and compilers of scholastic programmes.

$$
\text { *** }
$$

If the author has dwelt upon the subject of the associations longer than may seem necessary, it has been with the object of furnishing proof, from this additional source, that every sensation provoked by the external world, as it excites one sense or several senses together and crosses the threshold of consciousness, induces an extensive movement of cerebration. The work of perception and judgment never remains circumscribed within the cortical field of the sense excited, but, through the fibres, the neurofibrils of the dendrites and of the axis-cylinder prolongation of each cell, by direct and indirect paths, it provokes a great amount of work, more or less intense, which involves the whole cerebral mantle, or a great part of it, according to the degree of development and culture of the individual.

Association between ideas has a bearing upon the formation of propositions, which include a judgment or a conclusion on the relations of two or more of these associations. Every proposition contains the elements of conviction, in so far as every conclusive judgment tends to affirm or to deny.

Reasoning, which is essentially made up of propositions, is nothing else than a more extensive and complex association of propositions, the associated components of which possess correlatives of time, place and number, from which arise the grammatical inflexions of words and the syntactical form, these being the product of a more advanced mental evolution.

Only a part of the mental capital of each man can be represented in consciousness, and that under specially determined circumstances and always according to the laws of association. Only a small number of representations can be brought together in the focal point of consciousness or even within its visual field, in a given unit of time (determined by Wundt); all the rest constitutes what we term the unconscious (or the subconscious), which furnishes all the elements necessary for the development of a particular

1 Dearborn. A Study of Imagination. Amer. Journ. of Psychol. 1901.

${ }^{2}$ Richerehe di Aschaffenburg o di Binct e Henry. La fatigue intellectuelle. Paris, 1898. 
thought or a particular line of conduct. It is evident that this development will be more or less felicitous (1) according as the unconscious holds a larger or smaller stock of cognitions ; (2) as its constituent elements are well connected with one another ; (3) as the nerve-cells, perfectly sound and active, furnish their memory-traces punctually and faithfully; (4) as the innumerable associative paths (fibres and neurofibrils) are pervious so as to permit the reawakening of the correlative mnemonic traces even in the deepest recesses of oblivion, according to the laws of association; (5) according to the extent to which all this material is offered to the creative power of the intellect.

The whole mental content may cross the focal point of consciousness according to the laws of succession and connection (the law of succession may be included with that of time in the psychic processes, and the law of connection with that of space, which is another condition affecting associations).

In the unconscious lie both the debris of mind and the elements of genius; there the former may ferment and conspire, eventually forcing the threshold of consciousness ; there, too, the latter may sparkle with unexpected light, beaming forth to illuminate new paths of life.

Considerable importance attaches to the former elements, those psychic complexes or constellations which sometimes conspire in the unconscious, (Freud) and determine particular emotional states.

The whole capital of sensory images contributes to the formation of the various categories in the development of thought. The categories are of diverse evolutionary grade according to their content of thought and sentiment. From all that has gone before, it seems clear that in our examination of intelligence we should inquire into the quantity and complexity of the ideas (whether simple or compound), their re-evocation, their associations and combinations, the capacity of the brain to make use of the thought-content of the social environment (judgments, beliefs), all this being reconstructed into language and resolved into conduct. Thus, the scientific conception of the mechanism of intelligence here put forward is inseparably connected with that of language in its more evolved extrinsications.

Language made its appearance as an emotional reflex, and has attained the highest rank amongst the cerebral functions, whilst at the same time its mechanism has become gradually more complicated. The study of language embraces a large part of the difficult problem that concerns the mechanism of the cerebral functions. The functional complication and the high psychic value it has attained had not been possible were it not for the specific differentiation of extensive regions of the cerebral cortex. Speech is the product of a long and laborious process in which numerous psycho-physiological factors co-operate. It is co-extensive with the anatomical basis. Language might be said to be an organism which has its own evolutionary history, closely bound up with the story of the evolution of thought, and is the result of many diverse constituent elements intimately connected with one another.

The law of evolution is here apparent as the supreme law of thought and of language, of content and of form. The development of speech marks the great trajectory described by human thought, detaching man from all other 
animal species, in the comprehension of his environment and of all nature. Just as, in the development of organisms, cells become multiplied and differentiated and assume different forms, so human language, by the development of thought and the constant differentiation and distinction of objects, qualities, states and relations has become constantly enriched with new forms.

Again, just as in the embryonic development of the human organism some elementary transitional forms disappear to give place to elements that are more stable and more adapted to life, in the struggle with external agents, so it is with the dead languages and possibly with others which will die, insufficient as they were or are for the purpose of their existence under the new conditions of intellectual life and the successive states of society. Just as the transitional tissues which have disappeared never again return to life, so the dead languages became dissipated in the genesis of the tongues which came after them, and the latter in those which presently exist. ${ }^{1}$

${ }_{1}^{1}$ The doctrine that language was revealed to man by a Divine Creator, and that the first human beings expressed their thoughts and emotions in well-formed words, finds no acceptance now except in the minds of theologians. The publications of Grimm and Renan, reviving the genial intuitions of Lueretius, have met with wide approval.

That language has an origin and an evolutionary history inseparable from that of thought is a hypothesis generally accepted to-day not only by naturalists, particularly as the result of the diffusion of the Darwinian doctrines, but also by philologists and glottologists. Their wonderful investigations have laid bare a long retrospective history buried under the upper layers of modern tongues, just as the geologist draws his deduetions regarding the suecessive formations and the great eataclysms which transformed the crust of the earth which we inhabit from the superimposition of strata of which the earth is formed and from the traces of petrified beings who lived in other times.

To biologists, and even to the generality of philologists, the question whether language has had one origin only, or several origins in different regions of the earth far distant from one another, is a matter of minor importance. In either case the primitive tongues must have arisen by branchings off (as the result of the intervention of a great number of coefficients, internal and external), from stems or roots which, notwithstanding their simplicity, contained the germs of the luxurious filiation of modern tongues, just as seeds contain all the elements of the new growths that arise from them. Language is the result of a slow evolution that had its commencement in the modulation of the inarticulate voice of beings who preceded primitive man, under the aetion of emotion-producing stimuli.

Leaving aside observations on animals, we can start out with the certain datum that the emotional states of man translate themselves into movements and attitudes which vary with ehanges in the eontent of emotion. Fear and courage, love and hatred, pleasure and pain, besides the appetites, all have their language, inasmueh as they tend to be converted into movements : they induce different tones in the muscular groups of the face, giving rise to changes in physiognomy, and they cause a number of movements of the trunk and limbs which in combination with the foregoing express changing states of mind. The emotional language of man finds its counterpart in analogous attitudes of the higher mammals. Let anyone notice the joy of a hunting dog when he sees his master put on his hunting suit, or the mortification of the same animal when, after he has stolen a piece of meat, he finds himself in front of his angry master and crouches down, dejected and suspicious, 
Language, expressing as it does emotions and ideas, should be examined from both the subjective and the objective sides. Besides conveying our ideas, it has the property of reawakening in other individuals who hear us and perceive our words the same emotion as stirs us. Indeed, there is good reason to believe that in its earliest development it has been almost exclusively emotional.

and let him compare the two attitudes of the dog with those of happy urchins who, on a fête-day, strut along in front of an instrumental band, cutting capers all the time, or with that of a mother who has just lost a loved one, then he can testify to the mute language of the emotions, as these may be read in physiognomy and in gesture.

The court paid by the male to the female cephalopod in an aquarium, the agitation of ants when their own nest is attacked by another colony which has exhausted its provisions, the vigilant and suspicious air of the old elephant as he comes out of the wood into the open to make sure that no danger threatens his companions when they seek the pasture, or the agitation of the swallow which finds its nest occupied by an insolent sparrow, all prove to us that there is an immediate and constant relation between emotions and movements and that a communication of these emotions takes place between individual animals forming one group. It is certainly a form of language. To feel and to act represent two terms of the emotions which man incarnates in a marvellous number of physiognomic expressions, and these are the first phases of language.

The dominating sentiment in each individual confers a prevalence upon the relative muscular attitudes, and a trace of this remains which does not escape the trained observer. It is in these traces that we read the mute language of the human heart; it is by their guidance that we penetrate into its recesses, and the mind is unconsciously led by these intuitions into the tepid or warm regions of sympathy, or into the grey, cold and repellent regions of antipathy.

Photographs of physiognomies compose a very interesting and instructive book on the language of the human heart. In it there are certainly enigmatic pages, but these belong to the icy, the torpid, and to simulators, including certain diplomats and politicians whose spoken words, governed by strong will and interest to be conserved, serve to hide their real thought and sentiment. This is the art of lying; it is the exercise of will over the muscles of the physiognomy, with a power that these cannot escape.

It is to be borne in mind that the vocal organs and buccal nervous centres in man are very highly developed, and it is, therefore, not out of the way to suppose that strong emotional states, besides betraying themselves in movements of the face, limbs and body, might provoke also movements of the vocal organs, combined with movements of the tongue and lips; and that thus were formed, in ages long gone past, the first articulate sounds of primitive man. The association between the object and the phenomenon provoked by the emotion in the form of an articulate vocal sound, its immediate reflex, indicates the symbolic character of articulate sounds, more especially because the same phenomenon would be induced under similar circumstances. It is a generally accepted opinion, as held in ancient times by Plato (in the Cratile), and afterwards by Leibnitz and De Brosses, and in recent times by Renan, Max Müller and other philologists and naturalists, that language had an imitative origin. This view finds support in the fact that the imitative reflex is very commonly found where intelligence is less developed. Children, savages and imbeciles imitate much more than normally developed adults. Again, when consciousness is put to sleep by the induction of hypnotic states, in which individual initiative is suppressed, the imitative capacity is enormously augmented.

In the infancy of humanity, imitation reproduced sounds and noises in articulate 
Later it has become more cognitive, inasmuch as its content is much more largely made up of notions.

Simple vowel sounds, and even mimicry alone, are more than sufficient for the expression of elementary emotional states, but one cannot conceive the possibility of transmitting notions to others except with the aid of words, which give form to thoughts. Emotional language always remains the mode

voices which were simply the reflex of analogous phenomena which struck the senses. The running of water with its sustained sound probably provoked in a reflex way the sound of $r$, which in itself indicates movement. This syllabic sound is found in a great number of words which have a content of movement accompanied by more or less noise. Words have been gradually formed by the addition of affixes and suffixes; in the Italian language we have rumore, rotolare, ruggire, scorrere, correre, rifare, tremare, fremare, which preserve the motor element in one form or another. Breaking, with the noise it produces, becomes expressed by the Sanscrit rug, which has produced the Celto-Bretonic rogan and the German brechen. The whistling of the wind has by imitation given rise to the sound of $s$. The Persian sarse, to slide, was applied to the serpent, owing to the noise it produced, gliding on the grass. Without multiplying examples one can say with good reason that onomatopœia contributed, along with the emotional determinism, to the formation of the first articulate sounds which eame to designate certain objects and phenomena. These sounds symbolise something outside of ourselves and internal conditions. Repeated and fixed by habit, they would be the roots of the Indo-European languages, according to Max Müller, or even the roots of all languages, according to the recent doctrine maintained in Italy by Trombetta.

The language of prehistoric man, who lived and struggled against the treach. erous and terrible elements of nature in the palæolithic epoch, more than one hundred and fifty thousand years ago, was very probably monosyllabic and indefinite. By slow degrees it eame to acquire a more definite character in subsequent epochs, and progressed by gradually assuming phonetic-articulate demonstrative forms of language.

Max Müller ${ }^{1}$ has reduced the number of monosyllabic words with differentiated meaning, made use of by the Indo-European races in their infancy, to 121 .

In any case, it seems safe to say that modern languages became developed from roots by a process of ramification, contemporancously with the development of human thought and actions. The syllable ma or am must have been one of the first. It is found in many primitive tongues. In some ma signified watcr, whence perhaps the word mare. In other cases it gave rise to many similar words all signifying " hand "; thus the Italian " mano" and the words " mara," " marra," " murra," " malla," " mulla," of the Australian tongues.

In others again it gave rise to the words " mitir," " mater," " madre," "mutter," "mother," all of which mean mother.

The ramification is demonstrated by the majority of the roots of the IndoEuropean languages. Thus from the root fac have come the branches facio, factio, facultas.

It is held that thoughts became expressed by these men not merely by gestures but also by monosyllables with different inflexions to express different things. Later, the monosyllables became associated or combined with one another to express other things, and so by agglutination other languages came to be derived from these. Contractions of these additions gave rise to tongues with inflexions, which belong to the more advanced races. Even to-day ono can detect, in the tongues of savage and semi-savage peoples, the various phases of development of

1 Max Müller. The Monist. 1891. 
of communication between animals (song, calls); it is understood by other members of the same species and is capable of reproducing identical emotions in them.

All auditory images which are not words (sounds, musical tones, etc.) are more emotional than cognitive. To become cognitive it is necessary that they be associated with a sensory image of the concrete object to which

the more advanced languages. Those races whose cerebral development has suffered arrest from one cause or another have idioms which present the characters of a primitive language. The clicking, and sometimes crackling, sounds of the Hottentots and Bushmen are a living demonstration of what the language of the first human beings may have been.

The development of intellect and language has taken an extraordinarily long time in the lower races. One has only to remember that many tongues and dialects are of comparatively recent origin. The Cuschitic tongues and the Berber dialects are devoid of literature, and do not yet possess any true writing. The uneducated peasants of Lithuania, until a few years ago, spoke a language which approximated to Sanscrit. The oldest documents of Lithuania do not go any further back than the sixteenth century. Tongues that owe their origin to agglutination are to be found in the inferior races of America, Africa, Oceania, and Asia (Mongolians and Uralo-Altatians). Groups of Copts and Berberians have tongues that are intermediate between the agglutinative and inflexive; these have all been arrested.

The new words which are formed with suffixes and affixes, to keep pace with the growth of science and industry and our capital of ideas, demonstrate the indefinite continuation of arborisation in every branch of the evolved tongues. Take, e.g., the root spac and its Greek derivative "scopos." One need only mention a few words formed in recent times in consequence of the advance of science-e.g. microscope, spectroscope, telescope. The whole series would indeed be a long one. There is no doubt that the tongues of civilised people have been enriched by new words with a truly marvellous rapidity.

According to a calculation of Max Müller the cuniform inscriptions of Persia contain scarcely 379 words. The hieroglyphics of the doctors of ancient Egypt amount to not more than 658 words. The old Testament is written with 5642 words and the plays of Shakespeare with no less than 15,000. The English language which, in the earlier editions of Webster's and Robertson's dictionary, amounted in all to 43,566 words, has increased to $250,000 .^{1}$

This development of language is almost parallel with that of the senses and intellect. It is very probable, as Geiger asserted, that in the period of formation of the Aryan tongues only one colour was known, the blue of the sky not being distinguished from the green of the meadows, the grey of the earth from the deep yellow of the rocks and the golden dawn from the red twilight. It is said that the presence of an intelligent human being on the face of the earth dates back about 200,000 years. The finding of identical utensils in the districts of the Seine, the Thames, in South Africa, in the Indies, in Spain, leads us to believe that human beings in the palæolithic or pre-palæolithic epochs already inhabited different parts of the earth. Then men must have attained a remarkable cerebral development in the mesolithic period which commenced eighty thousand years ago. Perception of colours was slow and gradual. Besides their imperfection in design and the irregularity of their lines, the paintings of savages show no blending of colours. Savages do not perceive the shades (nuances) and rsthetic intonations dependent

${ }^{1}$ Max Müller. The Science of Language. 2nd edit.; and Leçons sur la science du langage. 
each refers. The song of the nightingale or the sound of the violin is cognitive only when we have the visual or symbolic image of the nightingale or the visual and tactile image of the violin.

Whilst the visual and, to some extent, also the tactile zone furnish us with the immense objective material of nature, to be translated into language, the auditory zone provides us with emotional material, with which nature

on these. The author has seen Ethiopian paintings of the Madonna in which the abrupt change from blue to yellow and from green to red was very noticeable. Painting, introduced perhaps by Cleofante Corintio, was monochromatic. It became transformed into the polychromatic, with fundamental colours, in the Etruscan ceramies and in the paintings of the Pompeian period. Even the preRaphaelite school shows a relative poverty of tints. Perceptive insufficiency with regard to colours characterises the works of art of certain painters ; and Daltonism, now quite common, reveals the late evolution of the sense of colours, even in some individuals of more advanced races.

Vory much the same thing may be said with regard to hearing and music. The human voice, produced by the most perfect musical instrument, expresses in the articulate word the language of thought, and in song that of the emotions. Music and speech have sprung from one and the same stem - the modulation of the human voice. Rhythm, as it is in nature, contributes to the passional tone. The Adagio, Andante, Allegro, Presto, and the inflexions, depending as they do upon infinite variations of movements of the vocal cords, reflect in song all the human emotions -love, hatred, tenderness, contempt, jealousy, joy, anguish, disdain -in short, the whole gamut of pleasure and of pain, the two fundamental sentiments.

The songs that accompanied the dances of our ancient forefathers were monotonous, and had a good deal of the recitative about them. Even in our own day the songs of savages are impressed on monotonous motifs in which rhythm is the prevailing feature, accompanied by exaggerated gesticulation, leaping and shouting. Mythological and heroic legends werc expressed in rhythmic language full of metaphor, prone to awaken intense passional states, and were sung. The harp, the lyre and the flute of the Egyptians, the lyre of David which exercised such an influence over his people, the numerous ehoruses of Euripides which provoked veritable accessions of eestasy in women, and the musical triumphs of Nero, were very far removed from the eomplex harmonies and the infinite inflexions of modern musie, which reaches a marvellous sentimental height. One can notice to-day a tendeney of modern music to free itself from the human passional element in order to aequire an independent dominion, a new creation of the human spirit-a sort of musical sestheticism. Similar arguments are applicable to smell and taste.

Everything points to a slow evolution of the intellect and of language. It is reasonable to suppose that many roots were the same in all parts of the world where human beings were to be found, because the struetural conditions that gave rise to the first monosyllabic sounds were identical. There is no doubt that variety in external nature did not fail to exercise a great influence both on the development of the brain and on onomatopœia, but it is equally certain that the blowing of the wind, the crashing of lightning, the murmur of running water, wero the same everywhere. The analogies and the differences found by glottologists can be explained along these lines.

Tho law that ontogenesis (development of the individual) is a recapitulation of phylogenesis (development of the species) is confirmed by the evolution of language in the infant, for there we have a synthetic presentation of starting points that are common to all human languages. Briefly, the first linguistic manifestations of the infant are the sounds $a$ and $e$; in the adult $a$ is expressive of pleasure, and of suffering. The senso-muscular mechanies of $a$ are more simple than those of $e$, but 
plagues or soothes our senses, and with linguistic material which has gone on accumulating through a long process of evolution, facilitating the rapid and marvellous development of thought.

The content of language emanates from the field of the concrete images on the one hand (sensory zones) and from that of the abstractions, syntheses and logic on the other. The development of a train of thoughts, in logical

neither sound has any ideative content in the case of the infant; the consciousness is just beginning to dawn. Later on, the first labial sounds appear on the scene, $m$ and $p$, in combination with vowels, forming syllables which have no other significance than that of a reflex motor impulse and imitative activity as exhibited in the infant, which commences to find pleasure in a certain acoustic effect. Subsequently the infant pronounces the sounds $m a, a m, n l, m l$, ach and $b$. Before the end of the first year it recognises objects and some persons. There is a commencing distinction of the ego from the external world. The infant shows a distinct tendency to pronounce words, but the psycho-motor apparatus has not yet attained such development as will permit pronunciation of bisyllabic, trisyllabic or still more complicated words, and it therefore simplifies them. Thus, it does not say "mama" from the beginning, but simply " ma-a-a," the second syllable being vowelled. It has always seemed to the author that one might perceive in this sound, which is amongst the first utterances of our infants, the root $m a$ of man's infancy, from which thousands of words have been developed.

Features that are constant in the development of the language of the infant are syllabic shortening and vocalisation of words. Mothers, who (like Paola Lombroso), observe carefully and fondly the mental development of their children are well aware that the first words are monosyllables and that bisyllables come after these. The infant says "kolat" instead of " chocolate " (Taine) or says " amama" instead of "grandmama." It is not too much to suggest that one may detect, in this phase of language in the infant, some of the characters that belonged to the initial periods of languages that are now highly advanced, and are to be found even to-day in the present grade of development of language amongst some Hottentots and certain Australian tribes. In these tongues or dialects there is a prevalence of monosyllabic words and cries.

Even in Europe there may be found, here and there, small groups of men and women who have rema:ned in a poor and primitive condition, incapable of pronouncing well words of four syllables. The infant, again, changes vowels and consonants, and even substitutes syllables, and in this fashion presents a miniature view of the long course that has been followed in the period of formation of modern languages.

The vowel sounds are not decisive but at most intermediate-io, au, ae, iau, ou. The consonants are often changed, instead of "cream" the infant says "team." This may also serve to explain why the same roots may have acquired different sounds in passing through the dialects or primitive tongues of different peoples. It may also serve to explain how it came to be that the Sanscrit "spac" became "scep" in Greek. The evolutionary process is the same in the development of the language of humanity and in that of the infancy of modern man.

Another argument in favour of speech having lad its first beginnings in vowels and then in monosyllables is to be drawn from pathology. We meet with human beings whose brains have not reached the grade of development that is common to other contemporary men of the same race, but have been arrested at one of the lower levels. The general law is that degeneration retraces in a retrograde direction the various phases that have been passed through in the course of evolution. Thus it happens that we may detect, in the psycho-anthropological degradation of modern man, the various phases of dovelopment belonging to evolved tongues. 
order, is possible only by means of language. Language also translates emotions and sentiments, movements, conduct, and all states of consciousness into symbols. Despite opinions to the contrary, there is good reason to believe that language normally summarises intelligence, and that there exists an anatomical intellectual field distinct from the field of speech considered as such. Speech may be disturbed not only as the result of injury

Many of these patients can be arranged in series. Some show only a great poverty in their vocabulary, but are able to pronounce all words well, even although they do not understand their meaning. Others are incapable of pronouncing words of complicated construction. They can utter not more than three syllables with some syllabic shortening, or some syllabic sounds are uttered as vowels. Others, again, simplify several syllables, making them a vowel-e.g. maoni for macaroni, dottoe for direttore. Some express all the syllables as vowels without shortening the word. I have had under my care a classical micro-cephalic whose brain weighed about one quarter of the average normal brain weight. At twenty-four years he could make use of only two syllables, $m a$ and $p a$, and of some animal calls by which he expressed the fundamental emotional states of pleasure and of pain, and some few desires.

It should be noted, however, that in some modern languages, even the more evolved, like French and English, one notices a tendency to simplification of words and to syllabic contraction by a law of adaptation. Thus the Latin word episcopus becomes by a series of simplifications eveque in French; conoscere becomes in English "know." This is due to a selective capacity and a tendency to spare energy, and has nothing in common with the abbreviation of words that is met with in those whose nervous organs have not been developed to the extent requisite for the co-ordination of the movements necessary for a complete and precise articulation of the phonetic symbols of thoughts.

In some diseases, eonsisting in a slow and progressive degeneration of the brain, one ean trace the various steps in the involution of language, from mere verbal impoverishment to vowelisation and monosyllabism. The story of writing offers us truly interesting proofs regarding the question of the genesis of language. Man has been brought by his structure to reproduce his images, emotions and thoughts in a sensible form, no matter whether these have been got from the eternal and varied language of nature or whether he has formed them in his own mental world. If he imitates forms and figures, he initiates art; he carves out the lion-hunt on the Mycenæan dagger, and rises to perfection in Phidias; and when his imagination reaches the highest point of genius, he produces marvellous paintings such as those of the vault of the Sistine Chapel where, one might say, reality " $\grave{e}$ rifatta si come pianta norella." If he translates his thought in to graphic symbols, he creates writing; but this was preceded by other symbols-the mnemonic signs and the symbolic objects.

Symbolic objects are still in use amongst the Malays of Sumatra, and consist of packets containing pieces of salt or powder or smoking herbs which respectively signify love, hatred and jealousy. The message serves to express one of these sentiments. A system of mnemonic tokens had reached a high grade of perfection among the Wampums of the Redskins. They include a kind of beaded rosary made with shells of different colours, or embroideries on ribbons which serve also as belts, or message sticks, very common amongst the Malays, the Niam-Niam and other peoples. Incisions on trees or pieces of wood represent the first step towards writing, properly so ealled. In the sepulchral grottoes of the quaternal period at Aurignae (Dordogne) tablets have been found bearing symbolie signs like those actually prevalent amongst the Eskimos, the Yakuts, the Macousis of Guiana, the negroes of the West Coast of Afriea. Knotted eords are in use amongst the 
to the organs immediately concerned in speech-mechanism but also when the mechanisms that are formative of the mental content are injured (concrete images and abstract ideas), and in addition when there is interruption of the paths which connect the various fields of images and notions and render possible the logical process.

It is true that, in diagrams, we are compelled to represent the intellectual

Ostiakis, the Angolian Negroes, the Malgasci. The ancient Peruvians used to express happenings and ideas with the aid of knots differently made and variously disposed. To each knot on the cord there was attached a thin cord, and each thin cord had a different colour, all having several knots diversely arranged. The knots, the colours and the thin cords had a meaning, symbolic of thoughts and sentiments.

Afterwards came writing in the form of designs expressing a certain number of ideas, such as is found amongst the bushmen and the Australians, the so-called pictography.

From pictography was derived figurative writing in the form of the hieroglyphics of the Egyptians, Mexicans and other peoples. Hieroglyphics are designs of objects the first syllable of which forms a part of the word it is desired to express. These figures had thus a phonetic value, and one can follow the transformation of the Egyptian hieroglyphics and the cuniform writing of the ancient Assyrians. The first syllabic writing appears to have been made by the inhabitants of the island of Crete, this evidently being more ancient than that of the Phonicians which was derived directly from pictography.

With the appearance of writing we get a sign of distinction between barbarism and civilisation. When writing assumed a definite character and came to fix conventional and indelible signs of thought and action, the rate of human progress accelerated enormously, because writing transmitted to successive generations the thought, sentiments and knowledge of the generations that went before.

In retracing the story of human evolution, one of the things that strikes us most is the constantly increasing tendency to substitute movements of spoken and written speech for the coarser and grosser movements by means of which primitive men reacted upon the external world and exchanged with one another their thoughts and emotions. In proportion as the capital of cognitions increases and sentiments are renewed, we get a preponderance of the movements concerned in spoken and written speech. Writing affords a means whereby the conseiousness of young generations absorbs much more quickly, and becomes integrated with, the thought and work of those that preceded them. By means of writing man communicates with all his fellow-beings scattered throughout the world.

The old mechanism and simple methods of our predecessors would be insufficient for the needs of to-day. Modern man is not an isolated and independent unit but forms a part of the whole, to the life of which he gives his contribution, and from which he draws the elements of his existence. Every intellect, as it assumes its own character and form, detaches itself from the Milky Way of humanity, which is always in process of formation, and goes to form part of a planetary system, the laws of attraction and repulsion of which he must necessarily obey.

To-day the solitary man is either a primitive or a sick man. One can only look upon him as a discordant note, a hindrance to the evolution of the race.

Writing and, through it, the press form the great arterial system in which circulates the animating current of human solidarity. The old individualisin is ridding itself of the old futilities and is rising to the dawn of a new era in which new sympathies, embracing extraneous as well as personal interests, are being kindled in every conscience.

It is needless to discuss whether the first journal was that which appeared in Venice in 1536, according to Voltaire, or in 1760 ; the difference, with respect to the 
field as a circumscribed area, but it should be remembered that it really embraces all the sensory as well as the frontal cortex.

Intellectual complexes and empirical images are bound up with words. The words " pen," "inkstand," correspond each to its respective empirical image in the visual zone ; the words " puff," " creak," "whistle," to their predominant sensory content in the auditory centre; the words "rough," "smooth," " hectogram," clothe corresponding images formed by the tactile and muscular centres. The words "school," "war," "humanity," have their conceptual content in that part of the brain which synthetises the large number of concrete images, emotions and experiences that go to constitute the corresponding conceptions. The suppression of any one of the sources of images produces a mnemonic disturbance of speech and, if the image which has disappeared is not substituted by another connected with the same word, the word cannot be recalled.

Substitution is quite common. If, in the night-time, we hear the tolling of a bell, the word "bell" comes up before us, brought to consciousness by the anditory percept. The same word comes to our lips during the day when, passing near a belfry, our eyes light upon the bell; one of these images alone is sufficient to recall the auditory or kinæsthetic image of the word "bell."

The image of the word "rose" is awakened when we smell or touch a rose

time employed by human evolution, is a negligible quantity. It is certain that a journal was published in Antwerp in 1665 and in Germany in 1616. The Weekly News from Italy, Germany, etc. first saw the light in 1622, and the first French journal appeared in 1631. When we reflect that the number of letters posted throughout the world in 1888 was $1,700,000,000$, and that to-day this figure has been multiplied more thn fivefold, when we know that 800,000 tons of letters and newspapers were posted in Europe alone in 1883 and that after twenty years even this was more than doubled, we cannot help wondering what life will be like to-morrow, as regards human thought and sentiment, human labour and production.

Whilst primitive man had to conserve and defend his body only, modern man has to develop, conserve and defend a whole moral world of inestimable value.

Reflex movements have become greatly complicated and transformed into the very delicate mechanisms of articulate speech and writing which, though still movements, lead the eommunity to action that is always more complex and more multiform.

The spoken or written word synthetises and condenses the great potency of modern man. It possesses all the arms that are requisite for conquest and dominion; it is grief or joy, if it wounds or caresses; it is love, if it translates into articulate sounds the sympathy of two beings; it is suggestion, if it transmits thoughts, sentiments or actions to others; it exalts and humiliates, wounds and kills. It is music and song in the harmony and rhythm of the voice; it represents darkness and splendour, peace, tempest and hurricane; it penetrates nature and lays bare all its beauties, the sublimely awful and the terribly threatening. It is painting or sculpture when it presents to us the forms and colours of objective realities or of the products of phantasy in the infinite varicties in which genius coneeives them; it is scienec if it translates the language of nature and its forces; it is eonsciousness when it communicates to us the notions and emotions of our fellow-beings; it is the synthesis of the universe, in so far as the latter is perceived and spiritualised in the busy workshop of the brain, which perennially reproduces the world in the form of thought and speech. 
in the dark, or when we see it from a distance. Cases occur in which an extensive cerebral lesion destroys one or even two areas of images that refer to the same object, and yet the word persists. One single area may thus suffice to reawaken the word, especially in the developmental period, if the remaining healthy part of the brain attains a great compensatory development. Thus is explained the intelligence of some blind people, based as it is on tactile and muscular memory, as exemplified in the well-known cases of Laura Bridgmann and Helen Keller.*

In such cases the cortical centres of the injured sensory organs have already assumed relations with other cortical fields, so that the construction of mental products by means of the associative paths is more easy than it would otherwise have been.

Great elaboration and fusion of the various percepts depends not only on irradiation from one centre of perception to all the other sensory centres but also on convergence of the products of the various perceptive centres upon one common area, that of language and of the frontal lobes.

Most authorities who follow Broadbent and speak of an intellectual centre distinct from the area of language (Kussmaul, Charcot, Bernard, Ballet, Lichteim and several others) speak entirely from an abstract point of view, and the diagrams they have drawn are pure abstractions unaccompanied by adequate psycho-anatomical explanation.

The localising doctrine that those authorities put forward does not find favour with many other neurologists who speak with no less authority. Bastian, e.g., declared that he was unable to find from the clinical data any clear evidence proving the existence of a defect of speech which could be explained by supposing the existence of a lesion in the centre for the concepts or in the course of its afferent or efferent fibres. ${ }^{1}$

The existence of areas for images of objects distinct from those for images of speech is proved by the fact that the word-image is quite distinet from the image of the object itself, to which it refers. It often happens that we see an object or evoke its image without being able to name it, and,

* The case of Helen Keller (Giulio Ferreri. Lo sviluppo della intelligenza nella privazione simultanea dell'udito e della vista. International Congress of Psychology 1905), shows that the intellect can only be developed by means of the senses; that when some senses are suppressed their functions may be compensated by increased activity of other senses. What is of more importance, however, is the fact that Helen Keller became blind and deaf, and therefore also mute, at the age of nineteen months-i.e. at a time when the sensory centres of hearing and of sight had already attained a certain degree of development, when they had organised and stored a capital, small though it was, of auditory and visual images of things and of words, sufficient to furnish an important starting point for the recognition of the external world by means of other senses and subsequent mental constructions. I have often noticed that, in the case of the blind, the mind develops more readily in those who become blind some years after birth-i.e. after the cortical fields have attained a certain development and have actually exercised their functions.

${ }^{1}$ Bastian. Aphasia and other Speech Defects. London, 1898. 
similarly, we often remember the name of an object whose image we cannot recall.

Clinical observations prove that, in the case of certain lesions, the faculty of recognising common objects by means of sight, touch or hearing may be preserved although the name cannot be recalled. All this goes to show clearly that the two images are distinct, and furnished by two different anatomical fields, associated with one another. Further, the story of the development of thought and of speech provides additional proofs of the soundness of this view. The infant has already acquired a significant capital of images of persons and of objects before verbal images of these are formed. Animals, too, which do not speak, recognise a number of things and persons.

In this regard, the abstract concepts present a much more difficult problem. These cannot be recalled except by means of the sensible forms of the respective words with which they are indissolubly bound up. There is no doubt that we are able to produce the image of a man without need of the word " man," but we cannot in any way represent to ourselves the concept of humanity without the word "humanity." No doubt we can figure to ourselves humanity by dividing the abstract concept into concrete and separate images of different men and different human attributes of which it is made up, but here we no longer have an abstract conception. The coalescence of the word with the abstract concept and the impossibility of separating these do not warrant us in denying that each is formed in a different area. From another source we can draw good reasons for considering these two factors as separate, both in mechanism and in site. An imbecile, e.g., can learn to pronounce the word "humanity" without having any conception of " humanity," just as the infant calls everyone he sees "Dada" because he does not yet possess the abstract concept of "father," and does not perceive the difference between other men and his father, even though the latter caresses him much more. If, then, the word can be learned and pronounced, completely void of any conceptual content, both by infants and by imbeciles, and if it is true that even dements, when their intelligence is resolved into its elements, may pronounce many words that have no longer any meaning to them and are quite void of conceptual content, the concepts having been lost in the wreckage of mind, one must conclude that the words which clothe the concepts have a distinct seat of formation and that the concepts may disappear and the words remain. The abstract conception is the product of fusion of the concrete images into which it may be decomposed, if we seek to analyse it-images which all vibrate together on the threshold of consciousness and in the anatomical field of the word which symbolises all of them. If, however, the word disappears, owing to a lesion of the area in which verbal images are formed and conserved, the concept can no longer be reproduced. At most, it may remain in a latent state because all the material that goes to form it remains intact and capable of being reproduced. From all we have said on this subject it is clear that all the sensory areas of the cerebral mantle, besides that of the syntheses and of logic, with which we will afterwards deal, must be regarded as intellectual fields of language, 
inasmuch as they furnish concrete images of concepts which constitute the content of speech. This view facilitates our comprehension of the different varieties of amnesic aphasia.

The auditory and visual sensory images of speech are found to be composed of a series of sensory components which by their combination form at the outset the image of the object, and subsequently the verbal image. If these components are evanescent, so that they do not succeed in becoming combined and represented by virtue of their own inherent power, or if they lack vividness, as some psychologists say, the image of the word will not bo recalled. The concrete image, e.g., of a key and the image of the word "key" are made up of a series of sensory components-the form of the key (visual image), the weight and the tactile image of the key, and then the image of the iron of which the key is made, the noise of the key (acoustic image), when a door is locked, as when Dante makes Count Ugolino say: "Sentii chiavar l'uscio di sotto a l'orrible torre," the thermic sense of the key when taken in the hand, and especially the muscular sense of the movemients involved in the action of turning the key in the lock.

If these images are evanescent and almost beyond our grasp, if the nervous waves do not reach their destination owing to defect of initial energy and necessary intensity, the image of the key will not be capable of recall or, if it is represented, the word " key" will not arrive at the focal point of pronunciation. One can here invoke the physical law that resistance is in inverse ratio to the excito-motor force. If, in these cases, the memoryimages are intensified by actual stimuli (as when we put the key into the hand of the amnesic patient), the word in question soon becomes recalled and pronounced, especially if the amnesic person goes through the action of opening a door. We have here an example of asthenic amnesia.

If we admit the existence of distinct fields, consisting of specialised elements, where images of objects and verbal images are formed and registered (auditory, visual and kinæsthetic images), we must agree that a paretic condition, not to speak of actual destruction, of the various fields, must consequently bring about an incapacity to reproduce a more or less considerable number of the respective images. It is in this way we get various forms of amnesia or amnesic aphasia.

Sometimes it happens that individuals who formerly were ready and felicitous in their use of the store of words they had acquired during the period of education arrive at a time in life, often not very far advanced, when they lose that faculty that made them ready, subtle speakers, rich in linguistic resource, able to adapt to each thought the adequate word and give to each object the proper name.

There are some individuals who have a highly educated visual centre of speech, as the result of extensive reading which has enabled them to accumulate a rich store of notions, which is at the service of the whole intellectual and linguistic mechanism. If, however, they read so largely that they come to throw an excessive strain on the visual centre, they begin to experience 
difficulty and sometimes an incapacity to reproduce, with the same promptness as formerly, the images which render their language truly rich and incisive.

If these forms of verbal amnesia have hitherto escaped clinical analysis they are nevertheless of great value in helping us to a better understanding of the functional mechanism of the brain. Cerebral fatigue and depressive states of mind furnish numerous and various examples of the dynamic (physical) law which regulates the function of the brain.

The intrinsic constituent elements of speech, considered per se, now deserve some attention.

In the modern man who can read and write, these components are at least three in number: (1) the auditory image and (2) the visual image of speech, these being impressive components ; (3) the articulatory-kinæsthetic image and, according to many, the graphic-kinæsthetic image (expressive components). Authorities are not all agreed as to the relative importance to be attached to these components. While Charcot and the writer have recognised the greatest formative and regulative power of speech in the acoustic images, Stricker, Bastian and some others accord the highest importance to the articulatory-kinæsthetic.

Equal importance may be attached to both. No doubt the subjective method renders great service in the study of language, but its value should not be exaggerated.

In the case of those who read and write the visual verbal image comes into play, and in some men it plays a preponderant part.

These three or four categories of images have their cortical fields in the evolutionary zones of the respective fundamental sensory and motor areas, as mentioned in Chapter I.

These cortical fields are linked up with one another by means of associative fibres, and it is very natural that interruption of these paths, by breaking the communication between two or more groups of cortical units, should hinder the formation of the complex product which, as a matter of fact, results from the combination of elementary functional products of many cell-groups. A considerable amount of careful analysis has served to make clear the great importance of the association-paths, and has enabled us to distinguish a number of different clinical conditions which correspond with lesions in the various paths. These are the subcortical and transcortical aphasic disturbances.

The process of association which gives rise to speech considered per sei.e. independently of the content of thought-is governed by fundamental laws, amongst which the more evident are (1) the hierarchic preponderance of one over the other components of language; (2) the law of individual variety. It is a probable hypothesis that the phonetic or acoustic centre of articulate sounds is the first to be differentiated. Even in the infant the first articulate sounds are only fragments of the words heard. It is common knowledge that only at a much later period is the infant able to repeat and 
articulate a word, the phonetic image of which has already been registered for some considerable time. In this way there come to be established not only relations between the acoustic centre and the centre of articulation of the word, wherever that be, but also a species of regulating influence exercised by the former over the latter, the articulate sound manifesting itself, as it were, under the protection of the acoustic image of the word. Should the acoustic image fail to be represented, or should there be interruption of the associative paths, so that the acoustic nerve-waves fail to reach the cortical motor groups, the word will be entirely lacking, or will be altered in form.

The acoustic image exercises, in addition, a regulative influence over the visual image of speech. Under present-day scholastic methods the infant does not learn to read until after the acoustic-motor organ is functionally perfected. Only at a much later period are the visual images of letters, syllables and words modelled on the acoustic, and perhaps also on the kinæsthetic, images, and they will not be recalled except by the acoustic or kinæsthetic images, or both together.

This law has an important bearing upon questions concerning aphasia, as also upon questions of psychology. It serves to explain individual variations. It is a fact that word-blindness very often results from a lesion of the auditory area of speech, even when the visual area of speech has been spared.

The mechanism of language, as already mentioned, is much more simple in illiterate persons. In them, only the acoustic images come into play. These excite the motor images, which, in turn, are resolved into co-ordinate movements of the apparatus concerned with speech articulation.

In educated persons the mechanism becomes more complex, inasmuch as other factors, varying in importance and value in different cases, become associated with the two just mentioned.

If we follow the different stages in the education of the infant, as it learns to read and write, we can readily verify the fact that the graphic symbols (written syllables and words) have their correlatives in the auditory symbols with which the child is already acquainted. A relation is consequently established between the acoustic image and the visual image of the word, the former preponderating over the latter, because the graphic image has no significance for the child, apart from that given to it by the acoustic or phonetic image. So intimate does the association between the two become that it is difficult for the visual image to be represented alone, without determining the pronunciation of the respective word.

In the majority of those who are more or less practised in reading, the sight of an object does not recall the graphic symbol unless through the acoustic image, the paths of communication of the latter being more exercised and more pervious. Heredity, education, occupation and practice, besides other contingencies in life, may nevertheless reinforce the visual wordimages to such an extent that they acquire a very high value in the physioanatomical mechanism of speech.

Once the system is constituted and dynamic relations established between 
the various fields, it is clear that disappearance of one of these will induce a disorder in the function of all the others. It is clear, too, that this disturbance will be more pronounced the greater the regulative influence exercised by one over the others, and consequently over the movement of thought. Suppression of the acoustic image gives rise also, in the majority of men, to inhibition of reproduction of the visual, the articulatory-kinæsthetic and the graphic-kinæsthetic images.

Disappearance of the graphic-visual image leads to inhibition of the graphic kinæsthetic centre of speech. Not infrequently it gives rise at the same time to disorder of the acoustic eentre of speech which, as a rule, functions in concert with the visual centre in men accustomed to reading, and sometimes it causes also a significant disturbance of the kinæsthetic centre of articulation. This disturbance is more severe the higher the degree of evolution attained by the visual word-centre.

Sollier adopts the hypothesis of Flechsig to explain the mechanism of language. The auditory and visual word-images would not reside, as it were, in the centres of reception but in other localities. These centres would really rank as associative centres, and destruction of them would produce worddeafness and word-blindness, not because the formative organ of the linguistic images would be destroyed, but, rather, on account of destruction of the organ which has the function of transmitting the vibrations that determine a particular state corresponding to the excitation, external or internal. Reference has already been made (Chapters I. and II.) to the obscure nature of this hypothesis, which is not supported by any facts which would give it a claim to probability.

As explained in Chapter I., evolution of language has necessarily been accompanied by development of the sensory areas. The auditory (temporal) field extends its boundaries and develops an area which, though still auditory, is yet concerned particularly with the construetion of acoustic verbal images, or word-symbols, of all the thought that has been gradually elaborated in the other sensory areas, with material derived from the external world. It comprises a good part of the middle and posterior sections of the first and second temporal convolutions. Similarly, the visual area extends forwards, in front of the visuo-psychic area, for the construction of graphic wordsymbols of the images of objects and features that have been elaborated and conserved in the various sensory areas, including that of the acoustic wordsymbols. Thus it comes about that men who are near one another can understand each other by speaking, whilst men far removed from one another can understand each other by writing. So, too, the patrimony of one generation or epoch becomes transmitted to succeeding generations, to become material of transformation in the renewed mental mechanisms of those who come after, continuing the process of evolution. Every simple image, every complex image, every synthesis of thoughts, on a progressively complicated scale, becomes clothed in a linguistic, sensible form, and is thus 
rendered capable of being transmitted to other beings. The cerebral mantle becomes evolved and perfected with the transformation of old and the establishment of new workshops which every day accomplish an increased amount of work, owing to the more plentiful and varied material supplied to them, in consequence of the growth of interhuman relations. It is not the case, as Flechsig thinks, that the language-areas thus become the organs of transformation of external and internal realities because, correctly speaking, realities take their form in the sensory areas. What happens is that, in the linguistic mechanisms, facts become connected together into a logical series, for the purposes of human interchange of thought. All external and internal facts pass through the auditory field of language, and are thence transmitted to other men in a sensible form and in logical relations, in the guise of acoustico-motor and visuo-graphic verbal symbols. When the cortical organs of speech suffer any severe injury (well-known pathological processes) the whole mental capital becomes interdicted and its natural movement hindered. This capital, or, in other words, the accumulation of images which reflect reality, remains intact in the sensory areas but is not utilisable, because there are lacking not only the names which symbolise them but the material means of conjunction necessary for formulating complex judgments, in logical series. The aphasic, if not markedly hemiplegic, rises in the morning like any other man, puts on his clothes (he recognises them and their use), goes to the lavatory, washes himself, dresses himself, makes his way to the dining-room and takes his breakfast. If the coffee is bitter he recalls the image of sugar and procures some. He thus formulates immediate judgments- the coffee is bitter, sugar sweetens coffee. Judgments of this kind are possible to this man, just as they are to a dog. To formulate them it is quite sufficient that he have the images of the things connected with the immediate sensation of sweetness or bitterness. As, however, he does not possess the word-symbols of the coffee, the sugar and the persons, and, what is more important, the word-symbols of the relations between the things or their images (verbs and their inflexions), it is quite impossible for him to say: "The coffee is bitter. Bring me some sugar." If, at one time or another in his life, he has taken coffee with a family in Constantinople, he may remember the aroma of that coffee and compare it with what he gets at home (the centres of taste, smell and sight are perfectly capable of furnishing these particular images and records); but he cannot engage in conversation on this subject; he cannot formulate such a simple sentence as: "The coffee at Constantinople was very fragrant," because he lacks not only the words that express names and qualities (predicates) but also those that express relations between objects and relations as to time (verbs). The intellectual material (the concrete notions) is preserved but remains inactive and interdicted. This is a variety of dementia the feature of which the author has several times sought to differentiate. In $1887^{1}$ he had occasion to

${ }^{1}$ Bianchi. "Un caso di sordità verbale. Il metodo pedagogico nella cura della stessa." Riv. sper. di Fren. 1887. 
write: "Unless I am mistaken in my study of this patient, there is good ground for advancing the opinion that in severe word-deafness the intellect is only potentially sound inasmuch as there is no damage to its material constitution, such as we find in primary or in terminal dementias : its constituent elements, indefinite as they are, no longer awaken states of consciousness, but remain buried in the subconscious; from this they may be evoked into the field of consciousness whenever this is reached by the corresponding sensible word-symbol with all its associative relations." In much more recent times various hypotheses as to the relations between intellect and language have been put forward, but they are not all clear or convincing. Dejerine, ${ }^{1}$ e.g., maintains that the act of thinking may be effected in two ways-either with the images of things or with the images of words (internal language). Even this conception of Dejerine, however, does not meet the case ; it is not at all complete. Such thought as consists of the images of objects alone is a very simple, rudimentary form of thought, and is only possible when the word-symbols are not reproducible. It is only by the aid of word-symbols that thought flows freely through all the paths and follows all the rules of logic. Dupré is more exact when he says that the two syndromes, dementia and aphasia, are two intellectual deficits, but whilst the former concerns the operations of the mind in general (memory, association of ideas, reasoning, affective or voluntary reactions), the latter has to do with the process of verbal symbolism, compromising the existence and play of the mnemonic materials. ${ }^{2}$

We have already mentioned that the concrete images of objects are formed in their own respective seats, independently of the verbal images. This view has been excessively and even erroneously generalised. Mingazzini and Seglas ${ }^{3}$ hold that the word is an auxiliary of the image of the object (of the idea, says Seglas). The latter author writes: "The idea may exist without the word." One might agree with this view if we limit the signification of "ideas" to the concrete images of objects. It is to be observed, however, that abstract or general ideas, no matter what Dejerine and Seglas may think, cannot be represented in consciousness, unless fused with the verbal symbol. Sufficient has already been said on this subject in the preceding chapters, but it may be worth while to give an example. I can recall all the visual and auditory (phonetic) images of the Parliament Hall and the physiognomies of my colleagues, their attitudes when they speak, the calls of the President, the written sheets with the orders of the day, as things seen; but I cannot represent to myself the content of political thought of my colleagues which is made up of word-abstractions. If there fail me the words " parliament," " politics," " government," " presidency," etc., and the political syntheses of the parliamentary debate, which is entirely composed of words, the material belonging to the visual images remains almost inactive. One has also to take into consideration the

1 Dejerine. Pathologie générale de Bouchard. Séméiologie, tome v.

2 E. Dupré. " Révue policlinique des démences." Bulletin médical. 1907.

3 Seglas. Leçons cliniques sur les maladies mentales et nerveuses. 
great value of verbs and their inflexions in the logical process of thought.

Without verbs immediate judgments are possible, but not the formation of phrases and sentences immediately bound up with the logical development of thought. In the aphasic, what we call a train of thought is interfered with in its motion towards the focal point of consciousness so that it cannot reach it in logical connection with other thoughts, and hence there is no process of fusion into general ideas.

We have learned that images of objects are formed before images of words, as can be observed in infants, but, in the process of evolution of intellect, these images become blended with the word-symbols, and subsequently the word-symbols are conjoined with one another for the construction of phrases, when we get inflexion of words and the use of conjunctions. When once the capital of cognitions is greatly increased and associative relations are established between its component parts, when the mind embraces wider dominions, there commences the formation of sentences, and, later, we get narration-i.e. a series of sentences conjoined with one another around a central theme, which is detained in the focal point of consciousness. In this case the images of things remain on the first level, whilst around the theme there flow, from the different cortical fields, all the notions that have a bearing upon it and all the intellectual creations of which the mind, with its imaginative power, is capable, all in linguistic form. It is in the form of words that they cross the focal point of consciousness and contribute to the formation of thought. If we take the case of the parliamentary aphasic one may unhesitatingly affirm that he, being unable to recall nouns, predicates, and a very large number of verbs with their relative inflexions, will not be able to remember (reproduce) the content of the speeches made by his colleagues in the House when he was sound and well and able to participate in the parliamentary debates-speeches made up of abstract concepts, names of persons, concrete and general ideas, and of verbs. In such a case, what remains of intelligence? The interdicted material, and the images of the things by means of which immediate judgments may be formulated. The dog which displays exceeding joy when he sees his master put on his hunting boots and take up his gun may picture to himself the hare, the bird, the moor, the gun-shots, the dead game, etc., by a process of imagination (Romanes).

The judgments formulated by monkeys are much more complex, as in the case of the animal, mentioned by the writer, which, after being admonished, hid behind the couch and peeped out from time to time, when the author moved to another spot or had his attention fully occupied, so that it might seize an opportunity to jump upon the table and furtively snatch away the cherries of which it was fond.

In the aphasic patient the position of the intellectual process is much more serious because, whilst in the higher mammals the mechanism of the judgments is very simple owing to the absence of linguistic overlapping, in man, on the other hand, with his complicated mechanism interposed between 
the images of objects and all the forms of expression and of action, the latter lose that expressive fidelity which they derived, in varying proportions in different men (heredity, education), from the greater or less precision of the word-symbols. For this reason lesions affecting the cortical or subcortical areas of speech cause a conspicuous mental disorder. Thus, too, is explained the great frequency of amimia and apraxia.

In the case of single ideas of objects and immediate reactions, such as we find in the higher mammals, in infants, and even in normal man, there is some room for Seglas' notion (loc. cit.) that the image of the word is an auxiliary of the image of the object, or Mingazzini's ${ }^{1}$ notion that internal speech (representations of verbal images) is of great utility in the elaboration of concepts, although the association between ideas and verbal images is not indispensable. This would mean that both may have free course, independent of each other, without thought as a whole suffering thereby. There is here an evident lack of distinction between single images with simple reactions on the one hand and connected thought on the other, between single images and regular trains of co-ordinated ideas, characteristic of evolved human intellect, as manifested in talk and conduct.

This confusion of thought with images was and is the cause of many euphemisms ${ }^{2}$ which tend to hinder the correct interpretation of the facts. Some estimable neurologists unknowingly participate in the Platonic doctrine, maintained by realists and certain metaphysicians, to the effect that ideas are so many realities existing throughout eternity, that they emanate from God and are found in the world outside of us. Having made this allusion to doctrines of ancient time, the writer professes his partiality for the ideas of Aristotle who taught the peripatetics that words are signs of the concepts, which latter are derived from experience. More definite, though rather original, is the notion of Pierre Marie, who denies the existence of images of language and maintains that there do not exist any word-images either auditory, visual or motor. In the words of his pupil Moutier ${ }^{3}$ : " "Verbal images ' are words void of sense, they are metaphysical things which should be expurged from our vocabulary." If this were true we should be compelled to take refuge in the doctrine of Plato, or to entrust all these distinctions between images of things and verbal images of things to the science of physics, which might invoke the perennial motion and indestructibility of the electrons.

Any attempt to simplify the problems of mental life by the construction of diagrams is apt to be dangerous and to lead us into grave error. Nevertheless, the diagrams the writer here puts forward may serve to give a clearer understanding of the mechanism of thought and its reflexes in action and in speech. Let us consider the representation of the image of an object-e.g. an orange. The complete image is composed of several images formed in

1 Mingazzini. Lezioni di anatomia clinica dei centri nervosi. 1913.

${ }^{2}$ Brissot, G. A phasie dans ses rapports avec la démence. 1912.

${ }^{3}$ Moutier. Aphasie de Broca. Paris, 1908. 
different cortical fields: the visual, reflecting the form, size and colour, in the occipital lobe ; the tactile and thermic, in the post-Rolandic convolution ; the olfactory, in the limbic lobe; and the gustatory in the temporal pole. These four images reawaken one another, in a reciprocal fashion, in their respective cortical areas, by means of association fibres. In the representation, one image will prevail over the others, according as particular individuals (for various reasons, which it is needless to mention here) have developed one of the four senses to a greater extent than the others, and, therefore, one of the organs concerned in the production of these images. All four images

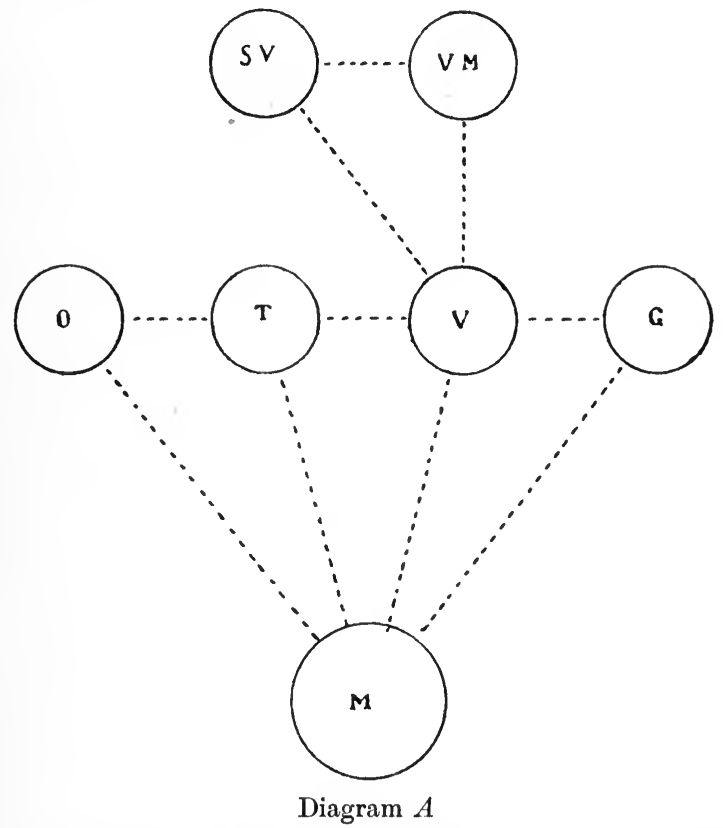

SV. Luminous image.-VM. Senso-motor elements necessary for the formation of the visual image.-V, O, T, G. Visual, olfactory, tactile and gustatory images.-M. Motor field for movements of advance and retreat

together give the concrete image of the orange. In the case of the infant, there are no images other than these, and we find them accompanied by a tendency to draw nigh or, perhaps, a movement of prehension.

Later on, the concrete image, or this group of images, becomes translated into the verbal symbol, the word "orange," which represents a fusion or synthesis of all the pre-existing memory-traces.

When we see, or picture to ourselves, an orange, all the four images, $\mathrm{O}, \mathrm{T}, \mathrm{V}, \mathrm{G}$, with the respective elementary components SV, VM, are awakened, and all tend to cross the threshold of consciousness. As the orange is a pleasing thing, a charge is determined in the motor area, accompanied by a tendency to approach and take the orange. The images $\mathrm{O}, \mathrm{T}, \mathrm{V}, \mathrm{G}$, acting through the mechanism of emotion and desire (about which we will talk elsewhere), arouse the image of the movement or movements necessary to reach and take it. To avoid complicating the diagram the circle $\mathrm{M}$ is made 
to represent the twofold physiological fact-the image of the movements and the motor discharge, the latter being given off from the motor centre and modelled in turn upon the images of the movements.

In the higher mammals and in the infant, $M$ is excited directly by $O, T, V, G$

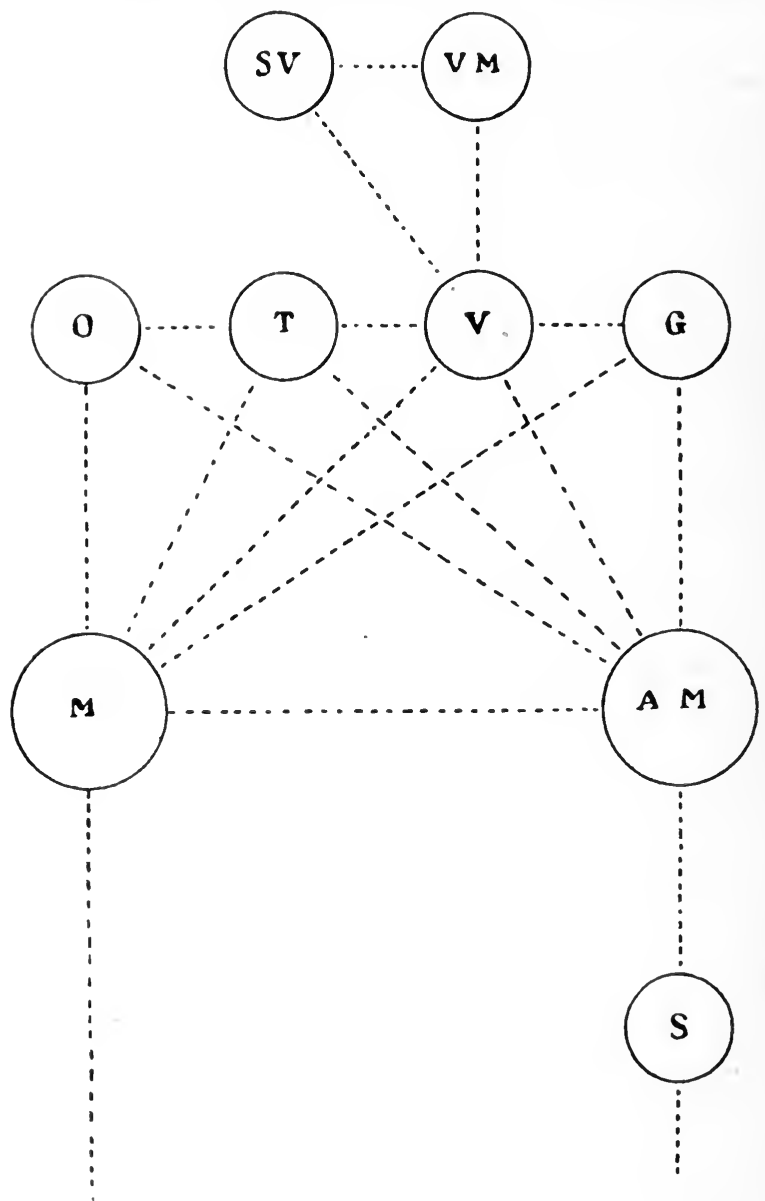

Diagram $B$

SV, VM, O, T, V, G. All as in preceding liagram.-M. Motor field for movements of approach animated by all or by one of the fields of the separate sensory images of the orange, O, T, V, G.-AM. Represents the field of the acoustic and motor images of the worl "orange" (shown as a single field in the diagram to facilitate comprehension of the mechanism, and to avoid complicating the diagram too much). $-\mathrm{S}$. Cortical and subcortical auatomical field for movements necessary in the articulation of the worl. Note that the new fields all assume associative relations with one another, and with the pre-existing

together, or it may be by one only of these, in this case the most strongly representative, or that most intimately connected with emotion and desire.

When language is developed the verbal symbol also assists in determining movements of locomotion and prehension. The mechanism is now more complicated (Diagram B). The representation of the verbal image does not 
really increase the impulse to approach, which rather arises from emotion (desire), but it is certainly a positive element which reinforces the image of the object and the determinism to advance or retire.

The separate images of the orange become inevitably associated with the verbal symbol, and all together arouse the function of M. At the same time, or a little later on, the acoustic verbal symbol in the infant becomes translated into the articulate word, a close association being established between the acoustic image and the motor image of the word. This new organ, which comes to be interposed in the mechanism, is represented by M placed in the same circle along with A, for simplicity's sake, in Diagram B. Subsequently, the human subject learns to read and write and so makes use not only of the acoustico-motor verbal symbol but also of the visual verbal symbol, thus adding still another organ to the mechanism, which is rather imperfectly represented in Diagram $C$.

All this work takes place in the sensory mantle, which includes the cortical fields of language or, in other words, all that part of the hemisphere lying posterior to the fissure of Rolando.

By degrees there is formed the abstract conception of citrus, which comprises a certain number of horticultural products which are very similar to one another, and as time goes on another concept, even more general, is arrived at, such as that of fruit. The word citrus synthetises as many different images similar to that of the orange as there are varieties of citrus-fruits, and sets in vibration all the corresponding fields, the respective images of which become directed towards the threshold of consciousness, although they may not actually cross it.

Suppose, now, that the modern evolved man studies to increase the size and aroma of the orange for commercial purposes. He is obliged to construct a series of judgments of similarities, analogies and differences between the known varieties of oranges, and to inquire into the chemical constitution of the soils, the influence of the climates, the exposure of the fields, etc. $\mathrm{He}$ obtains information regarding the markets on which he intends to place his goods and establishes the conditions necessary to attain his object. $\mathrm{He}$ calculates the various problems and then inquires into the means of transport, and gives attention to the question of preservation. All this work implies: (1) the possession of a considerable knowledge of agriculture, and hence of physics, chemistry and botany, and the power of increasing this by extending the inquiry into new fields (the experience of others which he obtains by means of reading and by verbal communications) ; (2) another series of cognitions concerning trade in oranges, the uses of oranges, and the tastes and habits of the various peoples who cannot produce these fruits, so as to select the best markets; (3) a third series of cognitions relating to methods of preservation during long or short transits by sea and land (all these cognitions are really series of mental syntheses, logically drawn from experience and imagination, and disposed in memory in accordance with personal observations and the observations of other people; (4) a capacity to detain the argument in consciousness or, in other words, the power of voluntary attention ; 
(5) a power to evoke and select cognitions stored, it may be, a considerable length of time, representing a long and varied experience in accordance with altered circumstances of time, place and persons ; $(6)$ a capacity to formulate ultimate logical conclusions - i.e. to form a synthesis of syntheses - as manifested in deliberation, determining action and conduct. The latter will be more or less successful, according to the capital of cognitions, the power of evocation and the associative and selective capacity, which together give rise to a series of actions best calculated to attain the object in view.

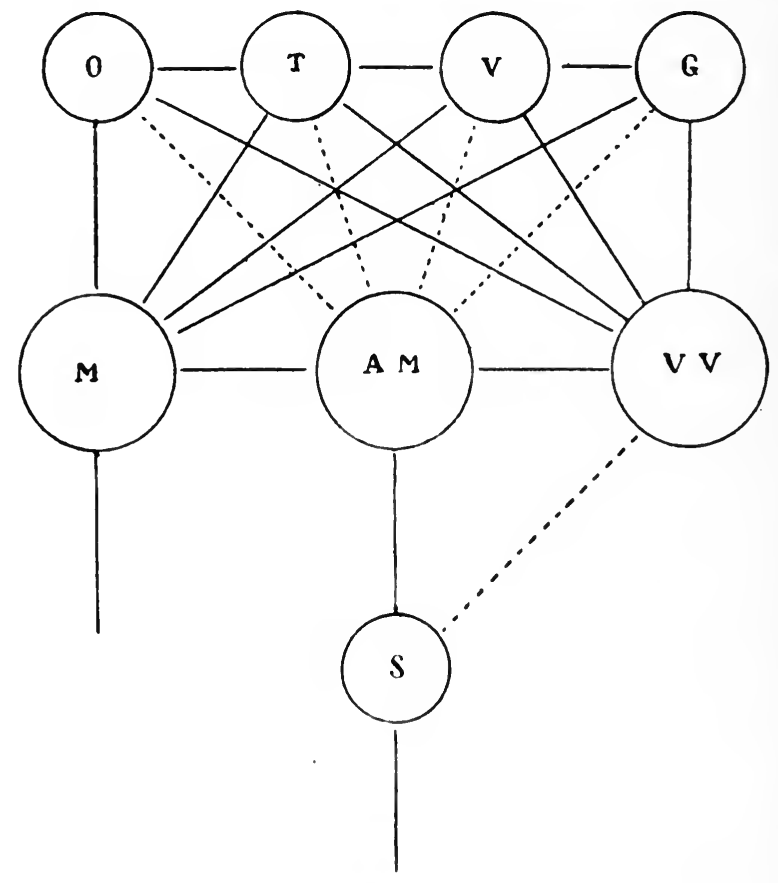

Diagram $C$

The diagram is the same as the preceding except for the fact that the primary tields, SV, VM, are not represented, whilst there is added a new field, VV, in those who have learned to read. VV, the graphical visual field, may also act directly on $\mathrm{S}$

All this immense work becomes translated into three words: citruscultivation, citrus-trade.

When we think of citrus-cultivation and trade in citrus fruits, there vibrates, more or less, throughout the whole brain, all that incalculable number of ideas represented by the three words in intensely synthetic forms which, in turn, are composed of other syntheses, no doubt less complicated but all inseparably combined with the respective words.

This last part of the cerebral work is performed with the aid of another extensive cortical field, that of the frontal lobes which are intimately connected with all the different sensory and linguistic fields. The latter furnish all the concrete images and the symbolic (linguistic) elements which allow of reasoning in the form of a complex train of thoughts, involving a long and 
logical process of development. The frontal field conceives the theme in a synthetic manner, detains it in consciousness and unfolds it by re-evoking cognitions relating thereto ; it exercises the power of selection, utilising the immense capital of concrete images and words, maintaining a logical connection between the various parts that compose the vast design and arriving

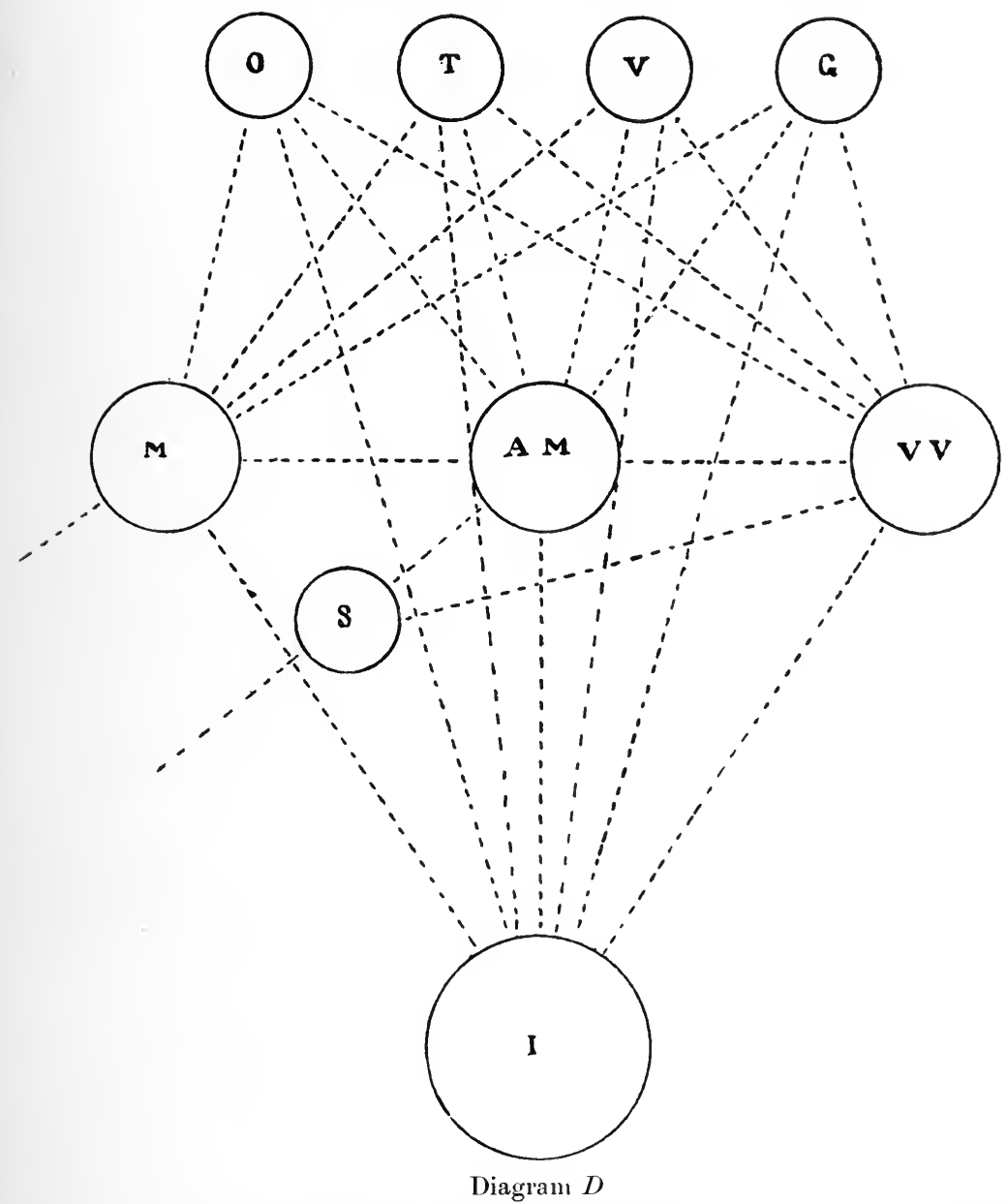

This diagram shows the field of the mental syntheses more fully than the preceding. I is the organ on which all the products of the other sensory and motor fields converge, for the construction of syntheses depending upon the power of re-evocation of the single images and groups of images

at a conclusion which becomes translated into a logical series of actions well adapted to achieve the end in view. So complicated is this mechanism that no diagram can give more than the faintest idea of it. In Diagram $D$ there is inserted a circle which is not present in the preceding diagram. It is intended to represent, in a diagrammatic and circumscribed manner, what cannot really be translated in a sensible form-the contemporary and successive operations of millions of cell-groups scattered throughout the cerebral mantle. 
"I" symbolises the frontal lobes-the organs of the ontogenetic and phylogenetic experience of the consciousness, nourished by records of the effects of previous actions, which undergo useful variations of adaptation: they are consequently, as already indicated, evocative and directive organs of thought and of action related to the end in view.

Experiments on the higher mammals and clinical observations of men who have suffered extensive lesions of the frontal lobes (foci, wounds, tumours) bring into evidence two orders of facts - $(a)$ the sensory cortical field continues to function normally so that individual perceptions are normal ; $(b)$ language and the simple reactions-i.e. those which do not involve any extensive reasoning-remain normal. The whole cycle of mental life in these cases is manifested in isolated actions to which the separate impressions give rise. There is an evident absence of mental co-ordination, whilst the representations are more or less isolated. There is nothing to indicate a true movement of thought, a co-ordinated process manifesting itself in speech and action, a preconceived objective and a capacity to fix this objective in consciousness and to select the requisite material from the mental capital.

The behaviour of monkeys that have suffered mutilation of the frontal lobes is strongly indicative of a suppression of all the manifestations of initiative and curiosity. This goes to prove that the experiment has resulted in suppression of the imaginative capacity, the evocative power and the determinism to think. The syndrome is complicated by irrational fear, errors of judgments, indifference towards persons and things, by a tendency to collect garbage and useless objects (like some idiots and dements) and by tics. Perceptive indifference, entire absence of initiative, lack of objective, incapacity to pursue a logical train of thought and pronounced emotional episodes (fear, anger, sometimes aggressiveness, leading to emotional reaction) are common features in the life of imbeciles. All this train of symptoms is fairly indicative of the function of the frontal lobes.

Anyone who has had an opportunity of examining a large number of patients suffering from the disturbances referred to, disturbances which exhibit themselves in all degrees of alterations of character, cannot have failed to be impressed by the fact that in every aspect of their intelligence, either in relation to the physical or to the social environment, the most prominent feature has been perceptive insufficiency in the sense that they are attracted only by the strongest stimuli, whilst their perceptive capacity fails to gather a number of the more specific features which are necessary for the complete cognition of objects and for the differentiation of one object from another that is similar or analogous. The idiot, like the mutilated monkey, takes a piece of chalk and, mistaking it for sugar, puts it in his mouth and does not decide to spit it out when he fails to detect the sweet taste of sugar, or, if he does reject it, does so grudgingly. One can easily play tricks upon him by offering him something resembling an object for which he has a predilection, and time after time he will obstinately attempt to get possession of it. Not only does he fail to perceive a number of features that are always detected 
by a normal person, but he is torpid and slow in his movements and shows an indifference to both persons and objects. He gathers up from the ground all sorts of useless articles, even filth, and puts them in his mouth; he will stop for a long time, without obvious reason, over a spot, a rag, or a piece of paper. Some idiots eat everything that is given to them. The absence of choice is frequent amongst them. Nearly all are easily frightened; they do not distinguish a pretended threat from a real danger, nor do they understand ridicule or appreciate the pungency of irony. They are unsociable and may or may not show an attachment to the person who gives them food and takes care of them. Rarely do they exhibit any spontaneous activity. They never take the initiative in talking, or even in some simple action; they follow no preconceived plan, not even when, as in the less pronounced imbeciles, speech is fairly well developed. They are egoistic and impulsive and often exhibit tics.

Their attention is weak and cannot be fixed on anything. They touch many objects one after another, and these they often break without exhibiting anything of that curiosity which is truly attentive and so characteristic of any normal child. Their memory is unfaithful and their action inconclusive, as also the content of their thought, which is evanescent. They hardly ever make use of past experience (even in the case of those few who possess a partial memory that is sometimes very remarkable). Association is very poor. The mental complexes are of surprising simplicity. They keep apart from others.

There can be no doubt that, just as the degree of mental insufficiency in the case of idiots is proportionate to the degree of subevolution of the layer of pyramidal cells (small and large), so, in cases of pathological or traumatic lesion of the frontal lobes and of experimental injury (in monkeys), the mental defect is proportionate to the extent of the lesion, provided it be bilateral. 


\section{APPENDIX TO CHAPTER VIII}

\section{Logic}

IN a previous chapter we have mentioned the fact that in the perceptive process is included a logical factor, inasmuch as every perception contains elements of judgment, and these reflect the reality perceived. The conclusion arrived at as the result of a perception becomes combined with the residual train of previous experiences of identical, analogous or different natures, also with the experience of the successive reactions provoked by the series of similar or analogous percepts, and with all the various judgments derived from notions with which the actual fact perceived has associative relations reflecting external reality or intellectual and emotional reality. I Logic, as a science, cannot be considered outside of the data and natural process of experience, based on memory and association. Individual or collective experience is wholly constructed of mnemonic residua, and it furnishes the content of phantasy (or imagination). Phantasy transports the consciousness into an analogous or different situation, in the future, a situation which is nothing more than a conclusion drawn from past experience and projected into the future, determining integrative or defensive attitudes or new creations. Foresight is the outcome of a logical process based on experience (memory, association and phantasy). The perceptive process furnishes an objective reality which becomes thought; the logical process reflects the association of objective realities in their relations of simultaneity, similarity, contrast and succession, in relation to the self which feels these realities, categorising and classifying them and reacting accordingly. It is from these relations that experience arises. Experience is essentially the outcome of series of reactions of the self, from the most simple to the most complex, either integrative or disintegrative, attractive or repulsive.

Logic, according to an old conception, is the science which examines, defines and concretes the condition and the procedure of exact knowing and correct thinking, laying down rules and laws which the intellectual operations must obey, if they are to be regarded as legitimate. It is unnecessary here to make acquaintance with these laws. It is of much greater importance that we should recognise clearly the reality itself, as it is revealed to us by experience.

Modern psychology seeks to discover and learn the laws of thought and the origin of the nexus between the parts of speech, not to lay down aprioristic laws of concrete reasoning. It is its business to examine the logical process of thought and of conduct in relation to the evolution of knowledge through the perceptive and reactive experience of life, and through the development of the capital of language, which is an essential condition of the logical process of speech. 
In this respect the evolution of logic coincides with the evolution of language. We must recognise an immediate logic corresponding to immediate judgments and manifesting itself in actions directed by the hedonistic law of life. Biophylactic coherence of conduct is logical but does not necessarily require language. My dog, e.g., has learned from experience in the diningroom that the same diner does not offer him two pieces of meat one immediately after the other, and so, as soon as he receives one piece, on he goes to make friends with another diner, from whom he obtains another piece of meat or some other eatable, and only later on, if time allows, does he return to the diner who gave him the first piece. In behaviour such as this one must recognise an immediate judgment, a summary form of reasoning which resolves itself into a series of rigorously logical actions. One cannot, however, conceive of reasoning such as falls within the category of successive knowledge (trains of thought), composed of syntheses connected one with another and with the object around which the reasoning process revolves, except with the aid of speech.

Even if we accept the evolutionist doctrine inaugurated by Darwin and Spencer it is needless here to dwell upon the various types of logic-formal, dialectic, genetic, etc. From our point of view we see no need for recognising differences in logical objects, as classified by Baldwin--e.g. hyperlogical objects (æsthetic) and extralogical objects (moral). Moral objects of knowledge are strictly logical because they represent a series of experimental facts, relating both to the individual and the environment, facts that are closely linked up with one another, whether regarded from a phylogenetic or from an ontogenetic point of view. We can recognise instances of departure from this law when, owing to habit in the case of particular, individual, reflexes (conduct), particular actions become withdrawn from the focal point of consciousness, or when, as sometimes occurs, a great effort is necessary in order to overcome egoistic impulsions in face of social obligations. One can understand why æsthetic objects are considered hyperlogical, because the sentiment of the beautiful is not subject to fixed laws. With respect, however, to the moral sentiment, it falls within experience and belongs in like degree to the individual and to an entire collection of people. Logic, as an object of study, reveals the laws observed by thought and conduct in their evolution, regarded from both individual and collective points of view. The immutable rules of logic are nothing more than a scientific creation derived from the facts of thought, just as the biologist creates a type of disease by deducing it from observation of a series of cases of the same affection.

Though life is constantly developing and expanding so that the elements of thought are changing, there is no alteration of the fundamental laws regulating the formation and manifestation of thought, these laws being those of perception, memory and association, on which logic, as a science, is reared. Logic draws its raison d'être from external truths and physioanatomical truths.

Logic does not create the norms and conditions of exact knowledge in 
the sense that, without an acquaintance with these norms, exactness and correctness of thought would not be possible. It may be said of logic what Bacon said of Moral Philosophy. One may be a professor of morals and yet not follow out moral precepts in life; whilst a country yokel, who possibly does not know the word "moral," may throw himself into an angry sea to save a drowning man. It does not create; rather does it investigate the laws by which thought is formed and by which it becomes expressed in spoken or written languages and in conduct. The actual mode of thinking is intimately connected with the mental structure of each individual and each social group, and this, in turn, draws its essence and its form from the structure of the brain, and from the physical and social environment in which the organ of thought is developed. I sogic may be regarded as a technique, the object of which is to perfect the structure of thought in its linguistic form (normative discipline of Wundt); but thought always draws its component elements from objective perception, either directly or indirectly, and also from the relations subsisting between these elements. The latter become combined with those of previous experience (memory) and with those of imagination or phantasy which in turn is based on heredity and upon the capacity to project experiences into the future.

It does not fall within the scope of this work to review the various opinions regarding the scientific conception of logic. The writer is unable to follow the pure intellectualists, but has no hesitation in accepting the notion of the evolutionists, that truth does not come from reasoning, however correct that may be, but from experience in the widest sense of the term.

Cerebral disturbances bring into evidence logical defects that are clearly dependent upon a mechanism of a biological character, in accordance with a law which can no longer be doubted. All errors of logic in thought and conduct are due either to defect of cerebral evolution or to a process of dissolution (disease) of the organ of thought and conduct. Mental pathology furnishes new data which, taken together, afford indisputable proof of the fact that thought is the result of a biological function, and logic, which reflects actual modes of thinking, is a biological phenomenon resulting from a series of more elementary phenomena linked up with one another and having an anatomical basis. These, as a whole, come within the domain of psychology, which cannot be considered as other than a branch of physiology and biology. From this point of view, logic becomes a biophylactic function. When the logical process loses its efficiency, the individual or social group loses its most certain weapon for protection in its struggle for existence in the physical and human environment. In the course of an address at the opening of the academic session in the University of Naples ${ }^{1}$ and in a series of lectures upon fixed ideas, ${ }^{2}$ the writer adopted the plan of applying the laws of biology to mental and social life, a conception more fully developed by $\mathrm{D}^{\prime} \mathrm{Ors},{ }^{3}$ who,

1 Bianchi. "Cervello e società." University Annual. Naples, 1891.

2 Bianchi. La Clinica Moderna. An. iv. 1899.

${ }^{3}$ D'Ors. International Congress of Psychology. Reported by Ingenieros. Loc. cit. 
in a study of logic, arrived at the conclusion that reason is a diastasis and logic an immunity.

The logical process, indeed, is founded on the power of assimilation and of rejection possessed by the mental products. An organism lives, evolves and adapts itself according to the extent to which it possesses the power of assimilating, dissimilating and eliminating promptly, in accordance with the norms regulating each species of living organism. To assimilate well signifies to incorporate the inorganic and organic elements that are useful or indispensable to life, and to eliminate well means to get rid of elements produced by dissimilation, and harmful to life:

If these conditions were not strictly observed, life would not be possible ; it would be a poor, abnormal, shortened existence. There is a considerable group of bodily diseases in which this fundamental biological function is altered, and the doctrine here referred to has had a very important influence upon the development and advancement of human pathology.

We meet with a similar state of affairs in mental life. A well-organised mind must select and assimilate, from amongst the numerous sensory products, those which are capable of serving for its development, becoming combined with other psychic elements (assimilation) and constituting more complex mental constellations-a process in which is summed up the conception of evolution and progress of the individual as a unit in harmony with the environment. This capacity is an essential factor of the logical process. The mind, by its own intrinsic force, eliminates everything which does not serve the ultimate object of reasoning and, generally speaking, the integrative, hedonistic process of the personality.

Of the millions of stimuli and impressions received by the senses and perceptive centres of an individual, stimuli which reach and cross the threshold of consciousness, not one is lost. No doubt, only a relatively small proportion of these becomes a utilisable capital, which can be re-evoked according to the circumstances of the varied and different psychic situations which continually succeed one another, but the reason is that the remainder becomes eliminated, or remains hidden in the depths of the unconscious, whence it does not rise again to the surface except under special circumstances, in certain storms and abnormal disturbances of mind. In the case of minds badly organised (owing to morbid heredity or other degenerative influences), it may happen that the psychic metabolism is disturbed, and that a psychic component which ought properly to fall into oblivion, after a very transitory passage through the field of consciousness, remains in consciousness and is not eliminated, reminding us of the poisons that are sometimes manufactured within the organism from substances introduced from without and, not being expelled, accumulate in the organism which has produced them. These psychic elements that are not eliminated are obsessions. An obsession is an illogical thing. That this is so is demonstrated by the fact that the psychic personality is very painfully aware of its presence or its irrational recurrence. The personality feels afflicted by this insistent visitor, recognises its helplessness in front of it, and even though it does react with the object of getting 
rid of it, it often happens that this endeavour only results in increasing the dominating influence.

Under normal conditions an idea may possess developmental potentiality and exercise a beneficial influence in polarising and directing the activity of the life of an individual towards a definite end. It sometimes assumes a directive power, drawing ever more and moreupon the resources of the intellect, becoming more expansive, courting the mind, illuminating the intellectual capital of a people, and, when transmitted from generation to generation, incarnating the principle that animates a period of history. Such ideas are biophylactic, they are like the regulating centre of a whole constellation, of a sidereal system; they are the outcome of a number of social and historical conditions, and at the same time the shining light which guides an individual or a country in the progress towards a happier existence. They have a wonderful capacity for assimilation; they draw freely from the intellectual capital, and in turn are assimilated with the personality, imparting to it their own particular warmth, and impressing on it the movement that is intrinsic in their nature. They possess an extraordinary logical and determinative power, because animated by sentiment. They are the stars which guide humanity through the paths of progress. All this work has its basis in logic.

Weakness of the mental organism, either congenital or acquired, forms the substratum of the obsessions, and serves in two ways to produce alogias and dyslogias. In the first place it allows only a scanty number of ideas or emotions of defence to be opposed to illogical emotions. In the second place, normal ideas are less active in these subjects, and hence are incapable of eliminating abnormal, useless products from consciousness, a condition of affairs which sums up the conception of obsession. In individuals with weak inheritance there is also a deficiency in the power of detaining the object of perception in the focal point of consciousness, so as to gather the greatest possible number of features that go to build up a more complete cognition. Not only so, but wider and fuller knowledge depends also on the capacity to evoke all the other features, similar, analogous or contrasting, from which is derived the perceptive differentiation of what the external world presents to us. This is the fundamental reason of multiplication of fields of knowledge, and so, too, of the ramification and growth of the sciences. The power of detaining an object of perception in the focal point of consciousness and of evoking its specific features so as to recognise the object in its differential characters constitutes the essence of the psychic fact which we call attention. This is defective or absent in idiots, and in monkeys that have suffered mutilation of the frontal lobes, and it is from this defect that the alogias and dyslogias which characterise the life of these beings arise.

Pathology thus lends additional confirmation of the views already expressed. Alogias and dyslogias result from definite processes, the mechanism of which is quite well known. From the dynamic and functional point of view, they also depend upon defects of perception, memory, imagination and association, and upon the intervention of extraneous elements in the reasoning 
process-emotions, delusions, hallucinations. Man is all the more logical in the sense already explained, or, in other words, he is the better provided with biophylactic power the more his perceptive force is able to penetrate things and situations and so arrive at precise knowledge. The work of perception is fundamental as regards both knowledge and logic. When perception is partial or superficial the conclusions are insufficient, and represent a deviation from that form of reasoning, or that logic in conduct and in speech, which bears the stamp of reality. Such conclusions are of no real value for the purposes of progress. All imbeciles and, to a greater extent, idiots fall into a number of errors, and are inconclusive and inefficient because they lack the means of penetrating reality, of apperceiving it, and of initiating action that is adequate in the biophylactic sense.

The perceptive process is thus closely bound up with attention. Defect of the one involves defect of the other. The psychic arcs in these cases are short, and terminate in inadequate actions or in phrases which, if not illogical, considered per se, are at least illogical as regards their insufficiency and their unsuitability for the needs of life. In all cases of this kind we have to deal either with pathological processes, general or partial, occurring in the period of development (infancy), and arresting the process of maturation of the brain, or else with a biological insufficiency of the organ of thought, which, from the outset, is incapable of reaching the normal degree of development. Almost the entire brain is affected by the evolutionary defect, no matter what its cause, but most of all the frontal lobe, and especially the layer of small and large (external and.internal) pyramidal cells (vide Chapter III.). Degenerative processes in the cells of the cerebral cortex, particularly in the frontal lobe, produce similar effects in adults, having due regard to the mental structure of each individual.

Every degenerated or degenerating cell represents a loss of energy to the cerebral dynamics. Just as an electric battery gives a lower voltage when the salts in the cells have been consumed and the relation between electromotor force and resistance altered, in the same way the intellectual power is curtailed and the relative logical disposition of thought disturbed when degeneration occurs in a certain number of nervous elements [of possible causes there are many-intoxications, autointoxications, overwork (leading to autointoxications), alterations of the vascular walls, insufficiency of the internal secretions, etc.].

The same factors produce defects of memory and of the associations, especially when there is degeneration or interruption, from one cause or another, of the associative paths. Apart from this, however, there exists a group of alogias or hypologias which depend upon a feeble capacity to detain a theme in the focal point of consciousness and to evoke and select the elements required in the process of reasoning. This evocative and selective power serves as a regulator of the function of the sensory cortex, and when it is weak the sensory areas fail to furnish the components which are necessary for the construction and logical course of thought, or give their 
products only under the compelling influence of strong internal and external excitement. In another group of cases the sensory products arise without any rule or order, and so we get a state of mental confusion. The detentive capacity in question is always found to be weakened in any degenerative or sub-evolutionary process affecting the frontal lobe.

The extraneous elements which intervene in the reasoning process are delusions, hallucinations and strong emotions. Primary delusions-those, that is to say, which are not the direct product of hallucinations-are conceptions which arise and thrive upon an abnormal emotional basis (fear, religion, vanity, pride) ; they have no counterpart in reality yet are upheld with arguments, and sometimes with strong dialectic power, in an apparently logical and connected fashion. The false conception, like a presupposition the reality of which is a matter apart, makes the cerebral powers subservient to it; all the apparently logical and pertinent arguments that the deluded individual, according to his mentality and culture, brings forward to support his thesis are furnished by unconscious cerebration, and not only do these lack entirely any basis in fact but often the very premises with which he sets out are obviously improbable.

In this case the attentive capacity is subjugated by a higher power (passional preconception), which pervades all the mental mechanisms and withstands any counter argument that the perceptive process might furnish in opposition, the perceptive process itself being enslaved by the delusion, which triumphs in a world of fancies.

The alogias that arise from hallucinations are the effect of an abnormal condition (inflammation, intoxication, congestion) of those same areas which we considered to be the organs for the construction of the images. The creative power of the brain is manifest not only in thought, in its wide and complete structure, but also in single images. These appear to consciousness as realities projected into the world without. There may be a single image or there may be a few or many, but in point of fact they neither reflect nor represent reality. They constitute a world of dreams with some features of reality, and are confounded with objective reality which the patient is still able to perceive in part. Confusion results from this, inasmuch as the hallucinated individual perceives real things along with the images of his dream, this state of affairs being aggravated by the fact that he has only an imperfect perception of the real things, whilst the phantasms take the place of reality in his consciousness, dysorientating it, decomposing it, and inciting to immediate reactions in speech and conduct, for hallucinations are furnished with a strong determinative power.

One can readily understand that the pathological process which determines the hallucinations will at the same time cause a marked disturbance of the perceptive process, because it is one and the same organ that is involved, and that it will interrupt the relations of the self with the preperceived real world ; for hallucinations, like dreams, transport the disturbed consciousness into a fantastic world which obeys none of the laws regulating the relations 
of consciousness with reality, and, in consequence, speech becomes inconclusive and conduct illogical.

There is a marked difference between these various alogias, hypologias, dyslogias and the conditions already mentioned as arising from lesions of the language-zone on the one hand, and alogia due to experimental ablations in monkeys or to severe bilateral lesions of the frontal lobes in man, on the other. In the latter, the evocative capacity is defective and, consequently, the activity designated as phantasy or imagination is suppressed or much reduced, even in its simplest form, such as that excited by desire and the appetites. Perceptive recognition of objects takes place, but, as in the case of the imbecile, perception is not rendered more perfect and complete by elements of apperception, because the evocation is simple. Comparisons are not arrived at, because association is defective, so that a monkey, perceiving the white colour and the form of a piece of chalk, mistakes it for sugar; he takes it and puts it in his mouth and, even after he perceives the taste of the chalk, which is not sweet, does not resolve to spit it out. That is a variety of dyslogia. The relations are simple, disjointed, varied, isolated, and so, in man, the discourse is poor and the conduct illogical. Past experience is not utilised-e.g. a monkey, tricked many times in succession, does not appreciate the fact, but stupidly repeats the same illogical actions which bring him no success. The resemblance between this and the mental state of imbeciles is very remarkable.

Profound imbeciles not only fail to arrive at the formation of general and categorical ideas, but are especially incapable of detaining a particular theme in the focal point of consciousness. The logical power that is a feature of normally developed men is, consequently, entirely beyond their attainment, and if the phrases which they sometimes pronounce seem to have something of a logical character about them, they nevertheless represent immediate reflex arcs and responses to single stimuli. Their conduct is disjointed and exhibits no succession of facts connected one with another.

Thus, then, psycho-pathology comes to our aid, helping us to complete and consolidate our conception of logic, regarded from the point of view of the function of the cerebral mantle. The various alogias and dyslogias that result from different pathological conditions furnish a positive proof of the physio-anatomical basis of logic and of the mechanism underlying the development and expression of human thought and conduct. 


\section{CHAPTER IX}

\section{Emotions and Sentiments}

Two orders of facts have their beginnings in, and develop from, sensation. One represents the intellect and its various grades of evolution; the other the degrees and forms of feeling and of emotion. The one has reference to the external world and its relations, translated into images with their respective associations; the other to modifications of the ego, provoked not only by stimuli from without but also by chemical and vital processes taking place within the organism, under the influence of these stimuli and of all the mental products, simple and complex, derived from them, in intimate relation with the cognitions of the external world. The one proceeds unceasingly in its development, and, with the constant aid of grafts from the external world, reaches the summit of knowledge, a summit which is ever becoming higher; the other evolves from the elementary emotions of pleasure and pain, originat. ing in the chemical changes that succeed one another, and broadens out to embrace, in synthetic fashion, the emotions and sentiments of other fellowbeings scattered over the face of the earth. The intellect finds its objective in cognition of the universe ; the sentiments, through the experiences of the ego in its changing elimatic and social relations, promote understanding of consciousness between human beings, in the general aspiration after a happier life, and the struggle against pain.

Emotion is inherent in the modifications of the organic being that take place under the action of stimuli. In the first representatives of animal life, this modification is of a chemical, physical or mechanical nature. The facts of tropism, already alluded to, contain a rudimentary emotional element. This gradually becomes more conspicuous as it becomes complicated with the psychic phenomena that appear in the successive stages of development of the nervous system. The behaviour of the amœba, when brought into proximity with an acineta, is a characteristic example of tropism. Electricity, light, heat, eold, are powerful modifiers of the organic processes and act either directly, or by means of chemical products, on the nervous system, from the moment that this makes its appearance; and they bring about conditions that form the basis of emotion.

Pressure and electric changes in the atmosphere are felt by many animals, which seek shelter from an approaching storm before it can be perceived by means of the ordinary organs of sense. ${ }^{1}$

That physical agents exert a remarkable influence upon the process of nutrition, and these again upon the psychic functions, are facts that have been known from antiquity. The influence of elimate and the seasons upon

${ }^{1}$ Hunter, Y. E'uvres complètes. 1841. 
the organic and psychic functions has been demonstrated by statistics relating to suicide, which is more frequent in certain seasons (Montesquieu, Bourbousson, Delaroche, Morselli, Massarotti). There are both old and recent observations connected with this subject, and amongst the latter may be mentioned the researches of Lombroso, ${ }^{1}$ Enrico Ferri, ${ }^{2}$ and Penta, ${ }^{3}$ in Italy, dealing with the influence of the seasons upon insanity and upon the frequency of crime.

Climate is the synthesis of a number of physical conditions-light, temperature, atmospheric electricity, direction and prevalence of certain winds, vicinity to the sea (independently of atmospheric pressure) and the chemicophysical constitution of the earth. We know that in the human subject, metabolism undergoes notable alterations in different climates, and with these we get certain changes in the humour and activity of the mind in individuals not adapted to the particular climate.

This has been confirmed particularly in the case of persons of exquisitely nervous temperament. In some climates neuropaths do not sleep, they are more irritable and more restless, whilst epileptics under identical conditions of life, so far as food and muscular exercise are concerned, and pursuing the same treatment as had obtained elsewhere, are subject to a much greater number of fits. It is strikingly suggestive that this phenomenon, if not of the same nature as, is at least fundamentally analogous to, that of tropism. When, again, we think of the great influence exercised upon the nervous system by factors connected with digestion and nutrition, it seems evident that the emotions are closely bound up with the organic processes taking place within the organism.

The fact, affirmed by Claude Bernard and definitely confirmed by Canalis and Morpurgo, that badly nourished animals are much more prone than others to infections, is a proof, from another source, of the intimate relations subsisting between all vital phenomena, emotions included, when the organisms are subjected to the action of external agents, in the changing conditions of existence.

The silence which night-time imposes upon many animals; the slowing of conversation, the difficulty or inhibition which we experience, as though taken by a vague fear or preoccupation, if the light goes out as we are talking ; and the gaiety which bright sunshine infuses into us after days of wet and wintry weather, are psychic equivalents, in the domain of the emotions, intimately connected with all the vital functions and hence with the organic processes. Moleschott, Fubini and Benedicenti have shown that the quantity of carbonic acid given off in darkness and in sunlight respectively is in the ratio of 3 to $5 .^{4}$ The amplitude of the respiratory movements under the influence of white light is different from that under coloured rays.

1 Lombroso. L'uomo delinquente, 1884 ; C. Lombroso and G. Ferrero. La donna delinquente. 1915.

2 E. Ferri. Atlante antropologico dell'omicidio. 1895.

3 Penta. I pervertimenti sessuali nell'uomo. 1893.

4 Moleschott, Fubini and Benedicenti. "Influence de la lumière sur le chimisme de la respiration." Arch. Ital. di Biol. 1891. 
Every-neuropathologist who has had piactical experience in the treatment or investigation of hysterical, neurasthenic or neuropathic patients is well aware of the fact that the humour of mind and behaviour of these patients, in the family and social relations, alter with atmospheric changes. The modifications undergone by the circulation under the influence of various external stimuli acting upon the different senses, modifications that have been clearly demonstrated since the introduction of Mosso's plethysmograph as an aid to physiological investigations, are now assured facts of physiology and psychology.

Clinical psychiatrists are familiar with the fact that some melancholics and neurasthenics suffer nocturnal anguish, in which the modifications of the circulation and respiration induced by darkness play a part; and that hallucinations with delusional representations and anxious states, which sometimes resolve themselves into suicidal impulses, are more frequent in the night-time and towards the morning hours.

These phenomena are but the reflex of chemical alterations, taking place within the organism, upon the humour of the mind and the psychic processes, in the sphere of the emotions, just like the tropisms of lower organisms but on a much higher scale, and embracing an order of facts of much greater complexity.

This complexity is caused by the intervention of the nervous system with its intricate structure, which is readily affected by all the chemicophysical endorganic modifications; many of these, when abnormal, are revealed to consciousness in the form of malaise accompanied by change of humour and greater excitability, along with a sense of ennui, fastidium and sometimes gloomy notions.

On other occasions these modifications reveal themselves by unusual irritability and intolerance in the family and social relations, or by a sense of depression, or by unfounded gaiety.

The humour of mind, be it gay or sad, and the psychic activity are closely connected, each in the same way, with the chemical and physical changes going on in the organism. It often happens that we are more or less disposed to act or not to act, to be generous or harsh, confident or diffident, optimistic or pessimistic, owing to modification of the kinæsthetic sense, and this in turn is influenced by the chemical and physical alterations which follow one another in the organism. Hunger, thirst, the sexual appetite, tasty substances, aromatic drinks, perfumes, contacts, all provoke emotion, either of pleasure or of disgust, hence a desire and attempt to approach or to retreat. A good dinner followed by healthy digestion makes a man generous; hunger and bad digestion render him pessimistic and intractable. The fundamental emotions can thus be traced back to their genesis-i.e. to the chemical alterations taking place within the organism, alterations which reverberate upon the kinæsthetic sense, which is the psychic foundation of emotion.

The two fundamental emotions, pleasure and pain, are, in man, intimately bound up with somatic phenomena. In proportion as the organs become 
more perfect and acquire more intimate relations with one another, or, in other words, as the organic machine becomes more complicated, the emotions become accompanied, as we shall see, by particular organic phenomena which, at bottom, are the most authentic expression of chemical modifications of the protoplasm under the action of external agents, such as we found to underlie the phenomena of tropism. In this notion is summed up the modern doctrine of energetics.

The continuous chemico-dynamic processes, the perennial action of the stimuli of the external world, generate in man, who is the most complex and perfect morphological machine, the sense of life and of self-existence, in association with the sense of reactive energy, or, in other words, the kinasthesis. This is but the reflex, on the threshold of consciousness, of all the inner work of the busy organic laboratories and of the incessant stimuli by which the outer world, in which we live, reveals itself to us. In 1889 the author wrote :

"The kinæsthetic sense is the synthesis of all the sensations in which the organic personality, the psycho-physical ego, is summed up. To it contribute not only the special senses by means of which we experience an infinite series of immediate relations with the external world, the ultimate resultant of which is the progressive comprehension of our own organism as distinct from the environment in which we live, but also the mnemonic reproduction of the physical or organic qualities of the organism, provided by all those other currents which establish uninterrupted relations between the organs innervated and the higher nerve-centres. The latter currents do not excite true states of consciousness, and therefore do not give rise to sensations in the strictly psychological sense of the word, but, along with the impressions from without, they are confluent in a chamber of harmonic resonance, the waves of which give rise to the sense of our own proper existence.

"It is this sense, which is known as the kincesthetic sense, that is the fundamental constituent element of consciousness, although it is one of the least representative.

"Any discordant note, generated in any of the organs and disturbing the harmony of the waves, is a cause of conflict in the chamber of resonance, and we become aware of it in the form of malaise." 1

It is the chief instrument of the emotions; it represents the soil in which they germinate; it furnishes the somatic elements of emotion, and prepares the way for the representations, which are the other constituent elements of emotion.

From all parts of the organism which are organic laboratories there is a continuous flow of nerve-waves, establishing relations between the organs and the higher nerve-centres. With these are conjoined all the special sensations through which we experience a series of mutations due to immediate contacts with the external world, the ultimate result being the progressive comprehension of our own organism, which becomes ever more distinct from the environment.

${ }^{1}$ Bianchi. Semiotica delle malattie del sistema nervoso. Milan, 1889. 
We have said that the kinæsthetic sense, though not a distinctly conscious element-i.e. distinctly represented in the waking consciousness as such-yet becomes an element of waking consciousness in all the more outstanding vicissitudes of the ego, in its external and internal environment; it becomes more prominent whenever there is functional difficulty, or a state of conflict, as the result either of excessive intensity of the external stimuli, these being so powerful as to be actually disintegrating (pain), or of insufficient intensity of these (anæsthesia, hypoæsthesia, paralysis), or because of an intensified general tone, or a dissonant note arising from one or other organ and disturbing the harmony in the chamber of organic resonance, where organic consciousness takes its form. In this case we get a sense of ill-being and a lowering of tone, and the result is a new attitude of consciousness. The kinæsthetic sense thus becomes at the same time a regulator of the emotional susceptibilities of the ego.

An intimate connection exists between the kinæsthesis and the sphere of the images, so that variations in the tone of the kinæsthesis tend to recall into the focal point of consciousness, through the law of association, determined categories of ideas, aspirations and tendencies. Thus are explained the particular inclinations, trends and emotional susceptibilities that are peculiar to separate individuals. Elements for the kinæsthesis are furnished by the larger part of the cerebral mantle, for even the specialised sensory areas have their motor centres and possibly also some tactile representation.

The cerebral cortex besides being the organ that gives rise to images, movements and the higher psychic functions, is, at the same time, an anatomical field, experimental excitation of which gives rise to modifications of the organic functions. The respiration, the circulation, the secretions and the movements of the internal organs are represented on the cortex of the brain. There is ample experimental proof of the fact that the cerebral mantle has acquired relations with all the viscera-i.e. with each unit of the vital workshop-and transforms their work into a psychic synthesis which, along with all the intellectual and emotional products, goes to form consciousness.

The literature dealing with these experiments is a very rich one, and only the most outstanding and assured facts can here be referred to.

Respiration is accomplished and modified in a mechanism that is extraneous to the focal field of consciousness, but it is also capable of modification by the will and by stimuli which cross the threshold of consciousness, as well as by representations furnished by memory. In support of the latter contention the fact may be mentioned that, leaving aside the examination of the lower, unconscious, respiratory arc, a cortical field has been discovered, experimental excitation of which gives rise to marked alterations of respiration.

Without dwelling upon the researches of Lépine, Bochefontaine, Munk and Danilewski, it may be said that recent investigations, especially those carried out in the physiological laboratory of Bechterew, ${ }^{1}$ prove that modi-

${ }^{1}$ Bechterew. “Ủber der Epilepsia." Sammlung Klinischer Vorträge. 1897. 
fications of respiration occur on exciting a very circumscribed area on the third primary convolution in the dog, in close proximity to the excitable area for the orbicularis palpebrarum. Electric stimulation at this point produces slowing and even arrest of respiration. Probrazènski obtained similar results in dogs. ${ }^{1}$

Horsley and Semon ${ }^{2}$ obtained modifications of respiration on exciting the cortical centre of the larynx (respiration is closely associated with the voice and with crying). They obtained acceleration and reinforcement of respiration on exciting a point in the vicinity of the lower extremity of the crucial sulcus.

Giannelli, ${ }^{3}$ in the course of his investigations, found two points where excitation gave rise to altered respiration; one on the sigmoid gyrus above and in front of the crucial sulcus, near the interhemispheric fissure, concerned with expiratory movements, and a second situated further forwards, at the upper extremity of the presylvian fissure.

Langelaan and Beyermann ${ }^{4}$ found a similar centre on the sigmoid gyrus. On exciting a small area near the crucial sulcus they obtained acceleration and greater depth of respiration, with a tendency towards inspiratory arrest of the thorax. Extirpation of this centre was followed by slowing and irregularity of the respiratory movements and by sighing.

Bechterew, in his later researches, seems to have defined with greater precision the points on the cerebral cortex of the dog where excitation causes modification of respiration. According to him, one point would be situated at the inferior extremity of the crucial sulcus, on the anterior branch of the sigmoid gyrus, and would have to do with the acceleration of respiration. A second point would be found at the anterior extremity of the sulcus which divides the second from the third external convolution, near the posterior branch of the sigmoid gyrus, and would be concerned with slowing of respiration, proceeding to arrest in expiration. A third point would be located at the upper extremity of the presylvian fissure, and would be concerned with deepened respiration and slowing of the movements, proceeding to arrest in inspiration. ${ }^{5}$

The objection has been raised that there are various points on the cerebral cortex where, according to the results of the experiments of different investigators, electric excitation produces alterations of respiration.

This diversity in localisation is due, at least in part, to the complicated mechanism of respiration, and to the relations of respiration with all the cortical functions. The assertion of Bochefontaine and François Frank that excitation of any point whatsoever of the cortex gives rise to modification

${ }^{1}$ Probrazènski. "Über das Atmungscentrum in der Hirnrinde." Wiener Klinische Wochenschrift. 1890.

${ }^{2}$ Horsley and Semon. "An Experimental Investigation of the Centre of Motor Innervation of the Larynx." Proc. Roy. Soc. of London. 1890.

${ }^{3}$ Giannelli. "L'influenza della corteccia cerebrale," etc. Ann. di Ne». 1900.

"Beyermann. "On the Localisation of a Respiratory Centre," etc. Brain. 1903.

${ }^{5}$ Bechterew. Die Functionen der Nervencentra. 1911. 
of respiration does not, however, seem probable, and is on the same level as the other statement that epilepsy can be provoked by exciting any area of the cerebral cortex. These cases of modifications of respiration and circulation, induced by electric excitation of cortical areas far removed from the sigmoid gyrus (or, in the monkey, from the Rolandic convolutions), find their explanation in the excessive intensity of the current employed in the experiments. In any case, the question remains sub judice. Even putting aside the complicating effects of all strong electric currents, it may be held that any excitation, even moderate, of the sensory areas reawakens images (hallucinatory) which give rise to modifications of respiration, varying according to the animals experimented upon and their emotional susceptibilities.

There is no question as to the relations subsisting between the musculature concerned with the respiratory movements and those of mimicry, phonation, deglutition and the movenents of the trunk. These motor centres are subordinate on one hand to the will, and on the other, quite apart from will; to the state of the sensory areas whence arise the associative reflexes (Pavlov, Bechterew) and the affective attitudes, which are almost always accompanied by modifications of the respiratory rhythm, of the circulation, the secretions, etc.

As a matter of fact, electric excitation of certain points of the motor area gives rise to marked modifications of the function of the heart, of the pulse and of the blood pressure. It seems evident that the cortex has an influence not only upon respiration but also upon the activity of the heart. Investigations carried out for some time by Tomasini, in the writer's clinique, confirmed these results in the main.

Researches both old and recent, amongst which may be mentioned those of Schiff, Danilewski, Bochefontaine, Eckard, Frank, Bechterew, Mislawski, and especially those of Cerevkov, ${ }^{1}$ have furnished ample evidence to support the contention that the cerebral cortex and the corresponding white substance exercise a decisive influence upon the function of the heart and vessels; the region indicated is the sigmoid gyrus (in dogs), the pre-crucial perhaps more than the post-crucial portion.

These facts have a very important bearing upon psychology. In the emotions we have modified not only respiration but also the circulation. Emotion-producing impressions from without and their representations or memories, the former much more than the latter, are accompanied by disturbances of the circulation that may go the length of pallor on the one hand and congestion on the other (constriction or dilatation of the vessels), along with acceleration or slowing of the heart's action. The activity of the heart and the behaviour of the vessels are, however, very intimately connected with muscular movements. In emotional states, physiognomic attitudes, agitated talk, gestures and circulatory disturbances together constitute one expressive whole, and accordingly the respective cortical fields are found situated close to one another in the same area, so close indeed that one almost runs into the other.

${ }^{1}$ Cerevkov. Über den Einfluss des Gefassystem. Karkov, 1892. 
Not only have we respiration and circulation but indeed all the viscera (the organs of life) represented in the cerebral mantle. The centre for deglutition has also very close relations with the centre of respiration. ${ }^{1}$

Bochefontaine, Vulpian, Fr. Frank, and more particularly Bechterew, in a series of very convincing researches, have demonstrated contractions of the spleen. Movements of the stomach and intestine can also be provoked by excitation of a small area of the cortex. As is known, movements of the cardiac end of the stomach are opposed to those of the pylorus, so that opening of the cardiac end coincides with contraction of the pylorus. When the stomach is full the cardium is inactive whilst the pylorus is active. In the cerebral cortex there exists, in the neighbourhood of the crucial sulcus, a centre, excitation of which opens the cardiac end and determines contraction of the pylorus. This area is situated on the posterior and external part of the sigmoid gyrus. Stimulation of the antero-external portion of this gyrus determines contraction of the cardium whilst the pyloric region remains in a state of rest.

Bechterew and Mislawslki ${ }^{2}$ detected points on the sigmoid gyrus (median surface) where excitation gave rise to movements of the vagina, to inhibition of these movements, and to movements of the uterus (median portion of the motor zone). Bechterew also defined a small area on the posterior part of the sigmoid gyrus excitation of which was followed by increased volume of the penis.

One cannot fail to recognise the great significance of these investigations when one reflects that the sexual function, although directly dependent upon the lower centres, has also its representation in the cerebral mantle. This anatomical and experimental fact may explain the influence exercised by the senses of sight, hearing and, more especially in the human subject, touch, which is the equivalent of smell in many animals, upon the excitation of the sexual appetite and upon the imagination. It certainly serves to explain certain symptoms observed by the author in some of the animals he experimented upon-e.g. prolonged erection in one of the dogs and attempts at copulation in several monkeys. The investigations in question prove that a lesion in the neighbourhood of the area for erection may cause irritation therein and so give rise to erection and that the cortical representation of the sexual functions is located behind the field of the experiments upon the frontal lobe. In the higher mammals and in man the sexual function is not a blind instinct but is subject to the control of the will and to conscious inhibition just like the vesical function, which Mosso and Pellacani maintain is also specially represented in the cerebral cortex.

Similarly, the movements of the sphincter of the anus (co-ordinated movements of defæcation) have their cortical centre. In the case of the monkey, Sherrington located it in the posterior part of the para-central lobule, for he there detected a small circumscribed area, 'excitation of which provoked

1 Bechterew and Ostankow. "Über den Einfluss der Gehirnrinde auf die Function des Schlückens und Atmens." Neurol. Centralblatt. 1894.

${ }^{2}$ Bechterew and Mislawski. Loc. cit. 
movements of the sphincter. Others have located the said centre in the posterior convolution of the sigmoid gyrus at some distance from the crucial sulcus.

There are other points on the cerebral cortex where excitation produces marked alterations of the principal secretions. We have already alluded to Pavlov's investigations which show how the sight, the sound and, even more, the taste of a food substance increases the secretion of saliva. We know that fear, on the other hand, dries up the mouth. Many orators, as they commence to speak, feel the mouth becoming parched. We are familiar, too, with sialorrhœa as a symptom not uncommon in dements. Experimental investigation has afforded an interpretation of these facts, for faradisation of the sigmoid gyrus provokes increased secretion of all the salivary glands, especially on the opposite side (Bochefontaine and Lépine), and this finding is in no way minimised by the fact that Bechterew and Mislawski point to a small area on the fourth external convolution, above and in front of the fissure of Sylvius, in the dog, as a centre of greater functional intensity for the secretion of the salivary glands. The influence of mental excitement upon secretion of saliva is undoubted, and interesting data in this connection have been furnished by Malloizel. ${ }^{1}$

Excitation of both branches of the sigmoid gyrus is very soon followed by abundant flow of tears from both eyes, but more especially from that on the side opposite to the excited hemisphere.

Less is known of the other secretions, but the researches of Bochefontaine, Pavlov, Gerver, Sollier, and especially those carried out throughout a period of years by a number of investigators in the laboratory of Bechterew, have furnished interesting data regarding the modification of the secretions of the liver, pancreas, stomach, intestines, kidneys and prostate, as the result of faradic excitation of various points in the sigmoid gyrus.

The secretion of the mammary glands is also represented on the sigmoid gyrus, a finding which confirms the old and popular view, supported by clinical observation, that powerful emotion arrests the secretion of milk in woman, or so modifies the chemistry of milk as to induce symptoms of intoxication. The researches of Nikitin ${ }^{2}$ dealing with the area of excitation of this secretion are very conclusive.

One can foresce that all the glands may have relations with the cerebral mantle, and their respective secretions must therefore possess some considerable significance in relation to the other functions of the mantle, in this respect, that the work of the glands is subject to the influence of emotional states, and, on the other hand, affects the condition and behaviour of the kinæsthesis, modifying what we call the humour of the mind (Stimmung of the Germans). It is a remarkable fact that the field of excitation of all the glands is almost entirely circumscribed within the tactile zone (of Flechsig) which comprises the two convolutions of the sigmoid gyrus, in

${ }^{1}$ Malloizel. Comptes rendus de la Société de Biologie. 1902.

${ }^{2}$ Nikitin. "Uber den Einfluss des Gehirn auf die Milchsekretion." Verhandl. des Wissens. Versamml. des Psych. u. Nervenk. St Petersburg, 1906. 
dogs, or the two Rolandic convolutions in monkeys and in man, along with the immediately surrounding parts-i.e. the same area as that which contains the points of excitation for the respiration and circulation as well as that of the sensibility and the musculature of the whole body.

From a functional point of view this region of the cerebral mantle is a very complicated one, its function being that of emotional motor reaction upon the external world.

The frontal lobe does not participate directly in this very fundamental function of life. Emotion is the psychic equivalent of the modifications (physical, chemical, mechanical) induced in organisms directly by external stimuli, or indirectly through the psychic self, in the varying vicissitudes of the physical and social environment. All the experimental investigations warrant us in excluding the frontal lobe from the complicated mechanism of those emotional manifestations that are intimately bound up with the kinæsthesis. Thus it happens that whilst the fundamental emotions, those of self-preservation and defence, closely associated with all the representations of the physical self, have their expressive mechanism in the tactile and motor zone (somæsthetic zone of Flechsig), the sentiments which arise from inter-human relations emanate, as we shall presently see, from another cortical field.

Hunger, thirst, the sexual need, the need of moving about, of sleeping, of avoiding high and low temperatures, are fundamental emotions. Hunger and thirst are intimately connected with material interchange and the chemical condition of the various cell-groups of which the organism is composed. The sexual appetite has probably also a chemical origin if the maturation of the ovule and the secretion of semen are to be considered chemical facts. Fxcessive heat and excessive cold are disintegrating, and so, in the course of various civilisations, a series of provisions for protection against these has arisen. Possibly some emigrations in prehistoric times were determined by climatic conditions unsuitable for existence. All sensations, with their respective appetites or repulsions and the determinism towards a series of actions calculated to satisfy them, may be included in what is termed by some the instinct of self-preservation, the pleasure of living, the will to live. When these attitudes of mind are analysed, we find in all of them the pleasure of satisfying the organic needs, more especially if delay or insufficiency in satisfying them bring us to the threshold of pain, which is an expression of chemical changes taking place within the organism owing to insufficiency of nourishment, dehydration, etc.

We cannot claim to know what chemical modifications of the organism, and more especially of the nerve cell, induced by prolonged rest, give rise to the impulse to move. There is little doubt, however, that this need, with the corresponding pleasure of satisfying it, is also of chemical origin.

The need for sleep, which is also of chemical origin, is to be included amongst the fundamental emotions, the chemical modifications here concerned being produced by the work of the senses and of the muscles, by 
suppression of light, etc. The satisfaction one experiences on awakening from a refreshing sleep is the conscious reflex of the restored chemical equilibrium throughout the body, especially in the nervous centres. The pain which follows nights of sleeplessness, taking, it may be, the form of an indefinite feeling of malaise and bad humour, and the despair that may go the length of suicidal emotion in individuals who suffer from severe agrypnia, are very significant proofs of the fact that the whole organism, but more especially the brain, takes part in sleep, which is a fact of organic integration.

After this fundamental group comes a second group of emotions of a higher order, such as fear, joy, anger, etc., which have a content of images that are to a much larger extent extra-organic. They are concerned with the ego in its relations with the outer world, and have a much richer content of images and a wider and more mutable field of actions and reactions, which are outside the domain of the fundamental organic needs, and reflect rather the ego in its hedonistic attitudes and impulses, such as those concerned with the avoidance of pain.

A constant feature of the more intense states of pleasure and of pain is the marked alteration of the circulation, respiration and secretions. Now, these functions are represented, as we have seen, in a very circumscribed area, and if we are not warranted in regarding that area as the cortical organ of the emotions, we are at least justified in speaking of it as the anatomical field of the organic reflexes of the emotions. Flechsig was of opinion that all the emotions and passions accompanying disturbances of the circulation and respiration must have their origin and seat in the tactile zone which, according to him, would be the cortical centre of the sentiments which become conscious.

Kirchhoff ${ }^{1}$ accepts this view with but slight modification. He limits the area for painful sensations to that tract of the gyrus fornicatus, on the internal aspect of the hemisphere, included by Flechsig within the somæsthetic zone.

According to Flechsig, the somæsthetic zone comprises the motor along with the tactile zone, and contains cells the axis-cylinder-prolongations of which either directly or indirectly reach the muscles of respiration, the circulatory apparatus and that of other organic functions, so that functional disturbances intimately connected with the emotions and instincts thus become conscious. It should, however, be pointed out that the mechanism and the content of the emotions are not wholly confined to the anatomophysiological circle of the tactile zone.

Evolved emotion is a state of consciousness concomitant with those reflex and co-ordinated phenomena which arise from perception or representation of definite objects containing elements either of integration or of disintegration of the organism, in the widest sense of the terms-i.e. in both the physical and the psychic sense. The two primordial forms of emotion

${ }^{1}$ Kirchhoff. "Neuere Ansichten ïber örtlichen Grundlagen geistiger Storungen. Halle, 1896. 
are those of pleasure, connected with all that leads to the integration or growth of the organism (physical and mental), and of pain, connected with all that tends towards its disintegration or diminution (under normal conditions of life). The fundamental law is that every stimulus which acts upon us modifies the ego in a pleasurable or disagreeable manner, according as it furnishes elements that are assimilable and favourable to existence or elements that are disintegrating or hurtful to the psycho-organic unity.

From this point of view we must conclude that the sensory mantle furnishes the psychic content of the emotions, whilst the tactile zone provides the organs of their cortical reflexes.

Broadly speaking, pain may be interpreted as an interference with the process of nutrition in the organ, ${ }^{1}$ an interpretation which corresponds, in general, with the fact that harmony and good adaptation are on the line of pleasure, whilst discord and faulty adaptation are in the direction of pain. Adaptation is a form of penetration of the organism into its environment, and is consequently favourable to its development.

Pleasure and pain, on the other hand, are intimately related with facility or difficulty in the biological processes. That feeling of indifference which all have experienced as the result of fatigue, has its origin, according to Hermann and Funke, in difficulty of the psychic and reactive processes. Féré has made reference to the uprising of the arcanistic, mystic sense of things in connection with depression of the muscular power. ${ }^{2}$

Successful psychic functioning (Meynert, Gilmann)-i.e. the ready representation of things, and facile internal motion or exteriorisation in order to meet the requirements of life-coincides with pleasure, whilst obstacles, delay and resistance are on the threshold of pain. A need, inclination or tendency always implies a motor innervation to some extent or another (Ribot). This motor innervation is pleasurable or disagreeable according as the movement is completed or hindered.

Hunger, which represents an organic need, is a pleasurable sensation if it can be satisfied. Many, indeed, are afflicted if they have no appetite and yet have the wherewithal to satisfy it, but in any case hunger becomes a painful sensation when there is any hindrance to its satisfaction.

The same psychological process has a painful or pleasant resonance in consciousness according as the accompanying circumstances are unfavourable or favourable.

Pain generates unrest and a tendency to flee from the cause of it, where circumstances permit, or to remove all that hinders or afflicts us. In this fact (the aversion of L. Miller) lies the mechanism of the motor reflex or, in other words, the reaction, which assumes as many forms and grades as there are degrees of aversions.

According to some authorities, amongst them Wundt and Ward, pleasure and pain are connected with a higher order of psychic phenomena, and are

${ }^{1}$ Mead. "A Theory of the Emotions from the Physiological Standpoint." Amer. Journ. of Psych. 1893-1895.

2Féré. "Pessimisme et impuissance." Rev. Phil. 1886. 
interpreted in terms of attention to the particular object. Any stimulus which fails to awaken some degree of attention is "indifferent." When attention is aroused, one of two things may happen: either it has full play without any hindrance, in which case we get a state of pleasure, or, it is disturbed by a conscious feeling of inhibition, as, e.g., in the case of obsessive ideas, and then we have a painful feeling. In other words, attention without hindrance or difficulty is on the threshold of pleasure, whilst pain coincides with obstructed attention (Sidney, E. Mezes). All states of intense attention which eventuate in clear thought or in efficacious work are pleasurable, whilst all states of internal conflict accompanied by hesitation and practical difficulty are inefficacious and unpleasant.

Physical or mental activity involving the expenditure of an amount of accumulated force, the potential of which when resolved into actual energy is in excess of what the stimulus ordinarily requires, is, according to Marshall, a cause of pleasure, whilst the psychic state connected with pain is got when the potential of energy is less than what the stimulus habitually requires, because of deficient capacity of the organs from which it emanates. Pleasure and pain would thus be primary qualities or psychic states determined by the relations between activity and capacity of the organs of consciousness, in its relations with the world.

H. Spencer puts forward the view that pleasure is concomitant with all medium activities. Wundt also declares that in all the sensory spheres excitations of moderate intensity are accompanied by feelings of pleasure, confirmation of this being afforded by the fact, already brought forward by Ribot, that pleasure carried to excess or over-prolonged is often transformed into the opposite condition. What best suits the nerve, says the eminent physiologist Richet, is a moderate excitation which brings its activity into play without tiring it. ${ }^{1}$

There are also sensations which are not pleasurable, yet at the same time are not really painful. These are ranged in a scale extending from indifference to detachment, and differ from true states of pain, such as those arising from unsatisfied desires, inasmuch as the psychic correlative, which is the experience previously acquired and brought to a high degree of tension by repeated attempts at satisfaction, betrays itself in obstructed psycho-physical functioning. Mention has been made of indifferent sensation with a nascent tendency to withdrawal or to approach (Kulpe, Marshall, Kalkins), but without any pleasant or disagreeable emotion. Strictly speaking, it is not so much a question of indifference, which generally is due to habit, but, rather, that we have here to deal with minimal or very low degrees of pleasure or of pain. The author is of opinion that in every psychic fact, perceptive or representative, there is always a pleasant or unpleasant emotional tone. The general law concerning the genesis of pain may be summed up in all those relations with the world which tend to disintegrate the nervous element to a greater extent than is necessary merely to overcome obstacles. This applies, in all probability, to every form of pain.

${ }^{1}$ Richet. Rev. scientifique. 1906. 
The author's experiments negative Kalkins' view ${ }^{1}$ that the frontal lobes are the chief organs of the fundamental emotions of pleasure and of pain. According to this psychologist, when the cells of the frontal lobes are well nourished and not fatigued - in other words, in a state of anabolism - they react more than adequately to the excitation which is transmitted from the Rolandic zone, and we get pleasure; when, on the contrary, the cells of the frontal lobes become ill-nourished and exhausted-i.e. when they are in a state of catabolism - they react inadequately to the excitation coming from the Rolandic zone, and we get pain.

It seems evident from a brief review of the evidence, even if we take into account only the two fundamental tones of emotion, that we are not warranted in localising the emotions in any definite part of the cerebral mantle, as, e.g. in the tactile zone. To this area we attribute no greater significance than that of being the cortical field of the somatic or reactive reflexes of emotion. Emotion must be regarded as emanating from the whole sensory field, so far as its representative content is concerned, and from the somæsthetic zone, so far as the respective organic reflexes are concerned.

We have seen that the humour of the mind has its roots in the kinæsthetic sense, and is good or bad according as all parts of the organism function harmoniously or otherwise. It may be said that emotion arises from any external or internal fact which disturbs the kinæsthetic equilibrium, and tends either to constrict or to increase the psychic personality, to oppose it or to arrest it in any of its tendencies or conative orientations in the organic, intellectual or affective spheres.

Somatic concomitants of pleasure are : increased circulation in the head, volumetric dilatation of the peripheral organs, ${ }^{2}$ heightening of the pulse, acceleration of the heart's action, radiant visage (or, as commonly expressed, face glowing with joy), increased depth of inspiration, with accelerated respiratory rhythm and increase of muscular power. Pleasure is dynamogenetic. $^{3}$

Somatic concomitants of pain are: diminution of the diameter of the vessels, through contraction of the vascular walls ; pallor of the skin, owing to ischæmia ; diminution of certain secretions--e.g. the mouth becoming dry and the milk disappearing, and increase of certain other secretions, such as tears; constriction of the pulmonary vessels, giving rise to that sense of oppression noticed by all who are under the tyranny of pain ; feeling of cold, want of tone in the voluntary muscles, whence the head bowed down (bent with sorrow, as Lange says), the face lengthened, and the lower jaw slightly drooping; weakness of the voice; enlargement of the eyes, with wider opening of the palpebral fissures. Pain is paralysing.

The fact that it is impossible to separate the mental representations from

${ }^{1}$ Kalkins. An Introduction to Psychology. New York, 1901.

${ }^{2}$ Lehmann. Die Hauptgesetzte des menschlichen Gefiihlslebens. Leipzig, 1892.

${ }^{3}$ Féré. Sensation et mouvement. Paris, 1887. 
the somatic concomitants of emotion has given rise to two opposite theories concerning the nature and seat of the emotions.

According to one theory, emotion consists in a psychic phenomenon determined by perceptions or by ideas, accompanied by a number of somatic phenomena-vaso-motor, muscular, secretory, respiratory-all of which are consecutive and reflex. According to the other theory, emotion is constituted essentially of vaso-motor, respiratory and general somatic phenomena, which immediately follow perception or representation, so that alterations in the various functions, transmitted to consciousness, give rise to emotion. According to the latter view emotion is only the consciousness of muscular and neuro-vascular variations taking place within the body: The names of James, Lange, Sergi, Pitres and Regis, Soury, Kulpe, Mercier, Kalkins, Irons, Baldwin, Dewey, and many others, are connected with this disputed question. Here is what James says : "Our natural way of thinking about these coarser emotions is that the mental perception of some fact excites the mental affection called the emotion, and that this latter state of mind gives rise to the bodily expression. My theory, on the contrary, is that the bodily changes follow directly the perception of the exciting fact, and that our feeling of the same changes as they occur is the emotion. Common-sense says : We lose our fortune, are sorry and weep; we meet a bear, are frightened and run; we are insulted by a rival, are angry and strike. The hypothesis here to be defended says that this order of sequence is incorrect, that the one mental state is not immediately induced by the other, that the bodily manifestations must first be interposed, and that the more rational statement is that we feel sorry because we cry, angry because we strike, afraid because we tremble, and not that we cry, we strike, or tremble because we are sorry, angry, or fearful, as the case may be. Without the bodily states following on the perception, the latter would be purely cognitive in form, pale, colourless, destitute of emotional warmth. We might then see the bear and judge it best to run, receive the insult and deem it right to strike, but we should not actually feel afraid or angry."

Further on he continues: "If we fancy some strong emotion, and then try to abstract from our consciousness of it all the feelings of its bodily symptoms, we find we have nothing left behind, no ' mind stuff' out of which the emotion can be constituted, and that a cold and neutral state of intellectual perception is all that remains. . . . I cannot help thinking that all who rightly apprehend this problem will agree with the proposition above laid down. What kind of emotion of fear would be left if the feeling neither of quickened heart-beats nor of shallow breathing, neither of trembling lips, nor of weakened limbs, neither of goose-flesh nor of visceral stirrings, were present, it is quite impossible for me to think. Can one fancy the state of rage and picture no ebullition in the chest, no flushing of the face, no dilatation of the nostrils, no clenching of the teeth, but in their stead limp muscles, calm breathing, and a placid face?"

If one could demonstrate that motor, vaso-motor, and secretory phenomena, those indeed that form the general characteristic of emotion, exist 
apart from that particular psychic state concerned in the emotions of pleasure and pain, whatever be their forms, the organic theory of the emotions would fall to the ground. When we consider the state of mind in a number of vaso-motor disturbances of longer or shorter duration, finding there nothing, or almost nothing, in the nature of true emotional states, we feel great distrust of the somatic theory. In hysteria we frequently observe cutaneous ischæmia, palpitation of the heart, respiratory anxiety, while the subject is utterly indifferent. In Basedow's disease we meet with a great increase in the cutaneous circulation, redness of the skin, clear and sparkling eyes, and, instead of joy or anger, a sense of anguish and fear accompanies those somatic phenomena. Hysterical women with anæsthetic skins, with ischæmia, and with paralysis of a great number of their muscles, are very far from showing signs of sadness.

The cutaneous ischæmia and the trembling, or even shaking, which we meet with in malaria do not give rise to any emotion, if we leave out of account the general feeling of malaise inevitably associated with the disorder.

Men afflicted with polyneuritis, with complete paralysis and muscular flaccidity, sometimes with disappearance of all the muscular masses, are for whole days in excellent humour, if only they are allowed to hope for recovery.

Grief and joy, along with their respective physiognomic attitudes and modifications of respiration and of the pulse, are capable of being simulated, but in this case the corresponding psychic factor is absent; indeed, the psychic content may be entirely different. We know of men endowed with great self-possession and inhibitory power who can suppress the reflex (organic) manifestations of great joy or profound grief.

The organic theory is no doubt seductive but that is because the experimental habit inclines one to favour the doctrine that is based upon objective and demonstrable facts.

It is certain that between the first psychic fact and the last fact of emotion there exist links that follow one upon another in consciousness, in the form of rapid reasonings and imaginative representations, of which the end always is that the ego appears to be either compromised or exalted.

In the writer's opinion the weak point of the somatic doctrine lies in the fact that it seeks to regard the somatic phenomenon as playing a very substantial part in the mechanism of emotion, whereas the truth is that in the disturbed consciousness there always co-exist two factors: (a) the representation, along with the internal participation of the ego itself; and $(b)$ the somatic factor. Even the melancholic in his grief probes his imagination for an explanation of the origin of his trouble. The two factors are inseparable.

In any study of the emotions one should also take into account the vital tone of the individual. There is no doubt a normal man, in perfect health, is not troubled at the thought of death. Owing to the uncertainty of the period of its happening, there is no feeling of dread, and for this reason sailors and miners, who are constantly risking their lives, have no fear of death, whilst old and weak persons think of it and fear it, because the organic 
sensation of the weakness of their bodies is in harmony with the idea of death.

The favourable acceptance of the James-Lange theory is due to the fact that we cannot demonstrate a subjective state of emotion dissociated from somatic phenomena, whilst only the latter can be put to experimental proof, as exemplified in the splendid work of Mosso, Lombroso and Patrizi in Italy. The modern scientific spirit attributes value only to such facts as it can control, estimate and measure objectively. The writer cannot get away from the conviction that the psychic components of emotion are not less important, but even more important, than the somatic. If the emotional tone is either pleasurable or painful that is because consciousness is directly informed of the emotion-producing fact which, on the other hand, induces the organic reflexes.

In a large number of emotions the element of surprise is highly important. If a soldier on the march comes in sight of the enemy and is assailed by fear of death and the pain of rapidly imagined wounds, or if a girl is terrified on seeing a snake close to her feet as she walks along a country path, the fear and the terror in these cases are the effects of a psychic factor, that of surprise, which interrupts the normal course of the representations and feelings, these being substituted in consciousness by others that have a disintegrating power. It is the imminent threat to the integrity of one's own physical and psychic personality that determines that complex psycho-physical state which we call fear.

If the girl sees the serpent from afar, and is not threatened, or if the soldier has already seen the enemy several times, so that there is no surprise, and danger appears less imminent, or, even should it be imminent, if the consciousness has previously been occupied by those images and dangers so often that the personality has become accustomed to them; if there is adaptation of the consciousness to the new order of images-as happens in the case of miners and sailors - the emotion "fear." either is insignificant or is a simple reminiscence of what it was on the first occasion.

Between perception or representation and the organic phenomena of emotion there exists a complex psychic factor, which in its essence can be reduced either to a threat of disintegration (pain), to interdiction, or, on the other hand, to instantancous exaltation of the personality. If a man striving for a fortune acquires it gradually, by adequate means and methods, he will not have any emotion of joy. His consciousness gradually integrates itself with the image of the million, and his internal satisfaction becomes a feature, a permanent attribute, of the progressive adaptation of his personality to the new conditions of existence. Such internal satisfaction is not an emotion, it is a state.

If, on the other hand, even a modest fortune should come to him suddenly - as, e.g., the winning of a prize in a lottery - his joy might be so intense that he would run the risk of a congestive attack or a cerebral apoplexy. In this case it is surprise that breaks up the habit of the consciousness and determines the emotion. 'Iwo factors-the person and the fortune-remain the 
same ; the third - the duration of the representation, which if brief gives rise to surprise, if prolonged or repeated creates a habit of consciousness--is variable. One might, in many cases, picture this phenomenon to oneself as a sudden and abrupt reversing of the machinery of the cerebral waves, after the mechanism is all ready to start, or has already acquired motion, in a particular direction. One may suggest that the high nervous tension produced by the surprise that interrupts the normal course of thought, while it interdicts the higher centres (reasoning and attention), discharges itself upon the lower organic centres, and determines the reflex organic phenomena of emotion. Let us eliminate surprise, give free course to thought, and direct our attention to the sensation that has produced the emotion, and, as Stratton has observed, the emotion ceases or becomes markedly lessened.

Where an individual places himself voluntarily in circumstances that occasion emotion, as in the case of the orator who has prepared his speech, or that of the traveller who passes along deserted ways by night, the real cause of the emotion is to be found in the conflict that takes place between the sentiments and impulses that determine him to speak or to travel, and other sentiments and impulses that threaten to compromise the ego. If the orator were sure of himself and of the opinion that others would express about him, and if he did not contemplate the possibility of compromising some of the components of his personality (self-esteem, ambition), then he would not be perturbed, just as the traveller would not be perturbed if he did not know that he was exposed to danger. In these cases the pain or fear arises from the contrast between the tendency of the ego to affirm itself and the danger of an opposite result. The respiratory anxiety and the tumultuous action of the heart are reflexes of these representations which compromise the ego, and do not in themselves constitute the state of conscious fear. In ereutophobia, or the fear of blushing, the principal and preponderating element is the idea of blushing; this it is which determines or provokes emotion. It is the fear and presumption that others are detecting in the redness of the face some hidden cause, and drawing inferences therefrom of a compromising nature. The situation becomes so desperate that finally the sufferer will only leave the house at night-time, so that the reddening may not be observed. Yet how often does it happen that flushings of the face and head take place without in any way disturbing the consciousness! The fact is that phenomena of vaso-constriction and vasodilatation, associated with disturbances of respiration, are observed in many debilitated persons, especially in young girls, without their being aware of any of those modifications which emotion induces in the consciousness. The young person who suffers from ereutophobia has been subject for some time past to reddening of the face and rush of blood to the head, but has not experienced any emotional fear of blushing until on some occasion she has been informed by others or has seen herself and begun to imagine that others are noticing the blush and indulging in suspicions which compromise her respectability.

Those who suffer from hemiplegia due to a lesion so situated that it 
withdraws the optic thalamus from the inhibiting influence of certain parts of the cerebral mantle, or allows abnormal stimulation of the thalamus, present the outward appearance of great emotivity. They weep, laugh, and cry out on the slightest impression; yet it is evident that there is no true emotion, only the semblance of it. ${ }^{1}$ The weeping and laughter of such sufferers are only simulacra of the real emotions and if there is any emotional resonance of these in the consciousness it has to be attributed to old and repeated association between the somatic phenomena and the respective emotional states.

In the ordinary chorea of Sydenham, if the affection is severe enough to involve the musculature of the face in the anarchy of the muscles of the trunk and limbs, the physiognomy assumes the strangest expressions of hatred, anger, menace, pain or joy, while the mind gives no evidence, through corresponding commotion, of sharing in the emotion expressed by the physiognomy.

We know how abundant secretion of tears, with red and swollen face, reddened eyes, and increased secretion of nasal mucus-all symptoms denoting dilatation of the vessels of the skin, the face and the mucous membranes - sometimes coincide with pain. Lange supposes that these symptoms are consecutive, of the nature of a reaction against the primary constriction - a relaxation of the small arteries after strong contraction. It remains an open question, however, whether pain and anguish are accompanied by vascular constriction, even of short duration, or whether it is not rather the case that the vascular reaction varies in different subjects. The writer's experience leads him to conclude that pain is not constantly associated with vascular constriction.

Rehmka ${ }^{2}$ in recent times has brought forward a wealth of facts in support of the view that sentiment is a subjective fact, while the representation is objective. The sentiment is determined by a group of images, either distinct or obscure, which constitute the objective feature of a particular consciousness. The mode of feeling, be it pleasant or disagreeable, thus depends on the image which falls into the focal point of attention.

Mantegazza, who undeniably was a keen observer, remarksin the Physiology of Pleasure and Pain ${ }^{3}$ that painful stimuli sometimes reduce the heart's action and sometimes increase it, whilst they cause respiration to be sometimes more frequent and shallow, sometimes slower and deeper. This statement holds good in the case of the movements that an animal makes under conditions that are painful_as in flight, for example. It is no doubt true that these movements are, as it were, spasmodic, in one sole direction, as remarked by Ribot, ${ }^{4}$ and that in its ultimate analysis pain is accompanied by diminution of muscular energy. Yet it is indisputable that the conscious fact of pain or fear sometimes coincides with phenomena that are opposite in nature to those upon which it has been said to depend.

${ }^{1}$ T. Senise. Il riso. Naples, 1914.

2 Rehmka. Zur Lehre vom Gemüt. Leipzig, 1911.

${ }^{3}$ Mantegazza. Fisiologia del dolore e del piacere. 1879.

${ }^{4}$ Ribot. Psychologie des sentiments. 1896. 
The assertion of Münsterberg that agreeable stimuli produce movements of extension and disagreeable stimuli movements of flexion has been only partly confirmed by the experiments of Dearborn and Spindler. ${ }^{1}$ These authors have found that the reaction varies according to the nature and temperament of each individual, some making movements of extension mostly, others those of flexion, whether the stimulus be pleasant or unpleasant. Under the influence of painful stimuli the flexions were found to be 66.6 per cent. and the extensions 33.3 per cent. On the other hand, under agreeable stimuli the extensions were 67.8 per cent. and the flexions 32.2 per cent.

Objective language is the physical component of thought just as the somatic phenomena described are the physical component of emotion. Take the case of an individual who has become aphasic as the result of a lesion of the cortical motor centre (so. that he suffers from true motor aphasia and represents to himself the images of things and the respective words, but is unable to translate them into motor images and hence into co-ordinated movements for the articulation of the words): if he does not express his thought because it lacks the physical component that is indispensable for its extrinsication, no one will think that thought rests upon that component of language, and that the anatomical basis of language, in its motor component, is the same as that of thought. Such a view is applicable to the sensory images of words, as mentioned in the preceding chapters.

If we adopt the formula preferred by the upholders of the somatic theory of emotion we shall have to say: "I see such a thing; I name it, therefore I perceive it." "I represent to myself certain images; these are reflected on the centres of speech, which then furnishes me with the notion of things." Surely reasoning such as this will not be adopted by any psychologist.

In some cases - e.g.degenerates-emotions of joy are expressed by mimicry of weeping. A lady mentioned by Sikorsky used to smile in such a way that it was always uncertain whether she were laughing or weeping. She had no other mimicry. In progressive paralysis and in hallucinatory paranoia we often find so-called indifferent mimicry associated with different emotional states, or we meet with laughter and weeping without emotional content.

Ferrari ${ }^{2}$ remarks appropriately that cognition of the organic modifications (vascular, glandular, etc.), as they are noticed by the consciousness, does not give rise, as James says, to emotion. He adopts Langley's view as to the sympathetic and its three divisions, the cerebral autonomic system, the sacral autonomic system, and also the spinal (visceral) system, and speaks of the emotional reaction as belonging to the sympathetic system. The emotional reflex becomes moderated by the progressive development of the cerebrum with its inhibitory powers (cognition).

Organic manifestations of emotion and consciousness are two factors that are closely bound up with one another, but, at the same time, one must

${ }^{1}$ Dearborn and Spindler. "Involuntary Motor Reaction to Pleasant and Unpleasant Stimuli." Psych. Rev. 1897.

2 Ferrari. "Le emozioni e la vita del subcosciente." Riv. di Psic. 1912. 
insist upon the fact that emotional manifestations may occur without any consciousness of emotion.

Another question presents itself to us at this point: "Do the fundamental emotions differ from sentiments? If so, wherein lies the difference between these two very important facts of mental life?"

Lange distinguishes the group of emotions from that of sentiments by the fact that sentiments are not accompanied by the organic phenomena attached to the emotions. One cannot altogether agree with this sharp distinction of Lange, as regards the essential features of the two groups, especially if it be remembered that he has been induced to make this distinction by the necessity of maintaining his theory of emotion. He says: "It is incontestable that sadness, joy, fear, anger, and other similar states constitute a group of phenomena distinct from love, hatred, contempt, and admiration, and from the psychological point of view it is necessary to separate them. It is only to the first group that I apply the term 'emotion'; the other states are passions, sentiments, or whatever one chooses to call them. . . . As a matter of fact it becomes absolutely necessary for anyone who studies the physiology of these phenomena to distinguish them as far as possible. We cannot assimilate things so different as terror, fury, joy, envy, love, and the passion for liberty." 1

It is difficult to demonstrate any distinction in the real nature of the two groups. Indeed, everything induces us to believe that the states of the second group represent only advanced degrees of those of the first. More advanced evolution means that new components have been united to and assimilated with the first, but that does not change their intimate nature or their origin. The same thing happens with emotions as with ideas. If from the simple images of herbs, fruits and flesh, with which we satisfy the sense of hunger and sustain ourselves, we have come to form the concept of food in association with that of preservation-it may be, perhaps, through the intervention of reflection, although this is very doubtful-that does not establish essential differences between separate foods and food in general.

So, too, the primitive satisfaction of hunger gradually becomes penetrated by the joy of labour, associated with the sentiment of the duty of working; into these there enter as components all the pleasures that arise from difficulties overcome and from the successful extrinsication of our increasing activities, as well as all those that come from the labour of others and from the beneficent current of solidarity that smooths away so many troubles in life, bringing additional comforts and giving fresh impulses to new and more harmonious forms of existence.

These new components, through which the primitive movements for securing food have been transformed into others, more complex and more combined, do not alter the intimate emotional nature of those states. Pleasure and pain are ever the watchful sentries which determine and co-ordinate the actions of human beings.

1 Lange. "Les émotions." Etude psychophysiologique. 
More complex representations, new desires, new motor tendencies, and new aspirations, corresponding to the ever-increasing capital of ideas and notions of the world and of the relations of each individual therewith, do not alter the real nature of the struggle for pleasure and against pain. On the other hand, with the suppression of many of the old motor tendencies the mode of satisfying the appetites has become complicated, but the essential nature of these has in no way been altered. Thus, through continuous grafting of emotional and intellectual components, the old emotion of hunger that formerly excited simple reflexes has given place to the elevated sentiment of the duty of labour, which in its turn generates reflexes of extreme complication in an endeavour to secure greater comfort in life.

Even the religious sentiment, patriotism, the moral sentiment, love, in the widest sense of the term, including love of liberty, of art and of science, all present the same fundamental characters. Analysis of these sentiments provides an incontestable proof of the tendency of the psychic ego towards progressive development, towards integration and towards the consummation of its aspirations. At one moment we behold it rising towards the sublime and divine; again, we see it penetrating the spirit of the ethnic group to which it belongs and, in the illusion of representing that group, seeking to synthetise in itself the emotions and sentiments of the group, bestirring itself for the happiness of all, even at the cost of self-sacrifice; and yet again, we find it assimilating the mind of another being whose vibrations increase, perhaps a hundredfold, its joy in life and its activities in any direction or form of labour. It is always the ego, in its hedonistic orientation, which exercises, consciously or unconsciously, a great power of assimilation both in the physical world (cognition) and in the social world.

The higher sentiments do not involve the field of the organic reflexes of emotions, of which we have spoken. They reflect the psychic ego more particularly, drawing their elements from the cosmic and social environment, and inciting mankind to actions which are to be considered as a wider reflex, the equivalent of the organic reflexes of the primitive emotions. Analysis shows the presence in their structure of numerous intellectual elements in logical connection. These intellectual components, associated as they are with various corresponding affective dispositions of the ego, do not bring into action, except in a very slight, perhaps imperceptible, degree, the centres of organic innervation which come into play in those primitive emotions more closely connected with the organic ego, but they are reflected, directly or indirectly (through other logical series of thoughts), in human conduct, considered in the widest sense of the term.

Sentiments, like the primary emotions, have their roots in the kinæsthesis, inasmuch as this is the essential nucleus of the personality. There is no sentiment that is not prepared in this subsoil. This it is which gives an individual his emotional sensibility, participating perhaps in the mechanism of expression and giving an emotional colouring and direction to the intellectual representations.

Emotions and sentiments, indeed, are merely degrees of states of 
consciousness, with more or less conscious reflexes. First in the series comes the fundamental sensation of pleasure or pain which simple stimuli produce when they come into contact with the body and penetrate the kinæsthetic sense. Last in the series are the higher sentiments, those which, in the moral and intellectual field, accompany the hazards of life within the environment. This evolution takes place by the progressive addition and fusion of new components, these being furnished by the relations of each individual with others, and by the experience of these relations.

The same evolutionary process is to be followed in the case of the sentiments as in that of intelligence.

In well-evolved beings thought and sentiment are in harmonious combination. This may be said both of men and of races. In some men there prevails that force which determines sentiment; in others that which is productive of calculating thought. Where cold thought prevails over sentiment, conduct is generally egoistic, and this is true both of the individual and of the group. Bergson, perhaps by way of protest against the Germanic mentality, asserted that life is not merely thought (Kultur) but something much more profound-sentiment. In the evolution of sentiment the altruistic element becomes more pronounced. Normal mental life, at its highest level, presents a harmony between thought and sentiment. Regnaud ${ }^{1}$ compares mental life with a straight line, the extremes of which represent thought and sentiment. The middle of the line coincides with equilibrium between the former and the latter. When sentiment prevails and assumes the control and direction of conduct, the regulating power of thought is encroached upon or made subservient. Where, however, thought or cold calculation, without any sentimental tone except the egoistic, directs or regulates human actions, conduct becomes directed towards the satisfaction and consummation of selfish aims, without consideration of the interests of others, and leads even to criminality, individual or collective.

Yet emotion or sentiment always does accompany the intellectual processes, inciting, encouraging and animating them, infusing into them the breath of interest. Even the most austere scientific research is imbued with the joy of truth, just as a social and political situation reveals the high national interest which pervades the soul of the statesman who courageously confronts the difficulties opposed to him. It is emotion that impels to new thoughts and gives rise to man's restless activity. That it is which promotes or creates interest, which exalts or depresses. Through this we are led to experience the whole gamut of pleasure and of pain; this it is which awakens all the hidden energies of the human spirit and lets fly from the anvil the spark of genius. The artist, the scientist, the hero, the politician, the man of the mart, the workshop or the field, men, indeed, engaged in all civil occupations, and works of progress, are inspired by ideas but moved by sentiment, which is the real goad that excites the ego, as man makes his essay in the struggle for life and victory. Emotion is like the sound of a stringed

\footnotetext{
${ }^{1}$ Regnaud. Origine et philosophie de langage. 1888.
} 
instrument from which the bow draws vibrations, ranging from the widest to the shortest and most acute, producing all the tones and symbolising the infinitely various states of the human mind, as it thinks, feels and acts. From timidity to bold assurance, from doubt to positive assertion, from fearful indifference to audacious desire, from æsthetic to moral pleasure, from love, which is penetration of minds, to repellent hatred, from fear which disarms to courage which confronts, from diffidence which hinders to faith which emboldens, from vanity to ambition, from need of the essential, which spurs one on, to enjoyment of accumulated fortune which allows its possessor to take life easily and permits the commencement of decadence, from love for others to the tyranny of an exacting and rapacious egoist-all these and other multiform states confer a particular tone and impress a particular directive upon life.

Desire, to some psychologists, is the turn-key of psychic life: it is fundamental; it stimulates thought, which in turn furnishes plans for its satisfaction.

At the same time it is true that it is often the intellect that discovers new horizons, opening up new fields to desire and human activity. Appetite, as Spinoza observed, is the very essence of man, and from it is derived all that seems to preserve him. Intellect offers motives but not the determinism, the impulse to action. Desire it is that urges to action, and that is emotion. Reason is inhibitive rather than propulsive and, from a psychological point of view, it would be absurd, as Ribot remarked, to suppose that an arid idea, a conception devoid of any emotional accompaniment, can have the slightest influence upon human conduct. Reason, says Schopenhauer, does not as a rule make use of the passions; rather do passions make use of reason to attain their ends.

Knowledge (culture) exalts man, placing him in a higher sphere in which he intuitively perceives a form of dominion with respect to others (ambition) (Sherrington).

The writer has no desire to discuss the question whether or not there are such things as ideas devoid of any emotional colouring, but he expresses the opinion that wherever there is an order of ideas which resolve themselves into actions, wherever the ego is caught in the motion of things and in the conflict of individual, social, scientific, religious, æsthetic, interests, it is always emotion that is the great multiplier of the energies, the vivifier of thought, the propulsive force which incites and determines.

It is not thought that controls sentiment, rather does the latter guide thought, and where it would appear that reason inhibits or controls, it will often be found that there is really another sentiment controlling the first, which is only in appearance the more propulsive. It is interest which gives value to the intellectual content of a man or a nation.

The exercise of intelligence and memory enlarges the field of the emotions and sentiments (desire) and, consequently, the field of action; but emotion and sentiment account for a large part of the story 
of humanity (all wars have had the way prepared for them by desire for dominion, for wealth, for liberty, or by religious sentiment or the influence of woman).

If emotion exceeds certain limits, the greater intensity is at the expense and to the detriment of intellect. The mental fields become restricted, the horizons obscure, the associative power enfeebled, and the flow of ideas retarded. Again, if emotion is so strong as to reach the degree of passion, this encroaches on the logical power and lays siege to the mental capital, paralysing the evocative and associative powers which are the factors of logic, and establishing the wasteful, static condition of a mind disarmed. Passion detains in the focal point of consciousness only those particular ideas and images from which it draws its origin: they are its objective. If other ideas do cross the field of consciousness, they are feeble and void of determinative capacity; as no interest animates them they succumb to the domination of ideas enlivened and protected by powerful passion. Passion is, at bottom, only an acute or chronic emotion which disturbs the intellect in various degrees, impairing its vigour, controlling it, rendering it subservient, or seeking from it arguments which, at most, cover up in an illusory fashion the depreciation of the consciousness, when this is not entirely obscured. Meanwhile, with reduction of the prophylactic power of logic, moral and economic interests become enfeebled; the harmony between the individual and his social environment is profoundly disturbed, because the action (conduct) of the passionate man is not a resultant of contrasting (propulsive and inhibitory) forces, but is determined by one single group of emotional ideas which has taken possession of consciousness, owing to special biological conditions in the development and function of the cerebral system. Passion lowers the human spirit from the elevated spheres of life to the domain of the primitive emotions. Thus it happens that noble ambition, if it becomes blended with poisonous vanity, abandons a man to a fatuous inebriety which consumes a quantity of energy that should be devoted to logic, all the more because the vain man never possesses sufficient energy to realise his dreams. So, too, it comes about that passional love becomes a tyrant dominating the intellect, which lays down its directive powers on the altar of love. Suspicion and distrust, if they exceed a certain measure, interdict intellectual action, whilst jealousy concentrates cerebral activity, in a wasteful manner, in a mono-idealistic sphere. Religion, when tempered by intelligent knowledge of life, is love, such as is reflected in the noble figures of Saint Francis of Assisi, Saint Catherine of Siena, Calvin, Bruno, and is then biophylactic; when it was perverted by fear of a devil roaming at large and ensnaring souls, it gave rise to epidemics of obsessions, of hysteria, of lycanthropy, of flagellation and the terrific political criminality of those times.

The moral sentiment is the highest expression of sentimentality, and is essentially constituted of ideas and experiences regulated by biophylactic laws, and it is the sentiment which has least connection with the field of the organic phenomena of the emotions. 
The author's experiments upon monkeys have shown that the fundamental and intermediate emotions are preserved (some altered) after removal of the frontal lobes, whilst the higher sentiments or emotions (as these are represented, in outline, in the monkey) are either absent or profoundly disturbed, corresponding with what is observed to follow severe injury of the frontal lobes in man. In the case of mutilated monkeys the conduct becomes reduced to single reflexes, dissociated and inconsequential, unfurnished, therefore, with biophylactic power, whilst at the same time there is a prevalence of the organic reflexes of the primitive emotions (fear, anger). In normal monkeys, on the other hand, the conduct is prevailingly more protective: it presents an evident affective intonation and is more logical, being based upon perceptions, experiences and judgment, whilst the organic reflexes of the primitive emotions are much reduced in proportion. The most striking evidence of this is afforded by the social sentiment, which becomes abolished in monkeys after removal of the frontal lobes. Sentimentality thus appears to be a function more particularly of the frontal lobes. Its very nature, consisting as it does of emotions, variously associated ideas, impulses and inhibitions, would give us grounds for presupposing the existence of organs that are different from those of the primitive emotions. This view is supported by the results of phylogenetic and ontogenetic investigations, dealing with the evolution of the brain, and of the frontal lobes in particular, as well as by clinical and experimental observations which are fairly constant and convincing. 


\section{APPENDIX TO CHAPTER IX}

\section{The Social Sentiment}

HighER consciousness is characterised, amongst other things, by the intervention of historical experience which co-operates with the impulses determined by actual sensations and the so-called instincts, so as to give rise to a form of conduct that is the resultant of both propulsive and inhibitive forces, involving the element of contrast. The resultant represents the intuitive synthesis of energies directed towards the more certain conservation of life and the development of the pleasure of existence, on a higher scale, and it always contains a utilitarian element derived from life in common, even when the community opposes inhibitory elements.

Many factors contribute in diverse manner and varying proportion towards its development. Amongst these the chief are :

1. The development of the posterior, sensory mantle, which includes all that part of cerebral cortex extending from the fissure of Rolando to the occipital pole, and from the interhemispheric surface to the inferior aspect of the cerebral hemisphere. From this development is derived the progressive perfecting of the perceptive and emotive capacity, and hence the progressive process of assimilation of the thoughts, emotions and conduct of the environment, and also of the language of nature, with the individual consciousness.

2. The development of the mnemonic activity attached to individual and collective experience, this impressing a certain orientation and directive upon conduct, which, to some extent, reflects the habits of life of the community, in accordance with the law of imitation and hedonistic adaptation (conservation and development), and in part manifests the creative power of the human brain, based upon association (phantasy, imagination).

3. The evolution of the social sentiments, which are intellectual-emotional syntheses of an experiential and historical order, and may be represented as the resonance, in one's own consciousness, of the emotions of other beings with whom one lives (reflex reproduction), along with the necessary observance of all social obligations imposed by the beliefs, sentiments, and needs of each individual in relation to the environment and to the customs of the social group,* customs which aim at defence from hurt and the promotion of well-being.

* In this connection, one may again refer to facts that are constantly observed in monkeys which have suffered mutilation of the frontal lobes-e.g. the complete indifference they manifest not only towards those who have been in the habit of tending them, and for whom they formerly showed affection, but also towards their 
The mnemonic process becomes daily richer in experiences and wealthier in content and this accelerates and intensifies the directive power over conduct, because of the greater coefficient furnished by the memory of past experience derived from life in common (reciprocal defence and protection, greater efficacy of life in common in the struggle for existence, beliefs, customs, etc.). The impulse given to this process by the accelerating power of writing is enormous as compared with the influence of traditions transmitted by word of mouth or in the form of customs (the enormous progress of mankind is due to the development of the printing press).

The emotions and thoughts of the social environment, when assimilated by an individual, take a prominent part in the structure of his consciousness and in the determinism of his conduct, which is the resultant of impulses provoked by ends in view, to the attainment of which there co-operate the individual and personal desires, sentiments and aspirations, tempered by the desires, sentiments and aspirations of others.

We cannot imagine a state of consciousness from which the conduct of every man is derived, apart from social relations. These give us the outline of the moral sentiment.

The social sentiment, or what may be called sociality, has been going on developing by degrees and in man has extended its domain from the cavern to the tribe, to groups of habitations, to communities, to regions, to the nation, to the race, to internationalism, in regular, inevitable fashion. The interhuman entente, which marks one of the aspects of human progress, though interrupted by periods of unheard-of ferocity or hidden by egoism (to-day we see it arrested at the more advanced stage of one race versus other races), uprises like a dawning sun, smiling upon many nations (internationalism), and will reach its zenith in the assured existence of the more evolved human groups. There is no longer any room for doubt on this point.*

Sociality has a long and slow evolution. We find it in the most varied forms in insects, birds and mammals. In middle-grade and even lower-grade monkeys, such as cercopithecus and cibus, it assumes rather human-like features. These may no doubt be rudimentary but they nevertheless present such manifestations as leave no doubt about its actual existence. Sociality is an integral element of mind and disappears entirely, so far at least as its symptomatic manifestations are concerned, after mutilation of the frontal lobes, and in almost all forms of mental disease in the human subject.

fellow-creatures, whose life in common is characterised by undoubted manifestations of the social sentiment.

* The author had already written an article on this subject (Ferrari's Rivista di Psicologia Applicata, 1917), when the notion regarding the League of Nations, so strongly supported by President Wilson, began to take shape. Wilson, who seems not to have had a full conception of the diplomatic prejudices of old Europe, has shown a marked incoherence, because, although he has given proof of a high degree of sociality, as this sentiment is conceived by psychologists and naturalistic sociologists, he has failed to meet the practical difficulties, and to free himself from the old formulæ of International politics. 
Reduction or suppression of sociality is a feature which the writer has constantly observed in monkeys which have suffered mutilation of the frontal lobes and also in imbeciles whom he has had under observation for years. No one who has had experience of the mental make-up of phrenasthenics, especially in cases of profound mental deficiency, can have failed to notice the isolation in which these afflicted subjects pass their existence. In the less degraded, one may certainly observe signs of attachment to some person or other, but this is for the immediate advantage to be derived therefrom, just as in the case of monkeys, dogs, and even birds, which exhibit pleasure when approached by persons who give them things to eat or otherwise show them attention. Sometimes this semblance of attachment to these persons is the corrective of fear, which predominates over all other emotions, in imbeciles and many inferior beings, and it promotes a sense of protection on the part of persons who are in the habit of taking care of these weaklings. Sometimes what we get is a mere imitation of attitudes, simulating an affection or sentiment which does not actually exist, as in the case of shame or religion, sentiments which find no place in the spirit of imbeciles. These beings live a life apart from their fellows and from other men. Their minds take no share in the griefs or joys of those amongst whom they pass their existence. If a piece of bad fortune overtake the family, the imbecile remains indifferent. If he weep, he merely imitates; if he laugh, it is only a mechanical reflex, a purely individual affair, void of any participation in the joy of the community. Egoistic and egocentric imbeciles are incapable of acts of generosity, and this is true even of the less degenerate. If, at school, they exchange some words with other scholars or give any sign of approach, it is nothing more than an imitative mechanism, without participation of that sentiment of joy that springs from companionship. If they beat their chests, at home or in church, it is not from religious sentiment; they are either imitating at the moment, or remembering what they have seen; their souls remain unthrilled by true sentiment. ${ }^{1}$

In monkeys, we have observed a number of manifestations which present, in a rudimentary or embryonic fashion, the features of human sociality. The histories of those animals, which the author had under observation for a long time, both before and after mutilation of the frontal lobes, have revealed facts of some considerable importance to anatomical psychology, to the furtherance of which these researches were directed. A cercopithecus, e.g., one day met in the garden a black dog which had had one of its frontal lobes removed. At first the monkey was afraid and took refuge in a tree. Several times it came down from its perch and cautiously approached the dog, but each time, as the dog came towards it, it made off again and ran for the tree. After many repetitions of this scene the monkey seemed to become persuaded that the dog was good-natured and so it cautiously drew near, retreating and advancing from time to time until it finally came near to the dog and, stretching out a hand, caressed it. Encouraged by this first success, it stood in front of the dog and with its other hand patted the other cheek,

${ }^{1}$ Maschka. L. Bianchi. Tratt. di Psich. 2nd edit. 1915. 
then stroked the head and passed its hand under the lower jaw. Coming still closer, it touched the dog all over with an air of solicitude and curiosity, as though wanting to make friends.

In order to keep this sympathetic cercopithecus under closer observation, the author decided to take it home. Signora Bianchi carried a small satchel suspended at the waist and in it were chestnuts, roasted and shelled, which she gave to the monkey. Soon it came about that, whenever she passed by, the monkey would jump up on her, paw her, and take hold of the satchel, which it soon learned to open. It was very jealous. One day Signora Bianchi entered the room, where the monkey was, carrying a friend's baby in her arms. It was fortunate that the monkey was chained, because as soon as it saw her with the baby in her arms it became wild and excited, such as it had never been before. It ground its teeth and made attempts to get hold of the infant and only quietened down when Signora Bianchi went away with the child and came back alone.

From the facts mentioned in the record of the experiments described in Chapter V. (apart from the fairly abundant literature dealing with the life and habits of monkeys-Garnier, Thorndike, Brehm) one can draw conclusions as to the degree of evolution of the emotions which, in monkeys, are seen to be taking on the character of sentiments, even granted these have mainly an alimentary or sexual content, sentiments, however, which pass beyond the limits of immediate interest and extend into the fields of friendship, gratitude and protection, which are the fundamental elements of sociality.

In every case of mutilation of the frontal lobes in monkeys, what was found to be suppressed was, without exception, that sentimentality or feeling for others that we designate as sociality. A similar condition exists in human beings who have suffered severe injury to both frontal lobes and in those whose frontal brains are imperfectly developed. The majority of the insane exhibit the same feature. Sociality is thus a psychic manifestation which comes with evolution. It varies in different individuals and races and becomes suppressed by mental disease and severe frontal lesions.

G. Pellacani ${ }^{1}$ declares that social conduct in animals is instinctive. This may be granted if we take instinct to mean the social attitude emanating from a psycho-anatomical structure in course of evolution. No one can fail to notice the gradual passage of the so-called instinct of lower animals to the sociality of monkeys. From the gregarious instinct of ants and bees, to that of birds (pigeons, swallows, cranes and a number of other birds of passage) ; from the latter to the gregarious instinct of the beaver and some of the higher mammals, especially the elephant, and finally to the gregarious instinct of the monkey, we have a graduated scale in which, step by step, new intellectual and sentimental elements make their appearance, and with the intervention of these the features of the so-called instinct become lost.

${ }^{1}$ Pellacani. I problemi della istintività nella condotta umana. Bologna, 1915. 
One of the characteristics of instinct* is its immutability, and if we subject members of any of the more evolved groups in the above-mentioned zoological scale to examination we find that the supposed instinct does not remain at the same level but exhibits individual variations and mutations which are determined by adaptation to a more assured (evolved) level of existence. The adaptation is brought about by a more perfect perception of the environment, and a consequent enlightenment of consciousness. This progression (and complication) of the primitive instinct is based upon associative memory and is apparently incited by the struggle for existence, bound up with the rising hedonism, which reaches its highest level in man.

Many writers make reference to conflict between the individual and the social environment. Yet, in the more advanced states of society, it is difficult to detect conflict between the individual and the social group, the reason evidently being that the group gives rise to a hedonistic influence which conforms the life of the individual to that of the environment, even from infancy: In the words of James: "With the child, life is all play and fairy tales and learning the external properties of things; with the youth it is bodily exercise of a more systematic sort, novels of the real world, boonfellowship, and song, friendship and love," and so the social spirit of the young person is formed. Other factors have to be taken into account: the suckling, the smiles, caresses and kisses of the mother and other members of the family; the solicitude manifested with regard to the satisfaction of the vital requirements; the trouble taken in promoting the development of the æsthetic sense of the proper self, which begins to spring up with the tidy dress, the curled hair, the sensual caressing and the praises bestowed upon the beauty and intelligence of the child; the toys, and the singing to sleep and so forth. Painful experience also plays its part, counterbalanced by the experience of comfort and protection that is derived from the family and, later on, from society.

In the contrast between experiences of a pleasant nature and those consequent upon denied satisfaction of desires, expectations and impulses: in the sum total of satisfactions and inhibitions experienced, even if the latter be the effect of coercions (education), hedonistic sensations always prevail, at least in natures normally conformed, and these, with the help of new sensory grafts and associative mental formations, give root to the gregarious sensations. Contrast and conflict belong to inferior natures, or depend upon violent methods of coercion, not calculated to attemper the nascent hedonism to stern reality, or, again, are due to misery which robs the environment of every happiness.

Under normal conditions in modern civilised life, progressive adaptation to the social environment is apparent from infancy. From the environment

* A discussion of instinct does not fall within the scope of this work, but the writer would maintain the term instinct in the nomenclature provided only it have a very different signification, such as that of aptitude, dependent upon special, inherited, anatomieal structure. We may have struetural evolution, but with that we get variations in attitude. 
there is an inflow of integrative currents whilst from the infant there is a reflow of actions which are constructive of the nascent personality, these returning as an unconscious homage to the social consciousness. The great joys in life spring from the social relations, whatever be their nature, more especially when man is adapted to his environment, and still more when his action is beneficent. It is only weak and sickly natures that adapt themselves with difficulty and have a painful consciousness of the flow of the human senses-painful because such individuals are incapable of a hedonistic assimilation of the social currents and of reaction which permits the individual ego to find its level in the environmental harmony.

With the development of the human psyche (psychogenesis) there disappears what, by way of euphemism, has been termed social instinct. This occurs not by suppression of the phylogenetic content, but by a slow transformation, in the structure of the personality, of all the more important and formal elements of antecedent life, and by assimilation of the impulses of the actual social environment. The innate impulses and tendencies which make man appear, to some, to be an instinctive being, are only complicate reflexes of innumerable stimuli coming from the environment and assimilated with the consciousness.

Waxweiler, ${ }^{1}$ after mentioning that the question of the instincts has now been submitted to experimental methods in the laboratory, remarks that he foresees the day when the word instinct will disappear from scientific terminology.

Liberty is not to be regarded as an invariable quality belonging to the individual but as a social compromise, according with the consciousness of all. It is, in a biological sense, a reflex of the social content, a transformation of receptive elements and fusion of these with the experiential elements of the individual life and of the gregarious life of the social group, resolved into conduct. The mechanism becomes increasingly complicated through the gradual dignification of the reflex and variation in the inherited substratum (family and ethnic) which manifests itself in a biological predisposition to feel the environment in a different way and to react in the most varied manner.

Language assists towards a more rapid development of sociality. To the sensory experience of the individual there is added, by degrees, that of the emotions and cognitions of other men, translated into phonetic articulate symbols, which afford a more perfect acquaintance with the states of mind of all the components of a social group. It participates in the emotions of the environment in proportion to the greater intensity of the interhuman impulses afforded by the increased number of paths and means of communication with the states of mind of other fellow-beings amongst whom the individual is spending his existence. The intellectual-emotional elements of the environment constitute a very rich material for the construction of human

${ }^{1}$ Waxweiler. "Sur la modification des instincts sociaux." Soc. d'Anthro. pologie de Bruxelles. 1907. 
mentality. The latter is an activity that is changeable in content, in methods, in intensity and results, and has nothing in common with instinct. In opposition to the notion expressed by Baldwin ${ }^{1}$ one may put forward the conception that there is nothing innate except the biological predisposition to react favourably in a hedonigenous environment. As, however, the reaction is variable, the reactive variability, even when set in motion by internal impulses, has not the character of instinct. It is, above all, the environment, with its numerous and varied currents of sentiments, with all its compulsive forces, its habits, its labour, its joys and its griefs, that nourishes the social sense and social habits, although the methods involved are very dissimilar-subjection and dependence, compulsion, imitation, suggestion, faith, and sympathy, which itself is rather a complex psychological factor capable of determining different forms of adaptation.

Boccardo ${ }^{2}$ has expressed the view that "goods" are the connective tissue of the social body. No doubt the economical factor possesses a great power of cohesion amongst the social components, but many other factors have to be taken into account.

Some of these forces, such as subjection and compulsion, act more strongly in the case of infants and feeble persons, whilst imitation, suggestion, sympathy, and interest, even in the guise of "goods," are the great forces which act upon the human mass and on its diverse and varied groups.

In the more highly evolved mammals, such as monkeys, we can detect a very rudimentary degree of ali these forces acting upon their sociality, on a hedonistic basis. Thus, we get protection in the case of the stronger, subjection in the case of the weaker; also the greater security which comes of number, the despair which arises from solitude in new surroundings (whence the comfort afforded by hearing the voices of distant companions), and sympathy. All these are based upon fundamental emotions and, along with a certain capital of mnemonic representations with a prevailingly protective, alimentary or sexual content and a significant degree of perceptive penetration, determine a behaviour which, though it still obeys, in the main, the vital laws of attraction and repulsion, reveals in the variety of attitudes a certain level of consciousness which affords a measure and means whereby we can estimate the effects of removal of the frontal lobes. In all these vital and reactive manifestations we find neither an invariable programme nor distinctly fixed rules, but momentary variations corresponding with a wider perception of the environment and more complex modes of adaptation thereto.

The marvellous mental and social development of mankind in the later millenniums, as compared with the slow and laborious evolution of the palæolithic and neolithic periods, is due, as already mentioned, to spoken language

${ }^{1}$ Baldwin. Mental Development in the Child and in the Race. Now York, 1906.

2Boccardo. Raccolta delle più pregiate opere moderne di economia politica. Vol. vii. 1871. 
in the first instance and subsequently to writing. Speech is the ocean in which all forms of consciousness become immersed, throwing off the last remnants of the instinctive preformations and emerging again to attain the mobility of human consciousness as we find it in the infinite variety of individuals and groups. The prelogic mentality to which Levy Bruhle ${ }^{1}$ refers probably corresponds to the first stages of the development of language, inasmuch as the logical process involved in lengthy reasoning is only possible with the help of language.

Writing, on the other hand, permits an enormous accumulation of material, consisting of human thought and experience, in the archives of the universal intellect, and from these are culled the norms for new social sensations, norms which correspond more fully and adequately with the new attitudes of life in common, in accordance with the pressing requirements of the individual and the social hedonistic consciousness. Hence comes about the marvellous activity displayed by mankind in the economic field, which daily becomes more and more controlled by scientific truth, for the intellect is ever striving anxiously after truth, in an endeavour to mould and direct human activity which, in turn, is becoming ever more disciplined and regulated by social sanction. With advance in civilisation man makes fuller use of intellect, whilst the intercourse and interchange between men is attended with more mutual advantage. Primitive societies, composed of poorly differentiated men, permit but a slow growth of inteilect and the use they make of what intellect they do possess is much less.

If we cannot agree with the notion that there exists a social instinct it is because the attitudes of each individual in his environment vary under the influence of an enormous number of stimuli and circumstances. They vary in accordance with the infinite variety of perceptions in the environment in which he is developed, and also according to individual and collective experience. Again, they vary with variations in the individual capacity for the reception of stimuli-i.e. with the degree of excitability. T'There is, however, something in all this which is stable, but it is not instinct nor is it characterised by a fixed mode of reacting: it is the predisposition to feel the social influx, which in turn determines conduct.

In this respect one can agree with Baldwin when he asserts that we inherit the physical variations of the cerebral plasticity, for this conforms to experience of the environment.

It matters little whether the human aggregates which constitute social groups are to be considered as organisms governed by laws pertaining to the life of individuals (biological), as maintained by upholders of the organic doctrine, Spencer, Worms, Schäfer, Novikow, and others, or whether they are governed by intuitions and economic determinism as maintained by Marx, Loria, De Molinari and others. In each case the guiding principle is the unconscious law of adaptation and natural selection aided by the accelerated evolutionary movement of the psychic activities caused by modifications 1910.

${ }^{1}$ Levy Bruhle. Sur la fonction mentale dans les sociétés inférieures. Paris, 
determined by the group. Comte, influenced by the notions of Saint-Simon, expressed a belief in the existence of one single law, in accordance with which hunan societies would develop. This conception was further developed by Spencer, who arrived at the conclusion that human societies are particular forms of existence shaped by the same laws as those which preside over the evolution of the universe.

There is a certain parallelism between the evolutionary movement of the mind to which reference has been made, and the total sum of stimuli, inasmuch as every acquisition and every variation, in the way of more advantageous adaptation on the part of each individual, becomes the patrimony of the group; division of labour which gives rise to mental and operative differentiation is another factor which must here be taken into account.

T'arde, ${ }^{1}$ indeed, maintains that all social facts are due to individual inventions propagated by imitation. To him invention is a supreme accident in which foresight plays no part, and it gives rise to that imitative well-being which is a constant feature of every social fact.

Economic organisation is the effect of a biological situation and it may promote evolutionary economic advances, which are regulated by a number of individual and collective psychic factors, mainly the following:- the different evolutionary capacity of different individuals belonging to one and the same group in the same environment; secondary variations of the environment and differentiation of labour, whence arise biological and psychological variations; differences in the mode of perceiving and feeling; differences in the amount of mental capital available and in the aptitude for utilising it and for acting.

If it is true that functional variations correspond with structural modifications and that morphogeny corresponds with psychogeny, we will take into account the fact that along with the general features, common to the social group, we meet with individual differences. The former are summed up in customs and creeds, the latter vary considerably because they depend upon a great variety of factors, amongst which are to be enumerated the following:- the complex and varied mental structure of different individuals and their genitors, their predominating emotions, their morphogeny-which may be altered under the influence and impulses of psychic states-the numerous eircumstances under which fecundation and gestation take place, intoxications of the nemasperm and the ovule (a product of civilisation), crossed matings, and differences in occupation, which in turn determine new morphological variations and new functional developments, and open up new fields of ideas and aspirations.

The social phenomenon is one of great complexity and almost all doctrines relating thereto contain some germs of truth. The structure of the family, religions, creeds, the organisations of labour, differentiation of which creates new social bonds, are amongst the more important factors. Labour organisations to-day are no longer the simple and elementary forms of social existence

\footnotetext{
${ }^{1}$ Tarde. Les Lois Sociales. 1898.
} 
mentioned by Le Play, ${ }^{1}$ but are complex organisations whose groups are bound together by the perfection of the product (the finished article), by economic interests, by sanitary interests, etc.

Apart from all this, however, there is no doubt as to the existence of the social sentiment. It is seen in love and hatred, faith and diffidence, cooperation and individualism, security and dread, help and treachery, exaltation and humiliation, victory and defeat, pride and humility -in short, in moral pleasure and moral pain, in every variety and every degree, as summed up in the fundamental law of contrast which regulates life, with its victory of hedonism over negativism. It is a product of collectivism, in the social sense, of intimacy, of co-operation and mutual protection, and, at the same time, of the consciousness of personal energy and personal contribution to the prosperity of the environment. In the sentiment of co-operation one can behold heterogeneity co-ordinated with the homogeneity of the social group. The social sentiment emanates from a combination of sentiments and ideas (the intellectual elements of the social sentiment), the latter more than the former, which together represent a complicate function, which in turn has determined an enlargement of the cerebrum and a differentiation of its parts, under the pressing stimulus of the hedonistic victories to which life inevitably aspires.

Language and sociality proceed pari passu. All emotions contain an expressive element. This gradually becomes transformed from the palpitation, the trembling and pallor of fear to the cry, the voice, the look, the caress, the first lispings, and to speech, in the more evolved emotions. We can detect this progressive transmutation in all the phases of evolution in the growing infant. To the primitive emotions of pleasure and of pain, with which organic changes are closely related, as already set forth, there are added the complex pleasures and pains that arise with inter-human relations, and these find their expressive paths and fields in speech and conduct. Patriotism, the spirit of self-denial, the sacrifice of life as a burnt offering upon the altar of the Motherland, love and religion in the purest expressions of art and of language, and in abstinence from organic pleasures, are nothing else than the sublimation of the primitive emotions.

With the development of their relations and means of communications, all societies which have attained a very high development tend to become like one another. Their legislation, civil rights and provisions, their scholastic institutions, the products of their lands and workshops, show a constantly increasing similarity. Democratic politics is daily extending and gaining ground; the humanitarian sentiment is insinuating itself into the popular consciousness, though still rather weak to combat successfully the old tendencies and old ideas of conquest and dominion that pertain to some races. The struggle for life and fortune between the social groups has assumed a different character : it is a struggle based on intellect, on industry and on courage.

${ }^{1}$ Le Play. Les Ouvriers Européens. 2nd edit. 1855. 
Differences between social groups dependent upon physical environment or upon the original nucleus of each race or upon social experiences or upon the organisation and differentiation of labour, as well as upon the political order of things, are becoming less noticeable. In proportion as civilisation is making rapid strides in the sciences, which every day unearth new sources of natural energies, subserving these with astounding rapidity to the individual and collective life, mysticism is dying out, and there lies before us a period, probably a long period, of indifference, in relation to spiritual habits and popular beliefs. Meanwhile there springs up, quivering and trembling, the sentiment of human liberty, individual and collective, giving more alluring promise in the free and unrestricted rivalry between all eivilised peoples as they vie with one another, with the products of their work, in the race for fortune. Crusades for religions, which no doubt represented an important stage in the progress of civilisation, are now being followed by crusades in the interest of health and well-being and crusades against the domination and conquest-making tendencies of any race which acts the part of a conspirator, concealing its treachery and lust for dominion under the plea of freedom to develop the activities and life of its people or of regard for its possessions. Interhuman contacts and exchanges tend towards uniformity in life and customs, and to a closer understanding, which resolves itself into the sentiment of human solidarity.

Whilst the sentiment of humanity and solidarity is always adding new constituent elements to consciousness, it observes the fundamental law of hedonism which assures new sources of pleasure and of power in the struggle for existence, as well as a larger and more reliable measure of protection to the individual and collective life.

After long and bloody struggles, the evolution of the sentiment of humanity and liberty has suppressed the tyranny of caste, kings and religions. Mankind is progressing towards a state of equilibrium in which there will be equal freedom for all in applying the personal energies to work and progress, allowance always being made for differences in intellectual and physical strength which are unavoidable individual prerogatives, along with their legitimate effects upon economic conditions, for these are inevitable and irreducible consequences of human differences, as regards both individuals and groups. It is from these that the new religion of culture and health takes its origin.

The evolutionary process in society may be summed up as the granting to each individual of a social group the fullest liberty to develop and utilise the personal energies, under the guidance of moral and juridical laws that are perfectly equal for every member of the group, along with the protection of labour, in any form, on the part of the group. The liberty (which is disciplined) and the protection, taken together, give the measure of solidarity of the social group and this furnishes elements for that strong social and humanitarian sentiment which is still in course of evolution, but is even now strong enough to play a part in the composition of every evolved personality. These elements consist essentially of the experience of the individual in his 
personal environment and the historical experience of the group (social ontogeny and social phylogeny).

The evolutionary process is not equal, nor is it equally progressive, in all peoples. It is often interrupted by periods of repose, by revolutions and regressions. Liberty, with co-operation and moral discipline, is one of the stars which guide the triumphal march of mankind. Liberty, however, may have obstacles put in its path. Labour may be enforced or it may be left without protection. Again, very unequal climatic or historical conditions (political institutions, religion, popular education, etc.) may so bring it about that too wide a gulf separates the most advanced elements of a people from the least progressive members who have had to live and struggle under unfavourable conditions of existence, with the result that one of two things happens. Either the people remains oblivious to the signs of the times, learning little or nothing of the forward march of other peoples and enduring the power of the rich and educated classes, in which case the character of the popular mass, blind and deaf as it were, puts a stamp of inferiority upon the whole social group, or, again, the populace feels the new impulses propagated from afar, like seeds borne upon the wind and falling upon receptive soil-and with what result ? Revolution, with all its horrors, all its excesses, in an endeavour to level outstanding inequalities, and often with a complete reversal of the order of things, which makes manifest the silence of the humanitarian sentiment, of too recent growth, in the evolution of the human understanding, to be capable of resisting the reactive impulses of the crowd, panting for the recognition of its rights! One cannot altogether agree with the assertion that a hierarchy of the lower as against that of the higher classes of a people is like comparing a savage and primitive race to a civilised people; not altogether, because when the mass provides a higher hierarchy, in that very fact lies the proof that it possesses the potentiality of a rapid evolution. All that is wanted is that it be placed in conditions favourable for development (education and protection of labour). This is the commanding duty of the higher hierarchy, though often it happens that it is not so understood-e.g. by those who rule over us. If, owing to defect or distortion of the sense of civil duty, the higher hierarchy fails to understand its social and political obligations, and presses upon the lower people, we will have the phenomenon of revolution, which at bottom is only the reactive impulse of the popular consciousness, aspiring, it may be somewhat blindly, to justice and more favourable conditions of existence, as against the tyranny of autocracy or of caste and against the stupid, ignorant presumption of those at the head of public affairs and their immediate and chief instruments.

It is different with savage and primitive peoples who do not feel the new impulses of the times. In their case the sentiment of sociality is but rudimentary and limited to a group of persons, generally not very numerous. Society is there maintained by traditions which fix determined customs and beliefs, making these, as it were, a social heredity, which remains unopposed by any extraneous element of advanced civilisation. If new elements come 
into opposition with, and become gradually substituted for, the old habits they are the fruits of a strong perceptive power which takes stock of the enviromment; in other words, they are the result of new acquisitions and a new determinism. Thus do innovations and variations come about. The old experience becomes substituted by the experience of the new adaptations, more in accord with the development of the individual and collective personalities and more promising of victories in the struggle for life.

It seems clear that the fundamental factor consists of an understanding between the components of the social group; it is the consonance of the manifestations and vicissitudes of the life of all in the mind of each ; it is painful experience of one reverberating in the minds of all; it is danger which concerns one individual yet is felt to be a threat to all; it is sentiment and imagination allowing the whole group to place and feel itself (less intensely, no doubt) in the same position as one member of the group ; it is the sharing of vicissitudes that affect the individual or are common to the lives of all ; in all of which there is a basis of mutual protection and co-operation in aspirations and in defence, which after all is hedonism.

One can readily understand that all the components of a group do not perceive and feel the impulses to a new situation in an equal degree. Truly evolved consciousness is the possession of few (Novikov), but two factors come into play which tend to make it universal-suggestion and imitation. Progress will be slow by evolution or by hasty bounds through revolution. According as the variations are useful so the new experiences are substituted for the old, and the social bonds are constantly being drawn closer together because the benefits obtained, benefits derived from the inventions of few and the co-operation of many, are utilised by the community and sometimes by the whole of humanity (Baldwin, Romanes, Tarde, Sergi, Max Nordau, E. Meyer, Linder).

This condition creates and promotes, as has been said, a sentiment which is still in course of evolution. It had its roots in prehuman and savage communities; it developed under the stimulus of some religions ; it received an extraordinary impulse from Christianity but it is to-day being transformed under our very eyes through the hedonistic impulse of labour and co-operation.

Everything tends to show that this sentiment takes shape along with the development of the frontal lobe and with the corresponding assimilation of numerous intellectual elements. The latter are intellectual syntheses which are essentially bound up with emotions possessing a high potentiality.

It has been said (by Sciamanna, vide Chapter II.) that the frontal lobes are the central organs of the emotions because, amongst other reasons, clectric excitation of these organs modifies the rhythm and frequency of the pulse, the blood-pressure in the arteries, the respiration, etc., and these are organic components of the emotions. In point of fact, there is nothing that is more amply contradicted by the results of experiments upon monkeys and of extensive lesions in the frontal lobes of the human subject. The truth of 
this has been amply demonstrated. The constant phenomenon presented by monkeys, and even dogs, which have suftered mutilation of the frontal lobes is the prototype of the emotions--viz. fear.

Dogs and monkeys have drenched themselves with urine as the result of a threatening impression which previously caused them no concern. This symptom coincides with the well-known fact that the centres of the organic phenomena of the fundamental emotions are localised in the sigmoid gyrus, in the dog, and in the Rolandic convolutions in monkeys and in man. Now if the emotions, and here we refer to the primary emotions, persist after removal of the frontal lobes, and if man becomes more excitable, more emotional and more impulsive, in consequence of severe and extensive lesions of the frontal lobes, this is to be explained by the fact that the seat of the organic phenomena of the emotions lies not in the frontal lobes but in the sigmoid and Rolandic areas, and that the latter have not been involved in the experimental lesion. This finding has been confirmed by clinical experience as well as by experimental observation.

The emotional excitability is increased simply because removal of the frontal lobes weakens the intellectual-sentimental powers comprised under the terms "associative perception" and "inhibition." If experimental excitation of the frontal lobes has sometimes produced organic symptoms of emotion there is a ready explanation. The strength of the current employed has been too great, with the result that there has been diffusion of the electric stimulus to the cortical zone in very close proximity, excitation of which produces precisely those modifications of the circulation, respiration, and of the bladder which accompany fear. What do become suppressed by extensive bilateral frontal lesions are, in man, social sentimentality, friendliness, social understanding, the sense of social obligations and therefore the moral sense, the sentiment of duty, true love, courage and the inhibitory and regulative power over conduct, and, in the case of mutilated monkeys, the rudimentary manifestations of some of these attributes.

There is no doubt that various criticisms that have been levelled at the doctrines of the functions of the frontal lobes have not been dispassionate. The critics have failed to make distinctions. They have evidently wished to believe, and to make others believe, that the functional localisation described was absolute and circumscribed, when, in point of fact, it was clearly stated that in its specific function the frontal lobe utilised the intellectual and emotional products of the entire brain. They allowed too much scope to prejudices and attached too little consideration to the immense difficulty that is involved in detecting and recognising the phenomena and in associating them together so as to interpret them in the light of psychology, aided in turn by psycho-pathology.

Sociality has constantly become suppressed after frontal mutilations. That semblance of friendliness and love, which appears in one form in the dog and in another in the monkey, is only the embryo of the mature condition which we find in modern civilised man. When both frontal lobes are severely injured in the human subject, or when these organs are not developed 
(idiots and imbeciles), similar conditions obtain. Love and friendship, which are fundamental sentiments of sociality based upon reciprocal protection, co-operation, etc., are not emotions which emanate directly from organic modifications, nor must they necessarily be accompanied by those changes that constitute the organic elements of the primary emotions. They are, rather, sentiments which, though they germinate and have their beginnings in these primary emotions, have nevertheless come to belong to the more purely spiritual aspects of mind-the light and heat of the higher consciousness. Even were we prepared to admit that excitation of the frontal lobes produces the fore-mentioned modifications of the organic functions (an admission that is quite at variance with the more reliable findings, summarised in the chapter on emotions) it would not necessarily follow that we should have to regard the frontal lobes ${ }^{1}$ as the " central organs of emotion." Bearing in mind the fact that there are points on the frontal area where excitations gives rise to movements of the eyes, pupil and ear, movements which are duplicates of those produced by excitation of corresponding sensory centres in the visual and auditory areas (a fact which led the writer to express the opinion that they are related to the processes of attention, inhibitory and evocative, which have their seat in the frontal lobe), one would be fully justified in advancing the hypothesis ${ }^{1}$ that the areas which give rise to the organic phenomena of emotion may likewise have duplicates which would furnish the kinæsthetic elements in a higher field of emotion-in other words, in the field of sentiments, especially the most synthetic of these-the social sentiment-with the whole train of intellectual syntheses incorporated with the emotions and consisting of renunciations, inhibitions, social obligations, protection, increased benefits, etc., all of which have their roots in, and draw their vital nourishment from, the kinæsthesis.

Suppression of interest and curiosity, leading to isolation from the group -in other words, disappearance of sociality, characterised by indifference towards the social environment and a melting away of all manifestations of friendship and attachment, and of that offshoot of sentimentality that displays itself in the courting of the sexual instinct-are facts that are constantly observed after removal of the frontal lobes. The primary emotions, the appetites and the instincts, remain.

This experimental condition of affairs finds a counterpart in human psycho-pathology.

If it is true that primary amentia (idiocy and imbecility) finds its explanation in defective evolution of the frontal lobes, especially the pyramidal layers, more than of any other part of the brain, we may institute a perfect parallelism between the mentality of the monkey that has suffered frontal mutilation, as manifested by its conduct in the simian environment, and that of the idiot in the human environment. Timidity, unsociability, selfishness, the absence of the sentiment of friendship, idleness, laziness, tics,

'Bianchi. "Sul significato dell'area corticale la cui eccitazione produce dilatazione della pupilla." Ann. di Nev., 1916; and Archives de Biologie, 1917. 
brutality of the sexual instinct (when present), are the most conspicuous features of idiocy in the human subject, regarded from the point of view of sentimentality.

This condition is closely linked with an analogous state of consciousness, more particularly moral consciousness.

\section{NOTE}

To the writer it has seemed not inopportune to add this appendix, which completes the picture of the function of the frontal lobes. It does not pretend to be an analysis of society, much less a dissertation upon the various doctrines relating to this subject that have been expressed from time to time. It is simply the expression of some thoughts which the author has entertained regarding the physio-psychological problem of sociality, a problem which he believes should find its most legitimate interpretation in the facts brought to light by researches in the fields of anatomy, experimental physiology and human pathology. This is a point upon which the author would lay stress, because of its bearing upon methods of research in psychology. Whilst certain exponents of social science betray great liberty of thought in their interpretation of social phenomena, the biologist must seek to come forward with the products of positive inquiry which bring social phenomena and, generally speaking, all psychic phenomena, as far as possible, within the domain of biology, more especially morphology, physiology, and cerebral cytotecture, controlled by psychopathology and experiment. 


\section{CHAPTER $\mathrm{X}$}

\section{Consciousness}

"'To us positivists," writes Morselli," "consciousness is an abstraction. Strictly speaking, there exist only phenomena and facts of our vital activity as conscious beings; i.e. we are conscious of them because we perceive them within ourselves." Consciousness is an attitude of psychic life which is stamped by the particular character of the notions that are constantly being grafted upon the kinæsthetic stem, and by the sentiments arising from these grafts and resolving themselves into actions the story of which is registered. The Ego is the synthesis of the life and the cognitions of the individual, it is a unifying activity which is constantly undergoing renewal, under the action of external agents and the relations that are ever being established with the consciousness of other beings with whom we live (social consciousness).

The anxious endeavour on the part of many naturalists and psychologists to settle the question where exactly in the scale of animal life the dawn of consciousness can be detected, or to fix the dividing line between the Umbewuste Denken and conscious thought, is not only futile but needless, like the vain endeavour to establish a line of separation between instinct and reason. Proceeding from phenomena of tropism up to reason, the nervous processes involved are all found to be of the same nature. The nervous system with its receptive mechanism, its centripetal paths, its transforming organs (ganglia), and its centrifugal or neuro-muscular paths, is built up on the same system in all creatures provided with a nervous system. In all animals complication of the reactions or adaptations is proportionate: (1) to the degree of perfection and differentiation of the receptive sensory organs ; (2) to the increased number of central organs which act as transformers and multipliers; and (3) to the greater possibilities of co-ordination of the motor organs by means of which living creatures react upon the world. The process is really always of the same nature. The relative invariability of what is called instinct has connection with the simplicity of the nervous apparatus whence emanates the life of relation. Nevertheless, variability of the instinctive reactions may be brought about by variations in the environment and, in the higher evolved beings, by methods of education which are capable of causing the evolution of the nervous system of the whole species to occur more rapidly, though the process always remains a slow one. Instinct is not invariable, nor is it probable that the mechanism which gives rise to it is immutable.

What has been called " unconscious thought," or, in the words of Lloyd

${ }^{1}$ Morselli. "I limiti della coscienza." Rivista di filosofia. 1913. 
Morgan, "the beginnings of a rational scheme," represents in the zoological series only a long dawn, the scanty and pallid light of which does not permit full and accurate observation. It is followed by the clear morning of intelligence in the higher mammals, more particularly the primates, then by full midday sunlight in the conscious intelligence of modern civilised man, which, too, has had to pass through phases of obscurity and penumbra. The stronger light is due to the larger number of nervous elements, and their greater development and differentiation, and is the more intense the greater the number of these and the more closely they act together.

It will, of course, be understood that this is merely a provisory formula, a tentative interpretation which may serve to provide a more intelligible conception of the phenomenon " consciousness," which, from its very nature; must ever remain shrouded in mystery. It is, further, to be observed that we know little or nothing of the life of relation of the lower creatures. McCabe reports an incident related by Jeannette Power, who observed an octopus in an aquarium carry a species of oyster with one tentacle and a stone with another, and wait for the oyster to open, whereupon the octopus immediately inserted the stone between the valves and thus was able to devour the mollusc. This action, if it took place as described, presented all the characters of reason. We may also speak of it as an adaptation, but the preparation, the waiting, the whole complexity of the reaction permit us to ascribe to it attributes of reason or, let us say, unconscious reason, if we may for the moment make provisional use of the latter term. In any case, we can agree that we have here a nervous mechanism educated by experience and productive of a result that is useful in life. There is no doubt that appropriate mechanical or visual stimuli give rise to actions that seem rational. Take, e.g., the selection of digestible from amongst indigestible material made by the limulus from which Loeb had removed the principal ganglion (supra-œsophageal). We have no standard or means by which we can estimate the extent, the lucidity or otherwise, of the consciousness of other creatures, because we have no counterpart other than our own consciousness, which itself is variable according to time and circumstances, and therefore cannot serve as a means of measurement.

All that can safely be said of such instances is that the behaviour is always logical, whether it be made up mostly of reflexes or mostly of psychism. The bond of coherence between actions is one of the most universal manifestations of life, extending from chemical affinities to tropisms, from these to adaptations occurring with unconscious thought, and finally to conscious logic, in the evolved mental world.

Consciousness, though not a faculty, is in continual evolution. It is a very variable and mutable condition pertaining to the psychic processes occurring in an evolved brain. Its field lies not in any special part of the cerebral mantle, but in the whole brain, perhaps in the entire organism. It evolves along with life, and has no origin other than in life. Its evolution has neither end nor limitation. Its components are the traces of the organic movements of the creature, the sensations, emotions, notions, sentiments 
and reactions in the most varied forms of adaptation. It may be restricted within a narrow circle of cognitions but expands with new perceptions of the physical environment and by assimilating the energies of the social as well as the physical environment, ever gaining in scope and power with advances in interhuman relations and understandings. All biological processes, be they chemical, physical or mechanical, play a part in its development, in so far as they represent the continual motion of matter, the ions of which become transformed into nervous waves from the very moment when the nervous system first makes its appearance in the evolution of living things. It keeps pace with the evolution and the complexity of living organisms and especially with the development and complexity of the nervous system.

Evolved consciousness has certain relations with time and space, two very important elements which serve to complete it. The conception of time and space is only arrived at through the intermediary of perceptive processes infinitely numerous in time, and through the immense number of small spaces measured by the innumerable movements of the body as a whole, of the limbs in particular, and also of the eyes. The consciousness of the ego in time and space relates but to one moment in one definite space. It is, however, susceptible of analysis by recalling into the focal point the whole succession of periods of time, in association with the various points of rendezvous in the story of the individual life - that is to say, the main incidents in which the ego was interested. All times and spaces cannot be represented separately at the same moment, but consciousness has the power to make them pass through its focal point in their infinite variety like a biographic film. All that is not represented in the focal point, in the same unit of time, constitutes the subconscious and this part of the mind is incomparably larger than the illuminated field.

Where and when, in the evolution of life and of the nervous system, consciousness begins, is, as already said, a futile question. To seek to find an answer is but to pursue a phantom which always vanishes as we step forward to grasp it. Psychologists and naturalists long made of this theme a debating ground for the most difficult and delicate mental exercitations; but it does not come within the scope of this work. In their endeavours to reconstruct the natural history of our planet and the story of life, naturalists and psychologists have no doubt taken advantage of all the newer and more delicate instruments of investigation which the modern scientific spirit has devised as the result of researches in the fields of Anatomy, Physiology, Comparative Physiology, as well as Geology and Palæontology ; but the problem seems no nearer to solution.

It is necessary that we come to an understanding as to the extension to be assigned to the conception "Consciousness." There are two main currents of opinion. According to one, consciousness is to be regarded as the light which emanates from the experience of the ego, in the acquisition and utilisation of cognitions obtained from the external world. If we accept this view we agree to recognise the dawn of consciousness in the mutable adaptations 
of living creatures as they react to the changing circumstances of the environment, guided by the past experiences of the individual and the species, and by the notions, be they few or many, which the creatures comprised within the particular category acquire through the perfecting of their perceptive instruments. According to the other view, and it has good reason to support it, we must conceive consciousness as including "the unconscious," which becomes organised with the vital experience of all beings submitted to the action of the forces of nature, which slowly but surely modifies them and impels them onwards to the light of consciousness. In this case the limits of consciousness would be confused with those of animal life - that is to say, with all those manifestations which to outward appearance are conscious and volitional, but in reality are nothing else than the effect of the chemicophysical action of external stimuli upon the protoplasm (vide Chapter I.). It is from the tout ensemble of the chemico-physical modifications that consciousness draws the elements of its first nucleus, and from the tout ensemble of these its raison d'être. In the human subject it draws its elements more directly from the unconscious, just as in the story of life the consciousness of the higher beings appears before us as the continuation of the physicochemical reactions of the lower creatures.

McCabe, ${ }^{1}$ in a recent publication, referring to the suggested possibility of the existence of the senses of pleasure and pain in protozoans, says that "we may speak of an organic analogue of feeling in the protozoa but there is no ground for saying that it is not a physico-chemical process." Max Verworn and Loeb refuse to recognise any true psychic manifestation in the various phenomena in the life of protozoans, cœlenterates and worms. ${ }^{2}$ R. S. Semon ${ }^{3}$ made an accurate study of a group of echinoderms and came to the conclusion that there is no trace of true psychic activity in these creatures. Even the selection of food from amongst digestible and indigestible material may be merely a mechanical response to chemical or physical stimuli rather than a matter of experience.

There is no doubt we cannot speak of consciousness and volition in these creatures, but at the same time we cannot refuse to recognise in the phenomena of reaction which they exhibit a slow transformation of the physico-chemical processes into unconscious mental processes.

According to Loeb, too, the circling of the moth around a lamp, sometimes resulting in burning, is a phenomenon of heliotropism like the bending of plants towards the light, just as the collecting of palomonetes at the anode, when subjected to the passage of an electric current, is a phenomenon of galvanotropism. It is neither instinct nor curiosity on the part of the moth that makes it fly towards the light but a chemical action of the light upon its protoplasm, with mechanical effect. ${ }^{4}$

${ }^{1}$ McCabe. The Evolution of the Mind. London, 1910.

${ }^{2}$ Loeb. Loc. cit.

${ }^{3}$ R. S. Semon. Beitrage zur Naturgeschichte der Synaptiden des Mittelmeers. Mittheilungen. Bd. vii.

${ }^{4}$ Loeb. Comparative Physiology of the Brain and Comparative Psychology. 
The similarity of the effects produced by physical agents on different organisms (animals and plants) would indicate that the latter must have some property in common. This condition would be fulfilled by the presence in all of them of some substance which undergoes a chemical change when subjected to the influence of light, this in turn giving rise to changes of tension in the contractile tissue. In the same way, what has been called instinct on the part of the females of certain insects, which always deposit their eggs on substances furnishing food for the larvæ, would be a phenomenon of chemiotropism - that is to say, the action of the insect would be the chemical effect of certain molecules which, by diffusion, act upon cutaneous elements and on the tension of the muscles, including those of the genital organs concerned in the depositing of the eggs.

The passage to a more illuminated and protective reaction is gradual. Thus, in the medusa, the rhythmical movements of the umbrella in locomotion are comparable with the movements of the heart and respiratory muscles, in which no one pretends to see the elements of consciousness; on the other hand, the movements of reaction which the medusa performs with the manubrium of the umbrella, when any point of it is touched with forceps, are markedly more complex and more protective.

Even did we admit that these movements are of a chemical nature, which is much more probable than one would imagine, although the chemical fact has not yet been established, we witness once again the slow and confused passage from the genuinely chemico-physical to the psychic process of life, and by degrees from the latter to the facts of consciousness. Were it suggested that in these reactions there is a dawn of consciousness, one could not strenuously deny it. Lloyd Morgan expresses the opinion that a dim consciousness probably accompanies those essentially physico-chemical facts.

In molluscs, e.g., we find a marked variety and complexity of movements, especially in the pursuit of prey, the avoidance of capture and the courting of the female, and at the same time more fully developed and perfected sense organs, and larger and more approximated nervous ganglia. Thus, in the octopus, the eye is highly developed, and the ganglionic and neuro-muscular apparatus makes clear the reason of the very complex manifestations, including even emotional attitudes, exhibited by this creature. Such behaviour as is observed in the octopus already presents the features of a more highly evolved intelligence. Not that this should signify consciousness in the human sense, but it certainly indicates something more than a simple reflex. Rather would it represent, in the words of Professor Lloyd Morgan, the commencement of the "utilisation of experience through the exercise of intelligence." Utilisation of experience coincides with enlargement of the nervous mass and the possibility of more numerous associations and, accordingly, of combined memory.

We have already mentioned, in Chapter III., that in the lowest vertebrates belonging to the fishes (e.g. the lamprey), the brain appears as a small bulbous expansion situated in front of and above the spinal medulla, whilst 
in the dog-fish it is a highly developed and complex organ. We saw, too, that a large and important olfactory organ communicates directly with the fore-brain, which is no more than represented by a thin layer-the humble precursor of the rich mantle of the higher vertebrates. ${ }^{1}$

We can now recognise in a general way a parallelism between the development of the nervous system and the evolution of intelligence and, accordingly, of that quality of mind which we call consciousness.

Many writers (Romanes, Brehm, Zell, Preyer, Hobhouse, Morgan) discuss the form and degree of intelligence in fishes and employ such words as these: "intelligence and consciousness," " obscure physiological processes," " organic memory," " unconscious memory," " unconscious intelligence," etc.

All this very varied nomenclature shows the great uncertainty of psychologists and naturalists in interpreting the actual psychological state of these inferior creatures, because the observer cannot always abstract himself from his own states of consciousness, in judging the movements and reactions of lower creatures subjected to the influence of external stimuli. Were it possible for us to abstract ourselves from our conscious and thinking ego, and could we picture to ourselves a diagram of the psychic complexes involved in the life of the different orders of animals, we would behold the perceptive line and the reactive line ascending regularly and keeping parallel with the growth and perfection of the sense-organs and the central nervous system, except for certain oscillations which cannot as yet be regularly explained.' In this succession of creatures in the evolutionary scale we can, in a manner, perceive two links of a long chain connecting the physical world with consciousness, as Lugaro ${ }^{3}$ puts it in his penetrating criticism of philosophic systems.

In the higher fishes, as the cerebral mantle develops and the nerve-cells increase in number, at the same time becoming more evolved and assuming more numerous relations with one another, the psychic life makes the mantle its organ, and there now appear on the scene emotional manifestations which, in their intensity and some other respects, resemble those displayed by mammals.

Many years ago the writer went out one day with fishermen, at a tunny fishery near Palermo. Before the fishermen began to haul in the net of the so-called "death-chamber," which that day held more than 700 fish, ali seemed to be quiet and peaceful amongst the captured fish. Immediately the crew commenced to haul in the net there followed a state of agitation which increased each moment and thrilled the spectator. The tunny-fish dashed madly about in all directions, knocked against one another, leaped out of the water, dived below again, the whole performance being one of

${ }^{1}$ Johnston. Nervous System of Vertebrates. 1907.

2 The line undergoes interruption and even inversion on passing from the higher invertebrates to the first vertebrates provided with a cerebral mantle. Indeed, the intelligence displayed by some insects is much superior to that of the cartilaginous fishes and even higher species. An explanation is set forth in Chapter III.

${ }^{3}$ Lugaro. Modern Problems of Psychiatry. 1907. 
convulsive agitation. Amongst the tunny-fish there happened to be a swordfish, which at first was calm, then became seized with like agitation, and as we looked we saw it, in an accession of fear or excitement, plunge its terrible weapon into the body of a large tunny.

How is this intense emotional agitation to be explained? We may possibly set it down to chemical action arising from difficulty in breathing (the restricted space of the meshed net) or to a physical factor dependent upon abnormally close contact with one another; but the writer cannot exclude an intuitive fear of danger in the new and strange situation in which the fish found themselves. Shall we ascribe it to intelligence in the human sense? No, because there is no proof of a clear perception of the situation; nor can we even speak of consciousness, because consciousness implies perception and memory. It is something which, though not entirely free of the chemical or physical or mechanical element, such as is found in the mental life of creatures with a more rudimentary nervous system, we can nevertheless figure to ourselves as a step in the ascending scale of development of intelligence and consciousness.

With the constant growth of the nervous system, organic becomes associative memory.

As we cannot conceive of intelligence without memory of associated images-for even the perceptive process (of recognition) is based upon memory-consciousness may be regarded as a synthesis of memories and their combinations.

If memory of the organic modifications, determined by the succession of stimuli acting upon the surface of the body and on the different senses, is an indispensable condition of intelligence, it also seems clear that associative memory is the basis of consciousness. At the level of instinct, associative memory is static and uniform, or almost uniform, and for this reason we exclude consciousness, which involves movement of the multiform, conflict and choice.

With multiplication of the organs of life, with their constantly increasing differentiation and perfection as we rise in the animal scale, we obtain the constituent elements of consciousness to which are added, in the human subject, the images of one's own body provided by visual sensations, by frequently repeated tactile sensations, by the sound of the voice, by the performance of movements and the experience of one's own force acting against resistance, by the feeling of well-being or ill-being, and other obscure components. Amongst the latter must be mentioned the chemical processes of the organic interchange, secretory movements, movements of the stomach and intestines, movements of circulation and respiration, etc. All these fundamental and vital functions, with their accompanying movements, furnish additional elements of consciousness in evolved organisms, elements that are not apparent but are none the less important. The nucleus of the organic ego enlarges with the more differentiated intuition of the physical ego and its own peculiar features, and with all the organic modifications caused by external stimuli, which at the same time furnish the objective material for associative memory. 
It should be quite clear that the phenomena of consciousness cannot be expressed in terms of a quantitative denominator which will serve as a standard for measurement, as in the other forces of nature. Any attempt in this direction based upon introspective methods of investigation yields at most some reflex bearing of the objective fact upon the subjective phenomenon, which it is quite impossible to submit to any process of measurement.

The writer would put forward the hypothesis that whilst consciousness has its nucleus in sensations and organic movements and doubtless may be fashioned in different manner in different beings, yet evolved consciousness emanates from the rencontre of the actual perception, or its representation, with other confirmatory or contrasting percepts furnished by the memory of previous states of consciousness, conserved in the unconscious.

In other words, it would be, as it were, the light which emanates from the fusion of actual images with mnemonic-traces that are analogous, allied, and at the same time confirmatory of the reactive determinism, or from the conflict between the determinism arising from actual sensations and images and an opposite determinism arising from contrasting memory-images which are recalled, in accordance with the law of resemblance and contrast (associations), from the capital of personal experience. Conflict and contrast are significant of a large store of utilisable mental capital, and appear to be the really characteristic features of an illuminated consciousness.

It would accordingly be wrong to compare the consciousness of animals, even the more highly evolved, with that of man, and to think that human consciousness is equal in all men. The capital of notions and experiences, the faculty of recalling these in the changing circumstances of life, the quantum of emotion accompanying a sensation or its representation, the effects of more or less vigorous reactions or of abstention from action, which is sometimes a result of interference, give the pitch of consciousness inasmuch as this is an expression of many factors, which either sum themselves together or come into conflict with one another, and of the quantity of light and heat (metaphorically speaking) which emanates from the process in question.

A great part of our mental life goes on outside the field of irradiation of that light, for the greater number of notions and experiences is conserved in the vast dominions of the unconscious memory. There is good reason to suppose that in the majority of lower animals conduct occurs without internal conflict, being determined by actual sensations, representations, and emotions that correspond with very simple nervous mechanisms, simple, i.e., as compared with the human nervous system. It is clear that with perfecting of the senses and growth of the nervous mass, the conduct (reaction upon the environment) of living beings becomes always more varied and multiform, more protective, and supported by a stronger framework of logic. As logic develops by a rigid process of assimilation, dissimilation and elimination of elements of thought, consciousness culminates in deliberation, just as this is derived from the judgment of convenience resulting from impulsions and inhibitions (conflict). If I prevent my dog, a very intelligent animal, from 
taking a piece of meat and then go away with forbidding and threatening air, leaving it beside the dog, he refrains from touching it. Such behaviour represents an inhibition resulting from the internal struggle between hunger, desire, the impulse to eat the piece of meat, and the prohibition received. It is conscious behaviour. Numerous representations take part in the particular state of consciousness involved therein; these, in conjunction, give rise to the sentiment of obedience, based upon fear, which in turn is an experiential biophylactic product, the outcome of relations with his master. If desire and hunger gain the upper hand and the dog seizes and eats the meat and then, very repentant and fearful, hides himself or runs a long way off, only to return home after some time, full of compunction and humiliation and imagining that he will have to confront his master's displeasure, this is consciousness, made up of memories, associations, imagination and sentiments (similar instances are recorded by Romanes).

If an elephant (case of Romanes) already laden for the journey, and tied to a tree by his conductor, who had cooked a large potful of rice to serve as food during the journey, undoes the knot of the rope with which it is tied to the tree, approaches the pot, takes off the lid, eats the rice, then, to deceive its master, returns to the tree and again ties the rope round it so that it is fixed as before, all this operation is conscious because it embraces perceptions, emotions, desire, imagination and elements of contrast drawn from past experiences of its relations with its master, and of the means and methods of correction employed by the latter during the period of its training. The elephant had a prevision (representation) of stern correction, but judged that it could avoid it, imagining that it might deceive its master by re-tying the rope to the tree.

It is only in some such fashion that we can arrive at a fairly probable conception of consciousness. If we eliminate the elements summation and conflict from the conception of the reactive determinism, we shall have to accept the doctrine of Romanes, Preyer and others, who assume that consciousness coincides with all psychic manifestations, no matter what their degree or their extent.

If one should feel repugnance in recognising the phenomenon consciousness in psychic acts which, though apparently motived and characterised by reason, are nevertheless derived from simple and almost invariable nervous mechanisms, one can fall back upon the hypothesis of two consciousnesses: (1) primary consciousness, which is coextensive with psychism in all its evolutionary phases, and (2) what may be called higher consciousness, the dawn of which coincides with the appearance of conflict between actual images and those furnished by the memory of past experience.

It is, of course, to be clearly understood that there is no clear demarcation between primary consciousness--which comprises all the lower mental manifestations, including those called instincts and tropisms-and higher consciousness. Both of these may be represented as situated on one line symbolising the evolution of animal life.

Parallel with this line we can picture to ourselves another, the segments of 
which represent nervous structures in all their grades from the simplest to the most complex-i.e. to those that are richest in nervous elements, those that represent the organ of the higher form of consciousness emanating from the sum of past experiences re-evoked and represented alongside of, and in comparison with, the actual situation, experiences which generally, or at least initially, contain an affirmative element or else one that is in conflict with the actual perceptions and correlative impulses.

The contrasting memory-images in conjunction with their respective emotional states represent both individual and collective experience. It is this that affords the true standard as regards adaptations of the individual to his environment for purposes of defence, maintenance of integrity, and further evolution. The writer cannot therefore agree with Exner when he asserts that consciousness is a group of representations pre-existing in the cortex, into which there enters a new representation (perception). Even lower animals, such as insects and birds, give undoubted evidence of possession of images and memories to which are added actual images, yet, notwithstanding, many naturalists and psychologists define the reactions resulting from these as an effect of instinctive processes dependent upon comparatively simple nervous structures which do not allow of variations.

Everything considered, consciousness in the narrowest sense shows progressive development with the evolution of the brain-i.e. with the constantly increasing number of image-records which, along with new perceptions and the emotional modifications accompanying them, constitute an organic experience. This is almost systematised where the nervous centres are still comparatively simple, offering no scope for new co-ordinations.

When the nervous centres become more complicate the perceptions are more perfect and more numerous, the memory more faithful and more certain, the associations more complex and more abundant, experience more extensive and further reaching; and, in addition to impulsive images and more ample and varied confirmatory associations, there appear upon the scene others of an inhibitory nature, these being derived from painful and disintegrative experience as opposed to that which makes for the integration of the organism. It is quite clear that consciousness in this case assumes another character, the mental processes become illuminated with a new light, and conduct, which is the reflex of consciousness, now appears as the resultant of impulsive and inhibitory forces.

Those reactions which are the outcome of impulsions to action on the one hand and contrasting inhibitions on the other, reactions which we can observe even in the higher mammals, warrant us in speaking of the dawn of a higher consciousness, and, if we trace the various stages in the evolution of the brain, we find that they first show themselves coincidentally with the first appearance of frontal lobes. All the information that has been gathered from observations and practical experiments goes to prove that the successive developments of the hind brain do not alone suffice for manifestations of 
higher consciousness. It seems evident that there are required the presence and collaboration of another cerebral organ of recent development which sums up, fuses, transforms and regulates the immense mental capital prepared by the hind brain. This organ, as may be inferred from what has hitherto been said in this volume, is the frontal lobe.

McCabe, ${ }^{1}$ after recounting examples of new intellectual manifestations in dogs, cats and other mammals, commonly noted for their astuteness and for acts which pass beyond the bounds of instinct, does not give a definite answer to the question whether or not lower animals have the power of reasoning. Evidently the same question has to be repeated in the case of reason as in that of consciousness - "Where does it commence?" We cannot shut our eyes to the all-important fact that stands out clearly in the story of the evolution of living creatures-viz. that reason is evolved from simple physico-chemical processes and is based on the same laws of affinity that apply to these. Reactions are but the reflex of stimuli. In that reflex lies the germ of reason. Just as the associations and the associative memory become more complicated with the evolution of the nervous system, so do the reasonings and the external responses of living creatures become more complex. All speculations on this subject on the part of a large number of observers, mostly English and American-e.g. Hobhouse and Thorndike-are but subtle and laborious exercitations in a field without limits, in which imagination, linguistic artifices and arbitrariness seem to present no obstacles. From the consciousness of the dog to that of the Tasmanian (such as the Sussex-man, now out of existence), from the Tasmanian to the Hottentot, from the latter to the modern uneducated European, and progressively to the average educated European and the man of exceptional talent, there is simply an ascending scale of consciousness in the wider sense. of the term.

It is apparent that the unity which we are apt at first to ascribe to consciousness is fictitious. Consciousness really varies with the intellectual content and mode of feeling in each individual and each social group at different periods of time. In the ever-changing content of consciousness we must recognise an inequality of the unity of the thinking being, which the individual recognises in himself and in his story from changes of sensations, emotions and images.

In highly evolved individuals or evolved social groups consciousness tends towards sublimation. In this fact lies the proof of its evolution. Examples have been provided by certain peoples in the present terrible period of history, but in expressing this opinion the writer will not venture one step beyond what is human. He can imagine the inventive spirit, the spirit of sacrifice, of heroism and of all the other virtues, to be the effect of a felicitous spiritual (cerebral) evolution which transports man beyond the limits and domains of personal interest into those recognised as social interests which are, amongst other things, the ideals of science, and should be likewise

${ }^{1}$ MeCabe, Loc, cit. 
the ideals of politics.* Such organisations evidently represent something that is superior to the ordinary higher consciousness; but neither imagination nor the author's positivistic conception of consciousness will allow him to accept the conception of Myers ${ }^{1}$ and of James ${ }^{2}$ as to the existence of a higher consciousness, in the sense of a sublimed ego, alongside the actual ego but quite without the human organism.

Here we enter into the realm of subjectivism and of creeds, which is a domain reserved for philosophical speculation.

In studying states of arrested cerebral evolution in man, as exemplified in the numerous class of idiots and imbeciles, we can retrace the story of the evolution of consciousness, laying bare, in a retrogressive fashion, all the various phases of development down to that of the lower monkeys. Further, it is easy to detect a very close relationship between the qualities and extent of consciousness on the one hand, and the number of cerebral cells, the greater or less degree of arrest of their structural evolution, and the number of their fibrillary relations on the other.

Human pathology, and more especially teratology, plays a part similar to that played by geology and palæontology. Pathology and teratology lay bare the various strata in the ancient morphological and psychic formations of man, whilst geology and palæontology bring into evidence the different phases in the formation of the earth and of the life which it supported for millions of years in very varied forms of organisms that have now entirely disappeared.

In studying idiots and imbeciles we can retrace the natural history of human and prehuman evolution by following the various phases of dissolution induced by disease. By psychological investigation of representatives of various degrees of imbecility and idiocy arising from defective or incomplete histo-morphological evolution of the brain (as distinguished from that, caused byinflammatory and destructive processes affecting different cerebral provinces), we come to form a naturalistic conception of consciousness as a mode of evolved mentality, a conception that is supported and

* In the course of a conversation with a great politician, little loved during his life, much regretted after his death, the author heard with his own ears these very words : "Don't talk to me of moral reasons in this or any other question; politics cannot obey moral laws." The writer, on the contrary, maintains that politics, which sums up the thought, sentiments and tendencies of a people, and illuminates the life of a country, cannot on any account claim exemption from moral and legal obligations. The fact is that the conceptions, tendencies and creative work of politics are often distorted by personal interests and ambitions.

Consider the social aggregate (which to the writer is primarily the State) "as a mere numerical sum of indifferent individuals" (worse still if egoistic) "who will be followed by other individuals, and heroism, sacrifice pure and simple, withcut possible or assignable compensations, can no longer be explained." 3

${ }^{1}$ Myers. Human Personality and its Survival of Bodily Death. 1902.

2 James. Philosophy of Experience.

${ }^{3}$ F. Orestano. "Un tragico esperimento di verità morali." Nuovo Convito. 1918. 
illuminated by the histo-morphological findings in different brains belonging to individuals who displayed different degrees of mind-power. Not only so, but we can detect arrests of evolution in all stages of the development of language, down to simple monosyllabic and vowel manifestations, and in the development of thought, if we have regard to its quality, its quantity, its structure, and its reactive reflexes, which are exceedingly simple, inconclusive and illogical.

Consciousness, where normally and happily constructed, varies as already indicated around a permanent and stable nucleus. This nucleus is characterised by a determinate manner of feeling, by a particular orientation towards certain objects, and by great resistance of the psychic compounds to the disintegrating action of the storms of life, whilst at the same time it confers a more rigid directive upon life, in its interhuman relations.*

This nucleus is indeed the framework of character. Great wealth of notions and images, with their corresponding emotional states, no doubt renders the consciousness unstable, but not its nucleus. Just as protoplasm is renewed within the cell without the latter losing its chemical, morphological and functional characters, so the renewal and even rapid flow of ideas in consciousness leave it unperturbed in its structure, its general tone, and its function, as an element of the social organism. Again, the more resistant and the more attuned to general principles is the collective consciousness of a people, the more fortunate is it for the country which produces it.

Movement of simple images and of ideas through the focal point of consciousness is one of the conditions of normal mental life.

Some psychologists have drawn a happy parallel between the movement of ideas and groups of ideas in the illuminated field of consciousness and the motions of planetary systems. Just as the stars describe their orbit, so the ideative systems move across the illuminated field of consciousness; as the greater and lesser stars maintain reciprocal relations with one another, regulated by the laws of gravity, so also do the ideative systems, which are regulated by the constant laws of association. Just as the planetary systems seem to rise and sink again into the infinity of space, so ideas rise into the light of consciousness, and, having described their orbit, sink again into the unconscious. The stars of greater magnitude and brilliancy have often their satellites, and, similarly, the more resplendent ideas attract around them a

* It is only in this sense that one can imagine unity and continuity of consciousness. "Each of us becomes aware of it," writes De Sarlo," " as something to which the whole of our experience is referred, as a unit common to a multiplicity of functions, as something which remains steady notwithstanding the flow of things. There is nothing of the transcendental in our conceptions. There is a historical continuity, in so far as the ego can follow and sum up all the variations of its existence, punctuated by infinite changes both of the kinæsthetic sense, owing to severe internal alterations, and of the external environment, which, too, is variable, mutable and discontinuous.

${ }^{1}$ De Sarlo. Psicologia e Filosofia. Florence, 1918. 
smaller or larger number of other ideas, and constitute associative systems or fields which give a particular intonation to the mental life, impressing a diverse direction on the wheel of fortune of each man or each human group.

We must thus recognise a continuous dynamism in consciousness, whereby the ideas and sometimes also the attitudes of mind are ever being renewed. We cannot conceive a static condition of mind except during periods of exhaustion or of ecstasy, or under conditions that are distinctly morbid, as in the case of stupor, catatonia, the somnambulistic condition, etc. It very often happens that a thought presents itself on the horizon of consciousness, dim and indistinct in outline. By degrees it assumes a concrete form, becoming always more illuminated, attracting around itself a larger or smaller number of notions and ideas and occupying consciousness for a certain period, only to fade and sink away again after a time. As the earlier notions are sinking into the ocean of oblivion new systems are arriving at their zenith, whilst others are springing up on the opposite horizon. The older notions may subsequently make their appearance again from time to time, some of them in a constantly rhythmic fashion, others at irregular intervals ; occasionally they flit through consciousness like comets, or they make their appearance only in the great cataclysms of the mind, or again they remain in the background of consciousness ready to show themselves anew, and even in the hiding-places of the unconscious they may conspire in secret and determine a correlative action.

The image which flatters and cajoles, the thought which takes possession of us and transports us into the infinity of the unknown, into the uttermost corners of the consciousness, into the bowels of the earth, or into the depths of the ocean, all owe their appearance in consciousness to that ceaseless dynamism that is concerned therein; nor is consciousness stable as regards emotional states, for these also follow one after another and evolve with experience of life, especially through the interhuman relations which each man assumes. With growth of the sentiment of understanding between different minds, many or few, near or distant, according to the constitution, degree of culture and affective capacity of each individual, and the nature of the work produced, there spring up emotions and sentiments which aid, vitalise and utilise that fundamental sentiment of sociality which is the turn-key of the conduct of modern civilised man, giving a particular tone to consciousness.

Desire which spurs one on, with its accompanying images of things, and sometimes makes one chafe and fret; feelings of attachment that beget enthusiasm and raise the potential of the spirits; passion which burns and subdues, disrupting the individual or collective system of life; fear with its morbid fancies, which chill the ardour as a frost nips the buds in springtime, dispelling Nature's sweet promises, all pregnant with beauty and throbbing with hope; pain which bows one down; pleasure which laughs with light and careless heart, enjoying every day of existence ; hatred which plots and undermines ; envy which in its impotence eats out the soul or makes hidden 
attack with the weapon of ealumny; love which transports one into the flowery fields of pleasure, enthusiasm and ardour, or raises one into the sublime spiritual sphere where is composed the joyous music-not understood by all--which acclaims the glory of existence, or, on the other hand, drags one into the foul pit which holds only fermenting debris of mind that nourishes evil germs and enzymes of crime; joy that comes of labour accomplished and rewarded by success; all these emotional states, associated as they are with the changes that come over the human mind, either abruptly or by degrees, only come about through the continuous dynamism that is in consciousness, a dynamism which resembles the constant motion of the stars in the firmament.

The dynamism and the movement of ideas do not destroy the intimate texture of the personality which, taking its tone from the kinæsthesis, maintains its own characteristic individuality in the constitution of which two factors, in addition to those already mentioned, play a part--viz. heredity and education during the developmental period.

It has been said that the consciousness of each individual preserves its own particular features because it assimilates the external world with its own structures, just as the different cells of the organism preserve their original morphologieal and chemical characters, notwithstanding the fact that they all receive the elements necessary for their material interchange from the same circulating lymph. Molecules are for cells what images are for consciousness. The images, although endowed with considerable dynamic power, pass into the unconscious after they have traversed the illuminated field of consciousness. "The unconscious" is the great bank wherein is deposited all the capital, be it large or be it small.

The unconscious is like the depth of the sea as contrasted with the surface, where the ship of life sails smoothly on or is tossed amongst the waves; it is like the bowels of the earth which furnish gold and coal for our existence; or like the immensity of space in which, even with the aid of the telescope, our eyes can see only a comparatively small number of stars, distant millions upon millions of miles from one another, yet all influencing each other by physical laws; it is like the power and wealth of a country prepared by peaceful spades delving deeply into the earth or by rasping files amidst the feverish activity of the workshop. What appears on the surface of mental life is but a small part of all that exists. The illuminated part of the mind is much less extensive than that which remains wrapped in darkness. The light of consciousness emanates from the inner working that goes on in the shades of the subconscious, just as electricity is developed from mysterious inner processes that take place in matter. It reminds us of the fact that the wealth of a country is prepared by the labours of thousands of arms or by the unobtrusive genius of a few. The whole mental capital is preserved, and to a very large extent organised, in the subconscious just as the external world has been spiritualised in the course of time and in diverse spaces. The work of composition of the mental products is not a prerogative that is vested solely in the illuninated field of consciousness, rather does it belong to the dominions 
of the subconscious. Morselli ${ }^{1}$ writes that, apart from very rare and exceptional products of some utility, the subconscious gives us only rejected products which we are constantly casting out of our mental life. There is truth in this statement if we apply it to dreams, somnambulism, automatisms and all mental diseases, etc.-in other words, to the subconscious of weak or diseased natures and to distinctly morbid conditions (trance, hypnotic, hysterical, etc., states). That apart, however, we must recognise in the illuminated ego a high evocative and selective power, in obedience to which the subconscious furnishes all the material required for the mental constructions, no matter whether these be mediocre, ordinary, or brilliant and original. It would even be justifiable to hold that in all higher living creatures sleep affects this selective, regulative and creative power in a particular manner; hence it is that organic, tactile and auditory stimuli arouse isolated, disconnected, extravagant representations either during sleep or in the course of disease-i.e. when the light of waking consciousness is spent.*

1 Morselli. "I limiti della coscienza." Riv. di filos. 1913.

* The author has used the words unconscious and subconscious without deliberate discrimination in meaning, and to these might be added the term conconscious, introduced by Morton Prince. An attempt will be made in this note to give a brief summary of the prevailing conception of to-day. It is not the author's intention to treat the subject in extenso, for that would exceed the limits he has put upon this work, nor does what follows pretend to be a detailed examination or criticism of the question of the unconscious and the subconscious. The unconscious is an integral part of mind, of which consciousness is the illuminated field in a given unit of time, and it is not quite correct to say, as some do, that it is completely insusceptible of analysis. All inquiries dealing with hypnotism, hysteria, somnambulism, and dreams, represent not unfruitful attempts at analysis. All the works of Freud, ${ }^{1}$ apart from exaggerations and certain points in his efflorescent production that are open to criticism, speak of the possibility of analysis of the unconscious by the methods of psycho-analysis, which have aroused keen enthusiasm in some countries. (See, e.g., the work of E. Jones. ${ }^{2}$ )

The uneonscious is made up of the same material, is constructed on the same plan, and follows the same laws as those which govern the evolution, structure and function of normal mind, and it emanates from the same anatomical substratum. We cannot recognise in it anything of the abstract, any extra-physiological power or any spontaneous activity, as asserted by Dwelshauvers. ${ }^{3}$

The unconscious is that part of nature which each individual has succeeded in spiritualising, in conjunction with all the mental constructions of which each individual is capable and with the memory-traces of all the reactions (individual and collective experience) determined by the action of an incalculable number of stimuli which have excited the nervous system of the individual and, for thousands of years, that of the species.

Mental working, which resolves itself into the construction of mental syntheses, is set in artion by external stimuli and by memory-traces, recent or old, which reach the illuminated field of consciousness; the latter detains a selected theme in the focal point, and thither directs a flow of the contents of the subconscious. The content of the subconscious is utilised by consciousness just as constructive material

1 Freud. Studien über Hysteria. 2nd edit. 1909. Die Traumdeutung. 3rd edit. 1912. Zur Psychopathologie des Alltagslebens. 4th edit. 1914.

${ }^{2}$ E. Jones. Papers on Psycho-Analysis. 1913.

${ }^{3}$ Dwelshauvers. L'Inconscient. Paris, 1916. 
The appearance of higher consciousness coincides with the integration of preformed psychic complexes by the addition of new-formed images, and with conflict between the dynamo-genetic power of sensations and ideas and the inhibitory power exercised by other image-records.

The development of inhibitory power in the psychic domain coincides with the appearance of the frontal lobes. It represents in the psychic field what resistance is in the physiological field. We know from the researches

is used by the artisan. When the power of evocation and selection becomes suppressed along with that special form of will which serves to detain in consciousness an argument towards which the work of the subconscious is directed, then the subconscious does not create any syntheses of real intellectual or social value.

Experiments in hypnotism such as carried on by Epinas and Azam, amongst the first, and subsequently by Binet, Janet, Bernheim, Bourgeois, Sidis, Morselli and Belfiore, have all demonstrated the poverty of the product of the unconscious, when the latter is not controlled and directed by the higher consciousness. Studies bearing upon hysteria - of great psychological significance because hysteria often presents the phenomenon of clouding of the higher consciousness, which permits the content of the unconscious and the subconscious to enter upon the scene ${ }^{1}$; inquiries and discussions upon spiritualismi of which the most complete synthesis is to be found in the two volumes of Morselli; the detailed investigation of the unconscious by the methods of psycho-analysis, introduced by Freud and Bleuler and continued with success by Freud and Jung who have written extensively on this subject, all these have not revealed any mental construction that is of service to life. The unconscious, when freed from the evocative and constructive influence of the higher consciousness, yields nothing but trivialities. Freud has found in the unconscious nothing more than the traces of sensory occurrences, belonging even to infancy, isolated psychic complexes which sometimes have acted the part of conspirators even from the period of childhood, and have been kept in submission by the eonsciousness, which exercises the right of censor. He has found none of those mental constructions which take a place in the intellectual architecture of the human subject, or which represent logical, and therefore biophylactic, reactions in the life of the individual or the community.

The artist is not surprised by the musical motif which creates enthusiasm in his auaience, because he has long been directing and dedicating his whole intellect and sentiment to the creation of that motif and the whole work of art, which is often the result of years of conscious labour and of will to select and detain the theme in conscionsness. The scientist creates, if at all, only after long and laborious researches, during which his mind is directed to the solution of the problem that has long eonfronted him, unless his trained perceptive capacity affords him occasion to observe some new fact which throws sudden light upon it. That is the function of the higher consciousness. Here we must recognise the power of the higher consciousness in directing the contents of the unconscious towards the illuminated ficld where the theme to be developed is detained, the subconscious furnishing the components that are necessary for the construction of the intellectual or intellectuosentimental syntheses. There seems no reason to doubt the existence of this power.

Mental pathology affords good proof in support of this view. The man who suffers from religious paranoia (paranoia to-day is regarded as a variety of dementia precox) for months on end directs his mind towards matters connected with God and religion, and if at length he observes some signs or movements in a figure of Christ that is on the wall of his room, or hears it speak (hallucinations) that is due to the evocative and expectant attention which for a long time has produced an

'Bianchi. "Contributo alla conoscenza dell'Isterismo."

Ann. di Nev.

1911. 
of physiologists, especially of C. Richet, ${ }^{1}$ that the time taken by a nervous current to reach a central point and produce a reflex is in proportion to the length of the nerve or nerves involved. In other words, the length of time required is in direct ratio to the distance or the resistance, and this corresponds with the universal law that velocity is in inverse ratio to resistance. To the time required for transmission along the nerves there falls to be added a latent time occupied in the centre (in the cell of the spinal medulla, in the case of

1 Loc. cit.

unusually high potential in the cortical and auditory fields, and so has resolved itself into the corresponding hallucinations. Some seven years ago the writer had in his clinique one who was obsessed by the memorising of words which she was afraid of forgetting. This condition caused a constant anguish during which she was constantly recalling and repeating the words in question. After years of this condition the patient began to have auditory hallucinations of dissociated words and phrases. Here, too, there must have been an excessively high potential in the cerebral acoustic field (and a corresponding histo-chemical alteration) giving rise to the hallucination.

I have grave doubts as to the automatism of the unconscious. When I cannot remember a name and it happens that this comes up spontaneously before me some time afterwards, perhaps the following morning on awakening, I do not look upon this as automatism, but rather as the effect of the direction given to the unconscious by the evocative action of my conscious will. I do not mean to say that the unconscious is totally lacking in constructive power, but it seems reasonable to suppose that any power of this kind is directed and manifested through the action of the conscious will, which often may be of very brief duration. The whole edifice erected to the unconscious from investigations, sometimes very commonplace, of mediums in hypnotic or trance-states impresses me as being a falsification of what should be comprised in the terms unconscious and subconscious. (I use these two words in the sense of degree of obscurity, the subconscious being regarded as the nearer to the threshold of consciousness.)

The subconscious, or what Sidis ${ }^{1}$ calls the secondary self, would not be a thing entirely by itself. It is a form of mental life in which one has to take into consideration the co-ordination of many series of moments-consciousness. One can understand this when one remembers that a part, perhaps the greatest part, of the content of the subconscious has at one time passed through the focal field of consciousness, and has consequently preserved some relationship with the elements of the waking consciousness, no matter how much it may appear to be completely isolated. Further, it is clear that without wishing it, or without the waking consciousness being aware of it, some, or perhaps many, elements of the subconscious are attracted, either separately or as complexes (preformed constellations), from the depths of the unconscious for the conscious structure of mental syntheses. Sometimes these elements, drawn from the unconscious, are recognised, at other times they are not. The content contributed by the activity of the senses, and preserved in the unconscious, is indestructible; a great number of reactions may be traced to the activity of impressions received during childhood, although they may have remained entirely forgotten and incapable of reaching the focal point of consciousness, except under particular circumstances. In the somnambulistic state thoughts and memories may be re-evoked which the waking consciousness has never been able to call up.

In the state of maniacal exaltation a number of memories that have been entirely obscured by time may come to the surface. Many delusions are psychic

\footnotetext{
1 Boris Sidis. The Psychology of Suggestion. 1899.
} 
spinal reflexes), for the metabolism or transformation of the sensory nervous waves into motor or centrifugal waves. To-day, our knowledge of the histology of the nerve-cell has been so enlarged by the researches of Golgi, Ramon y Cajal, V. Gehuchten, Donaggio, Iugaro, Fragnito, and numerous other investigators, that we can find a ready explanation of the long loss of time in the nervous centre (group of cells). We can understand it to be due to the enormous resistance offered by the great twisting of endocellular and

complexes that were preformed even in childhood, and kept removed from the field of the waking consciousness by the eliminating power of the latter (censor), because they were obviously contrary to fact, which is normally perceived as such.

Freud maintains that the whole mental life represents one continuous process. Apparent discontinuity is only an illusion due to the fact that what has gone before is not known. According to him, amnesia of this kind assumes a character of great importance, and is due in some cases to psychic repression resulting from education and upbringing in early age or from the hedonistic attitude in life and the influence of new sensory and intellectual acquisitions that come with the process of development. The repressed and forgotten images do not, however, always lose their dynamism.

These statements cannot all be confirmed by experimental methods, yet one cannot deny that the facts on which they are based demonstrate the great com. plexity and the great wealth of the subconscious, as well as the manifold relations that subsist between the subconscious and the illuminated consciousness.

There are many attitudes in the life of each individual that have their origin in the content of the unconscious without the higher consciousness being aware of it. The writer can take his own case as an example. He was educated in a naturalistic cnvironment by his father, who was a chemist and botanist. When he had com. pleted his coursc at the Lyceum he was commanded by his father to enrol in the Faculty of Jurisprudence. The writer objected, and was quite rebellious to the wish of his father, whom he really adored. Never before had he opposed his desire, but on that occasion be felt an irresistible attraction for the study of Natural Science and of Medicine which caused him many heart burnings owing to the strained relations with his father, who was a severe man and of strong will. The writer afterwards came to explain the reason of his choice as being due to the information about nature and naturalistic phenomena which he was constantly deriving from his father, and to the satisfaction he experienced in childhood from the little that he knew of nature-a fact of which he was quite unconscious when he chose his path in life.

Dreams undoubtedly offer a rich material for the study of the problem of the subconscious. Their origin is very complex and very varied in nature. One can give beed to the doctrine formulated by Freud concerning the origin of dreams which, in the case of children, he connects with a latent content, which would give rise to the imaginary satisfaction of a repressed wish. This is specially characteristic of the dreams of children. The wish repressed by the censuring consciousness cannot penetrate consciousness, but, during sleep, when the power of the waking consciousness is suppressed, the latent content would reveal itself. According to Freud, the presence of the repressed thought or wish finds confirmation in the fact that a reasoned and conscious wish is inadequate to produce a dream unless it be associated with another, repressed and unconscious, wish which would be the real instigator of the dream. If this is true of some dreams, there are yet many other causes of dreams, amongst them the internal and external corporeal impressions which, through unconscious association, provoke a number of dissociated and strange imaginary complexes (dreams), owing to the fact that sleep takes over in its own special way the directive and selective power of consciousness. (A more complete study of this subject will be found in the work of S. De Sanctis. "I. Sogni." 1899.) 
extra- or peri-cellular neuro-fibrils in a more or less extensive field of cells before the current assumes its centrifugal character in the motor paths.

As we cannot imagine inhibition to be a watchful, regulative power which is exercised from a distance, and much less think of it as an occult force, it is necessary to trace the phenomenon to its objective physical mechanism. There is no other way of understanding it.

It will help us to comprehend the phenomenon if we start out with the

The subconscious is revealed also by many acts performed by the generality of men; these, though apparently void of any psychic significance, are yet found through psycho-analysis to be determined by unconscious motives.

The genesis of many slips of the tongue (lapsus linguce) which even those well accustomed to speaking sometimes make, and slips of the pen (lapsus calami) may be traced to the unconscious. They are certainly not always due to inattention.

In still other cases we find evidence of the action of memory-traces preserved in the unconscious. For example, if we invite a child to mention a number, that chosen is often the vague memory of the same or an analogous number that has fallen into oblivion. The case mentioned by Adler ("Drei Psychoanalysen von Zahleneinfällen," etc. Psychiat. Neurol. Woch. 1905) is very suggestive in this regard.

We forget many things the memory of which is painful. Man assumes an attitude of.defence against painful memories. We are constantly finding ourselves confronted by the hedonistic law of life ("Zur Psyehologie des Vergessen," ete. Archiv. f. Kriminal-Anthropologie. 1905).

In the case of lapsus we can trace the influence of another train of ideas which has not crossed the limits of consciousness. Some of these errors are evidently of a mixed nature. The author is acquainted with a man who often makes slips in writing in that he interposes in the structure of a word a syllable or letter that belongs to the word that follows. In this ease the phenomenon has a double origin. There is the element of inattention, but there is also at work the influence of a preformed representation which comes forward of its own account. The latter phenomenon finds its explanation in the influence exercised by thoughts, images or wishes on the motor determinism, independently of the waking consciousness.

Thus, there exists a consciousness or vigilant self and a sub-vigilant consciousness (Boris Sidis ${ }^{1}$ ). A pen put between the anæsthetic fingers of a hysterical woman determines adapted movements of writing. If the outline of a letter be traced on the back of an anæsthetic finger of a hysterical patient, the letter may be reproduced by the hand making appropriate movements. A patient suffering from hysterical blindness may see an object of which her waking consciousness knows nothing (experiment of Binet ${ }^{2}$ ). The return to memory of things that were completely forgotten or were never actually apprehended by the waking consciousness because they affected only the senses, and did not bring voluntary attention into play, and the reawakened memory of things seen in infancy, accompanied by recognition of places and circumstances, are facts which reveal the existence of psychic processes which take place without us being fully conscious of them.

We are bound to agree that in the obseure domain of the mind there may be buried or eoncealed many memory-traces which never come into the illuminated field of consciousness, and that many reactions take place quite outside that field. Whether we have to deal with a secondary consciousness or whether, as the writer

${ }^{1}$ Boris Sidis. The Psychology of Suggestion. New York, 1899. Psycho. pathological Researches in Mental Dissociation. 1902.

${ }^{2}$ Binet. "Sur les altérations de la conscience. Rev. Philos. 1884. 
obvious fact that all the nervous organs together constitute a circulatory system for nerve-waves, which is much more complicated than that concerned with the circulation of the blood. No observer can have failed to be impressed by the fact that the circulation of the nerve-waves is regulated by the same laws as those pertaining to liquids in enclosed tubes and to the circulation of electric currents in conducting wires. This hypothesis has been confirmed by psycho-physical researches. We know that in the process

thinks, these facts descrve a different interpretation, this is not the place to discuss. All that is aimed at in this brief summary of the doctrine of consciousness is to establish the conception that what we call the waking or vigilant consciousness is only a part of our mind, that another part remains in the penumbra, and still another and larger part in completed arkness; but it is all one organisation, which manifests itself through the same anatomical substratum and by the same dynamism.

If any further proof were required to convince us that the whole corpus mensis is one single function, notwithstanding the distinction into an illuminated field (waking consciousness) and a more or less obscured field (the unconscious "and subconscious), it is furnished by the examination of the phenomenon of hallucination. The author must not here dwell upon the internal mechanism of hallucinations or upon the different interpretations put forward by Tamburini and Tanzi ${ }^{1}$ and others, but it is opportune to mention Ballet's view inasmuch as it touches the relations between hallucination and personality.

Ballet 2 holds that hallucination is the effect of disaggregation or dissociation of the personality. An unprejudiced examination of the facts and their mode of succession shows that hallucination is the effect of a morbid state of the sensory fields, whose abnormal product, the hallucination, surprises the consciousness and dissociates or decomposes the personality. The higher consciousness elaborates the material prepared and furnished by the sensory field. It is different when we come to deal with mediumistic states and induced somnambulism. In this case the waking consciousness is deposed whilst the sensory function is exalted as the result of the hypnotic or spiritualistic practices. The somnambulistic and trance phases, as practised by spiritualists upon the medium, are always accompanied by obscuration of the higher consciousness (superliminal consciousness of Myers), a kind of sleep, and at the same time by exaltation of the sensory field.

When the author occupied himself with hypnotism he was always able to observe these two facts. The slightest stimulus produces a brief movement of thought, and a reaction, or group of reactions, generally isolated. More complex actions and a wider movement of thought require to be suggested to the hypnotised person. There is no trace of any power which evokes and directs the intellectual function; no power which utilises the capital accumulated through the working of the brain on objective and subjective reality, and now lying dormant in the cerebral archives. On the other hand, it is very easy with the slightest stimuli, or by command, or simple suggestion, to awaken images or groups of images and even hallucinations.

The phenomenon of duplication of the consciousness or of the personality, as exemplified in the cases recorded by Camuset, Morton Prince, by the author, ${ }^{3}$ and several others, is more complex. What significance is to be attached in these cases to the bchaviour of the secondary (subliminal) self as manifested in logical actions,

1 Trattato di Psichiatra. Tanzi and Lugaro. Malattie Mentali. 2nd edit.

2 Ballet et Mallet. "Hallucinations et dissoeiations de la personnalité." L'Encéphale. 1913. Ballet. "La Psychose hallucinatoire chronique et dissooiation de la personnalité." L'Encéphale. 1913.

${ }^{a}$ Bianchi. T'rattato di Psichiatria. 2nd edit. 1915. 
of evolution of the nervous system new organs are developed and close anatomical relations are established between these and the pre-existing organs-in other words, the field of circulation of the nerve-waves becomes more extensive, and consequently a higher potential is required in order to overcome the increased resistances of the new paths, proportionately to the extent of the new field.

One can readily understand that if, for any reason whatsoever (artificial irritation, morbid processes, excessive functioning, etc.), one area receives an addition to its nervous charge, this is to the detriment of the other areas, in which the charge will be diminished by just so much as it is increased in the excited part of the nervous system. The former are inhibited through the hyper-functioning of the latter. In accordance with this view, any central nervous organ may inhibit or be itself inhibited as circumstances occur.

We get disturbance of the equilibrium of the nervous circulation and increase of the charge in certain parts of the nervous system, when any field of propagation in the brain becomes suppressed or cut off from the other organs, owing to interruption of the paths of communication between them.

Given the free circulation of the nervous currents through all the central areas of the system which are connected with the peripheral areas by means of centripetal paths (the conducting wires), we get a greater charge of nervous force in the lower cerebral organs when these are separated from the higher, and this gives rise in turn to an increased excitability which resolves itself into augmented reflex action. Exaggeration of the reflexes and diminution of the time of reaction are due to the simple physical facts that the field of propagation is diminished and that consequently the nerve-waves become concentrated in a more restricted field of distribution. The tonic contraction of certain groups of muscles (on the opposite side) that follows immediately on removal of one cerebral hemisphere or of both cerebral hemispheres (contractions on both sides), such as observed by Sherrington, ${ }^{1}$ is likewise an effect of suppression of an extensive field of propagation of the nervewaves which are constantly being aroused by external stimuli and transmitted in a centripetal direction. These, finding their paths obstructed, overrun the lower centres, augmenting their potential, which, in turn, becomes discharged along the motor paths, thus giving rise to tonic contraction of the muscles in the first instance and to exaggeration of the reflexes in the next. We know that in the cerebral mantle there exist distinct areas for groups of muscles which contract, and for their antagonists which relax. We also know that on exciting the area belonging to the first group

accompanied by a certain movement in thought but with unusual affective orientation, and how there arises, and what value one is to assign to, the symbolic diaphragm of Myers, ${ }^{2}$ are fields of discussion which lie beyond the limits assigned to this volume.

1Sherrington. "Decerebrate Rigidity." Journ. of Physiol. Cambridge and London, 1898.

2 Myers. Human Personality and its Survival to Bodily Death. 1902. 
the corresponding muscles enter into a state of contraction, whilst at the same time their antagonists become relaxed. In the writer's view this is due to the fact that the potential in the area belonging to the antagonistic group of muscles becomes diminished during excitation of the other area.

That is the most likely interpretation that can be offered of the experiments of Sherrington, of Herring and Sherrington, ${ }^{1}$ Topolanski, ${ }^{2}$ Libertini; Oddi, and Fano. ${ }^{3}$ The last-named physiologist found that removal of the whole frontal lobe, including the sigmoid gyrus, led to shortening of the reaction-time, whilst electric stimulation of the frontal lobes lengthened it.

This lengthening of the time of reaction we call inhibition, but it is clear that it is nothing else than the physiological effect of an experimental physical condition--viz. an increase of the quantity of nervous waves in the frontal lobes owing to the influx that takes place under the action of the electric stimulus, and a consequent diminution of the charge of nervous force in the remaining parts of the cerebro-spinal axis. So far as this question is concerned, it is very doubtful what value is to be attached to researches made with the object of demonstrating that the frontal lobes are a centre of inhibition. All points of the cerebral mantle may either be inhibitory organs or may themselves be inhibited, according as they are stimulated or as other neighbouring or distant areas with which they have functional relations are stimulated.

The inhibitory power is greater or less according to the degree of functional dignity of the cerebral area concerned and, one might add, according to the number of associative relations which it has with other parts of the central nervous system. As the frontal lobe does not send any fibres directly to the spinal medulla, nor receive any from it (vide Chapter VII.), its inhibitory power over the spinal medulla can only be exercised through an indirect path. It is sufficient to see the number of associative fibres that run between the frontal lobe and the Rolandic area to be convinced, without stretch of imagination, that any stimulation of the frontal lobes must induce such a flow of nerve-waves, especially from the Rolandic area, that the nervous charge becomes lowered in all the rest of the cerebro-spinal axis which therefore finds itself in a hypo-functional condition. Vice versa when the frontal lobes are removed, the nerve-waves which were destined to pass to the frontal lobes, whose field is now destroyed or obstructed, accumulate in other centres and this explains their greater excitability.

We find in Loeb's work ${ }^{4}$ a psycho-physical conception of inhibition similar to that here expressed. Most physiologists who have investigated this phenomenon seem to have confined their attentions to the effects of excitement or destruction of certain segments of the central nervous system (see Chapter IV.). The view which the writer here puts forward is one

${ }^{1}$ Herring and Sherrington. Arch. f. die Gesels. Physiologie, and Journ. of Physiology. 1898.

2 Topolanski. Arch. f. Ophtalm. Leipzig, 1898.

${ }^{3}$ Fano. Archives italiennes de Biologie. 1895. (See also Chapter II.)

4 Loeb. Loc. cit. 
which he clearly expressed twenty-five years ago in an article on the symptomatology of gait. ${ }^{1}$ 'The following is a quotation from that article:-

"The interpretation of this inhibitory action or action of arrest is one of the most difficult things in physiology. To appreciate it we must start from a datum of common observation. Fach person, by innate organisation, possesses an exponent of nervous force that depends upon the nutritive activities of his nervous centres, upon individual capacity to assimilate and dissimilate, and upon the particular congenital and hereditary molecular disposition. The quid which we call nervous force, in making itself manifest in different individuals, displays particular qualities that are inherently dependent upon the structural details of, and the central and peripheral relations between, the organs in which it is developed, in which it is modified and from which it emanates. It thus presents different characters in the form of various sensations, emotions and sentiments, as well as consciousness, perceptive attention, desire, will, movement, etc. The various faculties draw from a common source, are modifications of one and the same force, and they equalise and compensate one another. If, as the result of particular circumstances special to its own organ, one faculty acquires greater development than the others, under equal conditions, the latter become depressed by just so much as the former is exalted. This appears to be a general law and it is not belied by the behaviour of any of the modes of extrinsication of nervous properties, from the lowest to the highest. If we augment the sensibility of a particular area of skin on one side by the use of any æsthesiogenic body, the sensibility of the homonymous area of the other side is correspondingly reduced. Vice versa, if we lull the sensibility of any given area of the body by the use of a magnetic pole, the sensibility of the homonymous area of the other side is correspondingly increased. All strong sensations of pleasure or of pain arrest or retard other extrinsications of nervous energy, because every strong sensation represents an excessive consumption of nervous energy, to the detriment of other functions. If an individual engaged in deep meditation voluntarily determines to make an energetic movement, or rather an effort, a certain proportion of the reflective intensity is lost in that movement. A man who wishes and ardently desires anything does not allow himself to be much impressed by external or internal circumstances; that is another way of saying that organs which should be receptors and elaborators of new impressions from without are not equal to their task, because their nervous force has been reflected upon another territory and has left them thereby weaker. If, whilst one is suffering from a severe pain, another pain is induced, the intensity of the first diminishes proportionately to the intensity of the second. The reflexes which occur, sometimes in an intense form, in consequence of pain are no exception to the rule; all of them, from the simple, rapid movement of the hand to the site of the pain (somatic pain) up to raptus melancholicus of the agitated

${ }^{1}$ Bianchi. "Le andature." Giorn. Internaz. di scienze mediche. 1885; also Semiotica delle malattie nervose. Milan, 1879. 
melancholiac, are only realised at the expense of some other variety and mode of extrinsication of nervous energy ; indeed this may go so far as to result in loss of consciousness. There is no end to the examples that might be quoted in support of this fact. Good nervous organisation consists not only in the possession of a given quantity of nervous force which must not fall below certain limits, but also in the regular augmentation of that energy under the influence of education and other agents which operate upon us, in its due and proper distribution, permitting functional harmony between the various organs of the nervous system, and in its relative steadiness or not too great mobility so that it should be capable of being deflected now into one field, now into another of different functional dignity, in response to more or less adequate stimuli.

"These last two conditions in psychology form the basis of character and the tone of mind. If for any reason whatsoever the nervous energy of which we have been talking should discharge itself through any path with unusual rapidity, other conditions being equal, this will take place at the expense of consciousness and retentivity. We find examples of this in the movements of the maniac and in the volatile, irresponsible conduct of some people. Psychology follows the laws of physics.

"It is well recognised that, of all the functions of the nervous system, pain and attention are those which have most effect upon the consciousness, consuming the greatest amount of nervous force and depriving other organs of their fair share with the result that they remain in a condition of hypoactivity. Apart from the object which claims their attention or causes their pain or grief, the sufferers pay little or no heed to the attractions of the external world and do not react thereto or only feebly, if at all. This explains why those who suffer from pain or grief remain motionless, or walk with slow and difficult pace. On the other hand pleasure, regarded from the psychophysical point of view, consists essentially in facility of resolution of the psychic tensions. We cannot imagine pleasure where there is an obstacle. This ready flowing of the nervous currents brings it about that the pleasurable sensation impresses itself less upon the consciousness than would otherwise be the case, and is readily deflected into all the outgoing paths, in the form of a centrifugal current. As a rule, the man who has a happy disposition of mind moves about with lightness, facility and rapidity. The dispersal of nervous energy along the centrifugal paths occurs at the expense of the provinces of consciousness and reflection, and in all degrees from normal conditions to the most violent excitement of the maniac."

The above statement reaffirms in a different manner the well-founded view that one cannot conceive of a conscious phenomenon arising from stimulation of a very small number of cortical nerve-cells. Any conscious phenomenon presupposes the propagation of an external or internal stimulus to a large number of nerve-cells in the mantle. Tanzi and Lugaro ${ }^{1}$ express a similar view.

1 'Tanzi and Lugaro. Malattie mentali. 2nd edit. 1914. 
The fact that the cell-structure of the nervous system is incapable, so far as we know, of modification does not stand in the way of acceptance of the view expressed, because that power of starting, directing, evoking and selecting the nervous currents which we attribute to the frontal lobes would appear to be possessed also, in a minor degree, by all the cell-groups of the mantle, the so-called plejadi isodinamiche of Cajal.

Human actions are the resultant of impulsions and inhibitions. The inhibitory power is more active where, other conditions being equal, the mental field is more extensive and the capital of cognitions more conspicuous, or, in other words, when a larger number of isodynamic pleiads take part in the mental processes. Inhibition is less active where the mental field is more restricted and consciousness more obstructed.

In mental life, then, the phenomena of inhibition may be regarded as regulated by physical laws analogous to those which regulate the flow of liquids in closed tubes or of electric currents in conducting wires.

An internal or external stimulus A on reaching consciousness provokes an intuition of movement, accompanied by a charge of potential for the movement $X$. If at the same moment or immediately afterwards another stimulus $B$ reaches consciousness, one of two things occurs: the stimulus $B$ and the mnemonic residuum rearoused (from past experience) may be convergent and harmonious with $\mathrm{A}$, which in this case becomes reinforced by the determinative power of $B$, and we get $X=A+B$. This summation of potential reinforces the effort or accelerates the movement initiated by $\mathrm{A}$, or it transforms the simple tendency or intuition towards movement, which was induced by this stimulus $A$ alone, into actual movement. On the other hand, what may happen is this: the second stimulus $B$ may be of a contrasting nature and opposed to $A$. In this case it is evident that the movement $X$ will become $\mathrm{X}=\mathrm{A}-\mathrm{B}$. According to the strength of $\mathrm{B}$, we will have a proportionate decrease of the potential or charge that is necessary for realisation of the initial movement provoked by A. This dynamic situation is what is called inhibition. If $\mathrm{B}$, which is in contrast with $\mathrm{A}$, is very strong, $\mathrm{A}$ loses all its determinative efficacy because of the presence of another nervous charge and a different, sometimes opposite, tendency towards movement, so that the first movement will not now take place. It may even be substituted by another in a totally opposite direction and with a different objective. When we speak of stimuli in the mental field we mean actual perceptions or mnemonic traces of past experiences and the psychic complexes derived from these, with their corresponding emotional conditions (more or less extensivemental or associative fields that are capable of being utilised and of being brought into action in a given unit of time). Thus we get arrested or inhibited motor intuitions.

Certain delicate experiments have been made which lend support to this conception. Pavlov carried out a number of experiments which prove that the salivary secretion is very sensitive to the action of external stimuli and that these act and counteract, excite and inhibit, according to circumstances. 
In the higher mammals and in man the various actions become complicated with the memory of previous actions and reactions, and with all the reserves of experience in the life of each individual and of the social group. The nervous system is not a mere reflector of external agents ; it registers, conserves, and reproduces the images of external stimuli ; it combines them together and acts as a multiplier, a storehouse and an accumulator of energies; it is at the same time capable of new forms of reaction under the influence of educative processes (Pavlov, Cathcart). ${ }^{1}$

Wassiliew and Michtovte are mentioned by Bohn ${ }^{2}$ as having demonstrated the fact that any excitation whatsoever from the external world, or even anything that is in itself indifferent, may become an inhibiting agent, arresting some previously existing action. This inhibiting action is what Pavlov would call a conditional restraint. Orbelli, again, clearly establishes the fact that the two fundamental elements in the physiology of the nervous system which explain the great complexity of its functions in general, including psychic activity, are excitation and inhibition. With regard to inhibition Bohn expresses a view similar to that put forward by the writer in 1885 and again, in more distinct terms, in 1910 during the Congress of Scientists in Naples. In 1911, we find Bohn writing as follows :- "It now appears that the higher psychic activities may be traced to strictly determined laws." One must also take into account all that we inherit from generations of ancestors. "We are the slaves of our dead," wrote Comte.

If with this scheme in front of our minds we can imagine, on the one hand, a constant movement of ideas, and on the other hand, a continuous flow of diverse internal stimuli, desires and sentiments which are unceasingly invading the consciousness, and the incessant action of an infinite number of external stimuli which are ever impinging upon our nerves, and the various products which are formed from these in the busy workshops of the sensory (and emotional) centres, then we will form some idea of the great complexity of the mechanism of conduct both in individuals and in social groups, whose actions are always the resultant of impulsive and contending forces - that is to say of judgments, desires, motor impulses and inhibitions. If we have recourse to introspection and examine our consciousness, retracing our history (and that of our acquaintances), we feel that every day, every hour perhaps, our consciousness has been an open arena for combats of contending elements that surround us on every side, elements that are ever exciting desires and aspirations, and tending to become part and parcel of ourselves, entering into combination with the other intrinsic components of our minds. The latter may offer resistance if the new-comers are opposed to social interests, for they are the alert custodians of the mental organisation: they alone can outride the tempests of life; but, on the other hand, they may be drawn by alluring, deceptive appearances towards the mirage of rapid ill-gotten

\footnotetext{
${ }^{1}$ Catheart. "Psychic Secretion : The Influence of Environment." Journ. of Ment. Science. 1919.

"Bohn. La nouvelle psychologie animale. 1911.
} 
success, or, again, they may be disarmed by passionate caresses. In the midst of this contest between attractive (sometimes deceptive) elements and threatening elements, a contest that is ever being renewed under the most diverse circumstances, the well-organised ego proceeds with vigilant, selective power, in constant undisturbed harmony with the environment in which it lives (social consciousness) and with Nature, from which it derives its primordial elements. Will, after all, is only a compromise in the individual consciousness, a resultant of impulsions and inhibitions.

How many desires are aroused by the course of affairs around us, desires whose flame is extinguished by the chilling breath of education and upbringing, or stifled through the timely perception of danger! How many are the tendencies, how many the aspirations that are kept in check, as it were, by a morning mist which does not yet permit us to perceive the propriety, the opportunity, or the ways and means of realising them! How many timid and cautious explorations do we make before we turn back owing to insuperable difficulties, or to the veto imposed by the mere possibility of compromising the personal ego! Think of the impulses that fade away, chased completely out of consciousness by the strong sentiment of duty! And the taunts that chafe and fret the innermost soul, stayed by that inexorable power which governs the evolved man, mindful of his social obligations, holding up before him the threat to the integrity of the ego which cannot withdraw itself from the biophylactic law!

All these representations derived from the actual experience of the moment and from previous individual, family and environmental experience, constitute the real content of what we call inhibition, which finds its foundation in a wider association of ideas, embracing logic, memory, the more evolved sentiments with their accompanying notions, and all the complex structure of the cerebral mantle.

We will leave aside the inhibitions that concern the lower centres, to which physiologists have particularly devoted their attentions, and the reciprocal inhibition of the sensory areas, which can be reduced to the law of displacement of potential, and give our attention to this other form of conscious inhibition with a content of representations, contrasts, associations, memories, and logic. It is an integral and essential factor in human conduct.

Both impulsive and inhibitory forces are directed to the motor field of the cerebral mantle, where all sensory and intellectual representations resolve themselves into motor intuitions and subsequently into movements and, as a whole, into conduct. Motor impulsions are intimately bound up with the sensory functions and therefore with the areas which fulfil these functions. Every perception contains within itself the rudiment of a motor intuition. The motor area, in which all sensations and their intellectual products are translated into appropriate movements (according to the fundamental psychological law of attraction to that which is beneficial and pleasant and repulsion from that which is harmful or painful to the personality), is situated, as already shown, if not quite in the middle of the brain, at least on a line slightly in front of that which divides each hemisphere into two equal halves 
(the pre-Rolandic convolution and the intermediate motor area. Vide Chapters 1. and 11I.).

The Jarge extent of mantle lying behind the pre-Rolandic convolution constitutes the sensory field which is differentiated into as many areas as there are senses, these being veritable workshops dedicated to the transformation of the cosmic energies emanating from the world which surrounds us; it is at the same time the field of the emotions, which incessantly build up the kinxsthesis, the fundamental nucleus of the consciousness.

All that lies in front of the motor field, with the exception of a remnant of the old central organs of smell (in the orbital and internal portions of the hemisphere) is, as the writer has endeavoured to show in the preceding chapters, the organ where new and powerful conceptions are formed with the material provided by the sensory organs from individual and collective experience of life (interwoven with history, tradition and culture). It is also the ehamber of resonance of the somatic ego, which participates in all the mental processes, and it consequently serves as the meeting-place of the products of work of the sensory areas, variously associated and co-ordinated ; it is the throne-room of logic which draws its material from history, utilising the archives of the cognitions, sifting the emotions and impulses, and judging their effects in the light of individual and social experience. From this cerebral organ emanate the vigour and the dignity of the ego, which sails along more or less smoothly between the billows, the rocks and the shoals of life, emerging either victorious or shipwrecked; this is true whether we speak of the ego pervading the ranks of humble workers whose combined efforts give the measure of the activity of a country, or of the ego that raises humanity into a new and higher region of knowledge, impressing a more rapid movement along endless paths of progress.

With this anatomical disposition of the brain, which is supported by the most assured and controllable facts of embryology, comparative anatomy, experimental physiology and clinical anatomy, there correspond two grades of consciousness, a higher and a lower. The lower consciousness moves in the shorter circuits of the sensory fields where prevail sensations, images, relatively simple mental constructions, emotions, desires, acts of selfprotection, and instinets, which sometimes flame into passions. The higher consciousness moves in wider circuits, forming with the former a more extensive network of notions and experiences, being open to currents which come from all parts of the cerebral mantle. The pre-frontal portion of the mantle contributes to the higher consciousness with the weighty factors of reason and the more lofty and evolved sentiments, as summed up in the sentiment of sociality. The sensory consciousness tends preferably to immediate satisfaction of individual needs; the higher consciousness tends to temper the impulses of the other with social tendencies and instincts. Excessive predominance of the lower consciousness confers on man a more brutally egoistic character, which sometimes leads to criminality in the most diverse forms. Prevalence of the higher consciousness raises egoism to the higher sphere of human solidarity, leading sometimes to genius, which 
synthetises the story and the potentiality of the race, and at the same time opens up new horizons to thought and new fields to human activity.

This twofold form of consciousness may be followed step by step in the history of human evolution and human degeneration. The study of human disease lends confirmation in support of this twofold aspect of consciousness. If we trace the phylogenetic history of both modes of consciousness in relation to the two cortical fields, we arrive at conclusions that at present are difficult to combat. The sensory field is first to be developed: with it we have development of the perceptive capacity and the motor reactions, and whilst these assume in the main the character of fairly simple reactions, yet they show a progressive development, both in number and complexity, that keeps pace with the growth of the mass of the cerebral mantle. Step by step, from childhood to adolescence and maturity, we find the psychic manifestations becoming more varied and better co-ordinated, and reasoned inhibition coming into play.

These inhibitions coincide, in the zoological scale, with the development of the frontal lobe, and, in ontogenetic development, with the mature growth of that organ. Thəy assume the reins of government in life when the frontal lobes reach their maximum development in the evolved man of the more advanced races.

Although the frontal lobecomprises a great part of the cerebral hemisphere, it has no direct relation with the peripheral organs of sense, and for that reason cannot discharge sensory functions. That does not mean to say, however, that as this new part of the neopallium develops the sensory field remains stationary. On the contrary, a marvellous development takes place at the same time in the sensory field. We have already given examples in proof of this statement.

From the functional point of view, all the senses become finer and more developed with the progress of civilisation. Tacitle sensibility, e.g., becomes more delicate and highly differentiated. In the tactile and muscular sensibility of the blind we have an example of the development of which this sense is capable. The case is reported of a deformed girl whose four limbs were represented by the merest stumps, who had so developed the tactile sense in the tongue and mouth that she was able with her tongue and lips to work a thread in her mouth into a piece of lace. So far as the sense of hearing is concerned, we know that the song of the savage is monotonous and simple, consisting essentially of a few notes and beats in a deafening combination of stridulous noises in which, at most, some little respect is paid to rhythm. What a difference from the melancholy song of $L a$ Traviata or the voluptuous and seductive singing of Carmen, or the desperate and vindictive song of Othello, or the chattering of the chorus in Falstaff! What a variety of accents, what an inflexion of notes and motifs!

The compositions of Rossini, Verdi, Wagner, would not have been possible but for the marvellous development of the sense of hearing and the sentiments connected therewith. The music of Pergolesi, Rossini, Verdi, Beethoven and Chopin, reflecting, as it does, development and perfecting of 
the auditory ficld, stands in the same relation to primitive music as the capacity of civilised races and people who have been trained to distinguish the various shades and blendings of colours stands in relation to that of primitive peoples. This capacity has progressed pari passu with civilisation. The earliest men probably distinguished only the colours of wide vibrations such as red and orange. Other colours with shorter and weaker vibrations were not distinguished till a later period (Geiger).

There is thus no doubt that sensory evolution, with its anatomical substratum in the posterior regions of the cerebral hemispheres, also goes on indefinitely; but the life of interhuman relation, the tendency towards reciprocal understanding of minds, the great intellectual constructions, the logical pattern of thought and conduct based on attention, evocation, selection, imagination, co-ordination-in other words, the appraising of the marvellous products obtained by the work of Nature and natural forces on the sensory brain-are evidently dependent upon the frontal lobes, which evoke and utilise all these products in the construction of the great syntheses and at the same time moderate the impulsive or reflex activities inherent in each individual. In this way man proceeds, on the one hand, to perception of Nature and the infinite voices of the social environment; on the other hand, he attains to the most complex intellectual syntheses, and to an everincreasing inventive activity which is directed towards the overcoming of difficulties and the rendering of life more joyous and more certain, whilst at the same time he becomes more capable of adapting himself to the environment, tempering individual with social instincts. Thus it comes about that the primordial impulse of each man, as it evolves in contrast with the impulses of other men, obeying the fundamental biophylactic law, attains by degrees to that conduct which is characteristic of the truly evolved man, conduct that is the resultant of impulsions and inhibitions.

One may represent human conduct in the form of an equation into which there enter, in different proportions, the two forms of consciousness already alluded to. If we suppose conduct to be represented by $\mathrm{C}$, the sensory and emotional consciousness by $\mathrm{S}$, and the higher (logical and sentimental) consciousness by $\mathrm{I}$, the equation will be $\mathrm{C}=\mathrm{S}: \mathrm{I}$. The two terms $\mathrm{S}$ and I vary very much in different individuals and in different ethnic groups. $\mathrm{S}$ may be excessively developed (strong sensitivity and emotivity) so that this term will be represented by $\mathrm{S}^{\mathrm{n}}$, whilst at the same time $\mathrm{I}$ will be proportionately less, or vice versa.

The different combinations of numerator and denominator have their counterpart in the numerous varieties of, and outstanding differences in, individual and ethnic characters. Other factors, no doubt, enter into the structure of character, but all may be brought within the above formula, which comprises perceptivity, emotivity, associative capacity, impulsivity, inhibition, activity, sociality. The value of life is in proportion to the development of I and S, between which there should be a perfect, reciprocal understanding.

We still know but little of the obscure work of milliards of cells in the 
mantle which are grouped and connected with one another in a wonderful manner. Nevertheless it is safe to say that all the work of the human brain is the resultant of these two energies-impulse and inhibition. The products of this contrasting action are constantly being multiplied and renewed; and the work promoted by the constant renewal of the physical and social environment and by the changing circumstances of organic life always entails new co-ordinations and the co-operation of additional workers.*

It is clearly our duty to promote and encourage the development of the brain. The evolutionary progress of this very important organ coincides with a gradual perfecting of the sensory powers, which enables us more and better every day to grasp and appraise the world in which we live; it reveals itself in a progressive transformation of the products of the work of apprehension (more closely connected with the reflexes and instincts), into intellectual, sentimental and inhibitory power, which is based essentially upon more extensive association and co-operation.

In all grades of scholastic education it is necessary to develop the perceptive and retentive capacities on the one hand, and the associative, attentive and inhibitory powers on the other. Modern education should consist essentially in aiding and guiding the unceasing development of the general and special functions of the brain and in leading the individual into regions of wider and more effective relations with the physical, social and ethnic environment. Education which promotes the fullest possible development of all the functional capacities will lead not only to successful acquisition of knowledge of the world of nature, but also to comprehension of the responsibilities involved in discharging the duties of the family, social and national life. ${ }^{1}$

It is necessary, in other words, to gradually transform the primary ego (Meynert), which is prevailingly instinctive and egoistic, into the secondary

* This formula summarises the modern conception of the cerebral localisations. In the writer's opinion it is not made any clearer by the introduction of the term chronogenous, which expresses a very obvious fact, although it is insisted upon by Monakow (Les Localisations cérébrales. Wiesbaden, 1914). Time has reference to the evolutionary progress of the brain, and has little to do with the actual function of the evolved brain. In order to understand the cerebral function we must take cognisance of the spatial factor as explained in Chaps. I. and III. The chronogenous factor has reference to the evolutionary progress of the nervous system and of mind in the historical sense; the element of time in the psychic processes should not be brought into the question of the cerebral localisations. Physiologists, clinicians and histologists have long taken great pains and carried out many researches with the object of defining the various localities from which are derived the phenomena of the life of the nervous system. Apart from the still rather uncertain and contradictory results obtained from the study of cytotecture, everything goes to prove that the appearance of any new organ arising in the course of the development of the brain is counter-marked by the utilisation, on the part of that organ, of the products of the work of pre-existing organs. It is in this way that we get an extension of the field of localisation of the elementary products that go to make up the more complex psychic processes.

${ }^{1}$ L. Andrizen. "On the Bases and Possibilities of a Scientific Psychology." Journ. of Ment. Science. 1899. 
ego characterised by the development of morality and of higher consciousness, which assumes the government of life.

The fortune of an individual or an ethnic group is greater or less, according to the particular capacity to penetrate situations (apperception)-in other words, it depends upon the extent and strength of the associative and attentive faculties. Ability to penetrate the innermost sense of things provides the key to the efficacy of a single action or of the whole conduct, either of an individual or of an ethnic group.

To create the habit of superficiality in thinking and knowing, as happens in many of our schools, is but to prepare the way for conduct that is liable to be swayed by overtures and advances, because superficial knowledge leads to inconsistence of character, and to hypo-morality, and is linked with emotivity that is often egoistic, like the emotional explosions that we sometimes witness even in public assemblies, manifesting itself in enthusiastic applause to the more or less affecting speech of the orator, who, by oratorical artifice, appeals to the emotions, although the speech may be entirely void of any content of practical politics. From the school to the family, to the workshop, to public offices, and to the higher posts in the state and in the legislature, the character of the nation bears everywliere the stamp that was impressed upon it in school.

Consider a moment the attitude of Italy in the earlier stages of the Great War. The ignorance and consequent suggestibility of the masses, the flabby consciences of certain leaders, the lack of foresight (inattention and superficiality of judgment), the predominance of low emotions (vanity and pride in some cases, and fear, indifference and neglect of duty in others), injustice (falsehood, cruelty, moral weakness), etc., were able for the moment to place a veto upon the great and genuine virtues of the stock, subsequently revealed in the heroism of her soldiers, who smilingly sacrificed their lives for their country, and in the noble, dignified, patient resistance of a people enduring endless suffering, yet throbbing with the highest and purest patriotic sentiment, virtues which, regaining their proper dominion, made victory assured.

The excessively egoistic conception which another people had of life, the immeasurable, overbearing exclusiveness of the race, the aggressive, ferocious, insensible haughtiness in the exercise of the right of force, had much to do with the ruination of that people, who by perseverance and labour had succeeded in penetrating every region of the world with the products of its industry.

To-day, when psychology helps us to explain the phenomena of history, it is our duty to devote careful and weighty consideration to scholastic methods, the one aim and object of which should be to establish in each individual the sure foundations of a higher consciousness, embracing the larger conception of life, which is an aggregate of human values, associated, co-ordinated and acting in concert with one another. It behoves us to apply our minds assiduously to the solution of this problem which so intimately affects the life of the nation.

We must change our ways. Above all we should take care, in every grade 
of school, to get rid of methods and systems (perhaps even personnel) that favour slipshod and slovenly learning, that are concerned solely about the quantum necessary for the examination, drying up the springs of noble sentiments, and paying heed only to the expediencies of the moment, methods which arrest or hinder the difficult but enthusiastic work of integration and development of the higher consciousness and the powers of attention, which on the contrary should be developed, encouraged and brought to a high potential, for such is the source from which are derived the success of the individual and the fortune of the race. 



\section{INDEX OF SUBJECTS}

\section{A}

AfFectivity diminished by lesion of frontal lobes, 73

Agnosia, tactile, 46

Agrypnia, its psychic effects, 270

Alexia (word blindness) from lesions of parietal zone, 36,46

Alogias from experimental ablation in monkeys, 259

Amblyopia in monkeys and dogs from mutilation of frontal lobes, 195

Amimia, 244

Amnesia (See Aphasia), asthenic, 237

- from lesion of left inferior parietal lobe, 46

Amphibians, structure of nervous system of, 95

Angular gyrus, disturbances from lesions of, 34

Anthropoids, brain (frontal lobe) of, 104, 105,107

Aphasia, amnesic, 51, 234, 237

- compensation in, 126

- motor, and mental integrity, 119, 241

- optic, 32

- sensory, 34, 36. (See Word-Deafness)

Apperception (attentive recognition), and movements associated therewith, 193

- causes of failure of, 259

- centre of, in frontal lobes, $80,83,334$

Apraxia, from lesion of inferior parietal lobe, 36

- from lesion of tactile zone, 18

- in aphasics, 244

Archipallium, 11

Area, auditory (acoustic-sensory and acoustico-psychic), 18, 19

- for images of objects distinct from those for images of words, 235

- intermediate, precentral, 62

- motor, 16, 60

- - comparative limits of, in man and monkeys, 60, 71, 107, 110, 131

- - intermediate, 25, 112, 116

- of emotions and the tactile zone (Flechsig), 270

- of frontal evolution, 110

- of reception and area of perception, 30,48

- olfactory, 19

- pre-frontal, 62, 112

- pre-Rolandic, 116

- projection (Flechsig), 27

- somæsthetic (of Flechsig), 109, 270

- somatic (of Johnston), 11

- tactile, 16, 17

- visual, 18, 22, 23

Areas, associative (of Flechsig), 2.5

- evolutionary and intellectual evolu. tion, 23
Areas, myelogenetic, 26, 117

- posterior cerebral, importance of, in higher mental processes (Flechsig), 82

- sensory, in cerebrum, their distinction into sensory, intermediate or psycho-sensory, 19. (See Centre)

Arrest, centres of, 78

Assimilation, mental, 255

Association centres (Flechsig), 22, 25, 29

- paths, between frontal lobes and sensory area of cortex, 198

- - between various cells of cerebral cortex, 4

Associations and frontal lobes, 89

- between cortical centres and gustatory, acoustic, visual, etc., excitations, 128

- capacity for, reduced in monkeys mutilated in frontal lobes, 184, 251

- functional, between centres of movement of head, eyes, and ears, 133

- classification of, 219

Astereognosis from lesion of tactile zone, 18

Ataxia from lesion of frontal lobes (sig. moid gyrus), 87

Attention, and perceptive process, 257

- diminution of, in mutilated monkeys and in idiots, 251, 256

- expectant, 133

- expectant and hallucination, 318

- frontal lobes organ of, 70,83

- in relation to pleasure and pain, 272

- motor concomitants of, 194

Auditory cortical field, differentiation of, for phonetic images of things, 49

Automatisms and reflexes, 10

- and the unconscious, 319

- from lesions of frontal lobes, 73

- subcortical and cortical, 12, 13

\section{B}

BABINSKI's sign, absence of, in hemiparesis from lesion of inferior frontal lobe, 36

Biophylaxis, 256, 284

Birds, development of brain (frontal lobes) in, 95

Blindness from cortical lesion, 24

- psychic (of Munk), 24

- word (Alexia), 36

Brain. (See Cerebrum)

Bridgemann, Laura, 235

Bulbs, ocular. (See Eye)

Bundle, intellectual or psychie, 202

- fronto-occipital, associative, 203

- superior or areuate longitudinal or associative, 198 
Bundle, uncinate, connecting with frontal lobe, 199. (See Fibres)

Bundles, associative intrafrontal, between frontal lobe and sensory area of cortex, 198

- projection, effects of lesions of, 49

\section{C}

Capsule, internal, contains projection fibres of frontal lobe, 200. (See Monkeys)

Centre, acoustic or phonetic, of articulate sounds, 238

— auditory, of Wernicke, 34

- for somatic reflexes of the emotions, 270,273

- frontal, for dilatation of pupils, 194

- - for secondary and primary sensibility (Flechsig), 25

- - for secretions, 268

- - of sentiments which become conscious, 270

- - for respiration, 264

- - for viscera, 266, 267

- of centres, 21,79

- visual, 18

- visuo-psychic and visuo-sensory, 24. (See Area)

Centres, auditory, of language, 32, 47

- cortical, of association (Flechsig), 21, 22,25

- fictitious inhibitory, 78

- inhibitory, in frontal lobes, 85. (See Inhibition)

- of language, disturbances of, affect entire cerebral functioning, 49

- subcortical, their function, 12, 13. (See Areas)

Cerebrum, its morphological evolution and weight, 8,9

- intelligence and weight of, 9. (See Neopallium, Grey Substance, Weight, Intelligence, Hemispheres, Hæmorrhages, Convolutions, Cortex, etc.)

Character, individual, and frontal lobes, 71

- nucleus of, 314

Children, dreams and fibs of, 223

- earliest manifestations of language in, 230

- mechanism of reading and writing in, 239

- mental evolution in, 221

Chimpanzee, frontal lobe (third frontal convolution) of, 105

Chorea, Sydenham's, and emotional attitudes, 278

Chronogenous and spatial element in cerebral localisations, 333

Cibus, lower, brain and frontal lobe of, 106

Cingulum, association-bundle of rhinencephalon, 198

Cinocephalus, development of brain (frontal lobe) of, 106

Circulation, effect of cortical excitation upon, 266;
Circus movements, from lesions of sig. moid gyrus, 87

- not dependent upon inhibition, $78,87,191$

Claustrum, bundle of fibres in connection with frontal lobe, 206

Climate and the elements from which it results (light, temperature, electricity, winds, etc.). Its influence on the psychic function, on insanity, crimes, suicide, etc., 261

- influence of great heat and great cold, 269

Clinical histories of dogs, foxes and monkeys, deprived of frontal lobes, 169

Cold, disintegrating action of, 269

Colloids, importance of, in the vital phenomena, 3

Colours and evolution of vision, 332

- distinguished by monkeys, 130

Compensations, functional, in cerebral cortex, 22, 126, 142

Complexes, sensory and motor, 13

- psychic, 216, 318

Conceptions, abstract, formation of, 247

Concomitants, somatic, of pleasure and of pain, 273

Conconscious, conception of, 317

Conduct, logical character of, 303

- individual, and frontal lobes, 71

Consciousness, and images, 12

- and propagation of stimulus to a large number of nerve-cells, 326

- genesis and localisation of, 10, 15, 16, $82,302,311,312$

- higher and lower, evolution of, 330, 331

- successive mutations of, - analogous with physical and physiological facts, 2

- the unconscious or subconscious, 224,317

Contrast, psychic, in mental associations and in delusions and obsessions, 222, 256, 311, 329

Convolution, ascending parietal, disturb. ances from lesions of, 46

- supra-marginal, disturbances from lesions of, $\mathbf{3 4}$

Convolutions, and functions do not coincide, 108

- and sulci, frontal, 115

- temporal, physio-pathology of, 51

- temporal, disturbances from lesions of, 34

Corpus striatum, in fishes, 94

Cortex, cerebral, development of, in batrachians, 14

- - - in vertebrates, 9,11

- - - in the various zones and its histological structure in relation to function, 116

- degenerative dissolution of, in inverse order from evolution, 117

- differentiation and functional localisation in, 14

- sensory, and psychic content of the emotions, 271

- - association-paths between frontal lobe and, 198 


\section{INDEX OF SUBJECTS}

Crime, influence of climate upon, 261

Currents, electric, excessive strength of, a source of error in experimental investigations, 132

Cytotecture of cortex, 17, 20, 23, 30, 61, 89,90

- of frontal lobe, 117. (See Histotecture)

\section{D}

Deafness, word. (See Word-Deafness)

Decortication of frontal lobes left in situ, 135

Defæcation, cortical centre of, 267

Degeneration, anthropological, 113, 114, 127

- descending, in study of cerebral localisations, 29

Degenerative processes in cerebrum after destructive foci and experimental lesions, 127

Deglutition, cortical centre of, in relation with respiratory centre, 267

Deliberation, action and conduct, 248, 309

Delusions, extraneous elements in reasoning process, 258

- from associations by contrast, 223

Dementia and law of dissolution, 114

- associated with psychic blindness and word-deafness (auditory aphasia), 31, 44

- accompanied by degenerative histo. logical changes in pyramidal cells, 113

- post-epileptic : clinical case, 57

Destructions, experimental, in cerebrum, proximate and remote effects of, 125

Diaschisis, 35, 87, 90, 122, 142

- and bilateral homonymous hemianop. sia, 196

Differentiation, and co-operative association, law of, 15

- functional, in cerebral cortex, 15

Discipline, normative, of Wundt, 254

Disintegration and pain, 271

Dislogias, 256

Distance, perception of, in infants, 25

Dogs, clinical histories of experiments upon, 137

- development of brain (frontal lobes) of, 97

Dolphin, development of brain (frontal lobe) of, 96, 107

Double personality, 322

Dreams, and "Repressed Wish," 320

— of infants, 223

\section{E}

EARs, movements of, provoked by stimulation of certain excitable areas of frontal lobe, 191

Education, human, essence of, 333

- of animals in new associations for purpose of re-examination after excision of frontal lobes, 88

Ego, primary, secondary, Meynert, 333

Electrons, theory of, in relation to psyehology, $1,2,3,4,5$
Emotions, and kinæsthesis, 273

- and sentiments, 260, 280, 289

- and the sensory cortex, their psychic content, 271

- and the various "grades " of feeling, 213

- and touch, area of (Flechsig), 270

- as extraneous elements in reasoning, 258

- evolved, 270

- in monkeys deprived of frontal lobes, 185

- in relation to associative powers, 84

- organic basis of, not in frontal lobe, $65,83,84,89$

- the two theories of, as regards nature and seat, the somatic theory and the psychic factor, 274

Emotivity, after lesions of frontal lobes, 73,89

Energetics, 263

Energy, psychic, in relation to laws of natural energy, 213

Epileptic attack, dilatation of pupil in, 192

- from electric stimulation of frontal lobes, 75,76

Equivalents, biological, nervous and psychic, 8, 9

Ether, and electro-magnetic, luminous and nervous waves, $1,3,10$

Evolution, mental, in infants, 212, 221

- of neuro-muscular system, 6. (See Various Sensory Organs)

Excitable area of frontal lobe and its significance, 187,191

Experience, individual (ontogenetic) and inherited (phylogenetic), 215

- not made use of in mutilated monkeys, 251

- result of relations in simultaneity, resemblance, etc., 252

- utilisation of, 306

Extension, movements of, from pleasurable excitations, 279

Extra-logical (or moral) objects, 253

Eye, morphological evolution of, in relation to psychic development, 6,7

- movements of, provoked by stimulation of certain excitable areas of frontal lobe, 190,191

- visual disturbances in dogs and monkeys after frontal mutilations, 195

\section{F}

FEAR, genetic conditions of, 277

- in monkeys mutilated in frontal lobes, 185

Fibres, associative, 30

- associative, deficient in pre-frontal area, 112

- projection, of frontal lobe, 200. (See Bundles.)

Fibs of young ehildren, 22:3

Fields. (See Area and Centres)

Flexion, movements of, from unpleasant excitations, 279

Fishes, structure of encephalie nervous system in, 94 
Fissure of Rolando, correspondence between crucial sulcus and presylvian suleus of animals and, 97

- - - position of, in monkeys, 107

- - - (See Sulci and Convolutions)

Form, perception of, in infants, 25

Frontal lobe, a centre of association and co-ordination, 22

- - associative paths between, and sensory field of cortex, 198

- - evolution, morphology, and structure of, 94, 118

- - functional value of left and right (language, intelligence, etc.), 118, 119

\section{G}

GAIT, influence of inhibition on, 325

Ganglia, evolutionary story of, 5, 8

Glands, influence of cortex (tactile zone) on secretion of, 268

- lachrymal. (See Tears)

- mammary. (See Milk)

- salivary. (See Saliva)

Gorilla, frontal lobe and third frontal convolution in, 105. (See Monkeys)

Grey substance, relative development of, in man and animals, 10

Grief and joy, 273, 275, 279

Gustatory area, 19

Gyrus, angular, disturbances from lesions of, 34

\section{H}

HanorRHAGE, cerebral symptoms of transitory shock from, 125

Hallucinations, alogia resulting from, 256, 257,258

- and personality, 322

-.. visual, unilateral, 141

Hearing, cerebral localisation of auditory sensibility, 18

- disturbances of, from lesions of parietal lobe, 34

- - - - - temporal lobe, 51

- evolution of, 331

Heart, influence of cortex upon, 266

Heat, disintegrating action of, 269

Hedonism, 296

Hedonistic sensations, 290

Hemiagnosis, tactile, in parietal lesions, 46

Hemianasthesia from lesion of inferior parietal lobe, 36

Hemianopsia, bilateral homonymous, from lesion of inferior parictal lobe, 36

- - in dogs and monkeys mutilated in frontal lobes, I95

Hemiparesis. (See Paresis)

Hemiplegies, apparent emotivity of, 277, 278

Hemisplieres, cerobral, differentiation of right and left, 119,120

Hieroglyphics in evolution of writing, 233

Histotecture, accorling to Broclmann, 111

- geographic, of cerebral cortex, 111 . (See Cytotecture)
Horse, development of brain (frontal lobe) of, 100

Humanity, sentiment of, 296

Hunger and thirst, and pleasure and pain, 269,271

- - - fundamental emotions con. nected with material exchange and chemical conditions, 269

Hyperlogical, or æsthetic, objects, 253

Hypnotism and the unconscious, 317,318

Hypologia, 257

Hysteresis, associative, 3

Hysteria and the subconscious, 317

Hystological structure and psychological facts, 30

Hystotectural geography of cerebral cortex, 110

- type of Brodmann. (See Cytotecture)

IDEAS, general, and concrete images, 217

Idiots, behaviour of, compared - with mutilated monkeys, 250

Illiterate, effects of lesions of parietal lobe in the, 48, 50

Images, and consciousness, 12

- concrete, words, and general ideas, 217

- elaboration of, in sensory cerebral areas, 19,20

- elementary constitution of, 4

- memory, 237

- mnemonic, produced by perception and associated in the cerebral centres, 21 .

- visual, formation of, 24

- word (auditory, visual, kinæsthetic), 237

- word, Marie's views against, 244

Imagination, creative, 223

Imbeciles, lack of sociality in, 288

- natural history of human evolution reproduced in, 313

Imitation as a social law, 294

Impressions, varying effect of, in different individuals, 2

Impulses and contrast, $327,332,333$

Impulsions and inlibitions in human conduct, 309,327

Index of variability in frontal lobe, 119

Indifference, from tiredness, 271

- psychic, in monkeys from lesions of frontal lobes, 73

Indifferent sensations, 272

Inhibition, cerebral, absence of, in monkeys mutilated in frontab lobes, 185

- - and impulses in human conduct, 333

- - foundation of, 329,331

- - in the various cerebral regions, 324

- conscious, 329

- frontal lobes and, 318, 331

- reciprocal, of sensory areas, 329. (See Centres)

- centres of, 78

- cerchral, 309,310

Insomnia, psychic effects of, 270

Instinct of self-preservation, $26 !), 290$

Instincts and sociality, 289 
Intelligence, and emotions, 283, 284

- and language, 209

- and weight of brain, 9

- disturbances of, from lesions of left inferior parietal lobe, 46

- evolutionary phases of, 10

- in lower creatures, 307

- in man and animals, applicability of experiments relating to, 127

- localisation of $15,16,19,20$

Interference in mental associations, 223

Internationalism and sociality, 287

Interrupter, hand, in electrodes used in experiments, 136

Intestine, movements of, in relation to cortical stimulations, 267

Invariability and variability of reactions in progressive evolutions, 12

Irresistibility of movements from lesions of frontal lobes, 72,73

\section{J}

Joy and grief, genetic conditions of, 262, $275,276,278$

Judgment, and deliberation, 224, 248, 309

- defects of, from lesions of frontal lobes, 73

\section{K}

Keller, Helen, 235

Kinæsthesia, and emotions, sentiments and images, 262, 273

- and tactile sphere, 65

- centre of, according to Flechsig, in frontal lobes, 82

Kinæsthetic sense, 263

\section{L}

LAMB, development of the brain (frontal lobes) and motor zone in, 103

Language, and intelligence, 209

- and left cerebral hemisphere, 119, 120

- and phenomena of compensation after cerebral lesions, 126

- and sociality, 291

- cerebral localisation of, 19, 32

— internal, 242

- lesions in areas of, affect entire cerebral functioning, 49

- motor zone for, 63

- reconstruction of cerebral mechanism of, $19,28,29$

- visual and auditory cortical areas of, 47,48

- See Aphasia, Paraphasia, Paralogia, etc.

League of Nations and sociality, 287

Liberty, sentiment of, 291, 296

Light, formation of visual images, 24 . (See Climate)

Lobe, frontal, a centre of association and co-ordination, 22

- associative paths between, and sensory field of cortex, 198
Lobe, frontal, evolution, morphology, and structure of, 94

- - functional value of left and right (language, intelligence, et(.$), 118,119$

- inferior parietal, symptomatology of lesions of, 32

- left temporo-sphenoi(lal, destructive lesions and clinical cases, 5l (language), 59

- temporal, psycho-pathology of, 50

Localisation of intelligence and conscious. ness, $15,16,19,20$

Localisations, cortical, and post-operative phenomena, 154

- - linguistic, chronogenous and spatial elements in, 333

- - in relation to diaschisis, 122

- sensory, $15,16,18,19$

Logic, disturbances of, in pathological processes, 255

- extralogical (moral) and hyperlogical (æsthetic) objects, 253

- nature, function, and development of, 252

- obsessions, a disturbance of, 255

Lying, a phenomenon of creative imag. ination in children, 223

\section{M}

Memory, and perception, 30

- associative, a factor in variability of reactions, 8

- defects of, from lesions of frontal lobes, $73,88,251$

frontal lobes, 184

- motor, visual, etc., 219

- undisturbed after slight frontal lesions, 78

Mesencephalon, volumetric relations be. tween neencephalon and, 10

Methods of investigation of frontal lobes, 121

Milk, psychic influences and secretion of, 268

Mnemonic residua, emotional, in ideas, 84

- - or traces of previous sensations, 8, 16

Monkeys, development of brain (frontal lobe) and the question of a third frontal convolution in, 105

- effects of experimental lesions in trained as compared with untrained, 130,131

- experimental and clinical histories of, after mutilation of frontal lobes, 137

- extent of cerebral cortex in, as com. pared with man, 107

- psychology of, 183, 250

Morals and polities, 313

Motor function, restricted compensation of, after cerebral lesions, 126

Mutilation of cerebrum, proximate and remote effects of, 121,123

- - frontal cortex, effects vary according to extent of, 135 
Myelinisation and cvolution of intelligence, 29

- of cercbral fibres, 25

- of spinal bundles, 15

Myelotecture, cerebral, 117

Myxine, structure of pallium in, 9.5

\section{$\mathrm{N}$}

Neencerhaton, volumetric relations between mesencephalon and, 10

Neothalamus, 12

Nervous system, evolution of, 1,6

New nervous organs utilise functional products of pre-existing nervous organs, 14

Nucleus caudatus, relations of, with frontal lobe, 203

- lenticular, relations of, with frontal lobe, 204

\section{$\mathrm{O}$}

Orsessrons, illogical nature of, 255

Olfactory area, evolution and localisation of, 11,19

Ontogenesis and phylogenesis of nervous system, 9

- perceptive, 215

Operation, extent of cerebral mutilation in relation to effects of, 135

Operculum, Rolandic, projection fibres in, 201

Optic lobes in frogs, 14

Orang-utang, extent of cortex in, as compared with human cortex, 107

Organisation, economic, and society, 294

Organs of the senses, evolution of, 6

- of recent development utilise products of pre-existing nervous organs, 14

- sexual, cortical representation of, 267

Ox. (See Ruminants)

\section{$\mathbf{P}$}

PAIN, aversion to, 271

- and pleasure, elements from which they result, 271

Pallium membranosum in lower fishes, 94

Paralogia and lesions of temporal lobe, 58

Paraphasia and lesions of left inferior parietal and temporal lobes, 35, 37, 58

Paresis (hemi-) from lesion of left inferior parietal lobe, 35

Parietal syndrome, 32

Passion, emotions and, 284

Pathology, mental, and the subconscious, 318

Perception, a process of association of various components, 30

- defects of, in monkeys mutilated in frontal lobes, 183

- sensory, and apperception, 80

- involuntary (immediate), and voluntary perception with attention (apperception), movements associated with, 193

Perception and reception, relation between, 30, 48
Personality, duplication of, 322

- organic, 263

- psychic, alterations of, through paralysis of cortical field of kinasthesis, 66 - - growth of, 216

Petromyzon, cerebral structure of, 95

Phantasy, psychic elements of, 19, 218

Phrenasthenics, mode of language of, 231, 232

- structural and histological details in brains of, 113,117

Phylogenesis and ontogenesis of nervous system, 9

- social, 215

Pleasure and pain, integrative and disintegrative factors, as primordial forms of emotion, 262, 270, 271

Pleiads, isodynamic, of Cajal, 327

Plethysmograph, emotions and the, 262

Polarisation, psychic, and association through contrast, 222

Pole, frontal, a small part of pre-frontal lobe, 79

Politics and morals, 313

Precentral area, intermediate, 62

Pre-frontal area, 62

Pre-Rolandic area, 116

Processes, degenerative, cerebral, after destructive foci and experimental lesions, 127

- logical, in monkeys, 185

Projection fibres of frontal lobe, 200

Protoplasm, constitution and differentia. tion of, 5,8

Psychic function in man and animals, applicability of experimental findings to, 127

Psychism and tropism, 3, 7

- in lower creatures, 305

Psycho-analysis and the unconscious, 317

Psychology, comparative, and the application of experimental findings to the human subject, 129, 209

Psycho-reactive function. (See Association)

Pupils, dilatation of, in epileptic attack, 192

- - frontal centre for, 194

- mechanism of, 192

- movements of, provoked by stimulation of certain excitable areas of frontal lobe, 190, 191

- relations of, with facial nerve, 194

- state of, in relation with mental processes (attention, evocation, etc.), 195

Putamen, relations of, with frontal lobe, 204,207

Q

QUANTITY, in psychic phenomena, 212

$\mathbf{R}$

RABBIT, development of brain (frontal lobes) of, 96

Races, human, variation of frontal lobe in, 118,119 
Rat, clevelopment of brain (frontal lobes) of, 96

Reactions, chemical, physical, or mechanical nature of, in living creatures, 7

Reading, cortical area of visual images and graphic signs of words, 31

- mechanism of, in infants, 239. (See Alexia.

Reason, variability and invariability of, in various creatures, 12

- genesis of, 386

Reasoning, 224

Re-education, after cerebral mutilations, 124

Reflexes, automatism and, 10

- rapidity of, in relation to cerebral inhibitory action, $85,86,88$

Region. (See Area, Centre, Field, etc.)

Relation, life of, in lower creatures, 303

Religion, passion in, 284

Reptiles, structure of central nervous system of, 95

Respiration, cortical area associated with, 264

Restraints. (See Inhibition)

Retina, area of cortical projection of, 24

Ruminants, frontal lobes poorly developed in, 98

\section{S}

Saliva, secretion of, arising from cortical and psychic excitation, 268

Seasons, influence of, on psychic functions, 261

Secretions, influence of cerebral cortex upon, 268

Self, primary and secondary (Meynert), 333

Sensations, and cerebration, 224

- hedonistic, in education, 290

- indifferent, 272

Sense, kinæsthetic, 263. (See Kinæsthesis)

Senses, special, cerebral localisations of, $16,18,19$

Sensibility, auditory. (See Hearing)

- differential, a new factor of variability of reactions, 8

- tactile. (See Touch)

- visual. (See Vision)

Sensory field of cortex, associative paths between frontal lobe and, 198

Sentimentality, frontal lobes and, 285

Sentiments, emotional and intellectual components of, 281,282

- in monkeys deprived of frontal lobes, 186

Sex, variations in frontal lobe according to, 118,119

Sexual functions, their "representation" in cortex, 267

Sheep, development of brain (frontal lobes) in, 102, 104

Shock, in experimental investigations, 125

Sight, power of, in various animals, 6,7

- cerebral localisation of, 18

- development of, in infants, 25

Sledge, Du Bois-Reymond, in experimental investigations, 136
Sleep, chemical nature of conditions which induce, 269

Smell, cerebral localisation and evolution of, $11,1 !$

Social sentiment, 286, 295

Sociality and instincts, 289

- and League of Nations, 287

- disappearance of, in imbcciles and mutilated monkeys, 288

Somatic area of Johnston, 11

- concomitants of pleasure and of pain, 273

- phenomena, the physical components of emotion, 279

Sorrow and gladness, 275

Space and time in cerebral localisation, 333

- and spatial relations, perception of in infants, 25

Speech, central organ of, on foot of third frontal, 109

- internal, 242

- its intrinsic constituent elements (auditory, visual and kinæsthetic) and their associative inter-relations, 238. (See Language)

Sphincter, anal, cortical centre of, 267

Spleen, contractions of, in relation to cortical excitation, 267

Stereotropism, 7

Stereotypisms, from lesions of frontal lobes, 73

- in monkeys mutilated in frontal lobes, 186

Stimuli and functional differentiation, 9

Stomach, movements of, from cortical excitations, 267

Subconscious, the, and subcortical automatisms, 13

- products of, 317

Succession of ideas in content of consciousness, law of, 225

Suicide, influence of climate upon, 261

Sulcus, crucial, the analogue of fissure of Rolando, 97

- frontal and pre-frontal, 116

- presylvian, the analogue of fissure of Rolando, 97. (See Fissure and Convolutions)

Surprise, part played by, in emotion, 276

Sygmoid gyrus, and organic phenomena of emotion 84

- circus movements from lesions of, 87

- - proximity of, to frontal lobe, 129

Symbolic writing (pictography), 233

Symbols, word, of objects and of relations between objects and images, 241, 248

Sympathetic system and emotional reaction, 279

Syndrome, parietal, 32

Syntheses, psychic, and cerebral localisations, 21, 22

Synthesis of syntheses, 248

TACtILE agnosis, 46

- area, 16, 17 
Tapetum, symptomatology of lesions of, 42

Taste, cerebral area of, 19

Tears, secretion of, in relation to cortical stimulation, 268

Temperature. (Sce Climate)

Territories, cortical, embryological, 26

- - intermediate and terminal, 27

Thalamus opticus, development of, 15

- - psychic facts and, 12

- - relations of, with frontal lobe, 291 , 202

Third frontal convolution, question of, in monkeys, 105

Thirst and hunger, fundamental emotions connected with material exchange and chemical conditions, 269

Thought, association-centres the organs of, according to Flechsig, 81

- influence of temporal lobe upon, 59

- in mutilated monkeys and in idiots, 315

- laws and elements of, and their origin, $215,216,253$

- origin of, 10

- unconscious, 302

Thrombosis, cerebral, and transitory symptoms of shock, 125

Tics in monkeys mutilated in frontal lobes, 186

Time and space in cerebral localisations, 333

- of reaction, after removal of frontal lobe, 324

Tiredness, physical, and psychic indifference, 272

Tone, emotional, pleasant or unpleasant, in all psychic facts, 272

- vital, of individual, 275

Touch, cerebral localisation of sense of, 16

- disturbances of, from lesion of postcentral convolution, 34

- refinement and development of, 331

Trance and the subconscious, 319,322

Tropism, relation of, to colloidal substances, 3

- - to emotion, 260

- reflexes, automatism, and, 13, 14

\section{U}

Unconscious, the, and subcortical automatisms, 13, 317
Unconscious, the constitution of, 284, 316

Uniformity, perceptive, and the law of variation, 4

Uterus, movements of, and points of cortical excitation, 267

\section{V}

VAGINA, movements of, and points of cortical excitation, 267

Variability and invariability of reactions in progressive evolution, 12

- in frontal development, 118

Variations, law of, in relation to perceptive uniformity, 4

- - - kinetogenetic, in evolution, 7 evolution, 7

Vascular alterations in emotion, 266

Vasomotor phenomena and emotions, 277, 278

Vesical movements represented in cortex, 267

Viscera, representation of, in cerebral cortex, 267

Vision, acuteness of, in various animals, 7

- cerebral localisation of, 18, 23

- development of, in infants, 25

- disturbance of, in dogs and monkeys after mutilation of frontal lobes, 195

\section{W}

WEIGHT of brain, intelligence and, 9

Will, and frontal associative centres, 83, 329

- attentive, 247

Word-deafness from temporal lesions, 51

- - reciprocal relations between, and dementia, 50

- - (sensory aphasia), 32, 35, 242

Word-images. (See Images)

Writing, centre for, $61,62,109$

- mechanism of, in children, 239

- relations between, and centre for hand or arm, 63

- - - and language (symbolic, pictographic, syllabic), 232

\section{Z}

Zone. (See Area and Centre) 


\section{INDEX OF AUTHORS}

A

AdLer, 396

Andrizen, 333

Anton, 200

Aristotle, 244

Aschaffenburg, 220, 224

Azam, 318

B

BABINSKI, 36

Bacon, 254

Bain, 210, 221

Baldwin, 253, 292, 293, 298

Ballet, 235, 322

Basedow, 295

Bastian, 235, 238

Baum, 98

Bechterew, 8, 75, 84, 92, 126, 188, 191, $192,194,264,265,266,267$

Beduschi, 32

Beethoven, 331

Beevor, 105, 189, 199

Belfiore, 318

Benedicenti, 261

Bergson, 212, 282

Bergstrom, 223

Bernard, 235, 261

Bernheim, 318

Bessau, 193

Betz, 117

Beyermann, 84, 265

Bianchi, L., 13, 22, 36, 54, 57, 63, 76, 83, $87,92,126,188,190,191,203,222,241$, $254,263,288,300,318,325$

Bianchi, V., 15, 96, 107, 127, 157

Binet, 224, 318

Bleuler, 318

Boccardo, 292

Bochefontaine, 84, 264, 265, 266, 267, 268

Bohn, 7, 8, 59, 129, 130, 328

Bolton, 25, 61, 113, 114, 116

Bonne, 117

Bourbousson, 261

Bourdon, 220

Bourgeois, 318

Braunstein, 193

Brehm, 289, 307

Brissot, 244

Broadbent, 235

Broca, 69, 83, 97, 108

Brodmann, 25, 33, 61, 62, 105, 110, 111 , $113,114,117,118$

Bruno, 221, 284

Bunke, 194

Burdach, 50, 68

Byron, 213

CAJAL, 203, 320

Calkins, 220, 272
Calvin, 284

Campbell, 25, 33, 61, 62, 105, 109, 110, $111,112,113,114,117$

Canalis, 261

Cantani, 126

Cantoni, 3

Catheart, 328

Cerevkov, 266

Charcot, 51, 61, 235, 238

Chopin, 331

Cleofante Corintio, 230

Cole, 107

Comte, 294, 328

D

DANILEWSKI, 264, 266

Dante, 237

Dantec, 8

Darwin, 253

David, 230

Dearborn, 224, 279

Deboyer, 61, 62

De Brosses, 227

Déjerine, 29, 61, 114, 127, 198, 199, 200, $201,202,207,242$

Delaroche, 261

De Molinari, 293

Di Sanctis, S., 221

De Sarlo, 314

Dewey, 13

Donaggio, 320

D'Ors, 254

Drzewina, 7

Du Bois-Reymond, 3, 136, 160

Dumas, 221

Dupré, 242

Dwelshauvers, 317

E

Eberstaller, 97

Eckard, 266

Ecker, 211

Edinger, 9, 11, 95, 201

Ellenberger, 98

Engelmann, 6

Espinas, 318

Euripides, 230

Exner, 311

FANO, 86,324

Féré, 271,273

Ferrari, 279

Ferreri, 235

Ferri, 261

Ferrier, 17, 31, 50, 51, 70, 71, 74, 75, 76, $92,96,132,187,189,191$

Ferrier et Yeo, 31 
Flechsig, 21, 22, 25, 26, 27, 28, 29, 30, 31 , $32,47,48,58,59,61,64,67,68,76,78$, $79,81,82,83,91,92,107,109,114,202$, $240,241,268,270$

Fleurens, 68, 73

Forel, 7, 199

Fragnito, 320

Franceschi, 52

François Frank, 84, 265, 267

Franck, 192

Freud, 222, 225, 317, 318, 320

Fubini, 261

Funke, 271

\section{G}

GALL, 68

Garnier, 130, 289

Gehuchten v., 320

Geiger, 332

Gerver, 268

Giannelli, 265

Gilmann, 271

Golgi, 320

Goltz, 14, 69, 74, 75, 76, 77, 88, 92, 128

Gratiolet, 69

Grimm, 285

Grossglick, 75, 92, 132

Grünbaum, 62, 71, 189

Grünhagen, 193

\section{H}

НАав, 194

Hammerburg, 117

Havet, 5

Helmoltz, 1

Henrick, 12

Henry, 224

Henschen, 23

Herrick, 14

Herring, 324

Herrmann, 271

Hertwig, O. and K., 5

Hertz, 3

Hitzig, 16, 20, 21, 22, 29, 31, 50, 59, 69, $70,73,74,76,81,92,130,132,187,196$

Hobhouse, 307, 312

Horsley, 18, 31, 78, 79, 92, 105, 123, 132, 188,265

Hufeland, 67

Hughlings, Jackson, 119

Hunter, 260

\section{I}

INGENIEROS, 130,215

Ircland, 68

$\mathrm{J}_{\text {ACOB }, 9,94,107,117}$

James, 80, 211, 223, 274, 276, 279, 290

Jennings, I:30

Johnston, 11, 307

Jones, 317

Jung, 318
K

KALISCHER, 79, 127

Kennedy, 58

Kinnamann, 130

Kirchhoff, 270

Kleinenberg, 5

Kocher, 63

Kölliker, 203

Kulpe, 272

Kussmaul, 34, 51, 235

L

LAMARCK, 8

Lancisi, 68

Lange, 273, 276, 278, 280

Langelaan, 84, 85, 265

Langley, 279

Lehmann, 273

Leibnitz, 227

Lelut, 69, 222

Lemesle, 223

Lépine, 264, 268

Le Play, 295

Leuret, 97

Levinsohn, 188

Levy Bruhle, 293

Libertini, 85, 324

Lichstein, 235

Linder, 298

Loeb, 3, 7, 9, 15, 78, 303, 305, 324

Lombro so, Cesare, 261, 276

Lombroso, Paolo, 231

Lorenz, 3

Loria, 293

Lubbock, 7

Luciani, 12, 31, 50, 51, 75, 78, 79, 84, 107, 132,187

Lugaro, 91, 307, 320, 322, 326

M

$\mathrm{McCABE}, 303,305,312$

Macci, 212

Mallet, 322

Malloizel, 268

Mantegazza, 278

Maragliano, 126

Marchand, 105

Marie, 24, 50, 63, 195, 244

Marinesco, 203

Marshall, 272

Marx, 293

Maschka, 288

Massarotti, 261

Matteucci, 3

Maxwell, 3

Mead, 271

Meyer, 298

Meynert, 81, 97, 108, 203, 271, 333

Mezes, 272

Michelangelo, 213

Michtovte, 328

Miller, 271

Mills, 119

Mingazzini, 242, 244

Miralliè, 61 
Mislawski, 84, 188, 266, 267, 268

Moleschott, 261

Monakow, 29, 32, 35, 87, 90, 91, 92, 98, $121,122,199,200,207,333$

Montesquieu, 261

Montier, 244

Moolmann, 77

Morelli, 223

Morgan, 303, 306, 307, 312

Moriceau, 201

Morpurgo, 261

Morselli, 214, 261, 302, 31 7, 318

Mosso, 84, 213, 262, 267, 276

Mott, 62, 109, 110, 189

Müller, 227, 228, 229

Munk, 20, 24, 31, 69, 70, 74, 75, 76, 78, 81, $82,84,86,92,97,128,129,132,196,264$

Münsterberg, 279

Muratow, 200

Myers, 322, 323

$\mathbf{N}$

Negrint, 98

Nero, 230

Nikitin, 268

Nordau, 298

Novikow, 293

$\mathrm{O}$

ODDI, 86, 324

Onufrowicz, 199

Orbeli, 328

Orestano, 313

Ostankow, 267

$\mathrm{P}$

PARker, 6

Patrizi, 213, 276

Paulham, 221

Pavlov, 128, 268, 328

Pearson, 188

Pellacani, 84, 267, 289

Penta, 76, 261

Pergolesi, 331

Pfeffer, 7

Pfungen, 84

Phelps, 119

Pieret, 7

Pilcz, 194

Pitres, 61, 62, 84, 192

Plato, 227

Polimanti, 78, 86, 87, 88

Potter, 97

Pouchet, 6

Power, 303

Preyer, 307, 310

Prince, 317, 322

Probazènski, 265

Q

QUensel, 202

RADT, 8

Ramon y Cajal, 203, 320
Regnaud, 282

Rehmka, 278

Renan, 227

Ribot, 211, 271, 272, 278

Richet, 272

Righi, 1, 3

Rignano, 211

Robertson, 229

Romanes, 243, 298, 307, 310

Roncoroni, 89, 90, 118, 127!

Röntgen, 3

Rossini, 331

Rossolimo, 92

Rutishauser, 92

S

SACHS, 203

Saint-Simon, 294

St Francis d'Assisi, 284

St Catherine of Siena, 284

Schäfer, 78, 79, 92, 117, 124, 132, 188, 189 , 293

Schiff, 87, 266

Schnopfhagen, 199

Schopenhauer, 283

Schrader, 14, 15

Schukowski, 202

Schuster, 62, 110

Sciamanna, $79,83,84,85,126,133,298$

Seglas, 242, 244

Semon, 265, 305

Senise, 278

Sergi, G., 298

Sergi, S., 83, 118, 119

Shakespeare, 229

Shepherd, I., 75, 88, 89, 119, 124, 125, 126,130

Sherrington, $62,71,84,110,123,189,323$, 324

Sidis, 318, 319, 321

Sidney, 272

Siemerling, 29

Smith, 84

Socrates, 222

Sollier, 240, 268

Soury, 50

Spencer, 84, 253, 272, 293, 294

Spindler, 279

Spinoza, 283

Spurzhein, 67, 68

Starr, 17, 119

Stewart, 188

Stratton, 277

Stricker, 238

Subbock, 128

Sydenham, 278

Sydney-Cole, 107

Sikorsky, 279

$\mathrm{T}$

TAINE, 231

Tamburini, 31, 50, 51, 83, 187, 322

Tanzi, 322, 326

Tarde, 294, 298

Tenchini, 98

Thorndike, 88, 129, 130, 289, 312

Thornton, 7

Tomasini, 266 
Topolanski, 324

Turner, 105, 113

\section{U}

UNVERRICHT, 75,76

\section{V}

Van Gehuchten, 198, 199

Vascide, 223

Verdi, 331

Verworn, 305

Vialet, 23

Vogt, 29, 117, 118

Voltaire, 233

Vulpain, 267

W

WARD, 271

Wasmann, 7
Wassiliew, 328

Watson, 113, 117

Waxweiler, 291

Webster, 229

Werner, 75

Wernicke, 34, 51

Westphall, 195

Wilson, 287

Winkler, 97

Worms, 293

Wundt, 80, 83, 193, 219, 220, 224, 254, 271,272

\section{Z}

Ż̀LIONY, 128

Zell, 307

Zimmerl,,99

Zingerle, 200

Zulowski, 93 


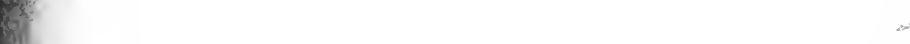

$$
\text { , }
$$$$
\begin{aligned}
& \lambda \\
& x \\
& +2 \\
& 2
\end{aligned}
$$

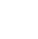

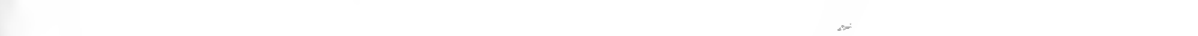




RETURN TO the circulation desk of any University of California Library

or to the

NORTHERN REGIONAL LIBRARY FACILITY

作

- Blda. 40Q RIIAY BE RECALE

- (510) 642-6753 may be renew AFTER 7 DAYS

- l-year loans may be recharewed by calling

- Rens to NRLF be rechargea by bringlow

days pris and recharges bringing

prior to due date.

4⿻ำ 1 ำ

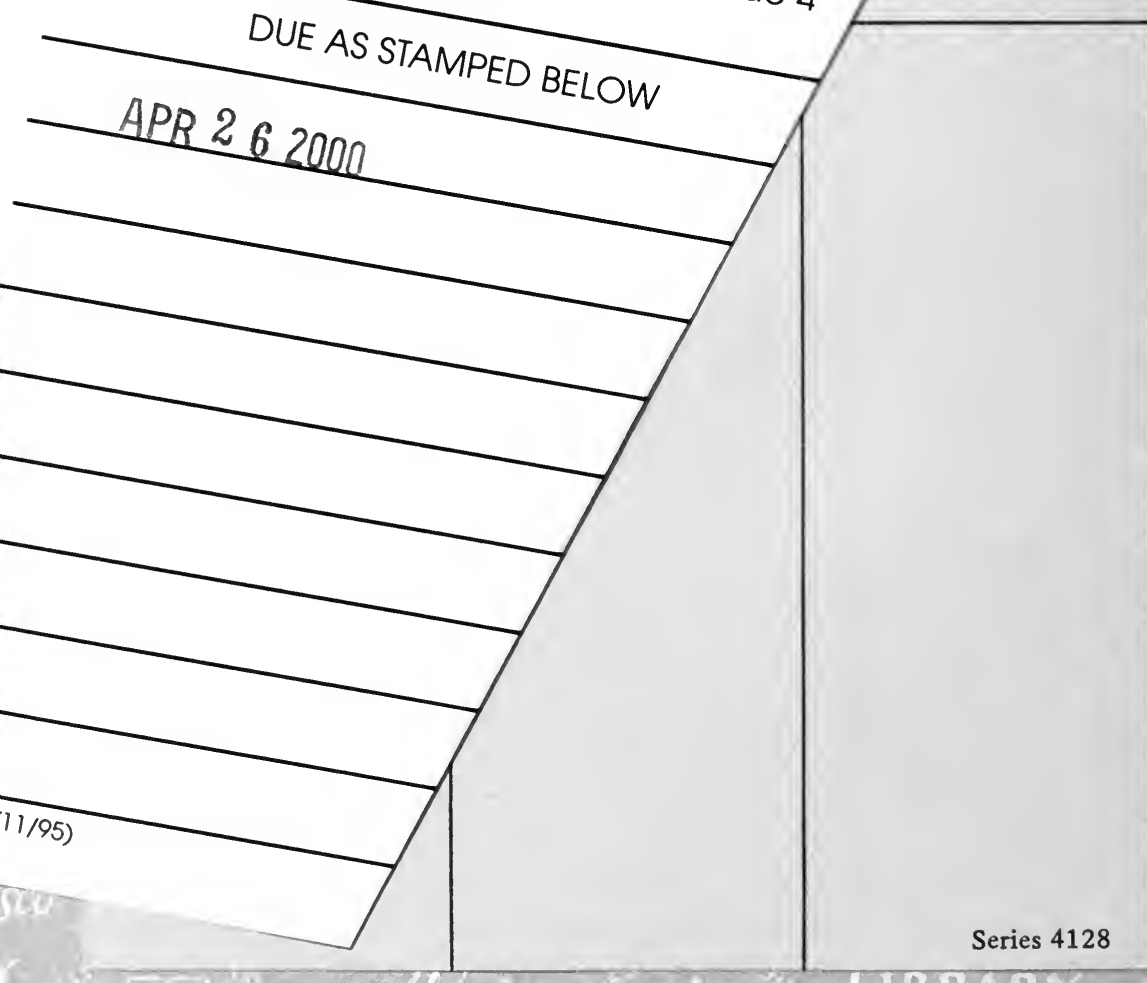


